\title{
Kostengünstige Maßnahmen an Unfallhäufungen im Vorher/Nachher-Vergleich
}

Reinhold Maier

Ralf Berger

Martin Schmotz

Martin Bärwolff

Martin Schimpf

Petra Butterwegge

Jörg Ortlepp

Unfallforschung der Versicherer 


\section{Kostengünstige Maßnahmen an Unfallhäufungen im Vorher/Nachher-Vergleich}

Reinhold Maier

Ralf Berger

Martin Schmotz

Martin Bärwolff

Martin Schimpf

Petra Butterwegge

Jörg Ortlepp

Unfallforschung

der Versicherer 


\section{Impressum}

Gesamtverband der Deutschen Versicherungswirtschaft e. V. Unfallforschung der Versicherer

Wilhelmstraße 43/43G, 10117 Berlin

Postfach 0802 64, 10002 Berlin

E-Mail: unfallforschung@gdv.de

Internet: www.udv.de

Facebook: www.facebook.com/unfallforschung

Twitter: @unfallforschung

YouTube: www.youtube.com/unfallforschung

ISBN-Nr.: 978-3-939163-70-1

Redaktion: Dipl.-Ing. Petra Butterwegge

Erschienen: 09/2016 


\section{Kostengünstige Maßnahmen an Unfallhäufungen im Vorher/Nachher-Vergleich}

Abschlussbericht Oktober 2016

Bearbeitet durch

Professur für Straßenverkehrstechnik und Theorie der Verkehrsplanung

TU Dresden

Prof. Dr.-Ing. Reinhold Maier

Dr.-Ing. Ralf Berger

Dr.-Ing. Martin Schmotz

Dipl.-Ing. Martin Bärwolff

Dipl.-Ing. Martin Schimpf
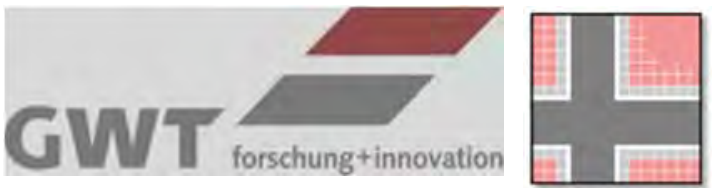

Bei der UDV betreut von:

Petra Butterwegge Jörg Ortlepp

Unfallforschung

der Versicherer 


\section{Kurzfassung}

Mit dem Forschungsvorhaben wurden kostengünstige Maßnahmen gegen Unfallhäufungen bestimmt. Dazu wurden unter enger Einbindung der Unfallkommissionen Maßnahmen erhoben, analysiert und kategorisiert.

Die Kontaktaufnahme und Datenerhebung bei Unfallkommissionen im Bundesgebiet bildete den Ausgangspunkt der Untersuchung. Um eine Vergleichbarkeit der Daten zu gewährleisten, wurden die erhaltenen Maßnahmen kategorisiert und plausibilisiert. Die Daten unterschieden sich in ihrer Qualität deutlich. Insgesamt lagen zur weiteren Untersuchung 1.812 Maßnahmen vor. Diese wurden in 82 Maßnahmenkategorien geordnet. Maßnahmenkategorien fassen (annähernd) identische Maßnahmen zusammen. Angesichts der Anzahl verschiedenen Maßnahmenkategorien wurde eine Auswahl 35 geeigneter Kategorien für die weitere Untersuchung getroffen. Das Augenmerk lag auf der Untersuchung von Maßnahmenkategorien, für die bisher nur ein geringer oder unzureichender Wissensstand vorlag.

Zwei Säulen der Maßnahmenbewertung waren Bestandteil der weiteren Untersuchung: Die erste Säule umfasste die Evaluation der Maßnahmenkategorien durch Mitglieder der Unfallkommissionen mittels eines Fragebogens. Ziel dieser Herangehensweise war der Erkenntnisgewinn darüber, wie Maßnahmen durch die Praxis bezüglich ihrer Wirksamkeit und Umsetzbarkeit eingeschätzt werden. Im Ergebnis konnten Erkenntnisse darüber gewonnen werden, welche Maßnahme als „kostengünstig“ und „wirksam“ angesehen wird.

Die zweite Säule beinhaltete die Unfalldatenanalyse der zur Verfügung gestellten Maßnahmenumsetzung. Aus dem Vergleich des Unfallgeschehens im Vorher- und Nachher-Zeitraum konnte die durchschnittliche Maßnahmenwirkung (Effektivität) bestimmt werden. Die Berücksichtigung der (Investitions-)Kosten erlaubten Aussagen zur Wirtschaftlichkeit (Effizienz) der Maßnahmen. Diese werden über das Nutzen-Kosten-Verhältnis beschrieben. Auf Basis beider Ergebnisse erfolgte eine Gesamteinschätzung der Maßnahmen. Insgesamt konnten zehn Maßnahmenkategorien für eine Anwendung als „empfehlenswert" eingestuft werden.

Die Ableitung (besonders) geeigneter kostengünstiger Maßnahmen baut auf den Erkenntnissen der Evaluation und der Unfalldatenanalyse auf. Diese Maßnahmen zeichnen sich durch eine hohe Wirksamkeit, hohe Nutzen-Kosten-Verhältnisse und homogene Wirkungen im Untersuchungskollektiv aus. Es wurden neun geeignete kostengünstige Maßnahmenkategorien identifiziert.

Die entwickelten Handlungsempfehlungen stellen ein Handwerkszeug für die Unfallkommissionen zum Auffinden und bei der Auswahl geeigneter kostengünstiger Maßnahmen gegen Unfallhäufungen dar. Sie sind mit ihrem thematischen und strukturellen Aufbau an den gängigen Empfehlungen und Literaturen zur Maßnahmenfindung orientiert.

Die Ergebnisse münden in der Bereitstellung von Faktenblättern für die untersuchten Maßnahmen, die Ableitung kostengünstiger Maßnahmen und die Bereitstellung von Handlungsempfehlungen zur deren Anwendung. 


\begin{abstract}
This research project was able to identify cost-effective measures for the prevention of accident blackspots. In close cooperation with accident commissions, these measures were compiled, analyzed and categorized.

Contact was first established with accident commissions across Germany, where data was collected for the outset of the investigation. The data differed significantly in quality. Therefore, in order to ensure data comparability, the compiled measures were carefully categorized and tested for plausibility, leaving a total of 1,812 measures available for continued evaluation. These were organized into 82 categories, which grouped the measures according to (approximate) similarity. Of these categories, 35 were suitable for further analysis. Priority was placed on the measure categories for which there was little or insufficient current scientific knowledge.
\end{abstract}

Two approaches were used to assess the 35 selected measure categories:

For the first approach, members of the accident commissions evaluated the selected measure categories, using a standardized set of questions. The goal was to evaluate the measures for effectiveness and feasibility in a practical setting. This provided insight into the conditions under which a measure could be considered "low-cost" and "efficient".

The second approach comprised the analysis of accident data gathered from the selected measures. The average effect of the selected measures (effectiveness) was determined by comparing the accident events within a set before-and-after timeframe. The (investment) costs were taken into account, thus ascertaining the economic efficiency (efficiency) of each measure. This is referred to as the cost-benefit ratio. On the basis of these results, the measures were then comprehensively assessed. As a result, ten measure categories were classified as "recommendable".

The deduction of (particularly) suitable, cost-effective measures was based on the findings of the measure-category evaluations and on the analysis of the accident events. These measures categories were characterized by high efficiency, high cost-benefit ratios, and homogeneous impact within the collected samples. Nine suitable cost-effective measure categories were identified.

The recommendations for action, developed from this project, provide accident commissions with tools for locating and selecting suitable low-cost measures for the remediation of accident blackspots. These recommendations adhere, thematically and structurally, to the format of the current recommendations and literature for accident blackspot management.

The results of this project will be supplied as fact sheets for the investigated measures, the description of low-cost measures and the recommendations for action and application. 


\section{Inhaltsverzeichnis}

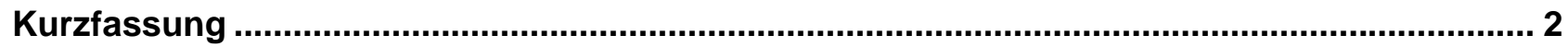

Abstract

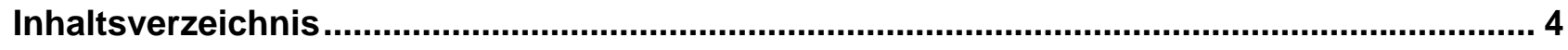

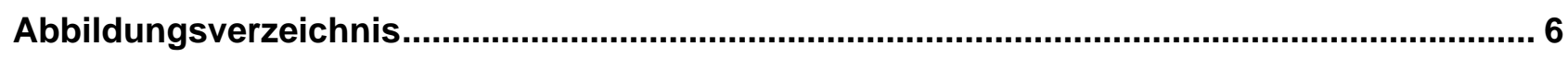

Tabellenverzeichnis............................................................................................................ 8

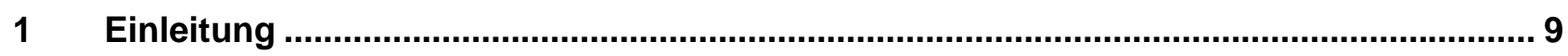

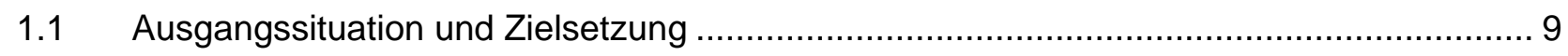

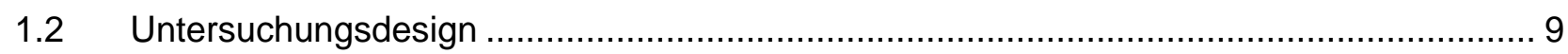

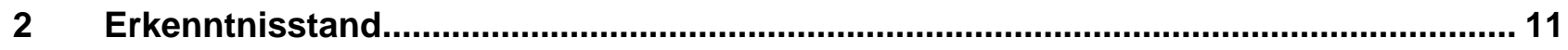

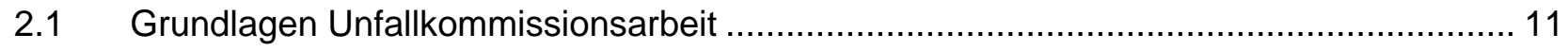

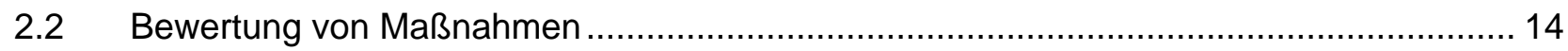

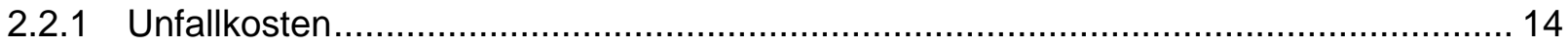

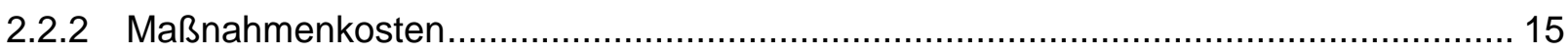

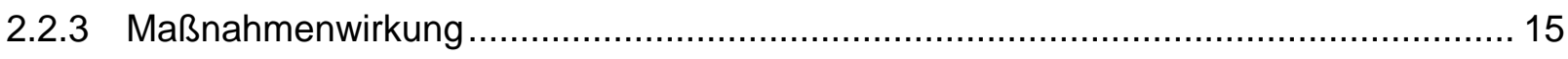

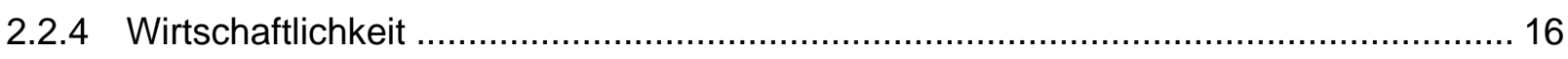

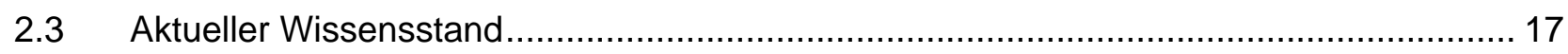

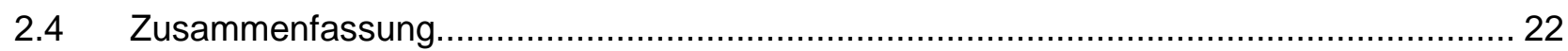

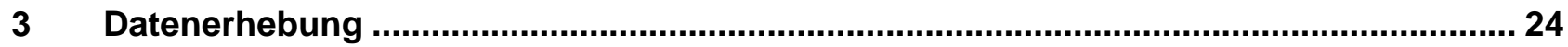

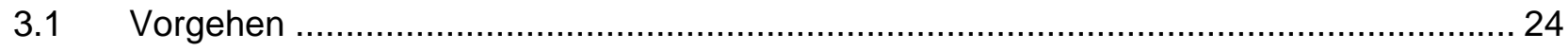

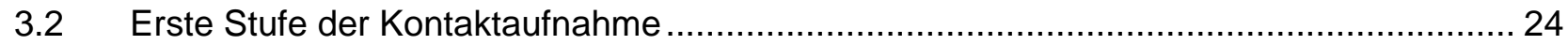

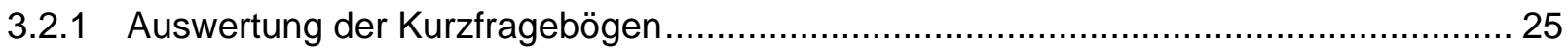

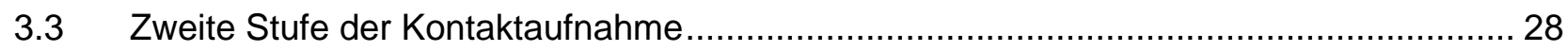

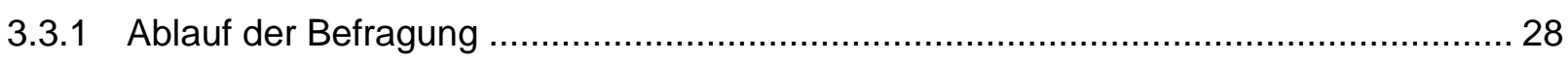

3.3.2 Auswertung der Telefoninterviews und der Datenübermittlung ................................ 29

3.3.3 Auswertung der Zusatzfragen aus den Telefoninterviews.................................... 31

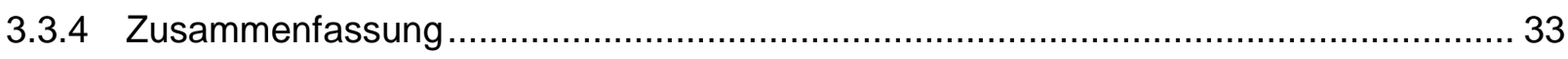

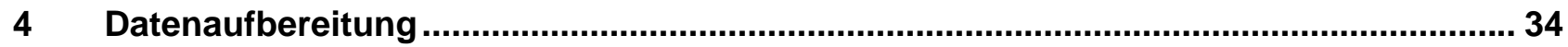

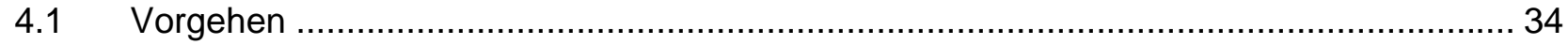

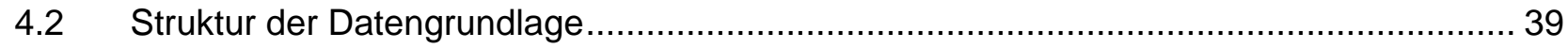

4.3 Methodik zur Auswahl geeigneter Maßnahmen(-pakete) zur Detailuntersuchung ........... 42

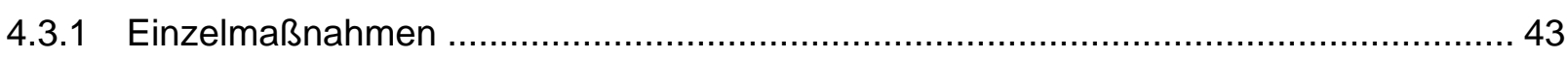

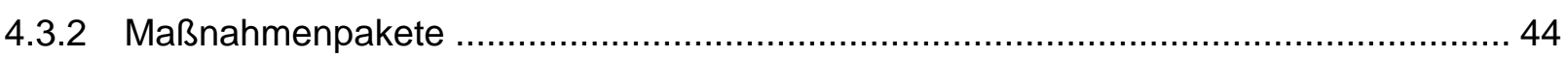

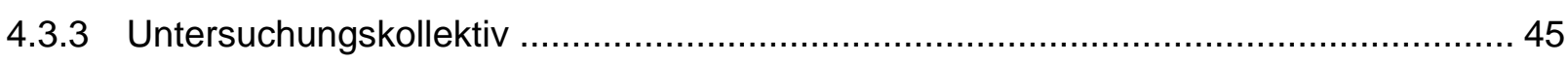

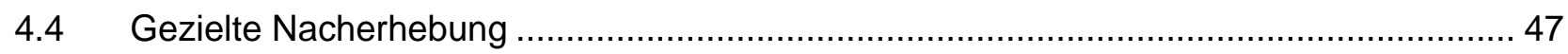

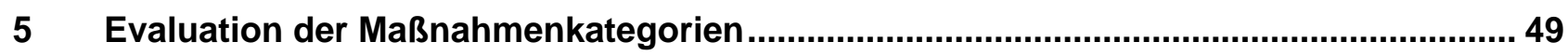

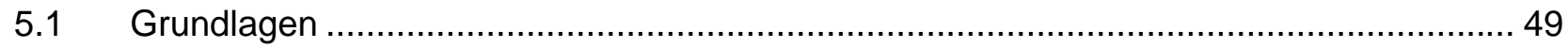




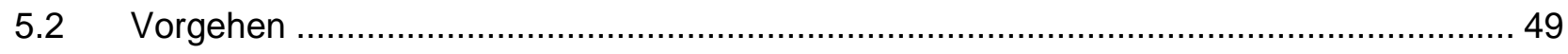

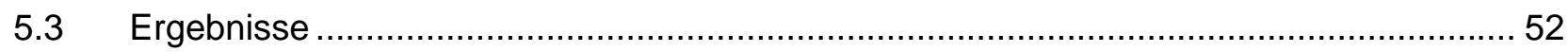

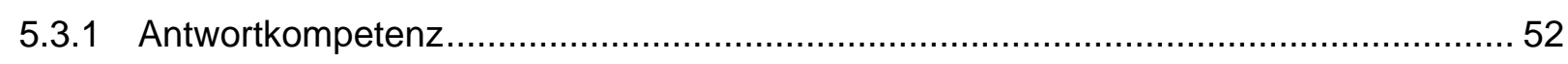

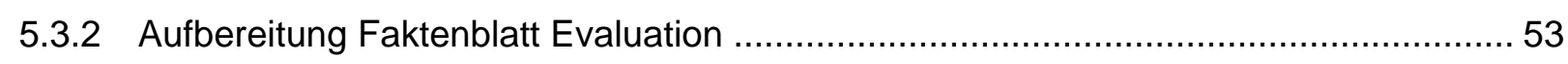

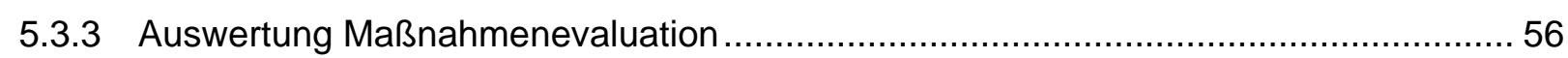

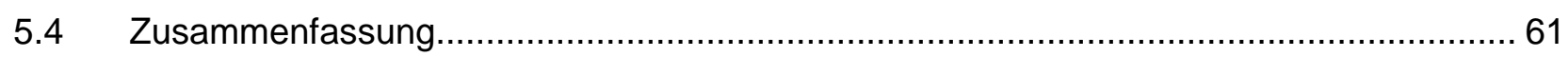

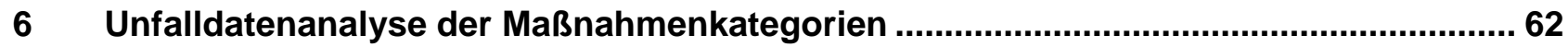

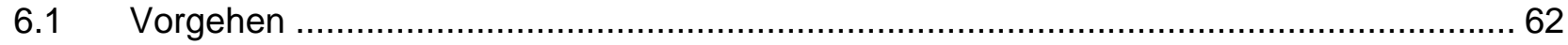

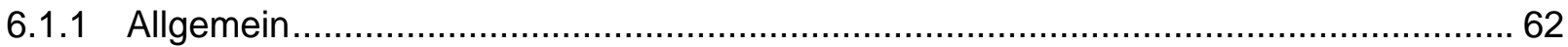

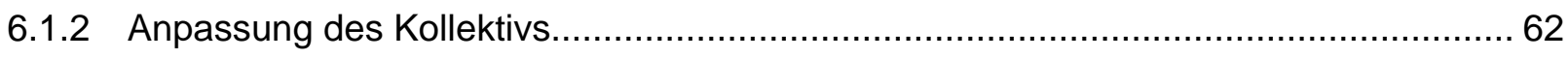

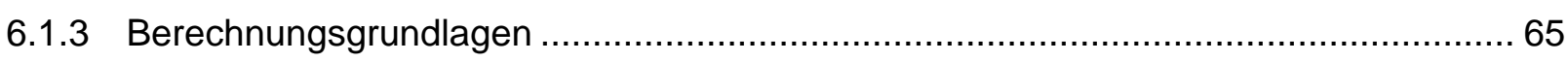

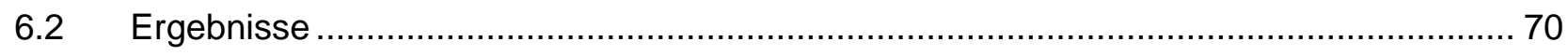

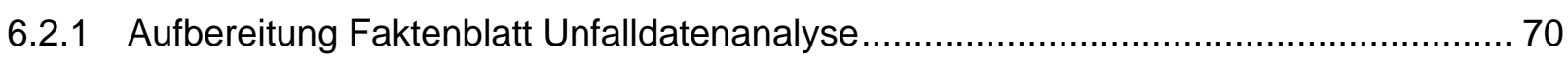

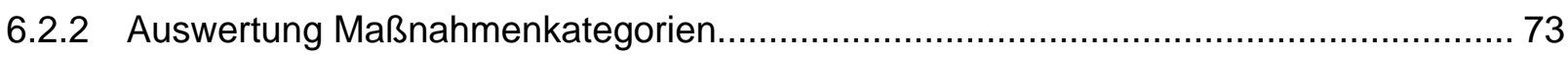

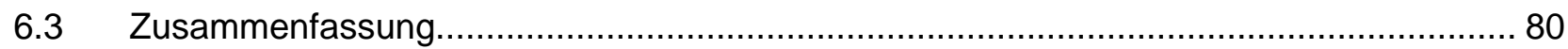

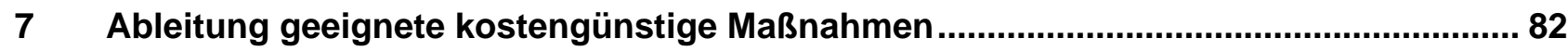

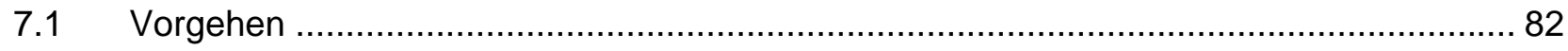

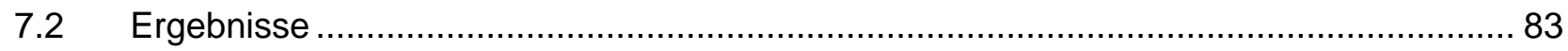

8 Handlungsempfehlung zur Umsetzung von Maßnahmen.......................................... 85

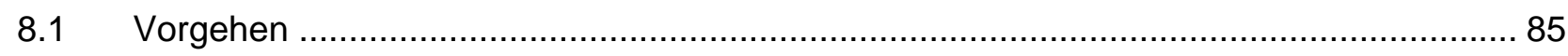

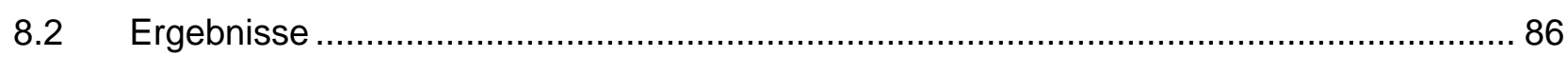

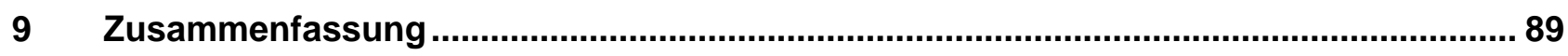

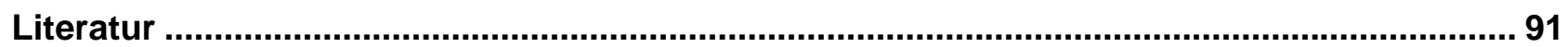

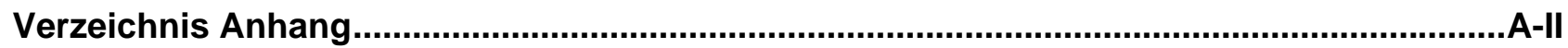




\section{Abbildungsverzeichnis}

Abbildung 1: Schematische Darstellung des Vorgehens.

Abbildung 2: $\quad$ klassifizierte Maßnahmenwirkung [\%] in Bezug auf die Reduzierung von

Unfallkosten baulicher und verkehrstechnischer Maßnahmen im Kollektiv

GERLACH et al. [2009]

Abbildung 3: Nutzen- und zielorientierte Gesamtbewertung der Maßnahmenwirksamkeit, Level of Effictiveness [SPAHN 2012] ....................................................................

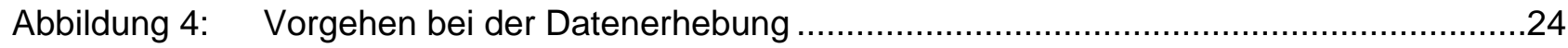

Abbildung 5: Verteilung der zurückgesendeten Fragebögen auf die einzelnen Bundesländer ...26

Abbildung 6: Anzahl der Maßnahmen und Sofortmaßnahmen aus den Kurzfragebögen nach

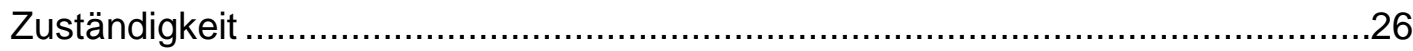

Abbildung 7: $\quad$ Box-Plot der Sofortmaßnahmen je Unfallkommission ........................................27

Abbildung 8: In den UKO vorhandene Unterlagen vor und nach Maßnahmenumsetzung..........27

Abbildung 9: Häufigkeit des Vorliegens von Nachher-Unterlagen in Abhängigkeit der VorherUnterlagen

Abbildung 10: Stand der Datenübermittlung nach dem Ablauf der zweiten Stufe der Kontaktaufnahme

Abbildung 11: Tabellarischer Aufbau der Datenbank zur Erfassung von Maßnahmen................36

Abbildung 12: Darstellung Arten von Gruppierungen des Maßnahmenkollektivs .......................38

Abbildung 13: Anteil der Maßnahmen(-pakete) in Abhängigkeit der Maßnahmen- bzw. Paketart39

Abbildung 14: Anteil der Maßnahmen(-pakete) in Abhängigkeit der Ortslage ...........................39

Abbildung 15: Anteil der Maßnahmen(-pakete) in Abhängigkeit des Netzelementes ..................40

Abbildung 16: Anteil der Maßnahmen(-pakete) nach Netzelement und Ortslage ......................40

Abbildung 17: Unfalldatenlage im Vorher- und Nachher-Zeitraum .........................................41

Abbildung 18: Kombination der Unfalldatenlage im Vorher- und Nachher-Zeitraum..................41

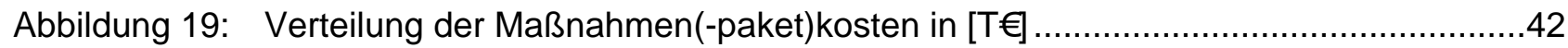

Abbildung 20: Darstellung der Präsentation einer Maßnahmenkategorie MK für die Evaluation durch die Teilnehmer...................................................................................51

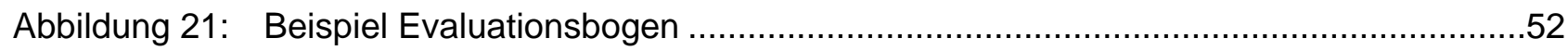

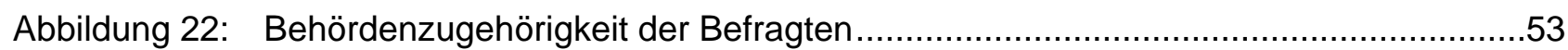

Abbildung 23: Faktenblatt zur Evaluation einer Maßnahmenkategorie MK (Die Nummern verknüpfen die Darstellung mit den inhaltlichen Erläuterungen zu den einzelnen Bereichen in Tabelle 10.)......

Abbildung 24: Evaluationsergebnisse allgemeine Einschätzung der Maßnahmenkategorien ......56

Abbildung 25: Klassifizierung für Prädikat „kostengünstige Maßnahme“ nach Kriterium „Kosten“59

Abbildung 26: Klassifizierung für Prädikat „kostengünstige Maßnahme“ nach Kriterium „zeitliche Umsetzbarkeit"

Abbildung 27: Klassifizierung für Prädikat „kostengünstige Maßnahme“ nach Kriterium „verwaltungstechnischer Aufwand“.... 
Abbildung 28: Klassifizierung für Prädikat „wirksam“ nach Kriterium „Reduzierung der Unfallschwere".... 60

Abbildung 29: Klassifizierung für Prädikat „wirksam“ nach Kriterium „Reduzierung der Unfallanzahl“...... 61

Abbildung 30: Aufbau Faktenblatt zur Unfallanalyse einer Maßnahmenkategorie MK .71

Abbildung 31: Kennwerte der einzelnen Maßnahmenkategorien MK, Örtlichkeit und Wirkung auf Unfallanzahl.

Abbildung 32: Kennwerte der einzelnen Maßnahmenkategorien MK, Wirkung auf Unfallkosten, NKV und Gesamtprädikat.

Abbildung 33: Verteilung der Prädikate nach Klassifizierung der Maßnahmenwirkung MWu (links) und $\mathrm{MW}_{\mathrm{UK}}$ (rechts).

Abbildung 34: Summenhäufigkeit der mittleren Investitionskosten (links) und des NKV-Median (rechts) für die Maßnahmenkategorien MK

Abbildung 35: Verteilung der NKV-Mediane, Gruppierung nach Art des Maßnahmeneingriffs, kompletter NKV-Bereich (links), Ausschnitt unterer Bereich (rechts) .78

Abbildung 36: Verteilung der NKV-Mediane, Gruppierung nach Primärdefizit, kompletter NKVBereich (links), Ausschnitt unterer Bereich (rechts)

Abbildung 37: Gegenüberstellung Median MWUK und Median NKV nach Art des Maßnahmeneingriffs (links) und Primärdefizit (rechts) .79

Abbildung 38: Verteilung der Maßnahmenkategorien MK nach Klassifizierung des Gesamtprädikats .80

Abbildung 39: Handlungsempfehlung zur Ableitung von Maßnahmen Innerorts 87

Abbildung 40: Handlungsempfehlung zur Ableitung von Maßnahmen Außerorts (Landstraße) ...88 


\section{Tabellenverzeichnis}

Tabelle 1: $\quad$ Kriterien für UHS und UHL nach M Uko FGSV [2012] ......................................12

Tabelle 2: $\quad$ Kriterien für UHS und UHL nach FGSV [2003] ...........................................12

Tabelle 3: $\quad$ Pauschale Unfallkostensätze der einzelnen Unfallkategorien FGSV [2012] ..........14

Tabelle 4: $\quad$ NKV für Maßnahmentypen nach WEINERT [1999] .......................................20

Tabelle 5: $\quad$ Verteilung der Zuständigkeiten der UKO mit Datenübermittlung.........................30

Tabelle 6: Beispielhafte Zuordnung von Maßnahmen zu drei Maßnahmengruppen ..............36

Tabelle 7: $\quad$ Besetzung der aus den Einzelmaßnahmen zur weiteren Untersuchung vorgesehenen Kategorien (grau).

Tabelle 8: Maßnahmenkategorien und Aufnahme der Kriterien Örtlichkeit und

Straßencharakteristik für Bewertungsansatz Evaluation UKO bzw.

Unfalldatenanalyse.

Tabelle 9: $\quad$ Termine für Schulungen und Tagungen von Unfallkommissionen zur Evaluierung mit Angabe der evaluierten Maßnahmenkategorie.

Tabelle 10: $\quad$ Erläuterung der Inhalte des Faktenblatts zur Evaluation einer Maßnahmenkategorie MK

Tabelle 11: $\quad$ Einteilung der Maßnahmenkategorien nach Einschätzung als „kostengünstig“ und als „wirksam“

Tabelle 12: Klassifizierungen der Unfallkategorien nach Datenlage.....

Tabelle 13: $\quad$ Skalierung/Einteilung Wirkungsgrade für die Entwicklung von Unfallanzahl und Unfallkosten

Tabelle 14: Prädikate für die mittlere Maßnahmenwirkung $M W_{\cup}$ und $M_{U K}$ einer Maßnahmenkategorie MK in Abhängigkeit der Einzelbewertung der Stellen.

Tabelle 15: Prädikat für die Wirtschaftlichkeit einer Maßnahmenkategorie in Abhängigkeit der Einzelbewertung der Stellen.

Tabelle 16: Gesamtprädikat für Maßnahmenkategorien MK.

Tabelle 17: Erläuterung der Inhalte [nummerierte Bereiche] des Faktenblatts zur Unfallanalyse einer Maßnahmenkategorie.................................................................... 72

Tabelle 18: $\quad$ Kennwerte des Kollektivs, Entwicklung $U(P)$ und Unfallkosten ..........................74

Tabelle 19: Kennwerte zur Abgrenzung „geeigneter“ und „besonders geeigneter“ kostengünstiger Maßnahmen

Tabelle 20: Übersicht geeigneter kostengünstiger Maßnahmen ......................................83

Tabelle 21: Übersicht besonders geeigneter kostengünstiger Maßnahmen ..........................84 


\section{$1.1 \quad$ Ausgangssituation und Zielsetzung}

Nach § 44 der Verwaltungsvorschrift zur Straßenverkehrs-Ordnung VwV-StVO [2015] ist die Örtliche Unfalluntersuchung als Instrument zur Verbesserung der Sicherheit des Straßenverkehrs an Unfallhäufungen (UH) Aufgabe der Unfallkommissionen (UKO). Wie in einer seitens der Unfallforschung der Versicherer (UDV) durchgeführten Studie aus der Befragung von UKO hervorgeht, leidet die Arbeit von UKO zunehmend unter mangelhafter bis ungenügender Finanzierung zur Realisierung von Maßnahmen zur Erhöhung der Verkehrssicherheit an Unfallhäufungen [UDV 2012]. Dies wirkt sich insbesondere bei der Umsetzung baulicher und verkehrstechnischer Maßnahmen aus. Daher kommt innovativen und kostengünstigen Maßnahmen aus Sicht der UKO eine wachsende Bedeutung zu. Um weiterhin eine erfolgreiche Arbeit der UKO sicherzustellen, ist es erforderlich, anhand einer möglichst validen Datengrundlage kostengünstige Maßnahmen an UH hinsichtlich ihrer Wirkung auf das Unfallgeschehen zu analysieren.

Ziel der Untersuchung ist die systematische Darstellung von kostengünstigen Maßnahmen an UH und deren Bewertung der Wirksamkeit. Am Ende des Projektes soll ein Beispielkatalog entstehen, in welchem Hinweise und Empfehlungen zum Einsatz kostengünstiger Maßnahmen an UH aufgeführt sind. Dieser kann den UKO für ihre Arbeit zur Verfügung gestellt werden und so im Umgang mit derartigen Maßnahmen sensibilisieren.

\section{$1.2 \quad$ Untersuchungsdesign}

Das Untersuchungsdesign ist schematisch in Abbildung 1 dargestellt. Es enthält sechs Arbeitspakete, welche im Allgemeinen aufeinander aufbauen.

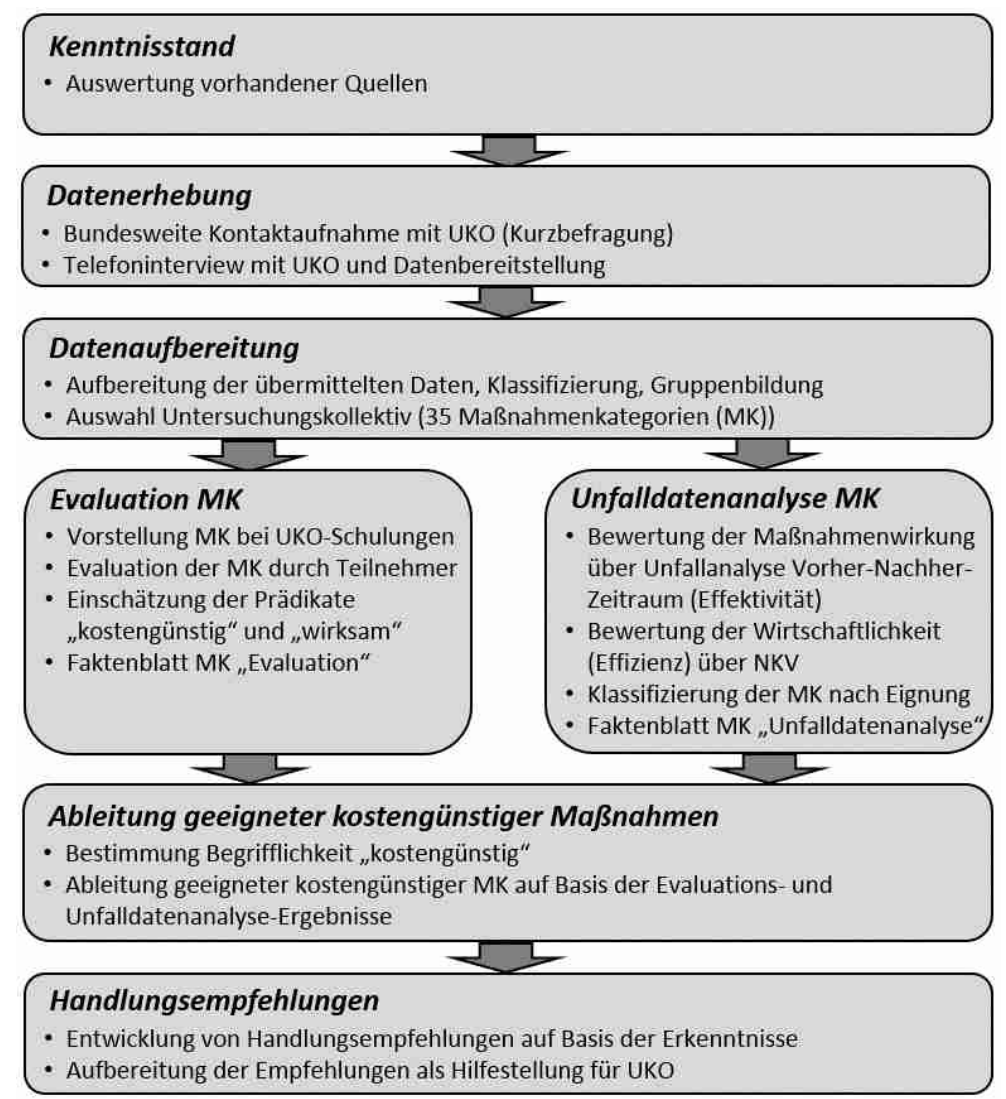

Abbildung 1: Schematische Darstellung des Vorgehens 
Zunächst wird ein Überblick über den aktuellen Kenntnisstand zum Thema Maßnahmen an UH gegeben. Basis hierfür sind neben den einschlägigen Merkblättern auch andere Forschungsarbeiten mit diesem Gegenstand, wobei insbesondere hier der Fokus auf kostengünstige Maßnahmen gelegt wird. Daraus abgeleitet werden sowohl Vorschläge für Kenngrößen zur Bewertung der Maßnahmenwirkung im Allgemeinen, aber auch erste Hinweise und Erfahrungen darauf, wie kostengünstige Maßnahmen wirken.

Parallel zu diesen Recherchen beginnt die Datenerhebung, bei welcher bundesweit UKO kontaktiert und um Unterstützung gebeten werden. Die Kontaktaufnahme umfasst mehrere Stufen, wobei die erste Stufe (in Form einer Kurzbefragung) zur Einordnung der zu erwartenden Datengrundlage als auch zur generellen Abklärung der weiteren Unterstützung durch die UKO dient. In der zweiten Stufe werden ausgewählte UKO telefonisch kontaktiert und der Ablauf der Datenübermittlung geklärt. Im Fokus stehen dabei Informationen zum Unfallgeschehen vor und nach der Maßnahmenumsetzung sowie zur Maßnahme selbst (Kosten, Umsetzungszeitraum etc.). Bei UKO mit einer besonders hohen Maßnahmendichte und einer (zu erwartenden) guten Datenverfügbarkeit wird unter Umständen eine Vor-Ort-Recherche durchgeführt, um den Arbeitsaufwand für die UKO möglichst gering zu halten.

Im dritten Schritt werden die von den UKO übermittelten Daten digitalisiert, kategorisiert und plausibilisiert, was maßgeblich der Systematisierung der Daten dient. Zunächst werden die Daten, digitalisiert und in einer Datenbank vereinheitlicht. Zu jeder Maßnahme werden Informationen zur Umsetzung und zum Detailierungsgrad der vorliegenden (Unfall-)Daten festgehalten. Anschließend werden die Maßnahmen kategorisiert. Dabei werden Maßnahmen, die oft oder ausschließlich gemeinsam eingesetzt werden oder die sich durch einen Oberbegriff beschreiben lassen, in unterschiedlichen Kategorien zusammengefasst. Dadurch werden die Maßnahmen systematisiert und die spätere Auswertung sowie die Auswahl geeigneter Maßnahmen zur Detailanalyse vereinfacht, ohne dass durch die Aggregation entscheidende Maßnahmencharakteristika vernachlässigt werden. Auf diese Weise können für das Projekt relevante Maßnahmen(-kategorien) herausgefiltert und für die weiteren Untersuchungen vorbereitet werden.

Die so selektierten Maßnahmen werden zunächst durch ausgewählte UKO evaluiert. Dies geschieht durch mehrere Befragungen im Rahmen von Aus- und Fortbildungsveranstaltungen in verschiedenen Bundesländern. Im Mittelpunkt der Befragung steht, wie die Mitglieder der UKO die vorgestellten Maßnahmen hinsichtlich ihrer Wirkung, Umsetzungsdauer und -kosten (subjektiv) beurteilen.

Parallel dazu werden die ausgewählten Maßnahmen einer (objektiven) Detailanalyse, der Unfalldatenanalyse unterzogen. Hierfür wird das Unfallgeschehen drei Jahre - soweit dies die Daten zulassen - vor sowie nach der Maßnahmenumsetzung analysiert. Aus den Differenzen im Unfallgeschehen sowie den Kosten der Maßnahmenumsetzung können anschließend verschiedene Kenngrößen zur Maßnahmenwirkung und Wirtschaftlichkeit bestimmt und zur Beurteilung herangezogen werden.

Die objektive und subjektive Bewertung der Maßnahme werden im vorletzten Arbeitsschritt zusammengeführt. Es soll geklärt werden, welche Maßnahmen als „kostengünstig“ gelten und daher besonders geeignet sind.

Aufbauend auf dem vorangegangenen Erkenntnisgewinn entsteht ein Beispielkatalog mit Hinweisen und Empfehlungen zum Einsatz kostengünstiger Maßnahmen an Unfallhäufungen, welcher den UKO zur Verfügung gestellt werden kann. Dabei werden die Maßnahmen nach ihrer Wirksamkeit kategorisiert, um die UKO im Umgang mit kostengünstigen aber ggf. gering wirksamen Maßnahmen zu sensibilisieren. 


\section{$2 \quad$ Erkenntnisstand}

\subsection{Grundlagen Unfallkommissionsarbeit}

Die UKO sind nach § 44 VwV-StVO [2015] mit der Aufgabe der Örtlichen Unfalluntersuchung betraut. Mitglieder sind die Straßenverkehrs-, Straßenbaubehörde und die Polizei. Das Ergebnis der Analyse von Unfallhäufungen (UH) dient den Straßenverkehrsbehörden als Grundlage für verkehrsregelnde, den Straßenbaubehörden für bauliche und der Polizei für überwachende Maßnahmen. Maßnahmen umschreiben dabei Eingriffe in den Status quo der Verkehrsanlage mit dem Ziel, eine Verbesserung der Verkehrssicherheit zu erzielen. Das „Merkblatt zur Örtlichen Unfalluntersuchung in Unfallkommissionen M Uko" [FGSV 2012] beschreibt die Methodik systematischer Unfallkommissionsarbeit. Es dient als Leitfaden und führt die relevanten Arbeitsschritte auf. Das Merkblatt stellt eine Weiterentwicklung und Anpassung von Verfahren dar, mit denen bereits seit den 1960er Jahren ein Beitrag zur Verbesserung der Verkehrssicherheit geleistet wurde [HUK 1961, PFUNDT 1972, 1991]. Zu Beginn des Jahrtausends veröffentlichte u. a. der Gesamtverband der Deutschen Versicherungswirtschaft (GDV) mit den Merkblättern „Auswertung von Straßenverkehrsunfällen" Teil 1 - Führen und Auswerten von Unfalltypensteckkarten - und Teil 2 - Maßnahmen gegen Unfallhäufungen - [FGSV 2003, 2002] erstmals ein methodisches Vorgehen, mit dem geeignete Maßnahmen auf Basis vorliegender Analyseergebnisse zu einer Unfallhäufung gewählt werden konnten. Während der Teil 1 des Merkblatts durch das M Uko ersetzt wurde, steht als Nachfolger des Teils 2 seit kurzer Zeit ein webbasierter Online-Katalog mit aktuellen Maßnahmen gegen UH, betreut durch die Bundesanstalt für Straßenwesen, zur Verfügung. Dieser gründet auf dem Forschungsprojekt von MAIER et al. [2015].

Die Arbeit der UKO beginnt mit dem Erkennen und Festlegen einer UH im Straßennetz. Grundlage bildet das polizeilich erfasste und in Unfalltypenkarten hinterlegte Unfallgeschehen. Als UH werden nach M Uko [FGSV 2012] Stellen (UHS) oder Linien (UHL) verstanden, an denen in einem festgelegten Untersuchungszeitraum, in einer festen räumlichen Ausprägung ein definierter Grenzwert, unabhängig der Verkehrsstärke, erreicht bzw. überschritten wird. Um eine Belastbarkeit der Aussagen zu gewährleisten, variieren die Untersuchungszeiträume und Grenzwerte in Abhängigkeit von der Ortslage sowie außerorts zusätzlich für einbahnige Landstraßen und Autobahnen. Das seltenere Auftreten von Unfällen mit schweren Unfallfolgen erfordert die Betrachtung mehrjähriger Zeiträume zur Festlegung von UH. Aufbauend auf der Untersuchung von MAIER et al. [2008] liegen im M Uko folgende Grenzwerte für UH, zum Teil in Gewichtung nach der Unfallschwere, vor (Tabelle 1). Darüber hinaus werden UHS Innerorts mit mindestens 15 Unfällen gleichen Typs als Massenhäufungsstellen erfasst. In der überwiegenden Anzahl der Bundesländer werden derzeit UH per Erlass noch nach den Grenzwerten aus Merkblatt „Auswertung von Straßenverkehrsunfällen, Teil 1" ermittelt (Tabelle 2).

In einigen Bundesländern liegen von dem Grenzwert des M Uko abweichende Regelungen zur Definition von UH vor. In Hessen kommen zwei Betrachtungsweisen, nach Unfalltyp und Unfallschwere, zur Identifikation von „Unfallpunkten“ zur Anwendung [HSVV 2006]. Für beide variiert der Grenzwert in Abhängigkeit der Verkehrsbelastung. Je höher die Verkehrsbelastung, desto höher ist die notwendige Unfallanzahl, welche einen Unfallpunkt definiert. Auch hier erhalten Unfälle mit schweren Unfallfolgen eine höhere Gewichtung, indem hier eine geringere Unfallanzahl zur Abbildung eines Unfallpunktes ausreicht. In Bayern sind UH auf Landstraßen durch eine Mindestdichte von 3 U(SP) auf einem Kilometer in drei Jahren definiert [BÖHM \& SPAHN 2008]. Auf Autobahnen variiert der Grenzwert nach der Unfallschwere und der Anzahl der Fahrstreifen. Für Ortsdurchfahrten gilt ein Grenzwert von mindestens $5 \mathrm{U}(\mathrm{P})$ in einem Zeitraum von drei Jahren.

Neben der Definition von UH nach dem M Uko [FGSV 2012] liegen mit den „Empfehlungen zum Schutz vor Unfällen mit Aufprall auf Bäume ESAB“ [FGSV 2006] und dem „Merkblatt zur Verbesserung der Verkehrssicherheit auf Motorradstrecken MVMot“ [FGSV 2007] weitere Regelwerke vor, 
welche unfallauffällige Bereiche mit Blick auf besondere Unfallumstände bzw. Verkehrsteilnehmerarten definieren. In einigen Bundesländern liegen weitere Bestimmungen vor, die „auffällige“ Bereiche in Abhängigkeit unterschiedlicher Randbedingungen beschreiben. In MecklenburgVorpommern betrifft dies die Unfälle mit Aufprall auf Bäume [VwV-U MV 2012], in Rheinland-Pfalz werden Unfälle mit Wild gesondert berücksichtigt [ÖU RP 2014].

Tabelle 1: $\quad$ Kriterien für UHS und UHL nach M Uko FGSV [2012]

\begin{tabular}{|c|c|c|c|c|c|}
\hline Art & Ort & Netzelement & Karte & Grenzwert & Ausdehnung \\
\hline \multirow{8}{*}{ UHS } & \multirow{4}{*}{10} & \multirow{2}{*}{ Knoten } & $1-J K$ & $\left.5 \cup_{\text {gтур }}{ }^{*}\right)$ & Fahrbahnrand = 25 m \\
\hline & & & 3-JKu(P) & $5 U$ & Fahrbahnachse $=50 \mathrm{~m}$ \\
\hline & & \multirow{2}{*}{ Freie Strecke } & $1-J K$ & $\left.5 \cup_{\text {gTyp }}{ }^{*}\right)$ & $\max .50 \mathrm{~m}$ \\
\hline & & & 3-JKu(P) & $5 U$ & (ab Knoteneinfluss) \\
\hline & \multirow{2}{*}{ Landstraße } & Knoten & $3-J K_{U}(P)$ & $\begin{array}{c}\mathrm{n}_{\mathrm{U}(\mathrm{SP})} \cdot 5+ \\
\mathrm{n}_{\mathrm{U}(\mathrm{LV})} \cdot 2 \geq 15\end{array}$ & $\max .300 \mathrm{~m}$ \\
\hline & & Freie Strecke & 3-JKu(P) & $\begin{array}{c}\mathrm{n} \cup(\mathrm{SP}) \cdot 5+ \\
\mathrm{n} \cup(\mathrm{LV}) \cdot 2 \geq 15\end{array}$ & $\begin{array}{c}\text { FB-Rand }=25 \mathrm{~m} \\
\text { Achse }=50 \mathrm{~m}\end{array}$ \\
\hline & \multirow{2}{*}{ Autobahn } & $\begin{array}{c}\text { Knoten } \\
\text { (Ri.-Fahrbahn) }\end{array}$ & 3-JKu(P) & $\begin{array}{c}\mathrm{n} \cup(\mathrm{SP}) \cdot 5+ \\
\mathrm{n} \cup(\mathrm{LV}) \cdot 2 \geq 15\end{array}$ & $\begin{array}{c}\max .1 .000 \mathrm{~m} \\
\left.(\mathrm{ESN}: 500 \mathrm{~m})^{\star}\right)\end{array}$ \\
\hline & & $\begin{array}{l}\text { Freie Strecke } \\
\text { (Ri.-Fahrbahn) }\end{array}$ & 3-JKu(P) & $\begin{array}{c}\mathrm{n} \cup(\mathrm{SP}) \cdot 5+ \\
\mathrm{n} \cup(\mathrm{LV}) \cdot 2 \geq 15\end{array}$ & $\begin{array}{c}250 \text { m vor Ausfahrbereich bis } \\
250 \text { m nach Einfahrbereich) }\end{array}$ \\
\hline \multirow{2}{*}{ UHL } & Landstraße & & 3-JKu(P) & 3u(Р)Тyр4 & $\max .300 \mathrm{~m}$ \\
\hline & Autobahn & & 3-JKU(SP) & $3 u(S P) / 3 a$ & $\max .600 \mathrm{~m}$ \\
\hline
\end{tabular}

Tabelle 2: $\quad$ Kriterien für UHS und UHL nach FGSV [2003]

\begin{tabular}{|c|c|c|c|c|c|}
\hline Art & Ort & Netzelement & Karte & Grenzwert & Ausdehnung \\
\hline \multirow{3}{*}{ UHS } & \multirow{3}{*}{$\begin{array}{c}\text { IO, AO, } \\
\text { BAB }\end{array}$} & \multirow{3}{*}{ Knoten oder Strecke } & $1-J K$ & 5 U Gleichartige $^{*}$ ) & \multirow{3}{*}{ geringe Längenausdehnung } \\
\hline & & & 3-JKu(P) & $5 U$ & \\
\hline & & & 3-JKu(SP) & $3 U$ & \\
\hline UHL & $\mathrm{IO}, \mathrm{AO}$ & elementübergreifend & 3-JKu(SP) & $3 U$ & $\begin{array}{l}\text { Optische Unfalldichte (min- } \\
\text { destens } 1 \mathrm{U}(\mathrm{SP}) / \mathrm{km})\end{array}$ \\
\hline
\end{tabular}

UH werden durch die UKO einer tieferen Untersuchung, der Unfallanalyse, unterzogen. Es werden die Merkmale der Einzelunfälle näher betrachtet, das Unfallgeschehen auf strukturelle Zusammenhänge untersucht und mögliche unfallbegünstigende Einflussfaktoren ermittelt. Mittel der Unfallanalyse stellen die

- Ausschnitte aller verfügbaren Unfalltypenkarten,

- Unfalllisten,

- Unfalldiagramme,

- Ortsbesichtigung und sofern notwendig,

- weitere Erhebungen dar. 
Neben den Darstellungen in den Unfalltypenkarten helfen Unfallisten, strukturelle Gleichartigkeiten im Unfallkollektiv aufzufinden. Diese geben erste Rückschlüsse auf mögliche Mängel der Verkehrsanlage. Aufschluss darüber geben die zu analysierenden Merkmale, wie zum Beispiel der Unfalltyp, die Unfallkategorie, die Unfallumstände oder die Beteiligungsart sowie weitere Merkmale [FGSV 2012]. Die Merkmale der Unfallliste sind zu aggregieren bzw. es sind die am häufigsten vorkommenden Merkmale zu benennen. Die Zusammenfassung gibt Auskunft darüber, wie stark einzelne Merkmale besetzt sind. Weiterhin können die Ausprägungen der Unfallumstände mit den gesamtdeutschen Durchschnittswerten gegenübergestellt werden. Ein deutliche Über- oder Unterschreitung dieser Durchschnittswerte gibt Hinweise für die weitere Analyse der Unfallhäufung.

Das Unfalldiagramm entspricht einer stilisierten Skizze der Örtlichkeit, in der die Einzelunfälle mit der Bewegungsrichtung der Unfallbeteiligten vor dem Unfall und weiteren Informationen - ggf. getrennt für unterschiedliche Unfalltypenkarten - dargestellt sind. Die Darstellung erfolgt mit Hilfe von Pfeilkombinationen [FGSV 2012]. Der wesentliche Vorteil des Unfalldiagramms stellt die grafische Zerlegung des Unfallgeschehens in die örtlichen Konfliktsituationen dar, welche sich aus den Unfalllisten nicht erschließen. Somit wird deutlich, welche Fahrtrichtungen bspw. vom Unfallgeschehen betroffen sind, bzw. wie die Unfallbeteiligten aufeinander getroffen sind.

Die Ortsbesichtigung dient primär zur Aufdeckung von Mängeln in der Örtlichkeit. Unfalliste und Unfalldiagramm dienen als Einkreisung und Hilfestellung zur deren Identifikation. Die Ortsbesichtigung ist nach Möglichkeit zu den Tageszeiten und Witterungsbedingungen durchzuführen, an denen sich die meisten Unfälle ereignet haben. Es sind dabei die Verkehrsbedingungen für alle Verkehrsbeteiligungsarten zu prüfen.

Auf die Erkenntnisse der Unfallanalyse baut die Maßnahmenfindung auf. Eine fachlich fundierte Analyse trägt zum Auffinden geeigneter Maßnahmenvorschläge wesentlich bei. Nach M Uko [FGSV 2012] wird zwischen

- Sofortmaßnahmen und

- mittel- und langfristige Maßnahmen

unterschieden. Sofortmaßnahmen stellen eine schnelle Reaktion auf die vorhandene Problemstellung dar. Sie sind meistens zeitlich zügig und ohne umfangreichen verwaltungstechnischen Aufwand nach dem Maßnahmenbeschluss umsetzbar. Sie sind unabhängig eventuell geplanter/genehmigter Umbaumaßnahmen zur sofortigen Erhöhung der Verkehrssicherheit umzusetzen. Mittel- und langfristige Maßnahmen hingegen, bedürfen meist einer längeren Planungs- und Realisierungsphase. In der Regel lösen sie die Sofortmaßnahmen ab. Vielfach stehen verschiedene Maßnahmen zur Umsetzung zur Auswahl. Vor der Festlegung auf eine Maßnahme sind daher die Kriterien der

- Eignung,

- Angemessenheit und

- Durchsetzbarkeit

zu prüfen. Das Kriterium der Eignung schließt den sicherheitsrelevanten Aspekt der Maßnahmen ein und zielt darauf ab, zu prüfen, ob möglicherweise weitere Maßnahmenvorschläge vorliegen, deren Umsetzung in einem stärkeren Maße zur Erhöhung der Verkehrssicherheit beiträgt. Sowohl beim Vorliegen gleichgeeigneter als auch bei unterschiedlich geeigneten Maßnahmen ist die Angemessenheit der Maßnahmen zu prüfen. Unter Angemessenheit ist zu verstehen, inwieweit die volkswirtschaftlichen Verluste des Unfallgeschehens finanzielle Aufwände bei der Maßnahmenumsetzung rechtfertigen. Allgemein gilt, dass gerade das Auftreten von Unfällen mit einer hohen Schwere auch finanziell aufwendigere Maßnahmen angemessen rechtfertigt. Sind mehrere Maßnahmen gleich gut geeignet und auch angemessen, sollte die Maßnahme verfolgt werden, die planerisch, finanziell oder politisch am schnellsten umgesetzt werden kann [FGSV 2012]. 
Umsetzungskontrollen und die Wirksamkeitsprüfung umgesetzter Maßnahmen stellen weitere Bestandteile der Unfallkommissionsarbeit dar. Die Umsetzungskontrolle umfasst die Dokumentation der Arbeitsschritte und somit den Status der UH. Ziel der Unfallkommissionsarbeit ist es, eine Verbesserung der Verkehrssicherheit zu erreichen. Die Wirksamkeitsprüfung stellt ein Mittel zur Kontrolle dieses Ziels dar und ist unter Kapitel 2.2.3 näher beschrieben.

\section{$2.2 \quad$ Bewertung von Maßnahmen}

\subsubsection{Unfallkosten}

Unfälle führen zu volkswirtschaftlichen Kosten, welche in den Unfallkostensätzen quantifiziert vorliegen. Die Höhe der Unfallkostensätze ist abhängig von der Unfallschwere und wird für die Unfallkategorien getrennt angegeben. Humanitäre Kosten, außermarktliche Wertschöpfung, Ressourcenausfall sowie direkte und indirekte Reproduktion stellen dabei die Kostenpositionen dar. Für Unfälle mit Personenschaden der Kategorie 1 bis 3 setzen sich die Unfallkostensätze aus einem Bestandteil für den reinen Personenschaden (Verunglücktenkostensatz) sowie einem reinen Sachschadensanteil zusammen. Letzterer liegt - dem Schadensbild angepasst - auch für die Unfallkategorien 4 bis 6 vor.

Die Höhe der Verunglücktenkostensätze wird durch die durchschnittliche Verletzungsschwere und Verunglücktenzahl nach den örtlichen Gegebenheiten (Ortslage, Straßenkategorie) bestimmt. Unfälle mit Personenschaden im Außerortsbereich weisen gegenüber Unfällen Innerorts eine im Mittel höhere Unfallschwere auf. Die Ermittlung der Schadenshöhe zur Festlegung der Unfallkostensätze erfolgt seit geraumer Zeit auf der Auswertung des gesamtdeutschen Unfallgeschehens in einem Fünfjahreszeitraum. Diese pauschalen Kostensätze werden turnusmäßig von der Bundesanstalt für Straßenwesen veröffentlicht. Im aktuellen M Uko [FGSV 212] finden sich die Kostensätze zum Preisstand 2009 (Tabelle 3).

Tabelle 3: $\quad$ Pauschale Unfallkostensätze der einzelnen Unfallkategorien FGSV [2012]

\begin{tabular}{|c|c|c|c|c|}
\hline \multirow[t]{2}{*}{ Schwerste Unfallfolge } & \multirow[t]{2}{*}{ Kategorie } & \multicolumn{3}{|c|}{$\begin{array}{c}\text { Unfallkostensatz KS [€/U] } \\
\text { Örtlichkeit }\end{array}$} \\
\hline & & BAB & $\mathrm{AO}$ & IO (Gesamt) \\
\hline $\begin{array}{l}\text { Unfall mit Getöteten oder Schwerverletzten } \\
\mathrm{U}(\mathrm{SP})\end{array}$ & 1 & 341.000 & 266.000 & 162.000 \\
\hline Unfall mit Leichtverletzten U(LV) & 2 & 43.500 & 24.700 & 14.600 \\
\hline Unfall mit Personenschaden U(P) & 3 & 113.000 & 100.000 & 41.500 \\
\hline $\begin{array}{l}\text { Schwerwiegender Unfall mit Sachschaden } \\
\mathrm{U}(\mathrm{SS})\end{array}$ & 4 und 6 & 23.900 & 17.900 & 15.100 \\
\hline Sonstiger Unfall mit Sachschaden U(LS) & 5 & 4.630 & 4.190 & 6.310 \\
\hline Unfall mit Sachschaden U(S) & - & 6.860 & 5.190 & 6.740 \\
\hline
\end{tabular}

Die Bezifferung der volkswirtschaftlichen Verluste durch Unfälle über Unfallkostensätze erlaubt auch einen Nutzen darzustellen. In Falle von UH entspricht dieser den vermiedenen Unfallkosten, die aus einer Verbesserung der Infrastruktur durch die Umsetzung von Maßnahmen resultieren. Zur Ermittlung der Größenordnung erfolgt in der Regel ein Vergleich der Unfallkosten im Vorherund Nachher-Zeitraum. In dieser Zeitspanne soll das betrachtete Netzelement den ceteris-paribus Bedingungen unterliegen. 


\subsubsection{Maßnahmenkosten}

Mit der Umsetzung von Maßnahmen in der Örtlichkeit entstehen Aufwände, die als Maßnahmenkosten bezeichnet werden. Diese umfassen die Investitions- und jährlichen Betriebskosten. Während die Investitionskosten die Herstellungskosten beschreiben, beinhalten Betriebskosten die jährlichen Aufwände für Unterhalt und Instandsetzung. Mit Hilfe von Annuitätenfaktoren kann der jährliche Tilgungsbetrag einer Maßnahme bezüglich eines definierten Abschreibungszeitraums ermittelt und die jährlichen Investitionskosten dargestellt werden. Die Kosten für eine Maßnahmenart variieren teilweise erheblich in Abhängigkeit von der Örtlichkeit, der räumlichen Ausdehnung einer UH sowie des Ausführungsumfangs und der Umgebung. Für UH auf Strecken bestimmt häufig die räumliche Ausdehnung den Kostenumfang, wie es z. B. für Fahrbahnsanierungen der Fall ist. Verkehrstechnische Maßnahmen, wie das Aufstellen von Z 625 StVO (Richtungstafeln in Kurven) wird maßgebend von der Geometrie der Kurve geprägt. An Knotenpunkten wirken sich häufig deren Größe und Ausstattung auf die Maßnahmenkosten aus. Größere Knotenpunkte erfordern stellenweise einen höheren Umfang an Markierungsarbeiten bzw. erfordern z. B. mehrstreifige Knotenpunktzufahrten an signalisierten Knotenpunkten einen höheren Mitteleinsatz bei einem Leuchtmittelwechsel der Signalgeber, als es bei kleineren Knotenpunktzufahrten der Fall wäre. Diesbezüglich sind Maßnahmenkosten immer auf einen Anwendungsfall bezogen und können nicht ohne weiteres übertragen werden. Bei der Zusammenfassung mehrere Maßnahmen gleicher Art in einer Gruppe, wird daher überwiegend der Mittelwert aller Maßnahmen zur Beschreibung der Maßnahmenkosten herangezogen [SPAHN 2012, MAIER et al. 2015].

\subsubsection{Maßnahmenwirkung}

Die Maßnahmenwirkung oder auch Wirkungsgrad (Effektivität) beschreibt die Eignung einer Maßnahme. In der Unfallkommissionsarbeit entspricht die Reduzierung des Unfallgeschehens/Unfallkosten dem Ziel, das Unfallgeschehen einer UH zu senken. Um die Wirksamkeit einer Maßnahme zu prüfen, wird nach FGSV [2012] das Unfallgeschehen des Vorher- und NachherZeitraums einer UH gegenübergestellt. Der Vorher-Zeitraum entspricht dem Analysezeitraum. In diesem ist die Örtlichkeit als Unfallhäufung in Erscheinung getreten. Der Nachher-Zeitraum soll nach Möglichkeit in seiner Zeitspanne dem Vorher-Zeitraum entsprechen. Er beginnt nach der Umsetzung der Maßnahme und einer Eingewöhnungszeit. Diese entspricht in der Regel ein bis drei Monaten.

Die Differenz aus dem Unfallgeschehen des Vorher- und Nachherzeitraums erlaubt eine Aussage über den absoluten Rückgang der Unfallzahl. Es können auch Unfallkosten herangezogen werden. Die Maßnahmenwirkung auf das Unfallgeschehen kann über eine relative Kenngröße, den prozentualen Wirkungsgrad, abgebildet werden. Dieser ermittelt sich aus dem Verhältnis von Vorher- und Nachher-Zeitraum (GI. 2-1). Für beide Zeiträume hat sich eine Zeitspanne von drei Jahren etabliert. Diese Zeitspanne ermöglicht zum einen eine verlässliche Aussage über die Entwicklung der Unfälle mit Personenschaden - diese treten i. d. R. seltener als Unfälle mit Sachschaden auf -, zum anderen fallen strukturelle Änderung in der Örtlichkeit und im Verkehrsablauf relativ gering aus. Eine hohe Maßnahmenwirkung geht mit einer hohen Eignung einher.

$\mathrm{MW}=\left(1-\frac{U N}{U V}\right) * 100$

mit:

MW Maßnahmenwirkung auf die Unfallanzahl [\%]

UN Unfälle Nachher-Zeitraum [-]

UV Unfälle Vorher-Zeitraum [-] 
Die Maßnahmenwirkung kann sowohl für absolute Unfallzahlen als auch für Unfallkosten ermittelt werden (GI. 2-2). Die Betrachtungsweise der Unfallkosten erlaubt gleichzeitig eine Bewertung der Unfallschwere, da die Unfallkategorien mit unterschiedlichen Unfallkostensätzen versehen sind.

$\mathrm{MW}=\left(1-\frac{U K N}{U K V}\right) * 100$

mit:

MW Maßnahmenwirkung auf die Unfallkosten [\%]

UKN Unfallkosten Nachher-Zeitraum [€]

UKV Unfallkosten Vorher-Zeitraum [€]

\subsubsection{Wirtschaftlichkeit}

Die Effizienz beschreibt das Maß der Wirtschaftlichkeit. Zur Bewertung der Wirtschaftlichkeit von Maßnahmen wird das Nutzen-Kosten-Verhältnis (NKV) berechnet (GI. 2-3). Der Nutzen entspricht hierbei den vermiedenen Unfallkosten, die sich aus der Differenz des Unfallgeschehens im Vorherund Nachher-Zeitraum ergeben. Als Kosten gehen die mit Annuitäten bestimmten jährlichen Investitionskosten ein. Erhöhen sich die Betriebskosten durch die Umsetzung von Maßnahmen, wird die resultierende Erhöhung ebenfalls bei den Maßnahmenkosten berücksichtigt. Da grundsätzlich alle Investitionen in das öffentliche Straßennetz einer wirtschaftlichen Rechtfertigung unterliegen, sollten die eingesetzten Aufwände (Kosten) den erwarteten Nutzen nicht übersteigen. Ziel ist es, ein NKV > 1 zu erreichen. In diesem Zusammenhang wird von der Angemessenheit der Maßnahme gesprochen. Diese stellt einen weiteren Aspekt im Prozess zur Bekämpfung von UH darstellt. Liegen alternative Maßnahmenvorschläge, die sich bezüglich Eignung und Durchsetzbarkeit nur marginal unterscheiden, vor, entscheidet die Angemessenheit (größter Nutzen) über die Umsetzung von Maßnahmen.

$\mathrm{NKV}=\frac{N U_{a}}{K_{a}}$

mit:

$\begin{array}{lll}\mathrm{NKV} & \text { Nutzen-Kosten-Verhältnis } & {[-]} \\ \mathrm{NU}_{\mathrm{a}} & \text { vermiedene Unfallkosten pro Jahr } & {[€]} \\ \mathrm{K}_{\mathrm{a}} & \text { jährliche Kosten der Maßnahme } & {[€]}\end{array}$

Das NKV lässt keine direkten Rückschlüsse auf die Wirkung einer Maßnahme zu. So können Maßnahmen, die nur eine geringe Wirkung erreichen, aufgrund sehr geringer Kosten (bspw. ein Verkehrsschild aufstellen) ein hohes NKV aufweisen. Diese Maßnahmen erscheinen wirtschaftlich sinnvoll, tragen aber nur unwesentlich zum Ziel der Unfallbekämpfung bei. Dementgegen haben kostenintensive Maßnahmen, trotz eines geringen NKV, häufig eine hohe Maßnahmenwirkung.

Es zeichnen sich die Maßnahmen als besonders empfehlenswert (geeignet und angemessen) aus, die an erster Stelle eine hohe Maßnahmenwirkung und an zweiter Stelle ein hohes NKV aufweisen. Die Durchsetzbarkeit von Maßnahmen steht in Zusammenhang mit möglicherweise auftretenden planerischen, finanziellen oder politischen Hindernissen. 


\section{$2.3 \quad$ Aktueller Wissensstand}

Seit seiner ersten Veröffentlichung im Jahr 2000 - damals noch mit Kostensätzen in D-Mark stellt das Merkblatt „Auswertung von Straßenverkehrsunfällen, Teil 2, Maßnahmen gegen Unfallhäufungen" des FGSV [2002] eine Sammlung auf praktischen Erfahrungen beruhend, geeigneter Maßnahmen dar. Verschiedene Ländererlasse zur Arbeit in den UKO verweisen aktuell auf dieses Merkblatt. Die Aufbereitung des Maßnahmenkatalogs liegt in mehreren Stufen vor und gewährleistet eine zielorientierte Auswahl von geeigneten Maßnahmen. Die einzelnen Stufen gliedern sich nach:

- Ortslage (Innerorts, Außerorts, Bundesautobahn)

- Straßenkategorie

- Straßenelement (Knoten, Strecke)

- Unfalltyp

Die Stufe des Unfalltyps enthält eine weitere Unterteilung. Diese richtet sich nach der Bewegungsrichtung der Unfallbeteiligten (in Anlehnung an den dreistelligen Unfalltyp) und besonderen Charakteristiken der Unfallstelle (bspw. abknickende Vorfahrtstraße). Diese Gliederung hilft, UH zu ordnen, in einzelne Konfliktsituationen aufzulösen und für jede Situation, einem Systembaukasten ähnlich, geeignete mögliche Maßnahmen zielgerichtet auszuwählen. Dabei wird jede als maßgebend eingeschätzte Konfliktsituation einzeln betrachtet. Die Verwendung des Unfalldiagramms unterstützt bei der räumlichen Zuordnung von Konfliktsituationen. Die Maßnahmen der jeweiligen Konfliktsituation sind mit entsprechenden Defiziten attribuiert. Während der Ortsbesichtigung soll die Verkehrsanlage auf Vorliegen dieser Defizite überprüft werden. Die Reihung der Maßnahmen je Konfliktsituation folgt keinem festen Kriterium. Angaben zu mittleren Kosten, Wirkungsgrad oder eine volkswirtschaftliche Bewertung der Maßnahmen liegen in FGSV [2002] nicht vor. Da dieser Maßnahmenkatalog auf praktischen Erfahrungen beruht, ist den Einzelmaßnahmen eine sicherheitsverbessernde Wirkung auf das Unfallgeschehen zuzuschreiben. Im Anhang des Merkblatts sind darüber hinaus ungeeignete, nicht wirkungsvolle Maßnahmen aufgeführt.

Die Norm „Strassenverkehrssicherheit Unfallschwerpunkt-Management" vom" Schweizerischen Verband der Straßen- und Verkehrsfachleute VSS“ [2013] beschreibt die Vorgehensweise zur Bekämpfung von UH in der Schweiz. Ähnlich wie in Deutschland werden UH über Grenzwerte - Gewichtung über die Unfallschwere - für Straßentypen und Ortslagen definiert. Aus der Analyse des Unfallgeschehens sind Maßnahmen zur Bekämpfung der UH abzuleiten. Dabei wird u. a. auf den Maßnahmenkatalog „Strassenraumgestaltung“ [bfu 2013] verwiesen. Maßnahmenkataloge sollen nach VSS [2013] idealerweise „...die durchschnittlichen Wirksamkeiten sowie überschlägliche Kosten" für die Maßnahmen enthalten. Maßnahmen sind bezüglich ihrer Eignung, Durchsetzbarkeit und Angemessenheit auszuwählen. Maßnahmen gelten hier als angemessen, wenn sie ein möglichst hohes NKV, aber mindestens ein NKV > 1 aufweisen.

GERLACH et al. [2009] untersuchten die umgesetzten Maßnahmen an $110 \mathrm{UH}$ bezüglich ihrer Effektivität und Effizienz. Die Effektivität wurde auf Basis der vermiedenen Unfallkosten, die Effizienz anhand von NKV ermittelt. Voraussetzung für diese Berechnung war die Einhaltung der ceteris-paribus-Bedingungen, welche erfordert, dass sich im Vorher- und Nachher-Zeitraum keine wesentlichen Randbedingungen verändert haben. Zwischen beiden Zeiträumen wurde eine Eingewöhnungszeit nach Umsetzung der Maßnahme von vier bis sechs Wochen berücksichtigt. Mit dem Poisson-Test und dem Test nach McNemar wurden die Maßnahmenwirkung und das NutzenKosten-Verhältnis jeder Maßnahme bezüglich ihrer statistischen Aussagekraft überprüft. Zeigen beide Tests einen signifikanten Unterschied an, so unterstellen GERLACH et al. [2009] der Maßnahme eine nachweisbare Wirkung auf das Unfallgeschehen. Für 87 Maßnahmen liegen Effektivitäts- und Effizienzbetrachtungen vor. Die Maßnahmen wurden bewertet und in drei Kategorien geordnet:

- (1) „geeignete“ Maßnahme/sehr gute Wirkung 
- (2) „bedingt geeignete“ Maßnahme/ausreichende Wirkung

- (3) „nicht geeignete“ Maßnahme/ungenügende bzw. keine Wirkung

In der vertiefenden Analyse der Maßnahmen beschränken sich GERLACH et al. [2009] auf einen Vergleich von baulichen und verkehrstechnischen Arten von Maßnahmen. Der Anteil baulicher Maßnahmen, die als geeignet eingestuft werden, übertrifft den Anteil verkehrstechnischer Maßnahmen. D. b., bauliche Maßnahmen werden häufiger zweckmäßig eingesetzt. In der Kategorie nicht geeigneter Maßnahmen überwiegen die verkehrstechnischen Maßnahmen. Aus der Bewertung der Maßnahmenwirkung - Klassierung in vier Gruppen - geht hervor, dass bauliche Maßnahmen häufiger gute bis sehr gute Wirkung aufweisen (Abbildung 2). Je geeigneter die Maßnahme, desto größer ist der Vorteil baulicher Maßnahmen bezüglich ihrer Wirkung.

Die Analyse der NKV beider Maßnahmenarten zeigen für verkehrstechnische Maßnahmen aufgrund ihrer geringen Kosten gegenüber den baulichen Maßnahmen, deutlich höhere NKV als auch der Streuung selbiger. Der Median des NKV für verkehrstechnische Maßnahmen übersteigt mit einer Größenordnung von 404 den Wert baulicher Maßnahmen um das 20-fache. Demzufolge sind verkehrstechnische gegenüber baulichen Maßnahmen zwar wesentlich effizienter, jedoch können auch bauliche Maßnahmen effizient eingesetzt werden. Die Einschätzung der Maßnahmen unterliegt dahingehend einer Einschränkung, dass jede Maßnahme nur an einer Stelle erhoben und bewertet wurde.

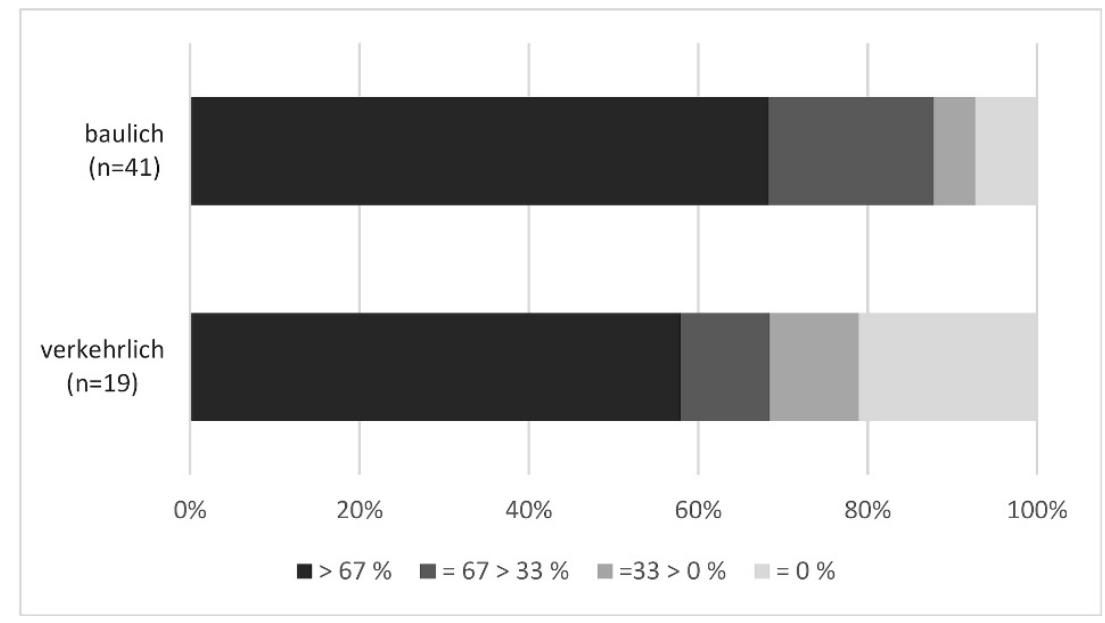

Abbildung 2: $\quad$ klassifizierte Maßnahmenwirkung [\%] in Bezug auf die Reduzierung von Unfallkosten baulicher und verkehrstechnischer Maßnahmen im Kollektiv GERLACH et al. [2009]

Des Weiteren befragten GERLACH et al. [2009] 65 Mitglieder von UKO zu ihrer Arbeit. Bezüglich des Einsatzes von Maßnahmenarten zeigte sich, dass ca. $30 \%$ aller umgesetzten Maßnahmen baulich und ca. 65 \%verkehrstechnischer Natur sind. Zur Frage, wo bauliche Maßnahmen umgesetzt werden sollen bzw. sinnvoll sind, sah der größte Anteil der Befragten das Einsatzspektrum solcher Maßnahmen an Stellen mit baulichen Mängeln. Überwiegend scheitern Beschluss und Umsetzung baulicher Maßnahmen an einer ungesicherten Finanzlage. Sind zum ursprünglichen Vorschlag alternative Vorschläge umzusetzen, wird bevorzugt und doppelt so häufig auf verkehrstechnische Maßnahmen zurückgegriffen. GERLACH et al. [2009] geben an, dass bei der Maßnahmenauswahl als erste die Effektivität (Maßnahmenwirkung) beurteilt und an zweiter Stelle das NKV zum Vergleich mit alternativen Varianten geprüft werden soll.

Auf der Grundlage von Maßnahmen an 2.790 außerörtlichen UH im Freistaat Bayern, bewertete SPAHN [2012] deren Wirksamkeit und Wirtschaftlichkeit. Die Maßnahmenbewertung setzt sich dabei aus einem nutzenorientierten (Effektivität) und einem zielorientierten (Effizienz) Bestandteil zusammen. Der nutzenorientierte Ansatz beruht auf einem volkswirtschaftlichen Vorher-NachherVergleich des Unfallgeschehens unter Berücksichtigung eines Auswahlfehlers. Der Auswahlfehler 
beschreibt die Differenz zwischen dem Unfallgeschehen im aktuellen Ermittlungs-/Analysezeitraum und dem langfristigen „Mittel“ dieses betrachteten Netzelements. Diese Differenz ist bei der Wirkungsermittlung einer Maßnahme vom Nutzen abzuziehen, da sich langfristig das Niveau wieder dem Mittelwert angleicht. Dieser Effekt ist bekannt unter dem Ausdruck „Regression zur Mitte“. Übersteigt die Differenz des Unfallgeschehens von Vorher- und Nachher-Zeitraum die Differenz des Auswahlfehlers, liegt eine effektive Verbesserung der Verkehrssicherheit vor, die Effektivitätskostenrate nimmt einen Wert größer Eins an. Der Auswahlfehler wurde aus Beobachtungen von UH bestimmt, die seit ihrer Identifikation über einen mehrjährigen Zeitraum keiner Maßnahme ausgesetzt waren und auch sonst den ceteris-paribus-Bedingungen unterliegen. Ob sich mit der Maßnahmenumsetzung an der betroffenen Stelle ein ausreichendes Sicherheitsniveau eingestellt hat, wird mit Hilfe des zielorientierten Ansatzes überprüft. Als ausreichendes Sicherheitsniveau - und damit gelungenen Maßnahmeneinsatz - definiert SPAHN [2012] Unfallkostenraten, die auf oder unter dem Niveau entsprechender Grundunfallkostenraten des Netzelements liegen. Die Differenz zwischen Unfallkostenrate im Nachher-Zeitraum und der Grundunfallkostenrate wird als Zielerreichungskostenrate definiert. Zur Ermittlung der fahrleistungsbezogenen Kenngrößen wurde auf die Verkehrsstärken aus Dauerzählstelldaten zurückgegriffen. Aus der Überlagerung des nutzer- und zielorientierten Ansatzes wird ein mehrstufiger „Level of Effectiveness" ermittelt (Abbildung 3). Mit der Berücksichtigung eines Auswahlfehlers und der Verwendung von fahrleistungsbezogenen Kenngrößen, unterscheidet sich dieser Bewertungsansatz von anderen Verfahren.

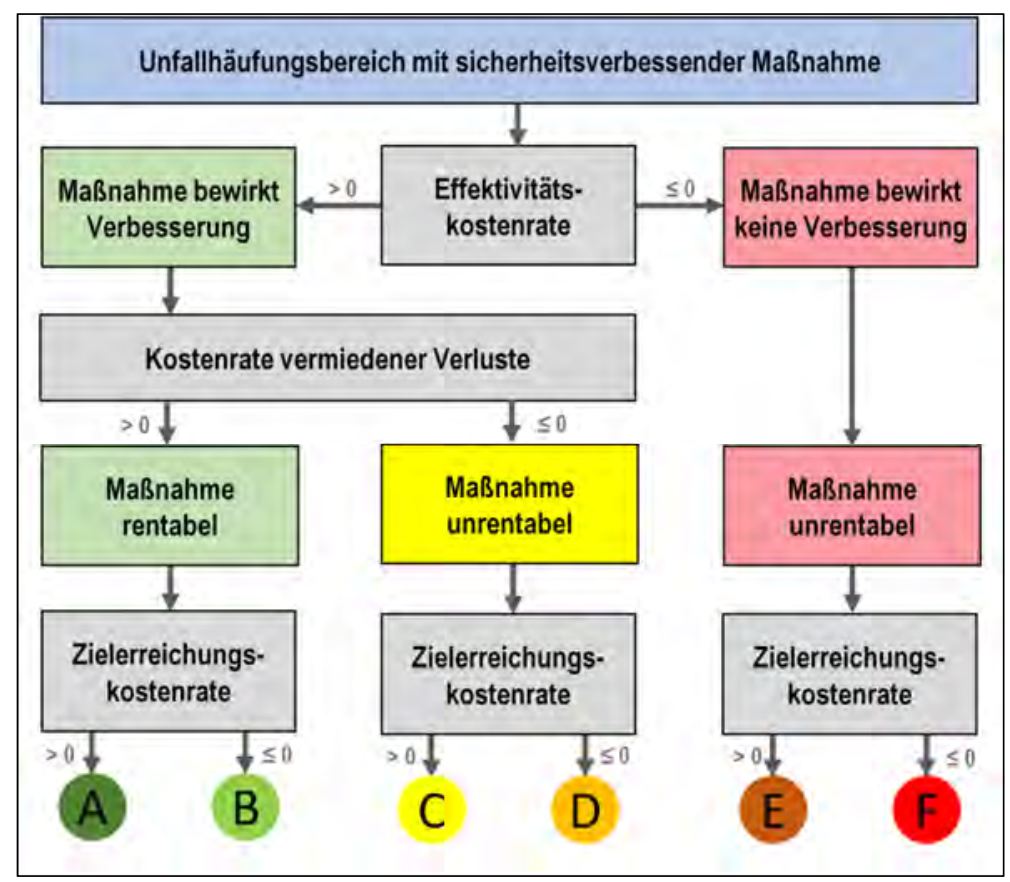

Abbildung 3: Nutzen- und zielorientierte Gesamtbewertung der Maßnahmenwirksamkeit, Level of Effictiveness [SPAHN 2012]

SPAHN [2012] gliedert die Einzelmaßnahmen anhand drei Kodierungsebenen in Maßnahmengruppen (z. B. Linienführung, Beschilderung) mit verschiedenen Eigenschaften. Die Ergebnisse der Untersuchung münden in einem Maßnahmenkatalog gegen Unfallhäufungen, in welchem - in ähnlicher Struktur zum Merkblatt Teil 2 [FGSV 2002] - mögliche Maßnahmen, kategorisiert nach Netzelement, ggf. Regelungsart des Knotenpunkts und der Konfliktsituation, aufgeführt werden. Den Maßnahmen sind Angaben zu Wirksamkeit und jährlichen mittleren Kosten zugeordnet. Somit ist ein Rückschluss auf preisgünstige wirksame Maßnahmen möglich. Dabei werden z. B. die Beseitigung von Unstetigkeiten im Straßenverlauf bzw. der richtliniengerechte Aus- und Neubau von Straßenabschnitten bei von Fahrunfällen dominierten UH uneingeschränkt empfohlen. Ähnlich rentabel erwiesen sich das Aufstellen von Z 274 StVO zur Beschränkung der zulässigen Höchstgeschwindigkeit an Knotenpunkten oder das warnen vor einem unerwarteten Straßenverlauf mit 
Z 105 StVO (Doppelkurve). Detaillierte Bewertung einzelner Maßnahmen sind SPAHN [2012] zu entnehmen.

Zur Entwicklung eines Expertensystems, mit dessen Hilfe Maßnahmen an UH mit Unterstützung durch die EDV abgeleitet werden sollen, analysierte WEINERT [1999] 128 innerörtliche UH. Die Maßnahmen wurden in Maßnahmenarten kategorisiert. Maßnahmen auf Basis verkehrsrechtlicher Eingriffe (bspw. Markierungsarbeiten) sind durch einen geringen finanziellen Aufwand gekennzeichnet. Im Rahmen der Untersuchung wurden die Maßnahmen mit einem Vorher-NachherVergleich der Kenngrößen Unfall(-kosten)rate und Verunglücktenzahl beurteilt. Zur Bewertung wirtschaftlicher Aspekte wurde das NKV herangezogen. Signifikanztests geben Auskunft über die Aussagekraft der Ergebnisse. Im Mittel weisen alle Maßnahmengruppen einen Rückgang der Unfall(-kosten)rate auf. Die Detailanalyse der Gruppen verdeutlicht, dass Ausreißer entgegen dem Trend sich in fast jeder Gruppe wiederfinden. WEINERT [1999] bemerkt, dass die Vielzahl an Maßnahmenmöglichkeiten, auch innerhalb einer Gruppe, bei geringen Stichprobengrößen nur eingeschränkt Vergleichsmöglichkeiten bieten und die statistische Aussagekraft einschränken.

Für die Berechnung des NKV erfolgt eine Gruppierung der Maßnahmen nach dem Arbeitsvorgang. 21 der 128 untersuchten Stellen wiesen ein negatives NKV auf. Erwartungsgemäß haben kostenintensivere Maßnahmen ein geringeres NKV (Tabelle 4). Weiterhin stellt WEINERT [1999] anhand einer Korrelationsanalyse fest, dass es keinen Zusammenhang zwischen der Unfallschwere an einer UH und den Maßnahmenkosten gibt. Dementsprechend werden an UH mit hohen Unfallkosten genauso häufig kostengünstige wie kostenintensive Maßnahmen umgesetzt. Das gleiche gilt auch für UH mit geringen Unfallkosten.

\begin{tabular}{c|c|c} 
Tabelle 4: NKV für Maßnahmentypen nach WEINERT [1999] & \\
Maßnahmentyp & Anzahl & NKV \\
\hline Baumaßnahmen & 16 & 4,92 \\
davon mit NKV > 0 & 14 & 6,34 \\
\hline Installation von LSA (Geräte) & 33 & 3,24 \\
davon mit NKV > 0 & 30 & 6,61 \\
\hline Überwachung & 5 & 12,90 \\
davon mit NKV > 0 & 4 & 19,07 \\
\hline Verbesserung Fahrbahnoberfläche & 4 & 9,97 \\
\hline Änderung Signalprogramm & 20 & 80,74 \\
davon mit NKV > 0 & 18 & 137,48 \\
\hline Beschilderung & 16 & 121,25 \\
davon mit NKV > 0 & 11 & 216,71 \\
\hline Markierung & 23 & 119,90 \\
davon mit NKV > 0 & 17 & 207,79 \\
\hline
\end{tabular}

In einer retroperspektiven Betrachtung des Sicherheitspotentials von Maßnahmen an UH analysierte LINKE [2014] 99 UH auf Landstraßen aus Sachsen und Sachsen-Anhalt. Die Maßnahmenbewertung orientiert sich an dem Verfahren von SPAHN [2012] und differenziert in die Beurteilung der Effektivität (Maßnahmenwirkung im Vorher-Nachher-Vergleich) und der Effizienz (NKV) der Maßnahmenumsetzung. In der Untersuchung wird in bauliche (Deckenbaumaßnahmen, Umbau Knotenpunkt) und verkehrstechnische Maßnahmen differenziert. Zur zweiten Kategorie zählen $u$. 
a. Maßnahmengruppen wie Markierungsarbeiten, Aufstellen von Verkehrszeichen zur Geschwindigkeitsreduzierung, oder Änderung der Signalisierung. Die Analyse der Unfallzahlen zeigt, dass bauliche gegenüber verkehrstechnischen Maßnahmen eine höhere Maßnahmenwirkung besitzen - es tritt im Schnitt eine höhere Reduzierung der Unfälle ein. Dies spiegelt sich in der durchschnittlichen Höhe vermiedener Unfallkosten beider Kollektive wider. Auch bei LINKE [2014] zeigen verkehrstechnische Maßnahmen im Mittel ein deutlich höheres NKV (452) und eine größere Streuung als bauliche Maßnahmen (Mittelwert NKV = 122). Im Kollektiv baulicher Maßnahmen hat keine Einzelmaßnahme ein negatives NKV. Es wird darauf verwiesen, dass die Wirkung von Maßnahmen auch innerhalb der Gruppen deutlich variiert. LINKE [2014] stellt fest, dass bauliche Maßnahmen aufgrund planerischer und kostenbezogener Aufwände in der Regel seltener und nur dann umgesetzt werden, wenn verkehrstechnische Maßnahmenumsetzungen nur mangelnden Erfolg zeigten. Inwieweit in den Beschlüssen der UKO von vornherein festgelegt wurde, schnell umsetzbare verkehrstechnische Maßnahmen langfristig durch bauliche Maßnahmen zu ersetzen, war aus den Daten nicht abzuleiten.

Auch MEULENERS et al. [2005] weisen in einer Analyse von ca. 150 UH in Australien nach, dass verkehrsrechtliche (kurzfristig umsetzbare) Maßnahmen, wie z. B. Verbot einer Abbiegebeziehung oder die Verdeutlichung der Verkehrsführung durch Verkehrszeichen, sowohl in Streuung als auch in absoluter Höhe größere NKV aufweisen als bauliche Maßnahmen.

Der webbasierte „Maßnahmenkatalog gegen Unfallhäufungen“ [MAIER et al. 2015] ermöglicht, die Arbeitsschritte der Analyse, Maßnahmenfindung und Wirkungskontrolle für UH durchzuführen. Die Vorgehensweise zur Auswahl geeigneter möglicher Maßnahmen folgt inhaltlich der Methodik des Merkblatts Teil 2 [FGSV 2002] und wird durch eine programminterne Analyse des Unfallgeschehens unterstützt. Dabei steht es dem Nutzer offen, mögliche Maßnahmen als Sofort- oder langfristige Maßnahmen zu definieren. In Abhängigkeit des vorliegenden Unfallgeschehens, einer erwarteten Maßnahmenwirkung und der im Programm hinterlegter mittleren Maßnahmenkosten, werden die NKV von Einzelmaßnahmen bzw. Maßnahmenpaketen in der Analyse geschätzt. Diese dienen zu einer vorausblickenden wirtschaftlichen Einschätzung/Bewertung von Maßnahmen. Im Arbeitsschritt der Wirkungskontrolle erhält der Nutzer die Möglichkeit, Maßnahmenwirkung und NKV auf Basis eines Vergleichs der Unfallzahlen im Vorher-Nachher-Zeitraum und der tatsächlichen Maßnahmenkosten zu evaluieren. Auch hier dienen die NKV als Kontrolle eines effizienten Mitteleinsatzes.

In der Broschüre des DVR [2002] „Mehr Sicherheit im Straßenverkehr, Bau- und verkehrstechnische Maßnahmen" wird der Vergleich der Unfälle/Unfallkosten von Vorher- und Nachher-Zeitraum als Verfahren zur Bewertung der Wirksamkeit benannt. Die Berechnung des NKV gibt einen Überblick über die Wirtschaftlichkeit der Maßnahme. Es wird darauf verwiesen, dass auch mit „billigen“, sogenannten „low-cost Maßnahmen“ durchaus deutliche Erhöhungen in der Verkehrssicherheit erzielt werden können. Diese sollen verstärkt zum Einsatz gebracht werden.

In einer Erörterung zum Unfallgeschehen mit Aufprall auf Bäume im Land Brandenburg bezeichnet VOLLPRACHT [2000] schnell realisierbare Maßnahmen, wie Beschilderungen, Markierungen, passive Schutzeinrichtungen oder Geschwindigkeitsüberwachungen, als „low cost measures“.

Im Auftrag der „Conference of European Directors of Roads" (CEDR) erfolgte auf europäischer Ebene die Zusammenstellung und Bewertung von kostengünstigen/rentablen Maßnahmen zur Verbesserung der Straßeninfrastruktur [CEDR 2008]. Insgesamt wurden 55 Investitionen näher bewertet, von denen mehr als die Hälfte geeignet ist, um auch an einzelnen Netzabschnitten diese entsprechen übertragen Maßnahmen gegen $\mathrm{UH}$ - eingesetzt werden können. Sowohl für Investitionskosten als auch Maßnahmenwirkung erfolgte eine dichotome Bewertung (u. a. mit NKV) und Zuordnung in die Klassen „hoch“ und „gering“. Im Vordergrund stand die Identifikation von kostengünstigen Investitionen, die in einem hohen Umfang zur Verbesserung der Verkehrssicherheit beitragen. 
Als besonders empfehlenswert werden die Investitionen

- Verbesserung der Seitenräume (hindernisfreie Seitenräume, Einsatz passive Schutzeinrichtungen),

- Geschwindigkeitsbeschränkung,

- Verbesserung der Knotenpunktgestaltung (Einsatz Kreisverkehr, Ordnung der Verkehrsströme),

- Einsatz von Lichtsignalanlagen und

- Maßnahmen zur Verkehrsberuhigung/Geschwindigkeitsreduzierung angesehen.

Für weitere Details wird auf CEDR [2008] verwiesen.

Eine Bewertung kommunaler Erfahrungen zu 50 „low-cost Maßnahmen“ in Tempo 30-Zonen enthält ADAC [1995]. Die Maßnahmen sind nach ihrer Art klassifiziert. Es liegen in den Datenblättern der Maßnahmen u. a. Informationen zu Kosten, Wirksamkeit (Vorher-Nachher-Vergleich), Akzeptanz bei den Verkehrsteilnehmern, Auswirkungen auf die Umwelt und eine allgemeine lediglich qualitative Beurteilung der Maßnahme durch den ADAC vor. Im Katalog fehlt jedoch eine typbezogene Einordnung der Maßnahmen.

\section{$2.4 \quad$ Zusammenfassung}

Das Regelwerk „Merkblatt zur Örtlichen Unfalluntersuchung in Unfallkommissionen M Uko“ [FGSV 2012] enthält die methodische Vorgehensweise zur Bekämpfung von UH. Der überwiegende Teil der Bundesländer verweist in Erlassen zur Arbeit der UKO derzeit noch auf den Vorgänger, das Merkblatt „Auswertung von Straßenverkehrsunfällen, Teil 1“ [FGSV 2003]. Darüber hinaus liegen länderspezifische Festlegungen zur Definition von UH-Grenzwerten vor. Dementsprechend variiert die Unfallanzahl, ab der ein Netzelement als unfallauffällig (Erreichen des Grenzwerts) eingeordnet wird. Dies wirkt sich auf die Bewertung der Kenngrößen Wirksamkeit und Wirtschaftlichkeit aus. Zur Maßnahmenfindung wird in den Erlassen der Länder überwiegend auf das Merkblatt „Auswertung von Straßenverkehrsunfällen, Teil 2“ [FGSV 2002] verwiesen.

Gegenüber seinem Vorgänger stellt das M Uko [FGSV 2012] deutlicher auf die Umsetzungskontrolle und Wirksamkeitsprüfung im Verfahren der Unfallkommissionsarbeit ab. Die Wirksamkeitsprüfung erfolgt primär durch eine Bewertung der Maßnahmenwirkung (Effektivität). Diese entspricht einem Vergleich der Unfälle bzw. Unfallkosten im Vorher-Nachher-Zeitraum. Vor dem Hintergrund beschränkter finanzieller Mittel und einer wirtschaftlichen Rechtfertigung öffentlicher Investitionen, sollen Maßnahmen gezielt eingesetzt werden. Die Überprüfung der Wirtschaftlichkeit von Maßnahmen (Effizienz) beruht i. d. R. auf einem Berechnungsnachweis mit dem NutzenKosten-Verhältnis.

Während im Merkblatt „Auswertung von Straßenverkehrsunfällen, Teil 2“ lediglich bewährte Maßnahmen gegen $\mathrm{UH}$, in Abhängigkeit von Örtlichkeit und weiteren Randbedingungen, ohne Wirkungsgrad aufgeführt werden, enthalten WEINERT [1999], GERLACH et al. [2009] und SPAHN [2012] umfangreiche Sammlungen von Maßnahmen gegen $U H$, welche auch bezüglich ihrer Wirksamkeit und Wirtschaftlichkeit näher untersucht wurden. Während in diesen Quellen zur Bewertung der Maßnahmenwirkung unterschiedliche Ansätze verfolgt wurden, fußt die Wirtschaftlichkeitsbetrachtung auf der Ermittlung von NKV. Dabei sind Bewertungen gering besetzter Kollektive (Maßnahmenarten) häufig durch wesentliche Streuungen und damit einer geringen Aussagekraft gekennzeichnet [LINKE 2014, WEINERT 1999]. Die Untersuchung von SPAHN [2012] erlaubt hingegen aufgrund ihres umfangreichen Kollektivumfangs fundierte Aussagen zu Wirkungen von Maßnahmen auf Landstraßen.

Maßnahmen mit geringen Kosten - häufig sind dies verkehrstechnische Maßnahmen - zeichnen sich gegenüber baulichen Maßnahmen durch im Mittel höhere NKV aber auch höhere Streuungen selbiger aus [MEULENERS et al. 2005, WEINERT 1999, GERLACH et al. 2009, LINKE 2014]. Das NKV allein lässt keine Rückschlüsse auf die Wirksamkeit einer Maßnahme zu. Überwiegend besit- 
zen bauliche Maßnahmen eine deutlich höhere Wirkung auf das Unfallgeschehen. Sie werden darüber hinaus zweckmäßiger eingesetzt. GERLACH et al. [2009] als auch LINKE [2014] stellen fest, dass bauliche gegenüber verkehrstechnischen Maßnahmen seltener eingesetzt werden. Häufig stellen bauliche Maßnahmen Folgemaßnahmen dar, die zum Einsatz kommen, wenn sich mit der Umsetzung der verkehrstechnischen Maßnahmen kein Erfolg erkennen lässt. Der häufige Einsatz kostengünstiger (verkehrstechnischer) Maßnahmen steht dabei im engen Zusammenhang mit der Beschränkung finanzieller Mittel in den Haushalten [GERLACH et al. 2009].

Der DVR [2009] verweist darauf, dass auch mit geringem Mitteleinsatz durchaus eine Erhöhung der Verkehrssicherheit erzielt werden kann. Dabei stehen die kostengünstigen (low-cost) und schnell umsetzbaren Maßnahmen im Vordergrund. Eine klare Abgrenzung zur Definition „kostengünstiger" Maßnahmen liegt nicht vor [ADAC 1995]. Jedoch ist zusammenfassend zu erkennen, dass sich dieser Kategorie überwiegend Maßnahmen

- mit einem sehr hohem NKV,

- (sehr) geringen Investitionskosten,

- verkehrstechnischen Charakter und

- kurzem Realisierungszeitraum

zuordnen lassen. 


\subsection{Vorgehen}

Grundlage einer aussagekräftigen Bewertung verschiedener kostengünstiger Maßnahmen ist eine möglichst umfangreiche Datensammlung. Hintergrund ist, dass zum einen die Anzahl möglicher Maßnahmen an UH sehr groß ist (vgl. MAIER et al. [2015]). Zum anderen ist es sinnvoll, für ein und dieselbe Maßnahme mehrere Umsetzungen in die Untersuchung einzubeziehen, um eine statistisch valide Bewertung der Maßnahmenwirkung zu gewährleisten.

Aus diesen Gründen wurden zunächst schriftlich alle UKO der Bundesrepublik Deutschland (ca. 500) um generelle Auskunft zu relevanten Aktivitäten und ihrer Bereitschaft zur Mitarbeit gebeten. In einer zweiten Stufe der Kontaktaufnahme wurden aus der schriftlichen Umfrage die zur Unterstützung bereiten Kommissionen gefiltert sowie um persönlich oder der UDV (Preis der Unfallkommissionen, Dozentenschulungen) bekannte UKO ergänzt. Mit diesen Kommissionen wurde fernmündlich Kontakt aufgenommen. Im Rahmen persönlicher Gespräche (telefonisch oder vor Ort) konnten in dieser dritten Stufe der Kontaktaufnahme konkret in den Jahren 2004 bis 2011 realisierte Maßnahmen sowie die entsprechenden Umsetzungskosten erfragt werden. Dadurch wird gewährleistet, dass seit Umsetzung der Maßnahme mindestens drei Jahre vergangen sind, um die Maßnahmenwirkung beurteilen zu können [FGSV 2012].

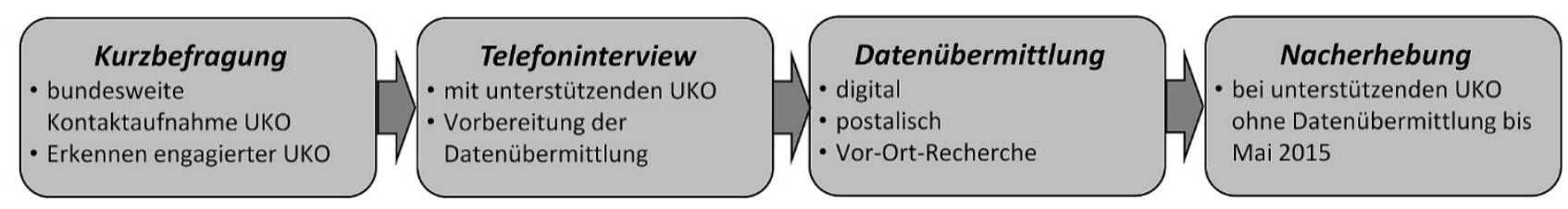

Abbildung 4: Vorgehen bei der Datenerhebung

\subsection{Erste Stufe der Kontaktaufnahme}

In der ersten Stufe der Kontaktaufnahme wurden alle UKO der Bundesrepublik Deutschland angeschrieben. Dazu wurden zunächst ausgehend von den Festlegungen in den Erlassen zur Arbeit der UKO in den einzelnen Bundesländern Vertreter in den Innenministerien (bei Leitung der UKO durch die Polizei) oder den für Bau bzw. Verkehr zuständigen Ministerien (bei Leitung der UKO durch die Straßenbaubehörde oder Straßenverkehrsbehörde) angeschrieben und um Unterstützung des Forschungsvorhabens gebeten. So konnte geklärt werden, auf welche Art und Weise der Kontakt zu den einzelnen UKO hergestellt bzw. die Befragung organisiert werden kann. Ergebnis war, dass

- in sieben Bundesländern die Befragung zentral mit Unterstützung der Ministerien veranlasst wurde,

- in vier Bundesländern die UKO direkt angeschrieben werden sollten,

- in zwei Bundesländern eine Befragung der einzelnen UKO vorerst abgelehnt wurde und

- in drei Bundesländern aus anderen Gründen die Befragung nicht erfolgte bzw. bereits Daten vorlagen.

Neben der formalen Abklärung des Ablaufs der Kontaktaufnahme diente diese erste Befragungswelle auch zum Verteilen eines Fragebogens. Dies geschah zum einen direkt durch den Auftragnehmer, zum anderen zentral durch die entsprechenden Behörden. Um die Anzahl der Rückläufe zu erhöhen, wurde neben der Möglichkeit, den ausgewählten Fragebogen postalisch oder per E-Mail zurückzusenden, auch die Option der webbasierten Befragung über eine entsprechende Online-Plattform angeboten.

Der Fragebogen (Vergleiche Anhang 2) gliederte sich in drei Teile. Im ersten Teil wurde das Anliegen kurz beschrieben. Im zweiten Teil wurde abgefragt, wieviel Maßnahmen innerhalb der letzten 
zehn Jahre durch die UKO umgesetzt worden sind und bei wieviel dieser Maßnahmen es sich um Sofortmaßnahmen handelte. Beide Angaben konnten frei eingetragen werden. Weiterhin wurde gefragt, mit welcher Häufigkeit Unterlagen zu den Maßnahmen vorliegen und eine Wirkungsanalyse durchgeführt wurde. Dabei konnte zwischen den drei Antwortmöglichkeiten

- immer,

- von Fall zu Fall und

- selten

gewählt werden. Diese Informationen wurden vor allem für die zweite Stufe der Kontaktaufnahme benötigt, um dort gezielt UKO mit möglichst vollständigem Datenbestand anzusprechen. Die letzte Frage im zweiten Teil des Fragebogens bezog sich auf die Zuständigkeit der UKO (Landkreis, Stadt etc.). Im dritten und letzten Teil des Fragebogens wurde das weitere Vorgehen innerhalb des Forschungsvorhabens kurz erläutert und gefragt, ob die UKO gegebenenfalls für weitere Rückfragen im Projektverlauf zur Verfügung steht. Im Anschluss folgte die Abfrage der Kontaktdaten der UKO bzw. der oder des Verantwortlichen. Der Fragebogen wurde bewusst kurz gehalten, um möglichst eine Vielzahl von Antworten zu erhalten.

Obwohl die Befragung in einem Großteil der Bundesländer zentral durch eine übergeordnete Einrichtung erfolgte, lag der Rücklauf ausgefüllter Fragebögen nur bei ca. $8 \%$. Dies entspricht 35 UKO. Aus diesem Grund wurde in Abstimmung mit der UDV eine zweite Befragungswelle gestartet. In dieser wurden zum einen UKO angeschrieben, die bisher noch nicht befragt worden waren und zum anderen wurden UKO angeschrieben, welche im Rahmen der ersten Befragungswelle nicht reagiert hatten. An der zweiten Befragungswelle beteiligten sich noch einmal 111 UKO, sodass der gesamte Rücklauf durch die zweite Befragungswelle um $23 \%$ auf $31 \%$ (146 UKO) gesteigert werden konnte.

\subsubsection{Auswertung der Kurzfragebögen}

Nach Abschluss der zweiten Befragungswelle lagen ausgefüllte Fragebögen von insgesamt 146 UKO zur Auswertung vor. Die Verteilung auf die einzelnen Bundesländer kann Abbildung 5 entnommen werden. Dabei machen die Stadtstaaten mit jeweils nur $1 \%$ einen sehr geringen Anteil aus, da in diesen Bundesländern in der Regel jeweils nur eine UKO tätig ist. Ein Großteil der UKO (115) gab an, auch für weitere Rückfragen zur Verfügung zu stehen. Im Folgenden sollen die Angaben dieser 115 UKO kurz dargestellt werden.

Der mit 53 \% (61 UKO) größte Teil der 115 unterstützenden UKO ist auf Landkreisebene tätig und somit sowohl für Innerorts- als auch für Außerortsstraßen zuständig. Der Anteil städtischer UKO in der Befragung liegt bei $43 \%$ (49), der sonstigen UKO (BAB und Stadtstaaten) bei nur 4 \% (5).

Nach den Angaben der 115 auch weiterhin unterstützenden UKO wurden in den letzten 10 Jahren 7.284 Maßnahmen an Unfallhäufungen umgesetzt. Davon entfallen etwa $39 \%$ auf UKO, deren Zuständigkeitsbereich im städtischen Bereich liegt. Der Großteil der Maßnahmen (56\%) wurde von UKO umgesetzt, welche in Landkreisen tätig und somit sowohl für innerörtliche als auch für außerörtliche Straßen verantwortlich sind. Nur etwa $5 \%$ der Maßnahmen entfallen auf Bundesautobahnen (vgl. Abbildung 6 links) ${ }^{1}$.

1 Bei den Stadtstaaten wurde angenommen, dass $80 \%$ der Maßnahmen auf Innerorts-Bereiche und $20 \%$ auf BAB entfallen. 


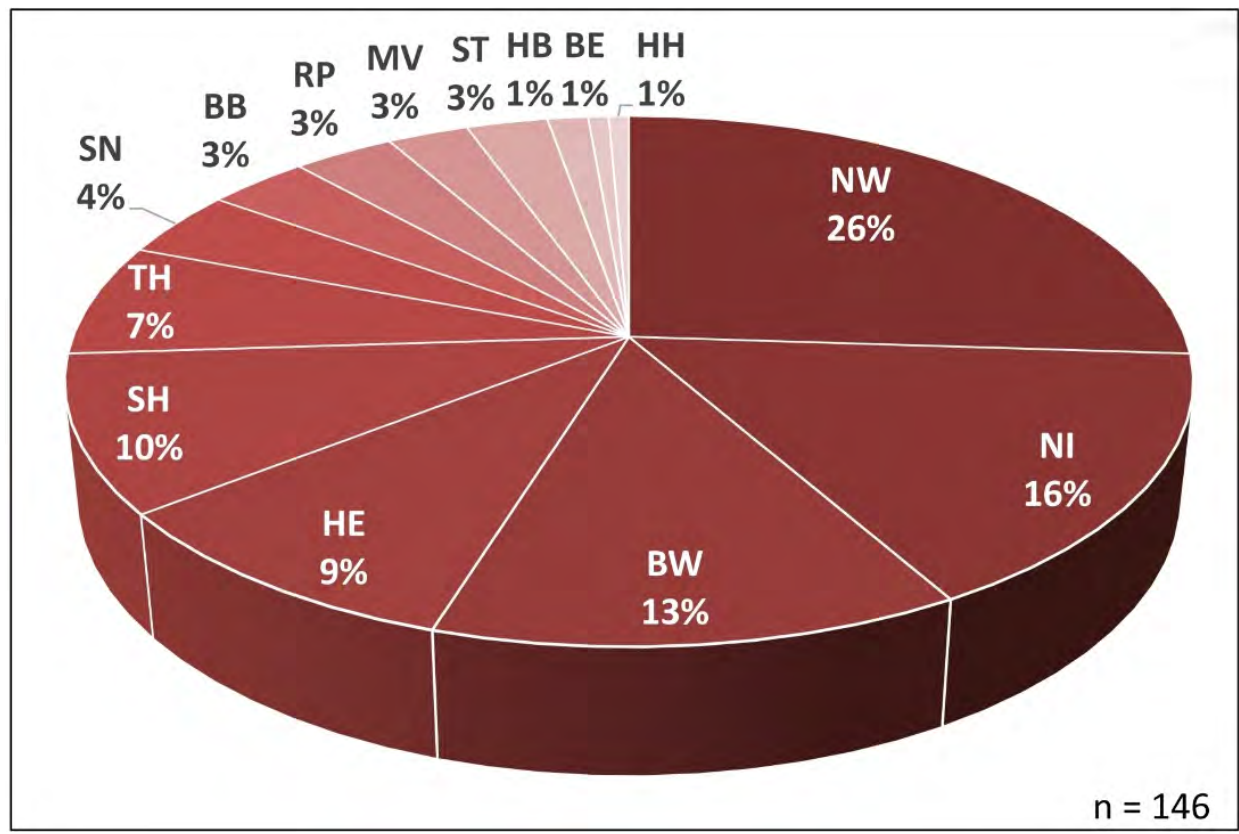

Abbildung 5: Verteilung der zurückgesendeten Fragebögen auf die einzelnen Bundesländer

Der Anteil der Sofortmaßnahmen ${ }^{2}$ an allen Maßnahmen liegt bei $57 \%$, wobei sich die Verteilung auf die Zuständigkeitsbereiche (innerorts, außerorts, BAB) nur unwesentlich ändert (Vergleiche Abbildung 6). Die Anzahl der je UKO umgesetzten Sofortmaßnahmen unterliegt dabei allerdings starken Schwankungen (Vergleiche Abbildung 7). Diese sind im Bereich der städtischen UKO am größten. Der Mittelwert über alle Zuständigkeitsbereiche liegt bei 42 umgesetzten Sofortmaßnahmen in den letzten 10 Jahren, wobei dieser mit durchschnittlich 34 umgesetzten Sofortmaßnahmen in 10 Jahren im Bereich der Bundesautobahnen am geringsten ist.

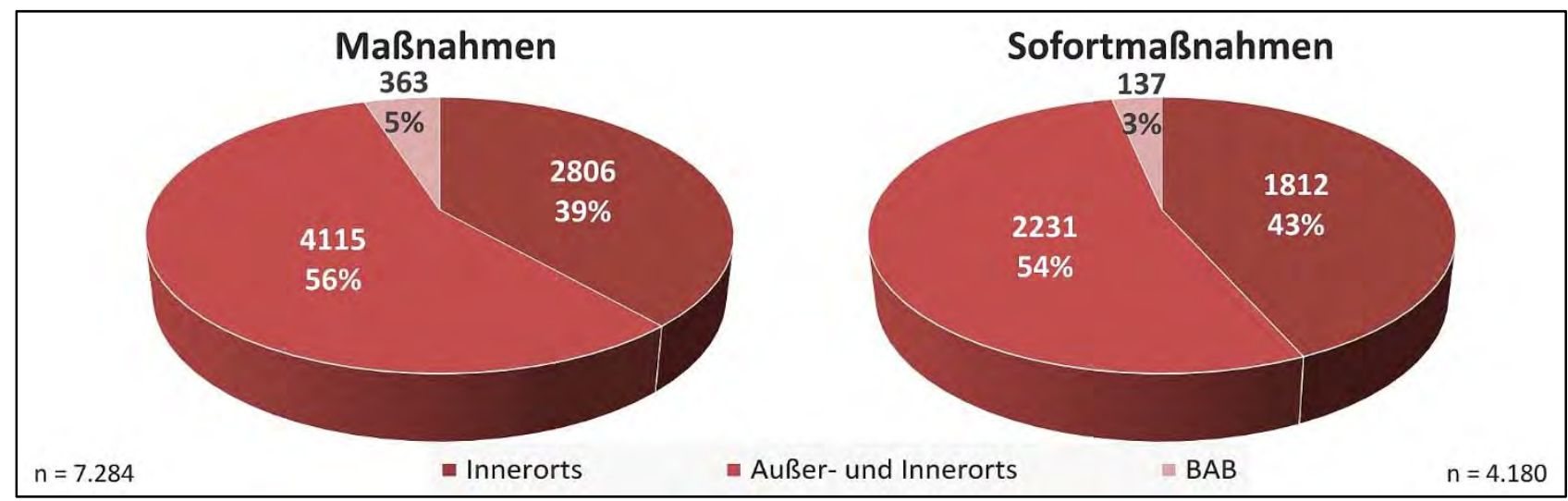

Abbildung 6: Anzahl der Maßnahmen und Sofortmaßnahmen aus den Kurzfragebögen nach Zuständigkeit

2 Sofortmaßnahmen sind Maßnahmen, die kurzfristig und ohne größeren Verwaltungsaufwand umgesetzt werden können. Es wird daher unterstellt, dass es sich bei Sofortmaßnahmen in der Regel um kostengünstige Maßnahmen handelt. 


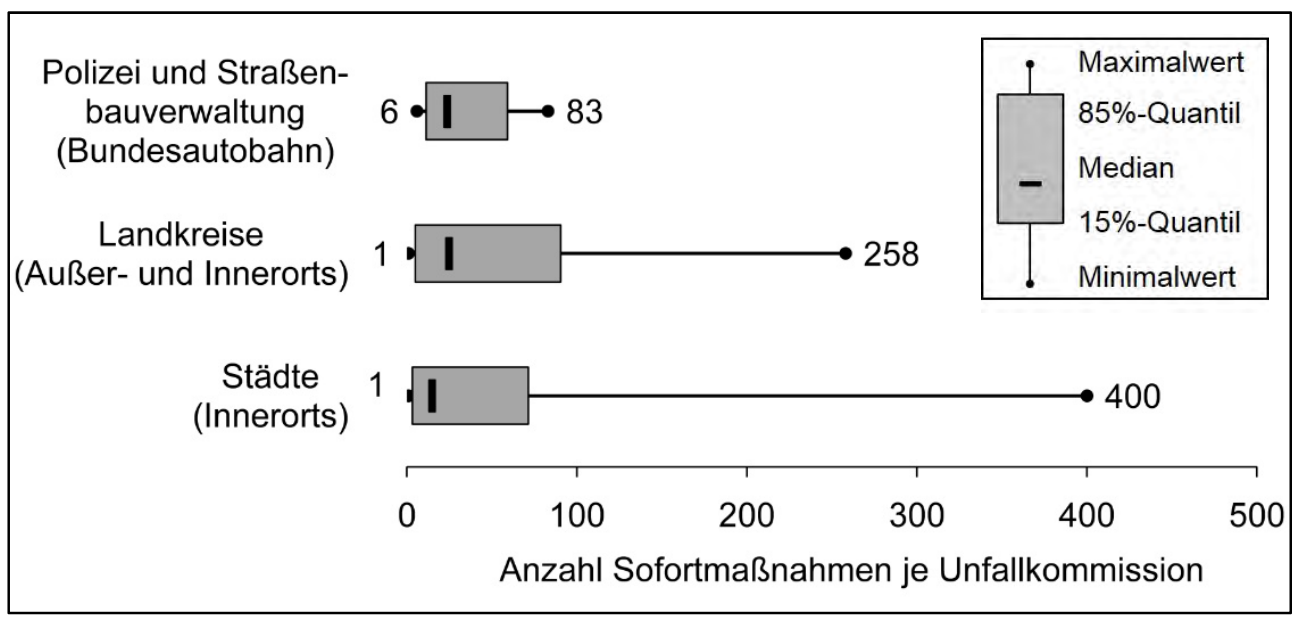

Abbildung 7: Box-Plot der Sofortmaßnahmen je Unfallkommission

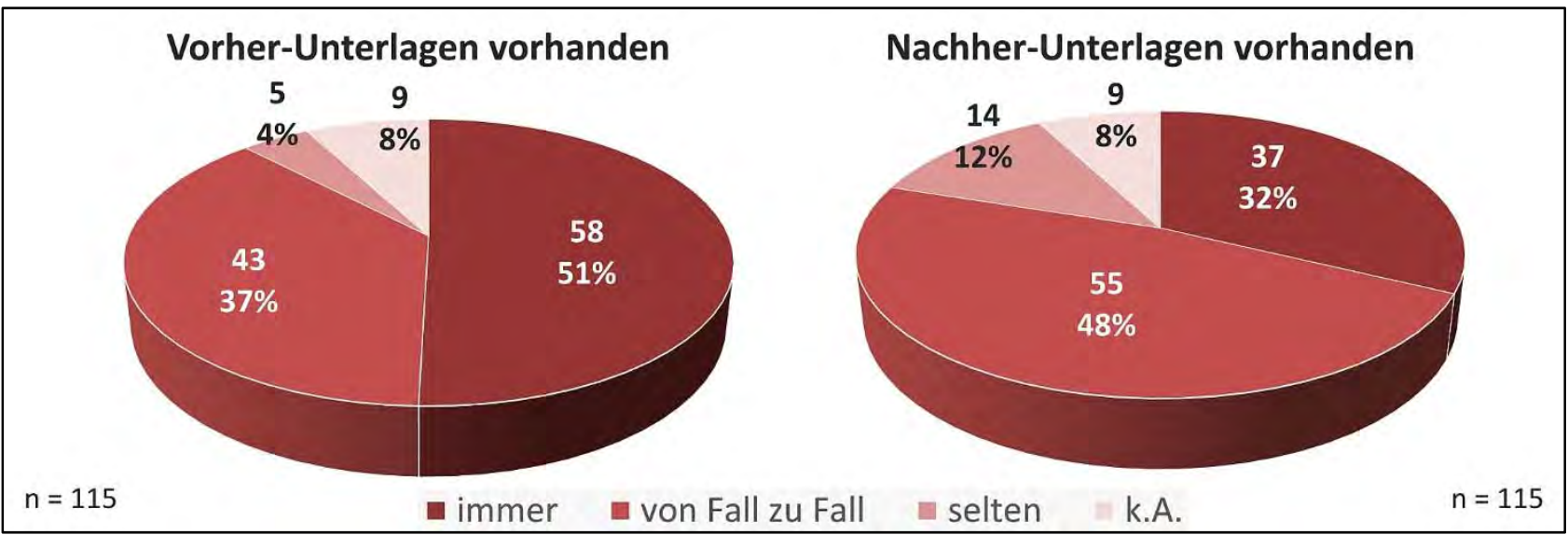

Abbildung 8: In den UKO vorhandene Unterlagen vor und nach Maßnahmenumsetzung

Abbildung 8 zeigt die Verteilung der vorhandenen Unterlagen vor und nach Maßnahmenumsetzung gemäß Aussage der befragten UKO. Vorher-Unterlagen sind Unterlagen, welche zur Maßnahmenfindung beigetragen haben (Analyse). Dies können neben Unfalldiagrammen und Unfalllisten auch Ausschnitte aus den Unfalltypen(steck)karten oder Protokolle der UKO-Sitzungen sowie Fotodokumentationen sein. Nachher-Unterlagen sind Unterlagen, welche nach Umsetzung der Maßnahme gesammelt werden, um die Wirkung der Maßnahme zu überprüfen. Diese beziehen sich in aller Regel ausschließlich auf das Unfallgeschehen nach Maßnahmenumsetzung und sind deswegen meist weniger umfangreich als Vorher-Unterlagen.

Wie Abbildung 8 zeigt, gaben $51 \%$ der UKO an, zu jeder Maßnahme über entsprechende VorherUnterlagen zu verfügen. Bei 43 UKO (37\%) liegen nur noch von Fall zu Fall Vorher-Unterlagen vor. Insgesamt 14 UKO (12\%) haben nur selten Vorher-Unterlagen bzw. haben keine Angaben dazu gemacht. Ein anderes Bild zeigt sich bei der Betrachtung der Nachher-Unterlagen. Hier gaben lediglich $32 \%$ der UKO an, immer über die entsprechenden Analysen zu verfügen. $4 \%$ der UKO führen solche Unterlagen nur von Fall zu Fall, wohingegen $20 \%$ der UKO solche Unterlagen nur selten führen bzw. keine Angaben machten. Dies deutet bereits auf das Problem hin, dass nicht zu jeder Maßnahme auswertbare Nachher-Unterlagen zur Verfügung stehen. In diesen Fällen ist eine Wirkungsanalyse nicht oder in Ausnahmefällen nur mit gezielten Nacherhebungen möglich.

Noch deutlicher stellt sich dieses Problem dar, wenn das Vorliegen von Daten im Vorher- und Nachher-Zeitraum kombiniert wird. Das Ergebnis dieser Kombination ist in Abbildung 9 dargestellt. Daraus lässt sich ablesen, dass nur $50 \%$ der UKO, welche immer Vorher-Daten vorliegen haben, auch in jedem Fall Nachher-Unterlagen zu den Maßnahmen angelegt haben. Dies entspricht einer Anzahl von 29 UKO und somit einem Anteil von lediglich etwa $25 \%$ an den 115 kooperativen UKO. Eine in etwa ebenso große Anzahl von UKO (28) führt sowohl für den Vorher- als auch für den 
Nachher-Zeitraum entsprechende Unterlagen nur von Fall zu Fall. Etwa $44 \%$ der UKO, welche immer über Vorher-Unterlagen verfügen, legen für den Nachher-Zeitraum nur von Fall zu Fall entsprechende Unterlagen an.

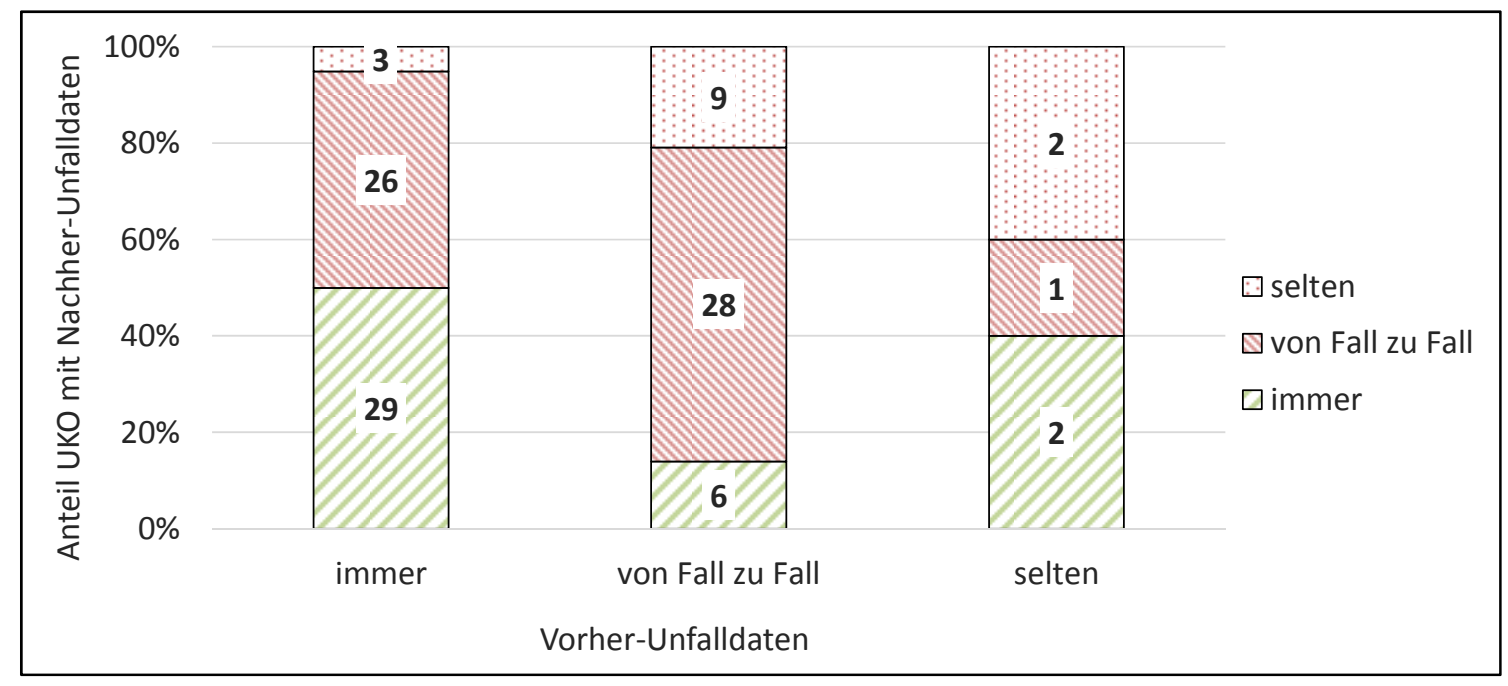

Abbildung 9: Häufigkeit des Vorliegens von Nachher-Unterlagen in Abhängigkeit der Vorher-Unterlagen

\subsection{Zweite Stufe der Kontaktaufnahme}

\subsubsection{Ablauf der Befragung}

In der ersten Befragungsstufe wurden alle UKO in Deutschland angeschrieben und mittels eines Kurzfragebogens um Informationen zu den von innen umgesetzten (Sofort-)Maßnahmen gebeten. Ferner wurde abgefragt, ob die jeweilige UKO für weitere Rückfragen im Projektverlauf zur Verfügung stehen wird. Von den 146 UKO, welche den Fragebogen ausgefüllt zurückgesendet haben, waren 115 bereit, auch im weiteren Fortgang der Untersuchungen für Rückfragen zur Verfügung zu stehen.

Zu diesen 115 Unfallkommissionen wurde fernmündlich Kontakt aufgenommen. Im Rahmen persönlicher Gespräche wurde der in der dritten Stufe vorgesehene Datenaustausch der Unterlagen zu den von den UKO in den Jahren 2004 bis 2012 realisierten Maßnahmen (Unfallgeschehen im Vorherzeitraum in Form der Unfalllisten bzw. -diagramme; Informationen zu Maßnahmen, z. B. aus den Protokollen der UKO-Sitzungen; Unfallgeschehen im Nachherzeitraum) vorbereitet und abgestimmt.

Um einen gleichbleibenden Standard zu gewährleisten, erfolgten alle Telefoninterviews nach einem festgelegten Ablauf. Das Ablaufdiagramm hierzu kann Anhang 3 entnommen werden. Nach einer kurzen Einleitung, in welcher für die bisherige Unterstützung gedankt wurde, wurde gefragt, ob in der UKO eine Liste aller umgesetzten Maßnahmen der letzten zehn Jahre existiert. Bei einer positiven Antwort wurde darum gebeten, diese Liste zu übermitteln. Ziel war es, die für das Forschungsprojekt relevanten Maßnahmen herauszufiltern, um anschließend gezielt Informationen zu diesen Maßnahmen bzw. dem Vorher- und Nachher-Unfallgeschehen zu erfragen. Auf diese Weise wurde der Aufwand für die UKO möglichst gering gehalten, da augenscheinlich kostenintensive Maßnahmen (z. B. Umbau zu Kreisverkehr, errichten einer LSA etc.) von vornherein aus den Untersuchungen ausgeschlossen werden konnten.

In den Fällen, in denen eine solche Liste nicht bei der UKO vorlag, wurde gefragt, ob eine Übermittlung von Daten zu allen Maßnahmen der UKO möglich ist. Bei einer positiven Antwort wurden mehrere Wege zur Datenübermittlung angeboten. Zum einen konnten die Daten postalisch (z.B. auf einer $C D$ oder in Papierform) übermittelt werden. Zum anderen wurde angeboten, die Daten digital entweder per E-Mail oder direkt auf einen gesicherten Server zu übertragen. Wenn eine Datenübermittlung nicht ohne weiteres möglich war, wurde angeboten, die Daten bei einer Vor-Ort- 
Recherche zu erheben. Dies war insbesondere dann der Fall, wenn die UKO in der ersten Befragungsstufe (Kurzfragebogen) angegeben hatte, in den letzten zehn Jahren eine Vielzahl von (Sofort-)Maßnahmen umgesetzt zu haben, für die Übermittlung der Daten bzw. die Recherche relevanter Maßnahmen jedoch nicht über ausreichende zeitliche oder personelle Ressourcen zu verfügen. Wenn einer Vor-Ort-Recherche zugestimmt wurde, wurden im Anschluss weitere organisatorische Fragen geklärt, wie beispielsweise Ort und Zeitpunkt der Datenaufnahme.

Unabhängig von der zuvor im Gespräch getroffenen Vereinbarung (Datenübermittlung, Vor-OrtRecherche, keine Unterstützung) wurden zum Abschluss des Gesprächs die folgenden fünf Fragen gestellt:

1. Welche Sofortmaßnahmen wenden Sie in Ihrer UKO regelmäßig an?

2. Wie definieren Sie in Ihrer UKO kostengünstige Maßnahmen?

3. Welche Erfahrungen haben Sie in Ihrer UKO mit Sofortmaßnahmen gemacht?

4. Welche Hilfsmittel nutzen Sie in Ihrer UKO zur Maßnahmenfindung?

5. Wie führen Sie Wirkungskontrollen durch bzw. wo sind Hemmnisse bei Wirkungskontrollen?

So konnte ein Einblick in die Arbeit der UKO gewonnen und überprüft werden, wie die UKO kostengünstige Maßnahmen definieren (Vergleiche Kapitel 3.3.3).

Im Anschluss an jedes der Telefongespräche wurde dem Ansprechpartner zudem eine E-Mail zugeschickt, in welcher die wichtigsten Inhalte des Telefonats sowie die verschiedenen Möglichkeiten der Datenübermittlung zusammengefasst wurden.

Die Antworten der UKO wurden in einer Datenbank gespeichert und mit weiteren Informationen (z.B. Zeitpunkt der neuerlichen Kontaktaufnahme) hinterlegt. Hatte die betreffende UKO im ersten Telefonat ihre Unterstützung in Form der Datenübermittlung zugesagt, jedoch innerhalb eines Vierteljahres nach dem Telefoninterview noch keine Unterlagen zugesendet, wurde erneut der Kontakt gesucht und ggf. Unterstützung bei der Recherche angeboten. Ähnlich war das Vorgehen bei UKO, bei welchen vor der Datenübermittlung eine interne Abstimmung erfolgen musste, um beispielsweise Belange der Zuständigkeiten oder des Datenschutzes zu erörtern. Auch diese UKO wurden nach Ablauf einer Frist von ca. 3 Monaten erneut kontaktiert.

\subsubsection{Auswertung der Telefoninterviews und der Datenübermittlung}

Es wurde mit den kooperativen 115 UKO (Vergleiche Kapitel 3.2) telefonisch in Kontakt getreten und die Datenübermittlung arrangiert.

Insgesamt konnte zu 110 der 115 UKO telefonisch Kontakt aufgenommen werden. Lediglich bei fünf UKO waren der auf dem Kurzfragebogen angegebene Ansprechpartner oder ein Vertreter nicht zu erreichen. Der Stand der Datenübermittlung zum Stichtag (31.05.2015) ist in Abbildung 10 dargestellt. Demnach haben 64 UKO Daten zu Maßnahmen übermittelt, wovon bei 5 UKO die entsprechenden Daten bei Vor-Ort-Recherchen beschafft wurden.

Die Verteilung der UKO, welche Daten übermittelt haben, auf die einzelnen Bundesländer und Zuständigkeiten zeigt Tabelle 5. Etwas weniger als die Hälfte der unterstützenden UKO (45 \% bzw. 29 UKO) ist in den Ländern Niedersachsen und Nordrhein-Westfalen tätig. Weiterhin zeigt sich, dass $75 \%$ der unterstützenden UKO in den alten Bundesländern angesiedelt sind. Mit $54 \%$ ist der Großteil der UKO den Landkreisen zugeordnet und somit für Innerorts- als auch für Außerortsstraßen zuständig. Der Anteil der für Innerortsstraßen zuständigen UKO liegt bei $41 \%$, während der Anteil der UKO mit BAB-Zuständigkeit bei lediglich $5 \%$ liegt. 


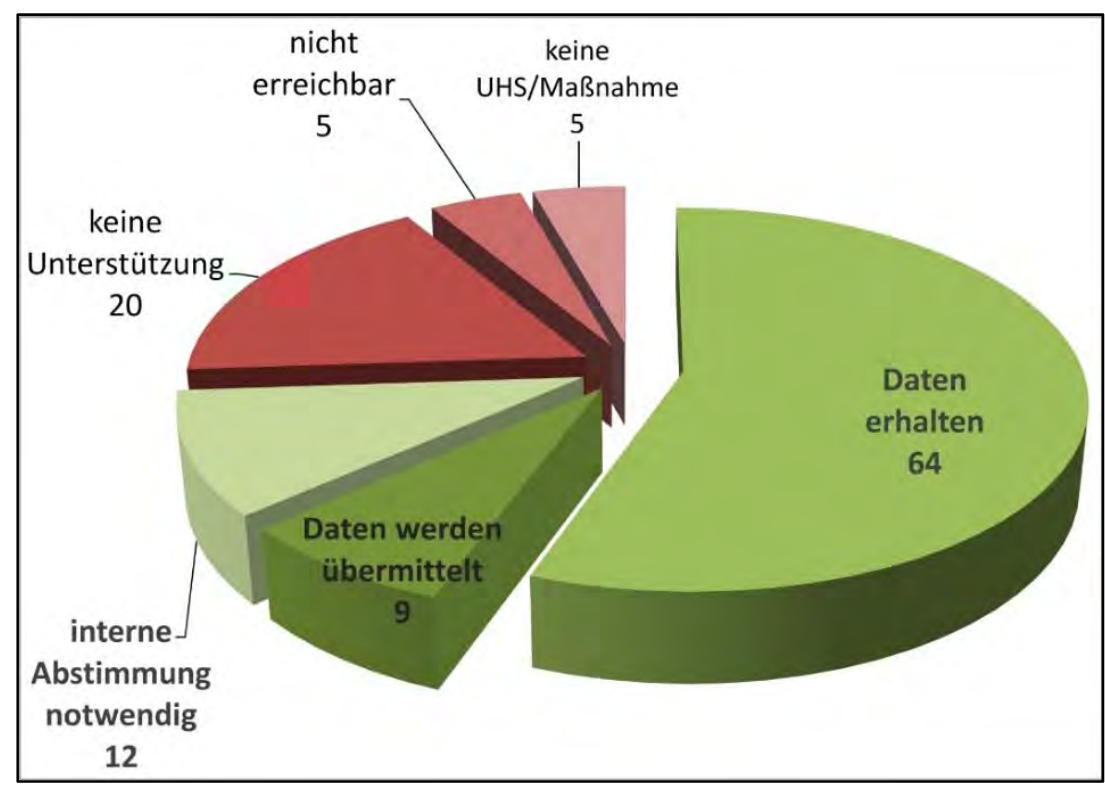

Abbildung 10: Stand der Datenübermittlung nach dem Ablauf der zweiten Stufe der Kontaktaufnahme

Tabelle 5: Verteilung der Zuständigkeiten der UKO mit Datenübermittlung

\begin{tabular}{|c|c|c|c|c|}
\hline \multirow[b]{2}{*}{ Bundesland } & \multicolumn{4}{|c|}{ Zuständigkeit } \\
\hline & Innerorts & $\begin{array}{l}\text { Außer- und } \\
\text { Innerorts }\end{array}$ & BAB & Gesamt \\
\hline Baden-Württemberg & 2 & 3 & - & 5 \\
\hline Berlin & 1 & - & (1) & $1(2)$ \\
\hline Brandenburg & 2 & - & - & 2 \\
\hline Bremen & 1 & - & - & 1 \\
\hline Hamburg & 1 & - & - & 1 \\
\hline Hessen & 1 & 4 & 1 & 6 \\
\hline Mecklenburg-Vorpommern & 1 & - & 1 & 2 \\
\hline Niedersachsen & 1 & 10 & 1 & 12 \\
\hline Nordrhein-Westfalen & 9 & 8 & - & 17 \\
\hline Saarland & 1 & 1 & - & 2 \\
\hline Sachsen & 2 & 1 & - & 3 \\
\hline Sachsen-Anhalt & - & 3 & - & 3 \\
\hline Schleswig-Holstein & 1 & 3 & - & 4 \\
\hline Thüringen & 3 & 2 & - & 5 \\
\hline Gesamt & 26 & 35 & $3(4)$ & 64 \\
\hline
\end{tabular}




\subsubsection{Auswertung der Zusatzfragen aus den Telefoninterviews}

Nach Abklärung der Art und Weise der Datenübermittlung wurden den Gesprächspartnern weitere fünf Fragen bezüglich der UKO-Arbeit gestellt. Diese sind bereits in Kapitel 3.3.1 aufgeführt und werden hier näher erläutert. Allerdings waren (in der Regel aus zeitlichen Gründen) nicht alle UKO bereit, diese Fragen zu beantworten. Die Ergebnisse dieser Befragung werden im Folgenden kurz dargestellt. Die detaillierte Auswertung ist in Anhang 4 zu finden.

\section{Regelmäßig angewendete Sofortmaßnahmen}

Die erste Frage bezog sich auf die von der UKO regelmäßig angewendeten Sofortmaßnahmen. Hier gaben 21 UKO (75\%) an, hauptsächlich Markierungsarbeiten zu empfehlen. Dies beinhaltet sowohl das Erneuern ausgeblichener oder abgenutzter Markierungen als auch das komplette Neuoder Um-Markieren von Knotenpunkten oder einzelner Fahrstreifen. 16 UKO (57\%) gaben an, häufig mit Beschilderungsmaßnahmen zu arbeiten. Dabei handelt es sich in der Regel um das Aufstellen einfacher Verkehrszeichen wie "Gefahrenstelle“ (Z 101 StVO), „Kurve“ (Z 103 StVO) oder „Schleudergefahr bei Nässe oder Schmutz" (Z 114 StVO). Häufig wurden auch Zusatzzeichen ergänzt, wie beispielsweise „Radfahrer kreuzen von rechts und links“ (Z 1000-32 StVO). Auch das Austauschen ausgeblichener Verkehrszeichen fällt in diese Kategorie. Lediglich sechs der befragen UKO (21\%) gaben an, an Unfallhäufungen oft auf die Beschränkung der zulässigen Höchstgeschwindigkeit zurückzugreifen. Diese Maßnahme wurde ausschließlich von UKO genannt, welche in den Landkreisen angesiedelt und somit auch für Außerortsstraßen zuständig sind. Das lässt darauf schließen, dass diese Maßnahme - ähnlich wie auch die Maßnahme „Kurvenverlauf verdeutlichen (Markierung oder Z 625 StVO)“ mit 4 Nennungen - häufiger im Bereich außerhalb geschlossener Ortschaften eingesetzt wird, als innerorts. Von sechs UKO wurde angegeben, häufig sichtverbessernde Maßnahmen anzuwenden. Davon wurde zweimal angegeben, Sichthindernisse im Allgemeinen zu entfernen (z.B. Werbetafeln, Maste etc.). Weitere dreimal wurde explizit der Grünschnitt als sichtverbessernde Maßnahme angegeben. Eine UKO gab an, häufig mit Pollern oder anderen „harten Maßnahmen“ zu arbeiten, um das Parken bzw. Halten im Kreuzungsbereich zu unterbinden und so die Sichtverhältnisse zu verbessern. Auch das Ausrichten von Verkehrszeichen oder Signalgebern (je zwei Nennungen) wird von den UKO als Maßnahme umgesetzt. Zwei UKO gaben an, auch gezielt Maßnahmen wieder zurückzunehmen, wenn sich diese keine Verbesserung oder sogar eine Verschlechterung im Unfallgeschehen bewirkt hat. Weitere von den UKO genannte Sofortmaßnahmen können der Tabelle in Anhang 4 entnommen werden.

\section{Definition kostengünstiger Maßnahmen}

Die zweite Frage bezog sich auf die Definition kostengünstiger Maßnahmen. Dabei sollten die UKO weniger einen konkreten finanziellen Betrag als vielmehr eine einfache Beschreibung geben, was für sie eine kostengünstige Maßnahme ausmacht. Diese Frage beantworteten elf UKO, wovon fünf den Umsetzungszeitraum einer Maßnahme als entscheidendes Kriterium angaben. Kurzfristig umsetzbare Maßnahmen werden demnach oft auch als kostengünstig empfunden. Dies zeigt, dass die weiter oben getroffene Assoziation, Sofortmaßnahmen seien in der Regel kostengünstige Maßnahmen, berechtigt ist. Auch der Vergleich mit den oft durch die UKO umgesetzten Sofortmaßnahmen (Frage 1) zeigt, dass am häufigsten Maßnahmen gewählt werden, welche sowohl finanziell kostengünstig als auch schnell umsetzbar sind. Bei der Beurteilung, ob eine Maßnahme kostengünstig ist, stellt der rein finanzielle Aufwand lediglich bei zwei UKO das entscheidende Kriterium dar. Eine ähnliche Definition treffen drei weitere UKO, bei denen ausschlaggebend ist, ob eine Maßnahme ohne Eingriffe in den Jahreshaushalt (der Gemeinde oder des Landkreises) umgesetzt werden kann. Eine UKO gab an, dass der verwaltungstechnische Aufwand, welcher für die Umsetzung einer Maßnahme notwendig ist, eine kostengünstige Maßnahme definiert. Allerdings kann davon ausgegangen werden, dass Maßnahmen mit einem geringen verwaltungstechnischen Aufwand auch vergleichsweise zeitnah umgesetzt werden können, weswegen unterstellt werden kann, dass auch hier die Umsetzungsdauern ein entscheidendes Kriterium darstellt. 


\section{Erfahrungen mit Sofortmaßnahmen}

Die dritte Frage zielte auf die Erfahrungen, welche die UKO mit Sofortmaßnahmen gemacht haben. $44 \%$ der 18 antwortenden UKO gaben an, mit Sofortmaßnahmen überwiegend positive Erfahrungen gemacht zu haben. Vier UKO berichten von ausschließlich positiven Erfahrungen, während weitere drei UKO gemischte Erfahrungen gemacht haben. Kostengünstige bzw. Sofortmaßnahmen werden jedoch häufig nur als Übergangslösung eingesetzt. Dies ist insbesondere dort der Fall, wo auf absehbare Zeit „größere Maßnahmen“ (z.B. Um- oder Ausbau Knotenpunkt) geplant sind, das Unfallgeschehen aber ein sofortiges Eingreifen erforderlich macht. Zwei UKO haben die Erfahrung gemacht, dass Sofortmaßnahmen häufig nicht oder nur kurzzeitig akzeptiert werden. Das liegt zu einem Großteil daran, dass - wie die Auswertung von Frage 1 gezeigt hat überwiegend „einfache, weiche Maßnahmen“ (Beschilderung, Markierung etc.) als Sofortmaßnahmen eingesetzt werden. So berichteten einige UKO, dass beispielsweise bei Beschilderungsmaßnahmen die Verkehrsteilnehmer nach einer kurzen Eingewöhnungsphase wieder in ihre alten Verhaltensmuster zurückfallen. Wenn ein solches Verhalten beobachtet wurde, haben die UKO weitergehende Maßnahmen angewendet. Diese waren aber in der Regel aufwändiger und wurden von den UKO nicht mehr als kostengünstig bezeichnet. Aus den Gesprächen ging hervor, dass viele UKO versuchen, durch ein solches „bottom-up-Prinzip“ zunächst einfache, kostengünstige Maßnahmen zu ergreifen. Erst wenn eine solche Maßnahme keine oder nicht die erwünschte Wirkung zeigt, werden "größere“ Maßnahmen getroffen, um das Unfallgeschehen positiv zu beeinflussen.

\section{Hilfsmittel zur Maßnahmenfindung}

Im Zuge der Telefoninterviews und der persönlichen Vor-Ort-Gespräche wurden die UKO auch gebeten, Angaben über die von ihnen zur Maßnahmenfindung verwendeten Hilfsmittel zu machen. Acht der zehn UKO gaben an, bei der Maßnahmenfindung auf ihren eigenen Erfahrungsschatz zurückzugreifen, wobei drei dieser UKO diesen als einzige Quelle nutzen. Das Merkblatt zur „Auswertung von Straßenverkehrsunfällen Teil 2 - Maßnahmen gegen Unfallhäufungen“ [FGSV 2002] nutzen lediglich vier Unfallkommissionen. Ebenso viele greifen bei der Maßnahmenfindung auch häufiger auf Expertenmeinungen zurück. Je nach UKO, Zuständigkeit und Charakteristik der Unfallhäufung können das Mitarbeiter der Straßenmeistereien, der Straßen- und Tiefbauämter oder anderer Behörden sowie von Universitäten sein. Diese werden in der Regel zu den jeweiligen Sitzungen der UKO eingeladen. Weitere Hilfsmittel wie Verkehrsschauen oder andere Merkblätter nutzen nur wenige UKO.

\section{Wirkungskontrolle}

Die abschließende Frage bezog sich auf die Durchführung von Wirkungskontrollen. Dabei sollten die UKO angeben, wie und mit welcher Häufigkeit sie Wirkungskontrollen durchführen. Es stellte sich heraus, dass der weitaus größte Teil keine Wirkungskontrolle im engeren Sinne durchführt. In den meisten Fällen (50\%) wird lediglich überprüft, ob die Unfallhäufung weiterhin existiert. In der Regel wird diese Kontrolle nach einem Jahr durchgeführt. Ist das Unfallgeschehen an der Stelle unauffällig, wird die Maßnahme als wirksam angesehen. Dies ist auch der Grund dafür, dass (wie in Abbildung 8 gezeigt) nur in wenigen Fällen zu einzelnen Maßnahmen Nachher-Daten vorliegen. Nur drei UKO gaben an, regelmäßig Wirkungskontrollen durchzuführen. Zudem gaben einige UKO an, Sofortmaßnahmen in der Regel ohne UKO-Sitzung und förmlichen Beschluss (,auf Wunsch“) umzusetzen, teilweise auch bereits bevor die Grenzwerte für Unfallhäufungen überschritten wurden. Somit werden diese Stellen nicht als Unfallhäufung geführt und es liegen kaum Unfalldaten für den Vorher-Zeitraum und noch seltener für den Nachher-Zeitraum vor. 


\subsubsection{Zusammenfassung}

Insgesamt wurden mit 110 UKO Telefoninterviews durchgeführt. Dabei wurde hauptsächlich der Ablauf der Datenübermittlung durch die UKO abgeklärt. Insgesamt haben 64 UKO Daten zu kostengünstigen Maßnahmen in ihrem Zuständigkeitsbereich zur Verfügung gestellt, was einem Rücklauf von $58 \%$ in der zweiten Befragungsstufe entspricht. Davon haben 59 UKO die Daten digital oder postalisch übermittelt. Bei fünf UKO wurden die benötigten Daten Vor-Ort recherchiert. Die Verteilung der Zuständigkeit der UKO mit Datenübermittlung ist mit $40 \%$ (innerorts) und $54 \%$ (inner- und außerorts) ausgeglichen.

Im Anschluss an die Abklärung der Datenübermittlung wurden den jeweiligen Ansprechpartnern fünf weitere Fragen zu Arbeit der UKO und zu Erfahrungen mit Sofortmaßnahmen gestellt. Diese haben allerdings lediglich 28 UKO vollständig oder teilweise beantwortet. Dabei stellte sich heraus, dass es sich bei den am häufigsten angewendeten Sofortmaßnahmen um Markierungs- und Beschilderungsarbeiten handelt. Auch die Beschränkung der zulässigen Höchstgeschwindigkeit, das Verdeutlichen des Kurvenverlaufs mittels Markierung oder Richtungstafel in Kurven (Z 625 StVO) sowie das Beseitigen von Sichthindernissen werden von den UKO an Unfallhäufungen eingesetzt.

Dabei hat sich neben vielen positiven Erfahrungen aber auch gezeigt, dass die Wirkung von Sofortmaßnahmen oft nur von kurzer Dauer ist. Dies ist auch ein Grund dafür, dass Sofortmaßnahmen häufig nur als Übergangslösungen eingesetzt werden, um das Unfallgeschehen kurzfristig positiv zu beeinflussen. Insbesondere ist das dann der Fall, wenn bereits "größere Maßnahmen“ geplant sind, das Unfallgeschehen aber ein sofortiges Eingreifen erfordern. Ein solches „bottom-up Prinzip“ („von der kleinen zur großen“ Maßnahme) wird von vielen UKO verfolgt.

Bei der Definition von kostengünstigen Maßnahmen gibt der überwiegende Teil der UKO die Umsetzungsdauer als maßgebendes Kriterium an. Kann eine Maßnahme schnell umgesetzt werden, wird diese in der Regel auch als kostengünstig angesehen. Rein finanzielle Aspekte werden nur von wenigen UKO genannt.

Auf die Frage, welche Hilfsmittel in der UKO bei der Maßnahmenfindung verwendet werden, gaben viele UKO an, sich nahezu ausschließlich auf ihre Erfahrung zu verlassen. Einschlägige Regelwerke werden indes seltener verwendet. Manche UKO greifen bei der Maßnahmenfindung auch auf Experten aus anderen Behörden, Ämtern oder universitären Einrichtungen zurück. Ein Grund hierfür ist unter anderem, dass viele UKO kleinere Maßnahmen häufig „auf Wunsch“ umsetzen. So wird beispielsweise aus den Straßenmeistereien eine Auffälligkeit im Unfallgeschehen gemeldet (beispielsweise, weil an bestimmten Stellen häufig Reparaturen durchgeführt werden müssen). Die zuständige Behörde veranlasst anschließend (ohne UKO-Beschluss) eine entsprechende Gegenmaßnahme. Das geschieht häufig auch bereits bevor die Grenzwerte für Unfallhäufungen überschritten wurden. Viele kleinere Maßnahmen werden so gar nicht in der UKO besprochen und auch kaum mit den entsprechenden Unterlagen (Unfalllisten etc.) dokumentiert.

Dies wiederum ist auch ein Grund dafür, weshalb viele UKO keine Wirkungskontrollen im eigentlichen Sinne durchführen. In aller Regel beschränkt sich die Wirkungskontrolle auf die Überprüfung, ob die Unfallhäufung auch im Folgejahr Auffälligkeiten im Unfallgeschehen zeigt. Ist dies der Fall, werden weiterführende Maßnahmen beschlossen. Andernfalls wird der Vorgang als abgeschlossen betrachtet. Kosten-Nutzen-Analysen wurden hingegen nur in seltenen Fällen angestellt.

Neben der Abklärung der Datenübermittlung bilden die Informationen zu den eingesetzten Sofortmaßnahmen und den damit gemachten Erfahrungen eine wichtige Grundlage im weiteren Verlauf des Forschungsprojektes. 
Parallel zu den Telefoninterviews wurden die Unterlagen gesichtet und in einem Datenbankformat aufbereitet. Grundlage hierfür waren zunächst die Daten, die in den Jahren 2003 bis 2014 von den UKO für den „Sicherheitspreis - Die Unfallkommission“ bei der UDV eingereicht und im Rahmen des Projektes von dieser zur Verfügung gestellt wurden. Aus diesen Daten konnten 155 Maßnahmen bzw. Maßnahmenpakete herausgefiltert werden, die zur Ergänzung der Daten, welche die UKO direkt übermittelten, herangezogen wurden. So entstand insgesamt eine Datenbank mit 1.899 Einträgen, wobei jeder Eintrag für eine Maßnahmen bzw. ein Maßnahmenpaketen steht.

\subsection{Vorgehen}

Die von den UKO und der UDV zur Verfügung gestellten Daten wurden gesichtet und in übersichtlicher Form zur weiteren Auswertung vorbereitet. Die Datenaufbereitung folgte einem dreistufigen Ablauf, welcher sich in die Arbeitsschritte

- Aufnahme,

- Kategorisierung und

- Plausibilisierung

gliedert. Alle drei Arbeitsschritte werden im Folgenden kurz erläutert.

Bei der Aufnahme der Daten war ein einheitliches und sorgfältiges Vorgehen äußerst wichtig, da die Daten in unterschiedlichster Form vorlagen. So bestanden die Unterlagen zum Teil aus Protokollen der UKO-Sitzungen, welche gelegentlich auch mit detaillierteren Angaben zum Unfallgeschehen (z. B. Unfallisten oder -diagrammen) versehen waren. In anderen Fällen wurden UKOeigene Tabellen- oder Unterlagenformate verwendet, sodass sich die Unterlagen zweier UKO kaum glichen. Angesichts der Datenmenge wurde beschlossen, zunächst auf eine detaillierte Auswertung jeder einzelnen Maßnahme bzw. jedes einzelnen Maßnahmenpaketes zu verzichten. Vielmehr sollten die vorliegenden Maßnahmen nur so genau wie nötig aber dennoch so gut wie möglich beschrieben werden. Dies geschah auch vor dem Hintergrund, dass im weiteren Verlauf der Auswertungen nur ein vergleichsweise geringer Teil der zur Verfügung gestellten Maßnahmen bzw. Maßnahmenpakete für eine Detailanalyse herangezogen wird. Die Auswahl dieser Maßnahmen erfolgt auf Basis der angelegten Datenbank. Im Folgenden wird das Vorgehen bei der Datenbankerstellung kurz beschrieben.

Zunächst wurde jeder UKO, welche Daten übermittelte, eine nummerische Identifikationsnummer (ID) zugewiesen, sodass die Maßnahme in der weiteren Auswertung der entsprechenden UKO zugeordnet werden kann. Weiterhin wurde jede aufgenommene Maßnahme ebenfalls mit einer ID versehen. Dabei konnte zum Teil auf die IDs, welche die UKO bereits selbst festgelegt hatten, zurückgegriffen werden. In anderen Fällen wurden aber auch eigene ID vergeben. Somit kann jeder Vorgang in der Datenbank genau einer UKO und genau einer bestimmten Maßnahme zugeordnet werden, was für die spätere Detailauswertung zwingend erforderlich ist.

Im Anschluss wurde die Örtlichkeit der Unfallhäufung bzw. der Maßnahme genauer beschrieben. Dabei wurden die Ortslage (innerorts, außerorts, BAB) und die Charakteristik der Örtlichkeit erhoben. Es wurde zwischen den folgenden Charakteristika unterschieden:

- Knotenpunkt mit Lichtsignalanlage (KP mit LSA)

- Knotenpunkt ohne Lichtsignalanlage (KP ohne LSA)

- Freie Strecke

- Motorradstrecke

- Unfallhäufungslinie (UHL) 
Sofern die Daten es zuließen, wurde für jede Unfallhäufung bzw. jede Maßnahme oder jedes Maßnahmenpaket der dreistellige Hauptunfalltyp bestimmt (vgl. FGSV [2003]). In manchen Fällen konnte dieser vergleichsweise einfach aus den Unfalldiagrammen oder Unfalllisten abgeleitet werden. In anderen Fällen wiederum konnte auf die Beschreibung des Unfallgeschehens in den Protokollen der UKO-Sitzungen zurückgegriffen werden. In wieder anderen Fällen konnte im Rahmen der Datenaufbereitung dazu keine Aussage getroffen werden, da die benötigten Daten nicht oder nur unzureichend vorlagen. Insbesondere an größeren Knotenpunkten mit LSA im Innerortsbereich kam es vor, dass in mehreren Knotenarmen Unfallhäufungen mit unterschiedlichen Hauptunfalltypen zu erkennen waren. In diesen Fällen wurde die Unfallhäufung „geteilt“ und im Folgenden als zwei unabhängige Unfallhäufungen behandelt. Weiterhin konnten in einer Kommentarspalte etwaige Besonderheiten der Örtlichkeit notiert werden, wie beispielsweise, dass sich das Unfallgeschehen hauptsächlich auf einen freien Rechtsabbieger (Knotenpunkt mit Dreiecksinsel) konzentriert.

In je drei weiteren Spalten wurde vermerkt, wie detailliert das Unfallgeschehen der 12 bzw. 36 Monaten vor der Maßnahmenumsetzung dokumentiert ist. Dabei wurde unterschieden, ob das Unfallgeschehen

- getrennt nach Unfalltyp und Unfallkategorie,

- getrennt nur nach Unfalltyp,

- getrennt nur nach Unfallkategorie oder

- gar nicht

vorliegt. Im Idealfall lag das Unfallgeschehen in Form von Unfalldiagrammen oder Unfallisten und somit sowohl nach Unfalltyp als auch nach Unfallkategorie vor. In vielen Fällen ist allerdings lediglich eine absolute Anzahl von Unfällen angegeben, welche sich weder sauber nach Unfalltyp noch nach Unfallkategorie trennen lässt. Auch hier wurden - wenn gegeben - in einer Kommentarspalte Auffälligkeiten wie beispielsweise eine hohe Beteiligungsrate von Fahrradfahrern am Unfallgeschehen notiert. Auf die gleiche Art und Weise wurde auch das Vorliegen von Unfalldaten in den 12 bzw. 36 Monaten nach Umsetzung der Maßnahme dokumentiert, sodass für jede Maßnahme bzw. jedes Maßnahmenpaket Informationen über den Detaillierungsgrad der vorhandenen Unterlagen für den Vorher- und Nachher-Zeitraum vorlagen.

Anschließend wurden die Informationen zu den getroffenen Maßnahmen selbst aufgenommen. Hierbei wurde zunächst notiert, ob es sich bei der Maßnahme um

- eine Einzelmaßnahme,

- ein synchron umgesetztes,

- ein sukzessiv umgesetztes Maßnahmenpaket oder

- ein Mischpaket

handelt. Maßnahmen, die augenscheinlich in direktem Zusammenhang stehen, wie beispielsweise das Markieren eines Radfahrstreifens mit der entsprechenden Beschilderung, wurden als Einzelmaßnahme aufgenommen. Maßnahmen, die nicht in einem solchen direkten Zusammenhang stehen aber gleichzeitig (oder mit nur wenigen Wochen Versatz) umgesetzt worden sind, wurden als synchrone Maßnahmenpakete aufgenommen. Ausschlaggebend für die Unterscheidung zwischen Einzelmaßnahmen und synchronen Maßnahmenpaketen war, ob die jeweiligen Maßnahmen sinnvoll auch als alleinige Maßnahme eingesetzt werden können. Ein Beispiel hierfür ist die Erneuerung der Markierung im Knotenpunkt mit einer gleichzeitig erfolgenden Installation von Schwellen zur Verdeutlichung von Abbiegeverboten. Beide Maßnahmen können auch als Einzelmaßnahme eingesetzt werden, weswegen die Gesamtmaßnahme den synchronen Maßnahmenpaketen zugeordnet wurde. Bei sukzessiven Maßnahmenpaketen verhält es sich ähnlich, nur das zwischen der Umsetzung der Maßnahmen ein größerer Zeitraum lag. Dieser betrug in der Regel mindestens 2-3 Monate, konnte sich aber auch über mehrere Jahre erstrecken. Für jede Stelle bzw. Unfallhäufung wurden ausschließlich die drei zuletzt umgesetzten Maßnahmen aufgenommen. Maßnahmenpakete, welche aus drei Maßnahmen bestanden, von denen zwei synchron und eine weitere in einem 
ausreichendem zeitlichen Abstand (min. 2-3 Monate) davor oder danach umgesetzt wurden, handelte es sich um ein Mischpaket. Zu jeder Maßnahme wurde zudem generell der Monat und das Jahr der Umsetzung notiert, sowie die Kosten der Gesamtmaßnahme, sofern diese angegeben waren. In Abbildung 11 ist der Aufbau der Datenbank noch einmal in Tabellenform dargestellt.

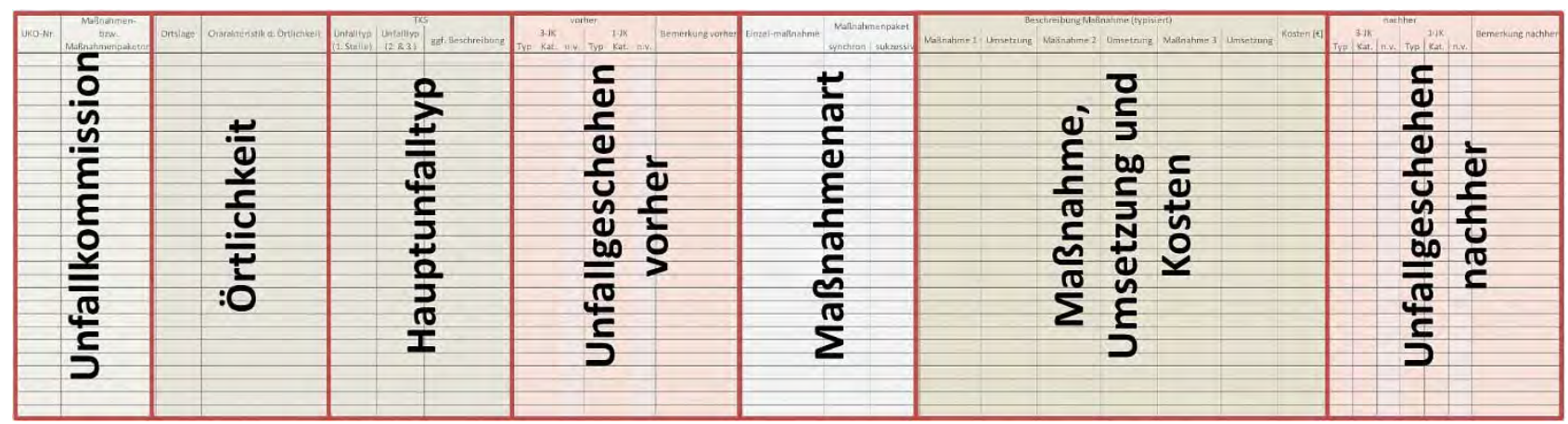

Abbildung 11: Tabellarischer Aufbau der Datenbank zur Erfassung von Maßnahmen

Eine Herausforderung bestand in der Bezeichnung der Maßnahmen selbst, da diese für die weitere Auswertung einheitlich gestaltet werden musste. Hierfür wurden zunächst Maßnahmengruppen definiert, welche sich an dem web-basierten Maßnahmenkatalog (vgl. MAIER et al. [2015]) orientieren. Allerdings wurden sowohl die Maßnahmengruppen selbst als auch die Zuordnung einzelner Maßnahmen zu diesen Gruppen auf die projektspezifischen Belange angepasst. Daraus entstanden ist ein Katalog mit 189 Maßnahmen, welche einer der 10 Maßnahmengruppen

- Markierung,

- Querungsanlagen,

- LSA-Steuerung,

- Verkehrszeichen,

- Geschwindigkeit/Überwachung,

- Fahrbahn,

- Erkennbarkeit/Sicht,

- Umbau/Anpassung,

- Radverkehrsanlagen und

- Passive Schutzeinrichtungen (PSE)

zugeordnet wurden.

Tabelle 6: Beispielhafte Zuordnung von Maßnahmen zu drei Maßnahmengruppen

\begin{tabular}{|c|c|c|}
\hline Querungsanlagen & Geschwindigkeit/Überwachung & $\begin{array}{c}\text { Passive Schutzeinrichtung } \\
\text { (PSE) }\end{array}$ \\
\hline Fußgänger-LSA einrichten & Beschränkung $\bigvee_{\text {zul }}$ & PSE aufstellen \\
\hline Mittelinsel einrichten & Tempo 30 Zone anlegen & PSE verlängern \\
\hline Fußgängerkap einrichten & Temporäre Beschränkung $V_{z u l}$ & PSE entfernen \\
\hline $\begin{array}{l}\text { Fußgängerüberweg (FGÜ) an- } \\
\text { legen }\end{array}$ & $\begin{array}{l}\text { Ortsfeste Geschwindigkeitsüber- } \\
\text { wachung (OGÜ) einrichten }\end{array}$ & PSE instand setzen \\
\hline FGÜ entfernen & Linienhafte OGÜ einrichten & $\begin{array}{l}\text { PSE mit Unterfahrschutz } \\
\text { aufstellen }\end{array}$ \\
\hline FGÜ verlegen & $\begin{array}{l}\text { Temporäre Geschwindigkeits- } \\
\text { überwachung }\end{array}$ & Unterfahrschutz ergänzen \\
\hline Umlaufgitter & Rotlichtüberwachung & $\begin{array}{l}\text { Hindernisse im Seitenraum } \\
\text { entfernen }\end{array}$ \\
\hline Fußgängerschutzgitter & $\begin{array}{l}\text { Sonstige Überwachungs- } \\
\text { maßnahmen }\end{array}$ & Errichten Schrankenanlage \\
\hline
\end{tabular}


Tabelle 6 zeigt die Zuordnung einzelner Maßnahmen am Beispiel von drei Maßnahmengruppen. Die Maßnahmengruppen dienen dabei der Systematisierung der 189 Maßnahmen und vereinfachen das Auffinden einzelner Maßnahmen innerhalb des Maßnahmenkataloges.

Unter Verwendung der Systematisierung und der weiter oben beschriebenen Merkmale entstand eine Datenbank mit 1.812 Zeilen, wobei jede Zeile eine Maßnahme bzw. ein Maßnahmenpaket repräsentiert.

Auf die Systematisierung der Maßnahmen, welche der einheitlichen Gestaltung der Datenbank diente, folgte die Kategorisierung der Maßnahmen. Dieser Schritt war notwendig, da unter den 189 Maßnahmen in den Maßnahmengruppen auch Maßnahmen zu finden waren, die namentlich unterschiedlich, inhaltlich jedoch identisch sind. Auch sollen im weiteren Projektverlauf gezielt Wirkungsanalysen (z. B. in Form von Nutzen-Kosten-Analysen) für ausgewählte, kostengünstige Maßnahmen durchgeführt werden. Aus Gründen der statistischen Sicherheit sollen dabei allerdings mehrere Umsetzungen ein und derselben Maßnahme betrachtet werden. Durch die Einteilung der Maßnahmen in (inhaltlich homogene) Maßnahmenkategorien, erhöht sich die Anzahl der Umsetzung einzelner Maßnahmen. Im Zuge dieser Kategorisierung konnten auch Maßnahmen aus der weiteren Untersuchung ausgeschlossen werden, bei denen es sich augenscheinlich nicht um kostengünstige bzw. Sofortmaßnahmen handelt. Dies waren in der Regel bauliche Maßnahmen wie der mehrstreifige Ausbau eines Streckenabschnittes oder der Bau eines Kreisverkehrs.

Die Kategorisierung erfolgte in zwei Stufen. Zunächst wurden die Maßnahmen zusammengefasst, welche oft oder ausschließlich gemeinsam eingesetzt werden. Ein Beispiel hierfür ist die Maßnahmenkategorie „HALT“, welche die Maßnahmen „Z 206 StVO aufstellen“ und „Markierung Haltlinie(n)" beinhaltet. Somit kann diese Stufe auch als "horizontale Zusammenfassung" verstanden werden, da sie hauptsächlich bei Maßnahmen Anwendung fand, welche in einem synchronen Maßnahmenpaket umgesetzt wurden. Dadurch konnte auch die Anzahl der Einzelmaßnahmen in der Datenbank erhöht werden. Die zweite Stufe der Kategorisierung betraf Maßnahmen, welche sich unter einem Oberbegriff zusammenfassen lassen. Ein Beispiel hierfür ist die Kategorie „Gefahrenzeichen Kurve“, welche das Aufstellen der StVO-Verkehrszeichen Z 103-10, Z 103-20, Z 105-10 und Z 105-20 beinhaltet. Diese sind im Maßnahmenkatalog noch als unterschiedliche Maßnahmen aufgelistet. Für die geplanten Auswertungen ist es jedoch unerheblich, ob es sich bei der Gefahrenstelle um eine Links-, eine Rechts- oder eine Doppelkurve handelt, da allein die Wirkung der Ankündigung einer gefährlichen Kurve oder Kurvenkombination untersucht werden soll.

Eine Besonderheit stellte dabei die Maßnahme „Beschränkung $V_{z u l}$ ", also die Beschränkung der zulässigen Höchstgeschwindigkeit $\left(V_{z u l}\right)$, dar. Diese wurde in die fünf Kategorien

- auf Strecken außerorts,

- auf Strecken innerorts,

- an Knotenpunkten innerorts,

- an Knotenpunkten mit LSA außerorts und

- an Knotenpunkten ohne LSA außerorts

unterteilt. Dies hat den Hintergrund, dass durch die unterschiedlichen Charakteristika der Netzelemente Knoten und Strecke sowie durch die Ortslage und den daraus resultierenden unterschiedlichen Geschwindigkeitsniveaus abweichende Wirkungsgrade der Maßnahme zu erwarten sind.

Der dritte und letzte Schritt der Datenaufbereitung bestand in der Plausibilisierung der Daten. Dabei wurde überprüft, ob die aufgenommenen Daten inhaltlich konsistent sind. Dieser Schritt war notwendig, da bei der Aufnahme und Digitalisierung der Daten u. U. Fehler auftreten können, welche eine anschließende Auswertung erschweren bzw. verfälschen. Dies geschah hauptsächlich unter der Verwendung bestimmter Algorithmen, welche eine Prüfung der eingegebenen Daten hinsichtlich ihres logischen Aufbaus ermöglichen. Als Beispiel zu nennen ist hier die Überprüfung der Maßnahmenpakete. So kann einer Einzelmaßnahme nie mehr als eine Maßnahme zugeordnet 
sein, einem Maßnahmenpaket hingegen nie weniger als zwei. Ein weiterer wichtiger Schritt innerhalb der Plausibilisierung lag in der Überprüfung der sukzessiven Maßnahmenpakete und der Mischpakete. Diese Pakete hatten die Eigenschaft, dass zwischen mindestens zwei einzelnen Maßnahmen ein Zeitraum von mindestens 2-3 Monaten lag, welcher sich in manchen Fällen aber auch auf mehrere Jahre erstreckte. Lag zwischen der Umsetzung zweier Maßnahmen ein vollständig auswertbarer 3-Jahres-Zeitraum, wurde dieses Maßnahmenpaket noch einmal geteilt. In solchen Fällen konnte beispielsweise in Mischpaketen die nicht synchron umgesetzte Maßnahme herausgelöst werden und fortan als Einzelmaßnahme betrachtet werden. So entstanden aus dem Mischpaket eine Einzelmaßnahme und ein synchrones Maßnahmenpaket. Aus einem sukzessiven Maßnahmenpaket hingegen konnten je nach Struktur zwei bzw. drei Einzelmaßnahmen oder eine Einzelmaßnahme und ein sukzessives Maßnahmenpaket mit zwei Maßnahmen hervorgehen.

Der Vorteil, der sich aus diesem Vorgehen ergab, war, dass die Anzahl von Einzelmaßnahmen in der Datenbank dadurch deutlich erhöht werden und die Anzahl von Misch- und sukzessiven Maßnahmenpaketen reduziert werden konnte. Dies hat den Hintergrund, dass bei Einzelmaßnahmen die Wirkung direkt der Maßnahme zugeordnet werden kann, was bei sukzessiven Maßnahmenpaketen nicht ohne weiteres möglich ist. Dahinter verbirgt sich die Überlegung, dass die gemessene Wirkung des gesamten Maßnahmenpaketes unter Umständen ausschließlich auf eine der Teilmaßnahmen zurückzuführen ist ${ }^{3}$. Da dieses Phänomen bei keinem Maßnahmenpaket auszuschließen ist, soll im weiteren Verlauf hauptsächlich die Wirkung von Einzelmaßnahmen untersucht werden.

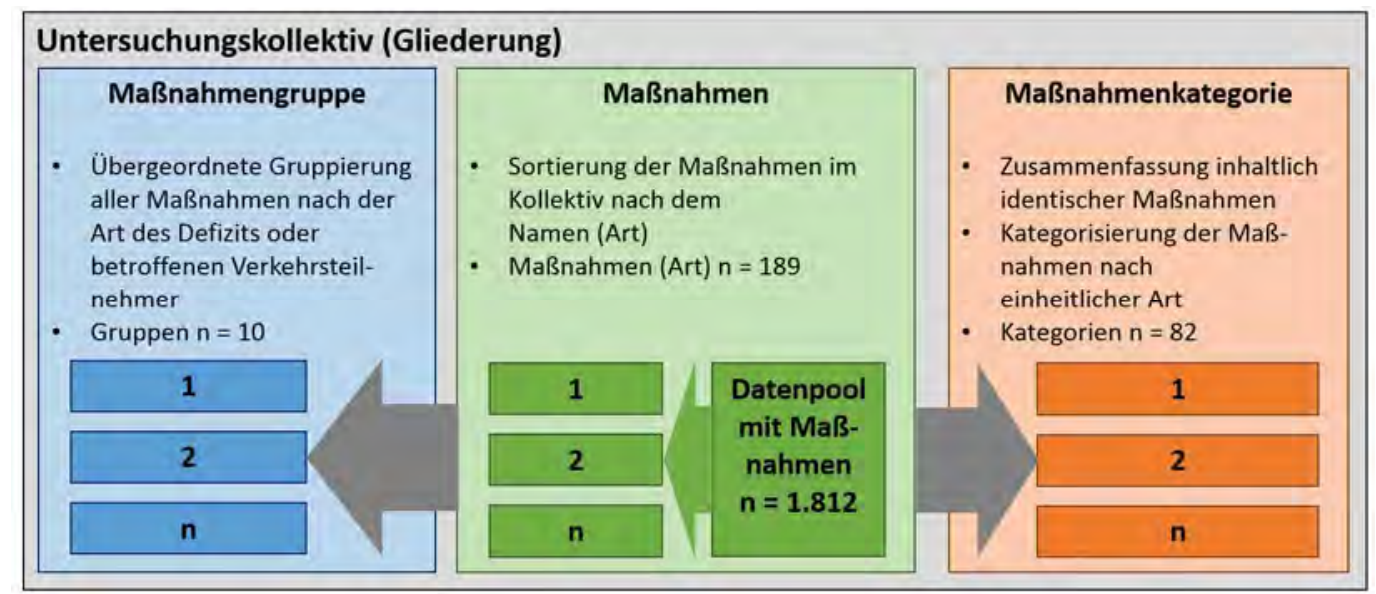

Abbildung 12: Darstellung Arten von Gruppierungen des Maßnahmenkollektivs

Mit endgültigen Abschluss dieses Arbeitsschrittes - inklusive der gezielten Nacherhebung (Vergleiche Kapitel 4.4) - war auch die Datenaufbereitung abgeschlossen. Bedingt durch den Wegfall augenscheinlich nicht kostengünstiger Maßnahmen und der Aufteilung von Maßnahmenpaketen ergab sich eine von der ursprünglichen Datenbankgröße abweichende Anzahl von Maßnahmen bzw. Maßnahmenpaketen. Die so überarbeitete Datenbank enthielt insgesamt 1.812 Maßnahmen(-pakete) ${ }^{4}$, welche einer von insgesamt 82 Maßnahmenkategorien zugeordnet wurden und als Grundlage für die weiteren Auswertungen dienten. Abbildung 12 verdeutlicht den Zusammenhang der verwendeten Begrifflichkeiten im Untersuchungskollektiv. Die Übersicht zu allen 82 Maßnahmenkategorien enthält Anhang 9. Die Struktur dieser Datengrundlage wird im folgenden Kapitel beschrieben.

3 Dies betrifft sowohl sukzessive als auch synchrone Maßnahmenpakete. Bei sukzessiven Paketen besteht die Möglichkeit, dass in der Zwischenzeit andere Randbedingungen (z.B. Verkehrsführungsformen in der Peripherie der Stelle) geändert haben und somit die ceteris paribus Bedingung verletzt wird.

4 Vor der gezielten Nacherhebung (Vergleiche Kapitel 4.4) lagen 1.776 Maßnahmen vor. In der weiteren Kollektivbetrachtung (Kapitel 4.2 und Kapitel 4.3) wurden nur Maßnahmen $(n=36)$ der gezielten Nacherhebung betrachtet, die auch maßgeblich zur Erweiterung der analysierten Datengrundlage (Maßnahmenkategorien) beitragen. 


\subsection{Struktur der Datengrundlage}

Abbildung 13 zeigt die Verteilung aller 1.812 Maßnahmen auf die einzelnen Maßnahmen- bzw. paketarten. Der mit ca. $61 \%$ weitaus größte Teil der Maßnahmen in der Datenbank sind Einzelmaßnahmen (1.099 Maßnahmen) ${ }^{5}$. Die Anzahl der synchronen Maßnahmenpakete ist mit 516 $(28,5 \%)$ nur etwa halb so groß. Die verbleibenden 10,7\% der Maßnahmen verteilen sich auf Mischpakete- bzw. sukzessive Maßnahmenpakete. Bei 24 Maßnahmenpaketen konnte aufgrund fehlender Zeitangaben keine Zuordnung getroffen werden. Der größte Teil der in der Datenbank vorhandenen Maßnahmen ist demnach als Einzelmaßnahme für die weitere Auswertung geeignet.

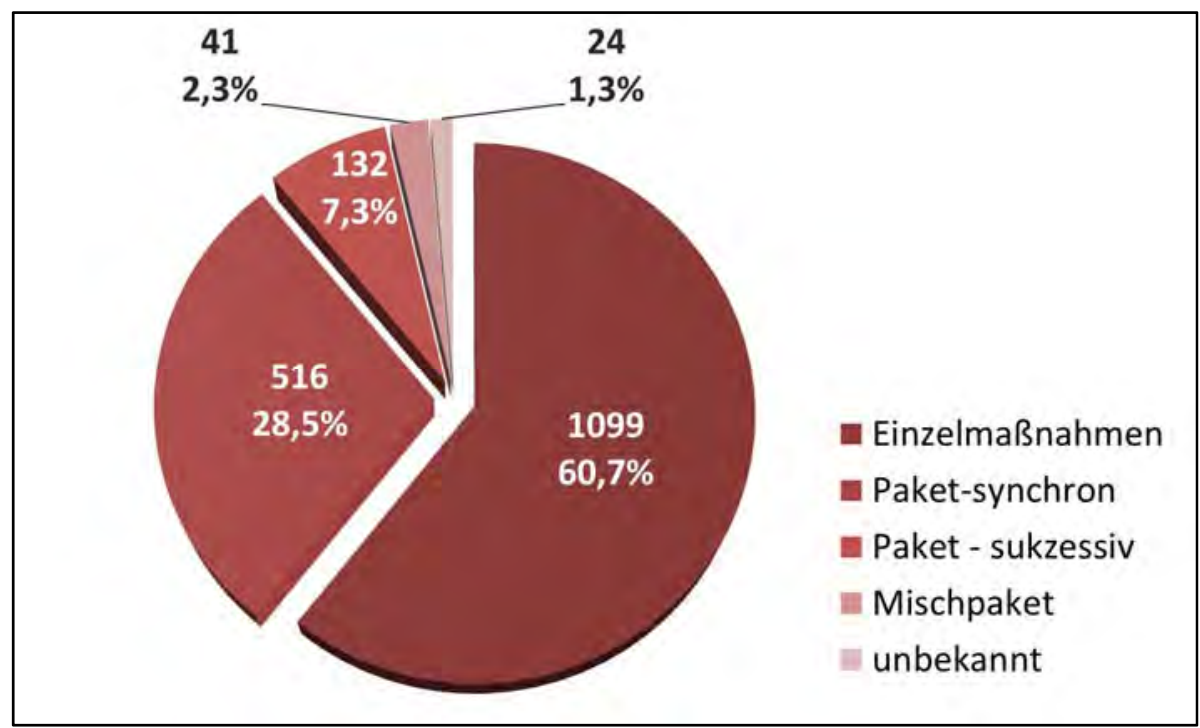

Abbildung 13: Anteil der Maßnahmen(-pakete) in Abhängigkeit der Maßnahmen- bzw. Paketart

Abbildung 14 zeigt die Anteile der Maßnahmen(-pakete) in Abhängigkeit der Ortslage. Der größte Teil der Maßnahmen(-pakete) befindet sich mit 886 Maßnahmen (48,9\%) im Außerortsbereich. Der Anteil der Maßnahmen(-pakete) im Innerortsbereich ist mit 858 Maßnahmen bzw. 47,4 \% nur unwesentlich kleiner. Lediglich 3,3\% der Maßnahmen liegen auf Bundesautobahnen. Bei neun Maßnahmen(-pakete) konnte auf Grund fehlender oder unvollständiger Angaben zur Ortslage keine Zuordnung erfolgen.

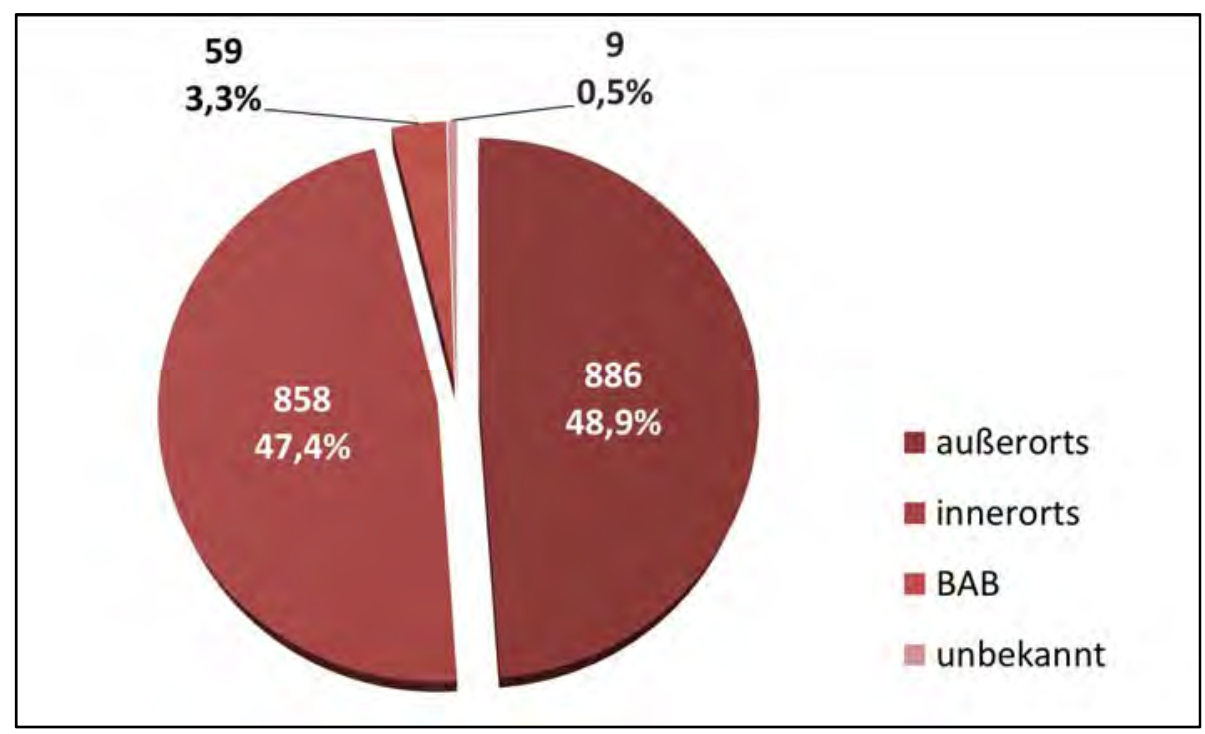

Abbildung 14: Anteil der Maßnahmen(-pakete) in Abhängigkeit der Ortslage

Vor der Plausibilisierung lag der Anteil der Einzelmaßnahmen bei etwa $56 \%$. 
In Abbildung 15 dargestellt sind die Anteile der Maßnahmen(-pakete) in der Datenbank in Abhängigkeit des Netzelementes, auf welchem sie zur Anwendung kamen. Dabei zeigt sich, dass mit 793 Maßnahmen(-pakete) (43,8 \%) der größte Teil im Bereich von Knotenpunkten ohne LSA umgesetzt wurde. Mit jeweils ca. $27 \%$ sind die Anteile der Maßnahmen(-pakete) an Knotenpunkten mit LSA und der freien Strecke in etwa gleich groß. Die Anteile von Maßnahmen(-pakete) im Zuge von Motorradstrecken und UHL sind hingegen mit 0,5\% bzw. 1,5\% nur sehr gering. Bei 10 Maßnahmen(-paketen) konnte aufgrund fehlender oder unvollständiger Angaben zur Charakteristik der Örtlichkeit keine Zuordnung erfolgen.

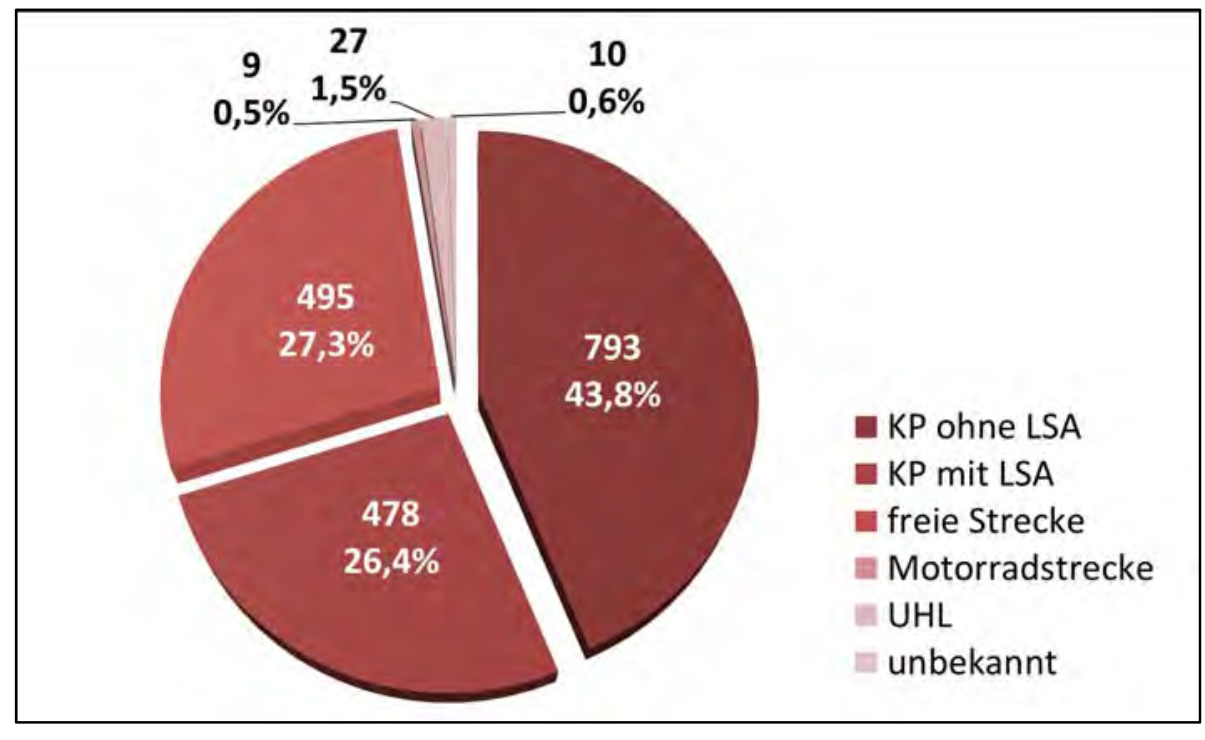

Abbildung 15: Anteil der Maßnahmen(-pakete) in Abhängigkeit des Netzelementes

Die Anteile der Maßnahmen(-pakete) nach Ortslage und Netzelement sind in Abbildung 16 dargestellt. Der mit 44,8\% größte Teil der Maßnahmen(-pakete) befindet sich an Knotenpunkten im Innerortsbereich, wohingegen ein Viertel der Maßnahmen(-pakete) an Knotenpunkten im Außerortsbereich umgesetzt wurden. Auf BAB wurden lediglich 3,3\% aller Maßnahmen(-pakete) umgesetzt. Der Anteil der Maßnahmen(-pakete) auf Strecken innerhalb geschlossener Ortschaften ist mit 2,4\% ähnlich gering. Insgesamt wurden erwartungsgemäß die meisten Maßnahmen(-pakete) (1.275 bzw. 70,4 \%) an Knotenpunkten (ohne BAB) umgesetzt.

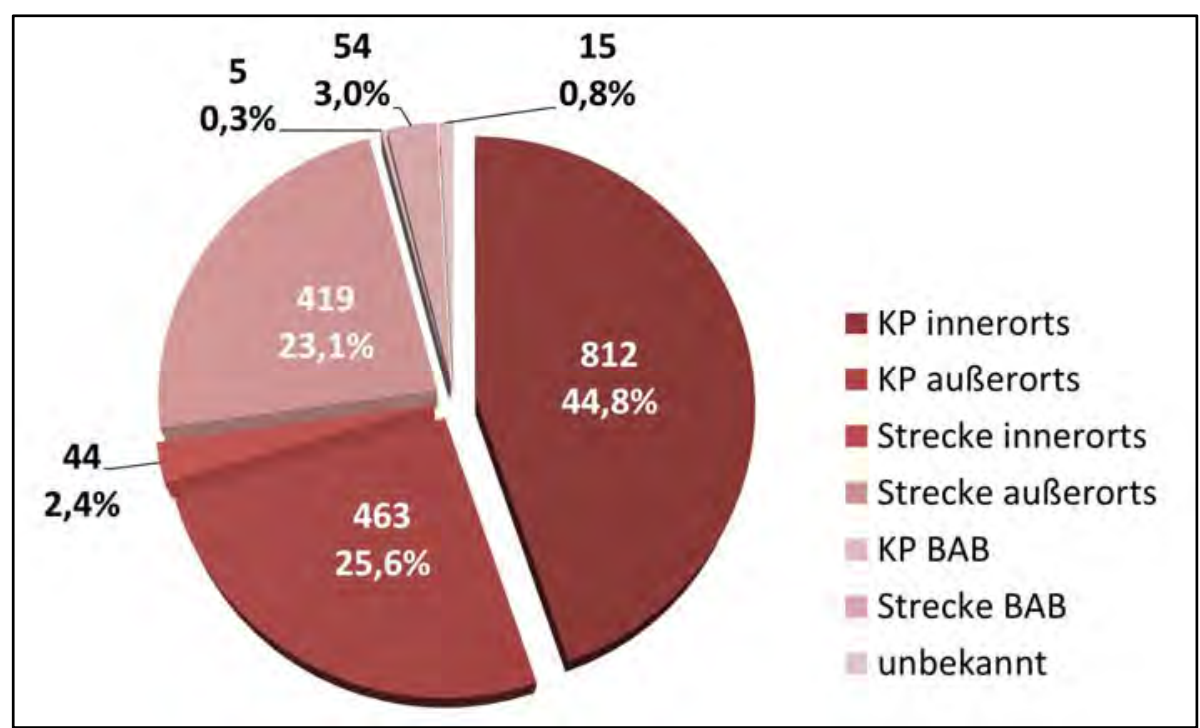

Abbildung 16: Anteil der Maßnahmen(-pakete) nach Netzelement und Ortslage 
Abbildung 17 zeigt die Unfalldatenlage im Vorher- und im Nachher-Zeitraum. Dabei ist generell festzustellen, dass im Vorher-Zeitraum für einen deutlich größeren Teil Unfalldaten vorliegen als im Nachher-Zeitraum. Während der Anteil an Maßnahmen(-pakete) ohne oder mit unvollständigen Unfalldaten im Vorher-Zeitraum bei $50,3 \%$ liegt, beträgt er im Nachher-Zeitraum $69,3 \%$, was maßgeblich auf die Verdopplung des Maßnahmen(-paket)anteils ohne Unfalldaten zurückzuführen ist. Auffällig ist auch, dass der Anteil der Maßnahmen(-pakete), für die ausschließlich das Unfallgeschehen der 3-Jahreskarte (3-JK) vorliegt, sowohl im Vorher- als auch im Nachher-Zeitraum mit 3,5 \% bzw. 0,1\% am geringsten ist. Der Anteil vollständig vorhandener 1-Jahreskarten (1-JK) ist im Vorher- und Nachher-Zeitraum mit 17,3\% bzw. 11,7\% in etwa gleich groß. Der Anteil der Maßnahmen(-pakete), bei denen beide Unfalltypenkarten vollständig vorliegen ist im VorherZeitraum mit 28,8 \% etwa $10 \%$ höher als im Nachher-Zeitraum (19,0 \%).

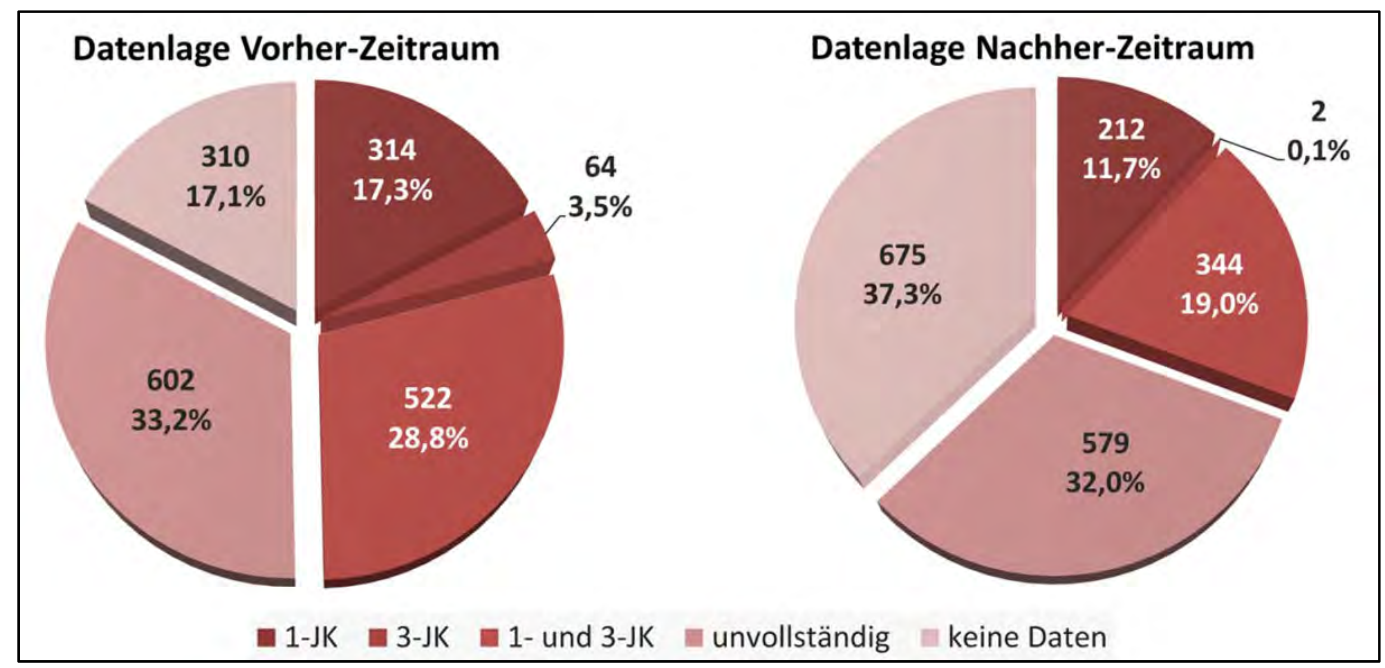

Abbildung 17: Unfalldatenlage im Vorher- und Nachher-Zeitraum

Aus der Kombination beider Zeiträume und der jeweiligen Datenverfügbarkeit entsteht das in Abbildung 18 dargestellte Diagramm. Es zeigt die Anzahl der Maßnahmen(-pakete), bei welchen Unfalldaten für den jeweiligen Nachher-Zeitraum vorliegen unter der Voraussetzung, dass auch Daten für einen der Vorher-Zeiträume vorliegen. So liegen beispielsweise bei 59 Maßnahmen(-pakete) Unfalldaten sowohl für die 12 Monate vor als auch für die 12 Monate nach der Maßnahmenumsetzung vor. Die Abbildung lässt erkennen, dass lediglich für 537 der 1.812 in der Datenbank enthaltenen Maßnahmen(-pakete) ein vollständiges Unfallgeschehen sowohl für den Vorher- als auch für den Nachher-Zeitraum vorliegt.

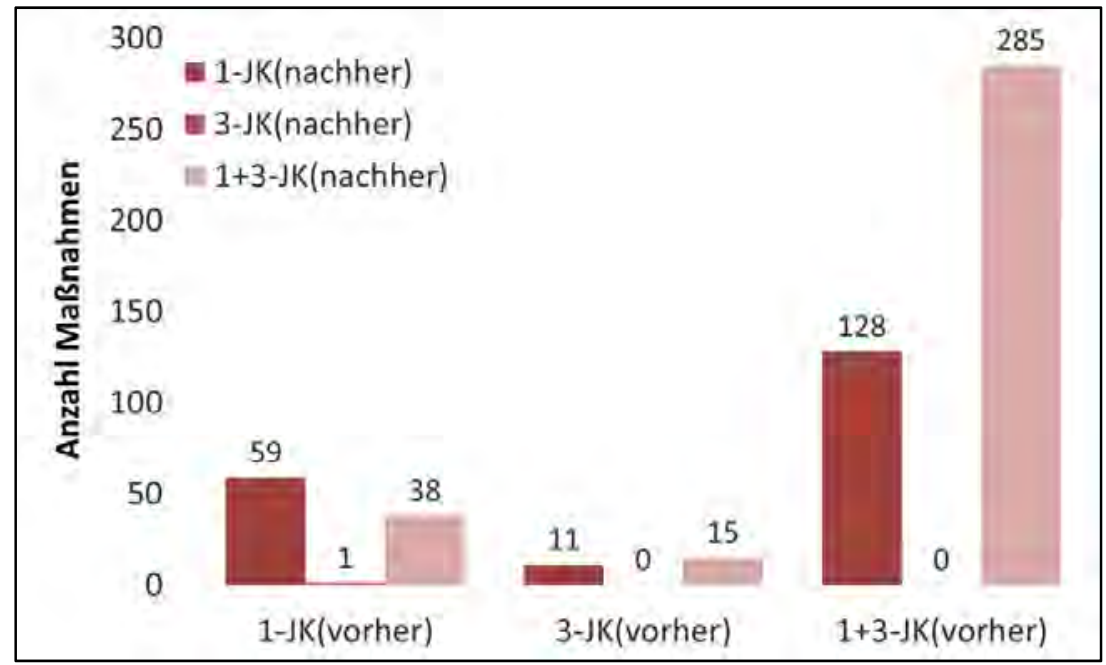

Abbildung 18: Kombination der Unfalldatenlage im Vorher- und Nachher-Zeitraum 
Dabei ist der Anteil der Maßnahmen(-pakete) mit Unfalldaten über 12 und 36 Monate vor der Maßnahmenumsetzung und über einen beliebigen Zeitraum nach der Maßnahmenumsetzung mit $77 \%$ (413 Maßnahmen(-pakete)) am höchsten. Davon sind bei 128 Maßnahmen(-paketen) (31 \%, bezogen auf die 413 Maßnahmen(-pakete)) auch Informationen zum Unfallgeschehen der 12 Monate nach der Maßnahmenumsetzung vorhanden. Bei den restlichen 69 \% (285 Maßnahmen(-pakete)) liegt das Unfallgeschehen differenziert nach 1- und 3-JK sowohl für den Vorher- als auch für den Nachher-Zeitraum vollständig vor.

Maßnahmenkosten sind nur für einen geringen Teil des Kollektivs hinterlegt. Für lediglich 120 Maßnahmen(-pakete) sind die Umsetzungskosten übermittelt worden, was nur etwa einem Anteil von $7 \%$ entspricht. Die Spanne der Umsetzungskosten reicht dabei von $200 €$ bis $983.000 €$, wobei der Mittelwert bei $84.351 €$ liegt.

Die Verteilung der Maßnahmen(-paket)kosten ist in Abbildung 19 dargestellt. Daraus kann abgelesen werden, dass $80 \%$ der in der Datenbank vorhandenen und mit Kosten hinterlegten Maßnahmen(-pakete) nicht mehr als $90.000 €$ gekostet haben. Der steile Anstieg des Graphen im vorderen Bereich deutet darauf hin, dass der Anteil der kostengünstigen Maßnahmen(-pakete) in der Datenbank sehr hoch ist. Der Kostenmedian liegt bei $10.000 €$. Nur etwa $26 \%$ der Maßnahmen(-pakete) wiesen Umsetzungskosten von mehr als $50.000 €$ auf, während der Anteil mit Umsetzungskosten von mehr als $100.000 €$ bei nur etwa $18 \%$ liegt. Auch hier müssen für die Detailanalyse und die Ermittlung des Wirkungsgrades weitere Daten erhoben werden. Allerdings ist zu erwarten, dass sich diese Erhebung weit weniger umfangreich gestaltet als die der Unfalldaten.

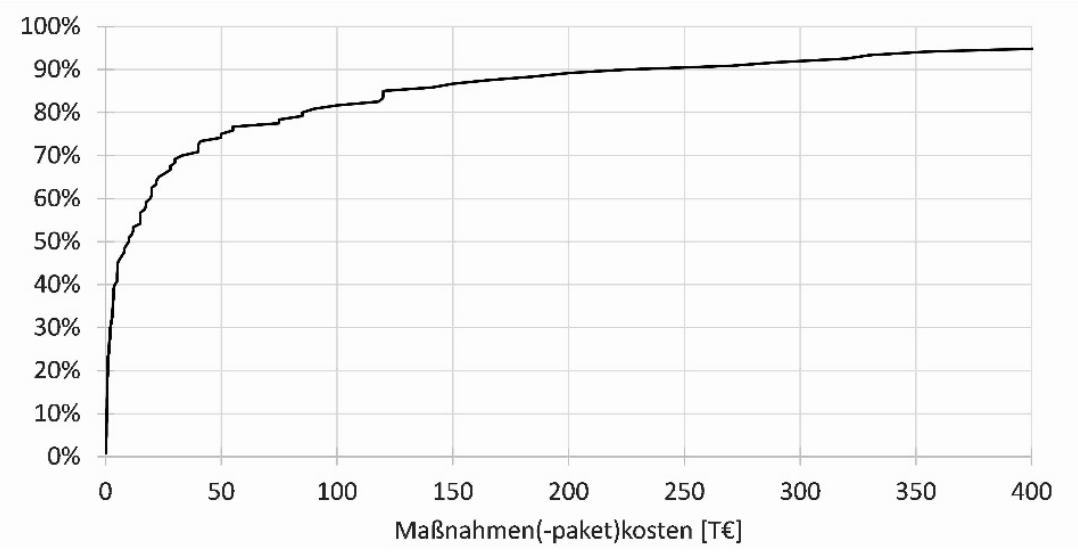

Abbildung 19: Verteilung der Maßnahmen(-paket)kosten in [T€]

\subsection{Methodik zur Auswahl geeigneter Maßnahmen(-pakete) zur De- tailuntersuchung}

Angesichts der Anzahl von 1.812 Maßnahmen(-paketen) und der 82 verschiedenen Maßnahmenkategorien (siehe Anhang 9) musste eine Auswahl geeigneter Untersuchungsbeispiele getroffen werden. Hierfür wurde zunächst untersucht, wie oft die unterschiedlichen Maßnahmenkategorien in der Datenbank aufgeführt waren. Die geschah separat sowohl für die Einzelmaßnahmen als auch für die Maßnahmenpakete. Bei den Maßnahmenpaketen erfolgte zudem eine Unterteilung in Pakete mit zwei und Pakete mit drei Maßnahmen.

In Abstimmung mit dem Auftraggeber wurde beschlossen, in den weiteren Untersuchungen überwiegend Einzelmaßnahmen zu betrachten, welche mit mindestens sechs Nennungen in der Datenbank aufgeführt sind. Die Beschränkung auf Einzelmaßnahmen wurde getroffen, da sich nur in diesen Fällen die gemessene Wirkung genau einer Maßnahme zuordnen lässt. Die Gesamtwirkung von Maßnahmenpaketen hingegen kann auch maßgeblich durch nur eine in dem Paket verwendete Maßnahme erzielt worden sein, während die anderen synchron oder sukzessiv umgesetzten Maßnahmen keine oder nur einen geringen Teil beigetragen haben. Diese Unsicherheit 
wird bei sukzessiven Maßnahmenpaketen durch den zeitlichen Versatz weiter verstärkt, da Gewöhnungseffekte der Verkehrsteilnehmer oder die Änderung der Randbedingungen nicht auszuschließen sind, was die ceteris paribus Bedingung unterläuft. Die Festlegung auf Maßnahmen mit mindestens sechs Nennungen erfolgte aus statistischen Überlegungen: Da es sich bei Unfällen um seltene, zufällige Ereignisse handelt, ist zu erwarten, dass die Wirkung ein und derselben Maßnahme an zwei verschiedenen aber charakterlich gleichen Stellen nur in Ausnahmefällen gleich groß zu beobachten ist, obwohl theoretisch die gleiche Wirkung unterstellt werden kann. Um dies zu berücksichtigen, sollen für jede ausgewählte Maßnahme nach Möglichkeit sechs Umsetzungen untersucht und daraus anschließend eine mittlere Wirkung der Maßnahme berechnet werden. ${ }^{6}$

\subsubsection{Einzelmaßnahmen}

Neben der oben beschriebenen Festlegung hinsichtlich der in die weiteren Analysen aufzunehmenden Maßnahmen bzw. deren Anzahl wurde für Einzelmaßnahmen zudem eine Unterteilung nach Ortslage (innerorts, außerorts ohne BAB) und Netzelement (Strecke, Knotenpunkt) vorgenommen. Eine Unterteilung nach Ortslage ist notwendig, da aufgrund der unterschiedlichen Verletztenstruktur der Verunglückten für den Innerorts- und den Außerortsbereich unterschiedliche Unfallkostensätze verwendet werden. Diese nehmen in Form der vermiedenen Unfallkosten Einfluss auf die Berechnung der Nutzen-Kosten-Verhältnisse und auf das Maß der Maßnahmenwirkung.

Die Unterscheidung der verschiedenen Netzelemente ist ebenso notwendig, da viele Maßnahmen sowohl an der Strecke als auch am Knotenpunkt eingesetzt werden können, dabei aber auf die Beseitigung unterschiedlicher Defizite oder unterschiedlicher Unfalltypen (Konfliktsituationen) abzielen?.

In der Datenbank sind insgesamt $1.108^{8}$ Einzelmaßnahmen aufgeführt, welche in 82 Maßnahmenkategorien (MK) eingeordnet wurden (Vergleiche Abbildung 12). Dabei sind 43 dieser 82 Kategorien mit mehr als sechs Nennungen vertreten, wobei die Häufigkeit der Nennung allein keine Auskunft über die Qualität der übermittelten Daten erlaubt. Eine Übersicht über die gewählten Kategorien und die jeweilige Besetzung gibt Tabelle 7.

In Abstimmung mit dem Auftraggeber wurden die 28 in Tabelle 7 aufgeführten MK zur weiteren Auswertung ausgewählt. Dabei wurde wie oben beschrieben vertieft nach Ortslage und Netzelement differenziert. Die Unterkategorien, welche zur weiteren Auswertung ausgewählt wurden, sind in Tabelle 7 grau hinterlegt ${ }^{9}$. Eine Besonderheit stellen die Kollektive „Beleuchtung anpassen/verbessern“ und „Rüttelstreifen aufbringen“ dar. Hier wird auf Grund zu geringer Umsetzungshäufigkeiten in den Einzelkollektiven keine Differenzierung nach Netzelement vorgenommen. Eine weitere Besonderheit sind die beiden Kollektive „Sichtschutzzaun aufstellen" und „Hindernisse im Seitenraum entfernen“. Diese werden aus inhaltlichen Gründen ebenfalls in die weitere Untersuchung aufgenommen, obwohl sie die Mindestanzahl von sechs Nennungen nicht erreicht haben. Die Auswahl der zu untersuchenden MK erfolgte in Abstimmung mit dem Auftraggeber und in Abhängigkeit der Güte der zur Verfügung stehenden Daten sowie der Maßnahmeneinschätzung durch die Mitglieder der UKO (Vergleiche Kapitel 5).

6 Diese Festlegung stellt einen Kompromiss dar. Da insgesamt ca. 180 Stellen näher betrachtet werden sollen, ergibt sich daraus eine Menge von ca. 30 verschiedenen Maßnahmenkategorien (vgl. Tabelle 7).

7 Ein Beispiel hierfür ist das Aufbringen von Längsschwellen auf der Fahrbahn, womit an Knotenpunkten ein Abbiegeverbot und auf der Strecke (z.B. in Kurven) eine Überholverbot durchgesetzt werden kann.

8 Inklusive der Maßnahmen aus der gezielten Nacherhebung

9 Die Maßnahmenkategorien mit mehr als sechs Nennungen, welche nicht farbig hinterlegt sind, bleiben in der Detailuntersuchung aus o. g. Bedingungen unberücksichtigt. 
Tabelle 7: Besetzung der aus den Einzelmaßnahmen zur weiteren Untersuchung vorgesehenen Kategorien (grau)

\begin{tabular}{|c|c|c|c|c|c|c|}
\hline \multirow{3}{*}{ Maßnahmenkategorie } & \multirow{3}{*}{ Gesamt } & \multicolumn{4}{|c|}{ Nennungen } & \multirow{3}{*}{$\begin{array}{c}\text { Anzahl } \\
\text { Auswertung }\end{array}$} \\
\hline & & \multicolumn{2}{|c|}{ davon innerorts } & \multicolumn{2}{|c|}{ davon außerorts } & \\
\hline & & Knoten & Strecke & Knoten & Strecke & \\
\hline Verbesserung Koordinierung & 8 & 8 & - & - & - & 1 \\
\hline $\begin{array}{l}\text { Änderung der Signalisierung (Änderung ohne neue } \\
\text { Signalbilder (kein separater Linksabbieger o. ä.)) }\end{array}$ & 58 & 45 & - & 13 & - & 1 \\
\hline „sonstige“ Gefahrenzeichen anbringen & 15 & 4 & 1 & 1 & 9 & 1 \\
\hline HALT Z 206 StVO (anstelle Z 205 StVO) & 61 & 30 & - & 31 & - & 2 \\
\hline Halte-/Parkverbot anordnen & 17 & 16 & - & 1 & - & 1 \\
\hline Z 625 StVO (Richtungstafel in Kurve) aufstellen & 42 & 1 & 2 & 5 & 34 & 1 \\
\hline $\begin{array}{l}\text { Zusatzzeichen ergänzen } \\
\text { (Z 1006-36 oder Z 1000-32) }\end{array}$ & 19 & 8 & - & 9 & 2 & 2 \\
\hline Verkehrszeichen anpassen & 24 & 11 & 1 & 9 & 3 & 2 \\
\hline Beschränkung $\mathrm{V}_{\text {zul }}($ Strecke, $\mathrm{AO})$ & 31 & - & - & - & 31 & 1 \\
\hline Beschränkung $\mathrm{V}_{\mathrm{zul}}(\mathrm{KP}-\mathrm{VZ}, \mathrm{AO})$ & 13 & - & - & 13 & - & 1 \\
\hline Überwachung $\vee_{\text {zul }}$ mit OGÜ & 18 & 1 & 2 & 9 & 6 & 2 \\
\hline Erneuerung Fahrbahnoberfläche & 87 & 7 & 2 & 13 & 65 & 1 \\
\hline zusätzlichen Signalgeber anordnen & 8 & 6 & - & 2 & - & 1 \\
\hline Leuchtmittel Signalgeber verbessern & 16 & 12 & - & 4 & - & 1 \\
\hline Sichthindernisse entfernen & 81 & 44 & 2 & 30 & 5 & 2 \\
\hline Beleuchtung anpassen/verbessern & 7 & \multicolumn{2}{|c|}{6} & 0 & 0 & 1 \\
\hline Sichtschutzzaun aufstellen & 4 & 1 & - & 3 & - & 1 \\
\hline Markierung anpassen/verbessern & 37 & 25 & - & 12 & - & 1 \\
\hline Markierung in Stand setzen & 68 & 45 & 4 & 16 & 3 & 1 \\
\hline Radverkehrsführung anpassen & 10 & 10 & - & - & - & 1 \\
\hline Radfahrfurt Rot einfärben & 17 & 14 & - & 3 & - & 1 \\
\hline Passive Schutzeinrichtung aufstellen & 12 & - & - & - & 12 & 1 \\
\hline $\begin{array}{l}\text { Unterfahrschutz an passiver } \\
\text { Schutzeinrichtung anbringen }\end{array}$ & 7 & - & - & - & 7 & 1 \\
\hline Hindernisse im Seitenraum entfernen & 3 & - & - & - & 3 & 1 \\
\hline Rüttelstreifen aufbringen & 7 & 1 & - & \multicolumn{2}{|c|}{6} & 1 \\
\hline Gummischwellen aufbringen & 11 & 4 & - & 7 & - & 1 \\
\hline Sperrfläche markieren & 8 & 6 & - & 1 & 1 & 1 \\
\hline $\begin{array}{l}\text { Verkehrszeichen versetzen } \\
\text { (wegen Erkennbarkeit) }\end{array}$ & 18 & 5 & - & 8 & 5 & 1 \\
\hline Anzahl (Auswertung) & - & 14,5 & 0,5 & 9,5 & 8,5 & 33 \\
\hline
\end{tabular}

\subsubsection{Maßnahmenpakete}

In der Datenbank sind insgesamt 231 verschiedene synchrone Maßnahmenpakete mit je zwei Maßnahmen und 117 mit je drei Maßnahmen aufgeführt. Von den Maßnahmenpaketen mit je zwei Maßnahmen erfüllen lediglich sieben das Kriterium von mindestens sechs Nennungen. Bei den Maßnahmenpaketen mit je drei Maßnahmen erfüllt keines dieses Kriterium. Unabhängig davon wurden in Abstimmung mit dem Auftraggeber dennoch zwei Maßnahmenpakete zur Detailanalyse ausgewählt. Die Gemeinsamkeit beider Maßnahmenpakete besteht in der Beschränkung der zulässigen Höchstgeschwindigkeit kombiniert mit einem Gefahrenhinweis. So wird

- die Wirkung einer Geschwindigkeitsbeschränkung im Zusammenspiel mit dem Z 625 StVO (Richtungstafel in Kurve) und 
- die Wirkung einer Geschwindigkeitsbeschränkung im Zusammenspiel mit dem Z 142 StVO (Wildwechsel) untersucht.

Dabei ist das erste Paket mit acht, das zweite Paket mit fünf Nennungen in der Datenbank vertreten. Weitere synchrone Maßnahmenpakete werden nicht einer Detailanalyse unterzogen. Gleiches gilt auch für sukzessive oder Mischpakete (Vergleiche Kapitel 4.3.1).

\subsubsection{Untersuchungskollektiv}

Nachstehend sind die zur weiteren Untersuchungen herangezogenen 35 MK mit entsprechender Maßnahmennummer dargestellt (Tabelle 8). Diese stützen sich auf die Angaben in Tabelle 7 (grau hinterlegte Felder) und sind um die zwei Maßnahmenpakete ergänzt

Für die Maßnahmeneinschätzung durch die Mitglieder der Unfallkommissionen (Evaluation) und die Unfalldatenauswertung (Analyse) sind die örtlichen und straßencharakteristischen Merkmale der MK aufgeführt. Zwischen beiden Bewertungsmethoden können sich bezüglich genannter Merkmale Differenzen ergeben. Diese beruhen u. a. darauf, dass mit der Evaluation eine allgemeine Einschätzung der MK erzielt werden soll, während sich die Analyse auf die zur Verfügung stehenden Nennungen je MK stützt. Das Design der Erhebungen und die detaillierten Ergebnisse der Evaluationen der UKO als auch der Unfalldatenanalyse sind Inhalt der Kapitel 5.3 und 6.2. 
Tabelle 8: Maßnahmenkategorien (MK) und Aufnahme der Kriterien Örtlichkeit und Straßencharakteristik für Bewertungsansatz Evaluation UKO bzw. Unfalldatenanalyse

\begin{tabular}{|c|c|c|c|c|c|c|}
\hline \multirow{2}{*}{\multicolumn{2}{|c|}{$\begin{array}{c}\text { Maßnahmenkategorie } \\
\text { Titel }\end{array}$}} & \multicolumn{2}{|c|}{ Evaluation } & \multicolumn{3}{|c|}{ Unfalldatenanalyse } \\
\hline & & $\begin{array}{l}\text { Örtlich- } \\
\text { keit }\end{array}$ & Charakteristik & $\begin{array}{l}\text { Örtlich- } \\
\text { keit }\end{array}$ & Charakteristik & Stellen \\
\hline 1 & Verbesserung der Koordinierung & 10 & KP-LSA & 10 & KP-LSA & 5 \\
\hline 2 & Änderung der Signalisierung & 10 & KP-LSA & 10 & KP-LSA & 6 \\
\hline 3 & "sonstige" Gefahrenzeichen anbringen & $\mathrm{AO}$ & Strecke & $\mathrm{AO}$ & Strecke & 2 \\
\hline 4 & HALT Z 206 StVO (anstelle Z 205 StVO) & \multirow{2}{*}{ IO/AO } & \multirow{2}{*}{$\mathrm{KP}-\mathrm{VZ}$} & 10 & $\mathrm{KP}-\mathrm{VZ}$ & 5 \\
\hline 5 & HALT Z 206 StVO (anstelle Z 205 StVO) & & & $\mathrm{AO}$ & $\mathrm{KP}-\mathrm{VZ}$ & 6 \\
\hline 6 & Halte-/Parkverbot anordnen & 10 & KP-VZ/r. v. I. & 10 & KP-VZ/r. v. I. & 4 \\
\hline 7 & Z 625 StVO (Richtungstafel in Kurven) aufstellen & $\mathrm{AO}$ & Strecke & $\mathrm{AO}$ & Strecke & 6 \\
\hline 8 & Zusatzzeichen Z 1006-36 StVO (Unfallgefahr) ergänzen & \multirow{2}{*}{ IO/AO } & \multirow{2}{*}{ KP-beliebig/Strecke } & 10 & $\mathrm{KP} /$ Strecke & 3 \\
\hline 9 & Zusatzzeichen Z 1006-36 StVO (Unfallgefahr) ergänzen & & & AO & $\mathrm{KP} /$ Strecke & 4 \\
\hline 10 & Verkehrszeichen anpassen & \multirow{2}{*}{ IO/AO } & \multirow{2}{*}{ KP-beliebig/Strecke } & 10 & $\mathrm{KP} /$ Strecke & 4 \\
\hline 11 & Verkehrszeichen anpassen & & & $\mathrm{AO}$ & $\mathrm{KP} /$ Strecke & 3 \\
\hline 12 & Beschränkung Vzul & $\mathrm{AO}$ & Strecke & $\mathrm{AO}$ & Strecke & 6 \\
\hline 15 & Beschränkung Vzul & $\mathrm{AO}$ & $\mathrm{KP}-\mathrm{VZ}$ & AO & $\mathrm{KP}-\mathrm{VZ}$ & 4 \\
\hline 16 & Überwachung zulässige Höchstgeschwindigkeit mit OGÜ & \multirow{2}{*}{$\mathrm{AO}$} & \multirow{2}{*}{ KP-VZ/Strecke } & $\mathrm{AO}$ & $K P-V Z$ & 2 \\
\hline 17 & Überwachung zulässige Höchstgeschwindigkeit mit OGÜ & & & $\mathrm{AO}$ & Strecke & 4 \\
\hline 18 & Erneuerung Fahrbahnoberfläche & $\mathrm{AO}$ & Strecke & $\mathrm{AO}$ & Strecke & 6 \\
\hline 19 & Zusätzlichen Signalgeber anordnen & 10 & KP-LSA & 10 & KP-LSA & 2 \\
\hline 21 & Leuchtmittel Signalgeber verbessern & 10 & KP-LSA & 10 & KP-LSA & 6 \\
\hline 22 & Sichthindernisse entfernen & \multirow{2}{*}{ IO/AO } & \multirow{2}{*}{$K P-V Z$} & 10 & $K P-V Z$ & 6 \\
\hline 23 & Sichthindernisse entfernen & & & $\mathrm{AO}$ & KP-VZ & 6 \\
\hline 24 & Beleuchtung anpassen/verbessern & 10 & KP-beliebig/Strecke & 10 & $\mathrm{KP} /$ Strecke & 6 \\
\hline 25 & Sichtschutzzaun aufstellen & $\mathrm{AO}$ & $\mathrm{KP}-\mathrm{VZ}$ & $\mathrm{AO}$ & $\mathrm{KP}-\mathrm{VZ}$ & 6 \\
\hline 26 & Markierung anpassen/verbessern & 10 & KP-beliebig & 10 & KP-beliebig & 6 \\
\hline 27 & Markierung in Stand setzen & 10 & KP-beliebig & 10 & KP-beliebig & 6 \\
\hline 29 & Radverkehrsführung anpassen & 10 & KP-beliebig & 10 & KP-beliebig & 5 \\
\hline 30 & Radfahrerfurt Rot einfärben & 10 & $K P-V Z$ & 10 & $K P-V Z$ & 4 \\
\hline 31 & Passive Schutzeinrichtung aufstellen & $\mathrm{AO}$ & Strecke & $\mathrm{AO}$ & Strecke & 6 \\
\hline 32 & $\begin{array}{l}\text { Unterfahrschutz an passiver Schutzeinrichtung } \\
\text { anbringen }\end{array}$ & $\mathrm{AO}$ & Strecke & $\mathrm{AO}$ & Strecke & 5 \\
\hline 33 & Hindernisse im Seitenraum entfernen & $\mathrm{AO}$ & Strecke & $\mathrm{AO}$ & Strecke & 2 \\
\hline 34 & Rüttelstreifen aufbringen & $\mathrm{AO}$ & Strecke & AO & Strecke & 5 \\
\hline 35 & Gummischwellen aufbringen & $\mathrm{AO}$ & KP-beliebig & $\mathrm{AO}$ & KP-beliebig & 5 \\
\hline 36 & Sperrfläche markieren & 10 & $K P-V Z$ & 10 & $\mathrm{KP}-\mathrm{VZ}$ & 4 \\
\hline 37 & Verkehrszeichen versetzen (Erhöhung Erkennbarkeit) & $\mathrm{AO}$ & KP-beliebig/Strecke & $\mathrm{AO}$ & $\mathrm{KP} /$ Strecke & 4 \\
\hline 38 & $\begin{array}{l}\text { Beschränkung Vzul und Z } 625 \text { StVO } \\
\text { (Richtungstafel in Kurve) }\end{array}$ & $\mathrm{AO}$ & Strecke & $\mathrm{AO}$ & Strecke & 6 \\
\hline 39 & Beschränkung Vzul und sonst. Gefahrenzeichen & $\mathrm{AO}$ & Strecke & $\mathrm{AO}$ & Strecke & 5 \\
\hline \multicolumn{7}{|c|}{$\begin{array}{l}\text { KP = Knotenpunkt, LSA = Lichtsignalanlage, VZ = Regelung durch Verkehrsze } \\
\text { beliebig = Maßnahme unabhängig von der Regelungsart des Knotenpunkts } \\
\text { Abkürzungen gelten auch für nachfolgende Auswertungen und Darstellunge }\end{array}$} \\
\hline
\end{tabular}




\subsection{Gezielte Nacherhebung}

Während der detaillierten Datenaufbereitung der ausgewählten MK war festzustellen, dass für einen Großteil der Nennungen das zur Verfügung stehende Datenmaterial unzureichend vorlag (Vergleiche Abbildung 17 und Abbildung 18 in Kapitel 4.2). Für jede MK wurden die einzelnen Nennungen auf ihre Eignung geprüft. Lagen für eine Kategorie mehr als sechs verwertbare Nennungen vor, wurden aus dem Kollektiv sechs Nennungen zufällig ausgewählt und zur Maßnahmenbewertung herangezogen. Mit dieser Vorgehensweise wurde eine unabhängige Auswahl von Maßnahmenumsetzungen und damit eine anhand der vorliegenden Daten objektive Bewertung der Maßnahmen gewährleistet.

Von den 35 näher untersuchten MK (inklusive der beiden Maßnahmenpakete) lagen nach Abschluss der Erhebungen für lediglich fünf Kategorien sechs oder mehr auswertbare Nennungen vor. Für 15 Kategorien lagen nur drei oder weniger Nennungen vor. Folgende Ursachen waren einzeln oder in Kombinationen Gründe für eine Nichtberücksichtigung der Nennungen:

- Unzureichende Datenlage in der Analyse; In diesen Fällen unterschritten die Vorher- bzw. Analysezeiträume den Zeitraum von einem Jahr oder es lagen keine Daten zum Unfallgeschehen vor. In einigen Fällen lagen nur verbal beschriebene Angaben zum Unfallgeschehen vor, welche aufgrund unzureichender qualitativer Beschreibung nicht berücksichtigt werden konnten.

- Unzureichende Nachbetrachtung; Hier gilt in gleicher Weise dass, was bereits für die Analyse beschrieben wurde. Jedoch war die Anzahl an Fällen, bei denen nur verbal beschriebene oder gar keine Angaben zum Unfallgeschehen für den Nachher-Zeitraum vorlagen, wesentlich höher.

- Ungenaue Angaben zur Maßnahmenumsetzung; In weiteren wenigen Fällen führten Unklarheiten in der Maßnahmenbeschreibung bzw. in der zeitlichen Abfolge der Maßnahmenumsetzungen (häufig in Kombination mit eher oder später erfolgten Maßnahmen) zur Relegation der Fälle. Mitunter konnte nicht verifiziert werden, ob eine Maßnahme nur beschlossen oder auch realisiert wurde.

Zur Erhöhung auswertbarer Fallzahlen erfolgte nach der oben beschriebenen Sichtung eine gezielte Nachfrage und -erhebung. Diese geschah auf verschiedene Art und Weise:

- Für einen Teil an Nennungen, bei den das Unfallgeschehen nur in kleinen Zeiträumen oder gar nicht vorlag, die Angaben zur Maßnahmenumsetzung aber bekannt waren, konnte zur Vervollständigung der Daten für ausgewählte Zeiträume und Bundesländer auf die Unfalldatenbank der UDV zurückgegriffen werden. In Absprache mit den UKO erfolgte die Abgrenzung der Zeiträume und die Aufbereitung des Unfallgeschehens. In ähnlicher Art und Weise konnten für das Bundesland Nordrhein-Westfalen Unfalldaten ergänzt werden, indem der „Landesbetrieb Straßenbau Nordrhein-Westfalen“ für den klassifizierten Teil des Straßennetzes die Unfalldaten nach gezielter Anfrage - Benennung der Stellen mit Angabe der Zeiträume - zur Verfügung stellte.

- Lagen Unterlagen zu Unfallhäufungen mit ungenauen Angaben zur Maßnahmenumsetzung vor, erfolgte eine Rücksprache mit den betroffenen UKO. Mit dieser Vorgehensweise konnten Lücken im Datenmaterial geschlossen und 14 Unfallhäufungen (Nennungen) vervollständigt werden.

- Trotz der erfolgten Ergänzungen konnte für einen Teil der MK kein vollumfänglicher Datensatz von sechs auswertbaren Nennungen erzielt werden. Um eine ausreichende Besetzung zu erzielen, erfolgte im Anschluss an die o. g. Vorgehensweise für unvollständige $\mathrm{MK}$ im Bereich Außerorts eine gezielte Anfrage bei der Zentralstelle für Verkehrssicherheit der Straßenbauverwaltung bei der Autobahndirektion Südbayern. Von den zur Verfügung gestellten Daten konnten 15 Unfallhäufungen in der Untersuchung berücksichtigt werden. Zur Abdeckung des innerörtlichen Straßennetzes erfolgte im gleichen Stil eine parallele Anfrage an 
die kreisfreien sächsischen Städte Leipzig, Dresden und Chemnitz. Aus dem zur Verfügung gestellten Maßnahmenpool einer Zeitspanne von ca. zehn Jahren wurden neun weitere Stellen den verschiedenen Maßnahmenkategorien zugeführt.

Die in der Nacherhebung gesammelten Erkenntnisse zu bereits bestehenden Nennungen und die Informationen zu den zusätzlichen Nennungen zu Unfallhäufungen sind Bestandteil der unter Kapitel 4.2 und Kapitel 4.3 beschriebenen Ausführungen. Die Mengengerüste der auswertbaren MK sind unter Kapitel 4.3.3 näher beschrieben.

Zusammenfassend ist festzustellen, dass die Datenzusammenstellung und Datenaufbereitung zu Unfallhäufungen zwischen den verschiedenen UKO deutlich differenziert und unterschiedliche Standards aufweist. Insbesondere bei der Nachbetrachtung (Wirksamkeitsanalyse) zeigte sich, dass sehr häufig nur verkürzte Zeiträume in die Betrachtung einflossen. Eine standardisierte und vereinheitlichte Aufbereitung der Daten und technische Unterstützung während der Bearbeitung von Unfallhäufungen, wie sie durch die Systeme und Hilfen wie:

- das Unfallhäufungsprogramm des Landes Bayern (UHP) [ZVS 2016],

- das Tool zur Effektivitäts- und Effizienzberechnung der BASt oder

- der „Maßnahmenkatalog gegen Unfallhäufung MaKaU“ der BASt ${ }^{10}$

seit geraumer Zeit angeboten werden, tragen wesentlich zu einer strukturierten und zielorientierten Arbeit bei der Bekämpfung von Unfallhäufungen bei.

${ }^{10}$ Der Maßnahmenkatalog gegen Unfallhäufungen (MaKaU) befindet sich bis Ende 2016 in der Erprobung und wird voraussichtlich ab Frühjahr 2017 den UKO vollumfänglich zur Verfügung stehen. 


\section{$5 \quad$ Evaluation der Maßnahmenkategorien}

\subsection{Grundlagen}

Bei der Bekämpfung von Unfallhäufungen folgt die Maßnahmenfindung auf die gründliche Unfallanalyse und Ortsbesichtigung. Bezugnehmend auf die erkannten Defizite und weiteren Randbedingungen sowohl der Verkehrsanlage als auch des Verkehrsablaufs sind Maßnahmenvorschläge zu erarbeiten, die geeignet, angemessen und durchsetzbar sind [FGSV 2012]. Die Maßnahmenvorschläge sind in der Unfallkommission zu diskutieren. Es wird in Sofortmaßnahmen - diese zeichnen sich durch eine zeitnahe Umsetzung und häufig geringe Kosten aus - und in Mittel- und langfristige Maßnahmen - bedürfen häufig längerer Planungs- und Realisierungsphasen - unterschieden. Für die Zusammenstellung geeigneter Maßnahmenvorschläge liegen verschiedene Berichte und elektronische Zusammenstellungen vor [GERLACH et al. 2009, FGSV 2002, SPAHN 2011, MAIER et al. 2015].

Das Wissen über die Möglichkeiten geeigneter Maßnahmen variiert bei den Mitgliedern der Unfallkommissionen in Abhängigkeit des Ausbildungsstandes, der Erfahrungswerte bzw. Berufserfahrung sowie abhängig von der Teilnahme an Fortbildungen, in denen relevante Inhalte transferiert werden [DEGENER/BUTTERWEGGE 2012]. Aufgrund geringer zeitlicher Ressourcen erfolgen Wirksamkeitsprüfungen nach der Umsetzung von Maßnahmen häufig mangelhaft und unvollständig. Erkenntnisse, in welcher Art und Weise eine Maßnahme ihre Wirkung entfaltet, werden selten eruiert. Dies schränkt eine objektive Auswahl von Maßnahmen, die eine hohe Eignung besitzen, ein. Vor dem Hintergrund beschränkter finanzieller Mittel in den öffentlichen Haushalten folgt die weitere Einschränkung, nach Möglichkeit Maßnahmen mit geringen Investitionskosten einzusetzen.

Vor diesem Hintergrund stellt sich die Frage, wie die Mitglieder der Unfallkommissionen Maßnahmen gegen Unfallhäufung bezüglich ihrer Wirksamkeit als auch ihrer Kosten bewerten. Aus der Evaluation der Maßnahmen lassen sich u. a. folgende Fragen beantworten:

- Ist die Maßnahme bekannt?

- Wie werden die Kosten, die zeitliche Umsetzbarkeit und der verwaltungstechnische Aufwand eingeschätzt?

- Welche Maßnahmen werden als kostengünstig angesehen?

- Wie wird die Wirkung der Maßnahmen auf die Entwicklung von Unfallanzahl und Unfallschwere eingeschätzt?

Die Überlagerung der Ergebnisse aus Evaluation und der Unfalldatenanalyse erlauben Rückschlüsse auf mögliche Fehleinschätzungen von Maßnahmen bezüglich ihrer Wirkung. So wäre es z. B. möglich, dass Maßnahmen von Unfallkommissionen nicht eingesetzt werden, weil deren Wirksamkeit als schlecht bewertet wird, während aus der Unfalldatenauswertung hervorgeht, dass diese wirksam sind. Demgegenüber kann aber auch der Fall auftreten, dass Maßnahmen vermeintlich „falsch" eingesetzt werden, weil deren Wirkung überbewertet wird. Neben der Wirkungseinschätzung gibt die Befragung auch Auskunft über den Bekanntheitsgrad von Maßnahmen. Dies ist mit Blick auf relativ neue Maßnahmen interessant - bspw. Sichtschutzzaun am Knotenpunkt oder Rüttelstreifen. Der Rückschluss aus einem fehlenden Bekanntheitsgrad zielt darauf, diese Maßnahmen in Schulungen von Unfallkommissionen oder anderen Veranstaltungen vorzustellen.

\subsection{Vorgehen}

Die Befragung der Mitglieder von Unfallkommissionen erfolgte im Rahmen von Fortbildungs- und Schulungsveranstaltungen. Sie wurden in verschiedenen Bundesländern durchgeführt. Ein zeitlich in den Ablauf des Projektes passender Schulungstermin war Randbedingung für die Befragung. Analog zu der ersten Stufe der Kontaktaufnahme bei den verschiedenen Bundesländern wurden 
deren Kontaktpersonen angeschrieben und gebeten, eine Vor-Ort-Befragung der Unfallkommissionmitglieder während der Schulungen zu arrangieren. Insgesamt konnte bei acht Veranstaltungen in sieben Bundesländern das Projekt vorgestellt und die Evaluation durchgeführt werden. Tabelle 9 stellt die Veranstaltungsorte mit den erhobenen Maßnahmenkategorien (MK) dar. Es konnten die Bögen von 217 befragten Teilnehmern zur Evaluation herangezogen werden.

Tabelle 9: Termine für Schulungen und Tagungen von Unfallkommissionen zur Evaluierung mit Angabe der evaluierten Maßnahmenkategorie

\begin{tabular}{|c|c|c|c|c|c|c|c|c|c|}
\hline \multirow{2}{*}{$\begin{array}{l}\text { Bundesland/ } \\
\text { Veranstaltung }\end{array}$} & \multirow{2}{*}{ Ort } & \multirow{2}{*}{ Datum } & \multirow{2}{*}{$\begin{array}{l}\text { Teilnehmer } \\
\text { (Rücklauf) }\end{array}$} & \multicolumn{6}{|c|}{ Nummer Maßnahmenkategorie } \\
\hline & & & & 1 & 2 & 3 & 4 & 5 & 6 \\
\hline Hessen & Darmstadt & Sep. 2015 & 80 & 15 & 26 & 27 & 29 & 34 & \\
\hline $\begin{array}{l}\text { 3. UDV-UKO- } \\
\text { Seminar }\end{array}$ & Münster & Sep. 2015 & 14 & 1 & 2 & $8 / 9$ & 27 & 21 & \\
\hline Sachsen & Bobritzsch & Sep. 2015 & 17 & 3 & $4 / 5$ & $16 / 17$ & $22 / 23$ & 31 & \\
\hline $\begin{array}{l}\text { Mecklenburg- } \\
\text { Vorpommern }\end{array}$ & Rostock & Nov. 2015 & 18 & 7 & $10 / 11$ & 19 & 32 & 37 & 12 \\
\hline Nordrhein-Westfalen & Neuss & Nov. 2015 & 15 & 25 & 32 & 38 & 39 & & \\
\hline Nordrhein-Westfalen & Neuss & Dez. 2015 & 23 & 35 & 30 & 36 & 24 & 18 & 6 \\
\hline Niedersachsen & $\begin{array}{l}\text { Hannoversch- } \\
\text { Gmünd }\end{array}$ & Apr. 2016 & 37 & 15 & 33 & 2 & 24 & $8 / 9$ & \\
\hline Schleswig-Holstein & Bad Malente & Jun. 2016 & 13 & 1 & 32 & 36 & 37 & 21 & 30 \\
\hline
\end{tabular}

Während der Projektvorstellung wurde besonders Wert auf die Sensibilisierung der Unfallkommissionen gelegt. Dies bedeutet, dass die Teilnehmer gezielt aufgefordert wurden ihre eigenen Erfahrungen bzw. Einschätzungen einfließen zu lassen. Dabei lag der Grundgedanke auf dem Schaffen einer Situation, bei der die Teilnehmer für eine beschriebene fiktive Unfallhäufung die vorgestellte Maßnahme für sich selbstständig bewerten sollten. Die Evaluation der MK beruhte auf einer Kurzvorstellung der jeweiligen Kategorie durch den Auftragnehmer. Dabei erfolgte die:

- Vorstellung der typischen Konfliktsituation (Unfalltyp/Beteiligungsart),

- Beschreibung der Örtlichkeit (Differenzierung zwischen Strecke und Knotenpunkt, bei letzterem Angabe der Art der Verkehrsregelung),

- Beschreibung der Maßnahme (konkrete Maßnahmenbeschreibung und Abgrenzung zu ähnlichen Maßnahmen) sowie

- die Darstellung und Erläuterung einer typischen Situation anhand geeigneter Bilder.

Die Abbildung 20 zeigt beispielhaft die grafische Aufbereitung einer MK, wie sie den Teilnehmern präsentiert wird. Während der Befragung wurde das Projekt sowie fünf bis sechs MK vorgestellt. Diese wurden einzeln durch jeden Teilnehmer der Veranstaltung evaluiert. Die Teilnehmer erhielten die Möglichkeit während der Präsentation unmittelbar Nachfragen zu stellen. Die Darstellung eines Evaluationsbogens, wie er von den Teilnehmern ausgefüllt wurde, enthält Abbildung 21. Der Evaluationsbogen setzt sich aus vier Teilen zusammen, dessen Aufbau und Inhalt eng mit dem Auftraggeber abgestimmt wurde. Weiterhin erfolgte im Vorfeld eine Beispielbefragung mit ausgewählten Teilnehmern in Sachsen, um die Praktikabilität der Befragung zu prüfen. Eine Übersicht der Aufbereitung aller MK enthält Anhang 8.

Der erste Teil umfasst Fragen zur Kenntnis über die Maßnahme und mögliche schon erfolgte Umsetzungen durch die Teilnehmer. Eine Einordnung der Maßnahme bezüglich der erwarteten Kosten, zeitlichen Umsetzbarkeit und des verwaltungstechnischen Aufwands erfolgt im zweiten Teil. Dieser Teil zielt neben der subjektiven Kosteneinschätzung darauf ab, eine Einschätzung darüber zu erhalten, ob die Maßnahme - wie im M Uko [FGSV 2012] gefordert - als „durchsetzbar" angesehen wird. Eine zielgerichtete Identifikation als kostengünstig und schnell umsetzbar angesehener Maßnahmen wird durch die gewählte Klassierung der Antwortmöglichkeiten unterstützt. Hier 
liegt der Fokus auf einer engeren Differenzierung der Wahlmöglichkeit im Bereich geringer Kosten und geringem zeitlichen Aufwand.

Die Einschätzung zur Wirkung der Maßnahme wird im dritten Teil der Evaluation aufgeführt. Zum einen soll die Wirkung der Maßnahme zur Reduzierung der Unfallschwere $-z$ B. bei Aufstellen von passiven Schutzeinrichtungen -, zum anderen die Wirkung der Maßnahme zur Reduzierung der Unfallanzahl - z. B. bei Entfernen von Sichthindernissen - eingeschätzt werden. Die Einteilung in jeweils vier Antwortmöglichkeiten wurde hier gewählt, um einer möglichen Antworttendenz „zur Mitte" vorzubeugen.

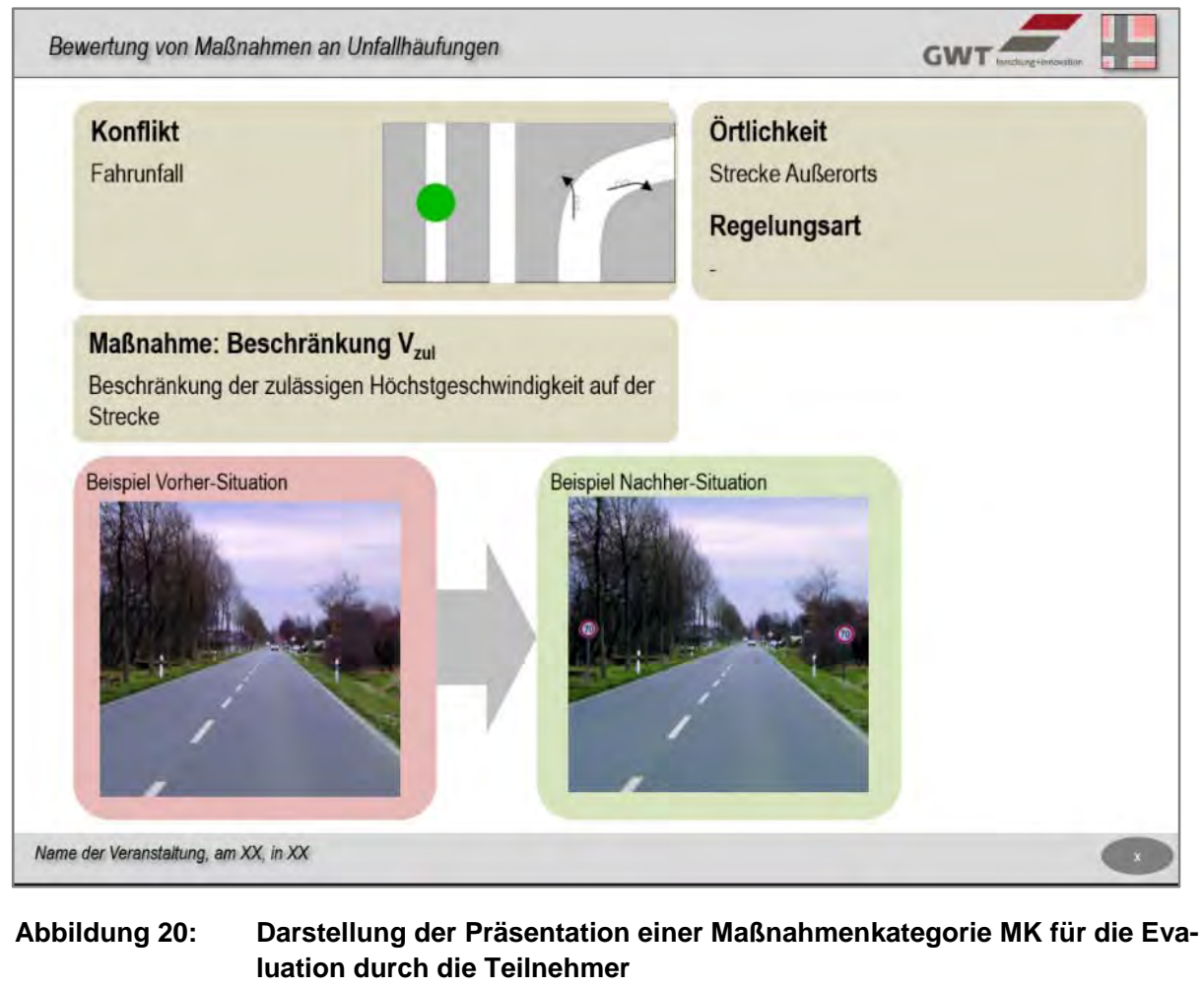

Die dichotome Fragestellung zu den Kriterien „kostengünstig“ und „wirksam“ im vierten Teil des Fragebogens erfordert eine Festlegung durch die Teilnehmer, ob sie eine Maßnahme als kostengünstig und/oder wirksam ansehen oder nicht. Diese Fragen sind bewusst am Ende des Bogens aufgeführt, weil sie einen direkten Rückschluss auf die Bewertung der Kosten und der Wirksamkeit im zweiten bzw. dritten Teil des Fragebogens ermöglichen. So kann zum einen die Aussage: „Ist die Maßnahme kostengünstig?" mit konkreten Kosten verknüpft werden. Zum anderen lässt die Verknüpfung der Frage: „Ist die Maßnahme wirksam?“ mit der Bewertung zur Reduzierung von Unfallanzahl und Unfallschwere den Rückschluss zu, bis zu welcher Größenordnung Maßnahmen als wirksam eingeschätzt werden. Mit der Auswertung aller Teilnehmerbögen lässt sich ein subjektives Bewertungsbild der Unfallkommissionen zu den vorgestellten Maßnahmen gewinnen.

Die Darstellung der Evaluationsergebnisse erfolgt zum einen in einer Zusammenfassung in Kapitel 5.3.3, welche eine Aufbereitung der MK bezüglich ihres Bekanntheitsgrades sowie der Einschätzung hinsichtlich der Aspekte „kostengünstig“ und „wirksam“ enthält. Zum anderen erfolgt eine detaillierte Darstellung der Befragungsergebnisse jeder einzelnen Maßnahmenkategorie in Form eines Faktenblattes zur Evaluation. Hierfür ist die Art der Aufarbeitung unter Kapitel 5.3.2 beschrieben, die Einzelergebnisse sind Kapitel 5.3.3 zu entnehmen. 


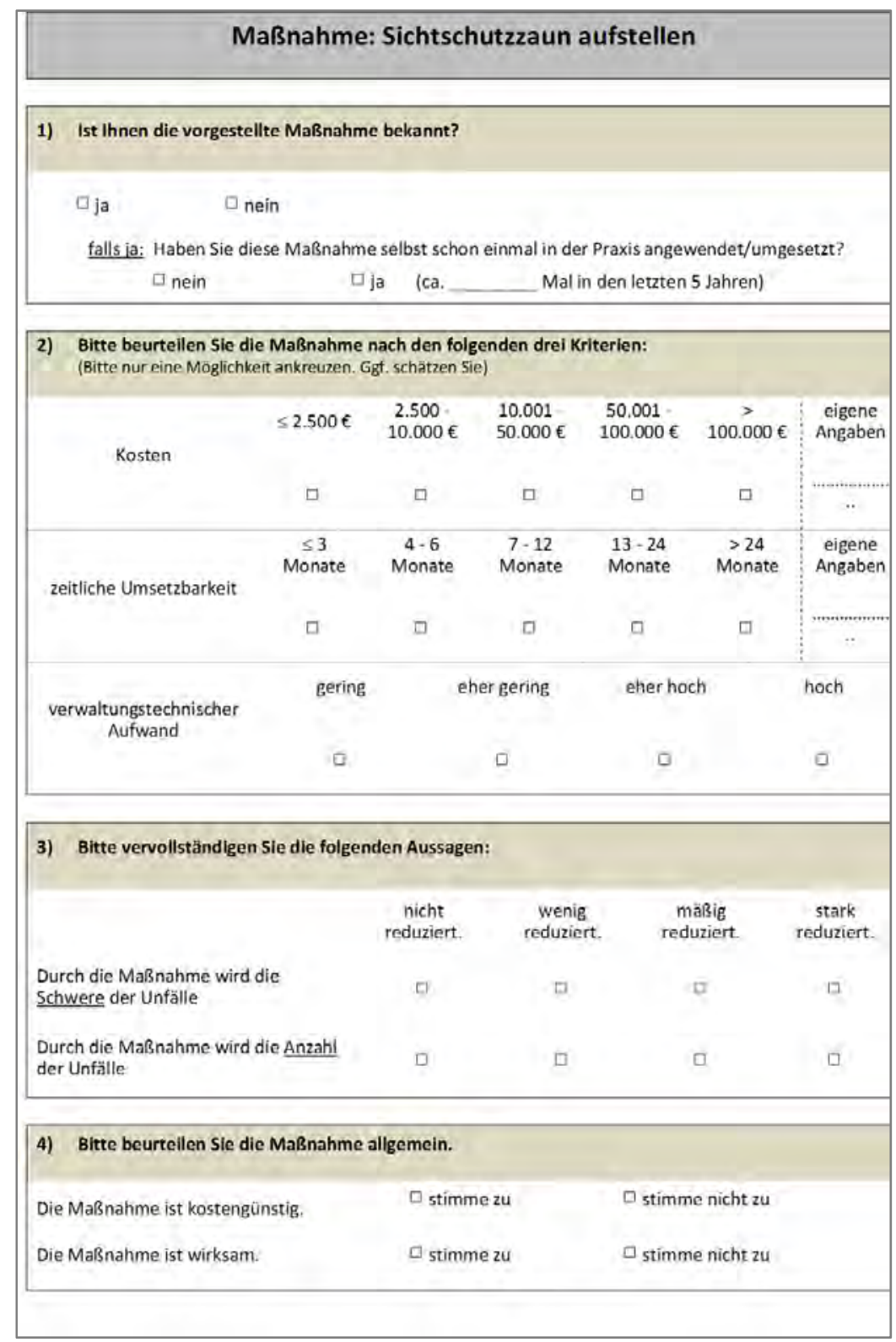

Abbildung 21: Beispiel Evaluationsbogen

\subsection{Ergebnisse}

\subsubsection{Antwortkompetenz}

Neben den Fragebogen zu den Einzelmaßnahmen wurden persönliche Daten zu den Teilnehmern in einem separaten Bogen erfasst. Diese dienen zur Ableitung einer Antwortkompetenz zu den verschiedenen Maßnahmen. Im Einzelnen wurden folgende Punkte anonymisiert befragt:

- Zugehörigkeit zu welcher Behörde

- Aktive Mitgliedschaft in einer Unfallkommission (wenn ja: wie viele Jahre)

- Zuständigkeitsbereich (Stadt, Landkreis oder Andere)

Bei allen acht besuchten Veranstaltungen handelte es sich um Schulungen der Unfallkommission. oder deren übergeordneten behördlichen Leitungen. Die Veranstaltung in Münster stellt dahingehend eine Besonderheit dar, dass hier städtische Unfallkommissionen zur Fortbildung im Rahmen eines UDV-Seminars aus verschiedenen Bundesländern geladen waren. Mit Blick auf das Audito- 
rium wurden die zu bewertenden Maßnahmen im Vorfeld mit den örtlichen Dozenten abgestimmt und ggf. angepasst. So wurden in Münster ausschließlich innerörtliche Maßnahmen evaluiert.

Eine sehr hohe Teilnehmerzahl lag in Hessen und Niedersachsen vor. Von den 217 befragten Teilnehmern gaben 159 (73 \%) an, aktive Mitglieder in einer Unfallkommission zu sein. Teilnehmer, die nicht Mitglieder der Unfallkommission sind, arbeiten auch häufig in anderen Behörden, die nicht direkt der Straßenverkehrsbehörde, Straßenbaubehörde oder Polizei zugeordnet sind. Diese wurden in der Gruppe „Andere" erfasst. Aus Abbildung 22 wird deutlich, dass der überwiegende Anteil der Teilnehmer bei der Polizei tätig ist, gefolgt von der Straßenverkehrsbehörde. In der Verteilung unterrepräsentiert ist die Kategorie der Straßenbaubehörde mit $9 \%$.

Bei der Angabe der territorialen Zuständigkeiten waren Mehrfachnennungen möglich. Knapp die Hälfte (46 \%) der Teilnehmer gab an, in einer städtischen Unfallkommission tätig zu sein. Der Anteil derjenigen, die im Landkreis tätig sind, ist mit $39 \%$ nur geringfügig niedriger. $18 \%$ der Teilnehmer sahen ihren Zuständigkeiten in „anderen Bereichen“.

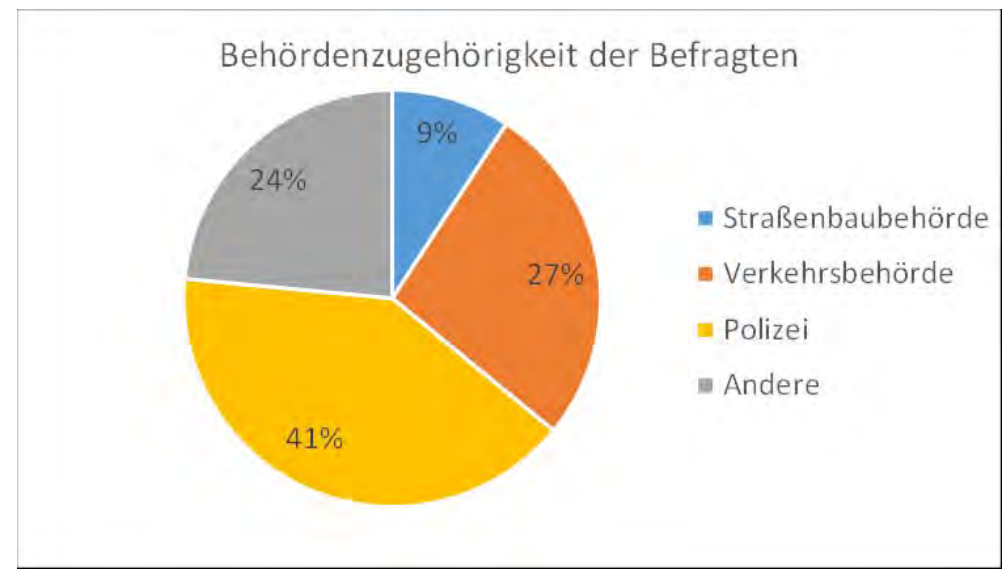

Abbildung 22: Behördenzugehörigkeit der Befragten

Die Tätigkeitsdauer der Teilnehmer, die aktiv in einer Unfallkommission arbeiten, beträgt im Mittel 7,5 Jahre. Dabei reicht die Spanne von 1 bis 27 Jahre. Die mittlere Tätigkeitsdauer variiert in Abhängigkeit der Schulungsart und Zielgruppe zwischen den Bundesländern erheblich. Von allen Schulungen wiesen die Teilnehmer im Land Mecklenburg-Vorpommern mit durchschnittlich 13,6 Jahren gegenüber allen anderen Bundesländern die mit Abstand längste Tätigkeitsdauer auf, während sie in Sachsen mit ca. 5 Jahren am geringsten ausfiel.

Es ist zusammenfassend festzuhalten, dass alle formal nach M Uko [FGSV 2012] in den Unfallkommissionen vertretenen Behörden auch an der Evaluierung teilgenommen haben. Dabei handelte es sich überwiegend um Teilnehmer, die unmittelbar mit der Unfallkommissionsarbeit vertraut sind und auch über einen gewissen Erfahrungsschatz verfügen.

\subsubsection{Aufbereitung Faktenblatt Evaluation}

Die Aufbereitung der Befragungen mündete in der Erstellung eines einheitlichen Faktenblattes. Darin enthalten sind die Ergebnisse der Evaluation zu den verschiedenen Fragen der einzelnen MK. Abbildung 23 zeigt den Aufbau des Faktenblattes. Die einzelnen Faktenblätter zu den MK enthält Anhang 6. 


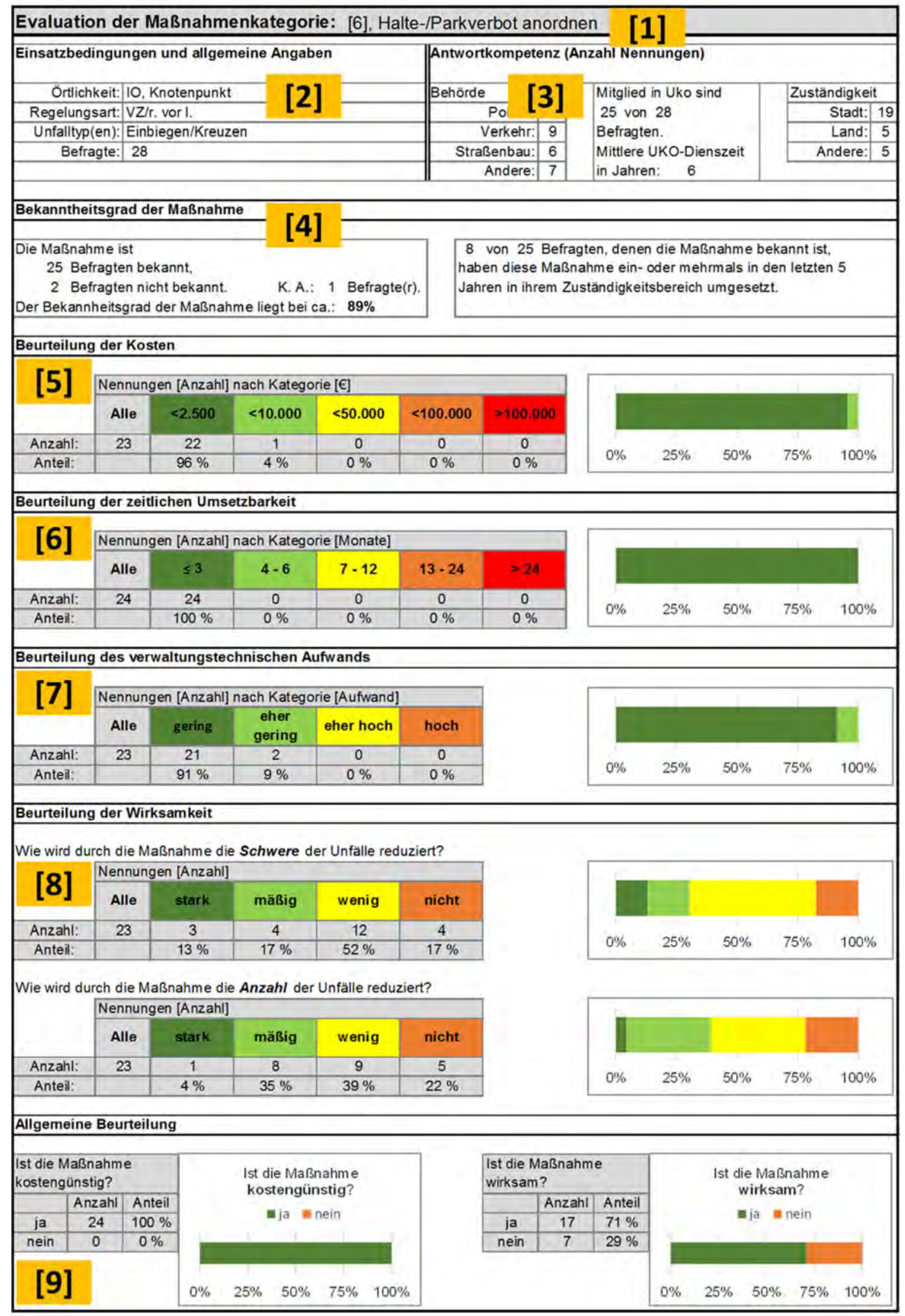

Abbildung 23: Faktenblatt zur Evaluation einer Maßnahmenkategorie MK (Die Nummern verknüpfen die Darstellung mit den inhaltlichen Erläuterungen zu den einzelnen Bereichen in Tabelle 10.) 
Tabelle 10: Erläuterung der Inhalte des Faktenblatts zur Evaluation einer Maßnahmenkategorie MK

\begin{tabular}{|c|c|c|}
\hline Nummer & Bezeichnung Bereich & Inhalt Bereich \\
\hline [1] & Bezeichnung MK & - Bezeichnung der Maßnahmenkategorie mit Nummer und Namen \\
\hline [2] & $\begin{array}{l}\text { Einsatzbedingungen und } \\
\text { allgemeine Angaben }\end{array}$ & $\begin{array}{l}\text { - Beschreibung zur Örtlichkeit, Regelungsart des Knotenpunkts (wenn gegeben) } \\
\text { und prägende Unfalltypen } \\
\text { - Angabe zur Anzahl der Befragten }\end{array}$ \\
\hline [3] & Antwortkompetenz & $\begin{array}{l}\text { - der Block zur Antwortkompetenz enthält Angaben zur Behördenzugehörigkeit, } \\
\text { Mitgliedschaft, Dauer der Mitgliedschaft in den UKO sowie Bereich der Zuständigkeit }\end{array}$ \\
\hline [4] & $\begin{array}{l}\text { Bekanntheitsgrad der Maß- } \\
\text { nahme }\end{array}$ & $\begin{array}{l}\text { - Angabe zur Verteilung des Bekanntheitsgrades der Maßnahme unter den Befragten } \\
\text { - Angabe, wieviel Befragte die Maßnahme selber schon umgesetzt haben }\end{array}$ \\
\hline [5] & Beurteilung der Kosten & $\begin{array}{l}\text { - Zuordnung der Angaben zur Kosteinschätzung in } 5 \text { Klassen, Angabe in [€] } \\
\text { - Darstellung der Verteilung der Kostenschätzung nach Klassen in einem } \\
\text { Balkendiagramm, Angabe in [\%] }\end{array}$ \\
\hline [6] & $\begin{array}{l}\text { Beurteilung der zeitlichen } \\
\text { Umsetzbarkeit }\end{array}$ & $\begin{array}{l}\text { - Zuordnung der Angaben zur zeitlichen Umsetzbarkeit in } 5 \text { Klassen, } \\
\text { Angabe in [Monaten] } \\
\text { - Darstellung der Verteilung der zeitlichen Umsetzbarkeit nach Klassen in einem } \\
\text { Balkendiagramm, Angabe in [\%] }\end{array}$ \\
\hline [7] & $\begin{array}{l}\text { Beurteilung des verwaltungs- } \\
\text { technischen Aufwands }\end{array}$ & $\begin{array}{l}\text { - Zuordnung der Angaben zum verwaltungstechnischem Aufwand in } 4 \text { Klassen, } \\
\text { ordinale verbale Skalierung, Angabe in [Aufwand] } \\
\text { - Darstellung der Verteilung des verwaltungstechnischen Aufwands nach Klassen in } \\
\text { einem Balkendiagramm, Angabe in [\%] }\end{array}$ \\
\hline [8] & Beurteilung der Wirksamkeit & $\begin{array}{l}\text { - Zuordnung der Angaben zur Wirksamkeit der Maßnahme bezüglich der Reduzierung } \\
\text { der Unfallschwere der Unfälle in } 4 \text { Klassen, ordinale verbale Skalierung, } \\
\text { Angabe in [Aufwand] } \\
\text { - Darstellung der Verteilung der Einschätzung zur Wirksamkeit auf die Unfallschwere } \\
\text { nach Klassen in einem Balkendiagramm, Angabe in [\%] } \\
\text { - Zuordnung der Angaben zur Wirksamkeit der Maßnahme bezüglich der Reduzierung } \\
\text { der Unfallanzahl der Unfälle in } 4 \text { Klassen, ordinale verbale Skalierung, } \\
\text { Angabe in [Aufwand] } \\
\text { - Darstellung der Verteilung der Einschätzung zur Wirksamkeit auf die Unfallanzahl } \\
\text { nach Klassen in einem Balkendiagramm, Angabe in [\%] }\end{array}$ \\
\hline [9] & Allgemeine Beurteilung & $\begin{array}{l}\text { - Angabe zur dichotomen Einschätzung, ob die Maßnahme kostengünstig ist } \\
\text { oder nicht, Darstellung der Antwortverteilung in einem Balkendiagramm, } \\
\text { Angabe in [\%] } \\
\text { - Angabe zur dichotomen Einschätzung, ob die Maßnahme wirksam ist } \\
\text { oder nicht, Darstellung der Antwortverteilung in einem Balkendiagramm, } \\
\text { Angabe in [\%] }\end{array}$ \\
\hline
\end{tabular}

Der Bereich mit den Ziffern 1 bis 4 bildet einen allgemeinen einführenden Teil des Faktenblatts, der die MK näher beschreibt und die Antwortkompetenz einordnet. Im oben dargestellten Beispiel handelt es sich um die MK 6 „Halte-/Parkverbot anordnen“. Die Maßnahme bezieht sich auf innerörtliche Knotenpunkte die entweder durch Verkehrszeichen oder rechts-vor-links geregelt sind. Den Hauptunfalltyp stellt der Einbiegen/Kreuzen-Unfall dar. Die Antworten von 28 Befragten fließen in die Evaluation ein, wobei 25 der Befragten Mitglied in einer UKO sind. Der Zuständigkeitsbereich der Befragten liegt überwiegend im Bereich der Stadt. Nur drei Befragten ist die Maßnahme nicht bekannt oder es fehlte eine Antwort. Acht Befragte haben die Maßnahme bereits mindestens einmal in den letzten zehn Jahren umgesetzt.

Die Bereiche der Ziffern 5 bis 9 beinhalten die Verteilungen der Antworten zu den einzelnen Fragen. Die Darstellung der Bereiche 5 bis zur Beurteilung der Kosten, der zeitlichen Umsetzbarkeit und dem verwaltungstechnischen Aufwand bei der Umsetzung einer Maßnahme, unterscheiden sich mit Ausnahme der Klassenanzahl nicht voneinander. Aufgeführt sind die jeweils die absoluten Anzahlen der Antworten als auch die Anteile. Letztere finden sich rechtsseitig in einem Balkendiagramm aufbereitet wieder. Im Falle der MK 6 schätzen nahezu alle Befragten die Kosten, zeitliche Umsetzbarkeit und den verwaltungstechnischen Aufwand als gering ein.

Bezüglich der Wirksamkeit unterscheidet sich die Einschätzung der Wirkung der MK 6 auf die Reduktion der Unfallschwere und Unfallanzahl nur gering. Jeweils ca. zwei Drittel der Befragten geben an, dass durch die MK die Unfallschwere (30\%) und Unfallanzahl (39 \%) mindestens "mäßig“" 
reduziert wird. Vier Befragte sind der Meinung, dass dies nicht der Fall ist. Der Bereich der allgemeinen Beurteilung (Ziffer 9) schließt den Fragebogen ab. Auch hier sind die Antworten nach absoluter Anzahl und Anteilen aufgeschlüsselt und mit einem Diagramm grafisch unterstützt aufgeführt. Jeweils 24 Antworten liegen zu den beiden Fragestellungen vor. Alle Befragten sind der Meinung, dass die MK 6 kostengünstig ist, aber nur 17 Befragte (ca. $71 \%$ ) schätzen die Maßnahme als wirksam ein.

\subsubsection{Auswertung Maßnahmenevaluation}

In Abbildung 24 ist die Einschätzung der MK bezüglich ihres Bekanntheitsgrades sowie der dichotomen Einschätzung zu den Kriterien „kostengünstig“ und „wirksam“ dargestellt. Die Aufführung enthält alle MK, die auch in der Maßnahmenbewertung analysiert werden konnten. Sie dient zur Übersicht und einem allgemeinen Vergleich der MK untereinander.

\begin{tabular}{|c|c|c|c|c|c|c|c|c|}
\hline \multirow{3}{*}{$\begin{array}{c}\text { Maß.- } \\
\text { nr. }\end{array}$} & Anzahl Kategorien: & 34 & & \multirow{3}{*}{\begin{tabular}{|l|} 
Art der \\
Verkehrs- \\
regelung
\end{tabular}} & \multirow{3}{*}{$\begin{array}{c}\text { Befragte } \\
\text { [n] }\end{array}$} & \multirow{3}{*}{$\begin{array}{c}\text { Bekannt- } \\
\text { heitsgrad } \\
\text { [\%] }\end{array}$} & \multicolumn{2}{|c|}{ Maßnahme wird als... } \\
\hline & \multirow{2}{*}{ Name } & Örtlichkeit & & & & & kostengünstig & wirksam \\
\hline & & Ort & Charakteristik & & & & $\begin{array}{c}\text { eingeschätzt von } \\
\text { [\%] }\end{array}$ & $\begin{array}{c}\text { eingeschätzt von } \\
{[\%]}\end{array}$ \\
\hline 1 & Verbesserung der Koordinierung & 10 & Knotenpunkt & LSA & 27 & 81 & 84 & 78 \\
\hline 2 & Änderung der Signalisierung & 10 & Knotenpunkt & LSA & 51 & 94 & 83 & 85 \\
\hline 3 & "sonstige" Gefahrenzeichen anbringen & $\mathrm{AO}$ & Strecke $(300 \mathrm{~m})$ & - & 18 & 78 & 100 & 33 \\
\hline $4 / 5$ & HALT Z 206 StVO( anstelle Z 205 StVO) & IO/AO & Knotenpunkt & vz & 18 & 94 & 100 & 88 \\
\hline 6 & Halte-/Parkverbot anordnen & 10 & Knotenpunkt & VZ/r. vor I. & 28 & 93 & 100 & 71 \\
\hline 7 & Z 625 Stvo (Richtungstafel in Kurven) aufstellen & $\mathrm{AO}$ & Strecke $(300 \mathrm{~m})$ & - & 18 & 100 & 100 & 88 \\
\hline $8 / 9$ & Zusatzzeichen ergänzen (Z 1006-36 Stvo) & IO/AO & beliebig & beliebig & 51 & 80 & 98 & 22 \\
\hline $10 / 11$ & Verkehrszeichen anpassen & IO/AO & beliebig & - & 18 & 88 & 100 & 76 \\
\hline 12 & Beschränkung Vzul & $\mathrm{AO}$ & Strecke $(300 \mathrm{~m})$ & - & 18 & 88 & 100 & 81 \\
\hline 15 & Beschränkung Vzul an Knotenpunkten (AO) & AO & Knotenpunkt & vz & 37 & 89 & 100 & 53 \\
\hline $16 / 17$ & Überwachung zulässige Höchstgeschwindigkeit mit OGÜ & $\mathrm{AO}$ & beliebig & - & 18 & 78 & 39 & 94 \\
\hline 18 & Erneuerung Fahrbahnoberfläche & $\mathrm{AO}$ & Strecke $(300 \mathrm{~m})$ & - & 14 & 100 & 18 & 92 \\
\hline 19 & Zusätzlichen Signalgeber anordnen & 10 & Knotenpunkt & LSA & 18 & 94 & 63 & 94 \\
\hline 21 & Leuchtmittel Signalgeber verbessern & 10 & Knotenpunkt & LSA & 27 & 81 & 83 & 92 \\
\hline $22 / 23$ & Sichthindernisse entfernen & IO/AO & Knotenpunkt & vz & 18 & 83 & 82 & 94 \\
\hline 24 & Beleuchtung anpassen/verbessern & 10 & beliebig & - & 51 & 94 & 52 & 89 \\
\hline 25 & Sichtschutzzaun aufstellen & AO & Knotenpunkt & vz & 23 & 36 & 83 & 70 \\
\hline 26 & Markierung anpassen/Verbessern & 10 & Knotenpunkt & beliebig & 80 & 73 & 92 & 93 \\
\hline 27 & Markierung in Stand setzen & 10 & Knotenpunkt & beliebig & 80 & 87 & 96 & 86 \\
\hline 29 & Radverkehrsführung anpassen & 10 & Knotenpunkt & beliebig & 80 & 71 & 72 & 84 \\
\hline 30 & Radfahrerfurt Rot einfärben & 10 & Knotenpunkt & Vz & 27 & 96 & 96 & 76 \\
\hline 31 & Passive Schutzeinrichtung aufstellen & AO & Strecke $(300 \mathrm{~m})$ & - & 18 & 83 & 39 & 94 \\
\hline 32 & Unterfahrschutz an passiver Schutzeinrichtung anbringen & $\mathrm{AO}$ & Strecke $(300 \mathrm{~m})$ & - & 31 & 75 & 41 & 91 \\
\hline 33 & Hindernisse im Seitenraum entfernen & AO & Strecke $(300 \mathrm{~m})$ & - & 60 & 92 & 88 & 74 \\
\hline 34 & Rüttelstreifen aufbringen & $\mathrm{AO}$ & Strecke $(300 \mathrm{~m})$ & - & 80 & 58 & 81 & 76 \\
\hline 35 & Gummischwellen aufbringen & AO & Knotenpunkt & beliebig & 94 & 73 & 74 & 84 \\
\hline 36 & Sperrfläche markieren & 10 & Knotenpunkt & Vz & 27 & 85 & 96 & 83 \\
\hline 37 & Verkehrszeichen versetzen (Erhöhung Erkennbarkeit) & AO & beliebig & beliebig & 31 & 93 & 100 & 85 \\
\hline 38 & Beschränkung Vzul und Z 625 StVO (Richtungstafel in Kurven) & $\mathrm{AO}$ & Strecke $(300 \mathrm{~m})$ & - & 23 & 96 & 96 & 74 \\
\hline 39 & Beschränkung Vzul und sonstige Gefahrenzeichen & AO & Strecke $(300 \mathrm{~m})$ & - & 23 & 100 & 96 & 70 \\
\hline
\end{tabular}

Abbildung 24: Evaluationsergebnisse allgemeine Einschätzung der Maßnahmenkategorien

Die geringste Anzahl auswertbarer Fragebögen weist die MK 18 „Erneuerung Fahrbahnoberfläche“ mit 14 Befragten auf, die höchste Anzahl, mit 94 Befragten, die MK 35 „Gummischwellen aufbringen".

Mit Ausnahme der MK 25 „Sichtschutzzaun aufstellen“ und MK 34 „Rüttelstreifen aufbringen“ sind die vorgestellten MK mehr als zwei Dritteln der befragten Teilnehmer vertraut. Die beiden eben genannten MK werden erst seit wenigen Jahren in der Praxis umgesetzt, daher erscheint der geringe Bekanntheitsgrad plausibel. Es zeigt aber auch, dass das Wissen zu neuen Maßnahmen sich nur langsam verbreitet, was nur zum Teil auf die Behördenzugehörigkeit (z. B. Polizei) zurückzuführen ist. 
Sechs der 35 vorgestellten MK werden von mindestens der Hälfte der Befragten als nicht kostengünstig eingeschätzt. Es sind dreimal Maßnahmen bei denen es sich um einen verkehrstechnischen Eingriff - Aufbau/Veränderung passiver Schutzeinrichtungen oder der Beleuchtung - handelt. Als wenig kostengünstig wird auch die Erneuerung der Fahrbahnoberfläche (MK 18) eingeschätzt, obwohl in der Vorstellung der Maßnahme explizit darauf verwiesen wurde, dass es sich nur um eine Erneuerung der Deckschicht (Verbesserung Griffigkeit) handelt. Ebenfalls als verhältnismäßig kostenintensiv wird das Einrichten einer Ortsfesten Geschwindigkeitsüberwachungsanlage (OGÜ) angesehen. In der Evaluation wurden die Kategorien 16 und 17 zusammengefasst. Beide MK unterscheiden sich lediglich hinsichtlich der Örtlichkeit der OGÜ an Knotenpunkten bzw. auf Strecken. Bei der Betrachtung des Gesamtkollektivs wird deutlich, dass alle MK, die in Zusammenhang mit dem Verändern, Versetzten oder Anbringen von Verkehrszeichen stehen, von nahezu allen Befragten als kostengünstig angesehen werden. Ähnlich hohe Anteilswerte erzielen MK, die den Markierungsarbeiten zuzuordnen sind.

Maßgebend für den möglichen Einsatz einer Maßnahme ist deren erwartete bzw. bekannte Wirksamkeit. Dieser kann sich sowohl auf die Reduktion der Unfallanzahl als auch auf die Unfallschwere beziehen. Drei der vorgestellten MK wurden von der ca. zwei Dritteln oder mehr Befragten als nicht wirksam eingeschätzt. Dazu zählen die MK 3 „sonstige Gefahrenzeichen anbringen (hier keine Gefahrenzeichen mit Hinweis zum folgenden Streckenverlauf)“ und die MK 8/9 „Zusatzzeichen ergänzen (Z 1006-36 StVO Unfallgefahr)“. MK 8 bezieht sich auf den Einsatz Innerorts, MK 9 auf den Außerortsbereich. Interessant erscheint, dass die Beschränkung der $\mathrm{V}_{\mathrm{zul}}$ an durch Verkehrszeichen geregelten Knotenpunkten Außerorts (MK 15) nur von der Hälfte der Befragten als wirksam angesehen wird, während jedoch der gleichen Maßnahme, eingesetzt im Bereich der freien Strecke, von ca. $80 \%$ der Befragten eine Wirkung attestiert wird. Allgemein werden die als nicht kostengünstig eingestuften MK von einem hohen Anteil der Befragten als wirksam eingeschätzt, während die Einschätzungen für $\mathrm{MK}$, die von nahezu allen Beteiligten als kostengünstig eingeschätzt wurden, bezüglich der Wirksamkeit deutlich differenzieren.

Folgende detaillierte Aussagen lassen sich zu ausgewählten MK treffen:

- Bezüglich der MK 3 „sonstige Gefahrenzeichen anbringen (hier keine Gefahrenzeichen mit Hinweis zum folgenden Streckenverlauf)" gaben drei Befragte an, diese Maßnahme auch schon umgesetzt zu haben. Alle drei beurteilen die Maßnahme als wirksam.

- Elf von 51 Befragten setzten während ihrer Tätigkeiten die MK 8/9 „Zusatzzeichen ergänzen (Z 1006-36 StVO Unfallgefahr)“ um. Sechs dieser Elf schätzen diese MK auch als wirksam ein.

- 16 von 37 Befragten gaben an, die MK 15 „Beschränkung $\mathrm{V}_{z u l}$ an Knotenpunkten (AO“) bereits umgesetzt zu haben. Neun von 16 Befragten - mehr als die Hälfte - schätzen die Maßnahme als wirksam ein. Dieser Anteil spiegelt das Antwortergebnis aller Befragten wider.

- Die Erneuerung der Fahrbahnoberfläche (MK 18) wurde von 9 der 14 Befragten als Maßnahme beschlossen. Acht dieser neun Befragten beurteilen diese MK als wirksam, aber nur zwei von innen sehen sie auch als kostengünstig an.

- Lediglich zwei von 23 Befragten haben bereits die MK 25 „Sichtschutzzaun aufstellen“ umgesetzt. Beide Befragte schätzen die MK als wirksam und kostengünstig ein.

Bei der Interpretation dieser Aussagen ist folgendes zu beachten: Es ist nicht bekannt, in welcher Art und Weise die Umsetzung der o. g. MK von Seiten der Befragten erfolgte. Es sind sowohl Umsetzungen als Einzelmaßnahmen als auch im Paket mit weiteren Maßnahmen möglich. Ein eindeutiger Rückschluss von den Einschätzungen der Befragten auf die tatsächliche Wirksamkeit der eruierten MK als Einzelmaßname ist somit nicht möglich.

Die Auswertung der Fragebögen dient darüber hinaus zur Beantwortung folgender Frage: Bis zu welcher Größenordnung der Kriterien 
- (Investitions-)kosten [€],

- zeitliche Umsetzbarkeit [Monaten] und

- verwaltungstechnischer Aufwand

schätzen UKO-Mitglieder Maßnahmen als „kostengünstig“ ein? Darüber hinaus soll geklärt werden, in welcher Dimension die Reduzierung der Kriterien Unfallschwere und/oder Unfallanzahl dazu führt, einer Maßnahme das Prädikat „wirksam“ zu verleihen. Die Evaluation aller vorgestellten MK erlaubt dahingehend eine tendenzielle Einschätzung.

Ausgangsbasis für die Beantwortung beider Sachverhalte bildet die dichotome Beantwortung der Fragen bezüglich der Prädikate „kostengünstig“ und „wirksam“. Für jede MK liegt der Anteil (A) darüber vor, wieviel Befragte die jeweilige MK als „kostengünstig“ und „wirksam“ bewertet haben. Die bewerteten MK wurden hinsichtlich beider Kriterien jeweils in drei Gruppen unterteilt (Tabelle 11). Die Einteilung der Gruppen folgt dabei pragmatischen Überlegungen. Beurteilt mehr als die Hälfte der Befragten eine MK als nicht kostengünstig oder nicht wirksam, ist davon auszugehen, dass diese Maßnahme in der Praxis im Allgemeinen nicht angewendet wird. Daher endet die unterste Klasse bei einer Zustimmungsquote von $A<50 \%$. Eine MK die vorherrschend als kostengünstig oder wirksam angesehen wird, erhält auch vom Großteil der Befragten dieses Prädikat. Als eine hohe Zustimmung gilt pragmatisch daher, wenn die Prädikate jeweils von mindestens vier von fünf Befragten $(A \geq 80 \%)$ verliehen werden. Daher wird bei diesem Anteil ebenfalls eine Grenze festgelegt. Differenziert nach den Prädikaten („kostengünstig“, „wirksam“) und den zugeordneten Kriterien erfolgt für jede Gruppe eine Darstellung der Antwortverteilung der Befragten.

Tabelle 11: Einteilung der Maßnahmenkategorien nach Einschätzung als „kostengünstig“ und als „wirksam“

\begin{tabular}{|c|c|c||c|c|c|}
\hline \multicolumn{2}{|c||}{$\begin{array}{c}\text { Anteil [A] Befragte, welche die MK als } \\
\text { kostengünstig ansehen }\end{array}$} & \multicolumn{3}{c|}{$\begin{array}{c}\text { Anteil [A] Befragte, welche die MK als } \\
\text { wirksam ansehen }\end{array}$} \\
\hline $\begin{array}{c}\text { Gruppen nach } \\
\begin{array}{c}\text { Anteil A (Zustimmende) } \\
{[\%]}\end{array}\end{array}$ & $\begin{array}{c}\text { Mittelwert } \\
\text { Kollektiv } \\
{[\%]}\end{array}$ & $\begin{array}{c}\text { Anzahl } \\
\text { MK } \\
{[\mathrm{n}]}\end{array}$ & $\begin{array}{c}\text { Gruppen nach } \\
\text { Anteil A (Zustimmende) } \\
{[\%]}\end{array}$ & $\begin{array}{c}\text { Mittelwert } \\
\text { Kollektiv } \\
{[\%]}\end{array}$ & $\begin{array}{c}\text { Anzahl } \\
\text { MK } \\
{[\mathrm{n}]}\end{array}$ \\
\hline $80 \leq \mathrm{A}$ & 93 & 22 & $80 \leq \mathrm{A}$ & 89 & 17 \\
\hline $50 \leq \mathrm{A}<80$ & 65 & 4 & $50 \leq \mathrm{A}<80$ & 71 & 11 \\
\hline $50>\mathrm{A}$ & 34 & 4 & $50>\mathrm{A}$ & 28 & 2 \\
\hline *Bewertung über alle vorgestellten MK, Zusammenfassung von MK wie unter Abbildung 24 dargestellt.
\end{tabular}

Abbildung 25 stellt die Klassifizierung der MK nach dem Prädikat „kostengünstige Maßnahme“ über das Kriterium „Kosten“ dar. Die Balkendiagramme lassen sich wie folgt interpretieren: Für die Gruppe A $\geq 80 \%$ (linker Balken) - in der MK vereinigt sind, die von 80 \% oder mehr der Befragten als „kostengünstig“ bewertet wurden - werden die Maßnahmenkosten von $94 \%$ der Befragten (im Mittel) mit einem Volumen von kleiner $10.000 €$ eingeschätzt. Kosten von unter $2.500 €$ vermuten $68 \%$ der Befragten. Für die Gruppe der vier Maßnahmenkategorien $\geq 50 \% A<80 \%$ (mittlerer Balken) schätzen $74 \%$ der Befragten die Kosten auf " $<10.000 €^{\prime \prime}$, jedoch nur $19 \%$ auf "<2.500 $€^{\prime \prime}$. Wird eine MK von weniger als der Hälfte der Befragten als „kostengünstig“ eingeschätzt (rechter Balken), ist auch der Anteil derjenigen Befragten kleiner, welche die Maßnahme in einem Kostenspektrum von „<10.000 €“ ansiedeln. Die im Diagramm angegebenen Mittelwerte beziehen sich auf den Mittelwert der Zustimmungen zum Prädikat „kostengünstig“. Bei der Interpretation ist zu beachten, dass in den Gruppierung Abhängigkeiten bestehen. Die Höhe der Anteilswerte in den einzelnen Gruppen ist abhängig von den in den Gruppen enthaltenen MK. Die Betrachtung anderer oder weiterer Maßnahmen kann zu einer Verschiebung der Anteilswerte führen.

Über alle MK korreliert der Anteil der Nennungen „kostengünstig“ für eine Maßnahme, mit dem Anteil der Nennungen für Kosten „<2.500 $€$ “ hoch $(r=0,83)$. Die Aggregation der Nennungen für Kosten "<2.500 $€$ " und " $<10.000 €$ " führt zu einer stärkeren Korrelation mit $r=0,95$. MK können daher annähernd als „kostengünstig“ eingestuft werden - unter Berücksichtigung der gewählten Kosteneinteilung -, wenn deren Kosten die Grenze von $10.000 €$ nicht überschreiten. 


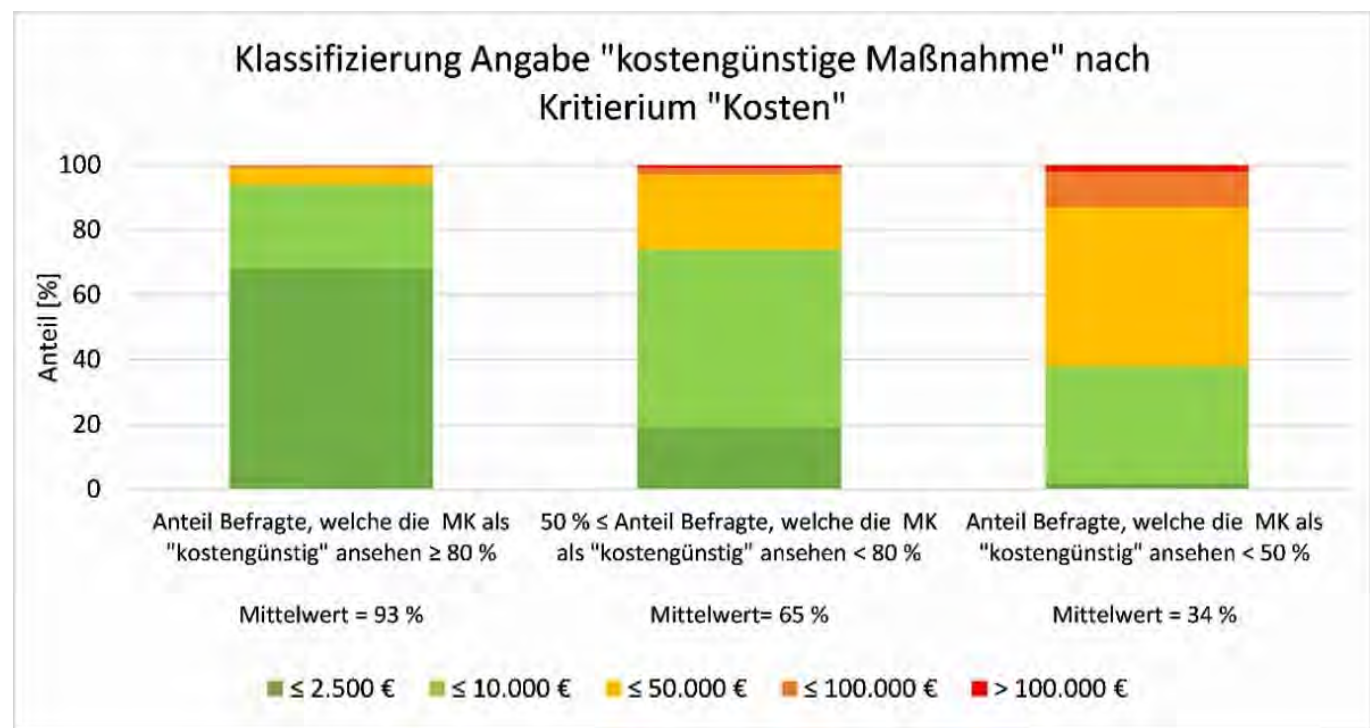

Abbildung 25: Klassifizierung für Prädikat „kostengünstige Maßnahme“ nach Kriterium „Kosten“

Für das Kriterium „zeitliche Umsetzbarkeit“ ergibt sich für die drei Gruppen ein ähnliches Bild (Abbildung 26). MK, die von mindestens $80 \%$ der Befragten als „kostengünstig“ bewertet wurden, werden mit einer schnellen „zeitlichen Umsetzbarkeit“ in Zusammenhang gebracht. Im Mittel geben $91 \%$ der Befragten an (grüner und hellgrüner Anteil), dass „die zeitliche Umsetzbarkeit" einen Zeitraum von „ $\leq 6$ Monaten“ nicht überschreitet. Der Anteil für Umsetzungsdauern „ $\leq 3$ Monate“ liegt bei $70 \%$. Je weniger die MK als „kostengünstig“ bewertet wurde, desto ausgeprägter ist der Anteil derjenigen, welche die Maßnahmenumsetzung mit einem höheren zeitlichen Aufwand in Verbindung bringen. Ähnlich den Kosten korreliert der Anteil der Nennungen „kostengünstig“ mit dem aggregierten Anteil der Nennungen für zeitliche Umsetzungen von „ $\leq 3$ Monate“ und „ $\leq 6$ Monate“ am deutlichsten $(r=0,92)$.

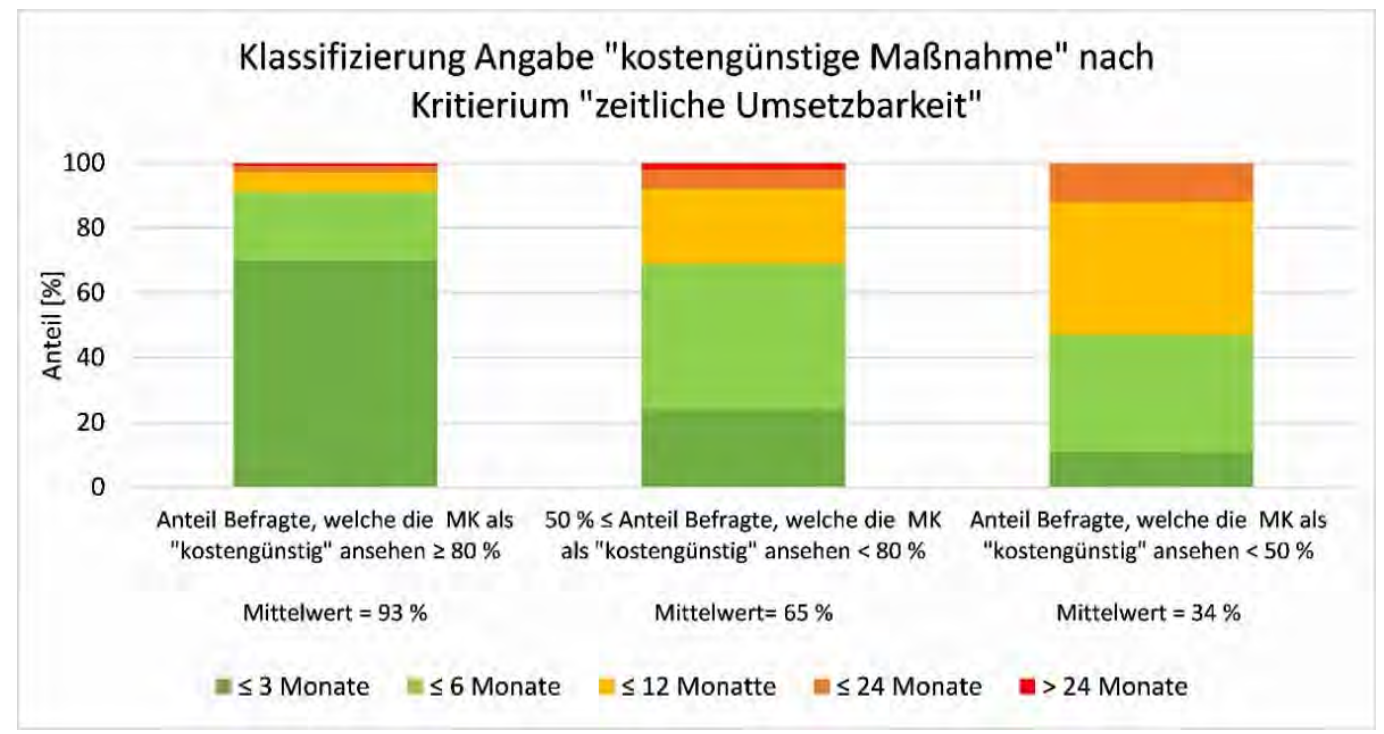

Abbildung 26: Klassifizierung für Prädikat „kostengünstige Maßnahme“ nach Kriterium "zeitliche Umsetzbarkeit"

Ein „geringer" bis „eher geringer" verwaltungstechnischer Aufwand ( $\Sigma 92 \%)$ wird von den Befragten für die Umsetzung von MK eingeschätzt, die von mehr als 80 \% der Befragten als „kostengünstig" eingestuft wurden (Abbildung 27). Gegenüber den beiden o. g. Kriterien bleibt der Anteil der tendenziell positiven Einschätzungen (Aufwand „gering“ und „eher gering") mit $56 \%$ auch für die Gruppe $A<50 \%$ relativ hoch, in der die MK vorliegen, die von mehr als der Hälfte nicht als „kostengünstig" eingeschätzt wurden. 


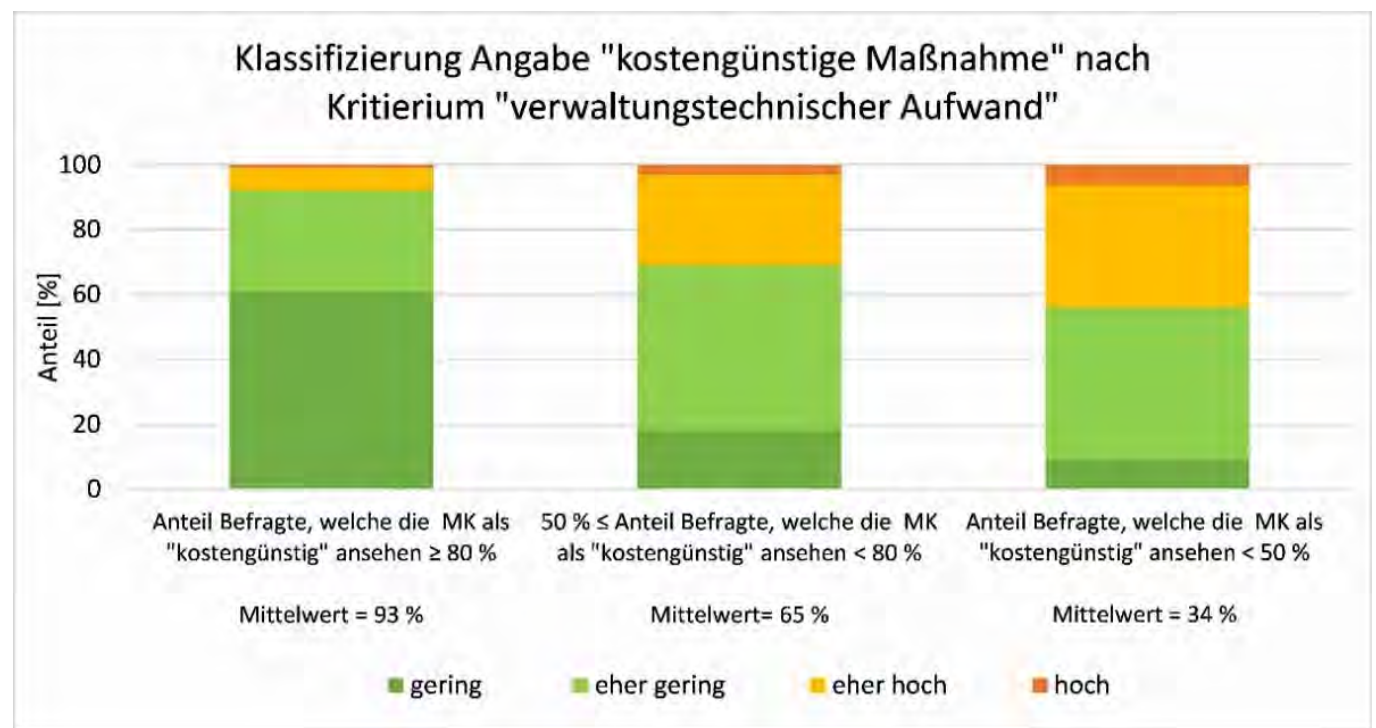

Abbildung 27: Klassifizierung für Prädikat „kostengünstige Maßnahme“ nach Kriterium „verwaltungstechnischer Aufwand“

Für das Prädikat „wirksam“ liegt die Antwortverteilung nach den Kriterien der Reduzierung der „Unfallschwere" und „Unfallanzahl“ vor. Für die Gruppe A $\geq 80 \%$ gaben in Mittel ca. $71 \%$ der Befragten an, dass die Unfallschwere mindestens stark oder mäßig reduziert wird (Abbildung 28). Dieser Anteil sinkt in der zweiten Gruppe (50\% $\leq \mathrm{A}<80 \%)$ geringfügig auf $62 \%$ und fällt erwartungsgemäß in der letzten Gruppe deutlich geringer aus. Für MK, die von über der Hälfte der Befragten als nicht wirksam eingeschätzt wurden, wird von ca. $81 \%$ der Befragten angegeben, dass die Abnahme der Unfallschwere nur wenig bis überhaupt nicht erfolgt.

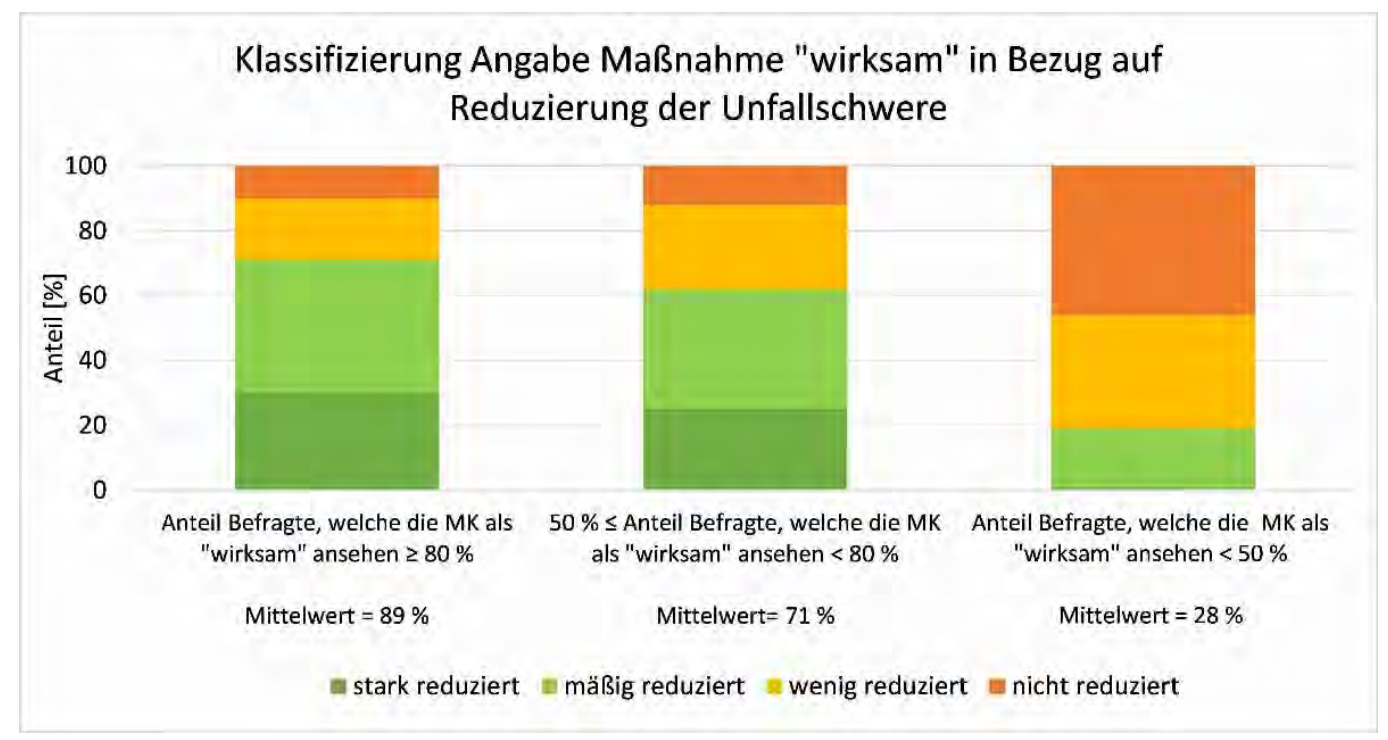

Abbildung 28: Klassifizierung für Prädikat „wirksam“ nach Kriterium „Reduzierung der Unfallschwere“

Der Anteil der Zustimmung zur Aussage: die Maßnahme sei „wirksam“, ist im Vergleich aller Antwortmöglichkeiten am stärksten mit dem Anteilswert der Klasse „die Unfallschwere wird stark reduziert" korreliert $(r=0,45)$. Der Korrelationskoeffizient ist niedriger, als es bei den Aussagen zum Prädikat „kostengünstig“ der Fall ist. Dies resultiert unter anderem daraus, dass die Wirkung einer Maßnahme auf die Reduzierung der Unfallschwere und/oder der Unfallanzahl abzielen kann. D. h., eine MK kann als wirksam eingeschätzt werden, jedoch beruht diese Einschätzung bspw. aber nur auf dem Rückgang der Unfallzahl. Daher beruht auch der relativ hohe Anteil bezüglich der Klassen „wenig reduziert" oder „nicht reduziert" auf der Gruppe A $\geq 80 \%$. 
Für das Kriterium „Reduzierung der Unfallanzahl“ unterscheiden sich die Antwortverteilungen nur geringfügig (Abbildung 29). Für die Gruppe $A \geq 80 \%$ liegt der Anteil derjenigen die mit den jeweiligen MK eine starke oder mäßige Reduzierung der Unfallanzahl in Zusammenhang bringen im Mittel bei $78 \%$. Dieser Anteil sinkt in der mittleren Gruppe auf $64 \%$, für die MK mit $A<50 \%$ auf im Mittel nur $26 \%$. Die Klasse „stark reduziert“ ist am stärksten mit dem Anteil der Zustimmung zur Aussage: „die Maßnahme ist wirksam“ korreliert $(r=0,48)$. Fasst man die Gruppen „stark reduziert" und "mäßig reduziert" zusammen, ergibt sich ein Korrelationskoeffizient von $r=0,64$.

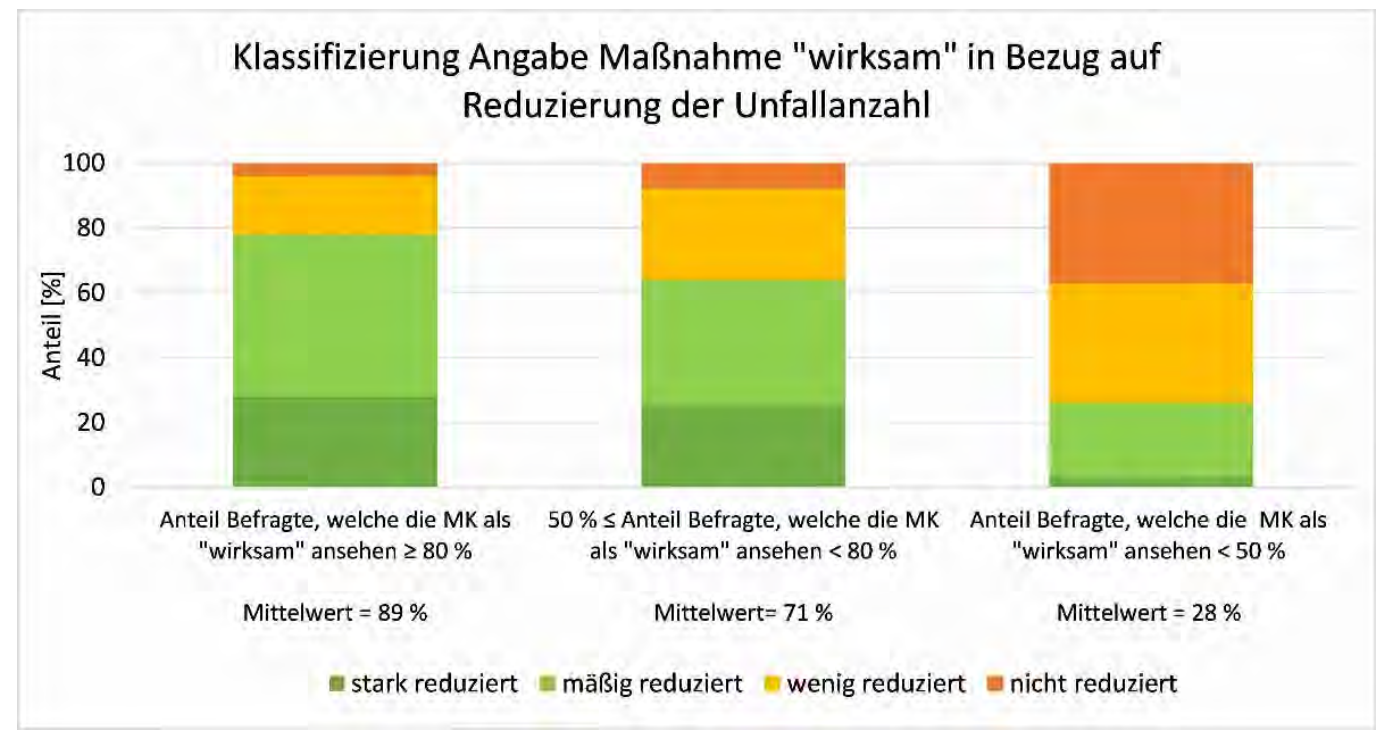

Abbildung 29: Klassifizierung für Prädikat „wirksam“ nach Kriterium „Reduzierung der Unfallanzahl“

\subsection{Zusammenfassung}

Die Evaluationen der vorgestellten MK durch die Mitglieder der UKO erlauben einen Einblick in die Einschätzung von Maßnahmen. Sie stellt neben der Unfalldatenanalyse eine Säule der Maßnahmenbeurteilung dar. Es konnten Aussagen darüber getroffen werden, unter welchen Größenordnungen von verschiedenen Aspekten Maßnahmen als kostengünstig und/oder wirksam eingeschätzt werden. Die Ergebnisse dieses Arbeitspakets liegen sowohl für jede MK einzeln in einem Faktenblatt zur Maßnahmenevaluation als auch aggregiert für alle MK vor.

Für den allgemeinen Vergleich der MK ist zusammenfassend festzustellen, dass die Ergebnisse weitgehend dem allgemeinen Kenntnisstand und Erwartungen in der Fachwelt folgen. Die Änderung oder Anpassung von lediglich Beschilderung wird in Zusammenhang mit geringen Kosten gestellt, allerdings differenziert die Einschätzung zum Aspekt der Wirksamkeit in Abhängigkeit der Inhalte der Beschilderung erheblich. Auch Markierungsarbeiten werden überwiegend als kostengünstig angesehen, während hingegen bauliche und verkehrstechnische Maßnahmen - wie im Bereich der passiven Schutzeinrichtungen - dieses Prädikat nur selten erhalten. Dementgegen werden diese MK vom überwiegenden Teil der Befragten als wirksam eingeschätzt. Relativ neue MK sind einem Teil der Befragten noch nicht bekannt.

Die vertiefende Analyse verdeutlicht, dass der überwiegende Teil der Befragten MK bis zu (Investitions-)kosten von ca. $10.000 €$ als kostengünstig bewertet. Kostengünstige Maßnahmen werden deutlich mit einer schnellen zeitlichen Umsetzbarkeit (ca. $\leq 6$ Monate) und tendenziell mit einem geringen verwaltungstechnischen Aufwand in Zusammenhang gebracht.

Aussagen zur Wirksamkeit sind aufgrund der geteilten Wirkung auf Unfallschwere und/oder Unfallzahl nur eingeschränkt möglich. Die Daten lassen dennoch den Schluss zu, dass Maßnahmen das Prädikat „wirksam“ unterstellt wird, wenn sie mindestens „mäßig“ zur Reduzierung von Unfallanzahl und/oder Unfallschwere beitragen. Die ordinalen Skalierungen des Evaluationsbogens zur Fragen der Wirksamkeit erlauben jedoch keine direkten Rückschlüsse auf absolute Größen. 


\section{$6 \quad$ Unfalldatenanalyse der Maßnahmenkategorien}

\subsection{Vorgehen}

\subsubsection{Allgemein}

Die Unfalldaten- und Kostenanalyse erlaubt eine objektive Beurteilung der Wirksamkeit der Maßnahmenkategorien anhand der Gegenüberstellung des Unfallgeschehens im Vorher- und NachherZeitraums. Auf Basis der übermittelten oder aus anderen Quellen übernommenen (Investitions-) kosten einerseits und der vermiedenen Unfallkosten andererseits lässt sich die Wirtschaftlichkeit der Maßnahmenkategorien über Nutzen-Kosten-Verhältnisse (NKV) annähernd beschreiben. Basis der Untersuchung bilden die 35 Maßnahmenkategorien (MK, Vergleiche Tabelle 8, Kapitel 4.3.3). Die Unfalldatenanalyse dient in Kombination mit der volkswirtschaftlichen Betrachtung als Grundlage der Bewertung und Empfehlungen der MK für den Anwender über ein Gesamtprädikat.

\subsubsection{Anpassung des Kollektivs}

Die Maßnahmen (Stellen), die in jeweiligen MK klassifiziert und berücksichtigt sind, müssen bezüglich ihrer Datengrundlage folgenden Ansprüchen genügen:

- Eindeutige Identifikation der Örtlichkeit

- Eindeutige Beschreibung der Maßnahme (als Einzelmaßnahme (Ausnahme stellen die Pakete der MK 38 und 39 dar))

- Kenntnis über das Umsetzungsdatum

- Unfallgeschehen im Vorher-Zeitraum liegt über 3 Jahre (mindestens aber 12 Monate) vor

- Unfallgeschehen im Nachher-Zeitraum liegt über 3 Jahre (mindestens aber 12 Monate) vor

Von den angestrebten Untersuchungszeiträumen von jeweils drei Jahren in der Vor- bzw. Nachbetrachtung wurde für MK mit allgemein geringer Datengrundlage dann abgewichen, wenn auch Maßnahmen vorhanden waren, bei denen auch aus kürzeren Zeiträumen eine eindeutige Entwicklung des Unfallgeschehens erkennbar wurde. Für jede MK sind die Eingangsgrößen in einem Faktenblatt dargestellt (Vergleiche Kapitel 6.2.1). Um die Vergleichbarkeit der untersuchten Stellen in einer MK als auch der MK untereinander zu gewährleisten, sind im Vorfeld verschiedene Anpassungen und Normierungen notwendig.

Das Unfallgeschehen der Vorher- und Nachher-Zeiträume ist auf ein Jahr normiert. Dies erlaubt den Vergleich von mehreren Stellen einer MK, wenn unterschiedliche Betrachtungszeiträume vorliegen. Die genaue Vorgehensweise ist nachfolgend beschrieben. Qualität und Umfang der aufgenommenen Unfalldaten unterscheiden sich zwischen den UKO, auch in Abhängigkeit der Ländererlasse. So liegen auch Daten von UH aus Bundesländern vor, in denen Unfälle der Unfallkategorie 5 (sonstiger Unfall mit Sachschaden) nicht oder nur bei dem Vorliegen eines Straftatbestands aufgenommen werden. Mitunter wurden von UKO in den Akten auch nur Unfälle mit Personenschaden $(\mathrm{U}(\mathrm{P}))$ übermittelt. Somit ergeben sich die in Tabelle 12 dargestellten Klassifizierungen in der Auswertung der Unfallkategorien.

Um die Aussagekraft über die Wirksamkeit einer MK zu erhalten, wäre die Aggregation unterschiedlicher Unfallkategorien - wenn sie denn so in einer MK vorliegen - nicht zielführend. Daher werden in der Bewertung einer MK die Unfallkategorien bewertet, deren Auswertung nach dem Prinzip des kleinsten gemeinsamen Nenners möglich ist. Liegen z. B. in einer MK nur U(P, SS, LS) oder $U(P, S)$ für alle Einzelfälle vor, erfolgt die Bewertung auf Basis der $U(P, S)$. Unfälle der Kategorie 4 bis 6 werden zusammengefasst ${ }^{11}$. Somit enthalten die MK je nach Datengrundlage unterschiedliche Unfallkategorien als Bewertungsgrundlage.

11 Für MK mit 6 Stellen wurde - wenn nur eine Stelle eine andere Kategorie aufwies und die Art der Berechnung als vertretbar eingeschätzt wurde - in der Bewertung in Einzelfällen auf die Unfallkategorien 
Tabelle 12: Klassifizierungen der Unfallkategorien nach Datenlage

\begin{tabular}{|c|c|c|}
\hline $\begin{array}{l}\text { Bezeichnung } \\
\text { (U.-Kateqorien) }\end{array}$ & \multicolumn{2}{|c|}{ Zuordnung der einzelnen Unfallkategorien } \\
\hline $\mathrm{U}(\mathrm{P})$ & - Kat. 1 bis 3 & Unfälle mit Personenschaden U(P) \\
\hline $\mathrm{U}(\mathrm{P}, \mathrm{SS})$ & $\begin{array}{l}\text { - Kat. } 1 \text { bis } 3 \\
\text { - Kat. } 4 \text { und } 6\end{array}$ & $\begin{array}{l}\text { Unfälle mit Personenschaden } U(P) \\
\text { Schwerwiegender Unfall mit Sachschaden, übrige Sachschadensunfälle mit be- } \\
\text { rauschenden Mitteln U(SS) }\end{array}$ \\
\hline $\mathrm{U}(\mathrm{P}, \mathrm{SS}, \mathrm{LS})$ & $\begin{array}{l}\text { - Kat. } 1 \text { bis } 3 \\
\text { - Kat. } 4 \text { und } 6 \\
\text { - Kat. } 5\end{array}$ & $\begin{array}{l}\text { Unfälle mit Personenschaden } U(P) \\
\text { Schwerwiegender Unfall mit Sachschaden, übrige Sachschadensunfälle mit be- } \\
\text { rauschenden Mitteln } U(S S) \\
\text { Sonstiger Unfall mit Sachschaden } U(L S)\end{array}$ \\
\hline $\mathrm{U}(\mathrm{P}, \mathrm{S})$ & $\begin{array}{l}- \text { Kat. } 1 \text { bis } 3 \\
\cdot \mathrm{U}(\mathrm{S})\end{array}$ & $\begin{array}{l}\text { Unfälle mit Personenschaden } U(P) \\
\text { Unfälle mit Sachschaden (Differenzierung nach Kategorien } 4 \text { bis } 6 \text { nicht möglich) }\end{array}$ \\
\hline
\end{tabular}

Die Berechnung der Wirtschaftlichkeit von Maßnahmenumsetzungen fußt auf dem Vergleich von (Investitions-)kosten und der Differenz der Unfallkosten von Vorher- und Nachher-Zeitraum, normiert auf ein Jahr. Die zugrunde gelegten (Investitions-)kosten ${ }^{12}$ werden über die für die Maßnahmen üblichen Annuitätenfaktoren, auf jährliche Kosten angepasst [GERLACH et al. 2009, SPAHN 2011, MAIER et al. 2015]. Zur Berechnung der Unfallkosten werden die aktuellen Unfallkostensätze des M Uko [FGSV 2012] getrennt nach den Ortslagen außerorts und innerorts herangezogen (Vergleiche Tabelle 3, Kapitel 2.2.1). Für den innerörtlichen Bereich wird auf die einheitlichen Kostensätze (KS) für alle Straßenkategorien zurückgegriffen. Zur besseren Beschreibung der Maßnahmenwirkung auf die Unfallschwere erfolgt für die $U(P)$ die Verwendung der Kostensätze für Unfälle mit Getöteten oder Schwerverletzten $\mathrm{KS}_{U(\mathrm{SP})}$ sowie für Unfälle mit Leichtverletzten $\mathrm{KS}_{U(\mathrm{LV})}$. Die Gruppe der Unfälle mit Sachschaden sind je nach Kategorie mit den entsprechenden Kostensätzen berechnet.

Jede Maßnahmenkategorie ist einer Maßnahmengruppe zugeordnet (Vergleiche Kapitel 4). Die Maßnahmengruppen dienen primär zur Ordnung des Kollektivs und geben erste Hinweise, auf welche Bereiche die Maßnahmenumsetzung abzielt bzw. in welcher Weise diese erfolgt. Die Gruppeneinteilung lässt in Einzelfällen offen, in welcher Art die Maßnahmenumsetzung erfolgt. Z. B. können geschwindigkeitsregulierende Maßnahmen sowohl durch das Aufstellen von Verkehrszeichen als auch durch ortsfeste Geschwindigkeitsüberwachungen erfolgen. Die technische Umsetzung einer Einzelmaßnahme wird hingegen besser durch die Art der Maßnahme beschrieben. Klassische Beispiele sind Markierungsarbeiten, Eingriffe in die Signalsteuerung oder Maßnahmenumsetzungen die sich auf das Aufstellen, Umstellen oder Verändern von Verkehrszeichen beschränken. Es soll geprüft werden, ob bestimmte Arten der Maßnahmensetzung besonders wirtschaftlich sind. Daher werdend die MK nach der Art des Maßnahmeneingriffs deklariert (Tabelle 13).

Maßnahmenumsetzungen haben das Ziel, Sicherheitsdefizite in der Verkehrsanlage zu beseitigen, mindestens aber ihrer Folgen auf das Unfallgeschehen zu minimieren. Unter Umständen lassen sich durch Maßnahmenumsetzungen mehrere Defizite (Ursachen) beseitigen. In der Regel stellt eine Maßnahme aber auf ein primäres Defizit ab. Defizite entstehen, wenn folgende Ansprüche an eine Verkehrsanlage nicht oder nur eingeschränkt erfüllt werden können:

- Erkennbarkeit

- Begreifbarkeit

- Sicht

- Befahrbarkeit/Begehbarkeit

zurückgegriffen, die am häufigsten in einer MK vorlagen. Diese Anpassungen sind im Faktenblatt unter "Allgemeine Hinweise" beschrieben.

12 "Sollten es Maßnahmenumsetzungen erforderlich machen, wurden in der Annuität auch die jährlichen veränderten Betriebskosten berücksichtigt. 
Mitunter lassen sich die Defizite nicht nur einem dieser Ansprüche zuordnen bzw. sind die Ansprüche zu präzisieren. Im Rahmen der Untersuchungen werden weitere Ursachen (Ansprüche) aufgenommen. Sie dienen als Beschreibung der Hauptursache - Mangel der Verkehrsanlage oder im Verkehrsablauf -, aus der ein Defizit resultiert. Dem entsprechend zielt die Maßnahme auf die Beseitigung dieser Ursache bzw. Mangels ab. Zu hohe "Geschwindigkeiten" stellen das Problem eines unangepassten Fahrverhaltens dar. Dies resultiert häufig aus der Kombination von mangelnder Erkennbarkeit, Begreifbarkeit und Sicht. Insbesondere an Knotenpunkten führen Mängel dieser Ansprüche zum Auftreten von UH. Maßnahmen im Untersuchungskollektiv, die sich nicht konkret einem dieser Ansprüche zuordnen lassen, zielen häufig auf eine Ordnung, Vereinfachung oder eindeutige Strukturierung (Standardisierung) der Verkehrsführung bzw. des Verkehrsablaufs ab. Diese MK werden unter der Ursache „Verkehrsablauf“ erfasst. Weiterhin existieren MK, deren primäres Ziel die Reduzierung der Unfallfolgen darstellt. Häufig stehen damit Maßnahmen in Zusammenhang mit der Veränderung oder Anpassung des Seitenraums. MK, mit dem Ziel eine Verbesserung des Zustands der Verkehrsanlage zu erreichen, werden ebenfalls unter der Ursache „Zustand/Umfeld“ aggregiert.

Tabelle 13: Maßnahmenkategorie MK, Art des Maßnahmeneingriffs und Primärdefizit

\begin{tabular}{|c|c|c|c|}
\hline Nr. & Maßnahmenkategorie & $\begin{array}{c}\text { Art des } \\
\text { Maßnahmen- } \\
\text { eingriffs }\end{array}$ & Primärdefizit \\
\hline 1 & Verbesserung der Koordinierung & LSA-Steuerung & Verkehrsablauf \\
\hline 2 & Änderung der Signalisierung & LSA-Steuerung & Verkehrsablauf \\
\hline 3 & "sonstige" Gefahrenzeichen anbringen & Vekehrszeichen & Begreifbarkeit \\
\hline 4 & HALT Z 206 StVO (anstelle Z 205 StVO) & Vekehrszeichen & Begreifbarkeit \\
\hline 5 & HALT Z 206 StVO (anstelle Z 205 StVO) & Vekehrszeichen & Begreifbarkeit \\
\hline 6 & Halte-/Parkverbot anordnen & Vekehrszeichen & Sicht \\
\hline 7 & Z625 StVo (Richtungstafel in Kurven) aufstellen & Vekehrszeichen & Erkennbarkeit \\
\hline 8 & Zusatzzeichen Z 1006-36 StVo (Unfallgefahr) ergänzen & Vekehrszeichen & Erkennbarkeit \\
\hline 9 & Zusatzzeichen Z 1006-36 StVo (Unfallgefahr) ergänzen & Vekehrszeichen & Erkennbarkeit \\
\hline 10 & Verkehrszeichen anpassen & Vekehrszeichen & Erkennbarkeit \\
\hline 11 & Verkehrszeichen anpassen & Vekehrszeichen & Erkennbarkeit \\
\hline 12 & Beschränkung Vzul & Vekehrszeichen & Geschwindigkeit \\
\hline 15 & Beschränkung Vzul & Vekehrszeichen & Geschwindigkeit \\
\hline 16 & Überwachung zulässige Höchstgeschwindigkeit mit OGÜ & Überwachung & Geschwindigkeit \\
\hline 17 & Überwachung zulässige Höchstgeschwindigkeit mit OGÜ & Überwachung & Geschwindigkeit \\
\hline 18 & Erneuerung Fahrbahnoberfläche & baul. Eingriff & Zustand/Umfeld \\
\hline 19 & Zusätzlichen Signalgeber anordnen & LSA-Steuerung & Erkennbarkeit \\
\hline 21 & Leuchtmittel Signalgeber verbessern & LSA-Steuerung & Erkennbarkeit \\
\hline 22 & Sichthindernisse entfernen & techn. Eingriff & Sicht \\
\hline 23 & Sichthindernisse entfernen & techn. Eingriff & Sicht \\
\hline 24 & Beleuchtung anpassen/verbessern & baul. Eingriff & Erkennbarkeit \\
\hline 25 & Sichtschutzzaun aufstellen & techn. Eingriff & Sicht \\
\hline 26 & Markierung anpassen/verbessern & Markierung & Begreifbarkeit \\
\hline 27 & Markierung in Stand setzen & Markierung & Begreifbarkeit \\
\hline 29 & Radverkehrsführung anpassen & Markierung & Verkehrsablauf \\
\hline 30 & Radfahrfurt Rot einfärben & Markierung & Erkennbarkeit \\
\hline 31 & Passive Schutzeinrichtung aufstellen & techn. Eingriff & Zustand/Umfeld \\
\hline 32 & Unterfahrschutz an passiver Schutzeinrichtung anbringen & techn. Eingriff & Zustand/Umfeld \\
\hline 33 & Hindernisse im Seitenraum entfernen & techn. Eingriff & Zustand/Umfeld \\
\hline 34 & Rüttelstreifen aufbringen & Markierung & Geschwindigkeit \\
\hline 35 & Gummischwellen aufbringen & techn. Eingriff & Verkehrsablauf \\
\hline 36 & Sperrfläche markieren & Markierung & Verkehrsablauf \\
\hline 37 & Verkehrszeichen versetzen (Erhöhung Erkennbarkeit) & Vekehrszeichen & Erkennbarkeit \\
\hline 38 & Beschränkung Vzul und Z 625 Stvo (Richtungstafel in Kurve) & Vekehrszeichen & Geschwindigkeit \\
\hline 39 & Beschränkung Vzul und sonst. Gefahrenzeichen & Vekehrszeichen & Geschwindigkeit \\
\hline
\end{tabular}

Das Ziel der Zuordnung übergeordneter bzw. primärer Defizite besteht darin, zu überprüfen, ob MK gegenüber einem Defizit als besonders wirksam oder wirtschaftlich in Erscheinung treten. Aus den 
Erkenntnissen lassen sich weitere Empfehlungen für den Anwender ableiten Die Zuordnung der MK enthält Tabelle 13.

\subsubsection{Berechnungsgrundlagen}

Die Maßnahmenwirkung kann sowohl auf die Reduzierung der Unfallanzahl als auch der Unfallschwere abzielen. Letztgenannte Wirkung spiegelt sich in einer Verschiebung der Unfälle in andere Kategorien wider. Um beide Wirkungsarten zu berücksichtigen, ist die Bewertung der Maßnahmenwirkung lediglich auf Basis der Unfallanzahl nicht ausreichend. Die Beurteilung der Wirkung einer MK beruht daher auf der Betrachtung von Veränderungen bei Unfallanzahl und Unfallkosten. Über die Unfallkosten(-sätze) wird die Schwere der Unfälle abgebildet und beziffert [FGSV 2012].

\section{Wirkungsgrade}

Während sich die Wirkung einzelner Maßnahmen (einzelne Stelle) direkt aus der Gegenüberstellung der Unfallzahlen und der Unfallkosten von Vorher- und Nachher-Zeitraum bestimmen lässt, so obliegt die Wirkungseinschätzung einer MK (mehrere Stellen) verschiedenen Randbedingungen. Die Wirkung einer Maßnahme ist immer abhängig von örtlichen Gegebenheiten. So ist es möglich, dass die gleiche Maßnahme, eingesetzt bei sehr ähnlichen $\mathrm{UH}$, unterschiedliche Wirkungen entfaltet. Dies begründet sich zum einen aus den vorliegenden - mitunter in verschiedener Ausprägung auftretenden - Defiziten der Verkehrsanlage als auch über das zeitliche Auftreten von Unfällen als Zufallsereignis, welches über die Poisson-Verteilung beschrieben werden kann. Erst aus der Betrachtung mehrerer Maßnahmen (Stellen) lässt sich die mittlere zu erwartende Wirkung der Maß nahmen(-kategorie) beschreiben. Bei der Berechnung der Wirkung ist bei der gewählten Vorgehensweise zu beachten, dass kleine Kollektive maßgeblich von Ausreißern beeinflusst werden. Die Aggregation von Unfallzahlen oder Unfallkosten einer MK schließt den Einfluss von Ausreißern nicht aus. Sie hat überdies zur Folge, dass UH mit hohem Unfallgeschehen das Gesamtergebnis maßgebend prägen und die Entwicklungen von UH mit per se geringem Unfallgeschehen überlagern bzw. dominieren. Daher wird zur Berechnung der Maßnahmenwirkung einer MK auf die Wirkungsgrade (in Prozent) der einzelnen Stellen zurückgegriffen (GI. 6-1). Somit werden im Vorfeld die unterschiedlichen Unfallniveaus normiert. Gleiches gilt bei der Berechnung der Wirkung auf die Unfallkosten (Gl. 6-2).

$\mathrm{MW}_{U}=\frac{\sum_{i}\left(\left(1-\frac{U N}{U V}\right) * 100\right)}{i}$

mit:

MW $\quad$ Maßnahmenwirkung auf die Unfallanzahl [\%]

UN Unfälle Nachher-Zeitraum [-]

UV Unfälle Vorher-Zeitraum [-]

i Betrachtete Stellen

Die Größenordnung der berechneten Mittelwerte $\mathrm{MW}_{\cup}$ und $\mathrm{MW}_{\mathrm{Uk}}$ kann ebenfalls noch von Ausreißern beeinflusst sein. Ist Z. B. für eine MK an vier Stellen ein Rückgang der Unfälle um 20 \%, jedoch an einer Stelle ein Zuwachs von $60 \%$ festzustellen, so ergibt sich eine mittlere MWu von $4 \%$, obwohl der positive Einfluss der Maßnahme an vier von fünf Stellen belegt ist. Zur Verdeutlichung dieses möglichen Effekts und zur Verbesserung der Interpretation wird, neben der MWU, der Median (50\%-Perzentil) über die Wirkungsgrade der einzelnen Stellen ermittelt. Dies gilt für MKKollektive mit einer Stellenanzahl $\mathrm{i} \geq 5$. Im betrachteten Beispiel beträgt der Median dementsprechend einer Maßnahmenwirkung von $20 \%$. 
$\mathrm{MW}_{U K}=\frac{\sum_{i}\left(\left(1-\frac{U K N}{U K V}\right) * 100\right)}{i}$

mit:

MW $\quad$ Maßnahmenwirkung auf die Unfallkosten $\quad[\%]$

UKN Unfallkosten Nachher-Zeitraum [€]

UKV Unfallkosten Vorher-Zeitraum [€]

i Betrachtete Stellen

Die Angabe eines mittleren Wirkungsgrades und dem entsprechenden Median einer MK erlauben keinen Rückschluss auf eine homogene oder heterogene Maßnahmenwirkung. Dies bedeutet, die Streuung der Einzelwerte wird nicht mit abgebildet und es ist unklar, ob eine homogene - geringe Streuung - oder eine heterogene - hohe Streuung - Maßnahmenwirkung vorliegt. Auch dieser Effekt spielt sowohl bei der Entwicklung der Unfallanzahl als auch den Unfallkosten eine Rolle. MK mit einem positiven homogenen Wirkungsgrad können als stabil bezüglich ihrer Wirkung für gegebene Einsatzorte bezeichnet werden. Hingegen sind für den Einsatz von MK mit heterogenen Wirkungsgraden die Randbedingungen (Örtlichkeit) besonders zu prüfen. Deshalb wird bei der Darstellung der Wirkungsgrade die Standardabweichung von $\mathrm{MW}_{\cup}$ und $\mathrm{MW}_{\mathrm{Uk}}$ mit angegeben. Eine skalierte Einteilung und grafische Aufbereitung der einzelnen Wirkungsgrade aller Stellen einer MK für Unfallanzahlen und Unfallkosten erlaubt eine zielgerichtete und schnelle Interpretation der Maßnahmenwirkung einer MK. Tabelle 14 zeigt die gewählte Skalierung. Die Größenordnung der einzelnen Klassen ist u. a. am Maßnahmenkatalog gegen Unfallhäufungen [MAIER et al. 2015] angelehnt. Dieser enthält eine dreiteilige Klassierung wirksamer Maßnahmen:

- „niedrigwirksam“ bis zu einem Wirkungsgrad von $33 \%$,

- „mittelwirksam“ bis zu einem Wirkungsgrad von $66 \%$ und

- "hochwirksam“ ab einem Wirkungsgrad von > $66 \%$ und höher.

Tabelle 14: Skalierung/Einteilung Wirkungsgrade für die Entwicklung von Unfallanzahl und Unfallkosten

\begin{tabular}{|c|c|c|c|c|}
\hline \multicolumn{2}{|r|}{ Klassen } & \multicolumn{2}{|c|}{ Grenzen } & \multirow{2}{*}{$\begin{array}{l}\text { Grafische Darstellung } \\
\text { für eine MK } \\
\text { (Beispiel Unfallanzahl U(S)) }\end{array}$} \\
\hline Farbe & Prädikat & $\begin{array}{c}\text { obere Grenze } \\
\text { in [\%] }\end{array}$ & $\begin{array}{c}\text { untere Grenze } \\
\text { in [\%] }\end{array}$ & \\
\hline & bedeutend & 100 & 60 & \multirow{5}{*}{$\mathrm{U}(\mathrm{S}), 6$ Stellen } \\
\hline & erheblich & $<60$ & 30 & \\
\hline & gering & $<30$ & 10 & \\
\hline & neutral & $<10$ & -10 & \\
\hline & nachteilig & $<-10$ & offen & \\
\hline
\end{tabular}

Ausgangspunkt für die in der Untersuchung festgelegte Klassenbildung ist der "neutrale“ Wirkungsbereich. Hier zeigen sich weder negative noch positive Entwicklungen nach der Maßnahmenumsetzung. Ermittelte Wirkungsgrade von $<-10 \%$ werden als „nachteilig“ eingestuft. Oberhalb der „neutralen“ Klasse schließen sich drei weitere Klassen an. Die letzte Klasse („bedeutend“) weist gegenüber den anderen Klassen die höchste Spannbreite auf. Dahinter steht der Gedanke, dass ab einer bestimmten Größenordnung des Wirkungsgrades Maßnahmen aus praktischer Sicht allgemein als sehr geeignete Maßnahmen beschrieben werden. In der Untersuchung wird ein deutlicher Rückgang der Unfallzahlen oder Unfallkosten von mehr als der Hälfte mit einer „bedeutenden" Maßnahmenwirkung in Zusammenhang gebracht. 


\section{Nutzen-Kosten-Verhältnis}

Während die Wirkungsgrade $\mathrm{MW}_{\cup}$ und $\mathrm{MW}_{\cup k}$ die Effektivität der Maßnahme beschreiben, lässt sich mit Hilfe einer Wirtschaftlichkeitsbetrachtung die Effizienz bestimmen. Zur Bewertung der Wirtschaftlichkeit wird in der Untersuchung das Nutzen-Kosten-Verhältnis (NKV) herangezogen Die Grundlage des Vorgehens bildet die Beschreibung unter Kapitel 2.2.4. Aus volkswirtschaftlicher Sicht sind NKV > 1 wirtschaftlich gerechtfertigt, der Nutzen (vermiedene Unfallkosten) fällt höher als die Kosten (Investition und ggf. Betriebskosten) aus.

Die Kosten für identische Maßnahmen variieren erheblich in Abhängigkeit der örtlichen Randbedingungen einer UH. Dazu zählt die Größe der Verkehrsanlage, deren Lage im Netz, die bisherige Ausstattung sowie die zugrunde liegenden Konfliktsituationen. Die Gesamtkosten einer Maßnahme lassen sich auf Basis des eingesetzten Materials nur überschlägig abschätzen, während die realen Kosten eine erhebliche Spannbreite aufweisen. Diese Eigenschaften übertragen sich auch auf die volkswirtschaftliche Bewertung einer MK, die auf der Analyse mehrerer Maßnahmen (Stellen) fußt.

Ähnlich den Angaben zur Entwicklung der Unfallzahlen und Unfallkosten unterliegen die Maßnahmenkosten einer hohen Spannbreite, Einzelfälle können den Mittelwert der Maßnahmenkosten einer MK maßgebend beeinflussen. Zur Angabe der mittleren Maßnahmenkosten wurde dennoch der Mittelwert aller betrachteten Stellen herangezogen (GI. 6-3). Für jede Stelle einer MK erfolgte die Berechnung des NKV (GI. 6-4). Dem NKV der MK liegt der Median der Einzelfälle zu Grunde. Somit werden Einflüsse aufgrund von Ausreißern eingeschränkt. Zur besseren Interpretation wird die Spannbreite der NKV der einzelnen Stellen im Faktenblatt mit dargestellt.

$\mathrm{NKV}=\frac{\sum_{i}\left(\frac{U K V_{a}-U K N_{a}}{M K_{a}}\right)}{i}$

mit:

$\begin{array}{llc}\text { NKV } & \text { Nutzen-Kosten-Verhältnis } & {[-]} \\ \mathrm{UKV}_{\mathrm{a}} & \text { jährliche Unfallkosten Vorherzeitraum } & {[€]} \\ \mathrm{UKN}_{\mathrm{a}} & \text { jährliche Unfallkosten Nachherzeitraum } & {[€]} \\ \mathrm{MK}_{\mathrm{a}} & \text { jährliche Maßnahmenkosten } & {[€]} \\ \mathrm{i} & \text { Stellen i } & \end{array}$

$\mathrm{NKV}_{i}=\frac{\left(U K V_{a}-U K N_{a}\right)}{M K_{a}}$

mit:

$\mathrm{NKV}_{\mathrm{i}} \quad$ Nutzen-Kosten-Verhältnis der betrachteten Stelle i $\quad[-]$

$\mathrm{UKV}_{\mathrm{a}}$ jährliche Unfallkosten Vorherzeitraum [€]

$\mathrm{UKN}_{\mathrm{a}}$ jährliche Unfallkosten Nachherzeitraum [€]

$\begin{array}{ccc}\mathrm{MK}_{\mathrm{a}} & \text { jährliche Maßnahmenkosten } & {[€]}\end{array}$

\section{Statistische Sicherheit}

Ob sich der Beitrag einer Maßnahme zur Erhöhung der Verkehrssicherheit nicht nur auf einen zufälligen Zusammenhang zurückführen lässt, kann mittels statistischer Signifikanztests überprüft werden. Als geeignete Tests zur Überprüfung, ob sich der Rückgang der Unfallzahl auf den Maßnameneinsatz gründet, kommen der Test nach McNemar und der Test auf Poisson-Verteilung in Frage [FGSV 1991, GERLACH et al. 2009]. Beide Tests beruhen auf dem Vergleich der Unfallan- 
zahlen im Vorher- und Nachher-Zeitraum, es gilt die ceteris paribus Bedingung. Der Test auf Poisson-Verteilung unterliegt weiterhin der Einschränkung, dass Vorher- und Nachher-Zeitraum die gleiche Größe aufweisen müssen. Die Aussagekraft der Testverfahren ist für Kollektive mit sehr kleinen Unfallzahlen beschränkt. Für beide Testverfahren wird ein Signifikanzniveau von $\alpha=0,05$ verwendet.

Ein signifikanter Unterschied liegt nach dem McNemar-Test im Unfallgeschehen vor, wenn die Prüfgröße T den Wert von 3,84 überschreitet (GI. 6-5). Dieser ergibt sich aus den vorhandenen Freiheitsgrade und dem Signifikanzniveau. Er lässt sich aus der $\mathrm{X}^{2}$-Verteilung ableiten.

$\mathrm{T}=\frac{\left(n_{1} * t_{2}-n_{2} * t_{1}\right)^{2}}{t_{1} * t_{2} *\left(n_{1}+n_{2}\right)}$

mit:

T Prüfgröße $\quad[-]$

$\mathrm{n}_{1}, \mathrm{n}_{2} \quad$ Unfälle im Vorher- bzw. Nachher-Zeitraum [-]

$\mathrm{t}_{1}, \mathrm{t}_{2} \quad$ Länge des Vorher- bzw. Nachher-Zeitraums [Monate]

Beim Poisson-Test dient $P$ als Prüfgröße und quantifiziert die Wahrscheinlichkeit, dass eine gegebene Änderung der Unfallanzahl zufällig auftritt (Gl. 6-6). Bei gewähltem Signifikanzniveau muss $P$ demzufolge einen Wert von $\leq 0,05$ für eine signifikante Änderung annehmen [GERLACH et al. 2009].

$\mathrm{P}=\sum_{X=0}^{E_{N}} e^{-m} \frac{m^{x}}{x !}$

mit:

P Wahrscheinlichkeit der Ereignisse $\geq$ Unfallanzahl $\quad[-]$

Nachher-Zeitraum $\quad[-]$

M Mittelwert Unfallanzahl Vorher-Zeitraum [-]

X Mittelwert Unfallanzahl Nachher-Zeitraum [-]

Die Testverfahren wurden in der Untersuchung als Indikator für die Aussagekraft bezüglich der Wirksamkeit der $\mathrm{MW}_{\cup}$ herangezogen, bildeten aber aufgrund ihrer Einschränkungen keinen Bestandteil einer allgemeinen Auswertung.

Allgemein unterliegt die Aussagekraft der Ergebnisse (Wirkungsgrad von einzelnen Stellen) u. U. Einschränkungen aufgrund der unterschiedlichen Grenzwertdefinition von UH in den Bundesländern bzw. dem Eingreifen zur Bekämpfung von unfallauffälligen Stellen durch die UKO. In einem Fall wurden z. B. Maßnahmen bereits beim Vorliegen von 2 U(SP) in drei Jahren ergriffen. Dementsprechend ist die Angabe zum Wirkungsgrad der Maßnahme durch die allgemeine Zufälligkeit des Auftretens von Unfällen wesentlich beeinflusst.

\section{Gesamtbewertung}

Ziel der Unfallanalyse ist es, aus der Bewertung der MK eine Empfehlung für ihren Einsatz abzugeben. Entscheidend für den Einsatz einer Maßnahme ist neben einem erwarteten hohen Wirkungsgrad (Effektivität) ihre Wirtschaftlichkeit (Effizienz). Maßnahmen, deren Kosten den Nutzen übersteigen, sind nicht wirtschaftlich. Das NKV stellt ein Maß zur Beurteilung der Wirtschaftlichkeit dar. Der Wirkungsgrad der Maßnahme hat maßgeblichen Einfluss auf den Nutzen der Maßnahme. 
Dieser kann sowohl aus der Reduzierung der Unfallanzahl (MWU) als auch der Unfallschwere/Unfallkosten (MW $\mathrm{MK}_{\mathrm{K}}$ ) resultieren. Die Bewertung und Einschätzung der drei Merkmale

- mittlerer Wirkungsgrad bezüglich der Unfallzahl (MWU),

- mittlerer Wirkungsgrad bezüglich der Unfallkosten (MWUK) und

- das Nutzen-Kosten-Verhältnis (NKV)

erlauben eine Gesamtbeurteilung der MK und die Abgabe eines Prädikats für die weitere Anwendung. Demgemäß sind Bewertungsvorlagen für die drei Merkmale zu entwickeln, die Grundlage einer Gesamtbewertung bilden.

Das Bewertungsschema der $M W_{U}$ und $M W_{U K}$ ist identisch und fußt auf einer Beurteilung der einzelnen Stellen in einem MK-Kollektiv. Für MW $\mathrm{MW}_{\cup}$ und $\mathrm{MW}_{\cup K}$ gilt jeweils: Eine Maßnahmenumsetzung ist wirksam bzw. erfolgreich wenn eine positive Entwicklung (deutliche Abnahme) der Unfallanzahl und/oder Unfallkosten eintritt. Als "positive" Entwicklung einer einzelnen Stelle (Stelle positiv$_{\text {) }}$ werden eine $M W_{\cup}$ bzw. MW $W_{u k}$ von $\geq 30 \%$ und damit ein als „erheblich“ bzW. „bedeutend" eingeschätzter Wirkungsgrad angesehen (Vergleiche Tabelle 14). Für die Bewertung einer MK ergeben sich die in Tabelle 15 dargestellten drei Prädikate in Abhängigkeit der Einzelbewertung der Stellen.

Die Anzahl der zugrunde liegenden Stellen i einer MK stellt die erste Stufe des Bewertungsschemas dar. Für MK, denen 4 bis 6 Einzelstellen zugrunde liegen $(i \geq 4)$, stehen drei Prädikate zur Auswahl. In Abhängigkeit der Anzahl von Stellen mit einer positiven Entwicklung (Stelle positiv) erfolgt

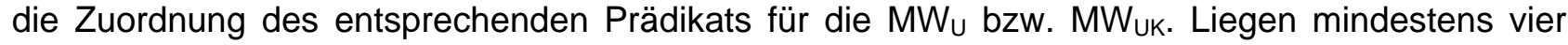
Stellen mit einer positiven Entwicklung vor, so erhält die MK das Prädikat „wirksam“. Für MK mit drei oder weniger betrachteten Stellen ( $\mathrm{i} \leq 3$ ) ist die Verlässlichkeit der Aussage, ob die MK wirksam ist oder nicht, aufgrund der geringen Fallzahl deutlich eingeschränkt. Für die Prädikatvergabe wird eine konservative Betrachtung gewählt. Das Prädikat „wirksam“ wird für diese MK nicht vergeben.

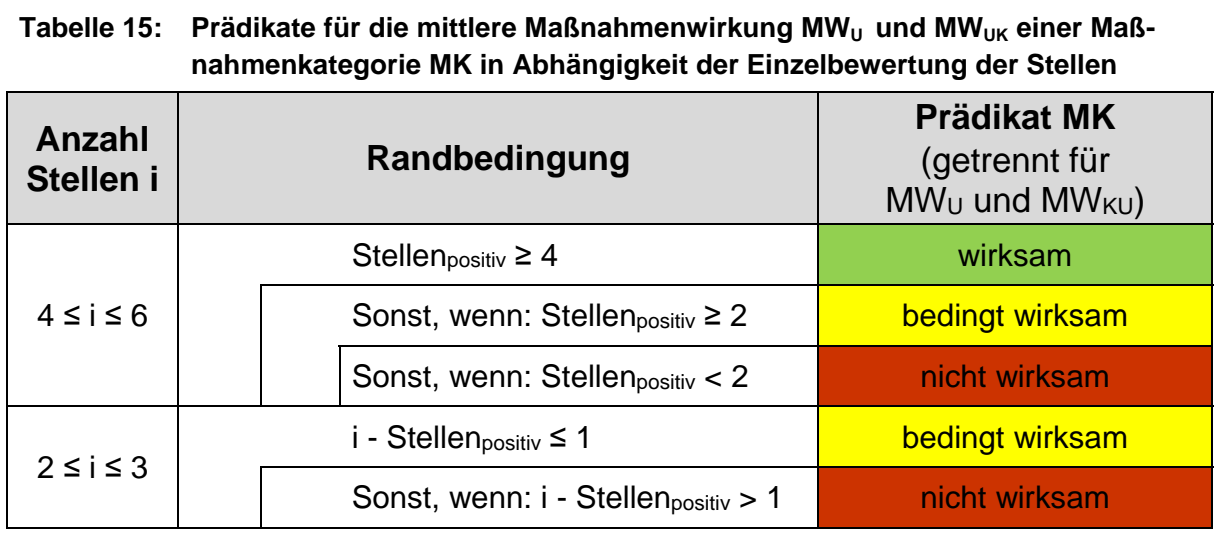

Die NKV der einzelnen Stellen einer MK können sich deutlich voneinander unterscheiden. Es erfolgt für die Wirtschaftlichkeitsbetrachtung einer MK eine zweiteilige Prüfung aus der die Bewertung resultiert. Es fließen sowohl der NKV-Median der MK als auch die Analyse der NKV der einzelnen Maßnahmen (Stelle i) in die Bewertung ein. Die gesamte Vorgehensweise ist Tabelle $16 \mathrm{zu}$ entnehmen.

Für Maßnahmen mit einem $0<$ NKV $\leq 1$ übertreffen die Maßnahmenkosten einen vorhanden Nutzen (positive $M W_{u k}$ ), es ist kein volkswirtschaftlicher Gewinn vorhanden. Treten derartige Fälle auf, wird die MK als „nicht wirtschaftlich“ eingestuft (Prüfung 1). Weiterhin haben Maßnahmen mit einer verfehlten Maßnahmenwirkung eine negative $\mathrm{MW}_{\mathrm{UK}}$ (negativer Nutzen) und damit per se ein negatives NKV. Dennoch kann die Maßnahme als solche - wenn sie unter den richtigen Randbedingungen eingesetzt wird - wirtschaftlich sein. Daher erfolgt eine zweite Prüfung, welche die Güte der MK bewertet. Liegt für eine MK ein Median des NKV $>1$ vor, so stehen für mindestens der 
Hälfte der betrachteten Stellen den Kosten ein höherer Nutzen gegenüber. Die MK gilt als „rentabel“. Überwiegt der Anteil an Stellen mit einem negativen Nutzen, so sinkt der Median auf eine Größenordnung < 1. Hier gilt, dass die Maßnahme nicht zwingend unwirtschaftlich ist, eher ist ein verfehlter Einsatz zu vermuten. Daher gelten die MK in diesem Falle als „bedingt rentabel“.

Tabelle 16: Prädikat für die Wirtschaftlichkeit einer Maßnahmenkategorie in Abhängigkeit der Einzelbewertung der Stellen

\begin{tabular}{|c|c|c|c|c|}
\hline 1. Prüfung & Randbedingung & 2. Prüfung & Randbedingung & $\begin{array}{c}\text { Prädikat MK } \\
(\mathrm{NKV})\end{array}$ \\
\hline \multirow{3}{*}{ Stelle i } & \multirow{2}{*}{$\begin{array}{c}\text { Stellen mit positivem MWUK } \\
= \\
\text { Stellen mit NKV }>1\end{array}$} & \multirow{2}{*}{$\begin{array}{l}\text { Median } \\
(\mathrm{MK})\end{array}$} & $>1$ & rentabel \\
\hline & & & $\leq 1$ & bedingt rentabel \\
\hline & $\begin{array}{c}\text { Stellen mit positivem MWUK } \\
> \\
\text { Stellen mit NKV > } 1 \\
\end{array}$ & & & nicht wirtschaftlich \\
\hline
\end{tabular}

Die Zusammenführung der drei einzelnen Prädikate führt zur Gesamtbewertung einer MK (Tabelle 17). Grundsätzlich werden die beiden Prädikate „empfehlenswert“ oder "bedingt empfehlenswert" vergeben. Nicht wirtschaftliche Maßnahmen sind für den praktischen Einsatz nicht empfehlenswert. Diese MK erhalten kein Prädikat, jedoch wird im Feld „Allgemeine Hinweise“ auf die vorgefundenen Einschränkungen und Defizite verwiesen. Die Vergabe des Prädikats, die MK ist „empfehlenswert", folgt einem konservativen Ansatz. Dieser beruht auf den Gedanken, dass das primäre Ziel der Unfallkommissionsarbeit die Beseitigung von UH und damit i. d. R. die Reduzierung der Unfallanzahl darstellt. Maßnahmen die nur zur Reduzierung der Unfallschwere beitragen - bspw. das Anbringen eines Unterfahrschutzes an passive Schutzeinrichtungen -, leisten zwar einen wesentlichen Beitrag zur Erhöhung der Verkehrssicherheit, tragen aber nicht zur Bekämpfung der Unfallursachen und damit der Unfallanzahl13 ${ }^{13}$ bei. Daher werden diese Maßnahmen nur als „bedingt empfehlenswert" eingestuft.

Tabelle 17: Gesamtprädikat für Maßnahmenkategorien MK

\begin{tabular}{|l|c|}
\hline \multicolumn{1}{|c|}{ Randbedingung } & Gesamtprädikat MK \\
\hline $\begin{array}{l}\text { Die Maßnahme ist wirksam bezüglich auf Rückgang Unfallanzahl und Unfallkosten, } \\
\text { die Maßnahme ist rentabel }\end{array}$ & empfehlenswert \\
\hline $\begin{array}{l}\text { Die Maßnahme ist bedingt wirksam bezüglich auf Rückgang Unfallanzahl und/oder } \\
\text { Unfallkosten, die Maßnahme ist (bedingt) rentabel }\end{array}$ & bedingt empfehlenswert \\
\hline $\begin{array}{l}\text { Die Maßnahme ist nicht wirksam bezüglich auf Rückgang Unfallanzahl und/oder } \\
\text { Unfallkosten, unabhängig der Rentabilität }\end{array}$ & $\begin{array}{l}\text { (kein Prädikat, Erläuterung } \\
\text { im Feld „Allgemeine Hin- } \\
\text { weise“ des Faktenblatts) }\end{array}$ \\
\hline $\begin{array}{l}\text { Die Maßnahme ist nicht wirtschaftlich, } \\
\text { unabhängig der Wirksamkeit auf Unfallanzahl oder Unfallkosten }\end{array}$
\end{tabular}

\subsection{Ergebnisse}

\subsubsection{Aufbereitung Faktenblatt Unfalldatenanalyse}

Ähnlich dem Faktenblatt zur Evaluierung der Maßnahmenkategorien MK fließen die Ergebnisse der Unfalldatenanalyse in ein eigenes Faktenblatt ein. Für jede MK liegen somit die Informationen zu den Einsatzbedingungen, zur Datengrundlage, dem Wirkungsgrad der Maßnahme, dem NKV als auch eine Gesamteinschätzung mit allgemeiner Empfehlung vor (Abbildung 30). Die in Abbildung 30 enthaltenen Nummern verknüpfen die Darstellung mit den inhaltlichen Erläuterungen zu den einzelnen Bereichen in Tabelle 18. Die einzelnen Faktenblätter zu den MK enthält Anhang 7.

13 Z. B. werden UH auf Landstraßen nach M Uko [FGSV 2012] auf Basis der 3 JK U(P) definiert. Hier ist es möglich, das oben geschilderte Maßnahmenumsetzung dazu beitragen kann, dass durch die Abnahme der Unfallschwere - Verschiebung der Unfälle in die Kategorien der Sachschadenunfälle -, eine entsprechende UH nicht mehr unfallauffällig ist. Ziel bleibt aber die Bekämpfung der Unfallursachen durch geeignete Maßnahmen. 


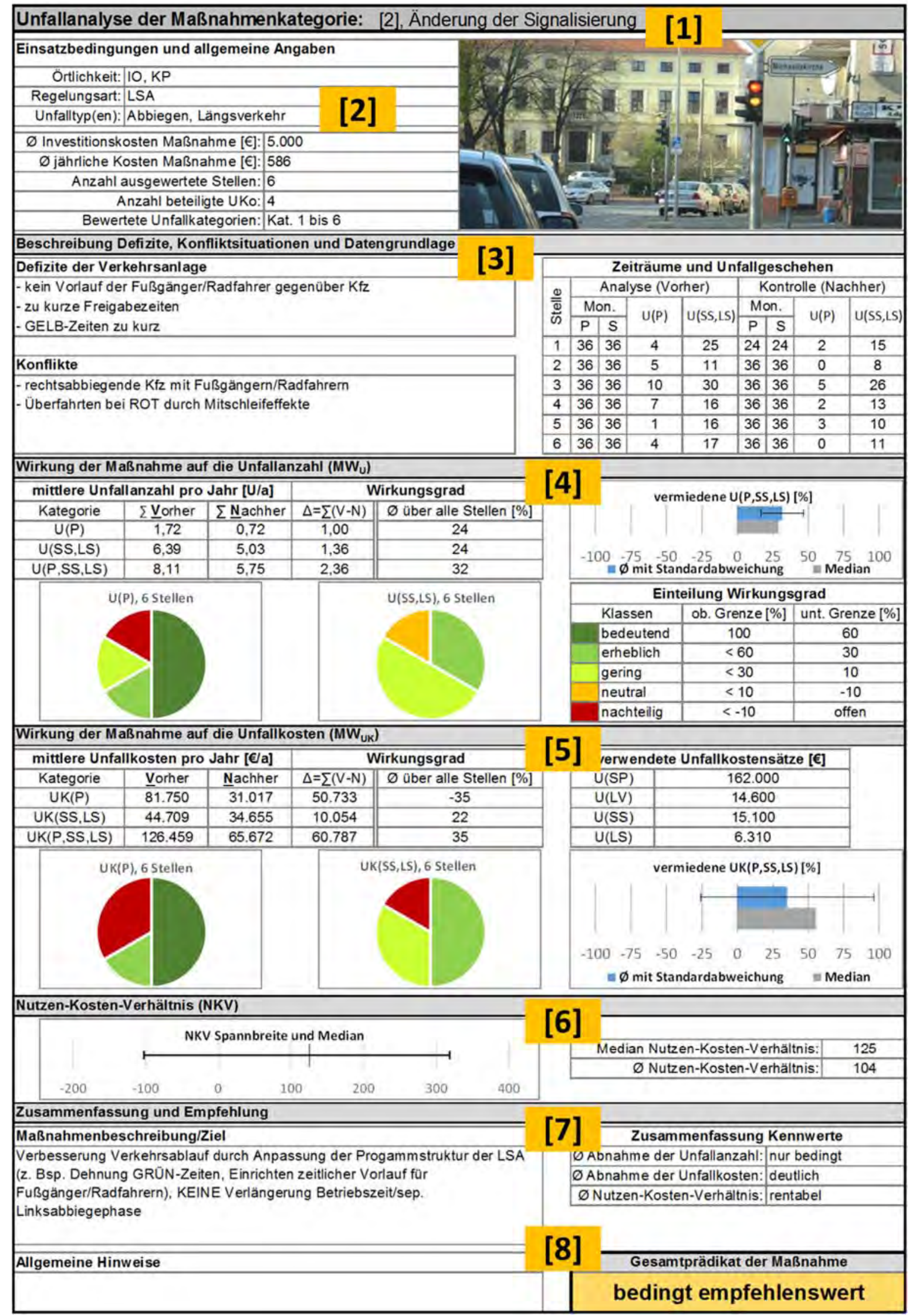

Abbildung 30: Aufbau Faktenblatt zur Unfallanalyse einer Maßnahmenkategorie MK 
Tabelle 18: Erläuterung der Inhalte [nummerierte Bereiche] des Faktenblatts zur Unfallanalyse einer Maßnahmenkategorie

\begin{tabular}{|c|c|c|}
\hline Nummer & Bezeichnung Bereich & Inhalt Bereich \\
\hline [1] & Bezeichnung MK & - Maßnahmenkategorie mit Nummer und Namen \\
\hline [2] & $\begin{array}{l}\text { Einsatzbedingungen und } \\
\text { allgemeine Angaben }\end{array}$ & $\begin{array}{l}\text { - Beschreibung zur Örtlichkeit, Regelungsart des Knotenpunkts (wenn gegeben), } \\
\text { prägende Unfalltypen und Beispielbild (allgemeine Situation) } \\
\text { • Angabe zu Investitionskosten und durchschnittlichen jährlichen Kosten (inkl. } \\
\text { Veränderung Betriebskosten } \\
\text { • Erläuterungen zu Kollektivgröße, Kollektivbeschaffenheit, bewertete Unfallkategorien }\end{array}$ \\
\hline [3] & $\begin{array}{l}\text { Beschreibung Defizite, Kon- } \\
\text { fliktsituationen und Daten- } \\
\text { grundlage }\end{array}$ & $\begin{array}{l}\text { - Defizite der Verkehrsanlage und der daraus resultierenden Konflikte } \\
\text { - Tabellarische Übersicht zum Unfallgeschehen der betrachteten Stellen, Angabe der } \\
\text { betrachteten Monate und Unfallanzahlen für U(P) und die betrachteten Kategorien } \\
\text { der Unfälle mit Sachschaden für Vorher- und Nachher-Zeitraum }\end{array}$ \\
\hline [4] & $\begin{array}{l}\text { Wirkung der Maßnahme auf } \\
\text { die Unfallanzahl }(U)\end{array}$ & 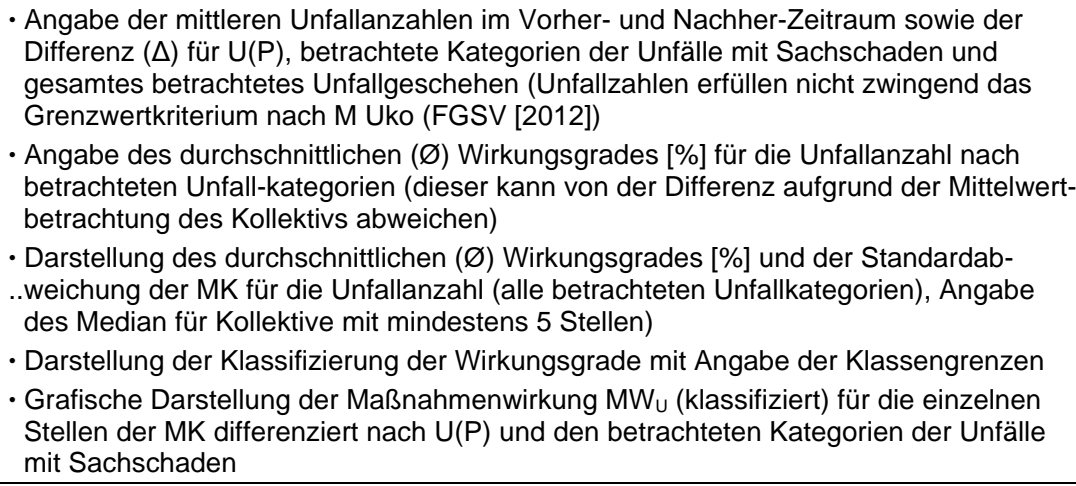 \\
\hline [5] & $\begin{array}{l}\text { Wirkung der Maßnahme auf } \\
\text { die Unfallkosten (UK) }\end{array}$ & $\begin{array}{l}\text { - Angabe der mittleren Unfallkosten im Vorher- und Nachher-Zeitraum sowie der } \\
\text { Differenz }(\Delta) \text { für } U(P) \text {, betrachtete Kategorien der Unfälle mit Sachschaden und } \\
\text { gesamtes betrachtetes Unfallgeschehen } \\
\text { - Angabe des durchschnittlichen }(\varnothing) \text { Wirkungsgrades [\%] für die Unfallkosten nach } \\
\text { betrachteten Unfall-kategorien (dieser kann von der Differenz aufgrund der Mittelwert- } \\
\text { betrachtung des Kollektivs abweichen) } \\
\text { - Darstellung des durchschnittlichen }(\varnothing) \text { Wirkungsgrades [\%] und der Standardab- } \\
\text { weichung der MK für die Unfallkosten (alle betrachteten Unfallkategorien), Angabe } \\
\text { des Median für Kollektive mit mindestens } 5 \text { Stellen) } \\
\text { - Tabellarische Darstellung der verwendeten Unfallkostensätze [€] } \\
\text { - Grafische Darstellung der Maßnahmenwirkung MWKu (klassifiziert) für die einzelnen } \\
\text { Stellen der MK differenziert nach U(P) und den betrachteten Kategorien der Unfälle } \\
\text { mit Sachschaden }\end{array}$ \\
\hline [6] & Nutzen-Kosten-Verhältnis & $\begin{array}{l}\text { - Grafische Darstellung Median des NKV (Kollektiv) und Spannbreite der Einzelwerte } \\
\text { - Angabe Median und Mittelwert ( } \varnothing \text { ) für NKV der Maßnahmenkategorie }\end{array}$ \\
\hline [7] & $\begin{array}{l}\text { Zusammenfassung und } \\
\text { Empfehlung }\end{array}$ & $\begin{array}{l}\text { - Beschreibung der konkreten Maßnahmenumsetzung (Ziele der Maßnahme) } \\
\text { - Angabe von allgemeinen Hinweisen, diese betreffen z. B. Einschränkungen bei der } \\
\text { Interpretation der Ergebnisse oder Besonderheiten des Kollektivs } \\
\text { - Zusammenfassung und Einschätzung der maßgebenden Kennwerte; Wirkung der } \\
\text { Maßnahme auf Unfallanzahl und Unfallkosten sowie Bewertung des NKV } \\
\text { (Da die Größe der mittleren Wirkungsgrade unter Ziffer } 4 \text { und Ziffer } 5 \text { durch } \\
\text { Ausreißer geprägt sein können, richtet sich die Vergabe des Prädikates für die } \\
\text { Abnahme der Unfallanzahl und Unfallkosten nach der Bewertung der MK nach } \\
\text { Tabelle 15.) }\end{array}$ \\
\hline [8] & $\begin{array}{l}\text { Gesamtprädikat der Maß- } \\
\text { nahme }\end{array}$ & •Vergabe des Gesamtprädikats der Maßnahme \\
\hline
\end{tabular}

Die als Beispiel aufgeführte MK 2 „Änderung der Signalisierung“ wurde für den innerörtlichen Bereich bewertet. Die MK zielt primär auf die Beseitigung von Abbiegen- und Längsverkehrs-Unfällen $\mathrm{ab}$, die auf Defizite aufgrund zur kurzer Freigabezeiten, zu kurzer oder fehlender GRÜN-Vorläufe für Fußgänger/Radfahrer an den Furten oder zur kurzer GELB-Zeiten an Kfz-Signalgebern zurückzuführen sind. Die daraus resultierenden Konflikte sind im Bereich der Ziffer 3 näher beschrieben. Die Unfallanalyse dieser MK stützt sich auf die Auswertung von sechs Stellen. Für alle dieser Stellen liegt das Unfallgeschehen vollumfänglich vor - alle Unfallkategorien für jeweils drei Jahre im Vorher- und Nachher-Zeitraum. 
Die mittlere $\mathrm{MW}_{\cup}$ der $\mathrm{MK}$ über alle betrachteten Unfallkategorien beträgt $32 \%$, der Median weist eine Größenordnung von 29 \% (Grafik) auf. Die differenzierte Betrachtung der U(P) und U(SS, LS) weist gegenüber dem gesamten Unfallgeschehen nur eine mittlere $\mathrm{MW}_{\cup}$ von jeweils $24 \%$ auf. Der Unterschied zum Gesamtwert der MK ergibt sich aus den verschiedenen Wirkungsgraden der Maßnahme für die Einzelstellen. Sie verdeutlicht auch den Einfluss von Ausreißern auf den Mittelwert kleiner Kollektive. Die Zusammenführung aller Unfallkategorien liefert stabilere Ergebnisse. Die Differenzierung des Unfallgeschehens in $U(P)$ und die Kategorien der Unfälle mit Sachschäden gibt $u$. a. Auskunft, ob sich ein allgemeiner Rückgang der Unfallzahlen oder die Verlagerung in andere Kategorien einstellt. Aus den Diagrammen im Bereich der Ziffer 4 ist zu entnehmen, dass die Maßnahme an 5 von 6 Stellen mindestens einen geringen positiven Wirkungsgrad aufweist. Bei der Interpretation der Ergebnisse ist zu beachten, dass sich der tendenziell stärkere Einfluss der Maßnahme auf die $U(P)$ auch aus den kleinen Unfallanzahlen der $U(P)$ ergibt.

Insbesondere die Betrachtung der $\mathrm{MW}_{\mathrm{UK}}$ für die $\mathrm{U}(\mathrm{P})$ untermauert den unter Kapitel 6.1.3 diskutierten Interpretationsspielraum der Ergebnisse (Bereich Ziffer 5). Für die Stelle 1 des Kollektivs ist durch die Maßnahme ein Rückgang der Anzahl der $U(P)$, aber auch eine Verschiebung von $U(L V)$ zu U(SP) zu verzeichnen. Dies zeigt die tiefere Analyse des Unfallgeschehens. Stelle 5 weist eine Zunahme der $U(P)$ auf. Die beiden negativen Entwicklungen spiegeln sich im Diagramm zu den Unfallkosten $U(P)$ wider. Die Entwicklungen haben zur Folge, dass die $M_{U K}$ der $M K$ für $U(P)-$ maßgebend durch die beiden Stellen beeinflusst - einen negativen Wert aufweist (- $35 \%)$. Jedoch die Differenz der aggregierten jährlichen Unfallkosten aller Stellen aus Vorherzeitraum abzüglich des Nachherzeitraums besitzt eine positive Größenordnung (50.733 €). Dies begründet sich aus dem wesentlich höheren Unfallkostenbeträgen der anderen Stellen im Kollektiv.

Der Mittelwert (35\%), dessen Standardabweichung und der Median (56\%, grauer Balken in der Grafik) für die MW ú über alle betrachteten Unfallkategorien belegen die allgemeine Wirkung der Maßnahmenkategorie. In Einzelfällen war die Maßnahme unzureichend, für den Großteil der betrachteten Stellen ist jedoch eine positive Wirkung festzustellen.

Die Spannbreite des NKV im Bereich der Ziffer 6 stellt den unterschiedlichen Erfolg des Maßnahmeneinsatzes aus volkswirtschaftlicher Sicht dar. Aus der Zunahme der Unfallkosten im NachherZeitraum für Stelle 1 ergibt sich unabhängig von den Kosten ein negatives NKV. Der Median der MK 2 beträgt 125, der Mittelwert 104. Der aus volkswirtschaftlicher Sicht größte Nutzen der Maßnahmenumsetzung, mit einem NKV von 317, ist an Stelle $3^{14}$ zu verzeichnen.

Die Beschreibung und Ziele, welche Veränderungen an der Verkehrsanlage durch die MK vorgenommen werden sollen, findet sich Bereich der Ziffer 7. Allgemeine Hinweise liegen für die MK 2 nicht vor. Die Zusammenfassung der Kennwerte und das Gesamtprädikat der MK schließen das Faktenblatt ab.

\subsubsection{Auswertung Maßnahmenkategorien}

\section{Deskriptive Statistik}

Die Tabelle 19 enthält deskriptive Kennwerte des gesamten Kollektivs. Es wurden in Summe 165 Maßnahmen (Stellen) den 35 MK zugeordnet, das sind im Mittel 4,7 Stellen je MK. 14 MK enthalten sechs bewertete Stellen, 6 MK nur drei oder zwei Stellen. Mit der Abnahme der Stellenanzahl in einer MK sinkt auch die Aussagekraft der Ergebnisse. In Summe aller Vorher-Zeiträume fließen $911 \mathrm{U}(\mathrm{P})$ in die Untersuchung ein, dass sind durchschnittlich 2,2 U(P) je Jahr und Stelle. Durch die Maßnahmenumsetzung konnte die durchschnittliche Anzahl nahezu halbiert werden. Eine ähnliche Entwicklung zeigen die Unfallkosten auf. Die Größenordnung beruht auf der Angabe aller der in den jeweiligen MK bewerteten Unfallkategorien. Davon ausgehend sanken die Unfallkosten im 
untersuchten Kollektiv von jährlich 7,5 Mio. € auf 3,5 Mio. € - entspricht $47 \%$ des Ausgangswerts - nach der Maßnahmenumsetzung.

Abbildung 31 und Abbildung 32 stellen die Einzelwerte der MK dar. Die Beschreibung der Örtlichkeit jeder $\mathrm{MK}$, die bewerteten UK und der Wirkungsgrad (MWU) auf die Unfallanzahl enthält Abbildung $31^{15}$. Abbildung 32 enthält das Pendant zu den Unfallkosten (MW $\mathrm{MK}_{\mathrm{UK}}$ ), die Angaben zum NKV und zum Gesamtprädikat. Die Faktenblätter zu den einzelnen MK enthält Anhang 7.

Tabelle 19: Kennwerte des Kollektivs, Entwicklung U(P) und Unfallkosten

\begin{tabular}{|c|c|c|}
\hline \multirow{2}{*}{ Kennwerte } & \multicolumn{2}{|c|}{ Zeitraum } \\
\hline & Vorher & Nachher \\
\hline$\sum$ Anzahl U(P) [n] & 911 & 473 \\
\hline$\varnothing$ Anzahl U(P)/(Jahr und Stelle) [n] & 2,2 & 1,1 \\
\hline$\sum$ Unfallkosten/Jahr* $[€]$ & 7,4 Mio. & 3,5 Mio. \\
\hline$\varnothing$ Unfallkosten/(Jahr und Stelle) ${ }^{\star \star}[€]$ & 211.600 & 101.100 \\
\hline \multicolumn{3}{|c|}{$\begin{array}{l}\text { * Die Aggregation der Unfallkosten beruht auf den bewerteten Unfallkategorien der } \\
\text { einzelnen MK } \\
\text { ** Angabe nicht als Mittelwert repräsentativ }\end{array}$} \\
\hline
\end{tabular}

\begin{tabular}{|c|c|c|c|c|c|c|c|c|c|c|}
\hline \multirow[b]{2}{*}{ Nr. } & \multirow{2}{*}{$\begin{array}{l}\text { Maßnahmenkategorie } \\
\text { Name }\end{array}$} & \multicolumn{3}{|c|}{ Örtlichkeit } & \multirow[b]{2}{*}{$\begin{array}{c}\text { Stellen } \\
{[n]}\end{array}$} & \multirow[b]{2}{*}{$\begin{array}{c}\text { bewertete } \\
\text { U.-Kat. }\end{array}$} & \multicolumn{4}{|c|}{ Unfallanzahl [n] } \\
\hline & & Ort & $\begin{array}{l}\text { Charakter- } \\
\text { isitik }\end{array}$ & $\begin{array}{l}\text { Art der } \\
\text { Verkehrs- } \\
\text { regelung }\end{array}$ & & & Wirk & $\begin{array}{l}\text { ngsgrad } \\
\text { IW }_{y} \\
{[\%]}\end{array}$ & $\begin{array}{l}\text { mindestens } \\
\text { deutliche } \\
\text { positive } \\
\text { Entwicklung } \\
\text { an... }\end{array}$ & $\begin{array}{l}\text { Prädikat } \\
\varnothing \text { Abnahme } \\
\text { Unfallzah! }\end{array}$ \\
\hline 1 & Verbesserung der Koordinierung & 10 & $\mathrm{KP}$ & LSA & 5 & Kat. 1 bis 6 & -7 & -4 & 0 von 5 Stellen & nicht wirksam \\
\hline 2 & Anderung der Signalisierung & 10 & KP & LSA & 6 & Kat. 1 bis 6 & 32 & 29 & 3 von 6 Stellen & bedingt wirksam \\
\hline 3 & "sonstige" Gefahrenzeichen anbringen & AO & Strecke $(300 \mathrm{~m})$ & - & 2 & Kat. 1 bis 4 & 51 & 51 & 1 von 2 Stellen & bedingt wirksam \\
\hline 4 & HALT Z 206 StVO (anstelle Z 205 StVO) & 10 & $\mathrm{KP}$ & $\mathrm{vz}$ & 5 & Kat. 1 bis 6 & 37 & 50 & 3 von 5 Stellen & bedingt wirksam \\
\hline 5 & HALT Z 206 StVO (anstelle Z 205 StVO) & AO & KP & $\mathrm{VZ}$ & 6 & Kat. 1 bis 4 & -7 & 21 & 2 von 6 Stellen & bedingt wirksam \\
\hline 6 & Halte-/Parkverbot anordinen & 10 & KP & Vz/r.v.l. & 4 & Kat. 1 bis 3 und $\cup(S)$ & 50 & 52 & 4 von 4 Stellen & wirksam \\
\hline 7 & Z 625 StVO (Richtungstafel in Kurven) aufstellen & AO & Strecke $(300 \mathrm{~m})$ & - & 6 & Kat. 1 bis 6 & 49 & 50 & 5 von 6 Stellen & wirksam \\
\hline 8 & Zusatzzeichen Z 1006-36 StVO (Unfallgefahr) ergänzen & 10 & $\mathrm{KP} /$ Strecke & - & 3 & Kat. 1 bis 4 & 42 & 40 & 2 von 3 Stellen & bedingt wirksam \\
\hline 9 & Zusatzzeichen Z 1006-36 StVO (Unfallgefahr) ergänzen & AO & $\mathrm{KP} / \mathrm{Strecke}$ & - & 4 & Kat, 1 bis 4 & -17 & 7 & 1 von 4 Stellen & nicht wirksam \\
\hline 10 & Verkehrszeichen anpassen & 10 & $\mathrm{KP} /$ Strecke & - & 4 & Kat. 1 bis 4 & 59 & 73 & 3 von 4 Stellen & bedingt wirksam \\
\hline 11 & Verkehrszeichen anpassen & AO & $\mathrm{KP} /$ Strecke & - & 3 & Kat. 1 bis 6 & 13 & 29 & 1 von 3 Stellen & nicht wirksam \\
\hline 12 & Beschränkung Vzul & AO & Strecke $(300 \mathrm{~m})$ & - & 6 & Kat. 1 bis 4 & 50 & 74 & 5 von 6 Stellen & wirksam \\
\hline 15 & Beschrănkung Vzul & AO & $\mathrm{KP}$ & $\mathrm{Vz}$ & 4 & Kat. 1 bis 4 & 33 & 16 & 1 von 4 Stellen & nicht wirksam \\
\hline 16 & Überwachung zulässige Höchstgeschwindigkeit mit OGÜ & AO & KP & $\mathrm{VZ}$ & 2 & Kat. 1 bis 4 & 55 & 55 & 1 von 2 Stellen & bedingt wirksam \\
\hline 17 & Überwachung zulässige Höchstgeschwindigkeit mit OGÜ & AO & Strecke $(300 \mathrm{~m})$ & - & 4 & Kat. 1 bis 3 & 48 & 60 & 3 von 4 Stellen & bedingt wirksam \\
\hline 18 & Erneuerung Fahrbahnoberfläche & $\mathrm{AO}$ & Strecke $(300 \mathrm{~m})$ & - & 6 & Kat. 1 bis 6 & 49 & 52 & 5 von 6 Stellen & wirksam \\
\hline 19 & Zusätzlichen Signalgeber anordnen & 10 & KP & LSA & 2 & Kat. 1 bis 4 & 38 & 38 & 1 von 2 Stellen & bedingt wirksam \\
\hline 21 & Leuchtmittel Signalgeber verbessern & 10 & KP & LSA & 6 & Kat. 1 bis 3 und $U(S)$ & 43 & 42 & 5 von 6 Stellen & wirksam \\
\hline 22 & Sichthindernisse entfernen & 10 & KP & vz & 6 & Kat. 1 bis 3 und $U(S)$ & 71 & 76 & 5 von 6 Stellen & wirksam \\
\hline 23 & Sichthindernisse entfernen & AO & $\mathrm{KP}$ & $\mathrm{VZ}$ & 6 & Kat. 1 bis 3 und $U(S)$ & 32 & 38 & 5 von 6 Stellen & wirksam \\
\hline 24 & Beleuchtung anpassen/verbessern & 10 & $\mathrm{KP} /$ Strecke & - & 6 & Kat. 1 bis 6 & 32 & 53 & 4 von 6 Stellen & wirksam \\
\hline 25 & Sichtschutzzaun aufstellen & AO & $\mathrm{KP}$ & $\mathrm{Vz}$ & 6 & Kat, 1 bis 4 & 31 & 48 & 4 von 6 Stellen & wirksam \\
\hline 26 & Markierung anpassen/verbessern & 10 & $\mathrm{KP}$ & beliebig & 6 & Kat. 1 bis 6 & 39 & 27 & 2 von 6 Stellen & bedingt wirksam \\
\hline 27 & Markierung in Stand setzen & 10 & $\mathrm{KP}$ & beliebig & 6 & Kat. 1 bis 6 & -11 & -10 & 1 von 6 Stellen & nicht wirksam \\
\hline 29 & Radverkehrsführung anpassen & 10 & KP & beliebig & 5 & Kat. 1 bis 3 und U(S) & 57 & 67 & 4 von 5 Stellen & wirksam \\
\hline 30 & Radfahrfurt Rot einfärben & 10 & $\mathrm{KP}$ & $\mathrm{Vz}$ & 4 & Kat. 1 bis 3 & 64 & 68 & 3 von 4 Stellen & bedingt wirksam \\
\hline 31 & Passive Schutzeinrichtung aufstellen & AO & Strecke $(300 \mathrm{~m})$ & - & 6 & Kat. 1 bis 3 und U(S) & 45 & 65 & 4 von 6 Stellen & wirksam \\
\hline 32 & Unterfahrschutz an passiver Schutzeinrichtung anbringen & AO & Strecke $(300 \mathrm{~m})$ & - & 5 & Kat. 1 bis 6 & 33 & 67 & 3 von 5 Stellen & bedingt wirksam \\
\hline 33 & Hindernisse im Seitenraum entfernen & $\mathrm{AO}$ & Strecke $(300 \mathrm{~m})$ & - & 2 & Kat. 1 bis 6 & 46 & 46 & 1 von 2 Stellen & bedingt wirksam \\
\hline 34 & Rüttelstreifen aufbringen & AO & Strecke $(300 \mathrm{~m})$ & - & 5 & Kat. 1 bis 6 & 56 & 71 & 3 von 5 Stellen & bedingt wirksam \\
\hline 35 & Gummischwellen aufbringen & AO & $\mathrm{KP}$ & beliebig. & 5 & Kat. 1 bis 3 und $U(S)$ & -10 & 40 & 3 von 5 Stellen & bedingt wirksam \\
\hline 36 & Sperrfläche markieren & 10 & $\mathrm{KP}$ & $\mathrm{VZ}$ & 4 & Kat. 1 bis 6 & 63 & 71 & 3 von 4 Stellen & bedingt wirksam \\
\hline 37 & Verkehrszeichen versetzen (Erhöhung Erkennbarkeit) & AO & $\mathrm{KP} / \mathrm{Strecke}$ & - & 4 & Kat. 1 bis 4 & 12 & 10 & 1 von 4 Stellen & nicht wirksam \\
\hline 38 & Beschränkung Vzul und Z 625 Stvo (Richtungstafel in Kurve) & $\mathrm{AO}$ & Strecke $(300 \mathrm{~m})$ & - & 6 & Kat. 1 bis 3 und $U(S)$ & 49 & 42 & 6 von 6 Stellen & wirksam \\
\hline 39 & Beschränkung Vzul und sonst. Gefahrenzeichen & $\mathrm{AO}$ & Strecke $(300 \mathrm{~m})$ & - & 5 & Kat. 1 bis 3 und $U(S)$ & 30 & 25 & 2 von 5 Stellen & bedingt wirksam \\
\hline
\end{tabular}

Abbildung 31: Kennwerte der einzelnen Maßnahmenkategorien MK, Örtlichkeit und Wirkung auf Unfallanzahl 


\begin{tabular}{|c|c|c|c|c|c|c|c|c|c|}
\hline & Maßnahmenkategorie & & & Infallkosten [ & & & & & \\
\hline Nr. & Name & & $\begin{array}{l}\text { ggsgrad } \\
N_{\mathrm{UK}} \\
\%]\end{array}$ & $\begin{array}{l}\text { mindestens } \\
\text { deutliche } \\
\text { positive }\end{array}$ & $\begin{array}{l}\text { Prädikat } \\
\emptyset \text { Abnahme }\end{array}$ & $\begin{array}{c}\emptyset \\
\text { Investitions - } \\
\text { kosten }\end{array}$ & & NKV & Gesamtprädikat \\
\hline & & $\varnothing$ & Median & $\begin{array}{l}\text { Entwicklung } \\
\text { an... }\end{array}$ & Unfallikosten & & Median & Prädikat & \\
\hline 1 & Verbesserung der Koordinierung & -31 & -33 & 2 von 5 Stellen & bedingt wirksam & 5.000 & -60 & bedingt rentabel & \\
\hline 2 & Änderung der Signalisierung & 35 & 56 & 4 von 6 Stellen & wirksam & 5.000 & 125 & rentabel & bedingt empfehlenswert \\
\hline 3 & "sonstige" Gefahrenzeichen anbringen & 70 & 70 & 2 von 2 Stellen & bedingt wirksam & 1.000 & 2.241 & rentabel & bedingt empfehlenswert \\
\hline 4 & HALT Z 206 StVO (anstelle Z 205 StVO) & 67 & 67 & 4 von 5 Stellen & wirksam & 1.800 & 349 & rentabel & bedingt empfehlenswert \\
\hline 5 & HALT Z 206 StVO (anstelle Z 205 StVO) & 39 & 52 & 4 von 6 Stellen & wirksam & 1.800 & 674 & rentabel & bedingt empfehlenswert \\
\hline 6 & Halte-/Parkverbot anordnen & -14 & 33 & 3 von 4 Stellen & bedingt wirksam & 2.000 & 194 & rentabel & bedingt empfehlenswert \\
\hline 7 & Z 625 StVo (Richtungstafel in Kurven) aufstellen & 82 & 94 & 5 von 6 Stellen & wirksam & 3.000 & 698 & rentabel & empfehlenswert \\
\hline 8 & Zusatzzeichen Z 1006-36 StVO (Unfallgefahr) ergänzen & 46 & 40 & 2 von 3 Stellen & bedingt wirksam & 2.000 & 217 & rentabel & bedingt empfehlenswert \\
\hline 9 & Zusatzzeichen Z 1006-36 StVO (Unfallgefahr) ergänzen & 20 & 45 & 2 von 4 Stellen & bedingt wirksam & 2.000 & 632 & rentabel & \\
\hline 10 & Verkehrszeichen anpassen & 52 & 84 & 3 von 4 Stellen & bedingt wirksam & 1.000 & 678 & rentabel & bedingt empfehlenswert \\
\hline 11 & Verkehrszeichen anpassen & 68 & 66 & 3 von 3 Stellen & bedingt wirksam & 1.000 & 2.240 & rentabel & \\
\hline 12 & Beschränkung Vzul & 68 & 74 & 6 von 6 Stellen & wirksam & 2.000 & 963 & rentabel & empfehlenswert \\
\hline 15 & Beschränkung Vzul & -61 & 15 & 1 von 4 Stellen & nicht wirksam & 2.000 & 218 & rentabel & \\
\hline 16 & Überwachung zulässige Höchstgeschwindigkeit mit OGU & 87 & 87 & 2 von 2 Stellen & bedingt wirksam & 70.000 & 17 & rentabel & bedingt empfehlenswert \\
\hline 17 & Überwachung zulässige Höchstgeschwindigkeit mit OGÜ & 32 & 47 & 3 von 4 Stellen & bedingt wirksam & 70.000 & 15 & rentabel & bedingt empfehlenswert \\
\hline 18 & Erneuerung Fahrbahnoberfläche & 52 & 45 & 4 von 6 Stellen & wirksam & 22.500 & 60 & rentabel & empfehlenswert \\
\hline 19 & Zusätzlichen Signalgeber anordnen & 37 & 37 & 1 von 2 Stellen & bedingt wirksam & 4.000 & 81 & rentabel & bedingt empfehlenswert \\
\hline 21 & Leuchtmittel Signalgeber verbessern & 63 & 64 & 6 von 6 Stellen & wirksam & 8.000 & 206 & rentabel & empfehlenswert \\
\hline 22 & Sichthindernisse entfernen & 86 & 87 & 6 von 6 Stellen & wirksam & 967 & 736 & rentabel & empfehlenswert \\
\hline 23 & Sichthindernisse entfernen & 71 & 76 & 5 von 6 Stellen & wirksam & 5.183 & 1.517 & rentabel & empfehlenswert \\
\hline 24 & Beleuchtung anpassen/verbessern & 51 & 52 & 5 von 6 Stellen & wirksam & 10.000 & 176 & rentabel & empfehlenswert \\
\hline 25 & Sichtschutzzaun aufstellen & 65 & 91 & 4 von 6 Stellen & wirksam & 2.833 & 1.149 & rentabel & empfehlenswert \\
\hline 26 & Markierung anpassen/verbessern & 50 & 54 & 5 von 6 Stellen & wirksam & 5.000 & 47 & rentabel & bedingt empfehlenswert \\
\hline 27 & Markierung in Stand setzen & 13 & 13 & 3 von 6 Stellen & bedingt wirksam & 5.000 & 19 & rentabel & \\
\hline 29 & Radverkehrsführung anpassen & 28 & 61 & 3 von 5 Stellen & bedingt wirksam & 15.800 & 10 & rentabel & bedingt empfehlenswert \\
\hline 30 & Radfahrfurt Rot einfärben & 23 & 88 & 3 von 4 Stellen & bedingt wirksam & 660 & 621 & rentabel & bedingt empfehlenswert \\
\hline 31 & Passive Schutzeinrichtung aufstellen & 76 & 75 & 6 von 6 Stellen & wirksam & 27.817 & 309 & rentabel & empfehlenswert \\
\hline 32 & Unterfahrschutz an passiver Schutzeinrichtung anbringen & -17 & 52 & 4 von 5 Stellen & wirksam & 13.300 & 120 & rentabel & bedingt empfehlenswert \\
\hline 33 & Hindernisse im Seitenraum entfernen & 94 & 94 & 2 von 2 Stellen & bedingt wirksam & 4.000 & 872 & rentabel & bedingt empfehlenswert \\
\hline 34 & Rüttelstreifen aufbringen & 87 & 96 & 5 von 5 Stellen & wirksam & 2.400 & 558 & rentabel & bedingt empfehlenswert \\
\hline 35 & Gummischwellen aufbringen & -187 & 81 & 3 von 5 Stellen & bedingt wirksam & 880 & 288 & rentabel & bedingt empfehlenswert \\
\hline 36 & Sperrfläche markieren & 69 & 70 & 4 von 4 Stellen & wirksam & 7.500 & 55 & rentabel & bedingt empfehlenswert \\
\hline 37 & Verkehrszeichen versetzen (Erhöhung Erkennbarkeit) & 22 & 3 & 1 von 4 Stellen & nicht wirksam & 1.300 & 31 & rentabel & \\
\hline 38 & Beschränkung Vzul und Z 625 Stvo (Richtungstafel in Kurve) & 79 & 92 & 6 von 6 Stellen & wirksam & 3.417 & 1.196 & rentabel & empfehlenswert \\
\hline 39 & Beschränkung Vzul und sonst. Gefahrenzeichen & -83 & 67 & 3 von 5 Stellen & bedingt wirksam & 2.000 & 660 & rentabel & bedingt empfehlenswert \\
\hline
\end{tabular}

Abbildung 32: Kennwerte der einzelnen Maßnahmenkategorien MK, Wirkung auf Unfallkosten, NKV und Gesamtprädikat

Die MK unterscheiden sich in Hinblick auf ihren Einsatz für die Netzelemente Knotenpunkt (KP) oder Strecke. Es gilt die Annahme, dass UH auf Strecken eine räumliche Ausdehnung von ca. $300 \mathrm{~m}$ aufweisen. Diese Festlegung entspricht dem Grundsatz zur Identifikation von UH nach dem M Uko [FGSV 2012]. Dementsprechend sind Maßnahmen, deren Kosten eine räumliche Abhängigkeit besitzen, an dieser Größenordnung orientiert. Folgende Knotenpunktregelungen bzw. Knotenpunktarten sind Bestandteil des Kollektivs:

- Regelung durch Lichtsignalanlage (LSA)

- Regelung durch Verkehrszeichen (VZ)

- rechts-vor-links Regelung (r. v. I.)

- Kreisverkehr (KV)

Kommt eine MK unabhängig der Regelungsform für einen Knotenpunkt in Betracht, sind die verschiedenen Möglichkeiten der Regelungsarten unter dem Begriff „beliebig“ zusammengefasst.

Für 13 MK liegt die Auswertung aller Unfallkategorien vor, in elf Fällen für die Unfallkategorien 1 bis 4. Eine fehlende Trennung der $U(S)$ weisen neun MK auf und für zwei MK konnten nur die $\mathrm{U}(\mathrm{P})$ zur Bewertung herangezogen werden (MK 17 und MK 30).

\section{Wirksamkeit}

Die unabhängig von der Ortslage identischen MK 4 und 5 (Z 206 anstelle Z 205 StVO) haben einen unterschiedlichen Rückgang der Unfallzahlen $\left(M_{\cup}\right)$ zur Folge. Die Maßnahme erscheint für 
den innerörtlichen Bereich gegenüber dem Einsatz auf Landstraßen besser geeignet. Ein mutmaßlich ähnlicher Zusammenhang zeigt sich ebenfalls im direkten Vergleich sowohl bei der Betrachtung der MK 8 und 9 (Z 1006-36 StVO) als auch der MK 10 und 11 (Verkehrszeichen anpassen). Jedoch beschränken Unterschiede bei den betrachteten UH-Netzelementen innerhalb der Kollektive die allgemeine Vergleichbarkeit. Weiterhin schränken in beiden letztgenannten Fällen die geringen Kollektivgrößen die Aussagekraft weiter ein.

Die Wirkung der Beschränkung der $\bigvee_{\text {zul }}(M K 12$ und 15) offenbart in Abhängigkeit des Einsatzortes erhebliche Unterschiede. Während sich aus den Unfalldaten für den Einsatz der Maßnahme im Bereich der Strecke außerorts eine deutliche $\mathrm{MW}_{\cup}$ und $\mathrm{MW}_{U \mathrm{uk}}$ ableiten lässt, so ist ein maßgebender Einfluss der Maßnahme an Außerorts-Knotenpunkten nicht nachweisbar. Mutmaßlich weisen diese Knotenpunkte noch weitere Defizite auf. Die Beschränkung der $\vee_{\text {zul }}$ stellt häufig nur einer Sofortmaßnahme dar, welche nicht zur vollumfänglichen Beseitigung der Defizite beiträgt. Im Bereich knotenpunktfreier Strecken wird wahrscheinlich durch die Beschränkung der $V_{z u l}$ eine ausreichende Verhaltensänderung erreicht, die sich in einer Reduzierung der Unfallkenngrößen manifestiert. Auffällig ist, dass die unterschiedliche Wirkung für beide MK durch die Ergebnisse der Evaluation (Vergleiche Kapitel 5.3.3) gestützt wird. Demgegenüber ist die Wirksamkeit der MK „Überwachung $V_{\text {zul }}$ mit OGÜ“ (MK 16 und 17) was die MWu anbelangt unabhängig vom Einsatzort auf ähnlich hohem Niveau. Dies lässt sich aus der mittelbaren Restriktion der Maßnahme (Verfolgung von Geschwindigkeitsüberschreitungen) ableiten. Beide MK sind u. a. aufgrund ihrer geringen Kollektivbesetzung als „bedingt wirksam“ eingestuft.

Die Maßnahme „Sichthindernisse entfernen“ (MK 22 und 23) zeichnet sich an durch Verkehrszeichen geregelten Knotenpunkten sowohl Innerorts als auch Außerorts durch eine annähernd gleich hohe $\mathrm{MW}_{\mathrm{UK}}$ aus. Die Unterschiede bezüglich der $\mathrm{MW}_{U}$ deuten darauf hin, dass die Maßnahme an UH Außerorts insbesondere ein Rückgang der Unfälle mit hoher Unfallschwere bewirkt.

Der Vergleich der MK 26, 27, 30 und 36 zeigt auf, dass das alleinige „Markierung in Stand setzen“ (MK 27) an UH keine ausreichend wirksame Maßnahme darstellt. Hingegen sind offensichtlich gezielte Veränderungen von Markierungen, sei es durch Anpassungen oder Verbesserungen (MK 26) oder in bestimmten Fällen das Einfärben der Radfahrerfurt in Rot (MK 39) sowie das Markieren von Sperrflächen (MK 36) geeignetere Maßnahmen zur Bekämpfung von UH.

Die Klassifizierungen des Kollektivs der $\mathrm{MK}$ für $\mathrm{MW}_{\cup}$ und $\mathrm{MW}_{\mathrm{UK}}$ nach den vergebenen Prädikaten zeigen leichte Abweichungen zueinander (Abbildung 33). Der Anteil an MK, die als "nicht wirksam“ bewertet wurden, ist für die $\mathrm{MW}_{\mathrm{U}}$ gegenüber den $\mathrm{MW}_{\mathrm{UK}} \mathrm{Zu}$ Lasten der Gruppe der „wirksamen“ MK höher. Für die MK der Untersuchung lässt dies den Schluss zu, dass die Erhöhung der Verkehrssicherheit häufiger aus einem Rückgang der Unfallschwere und weniger der Unfallzahlen resultiert. Auf Basis des Kollektivs weisen weder die Unfallanzahl ( $\mathrm{MW}_{U}$ ) noch die Unfallschwere (MW $\mathrm{MW}_{\mathrm{UK}}$ ) für die MK 15 „Beschränkung der $V_{\text {zul }}$ (VZ-KP, Außerorts)“ und MK 37 „Verkehrszeichen versetzen (Außerorts)" eine deutliche Reduzierung auf. Bei der Interpretation der Kenngrößen für die MK 1 ist zu beachten, dass die dem Kollektiv zugrunde liegenden Maßnahmen (Stellen) alle von einem Streckenzug stammen. Somit ist die Variabilität der Daten nicht gegeben und die Aussagekraft dieser MK eingeschränkt. Diese MK ist auch die einzige mit einem negativen Median des NKV und gilt daher als „bedingt rentabel“.

Die gesonderte Betrachtung der sechs MK mit Kollektivgrößen von zwei oder drei Stellen verdeutlicht folgende Merkmale: In keiner dieser MK tritt eine deutliche Reduzierung der Unfallanzahl an allen Stellen auf. Für vier der MK ist aber an allen betrachteten Stellen ein deutlicher Rückgang der Unfallschwere (MW $\mathrm{MK}_{\mathrm{H}}$ zu verzeichnen (MK 8, 11, 16 und 33). 


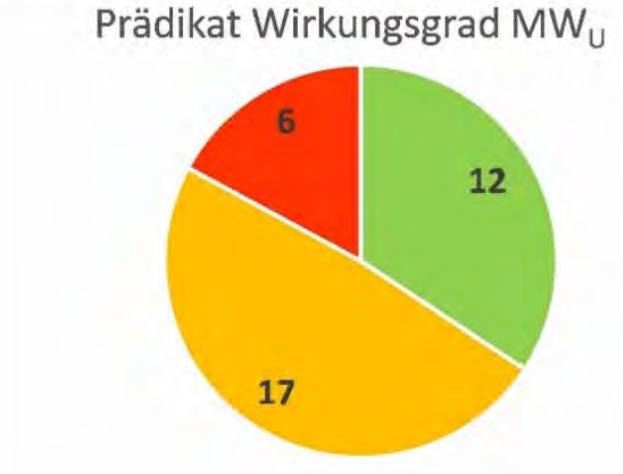

m wirksam $=$ bedingt wirksam $\mathbf{n}$ nicht wirksam
Prädikat Wirkungsgrad $\mathrm{MW}_{\mathrm{UK}}$

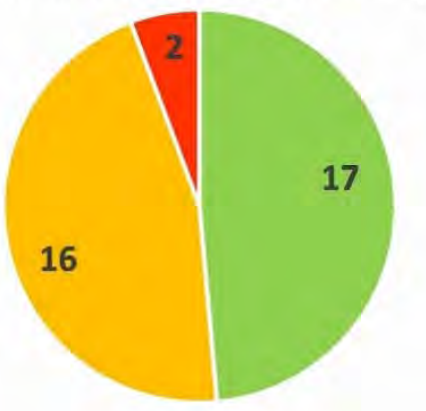

m wirksam $=$ bedingt wirksam $=$ nicht wirksam

Abbildung 33: Verteilung der Prädikate nach Klassifizierung der Maßnahmenwirkung $\mathrm{MW}_{U}$ (links) und $\mathrm{MW}_{\mathrm{UK}}$ (rechts)

\section{Wirtschaftlichkeit}

Die für die Berechnung herangezogenen mittleren Maßnahmenkosten (Investitionskosten) je MK unterscheiden sich deutlich (Abbildung 34, links). Der Anteil von MK mit mittleren Maßnahmenkosten $<10.000 €$ (Vergleiche Bereich 5 des Evaluationsbogens, Kapitel 5.3.2) liegt bei $80 \%$ aller MK. Die MK 22 „Sichthindernisse entfernen“, 35 „Gummischwellen aufbringen“ und 30 „Radfahrfurt Rot einfärben“ weisen mit unter $1.000 €$ die geringsten Kosten auf. Mit $70.000 €$ entfallen die höchsten Kosten auf die MK 16/17 - beide entsprechen der Überwachung der $\mathrm{V}_{\text {zul }}$ mit Ortsfester Geschwindigkeitsüberwachung. Nur bei lediglich zehn MK lagen Kostenangaben seitens der UKO vor. Für die restlichen Fälle wurde zur Kostenschätzung auf andere Quellen zurückgegriffen.
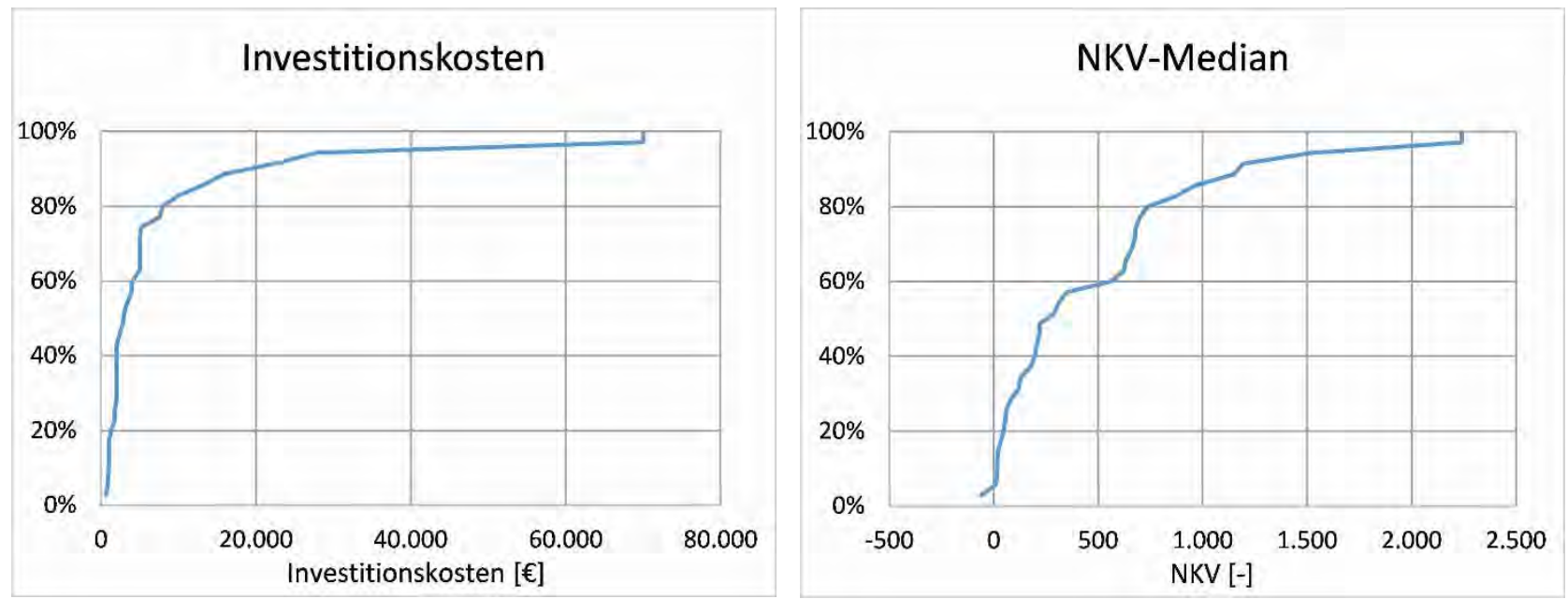

Abbildung 34: Summenhäufigkeit der mittleren Investitionskosten (links) und des NKV-Median (rechts) für die Maßnahmenkategorien MK

Für jede MK wurde der NKV-Median bestimmt. Lediglich eine MK hat einen negativen Medianwert. Die Kurve der Summenhäufigkeit (Abbildung 34, rechts) lässt eine dreiteilige Gruppierung der MK erkennen. Die erste Gruppe umfasst MK im linken unteren Teil des Kurvenverlaufs und damit MK mit einem NKV von 350 und niedriger. Auf diese Gruppe entfallen 20 MK (ca. $57 \%$ ). Daran schließt sich die Gruppe der MK mit einer NKV-Größenordnung von 550 bis 750 an (zweiter deutlicher Anstieg bis zu einer Häufigkeit von ca. $80 \%$ ). Die letzte Gruppe umfasst sieben MK mit sehr hohen NKV (> 850) und einer sehr breiten Streuung. Mit $r=-0,56$ korrelieren Investitionskosten und NKV-Median der MK gegengleich. D. h. tendenziell steigt mit Abnahme der Investitionskosten der NKV. Die NKV stellen aber keinen Indikator für die Wirksamkeit einer Maßnahme dar. Maßnahmen mit einem geringen Wirkungsgrad können aufgrund sehr geringer Maßnahmenkosten ein hohes NKV haben. Demgegenüber berechnet sich für Maßnahmen mit hohem Wirkungsgrad aus 
hohen Kosten u. U. ein geringes NKV. Mit der Einschränkung der MK 1 sind alle MK durchschnittlich als wirtschaftlich rentabel einzustufen. Die Gruppierung der MK nach der Art des Maßnahmeneingriffs verdeutlicht Unterschiede zwischen den einzelnen Gruppen (kompletter NKV-Bereich Abbildung 35 (links), Ausschnitt unterer NKV-Bereich Abbildung 35, (rechts)). Sehr hohe NKV weisen die beiden Eingriffsarten „Verkehrszeichen“ und „technischer Eingriff“ auf. Die breite Streuung in beiden Gruppen beruht unter anderem auf der Diversität der MK innerhalb der Gruppen. Da die Investitionskosten der MK in der Gruppe „Verkehrszeichen“ eine ähnliche Größenordnung aufweisen, ist die Streuung der Einzelwerte auf die unterschiedlichen Wirkungsgrade der Maßnahmen zurückzuführen. Auch die Streuung der Gruppe „Markierung“ ist primär von den verschiedenen Einsatzorten der MK geprägt. Demgegenüber sind für die Gruppe „technische Eingriffe" auch die Unterschiede bei den Investitionskosten bei der Interpretation zu berücksichtigen.

Die Größenordnungen der NKV der restlichen Gruppen fallen mit Ausnahme der Gruppe „Markierung" geringer aus. Erwartungsgemäß führen bauliche Eingriffe aufgrund tendenziell hoher Investitionskosten zu geringen NKV. Die Gruppe „Überwachung“ umfasst Maßnahmen, welche die Einrichtung von ortsfesten Geschwindigkeitsüberwachungsanlagen vorsehen. Demgemäß ziehen die hohen Kosten ebenfalls relativ geringe NKV nach sich. Die nicht wirtschaftliche MK 1 befindet sich in der Gruppe „LSA-Steuerung“.
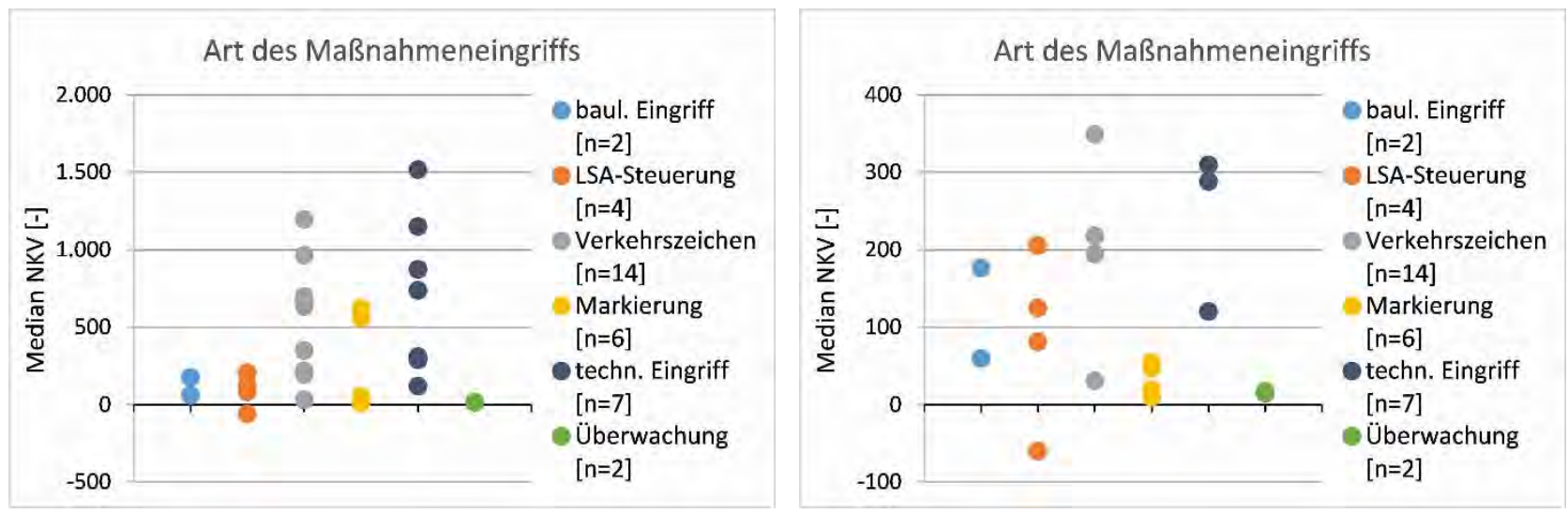

Abbildung 35: Verteilung der NKV-Mediane, Gruppierung nach Art des Maßnahmeneingriffs, kompletter NKV-Bereich (links), Ausschnitt unterer Bereich (rechts)

Die Einordnung der MK in Gruppen nach dem Primärdefizit enthält Abbildung 36. Auffallend ist die breite Streuung der Gruppen „Geschwindigkeit" und „Sicht“. Beide Gruppen sind überwiegend von MK mit NKV-Medianen > 200 geprägt. Ausnahme bilden in der Gruppe "Geschwindigkeit" die MK 16 und 17 - „Überwachung $\bigvee_{\text {zul }}$ mit OGÜ“ Außerorts an Knotenpunkt bzw. auf der Strecke. Für beide MK zeichnen zum überwiegenden Teil die hohen Investitionskosten für die niedrigen NKV verantwortlich. Die Streuung in den Gruppen „Begreifbarkeit“ und „Erkennbarkeit“ ist - aufgrund ähnlich hoher Investitionskosten aller MK - auf die vielseitigen Defizite und Rahmenbedingungen der UH zurückzuführen, bei denen die MK zum Einsatz kommen. Trotz des geringen Streubereichs und der Ausnahme von MK 1 sind die MK in der Gruppe „Verkehrsablauf“ mit NKV-Medianen > 10 als wirtschaftlich einzustufen. In ähnlicher Größenordnung sind den „Zustand/Umfeld“ verbessernde Maßnahmen einzuordnen. Aus der Gruppierung wird folgendes deutlich: Für UH mit Defiziten der Sichtbeziehung und/oder zu hoher gefahrener Geschwindigkeiten liegen aus wirtschaftlicher Sicht sehr rentable Maßnahmen vor. Für die anderen Gruppen gilt, dass die Wirtschaftlichkeit in Abhängigkeit der Randbedingungen stärker variiert. 

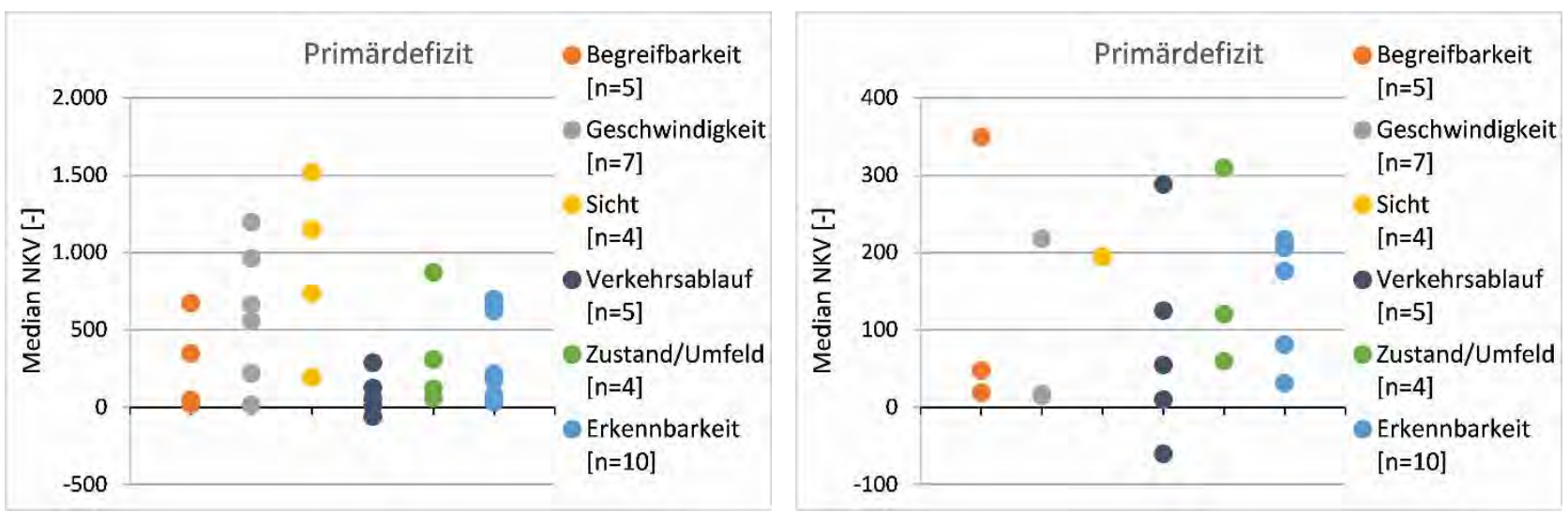

Abbildung 36: Verteilung der NKV-Mediane, Gruppierung nach Primärdefizit, kompletter NKV-Bereich (links), Ausschnitt unterer Bereich (rechts)

Ob die hohen NKV der MK ausschließlich aus geringen Investitionskosten und/oder aus hohen Nutzen - hohe Wirksamkeit der MK bezüglich des Rückgangs der Unfallschwer - resultieren, geht aus der Gegenüberstellung von MW $\mathrm{UK}_{\mathrm{UK}}$-Median und NKV-Median hervor. Abbildung 37 zeigt auf der linken Seite die Gegenüberstellung beider Größen gruppiert nach der Art des Maßnahmeneingriffs, auf der rechten Seite nach dem Primärdefizit.

Hinsichtlich der Art des Maßnameneingriffs gilt: Alle MK der Gruppe „technischer Eingriff“ weisen sowohl hohe $\mathrm{MW}_{\mathrm{UK}}(>50 \%$ ) als auch hohe NKV auf. MK der Gruppe „Überwachung" haben ebenfalls hohe Wirksamkeiten, jedoch ist das NKV auf niedrigem Niveau. Gleiches gilt mit Ausnahme der MK 1 für die Gruppen „LSA-Steuerung“ und „bauliche Maßnahmen“. Für die verbliebenen Gruppen „Verkehrszeichen“ und „Markierung" streuen die Werte überwiegend im Bereich hoher Wirksamkeiten (MW $\mathrm{UK}>50$ \%). Dies zeigt, dass einige MK beider Gruppen mitunter zur Bekämpfung von UH geeignet, stellenweise aber als Einzelmaßnahme in ihrer Wirkung nicht ausreichend sind.
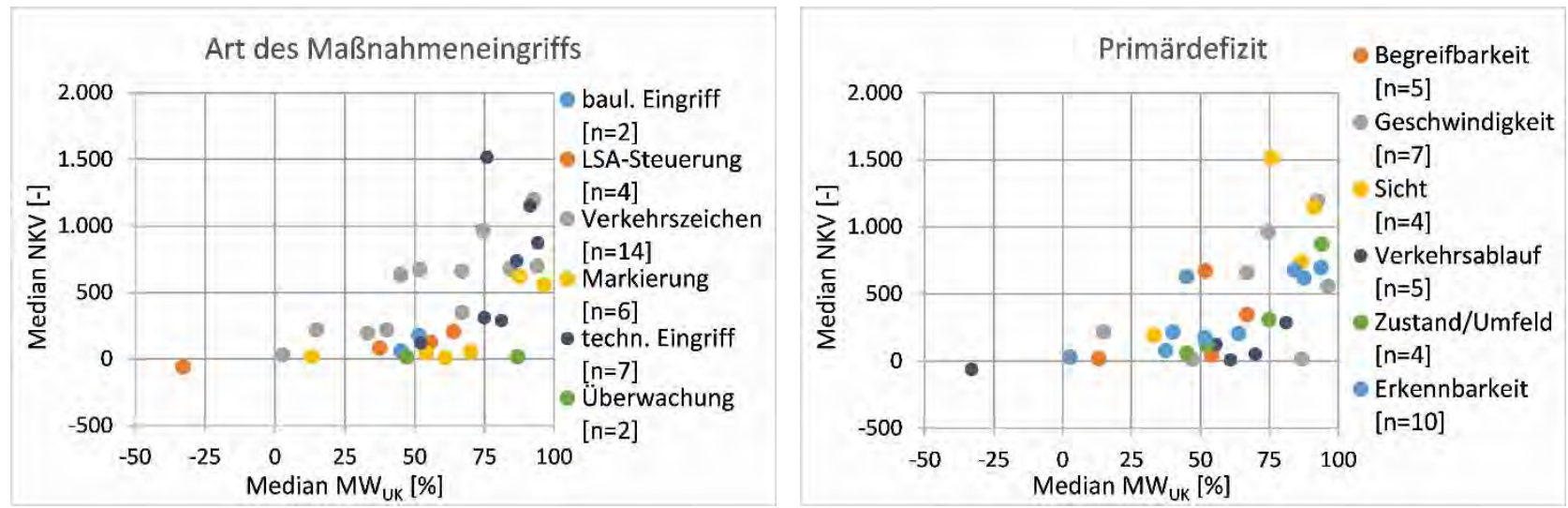

Abbildung 37: Gegenüberstellung Median $\mathrm{MW}_{\mathrm{UK}}$ und Median NKV nach Art des Maßnahmeneingriffs (links) und Primärdefizit (rechts)

Die Gruppierung der MK nach dem Primärdefizit offenbart die überwiegend hohe $\mathrm{MW}_{\mathrm{UK}}$ und Wirtschaftlichkeit von Maßnahmen die der Verbesserung von Defiziten bezüglich der "Sicht" dienen. Dabei handelt es sich ausnahmslos um MK, die an durch Verkehrszeichen oder rechts-vor-links geregelten Knotenpunkten zum Einsatz kommen. Der Gruppe „Verkehrsablauf“ zugehörige MK zeichnen sich in der Regel durch eine hohe $\mathrm{MW}_{\mathrm{UK}}$, im Verhältnis zu den restlichen MK aber geringeren NKV aus. Auch für die verbliebenen Gruppen gilt, dass die MK überwiegende hohe MWUK haben, aber bezüglich der NKV eine deutliche Streuung aufweisen.

\section{Gesamtprädikat}

Abbildung 38 stellt die Verteilung der MK nach dem Gesamtprädikat dar. Dieses setzt sich aus der Beurteilung von $\mathrm{MW}_{\mathrm{U}}, \mathrm{MW}_{\mathrm{Uk}}$ und NKV-Median zusammen. Das Prädikat „empfehlenswert“ haben 
zehn MK erhalten. Allen dieser MK liegen in der Auswertung sechs Stellen zugrunde. Mehr als die Hälfte (54\%) der MK trägt das Prädikat „bedingt empfehlenswert“. In dieser Gruppe befinden sich fünf MK mit einer Stellenanzahl $i<4$. Nach dem verwendeten Bewertungsansatz (Vergleiche Tabelle 15, Kapitel 6.1.3) können diese MK aufgrund der geringen Kollektivgröße per se kein Prädikat „empfehlenswert" erhalten. Hier gilt es, in weiteren Untersuchungen die allgemeine Wirkung zu evaluieren. Sechs MK erhalten überwiegend bezüglich einer nicht „deutlichen“ MWU kein Prädikat.

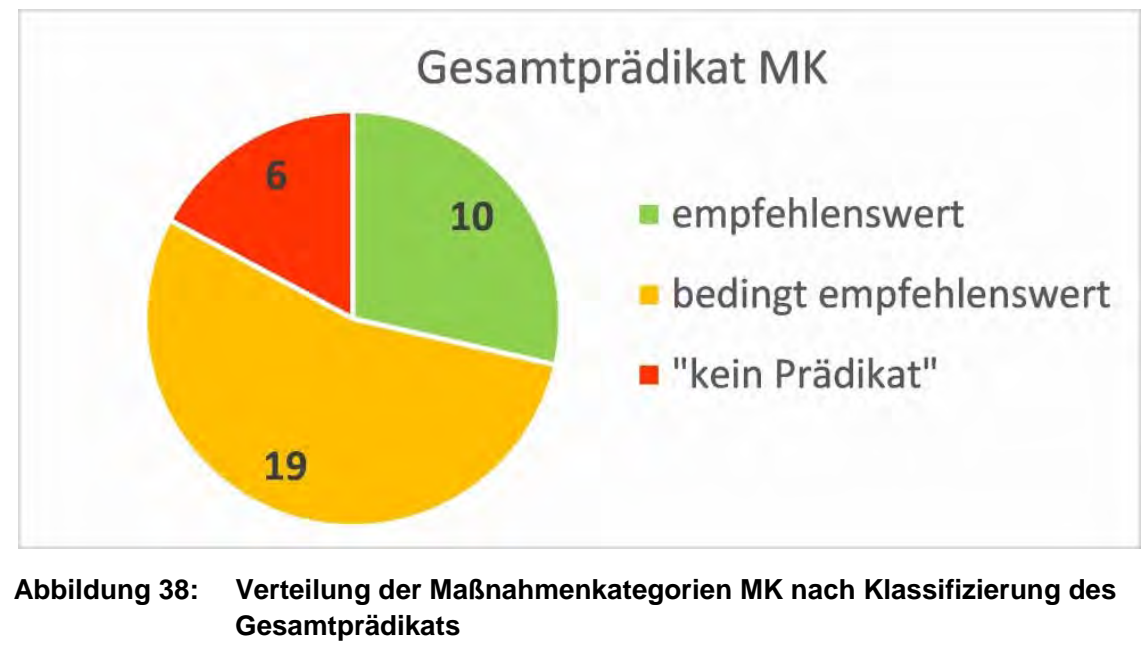

Das Gesamtprädikat gibt dem Anwender Auskunft über die Einsatzmöglichkeit der Maßnahme.

- Die MK mit dem Prädikat „empfehlenswert“ stellen allgemeingültige Optionen zur Umsetzung an UH dar, wenn die notwendigen Randbedingungen - Örtlichkeit, erkannte und zutreffende Defizite - erfüllt sind. Diese Maßnahmen versprechen eine hohe Erfolgswahrscheinlichkeit bei der Bekämpfung von UH.

- „Bedingt empfehlenswerte“ MK obliegen zum einen der Einschränkung, dass ihre Wirkung häufig nur auf die Reduzierung der Unfallschwere beschränkt oder die Kollektivgröße in der Untersuchung zu gering ist. Eine weitaus wichtigere Besonderheit dieser Gruppe stellt aber dar, dass eine positive Wirkung der MK nur für einige Stellen nachweisbar ist. Kommen diese MK für eine Umsetzung in Frage, sind die Bedingungen der Örtlichkeit (UH) besonders zu prüfen. Für eine erfolgversprechende Umsetzung sind diese Maßnahmen ggf. mit weiteren Maßnahmen im Paket zu kombinieren.

- MK ohne Prädikat sind nicht per se als mögliche Maßnahmen auszuschließen. Jedoch zeigen sich im Untersuchungskollektiv keine oder nur geringe Effekte, die zur Erhöhung der Verkehrssicherheit beitragen. Wird bei der Bearbeitung von UH festgestellt, dass die Umsetzung dieser MK zur Verbesserung/Aufwertung der Verkehrsanlage beiträgt - bspw. Verkehrszeichen versetzen (MK 37) oder Markierung in Stand setzen (MK 27) -, können diese Maßnahmen auch umgesetzt werden. Sie sind aber generell mit weiteren Maßnahmen zu flankieren. Ihre alleinige Umsetzung reicht i. d. R. nicht zur effektiven Bekämpfung einer UH aus.

\subsection{Zusammenfassung}

Die Unfalldaten- und Kostenanalyse als zweite Säule der Maßnahmenbeurteilung neben der Evaluation erlaubte eine objektive Bewertung der Wirksamkeit (Effektivität) und Wirtschaftlichkeit (Effizienz) der ausgewählten 35 Maßnahmenkategorien. Die Berechnung der Wirksamkeit stützte sich dabei auf einen Vergleich der mittleren jährlichen Unfallanzahlen und Unfallkosten im Vorher- und Nachher-Zeitraum. Die getrennte Betrachtung erlaubte eine differenzierte Bewertung der Wirkung der MK auf Unfallanzahl (MW $\mathrm{MW}_{\mathrm{U}}$ ) und Unfallschwere (MW $\mathrm{MK}_{\mathrm{UK}}$ ). Die Wirtschaftlichkeit der MK wurde über die Analyse des Nutzen/Kosten-Verhältnisses NKV (Median) bestimmt. Als Nutzen wurden die vermiedenen Unfallkosten, als Kosten die Investitionskosten der Maßnahme erfasst. 
Aus den über das Bundesgebiet gesammelten Daten zu bearbeiteten UH konnten165 Stellen berücksichtigt werden. Eine MK umfasst nach Datenlage mindestens zwei, maximal sechs Stellen. Die Aussagekraft der MK ist von der beinhalteten Stellenanzahl abhängig. Für das Gesamtkollektiv der bewerteten Maßnahmen ist eine Halbierung der $U(P)$ und Unfallkosten (bewerte Unfallkategorien) festzustellen. Neben einer Aggregation der Ergebnisse über alle MK wurde für jede MK ein Faktenblatt der Unfallanalyse angelegt. Dieses enthält maßgebende Informationen und Kennziffern zu Beurteilung der MK und final ein Gesamtprädikat der jeweiligen MK. Das Gesamtprädikat stützt sich auf die Beurteilung von $\mathrm{MW}_{U}, \mathrm{MW}_{\mathrm{UK}}$ und NKV einer $\mathrm{MK}$ in unterschiedlichen Abstufungen.

Für den überwiegenden Teil der MK liegen die Investitionskosten unter $10.000 €$. Geringe Investitionskosten stehen i. d. R. mit höheren NKV in Zusammenhang. Auf Basis der Größenordnung des NKV lassen sich die MK in drei Gruppen gliedern. Es wurde festgestellt, dass die Wirksamkeit der MK tendenziell häufiger aus dem Rückgang der Unfallschwere resultiert. Aus der differenzierten Analyse der NKV und Wirksamkeit der MK geht hervor, dass - für gegebene Randbedingungen Maßnahmenumsetzung über technische Eingriffe und Maßnahmen zur Bekämpfung von Sichtdefiziten an Knotenpunkten sowohl eine hohe Wirksamkeit als auch eine hohe Wirtschaftlichkeit aufweisen. Der überwiegende Teil der MK hat hohe $\mathrm{MW}_{\mathrm{UK}}$, während die NKV der MK größeren Streuungen unterliegen. Nur für die MK 1 „Verbesserung der Koordinierung“ sind - hinsichtlich des Untersuchungskollektivs - keine Wirksamkeit und damit keine Rentabilität nachgewiesen.

Für sechs der MK wird keine Empfehlung zur Umsetzung als Einzelmaßnahme ausgesprochen. Dies begründet sich i. d. R. aus einem nicht vorhandenen oder zu geringen Rückgang der Unfallanzahl. Das Prädikat „empfehlenswert“ erhielten zehn MK. Für diese MK ist bei richtigem Einsatz ein hoher Wirkungsgrad bei der Bekämpfung von UH zu erwarten.

Die Bewertung der MK und die Zuweisung eines Prädikats bilden die Grundlagen der Einordnung der Maßnahmen bezüglich der Auswahl geeigneter MK für die Handlungsempfehlungen. 


\section{$7 \quad$ Ableitung geeignete kostengünstige Maßnahmen}

\subsection{Vorgehen}

Aus volkswirtschaftlicher Sicht stellen Maßnahmenumsetzungen mit Nutzen-Kosten-Verhältnissen mit einem Wert von NKV>1 sinnvolle Investitionen dar, da der generierte Nutzen den Kosten überwiegt. Ein NKV > 1 lässt sich jedoch nicht unmittelbar mit dem Prädikat „kostengünstig“ verbinden. Von den Anwendern werden primär als kostengünstig die Maßnahmen angesehen, denen i. d. R. geringe Investitionskosten und eine schnelle zeitliche Umsetzbarkeit zugrunde liegen (Vergleiche Kapitel 5.3.3). Dass ein hohes NKV dabei nicht zwangsläufig aus einem deutlichen Rückgang der Unfallanzahl und damit der Unfallkosten, sondern mitunter aus den sehr geringen Investitionskosten resultiert, wurde hier aufgezeigt (Vergleiche Kapitel 6.2.2).

Für die praktische Anwendung kostengünstiger Maßnahmen ist es daher sinnvoll, solche Maßnahmen(-kategorien) zu identifizieren und zu klassifizieren, die aus volkswirtschaftlicher Sicht einen hohen Nutzen generieren. Diesem hohen Nutzen sollen zum einen geringe Investitionskosten, zum anderen auch eine hohe Wirksamkeit - Reduzierung Unfallanzahl (MW $\mathrm{MW}_{\mathrm{U}}$ ) und Unfallschwere (MW $W_{\cup K}$ - der MK zugrunde liegen. Grundlage der Bestimmung bilden die Erkenntnisse der Maßnahmenevaluation und Unfalldatenanalyse. Die Ergebnisse münden in einer Aufstellung „geeigneter" kostengünstiger Maßnahmen. Die gezielte Umsetzung dieser Maßnahmen führt zu einem effizienten Einsatz begrenzter finanzieller Haushaltsmittel.

Folgende Überlegungen führen zur Abgrenzung und Identifikation „geeigneter“ kostengünstiger Maßnahmen:

- Aus den Evaluation der MK geht hervor, dass die Mitglieder der UKO Maßnahmen mit Investitionskosten von $\leq 10.000 €$ als kostengünstig ansehen. Diese Einschätzung und Einstufung beruht auf den Erfahrungswerten der Praxis und dient nachfolgend als Indikator für die Definition „kostengünstig“ bezüglich der Investitionskosten einer MK. Diese Festlegung ermöglicht eine hohe Akzeptanz der gewählten Größenordnung durch die praktischen Anwender.

- Grundlegende Voraussetzung für eine gelungene Bekämpfung von UH ist die Umsetzung wirksamer Maßnahmen. Dabei gründet sich der Erfolg auf einen maßgebenden Rückgang der Unfallzahl ${ }^{16}$ und damit der Unfallschwere. Als maßgebend wird ein Rückgang der Unfallanzahl $\left(\mathrm{MW}_{\mathrm{U}}\right)$ und Unfallschwere (MWUK) einer MK von mindestens $30 \%$ (bezogen auf den Median) angesehen. Nach der in Tabelle 14, Kapitel 6.1.3 getroffenen Einteilung, setzt diese Forderung einen „erheblichen“ oder „bedeutenden“ Rückgang der Unfallanzahl und Unfallschwere (Unfallkosten) voraus.

- Maßgebend für die Umsetzung von Maßnahmen durch die UKO ist die Erwartung an eine hohe Erfolgswahrscheinlichkeit. Die Größenordnung von durchschnittlichen Wirkungsgraden einer MK in der Untersuchung kann jedoch durch Ausreißer beeinflusst sein. Die Angabe des auf den Median bezogenen Wirkungsgrades einer MK allein reicht zur Identifikation geeigneter Maßnahmen nicht aus. Zur Sicherung einer hohen Erfolgswahrscheinlichkeit sind daher nur die MK zu berücksichtigen, die neben den hohen Wirkungsgraden auch eine homogene Wirkung im Kollektiv haben. Diese sind nach Tabelle 15 Kapitel 6.1.3 sowohl für den Rückgang der Unfallanzahl als auch der Unfallschwere mit dem Prädikat „wirksam“ gekennzeichnet. Aus dieser Bedingung leitet sich ab, dass lediglich MK mit einer Stellenanzahl $\geq 4$ als geeignete Maßnahmen in Betracht kommen.

- Wie oben erwähnt, gelten Maßnahmenumsetzungen mit einem NKV $>1$ als wirtschaftlich sinnvoll. Zur Betrachtung der Wirtschaftlichkeit von MK wird der NKV-Median herangezo-

16 Es wird das maßgebende Ziel, die Verringerung der Unfallanzahl, als Bewertungsmaßstab zugrunde gelegt. 
gen, da er gegenüber dem Mittelwert weniger sensibel auf Ausreißer reagiert. MK mit einem NKV-Median > 1 kommen als geeignete Maßnahme in Betracht.

Aus der Betrachtung der NKV aller MK (Vergleiche Abbildung 34 (rechte Seite), Kapitel 6.2.2) geht eine Gruppierung der MK nach der Höhe des NKV-Median hervor. Besonders MK mit einem NKV > 500 zeugen von einer hohen Wirtschaftlichkeit. Des Weiteren liegen im Kollektiv MK mit sehr hohen $\mathrm{MW}_{U}$ und/oder MWUk vor. Damit ist es möglich, aus der Gruppe "geeigneter" kostengünstiger Maßnahmen „besonders geeignete“ kostengünstige Maßnahmen hervorzuheben. Sie unterscheiden sich gegenüber den „geeigneten“ kostengünstigen Maßnahmen durch ein besonders hohes NKV von $>500$ und einem bedeutenden $M_{U}$ oder MWUK $(\geq 60 \%)$. Tabelle 20 enthält die Kenngrößen zur Abgrenzung beider Arten der MK.

Tabelle 20: Kennwerte zur Abgrenzung „geeigneter" und „besonders geeigneter“ kostengünstiger Maßnahmen

\begin{tabular}{|c|c|c|}
\hline Kennwert & $\begin{array}{c}\text { Geeignete } \\
\text { kostengünstige MK }\end{array}$ & $\begin{array}{c}\text { Besonders geeignete } \\
\text { kostengünstige MK }\end{array}$ \\
\hline NKV & $>1$ & $\leq 10.000 €$ \\
\hline Investitionskosten & \multicolumn{2}{|c|}{$\geq 4$} \\
\hline Stellen i im Kollektiv & $30 \% \leq$ MWu und MWUK <60\% & $\begin{array}{c}\text { MWu und/oder MWUK } \geq 60 \% \\
\text { anderer Wert mindestens } 30 \%\end{array}$ \\
\hline Median Wirkungsgrade & \multicolumn{2}{|c|}{ MWu und MWUK mit Prädikat „wirksam“ } \\
\hline $\begin{array}{c}\text { Prädikat bzgl. } \\
\text { Wirkungsgrade }\end{array}$ & \multicolumn{2}{|c|}{ MW } \\
\hline
\end{tabular}

\subsection{Ergebnisse}

Die drei „geeigneten“ kostengünstigen Maßnahmen sind in Tabelle 21, die sechs „besonders geeigneten" Maßnahmen gesondert in Tabelle 22 dargestellt.

Die erstgenannte Gruppe enthält zwei innerörtliche Maßnahmen für Knotenpunkte mit Lichtsignalanlage. Die MK 24 ist für verschiedene Netzelemente als Maßnahme geeignet. Diese MK wurde in der Evaluation von nur der Hälfte der Befragten als kostengünstige Maßnahme bewertet. Hier spiegelt sich die Einschätzung wider, dass Maßnahmen mit Investitionskosten $>10.000 €$ nicht mehr als kostengünstig angesehen werden. Interessant ist, dass die MK 21 von nur ca. der Hälfte der Befragten als wirksame Maßnahme eingeschätzt wurde, während bei den beiden anderen MK ca. 9 von 10 Befragten die MK als wirksam einschätzen. Die Einschätzung der MK 21 belegt den Stellenwert des Forschungsprojektes und den zu entwickelten Handlungsempfehlungen. Hier kann den praktischen Anwendern der Einsatz von Maßnahmen - unter den gegebenen Rahmenbedingungen - vermittelt werden, die von einem Teil der Anwender womöglich bisher nicht als Optionen in Betracht gezogen wurden. Die MK 21 und 24 stehen mit Defiziten der Erkennbarkeit, MK 2 mit Defiziten bezüglich des Verkehrsablaufs in Zusammenhang.

Tabelle 21: Übersicht geeigneter kostengünstiger Maßnahmen

\begin{tabular}{|c|c|c|c|c|c|c|c|c|c|}
\hline \multicolumn{2}{|r|}{ Maßnahmenkategorie } & \multirow{2}{*}{ Ort } & \multirow{2}{*}{$\begin{array}{l}\text { Straßen- } \\
\text { Charakt. }\end{array}$} & \multicolumn{2}{|c|}{$\begin{array}{l}\text { Median Wir- } \\
\text { kungsgrad [\%] }\end{array}$} & \multirow{2}{*}{$\begin{array}{l}\text { NKV } \\
\text { Median }\end{array}$} & \multirow{2}{*}{$\begin{array}{c}\varnothing \text { Inv.- } \\
\text { Kosten } \\
{[€]}\end{array}$} & \multicolumn{2}{|c|}{$\begin{array}{c}\text { In Evaluation einge- } \\
\text { schätzt als... }\end{array}$} \\
\hline Nr. & Name & & & MWu & & & & $\begin{array}{c}\text { low-cost } \\
{[\%]}\end{array}$ & $\begin{array}{c}\text { wirksam } \\
\text { [\%] }\end{array}$ \\
\hline 2 & Änderung der Signalisierung & 10 & KP-LSA & 29 & 56 & 125 & 5.000 & 83 & 85 \\
\hline 21 & $\begin{array}{l}\text { Leuchtmittel Signalgeber } \\
\text { verbessern }\end{array}$ & 10 & KP-LSA & 42 & 64 & 206 & 8.000 & 75 & 52 \\
\hline 24 & $\begin{array}{l}\text { Beleuchtung } \\
\text { anpassen/verbessern }\end{array}$ & 10 & beliebig & 53 & 52 & 176 & 10.000 & 52 & 89 \\
\hline
\end{tabular}

Gegenüber der Gruppe der „geeigneten“ kostengünstigen Maßnahmen resultiert die Zuordnung der MK in die Gruppe der „besonders geeigneten“ Maßnahmen allein aus einem NKV > 500. Das 
Einsatzgebiet von fünf der sechs „besonders geeigneten“ kostengünstigen Maßnahmen liegt im Bereich von Landstraßen. Die MK „Sichthindernisse entfernen“ ist sowohl für den Einsatz Innerorts als auch Außerorts besonders geeignet. Die Maßnahmen für UH an Knotenpunkten beschränken sich auf die Regelungsart „durch Verkehrszeichen geregelt“. Allen drei dieser MK liegen Defizite in den Sichtbeziehungen am Knotenpunkt zugrunde. Die MK, die für den Einsatz an UH auf der Strecke in Frage kommen, zielen auf eine Veränderung des Fahrverhaltens bzw. Erhöhung der Er-

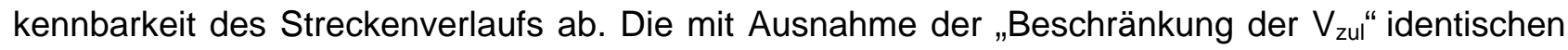
MK 7 und MK 38 zeigen eine sehr ähnliche $M W_{U}$ bzW. MW $W_{U k}$. Die zusätzliche Beschränkung der $V_{z u l}$ in der MK 38 führt zu keiner höheren Wirkung ${ }^{17}$.

Tabelle 22: Übersicht besonders geeigneter kostengünstiger Maßnahmen

\begin{tabular}{|c|c|c|c|c|c|c|c|c|c|}
\hline \multicolumn{2}{|r|}{ Maßnahmenkategorie } & \multirow{2}{*}{ Ort } & \multirow{2}{*}{$\begin{array}{l}\text { Straßen- } \\
\text { charakt. }\end{array}$} & \multicolumn{2}{|c|}{$\begin{array}{l}\text { Median Wir- } \\
\text { kungsgrad [\%] }\end{array}$} & \multirow{2}{*}{$\begin{array}{l}\text { NKV } \\
\text { Median }\end{array}$} & \multirow{2}{*}{$\begin{array}{c}\varnothing \text { Inv.- } \\
\text { Kosten } \\
{[€]}\end{array}$} & \multicolumn{2}{|c|}{$\begin{array}{c}\text { In Evaluation einge- } \\
\text { schätzt als... }\end{array}$} \\
\hline Nr. & & & & MWu & MWUK & & & $\begin{array}{c}\text { low-cost } \\
{[\%]}\end{array}$ & $\begin{array}{c}\text { wirksam } \\
{[\%]}\end{array}$ \\
\hline 7 & $\begin{array}{l}\text { Z } 625 \text { StVO (Richtungstafel } \\
\text { in Kurven) aufstellen }\end{array}$ & $\mathrm{AO}$ & Strecke & 50 & 94 & 698 & 3.000 & 100 & 88 \\
\hline 12 & Beschränkung $\mathrm{V}_{\text {zul }}$ & $\mathrm{AO}$ & Strecke & 74 & 74 & 963 & 2.000 & 100 & 81 \\
\hline 22 & Sichthindernisse entfernen & 10 & $\mathrm{KP}-\mathrm{VZ}$ & 76 & 87 & 736 & 967 & 82 & 94 \\
\hline 23 & Sichthindernisse entfernen & $\mathrm{AO}$ & $\mathrm{KP}-\mathrm{VZ}$ & 38 & 76 & 1.517 & 5.183 & 82 & 94 \\
\hline 25 & Sichtschutzzaun aufstellen & $\mathrm{AO}$ & $\mathrm{KP}-\mathrm{VZ}$ & 48 & 91 & 1.149 & 2.833 & 83 & 70 \\
\hline 38 & $\begin{array}{l}\text { Beschränkung } V_{\text {zul }} \text { und } Z \\
625 \text { StVO (Richtungstafel in } \\
\text { Kurven) aufstellen }\end{array}$ & $\mathrm{AO}$ & Strecke & 42 & 92 & 1.196 & 3.417 & 96 & 74 \\
\hline
\end{tabular}

Für alle MK liegen die mittleren Investitionskosten deutlich unter $10.000 €$. Die Unterschiede in den mittleren Investitionskosten zwischen den MK 22 und MK 23 beruhen auf der Spannbreite der von den UKO übermittelten Kosten. Mehr als $80 \%$ der Befragten schätzen die Maßnahmen als „lowcost Maßnahme" ein. Von allen MK sticht die MK 25 noch einmal hervor, da sie im Vergleich mit einem relativ geringen Anteil von $70 \%$ als wirksam eingestuft wird. Es ist zu beachten, dass von 23 Befragten, welche die MK evaluierten, nur acht die Maßnahme auch bekannt war. Nur drei Viertel der Befragten sehen in der MK 38 eine wirksame Maßnahme, wo hingegen die MK 7 - lediglich Aufstellen von Z 625 StVO ohne Beschränkung der $V_{\text {zul }}$ - neun von zehn Befragten die MK als wirksam prädikatisieren. Diese Unterschiede sind mutmaßlich auch auf die verschiedenen befragten Gruppen zurückzuführen.

Aufbauend auf den festgelegten Kriterien, welche geeignete kostengünstige Maßnahmen definieren, konnten aus dem Untersuchungskollektiv neun geeignete MK identifiziert werden, sechs davon als besonders geeignet. Allen diesen MK sind niedrige Investitionskosten, hohe NKV (>100) und homogene sowie mindestens deutliche Maßnahmenwirkungen eigen. Diese Maßnahmen tragen unter gegebenen Randbedingungen zur effektiven und effizienten Bekämpfung von UH bei.

17 Diese Einschätzungen sind auf die in der Untersuchung analysierten Fälle beschränkt. 


\section{$8 \quad$ Handlungsempfehlung zur Umsetzung von Maßnahmen}

\subsection{Vorgehen}

Die Entwicklung der Handlungsempfehlungen zur Umsetzung von Maßnahmen gründet auf den Ergebnissen und Erkenntnissen der Unfalldatenauswertung und Wirtschaftlichkeitsuntersuchungen der bewerteten 35 Maßnahmenkategorien (MK) unter Kapitel 6. Die Ergebnisse der Evaluation fließen hier nicht ein, sie geben lediglich Auskunft über das Grundverständnis und die Akzeptanz von Maßnahmen. Ziel der Handlungsempfehlung ist es, die UKO bei der Auswahl von Maßnahmen zu sensibilisieren und mit einem Beispielkatalog empfehlenswerte Maßnahmen zur Verfügung zu stellen.

Für die schnelle zielgerichtete Auswahl von geeigneten Maßnahmen in Abhängigkeit des Unfallgeschehens an einer UH wird die Vorgehensweise der Maßnahmenfindung vergleichbar dem Merkblatt „Auswertung von Straßenverkehrsunfällen Teil 2 - Maßnahmen gegen Unfallhäufungen“ [FGSV 2002], bzw. „Maßnahmenkatalog gegen Unfallhäufungen MaKaU“ [MAIER et al. 2015] aufbereitet. Das Auffinden geeigneter Maßnahmen orientiert sich auf einer hierarchischen top-down Gliederung folgender Merkmale der UH:

- Ortslage

- Straßencharakteristik

- Unfalltyp/typisierter Konflikt

- vorliegende Defizite

Die MK werden anhand ihrer Randbedingungen und der vorliegenden Unfalldaten nach diesen Merkmalen klassifiziert und geordnet. Da die Datengrundlage der MK keine abschließende Differenzierung nach dem typisierten Konflikt (3-stelliger Unfalltyp) zulässt, sind die Ebenen Unfalltyp und typisierter Konflikt in einer Stufe zusammengefasst. Die letzte Stufe sieht einer Zuordnung der Maßnahmen in Abhängigkeit der vorliegenden Defizite einer UH vor. Einige MK sind keiner bestimmten Straßencharakteristik zuzuordnen, ihre Einsatzmöglichkeiten im Straßennetz sind sehr heterogen. Ihrer Wirkung zielt häufig auf mehrere Unfalltypen/Konfliktsituationen ab. Dies gilt bspw. für die Verbesserung der Beleuchtung oder das Anbringen „sonstiger" Gefahrenzeichen. Da diese MK nicht eindeutig zuzuordnen sind, erfolgt eine Gruppierung der vorliegenden MK. Die MK, die zur Bekämpfung einer Vielzahl unterschiedlicher Konfliktsituationen zuzuordnen sind, werden in der Gruppe der divers konfliktbezogenen Defizite und Maßnahmen geführt. Die MK, die gezielt auf bestimmte Konfliktsituationen abstellen, sind in der Gruppe der direkt konfliktbezogenen Defizite und Maßnahmen(-kategorien) zusammengefasst.

Liegen dem Anwender mehrerer Maßnahmenmöglichkeiten zur Bekämpfung eines Defizites vor, so sind diese nach den Kriterien Eignung. Angemessenheit und Durchsetzbarkeit zu diskutieren, zu prüfen und gegeneinander abzuwägen [FGSV 2012]. Die erfolgversprechendste Maßnahme (nach diesen Kriterien) soll durch den Beschluss der UKO zur Umsetzung empfohlen werden. Für keine der in der Untersuchung analysierten MK sind umfangreiche Planungs- oder Realisierungsphasen erforderlich. Aus den Evaluationen geht hervor, dass der Großteil der Befragten für keine der MK Realisierungszeiträume von mehr als 12 Monaten einschätzt. Demgemäß werden die MK nicht als langfristige Maßnahme angesehen. Eine Unterscheidung nach kurz- und mit tel-/langfristigen Maßnahmen erfolgt in der Handlungsempfehlung daher nicht.

In den Handlungsempfehlungen werden nur MK berücksichtigt, die das Gesamtprädikat „empfehlenswert“ oder „bedingt empfehlenswert" tragen. Eine Ausnahme stellt die MK 1 (Verbesserung der Koordinierung) dar. Es wird angenommen, dass diese MK bei konkret defizitbezogener und zielgerichteter Umsetzung ebenfalls eine wirksame Maßnahme darstellt.

Der „Maßnahmenkatalog gegen Unfallhäufungen MaKaU“ [MAIER et al. 2015] enthält eine dreistufige Kategorisierung der Maßnahmen nach ihrem Wirkungsgrad auf die Entwicklung der Unfallkos- 
ten. Dieser bietet den UKO eine Hilfe bei der Auswahl geeigneter wirksamer Maßnahmen. Die in dieser Untersuchung durchgeführte Einschätzung der Maßnahmen zielt jedoch auf die Benennung wirksamer kostengünstiger Maßnahmen ab und beinhaltet die Bewertungskriterien $\mathrm{MW}_{\mathrm{U}}, \mathrm{MW}_{\mathrm{UK}}$ und NKV. Um diesem in der Untersuchung gewählten Bewertungsansatz Rechnung zu tragen, erfolgt in der Darstellung der Handlungsempfehlung lediglich eine Differenzierung der MK nach ihrem Gesamtprädikat. Durch die Auflistung der Kriterien $\mathrm{MW}_{U}, \mathrm{MW}_{\mathrm{UK}}$ und NKV in den Faktenblättern der MK wird eine mögliche spätere digitale Umsetzung der MK im Maßnahmenkatalog berücksichtigt.

\subsection{Ergebnisse}

Abbildung 39 enthält die Vorgehensweise zur Auswahl geeigneter Maßnahmen gegen UH Innerorts. Direkt konfliktbezogene Defizite und diesbezügliche Maßnahmen liegen nur für Knotenpunkte, differenziert nach den Arten der Regelung

- mit Lichtsignalanlage,

- rechts-vor-links und

- durch Verkehrszeichen vor.

Die Matrix wird von links nach rechts gelesen und schränkt mit jeder Stufe die Auswahlmöglichkeiten ein. Den Pfeilen folgend führt das Schema zur Aufführung von verschiedenen Defiziten eines Unfalltyps. Anhand der vorliegenden Defizite an einer UH kann der Anwender eine entsprechende Maßnahme (Nummer) ablesen und auswählen. Die Detailinformationen zur Maßnahme sind den jeweiligen Faktenblättern - den Bezug stellt die MK-Nummer her - zu entnehmen.

Für Knotenpunkte mit Lichtsignalanlage liegen Maßnahmenkategorien für Defizite vor, die sich auf drei verschiedene Unfalltypen zurückführen lassen. Hier am häufigsten vertreten sind Maßnahmen gegen Unfälle im Längsverkehr. Maßnahmen gegen UH an durch Verkehrszeichen geregelten Knotenpunkten beschränken sich in der Untersuchung auf die Unfalltypen Abbiegen (Typ 2) und Einbiegen/Kreuzen (Typ 3). Lediglich die MK 21 und 22 sind im Untersuchungskollektiv für die Knotenpunkte Innerorts empfehlenswerte Maßnahmen. Es liegen vier MK vor, deren Anwendung nicht auf einzelne Konflikttypen beschränkt ist. Diese sind mit den entsprechenden maßgebenden Defiziten unter den divers konfliktbezogenen Maßnahmen aufgeführt.

Die Vorgehensweise zur Auswahl geeigneter Maßnahmen gegen UH auf Landstraßen enthält Abbildung 40 und ist vom Ablauf mit dem Schema für Innerorts identisch. Direkt konfliktbezogene Maßnahmen liegen für die Streckencharakteristiken „Strecke“ und „Knotenpunkt (durch Verkehrszeichen geregelt)“ vor. Eine erhebliche Maßnahmenauswahl besteht für UH auf Strecken mit dem Hauptunfalltyp Fahrunfall. Für durch Verkehrszeichen geregelte Knotenpunkte liegen für die beiden knotenpunkttypischen Unfalltypen 2 und 3 Maßnahmen vor. Insbesondere für den Typ 3 bietet die Handlungsempfehlung eine breite Palette an Maßnahmen in Abhängigkeit der Defizite. Für UH auf der Strecke stehen zwei MK zur Verfügung, deren Anwendung sich nicht auf spezielle Unfalltypen beschränkt. Die MK 16 und 17 sind als „empfehlenswert“ berücksichtigt, obwohl sie nur das Prädikat „bedingt empfehlenswert"18 besitzen. Jedoch ist aus [MAIER et al. 2011] abzuleiten, dass diese Maßnahmen(-kategorien) einen hohen Wirksamkeitsgrad besitzen und daher in der Handlungsempfehlung als „empfehlenswert" eingeschätzt werden.

Die Datengrundlage der Unfallanalyse war ausschlaggebend, welchen Defiziten die MK zugeordnet wurden. Es besteht theoretisch die Möglichkeit einige MK weiteren Defiziten zuzuordnen. Aufgrund einer gesicherten Vorgehensweise wurde darauf verzichtet. Es gilt für Innerorts und Außerorts, dass alle - ohne besondere Kennzeichnung - aufgeführten „empfehlenswerten“ Maßnahmen gleichzeitig auch Maßnahmen darstellen, die bezüglich der Betrachtung als kostengünstige Maßnahme als „geeignet“ oder „besonders geeignet“ gelten.

${ }^{18}$ Das Prädikat „bedingt empfehlenswert“ begründet sich aus der geringen Anzahl betrachteter Stellen. 


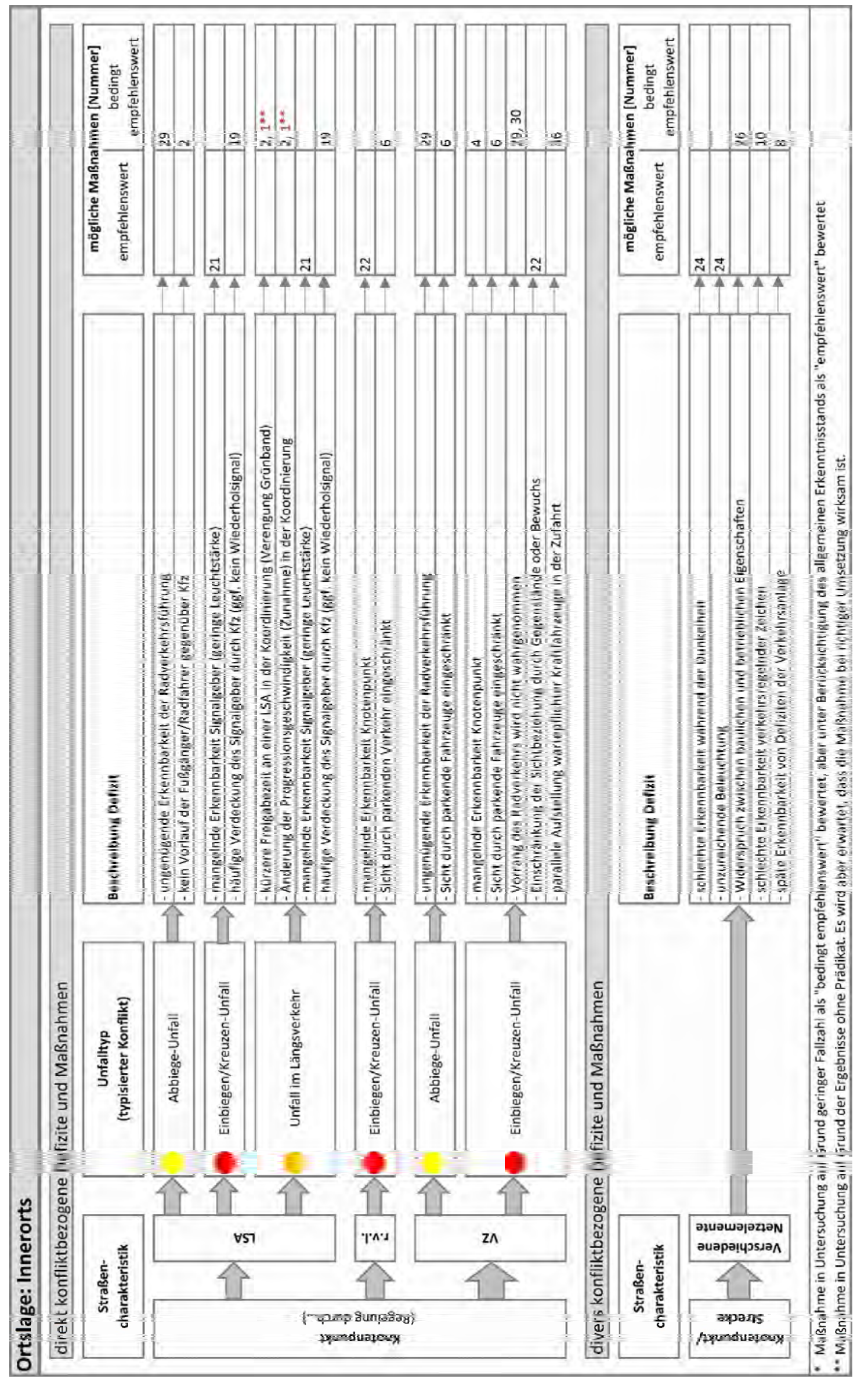

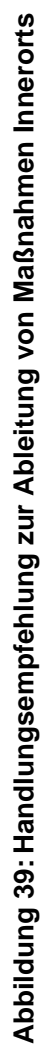




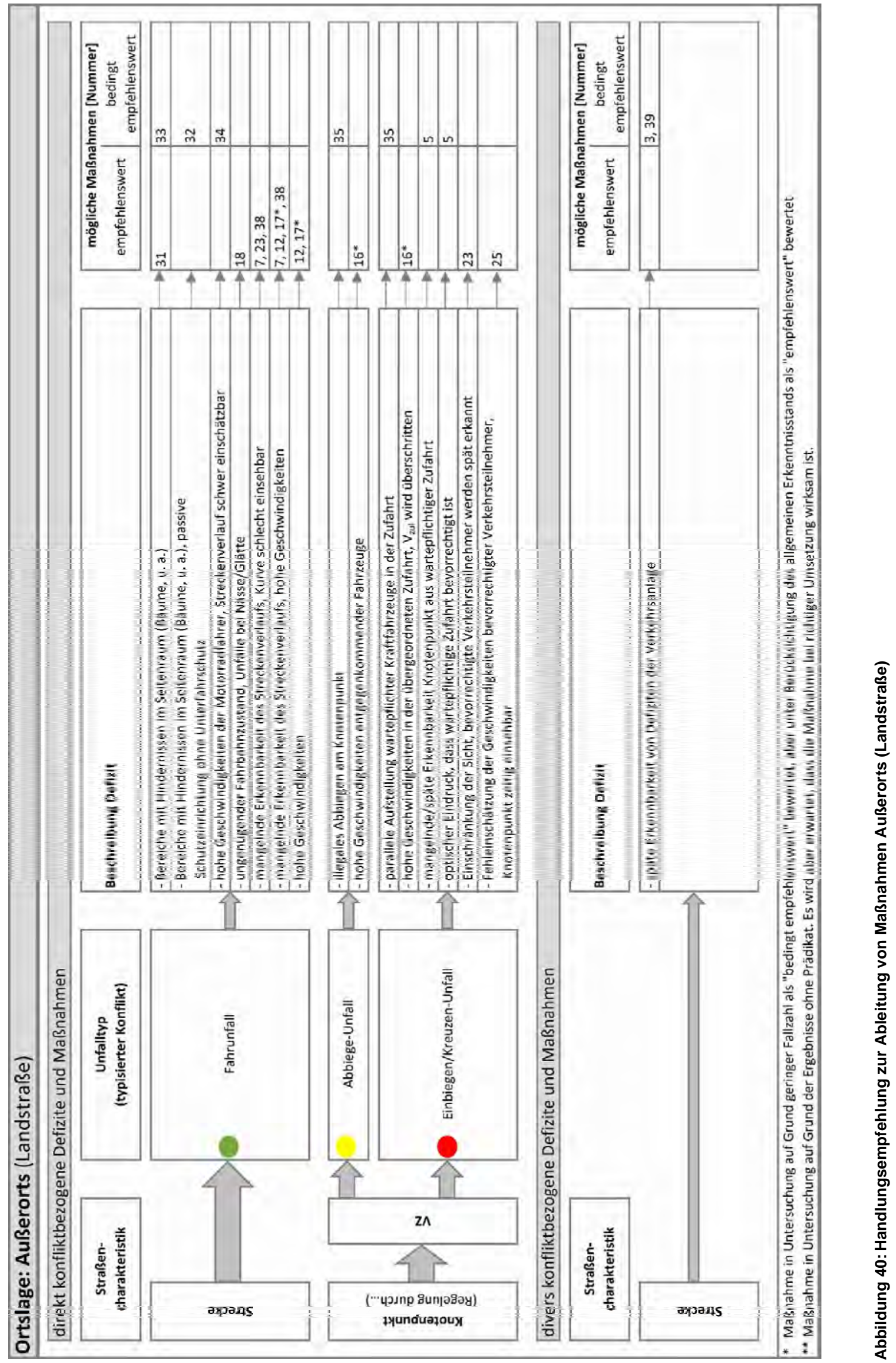


Ziel der Untersuchung war die systematische Darstellung von kostengünstigen Maßnahmen an Unfallhäufungen und der Bewertung deren Wirksamkeit. Dazu wurden unter enger Einbindung der Unfallkommissionen Maßnahmen erhoben, analysiert und kategorisiert. Ein ausgewähltes Kollektiv an Einzelmaßnahmen wurde durch Unfallkommissionsmitglieder und Experten evaluiert und die Wirksamkeit und Wirtschaftlichkeit auf Basis der Unfalldaten berechnet. Die Ergebnisse mündeten in der Bereitstellung von Faktenblättern für die Einzelmaßnahmen, die Ableitung kostengünstiger Maßnahmen und die Bereitstellung von Handlungsempfehlungen zu deren Anwendung. Damit steht den Unfallkommissionen eine konkrete Hilfe bei der Auswahl geeigneter und wirkungsvoller kostengünstiger Maßnahmen gegen Unfallhäufungen zur Verfügung, die auf abgesicherten Erkenntnissen beruht.

Ausgangspunkt der Untersuchung bildete eine mehrstufige Kontaktaufnahme und Datenerhebungen bei Unfallkommissionen im Bundesgebiet. Von 110 Unfallkommissionen, die sich an einer ersten Befragung zur Arbeit in Unfallkommissionen beteiligten, haben 64 Unfallkommissionen Daten (aus Maßnahmenumsetzungen) in ihrem Zuständigkeitsbereich zur Verfügung gestellt. Das Datenmaterial wurde zur Vereinheitlichung und Vergleichbarkeit aufbereitet und bezüglich Örtlichkeit, Art der Maßnahme, Datenqualität und weiterer Merkmale in einer Datenbank kategorisiert und plausibilisiert. Inklusive einer später erfolgten gezielten Nacherhebung lagen 1.812 Maßnahmen, geordnet in 82 Maßnahmenkategorien vor. Maßnahmenkategorien fassen (annähernd) identische Maßnahmen zusammen. Nahezu alle Maßnahmenumsetzungen beziehen sich auf die Bereiche Innerorts sowie Landstraßen. Die Analyse der Datenqualität zeigte deutliche Unterschiede auf. Häufig fehlen ausreichende Vorher- und/oder Nachher-Zeiträume zur Unfallanalyse. In nur 285 Fällen konnte bei der Auswertung auf die 3-Jahreskarte $U(P)$ und 1-Jahreskarte mit dem Gesamtunfallgeschehen in beiden Zeiträumen zurückgegriffen werden.

Angesichts der Anzahl verschiedenen Maßnahmenkategorien wurde eine Auswahl 35 geeigneter Kategorien für die weitere Untersuchung getroffen. Das Augenmerk lag auf der Untersuchung von Maßnahmenkategorien, für die bisher nur ein geringer oder unzureichender Wissensstand vorlag und die Maßnahme in der Regel einzeln, ohne weitere begleitende Maßnahmen, umgesetzt wurde. Die Betrachtung von sechs Einzelstellen je Maßnahmenkategorie diente zur Kräftigung der Aussage und Verringerung zufälliger Einflüsse bei der Bewertung.

Zwei Säulen der Maßnahmenbewertung waren Bestandteil der weiteren Untersuchung. Die erste Säule umfasste die Evaluation der Maßnahmenkategorien durch eine Gruppe von Experten und Mitgliedern der Unfallkommission mittels eines Fragebogens. Die Evaluationen wurden im Rahmen von acht Schulungen in mehreren Bundesländern durchgeführt. Primäres Ziel dieser Herangehensweise war der Erkenntnisgewinn darüber, wie Maßnahmen durch die Praxis bezüglich ihrer Wirksamkeit und Umsetzbarkeit eingeschätzt werden. Die Ergebnisse dienten unter anderem als Indikatoren zur Ableitung der Quantifizierung des Prädikats „kostengünstig“ und „wirksam“. Demnach schätzen Unfallkommissionen Maßnahmen bis zu Investitionskosten von ca. $10.000 €$ und mit einer zeitlichen Umsetzbarkeit kleiner sechs Monate als kostengünstig ein. Maßnahmen werden als „wirksam“ ab einer mäßigen Reduzierung von Unfallanzahl oder Unfallschwere eingestuft. Für jede Maßnahmenkategorie liegt ein Faktenblatt zu den Ergebnissen der Evaluation vor.

Die zweite Säule der Untersuchung beinhaltete die Analyse der zur Verfügung gestellten Maßnahmenumsetzung. Aus dem Vergleich der Unfallanzahl bzw. Unfallkosten im Vorher- und Nachher-Zeitraum konnte die durchschnittliche Maßnahmenwirkung und unter Berücksichtigung der (Investitions-)Kosten die Wirtschaftlichkeit - abgebildet über das Nutzen-Kosten-Verhältnis - der einzelnen Maßnahmenkategorien bestimmt werden. Die Berechnung stützte sich auf die größtmögliche Anzahl an Unfallkategorien, die je Maßnahmenkategorie zur Verfügung standen. Auf Grund fehlender Angaben mussten bei der Wirtschaftlichkeitsberechnung häufig Maßnahmenkos- 
ten aus anderen Quellen übernommen werden. Die Bewertung von Effektivität und Effizienz münden in einer Gesamteinschätzung der Maßnahmenkategorie für die weitere Anwendung. Zehn der Kategorien erhielten das Prädikat „empfehlenswert“, 19 Kategorien das Prädikat „bedingt empfehlenswert". Die aggregierte Betrachtung aller Maßnahmenkategorien offenbarte, dass mit der Maßnahmenumsetzung die Unfälle mit Personenschaden als auch die mittleren Unfallkosten halbiert werden konnten. Die Ergebnisse der Unfallanalyse sind ebenfalls für jede Maßnahmenkategorie in einem Faktenblatt zusammengefasst.

Die Ableitung (besonders) geeigneter kostengünstiger Maßnahmen baut auf den Erkenntnissen der Evaluation und der Unfalldatenanalyse auf. Zur Einstufung der Maßnahmenkategorien werden Kriterien der Wirksamkeit, der Kollektivzusammensetzung und der Wirtschaftlichkeit der Maßnahmenkategorien herangezogen. Als besonders geeignet stellten sich insbesondere Maßnahmen heraus, die zur Verbesserung der Sichtbeziehung an Knotenpunkten beitragen. Diese kombinieren hohe Wirksamkeit mit geringem Kosteneinsatz. Weiterhin stellt auch der Einsatz von Z 625 StVO (Richtungstafel in Kurven) eine sehr kostengünstige und gleichzeitig deutlich wirksame Maßnahme mit hoher Erfolgswahrscheinlichkeit dar. Für den innerörtlichen Bereich bilden zwei Maßnahmen für Defizite an LSA geregelten Knotenpunkten sowie die allgemein einsetzbare Maßnahme „Beleuchtung verbessern/anpassen" das Kollektiv geeigneter kostengünstiger Maßnahmen.

Die finale Entwicklung der Handlungsempfehlung rundet die Untersuchung ab und stellt ein Handwerkszeug für die Unfallkommissionen zum Auffinden und bei der Auswahl geeigneter kostengünstiger Maßnahmen gegen Unfallhäufungen dar. Sie ist mit ihrem thematischen und strukturellen Aufbau an den gängigen Empfehlungen und Literaturen zur Maßnahmenfindung orientiert. Die Verknüpfung der als empfehlenswert bzw. als geeignet kostengünstig eingestuften Maßnahmen in den „Maßnahmenkatalog gegen Unfallhäufungen MaKaU“ ist durch die Aufbereitung der Daten gewährleistet.

Die Untersuchung verdeutlichte Unterschiede in der Bearbeitung von Unfallhäufungen zwischen den einzelnen Unfallkommissionen. Insbesondere die Art und der Umfang der Datenhaltung zeigte eine weite Spannbreite. Häufig beruhen Analysen, aber insbesondere Wirksamkeitskontrollen auf unzureichenden Zeiträumen. Dies verdeutlicht die Notwendigkeit der kontinuierlichen Schulung und Fortbildung von Unfallkommissionen und die Unterstützung durch geeignete Materialien und Programme.

Die Untersuchung untermauert den hohen gesamtgesellschaftlichen Wert, den Unfallkommissionen bei der Bekämpfung von Unfallhäufungen mit geeigneten Maßnahmen liefern. Durch die Bereitstellung einer Handlungsempfehlung zum Einsatz geeigneter kostengünstiger Maßnahmen, in Kombination mit den Faktenblättern der einzelnen Maßnahmenkategorien, leistet die Untersuchung einen wichtigen Beitrag zur Verkehrssicherheitsarbeit. Die Handlungsempfehlungen stellen ein Hilfsmittel für Unfallkommissionen zur gezielten Maßnahmenauswahl vor dem Hintergrund beschränkter öffentlicher finanzieller Mittel dar. 


\section{Literatur}

Allgemeine Deutscher Automobil-Club e. V. (ADAC) (1995): Tempo 30 Low-Cost-Maßnahmen für die Praxis, München

Beratungsstelle für Unfallverhütung (bfu) (2013): Straßenraumgestaltung, bfu-Fachdokumentation 2.048, Beratungsstelle für Unfallverhütung, Bern

Böhm, L. Spahn, V. (2009): Die Definition von Unfallhäufungen als Basis für sicherheitsverbessernde Maßnahmen im Straßennetz, in: Straßenverkehrstechnik 11/2008, Bonn

Bundesministerium für Verkehr, Bau und Stadtentwicklung (BMVBS) (2009): Allgemeine Verwaltungsvorschrift zur Straßenverkehrs-Ordnung (VwV-StVO), Berlin

CEDR (2008): Best Practice for Cost-Effective Road Safety Infrastructure Investments, Conference of European Directors of Roads, Paris

Degener, S., Butterwegge, P. (UDV) (2012): Status quo Bericht zur Lage der Unfallkommissionen in Deutschland, Präsentation UDV, Zugriff www.udv.de, 07.08.2013

Deutscher Verkehrssicherheitsrat e. V. (2002): Mehr Sicherheit im Straßenverkehr - Bau- und verkehrstechnische Maßnahmen, Bonn

Forschungsgesellschaft für Straßen- und Verkehrswesen (FGSV) (1991): Hinweise zur Methodik der Untersuchung von Straßenverkehrsunfällen, Köln

Forschungsgesellschaft für Straßen- und Verkehrswesen (FGSV) (2002): Auswertung von Straßenverkehrsunfällen Teil 2 - Maßnahmen gegen Unfallhäufungen, Köln

Forschungsgesellschaft für Straßen- und Verkehrswesen (FGSV) (2003): Auswertung von Straßenverkehrsunfällen Teil 1 - Führen und Auswerten von Unfalltypen-Steckkarten, Köln

Forschungsgesellschaft für Straßen- und Verkehrswesen (FGSV) (2006): Empfehlungen zum Schutz vor Unfällen mit Aufprall auf Bäume ESAB, Köln

Forschungsgesellschaft für Straßen- und Verkehrswesen (FGSV) (2007): Merkblatt zur Verbesserung der Verkehrssicherheit auf Motorradstrecken MVMot, Köln

Forschungsgesellschaft für Straßen- und Verkehrswesen (FGSV) (2012): Merkblatt zur Örtlichen Unfalluntersuchung in Unfallkommissionen M Uko, Köln

Gerlach, J., Kesting, T., Thiemeyer, E.-M. (2009): Möglichkeiten der schnelleren Umsetzung und Priorisierung straßenbaulicher Maßnahmen zur Erhöhung der Verkehrssicherheit, Berichte der Bundesanstalt für Straßenwesen, Heft V185, Bergisch-Gladbach

Hessische Straßen- und Verkehrsverwaltung (HSVV) (2006): Handbuch für die Verkehrssicherheit und Verkehrstechnik, HSVV-Schriftenreihe Heft 53, Wiesbaden

HUK (1961): 10 Jahre im Dienste der Schadensverhütung, Beratungsstelle HUK, Verkehrsblatt-Verlag, Dortmund

Linke, T. (2014): Retroperspektive Bewertung des Sicherheitspotentials verkehrsplanerischer Maßnahmen anhand des sächsischen Unfallgeschehens, Diplomarbeit am Lehrstuhl für Straßenverkehrstechnik der TU Dresden, unveröffentlicht, Dresden

Maier, R., Körner, M., Schüller, H. (2008): Überprüfung und Verbesserung der Grenzwerte zum Erkennen von Unfallhäufungen im Straßennetz, Schlussbericht FE 03.423/2007/FGB, unveröffentlicht, Dresden

Maier, R., Berger, R., Kollmus, B. (2015): Weiterentwicklung der Verfahren zur Entwicklung von Maßnahmen gegen Unfallhäufungen, Schlussbericht FE 03.0504/2012/FRB, unveröffentlicht, Dresden 
Meuleners, L., Hendrie, D., Legge, M., Cercarelli, L. R. (2005): An Evaluation of the Effectiveness of the Black Spot Programs in Western Australia, 2000-2002, School of Population Health, The University of Western Australia, Crawley

ÖU RP (2014): Örtliche Untersuchung der Straßenverkehrsunfälle, Erlass des Ministierum des Innern, für Sport und Infrastruktur, Rheinland-Pfalz, Mainz

Pfundt, K. (1972): Studienkursve für Verkehrssachbearbeiter, Beratungsstelle HUK (3. Auflage), Köln

Pfundt, K. (1991): Handbuch der verkehrssicheren Straßengestaltung, Verkehrsblatt-Verlag, Dortmund

Schweizerischer Verband der Strassen- und Verkehrsfachleute VSS. (2013): Strassenverkehrssicherheit Unfallschwerpunkt-Management, Schweizer Regel SNR 641 724, VSS, Zürich

Spahn, V. (2011): Standardisierte Wirksamkeitsanalyse von sicherheitsverbessernden Maßnahmen an Unfallhäufungen auf Außerortsstraßen, Schriftenreihe des Instituts für Verkehrswesen und Raumplanung, Universität der Bundeswehr München, Heft 56, München

StVO (2015): Straßenverkehr-Oordnung vom 6. März 2013 (BGBI. I S. 367), die durch Artikel 2 der Verordnung vom 15.September 2015 (BGBI. I S. 1573) geändert worden ist

Vollpracht, H.-J. (2000): „low-cost-measures“ gegen Baum-Unfälle in Brandenburg, Straßenverkehrstechnik Heft 12/2000, Köln

VwV-StVO (2015): Allgemeine Verwaltungsvorschrift zur Straßenverkehrsordnung vom 22. Oktober 1998 in der Fassung vom 22. September 2015

VwV-U MP (2012): Verwaltungsvorschrift zur Bekämpfung der Straßenverkehrsunfälle durch örtliche Untersuchungen und daraus abgeleiteter Maßnahmen durch Unfallkommissionen, Ministerium für Energie, Infrastruktur und Landesentwicklung und des Ministeriums für Inneres und Sport, Mecklenburg-Vorpommern, Schwerin

Weinert, R. (1999): Entschärfung innerörtlicher Unfallstellen mit einem fallbasierten Expertensystem, Schriftenreihe des Lehrstuhls für Verkehrswesen der Ruhr-Universität Bochum, Heft 21, Bochum

Zeitschrift für Verkehrssicherheit (ZVS) (2016): Expertensystem zur Unterstützung der Unfallkommissionen in Bayern, ZVS-Schriftenreihe Heft 2/2016, S. 25-32, Bonn 
Anhang 
Verzeichnis Anhang

Anhang 1 Übersicht über die UKO-Anzahl in Deutschland..............................................III

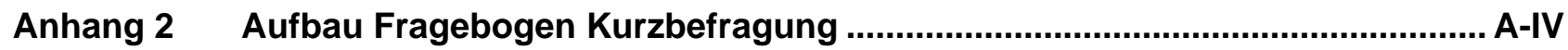

Anhang 3 Ablaufdiagramm Telefoninterview ............................................................. A-V

Anhang 4 Auswertung der Zusatzfragen .................................................................... A-VI

Anhang 5 Aufbau Fragebogen zur Evaluation von Maßnahmen................................. A-VIII

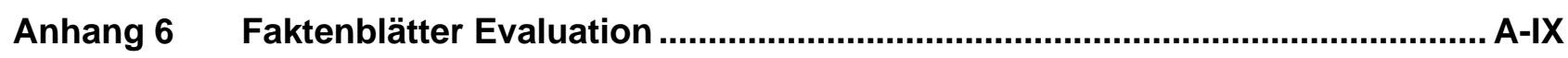

Anhang $7 \quad$ Faktenblätter Unfalldatenanalyse ........................................................... A-XLII

Anhang 8 Darstellung Präsentationsbeispiele Maßnahmenkategorien.................... A-LXXX

Anhang 9 Übersicht alle Maßnahmenkategorien ....................................................... A-XCVI 


\section{Anhang 1 Übersicht über die UKO-Anzahl in Deutschland}

\begin{tabular}{|c|c|c|}
\hline Bundesland & Federführung & Anzahl UKO* \\
\hline Baden-Württemberg & Straßenverkehrsbehörden & 124 \\
\hline Bayern & Straßenbaubehörden & 151 \\
\hline Berlin & Verkehrslenkung Berlin & 1 \\
\hline Brandenburg & Straßenverkehrsbehörden & 26 \\
\hline Bremen & Polizei & 2 \\
\hline Hamburg & Verkehrsdirektion & 1 \\
\hline Hessen & Straßenverkehrsbehörden & 34 \\
\hline Mecklenburg-Vorpommern & Straßenverkehrsbehörden & 13 \\
\hline Niedersachsen & Polizei & 36 \\
\hline Nordrhein-Westfalen & Straßenverkehrsbehörden & 109 \\
\hline Rheinland-Pfalz & Landesbetrieb Mobilität / Diverse & 100 \\
\hline Saarland & Straßenverkehrsbehörden & 10 \\
\hline Sachsen & Straßenverkehrsbehörden & 43 \\
\hline Sachsen-Anhalt & Polizei & 19 \\
\hline Schleswig-Holstein & Polizei & 27 \\
\hline Thüringen & Straßenverkehrsbehörden & 40 \\
\hline Gesamt & - & 736 \\
\hline
\end{tabular}




\section{Anhang 2 Aufbau Fragebogen Kurzbefragung}

\section{TECHNISCHE \\ UNIVERSITÄT \\ DRESDEN}

Unfallforschung

der Versicherer

\section{„Neue und kostengünstige Maßnahmen an Unfallhäufungen“}

Sehr geehrte Damen und Herren,

vielen Dank für Ihre Bereitschaft an der Kurzbefragung teilzunehmen. Damit unterstützen Sie das im Auftrag der Unfallforschung der Versicherer (GDV) durch die TU Dresden bearbeitete Forschungsvorhaben "Neue und kostengünstige Maßnahmen an Unfallhäufungen". Den Fragebogen können Sie auch online unter www.soscisurvey.de/uko beantworten.

Im Mittelpunkt des Forschungsvorhabens steht die Recherche von Maßnahmen an Unfallhäufungen bei den Unfallkommissionen in Deutschland. Bezüglich möglicher Maßnahmen Ihrer Unfallkommission ergeben sich folgende Fragen:

Anzahl der in den vergangenen 10 Jahren umgesetzten Maßnahmen:

davon Sofortmaßnahmen (auch wenn diese mittlerweile durch langfristige Maßnahmen ersetzt wurden):

Unterlagen zu den Maß̣nahmen vorhanden: $\square$ immer

von Fall zu Fall

selten

Durchführung von Wirkungsanalysen für die Maßnahmen:

immer

$\square$ von Fall zu Fall

selten

Zuständigkeitsbereich der UKO:

(z.B.: Landkreis Mittelsachsen oder Stadt Dresden o.ä.)

Im weiteren Verlauf werden durch uns beispielhaft Maßnahmen ausgewäh/t und deren Wirkung analysiert. Daraus sollen Empfehlungen speziell für kostengünstige Maßnahmen abgeleitet werden. Die ausgewählten Beispiele werden anonymisiert, so dass keine Rückschlüsse zu den Unfallkommissionen möglich sind.

Dazu würden wir uns gegebenenfalls gern mit Rückfragen an Sie wenden. Sofern Sie damit einverstanden sind, würden wir das konkrete Vorgehen (bezüglich Ihrer Erfahrungen mit Sofortmaßnahmen und der Übermittlung der Unterlagen zu Maßnahmen Ihrer Unfallkommission) telefonisch so mit Ihnen abstimmen, dass der Aufwand für Sie möglichst gering gehalten wird.

Stehen sie gegebenenfalls für Rückfragen zur Verfügung? $\quad \square$ ja / $\square$ nein

Ihre Kontaktdaten:

Name, Vorname:

Dienststelle:

Anschrift der Dienststelle:

E-Mailadresse:

Telefonnummer:

\begin{tabular}{|c|c|c|}
\hline Bitte senden Sie den Fragebogen & als E-Mail an & Martin.Schmotz@tu-dresden.de \\
\hline & auf dem Postweg an & $\begin{array}{l}\text { TU Dresden, Fakultät Verkehrswissenschaften } \\
\text { Professur für Straßenverkehrstechnik } \\
01062 \text { Dresden }\end{array}$ \\
\hline
\end{tabular}




\section{Anhang 3 Ablaufdiagramm Telefoninterview}

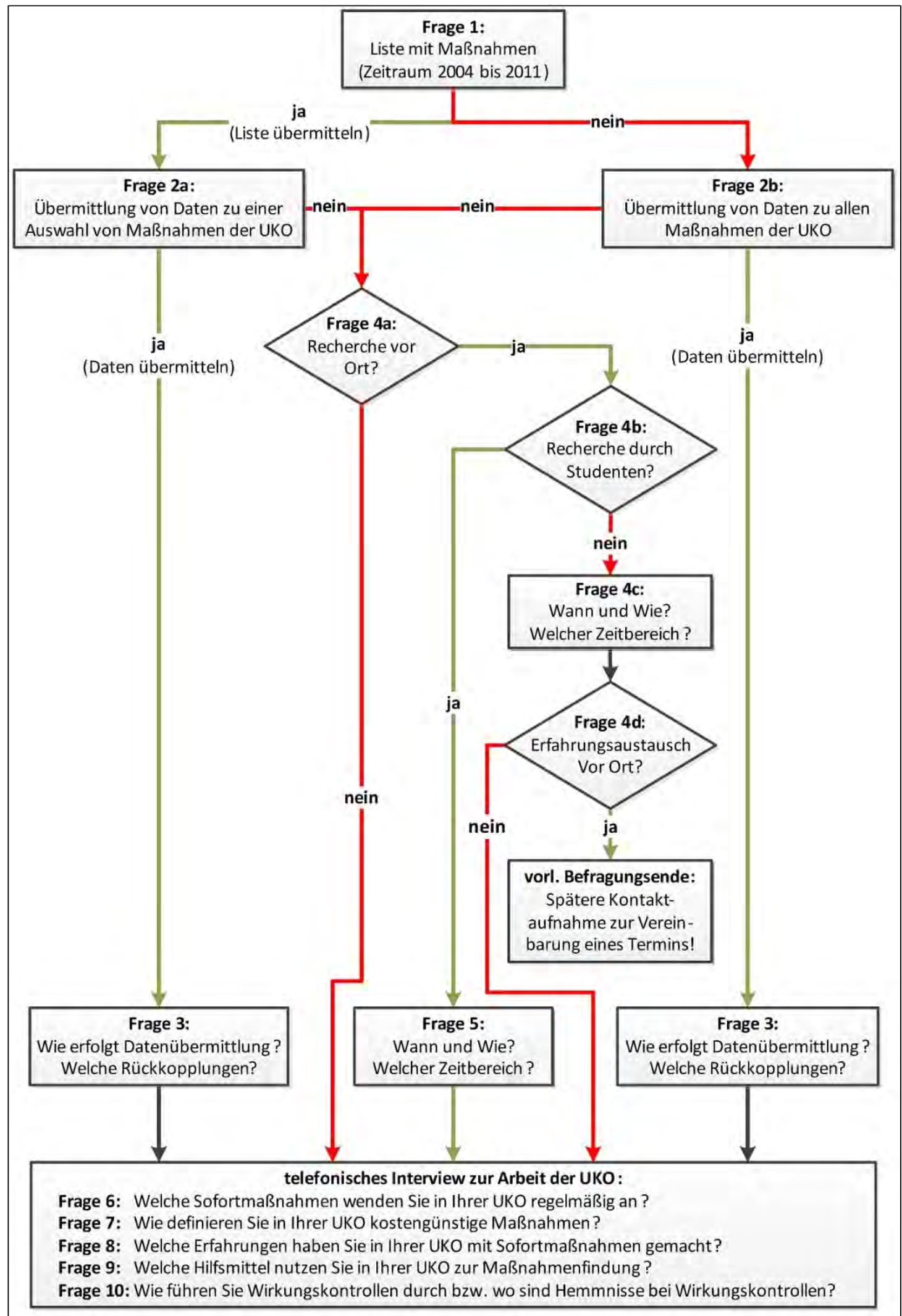




\section{Anhang 4 Auswertung der Zusatzfragen}

1. Welche Sofortmaßnahmen wenden Sie in Ihrer UKO regelmäßig an? (z.B.: Beschilderung, Markierung, Sichtfelder freischneiden/-halten, Ausrichtung Verkehrszeichen/Signalgeber, Vergrößern der Signalgeber, passive Schutzeinrichtungen, Fahrbahndecke, mobile/temporäre Geschwindigkeitsüberwachung)

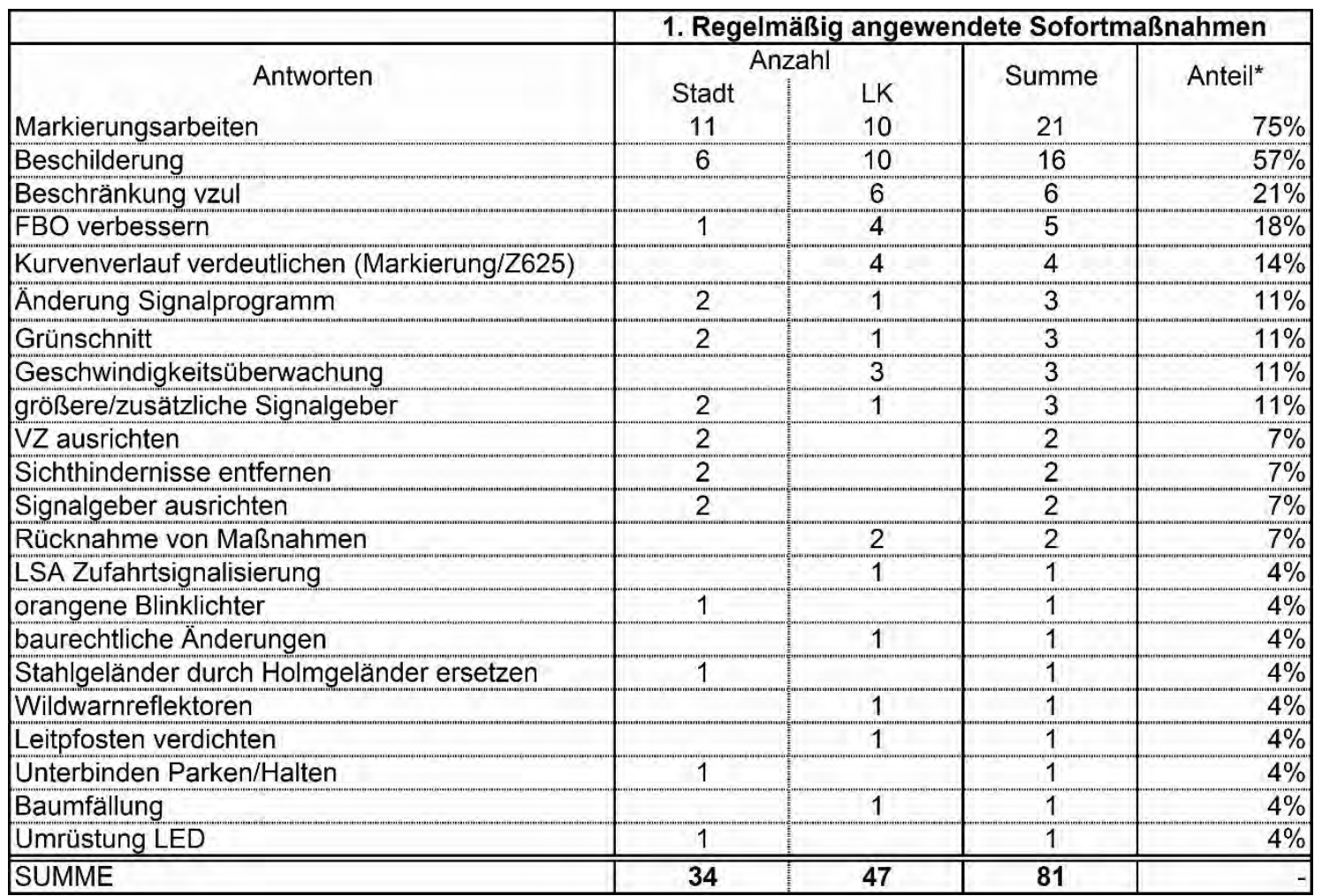

2. Wie grenzen Sie in Ihrer UKO kostengünstige Maßnahmen ab (nach baulichen Kosten, verwaltungstechnischem Aufwand, Umsetzungsdauer)?

\begin{tabular}{|l|c|c|r|r|}
\hline \multicolumn{1}{|c|}{ Antworten } & \multicolumn{3}{c|}{ 2. Definition kostengünstiger Maßnahmen } \\
\hline \multicolumn{1}{|c|}{ Anzahl } & \multicolumn{2}{|c|}{ Summe } & Anteil \\
Umsetzungszeitraum & Stadt & LK & Summe & $45 \%$ \\
\hline ohne Eingriffe in Jahreshaushalt & 3 & 2 & 5 & $27 \%$ \\
\hline reine fin. Umsetzungskosten & 1 & 2 & 3 & $18 \%$ \\
\hline verwaltungstechnischer Aufwand & 1 & 1 & 2 & $9 \%$ \\
\hline \hline SUMME & $\mathbf{5}$ & $\mathbf{6}$ & $\mathbf{1 1}$ & \\
\hline
\end{tabular}


3. Welche Erfahrungen haben Sie in Ihrer UKO mit einfachen sogenannten Sofortmaßnahmen gemacht (z.B.: Beschilderung, Markierung, Sichtfelder freischneiden/-halten, Ausrichtung Verkehrszeichen/Signalgeber, Vergrößern der Signalgeber, passive Schutzeinrichtungen, Fahrbahndecke)?

\begin{tabular}{|c|c|c|c|c|}
\hline \multirow{3}{*}{ Antworten } & \multicolumn{4}{|c|}{ 3. Erfahrungen mit Sofortmaßnahmen } \\
\hline & \multicolumn{2}{|c|}{ Anzahl } & \multirow{2}{*}{ Summe } & \multirow{2}{*}{ Anteil } \\
\hline & Stadt & LK & & \\
\hline überwiegend positiv & 2 & 6 & 8 & $44 \%$ \\
\hline positiv & 2 & 2 & 4 & $22 \%$ \\
\hline nur als Übergangslösung & 2 & 1 & 3 & $17 \%$ \\
\hline $50 / 50--3=$ & 2 & 1 & 3 & $17 \%$ \\
\hline wenig Akzeptanz & 1 & & 1 & $6 \%$ \\
\hline nur kurzfristig & & 1 & 1 & $6 \%$ \\
\hline SUMME & 9 & 11 & 20 & \\
\hline
\end{tabular}

4. Welche Hilfsmittel nutzen Sie in Ihrer UKO zur Maßnahmenfindung (z.B. Merkblatt für die Auswertung von Straßenverkehrsunfällen)?

\begin{tabular}{|c|c|c|c|c|}
\hline \multirow{3}{*}{ Antworten } & \multicolumn{4}{|c|}{ 4. Hilfsmittel zur Maßnahmenfindung } \\
\hline & \multicolumn{2}{|c|}{ Anzahl } & \multirow{2}{*}{ Summe } & \multirow{2}{*}{ Anteil } \\
\hline & Stadt & LK & & \\
\hline Erfahrung & 3 & 5 & 8 & $80 \%$ \\
\hline Merkblatt Teil 2 & 3 & 1 & 4 & $40 \%$ \\
\hline Experten & 1 & 3 & 4 & $40 \%$ \\
\hline andere Merkblätter & & 1 & 1 & $10 \%$ \\
\hline V 185 & & 1 & 1 & $10 \%$ \\
\hline SUMME & 7 & $\overline{11}$ & 18 & 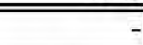 \\
\hline
\end{tabular}

5. Ihrer Antwort auf den Fragbogen ist zu entnehmen, dass Sie (immer/ von Fall zu Fall/ selten) Wirkungskontrollen zu den von Ihrer UKO umgesetzten Maßnahmen durchführen. Wie gehen Sie bei der Durchführung solcher Wirkungskontrollen vor bzw. wo sehen Sie Hemmnisse bei der Durchführung solcher Wirkungskontrollen?

\begin{tabular}{|c|c|c|c|c|}
\hline \multirow{3}{*}{ Antworten } & \multicolumn{4}{|c|}{ 5. Wirkungskontrollen } \\
\hline & \multicolumn{2}{|c|}{ Anzahl } & \multirow{2}{*}{ Summe } & \multirow{2}{*}{ Anteil } \\
\hline & Stadt & LK & & \\
\hline Prüfung, ob beseitigt & 4 & 4 & 8 & $50 \%$ \\
\hline Wirkungskontrollen & 1 & 2 & 3 & $19 \%$ \\
\hline Unfallanalyse durch Polizei & 2 & 1 & 3 & $19 \%$ \\
\hline Analyse $1-\mathrm{JK}$ nachher & 1 & 1 & 2 & $13 \%$ \\
\hline Protokollkontrolle & 1 & & 1 & $6 \%$ \\
\hline SUMME & 9 & 8 & 17 & \\
\hline
\end{tabular}




\section{Anhang 5 Aufbau Fragebogen zur Evaluation von Maßnahmen}

\section{Maßnahme: Sichtschutzzaun aufstellen}

1) Ist Ihnen die vorgestellte Maßnahme bekannt?
$\square$ ja
$\square$ nein

falls ja: Haben Sie diese Maßnahme selbst schon einmal in der Praxis angewendet/umgesetzt?
$\square$ nein
$\square$ ja $\quad$ (ca
Mal in den letzten 5 Jahren)

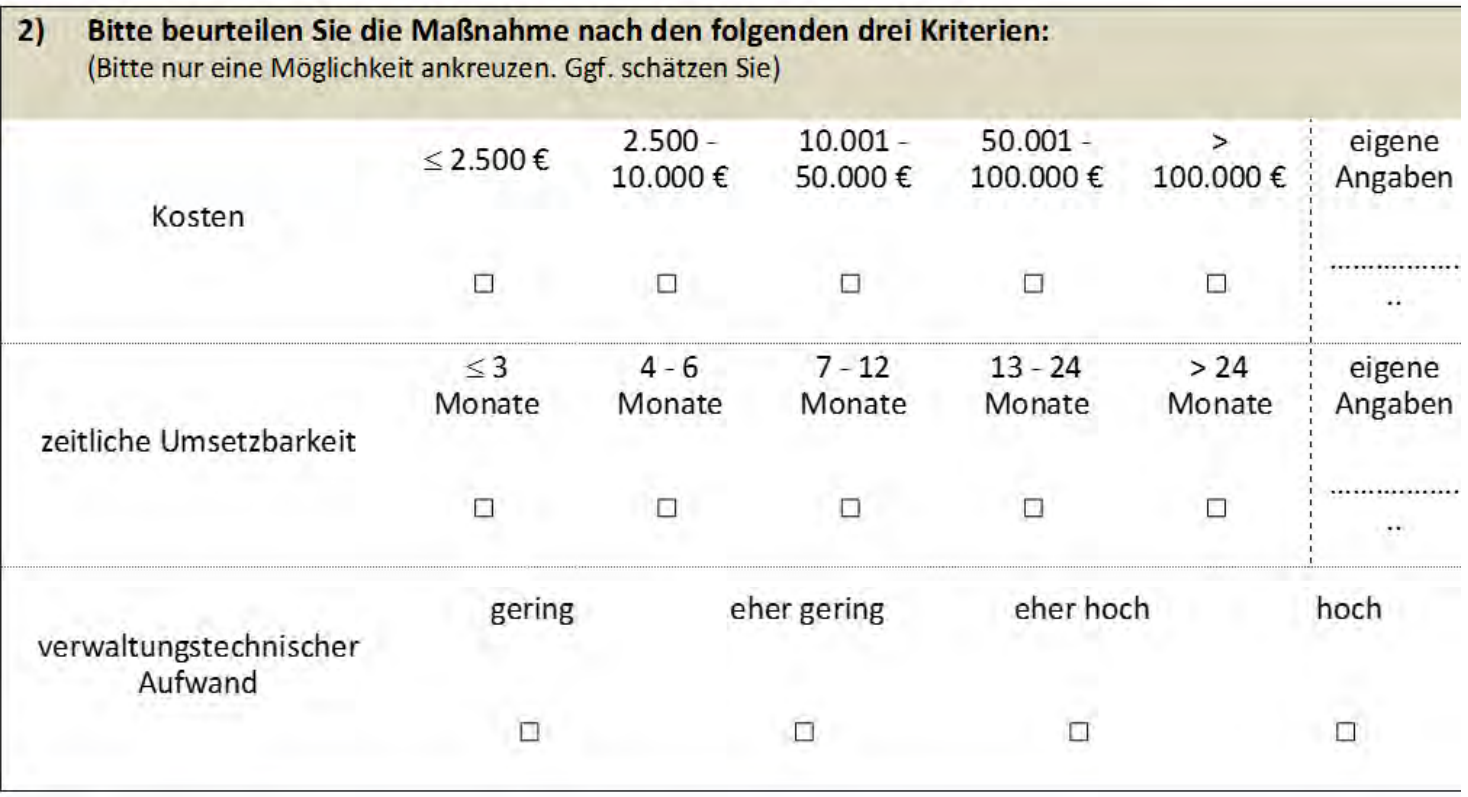

3) Bitte vervollständigen Sie die folgenden Aussagen:

$\begin{array}{cccc}\begin{array}{c}\text { nicht } \\ \text { reduziert. }\end{array} & \text { wenig } & \text { mäßig } & \text { stark } \\ \text { reduziert. } & \text { reduziert. } & \text { reduziert. }\end{array}$

Durch die Maßnahme wird die Schwere der Unfälle

$\square$

Durch die Maßnahme wird die Anzahl der Unfälle

4) Bitte beurteilen Sie die Maßnahme allgemein.

$\begin{array}{lll}\text { Die Maßnahme ist kostengünstig. } & \square \text { stimme zu } & \square \text { stimme nicht zu } \\ \text { Die Maßnahme ist wirksam. } & \square \text { stimme zu } & \square \text { stimme nicht zu }\end{array}$




\section{Anhang 6 Faktenblätter Evaluation}

Nachfolgend sind die Faktenblätter zur Evaluation der einzelnen Maßnahmenkategorien aufgeführt. Die Beschreibung des inhaltlichen Aufbaus ist Kapitel 5.3.2 zu entnehmen. Die Kollektivzusammensetzung beinhaltet Tabelle 8 in Kapitel 4.3.3. Die nachstehende Tabelle verweist auf die Seitenzahl der Maßnahmenkategorien. Der beispielhafte Aufbau des Faktenblattes ist Seite A-X dargestellt, die Erläuterung der Inhalte finden sich auf Seite A-XI.

\begin{tabular}{|c|c|c|}
\hline \multicolumn{2}{|r|}{ Maßnahmenkategorie } & \multirow{2}{*}{$\begin{array}{l}\text { Seite im } \\
\text { Anhang }\end{array}$} \\
\hline Nr. & Name & \\
\hline 1 & Verbesserung der Koordinierung & XII \\
\hline 2 & Änderung der Signalisierung & XIII \\
\hline 3 & "sonstige" Gefahrenzeichen anbringen & XIV \\
\hline 4 & HALT Z 206 StVO (anstelle Z 205 StVO) & \multirow{2}{*}{$\mathrm{XV}$} \\
\hline 5 & HALT Z 206 StVO (anstelle Z 205 StVO) & \\
\hline 6 & Halte-/Parkverbot anordnen & $\mathrm{XVI}$ \\
\hline 7 & Z 625 StVO (Richtungstafel in Kurven) aufstellen & XVII \\
\hline 8 & Zusatzzeichen Z 1006-36 StVO (Unfallgefahr) ergänzen & \multirow{2}{*}{ XVIII } \\
\hline 9 & Zusatzzeichen Z 1006-36 StVO (Unfallgefahr) ergänzen & \\
\hline 10 & Verkehrszeichen anpassen & \multirow{2}{*}{$\mathrm{XIX}$} \\
\hline 11 & Verkehrszeichen anpassen & \\
\hline 12 & Beschränkung Vzul & $x X$ \\
\hline 15 & Beschränkung Vzul & $X X I$ \\
\hline 16 & Überwachung zulässige Höchstgeschwindigkeit mit OGÜ & \multirow{2}{*}{ XXII } \\
\hline 17 & Überwachung zulässige Höchstgeschwindigkeit mit OGÜ & \\
\hline 18 & Erneuerung Fahrbahnoberfläche & XXIII \\
\hline 19 & Zusätzlichen Signalgeber anordnen & XXIV \\
\hline 21 & Leuchtmittel Signalgeber verbessern & $\mathrm{XXV}$ \\
\hline 22 & Sichthindernisse entfernen & \multirow{2}{*}{$\mathrm{XXVI}$} \\
\hline 23 & Sichthindernisse entfernen & \\
\hline 24 & Beleuchtung anpassen/verbessern & XXVII \\
\hline 25 & Sichtschutzzaun aufstellen & XXVIII \\
\hline 26 & Markierung anpassen/verbessern & $\mathrm{XXIX}$ \\
\hline 27 & Markierung in Stand setzen & $x X X$ \\
\hline 29 & Radverkehrsführung anpassen & XXXI \\
\hline 30 & Radfahrfurt Rot einfärben & XXXII \\
\hline 31 & Passive Schutzeinrichtung aufstellen & XXXIII \\
\hline 32 & Unterfahrschutz an passiver Schutzeinrichtung anbringen & XXXIV \\
\hline 33 & Hindernisse im Seitenraum entfernen & $x X X V$ \\
\hline 34 & Rüttelstreifen aufbringen & $\mathrm{XXXVI}$ \\
\hline 35 & Gummischwellen aufbringen & XXXVII \\
\hline 36 & Sperrfläche markieren & XXXVIII \\
\hline 37 & Verkehrszeichen versetzen (Erhöhung Erkennbarkeit) & XXXIX \\
\hline 38 & Beschränkung Vzul und Z 625 StVO (Richtungstafel in Kurve) & $X L$ \\
\hline 39 & Beschränkung Vzul und sonst. Gefahrenzeichen & XLI \\
\hline
\end{tabular}




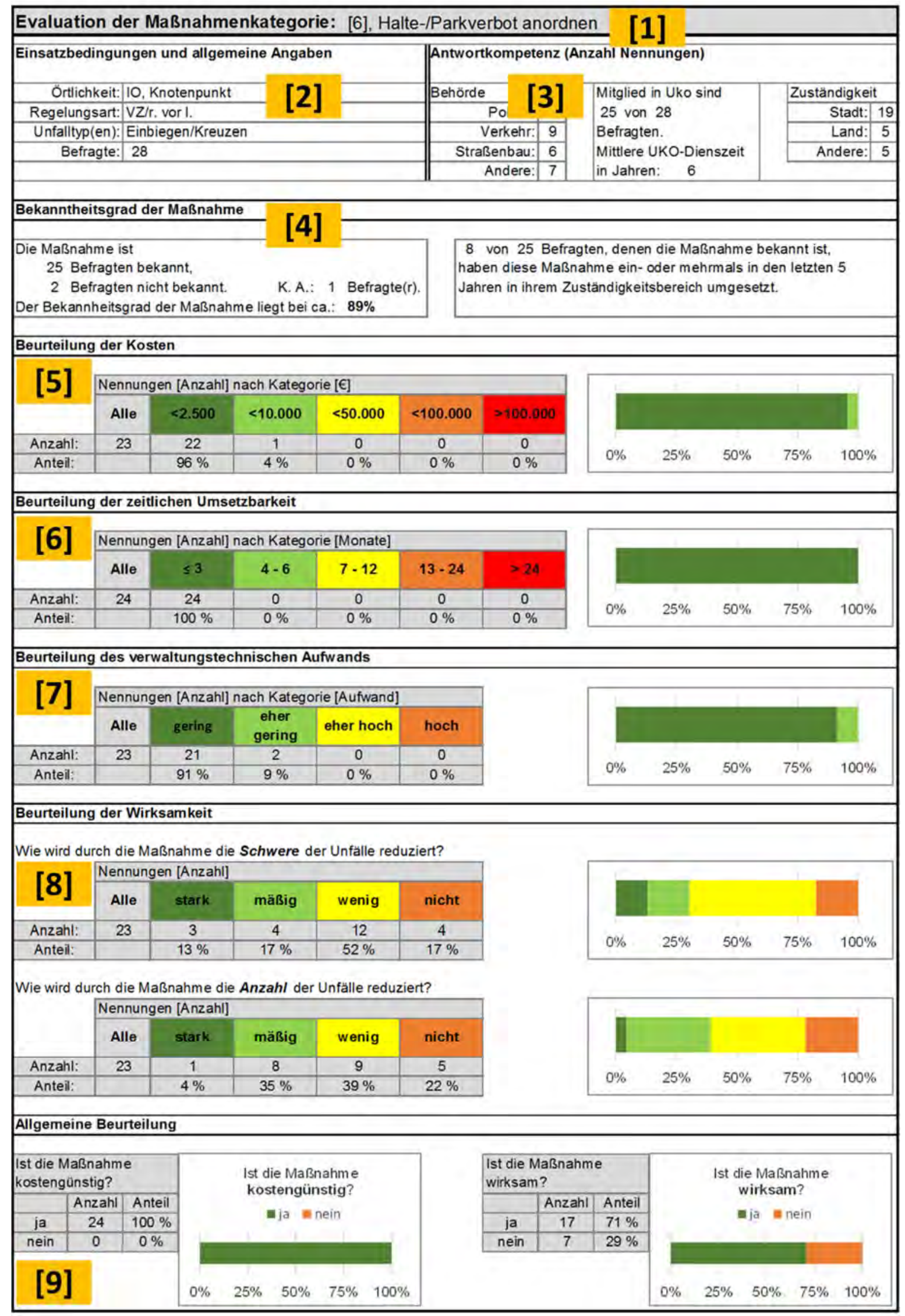

Faktenblatt zur Evaluation einer Maßnahmenkategorie MK, allgemeine Darstellung 


\begin{tabular}{|c|c|c|}
\hline Nummer & Bezeichnung Bereich & Inhalt Bereich \\
\hline [1] & Bezeichnung MK & - Bezeichnung der Maßnahmenkategorie mit Nummer und Namen \\
\hline [2] & $\begin{array}{l}\text { Einsatzbedingungen und } \\
\text { allgemeine Angaben }\end{array}$ & $\begin{array}{l}\text { - Beschreibung zur Örtlichkeit, Regelungsart des Knotenpunkts (wenn gegeben) } \\
\text { und prägende Unfalltypen } \\
\text { - Angabe zur Anzahl der Befragten }\end{array}$ \\
\hline [3] & Antwortkompetenz & $\begin{array}{l}\text { - der Block zur Antwortkompetenz enthält Angaben zur Behördenzugehörigkeit, } \\
\text { Mitgliedschaft, Dauer der Mitgliedschaft in den UKO sowie Bereich der Zuständigkeit }\end{array}$ \\
\hline [4] & $\begin{array}{l}\text { Bekanntheitsgrad der Maß- } \\
\text { nahme }\end{array}$ & $\begin{array}{l}\text { - Angabe zur Verteilung des Bekanntheitsgrades der Maßnahme unter den Befragten } \\
\text { - Angabe, wieviel Befragte die Maßnahme selber schon umgesetzt haben }\end{array}$ \\
\hline [5] & Beurteilung der Kosten & $\begin{array}{l}\text { - Zuordnung der Angaben zur Kosteinschätzung in } 5 \text { Klassen, Angabe in [€] } \\
\text { - Darstellung der Verteilung der Kostenschätzung nach Klassen in einem } \\
\text { Balkendiagramm, Angabe in [\%] }\end{array}$ \\
\hline [6] & $\begin{array}{l}\text { Beurteilung der zeitlichen } \\
\text { Umsetzbarkeit }\end{array}$ & $\begin{array}{l}\text { - Zuordnung der Angaben zur zeitlichen Umsetzbarkeit in } 5 \text { Klassen, } \\
\text { Angabe in [Monaten] } \\
\text { - Darstellung der Verteilung der zeitlichen Umsetzbarkeit nach Klassen in einem } \\
\text { Balkendiagramm, Angabe in [\%] }\end{array}$ \\
\hline [7] & $\begin{array}{l}\text { Beurteilung des verwaltungs- } \\
\text { technischen Aufwands }\end{array}$ & $\begin{array}{l}\text { - Zuordnung der Angaben zum verwaltungstechnischem Aufwand in } 4 \text { Klassen, } \\
\text { ordinale verbale Skalierung, Angabe in [Aufwand] } \\
\text { - Darstellung der Verteilung des verwaltungstechnischen Aufwands nach Klassen in } \\
\text { einem Balkendiagramm, Angabe in [\%] }\end{array}$ \\
\hline [8] & Beurteilung der Wirksamkeit & $\begin{array}{l}\text { - Zuordnung der Angaben zur Wirksamkeit der Maßnahme bezüglich der Reduzierung } \\
\text { der Unfallschwere der Unfälle in } 4 \text { Klassen, ordinale verbale Skalierung, } \\
\text { Angabe in [Aufwand] } \\
\text { - Darstellung der Verteilung der Einschätzung zur Wirksamkeit auf die Unfallschwere } \\
\text { nach Klassen in einem Balkendiagramm, Angabe in [\%] } \\
\text { - Zuordnung der Angaben zur Wirksamkeit der Maßnahme bezüglich der Reduzierung } \\
\text { der Unfallanzahl der Unfälle in } 4 \text { Klassen, ordinale verbale Skalierung, } \\
\text { Angabe in [Aufwand] } \\
\text { - Darstellung der Verteilung der Einschätzung zur Wirksamkeit auf die Unfallanzahl } \\
\text { nach Klassen in einem Balkendiagramm, Angabe in [\%] }\end{array}$ \\
\hline [9] & Allgemeine Beurteilung & $\begin{array}{l}\text { - Angabe zur dichotomen Einschätzung, ob die Maßnahme kostengünstig ist } \\
\text { oder nicht, Darstellung der Antwortverteilung in einem Balkendiagramm, } \\
\text { Angabe in [\%] } \\
\text { - Angabe zur dichotomen Einschätzung, ob die Maßnahme wirksam ist } \\
\text { oder nicht, Darstellung der Antwortverteilung in einem Balkendiagramm, } \\
\text { Angabe in [\%] }\end{array}$ \\
\hline
\end{tabular}

Erläuterung der Inhalte des Faktenblatts zur Evaluation einer Maßnahmenkategorie MK 
Evaluation der Maßnahmenkategorie: [1], Verbesserung der Koordinierung

\begin{tabular}{|c|c|c|c|c|c|}
\hline \multirow{3}{*}{$\begin{array}{l}\text { Einsatzbedingungen und allgemeine Angaben } \\
\text { Örtlichkeit: } \\
\text { Regelungsart: Knotenpunkt } \\
\end{array}$} & \multicolumn{5}{|c|}{ Antwortkompetenz (Anzahl Nennungen) } \\
\hline & \multicolumn{2}{|l|}{ Behörde } & \multirow{5}{*}{$\begin{array}{l}\text { Mitglied in Uko sind } \\
22 \text { von } 27 \\
\text { Befragten. } \\
\text { Mittlere UKO-Dienszeit } \\
\text { in Jahren: } 6\end{array}$} & \multicolumn{2}{|c|}{ Zuständigkeit } \\
\hline & \begin{tabular}{|r|r|} 
Polizei: 1 \\
\end{tabular} & 10 & & Stadt: & 17 \\
\hline Unfalltyp(en): Längsverkehr & Verkehr: & 7 & & Land: & 4 \\
\hline Befragte: 27 & Straßenbau: & 5 & & Andere: & 5 \\
\hline & Andere: & 5 & & & \\
\hline
\end{tabular}

\section{Bekanntheitsgrad der Maßnahme}

Die Maßnahme ist

22 Befragten bekannt,

5 Befragten nicht bekannt. $\quad$ K. A.: 0 Befragte( $r)$

Der Bekannheitsgrad der Maßnahme liegt bei ca.: $\mathbf{8 1 \%}$

13 von 22 Befragten, denen die Maßnahme bekannt ist, haben diese Maßnahme ein- oder mehrmals in den letzten 5 Jahren in ihrem Zuständigkeitsbereich umgesetzt.

\section{Beurteilung der Kosten}

\begin{tabular}{|c|c|c|c|c|c|c|}
\cline { 2 - 7 } & \multicolumn{7}{c|}{ Nennungen [Anzahl] nach Kategorie [€] } \\
\cline { 2 - 7 } & Alle & $<2.500$ & $<\mathbf{1 0 . 0 0 0}$ & $<\mathbf{5 0 . 0 0 0}$ & $<\mathbf{1 0 0 . 0 0 0}$ & $>100.000$ \\
\hline Anzahl: & 24 & 6 & 14 & 2 & 1 & 0 \\
\hline Anteil: & & $25 \%$ & $58 \%$ & $8 \%$ & $4 \%$ & $0 \%$ \\
\hline
\end{tabular}

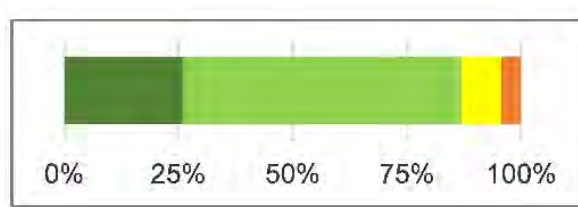

Beurteilung der zeitlichen Umsetzbarkeit

\begin{tabular}{|c|c|c|c|c|c|c|}
\hline & \multicolumn{2}{l|}{ Nennungen [Anzahl] } & nach Kategorie [Monate] \\
\cline { 2 - 7 } & Alle & $\leq 3$ & $\mathbf{4 - 6}$ & $\mathbf{7 - 1 2}$ & $\mathbf{1 3 - 2 4}$ & $>24$ \\
\hline Anzahl: & 25 & 10 & 10 & 4 & 1 & 0 \\
\hline Anteil: & & $40 \%$ & $40 \%$ & $16 \%$ & $4 \%$ & $0 \%$ \\
\hline
\end{tabular}

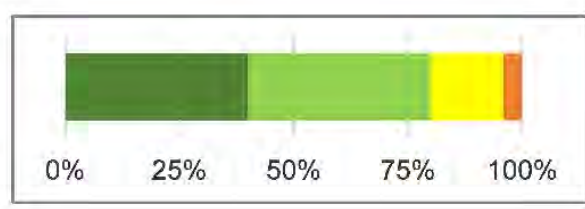

Beurteilung des verwaltungstechnischen Aufwands

\begin{tabular}{|c|c|c|c|c|c|}
\cline { 2 - 6 } & \multicolumn{6}{|c|}{ Nennungen [Anzahl] } & nach Kategorie [Aufwand] & \\
\cline { 2 - 6 } & Alle & gering & $\begin{array}{c}\text { eher } \\
\text { gering }\end{array}$ & eher hoch & hoch \\
\hline Anzahl: & 25 & 10 & 12 & 3 & 0 \\
\hline Anteil: & & $40 \%$ & $48 \%$ & $12 \%$ & $0 \%$ \\
\hline
\end{tabular}

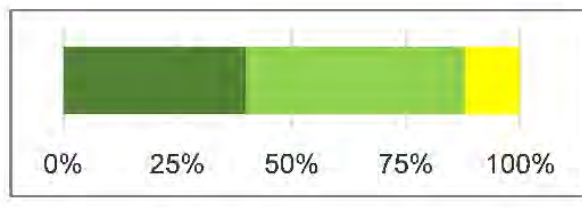

\section{Beurteilung der Wirksamkeit}

Wie wird durch die Maßnahme die Schwere der Unfälle reduziert?

\begin{tabular}{c|c|c|c|c|c|}
\cline { 2 - 6 } & \multicolumn{4}{|c|}{ Nennungen [Anzahl] } & \multicolumn{3}{l|}{} \\
\cline { 2 - 6 } & Alle & stark & mäßig & wenig & nicht \\
\hline Anzahl: & 25 & 4 & 7 & 7 & 7 \\
\hline Anteil: & & $16 \%$ & $28 \%$ & $28 \%$ & $28 \%$ \\
\hline
\end{tabular}

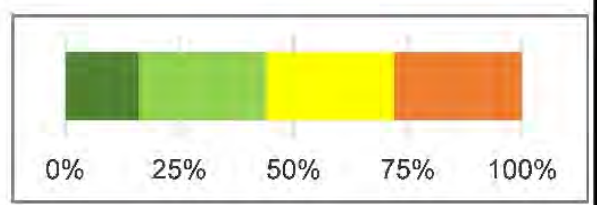

Wie wird durch die Maßnahme die Anzahl der Unfälle reduziert?

\begin{tabular}{|c|c|c|c|c|c|}
\cline { 3 - 6 } & \multicolumn{4}{|c|}{ Nennungen [Anzahl] } & \multicolumn{3}{l|}{} \\
\cline { 2 - 6 } & Alle & stark & mäßig & wenig & nicht \\
\hline Anzahl: & 22 & 0 & 2 & 7 & 13 \\
\hline Anteil: & & $0 \%$ & $9 \%$ & $32 \%$ & $59 \%$ \\
\hline
\end{tabular}

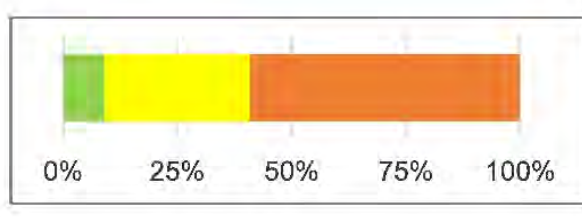

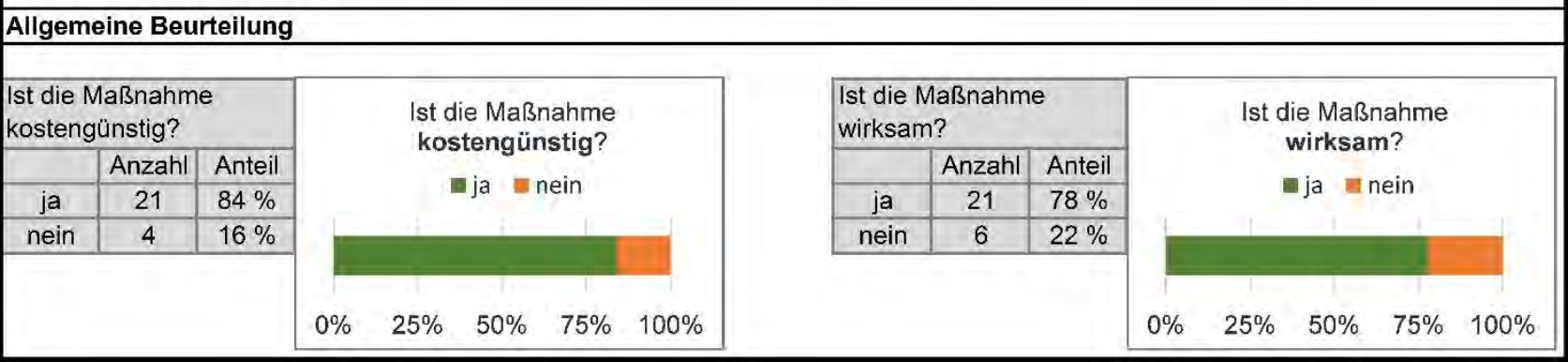


Evaluation der Maßnahmenkategorie: [2], Änderung der Signalisierung

\begin{tabular}{|c|c|c|c|c|}
\hline Einsatzbedingungen und allgemeine Angaben & \multicolumn{4}{|c|}{ |Antwortkompetenz (Anzahl Nennungen) } \\
\hline Örtlichkeit: 10, Knotenpunkt & Behörde & Mitglied in Uko sind & Zuständigkeit & \\
\hline Regelungsart: LSA & \begin{tabular}{|r|r|} 
Polizei: 38 \\
\end{tabular} & 43 von 51 & Stadt: & 36 \\
\hline Unfalltyp(en): Abbiegen, Längsverkehr & Verkehr: 3 & Befragten. & Land: & 23 \\
\hline Befragte: 51 & Straßenbau: & Mittlere UKO-Dienszeit & Andere: & 9 \\
\hline & Andere: & in Jahren: 7 & & \\
\hline
\end{tabular}

\section{Bekanntheitsgrad der Maßnahme}

Die Maßnahme ist

48 Befragten bekannt,

3 Befragten nicht bekannt. $\quad$ K. A.: 0 Befragte( $r)$

Der Bekannheitsgrad der Maßnahme liegt bei ca.: $\mathbf{9 4 \%}$

14 von 48 Befragten, denen die Maßnahme bekannt ist, haben diese Maßnahme ein- oder mehrmals in den letzten 5 Jahren in ihrem Zuständigkeitsbereich umgesetzt.

\section{Beurteilung der Kosten}

\begin{tabular}{|c|c|c|c|c|c|c|}
\cline { 2 - 7 } & \multicolumn{7}{c|}{ Nennungen [Anzahl] nach Kategorie [€] } \\
\cline { 2 - 7 } & Alle & $<2.500$ & $<\mathbf{1 0 . 0 0 0}$ & $<\mathbf{5 0 . 0 0 0}$ & $<\mathbf{1 0 0 . 0 0 0}$ & $>100.000$ \\
\hline Anzahl: & 47 & 26 & 15 & 5 & 0 & 0 \\
\hline Anteil: & & $55 \%$ & $32 \%$ & $11 \%$ & $0 \%$ & $0 \%$ \\
\hline
\end{tabular}

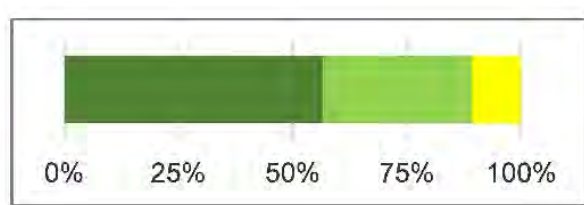

Beurteilung der zeitlichen Umsetzbarkeit

\begin{tabular}{|c|c|c|c|c|c|c|}
\hline & \multicolumn{8}{|c|}{ Nennungen [Anzahl] } & nach Kategorie [Monate] \\
\cline { 2 - 7 } & Alle & $\leq 3$ & $\mathbf{4 - 6}$ & $\mathbf{7 - 1 2}$ & $\mathbf{1 3 - 2 4}$ & $>24$ \\
\hline Anzahl: & 47 & 26 & 17 & 2 & 2 & 0 \\
\hline Anteil: & & $55 \%$ & $36 \%$ & $4 \%$ & $4 \%$ & $0 \%$ \\
\hline
\end{tabular}

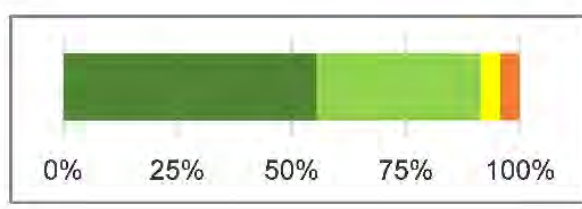

Beurteilung des verwaltungstechnischen Aufwands

\begin{tabular}{|c|c|c|c|c|c|}
\hline & \multicolumn{4}{|c|}{ Nennungen [Anzahl] nach Kategorie [Aufwand] } & \multirow[b]{2}{*}{ hoch } \\
\hline & Alle & gering & $\begin{array}{l}\text { eher } \\
\text { gering }\end{array}$ & eher hoch & \\
\hline Anzahl: & 49 & 21 & 23 & 4 & 1 \\
\hline Anteil: & & $43 \%$ & $47 \%$ & $8 \%$ & $2 \%$ \\
\hline
\end{tabular}

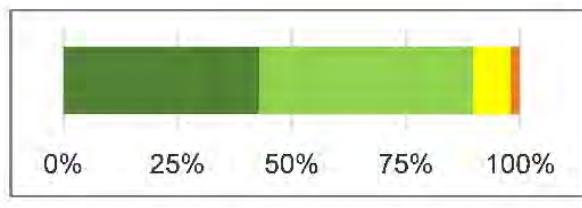

\section{Beurteilung der Wirksamkeit}

Wie wird durch die Maßnahme die Schwere der Unfälle reduziert?

\begin{tabular}{c|c|c|c|c|c|}
\cline { 2 - 6 } & \multicolumn{4}{|c|}{ Nennungen [Anzahl] } & \\
\cline { 2 - 6 } & Alle & stark & mäßig & wenig & nicht \\
\hline Anzahl: & 46 & 11 & 9 & 15 & 11 \\
\hline Anteil: & & $24 \%$ & $20 \%$ & $33 \%$ & $24 \%$ \\
\hline
\end{tabular}

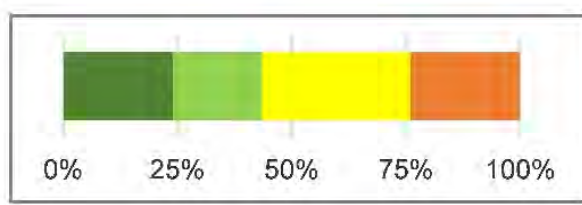

Wie wird durch die Maßnahme die Anzahl der Unfälle reduziert?

\begin{tabular}{|c|c|c|c|c|c|}
\cline { 2 - 6 } & \multicolumn{4}{|c|}{ Nennungen [Anzahl] } & \multicolumn{3}{c|}{} \\
\cline { 2 - 6 } & Alle & stark & mäßig & wenig & nicht \\
\hline Anzahl: & 44 & 2 & 8 & 22 & 12 \\
\hline Anteil: & & $5 \%$ & $18 \%$ & $50 \%$ & $27 \%$ \\
\hline
\end{tabular}

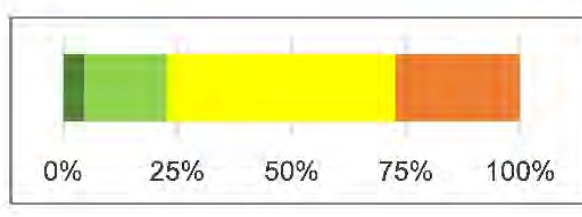

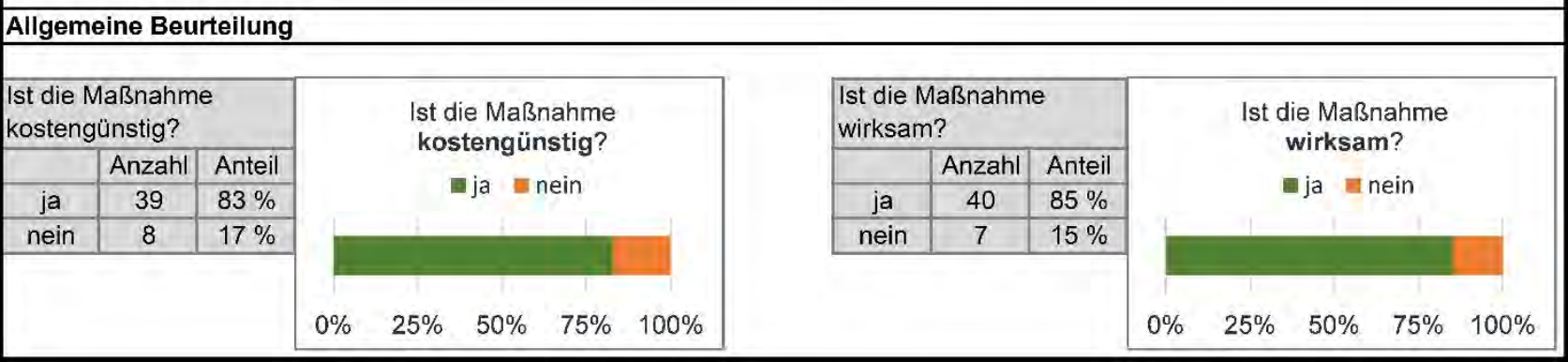


Evaluation der Maßnahmenkategorie: [3], "sonstige" Gefahrenzeichen anbringen

\begin{tabular}{|c|c|c|c|c|c|c|}
\hline \multicolumn{2}{|c|}{ Einsatzbedingungen und allgemeine Angaben } & \multicolumn{5}{|c|}{ |Antwortkompetenz (AnzahI Nennungen) } \\
\hline Örtlichkeit: & $A O$, Strecke $(300 \mathrm{~m})$ & \multicolumn{2}{|l|}{ Behörde } & \multirow{5}{*}{$\begin{array}{l}\text { Mitglied in Uko sind } \\
13 \text { von } 18 \\
\text { Befragten. } \\
\text { Mittlere UKO-Dienszeit } \\
\text { in Jahren: } 5\end{array}$} & \multicolumn{2}{|c|}{ Zuständigkeit } \\
\hline Regelungsart: & - & \begin{tabular}{|r|} 
Polizei: \\
\end{tabular} & 8 & & Stadt: & 10 \\
\hline Unfalltyp(en): & Fahrunfall & Verkehr: & 6 & & Land: & 6 \\
\hline Befragte: & 18 & Straßenbau: & 4 & & Andere: & 5 \\
\hline & & Andere: & 0 & & & \\
\hline
\end{tabular}

\section{Bekanntheitsgrad der Maßnahme}

Die Maßnahme ist

14 Befragten bekannt,

4 Befragten nicht bekannt. $\quad$ K. A.: 0 Befragte(r)

Der Bekannheitsgrad der Maßnahme liegt bei ca.: $\quad \mathbf{7 8 \%}$

11 von 14 Befragten, denen die Maßnahme bekannt ist, haben diese Maßnahme ein- oder mehrmals in den letzten 5 Jahren in ihrem Zuständigkeitsbereich umgesetzt.

\section{Beurteilung der Kosten}

\begin{tabular}{|c|c|c|c|c|c|c|}
\cline { 2 - 7 } & \multicolumn{7}{c|}{ Nennungen [Anzahl] nach Kategorie [€] } \\
\cline { 2 - 7 } & Alle & $<2.500$ & $<\mathbf{1 0 . 0 0 0}$ & $<\mathbf{5 0 . 0 0 0}$ & $<100.000$ & $>100.000$ \\
\hline Anzahl: & 18 & 18 & 0 & 0 & 0 & 0 \\
\hline Anteil: & & $100 \%$ & $0 \%$ & $0 \%$ & $0 \%$ & $0 \%$ \\
\hline
\end{tabular}

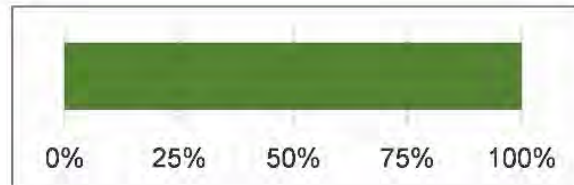

Beurteilung der zeitlichen Umsetzbarkeit

\begin{tabular}{|c|c|c|c|c|c|c|}
\hline \multicolumn{1}{c|}{} & \multicolumn{7}{c|}{ Nennungen [Anzahl] nach Kategorie [Monate] } \\
\cline { 2 - 7 } & Alle & $\leq 3$ & $\mathbf{4 - 6}$ & $\mathbf{7 - 1 2}$ & $\mathbf{1 3 - 2 4}$ & $>24$ \\
\hline Anzahl: & 18 & 16 & 2 & 0 & 0 & 0 \\
\hline Anteil: & & $89 \%$ & $11 \%$ & $0 \%$ & $0 \%$ & $0 \%$ \\
\hline
\end{tabular}

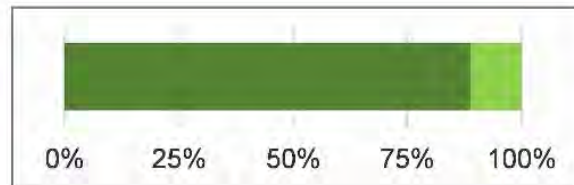

Beurteilung des verwaltungstechnischen Aufwands

\begin{tabular}{|c|c|c|c|c|c|}
\hline & \multicolumn{5}{c|}{ Nennungen [Anzahl] nach Kategorie [Aufwand] } \\
\cline { 2 - 6 } & Alle & gering & $\begin{array}{c}\text { eher } \\
\text { gering }\end{array}$ & eher hoch & hoch \\
\hline Anzahl: & 18 & 15 & 3 & 0 & 0 \\
\hline Anteil: & & $83 \%$ & $17 \%$ & $0 \%$ & $0 \%$ \\
\hline
\end{tabular}

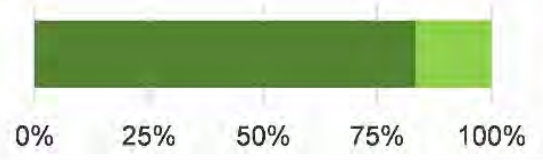

\section{Beurteilung der Wirksamkeit}

Wie wird durch die Maßnahme die Schwere der Unfälle reduziert?

\begin{tabular}{|c|c|c|c|c|c|}
\cline { 2 - 6 } & \multicolumn{3}{|c|}{ Nennungen [Anzahl] } & & \\
\cline { 2 - 6 } & Alle & stark & mäßig & wenig & nicht \\
\hline Anzahl: & 18 & 7 & 6 & 5 & 0 \\
\hline Anteil: & & $39 \%$ & $33 \%$ & $28 \%$ & $0 \%$ \\
\hline
\end{tabular}

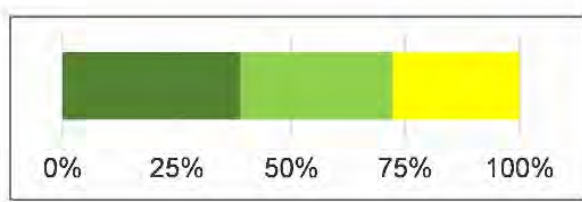

Wie wird durch die Maßnahme die Anzahl der Unfälle reduziert?

\begin{tabular}{|c|c|c|c|c|c|}
\hline & \multicolumn{5}{|c|}{ Nennungen [Anzahl] } \\
\hline & Alle & stark & mäßig & wenig & nicht \\
\hline Anzahl: & 17 & 5 & 6 & 6 & 0 \\
\hline Anteil: & & $29 \%$ & $35 \%$ & $35 \%$ & $0 \%$ \\
\hline
\end{tabular}

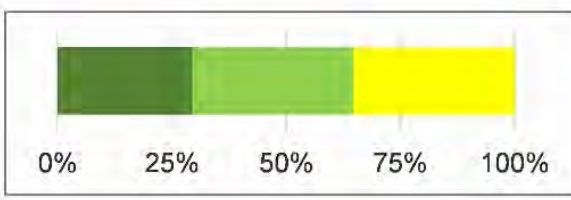

\section{Allgemeine Beurteilung}

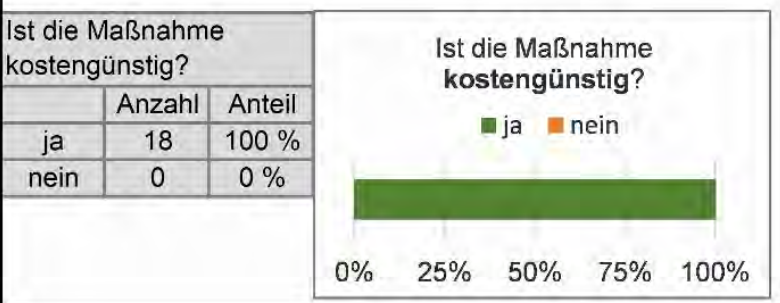

\begin{tabular}{|c|c|c|}
\hline \multicolumn{3}{|c|}{$\begin{array}{l}\text { Ist die Maßnahme } \\
\text { wirksam? }\end{array}$} \\
\hline & Anzahl & Anteil \\
\hline ja & 6 & $33 \%$ \\
\hline nein & 12 & $67 \%$ \\
\hline
\end{tabular}

Ist die Maßnahme wirksam?

m ja mein 
Evaluation der Maßnahmenkategorie: [4/5], HALT Z 206 StVO (anstelle Z 205 StVO)

\begin{tabular}{|c|c|c|c|c|c|}
\hline \multirow{2}{*}{$\begin{array}{c}\text { Einsatzbedingungen und allgemeine Angaben } \\
\text { Örtlichkeit: IO/AO, Knotenpunkt }\end{array}$} & \multicolumn{5}{|c|}{ |Antwortkompetenz (AnzahI Nennungen) } \\
\hline & |Behörde & & Mitglied in Uko sind & Zuständigkeit & \\
\hline Regelungsart: VZ & \begin{tabular}{|r|} 
Polizei: \\
\end{tabular} & 8 & 12 von 18 & Stadt: & 10 \\
\hline Unfalltyp(en): Einbiegen/Kreuzen & Verkehr: & 6 & Befragten. & Land: & 6 \\
\hline Befragte: 18 & Straßenbau: & 4 & Mittlere UKO-Dienszeit & Andere: & 5 \\
\hline & Andere: & 0 & in Jahren: 5 & & \\
\hline
\end{tabular}

\section{Bekanntheitsgrad der Maßnahme}

Die Maßnahme ist

17 Befragten bekannt,

1 Befragten nicht bekannt. $\quad$ K. A.: 0 Befragte(r).

Der Bekannheitsgrad der Maßnahme liegt bei ca.: $\mathbf{9 4 \%}$

Beurteilung der Kosten

\begin{tabular}{|c|c|c|c|c|c|c|}
\cline { 2 - 7 } & \multicolumn{2}{c|}{ Nennungen [Anzahl] nach Kategorie [€] } \\
\cline { 2 - 7 } & Alle & $<2.500$ & $<10.000$ & $<50.000$ & $<100.000$ & $>100.000$ \\
\hline Anzahl: & 18 & 14 & 4 & 0 & 0 & 0 \\
\hline Anteil: & & $78 \%$ & $22 \%$ & $0 \%$ & $0 \%$ & $0 \%$ \\
\hline
\end{tabular}

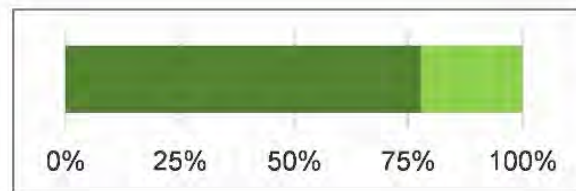

Beurteilung der zeitlichen Umsetzbarkeit

\begin{tabular}{|c|c|c|c|c|c|c|}
\hline \multicolumn{1}{c|}{} & \multicolumn{7}{c|}{ Nennungen [Anzahl] nach Kategorie [Monate] } \\
\cline { 2 - 7 } & Alle & $\leq 3$ & $4-6$ & $\mathbf{7 - 1 2}$ & $13-24$ & $>24$ \\
\hline Anzahl: & 18 & 13 & 4 & 0 & 1 & 0 \\
\hline Anteil: & & $72 \%$ & $22 \%$ & $0 \%$ & $6 \%$ & $0 \%$ \\
\hline
\end{tabular}

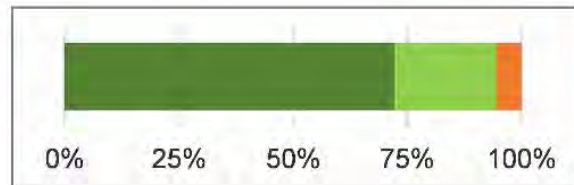

Beurteilung des verwaltungstechnischen Aufwands

\begin{tabular}{|c|c|c|c|c|c|}
\cline { 2 - 6 } & \multicolumn{6}{|c|}{ Nennungen [Anzahl] nach Kategorie [Aufwand] } \\
\cline { 2 - 6 } & Alle & gering & $\begin{array}{c}\text { eher } \\
\text { gering }\end{array}$ & eher hoch & hoch \\
\hline Anzahl: & 18 & 11 & 7 & 0 & 0 \\
\hline Anteil: & & $61 \%$ & $39 \%$ & $0 \%$ & $0 \%$ \\
\hline
\end{tabular}

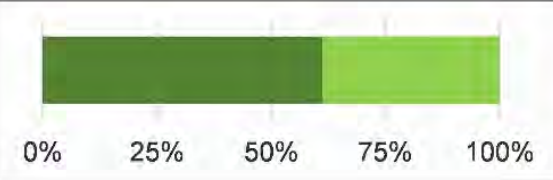

\section{Beurteilung der Wirksamkeit}

Wie wird durch die Maßnahme die Schwere der Unfälle reduziert?

\begin{tabular}{|c|c|c|c|c|c|}
\cline { 2 - 6 } & \multicolumn{2}{|c|}{ Nennungen [Anzahl] } & & \\
\cline { 2 - 6 } & Alle & stark & mäßig & wenig & nicht \\
\hline Anzahl: & 16 & 1 & 3 & 8 & 4 \\
\hline Anteil: & & $6 \%$ & $19 \%$ & $50 \%$ & $25 \%$ \\
\hline
\end{tabular}

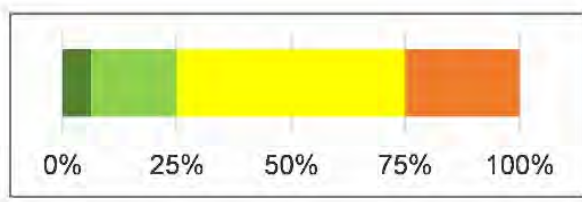

Wie wird durch die Maßnahme die Anzahl der Unfälle reduziert?

\begin{tabular}{|c|c|c|c|c|c|}
\hline & \multicolumn{5}{|c|}{ Nennungen [Anzahl] } \\
\hline & Alle & stark & mäßig & wenig & nicht \\
\hline Anzahl: & 16 & 0 & 2 & 8 & 6 \\
\hline Anteil: & & $0 \%$ & $13 \%$ & $50 \%$ & $38 \%$ \\
\hline
\end{tabular}

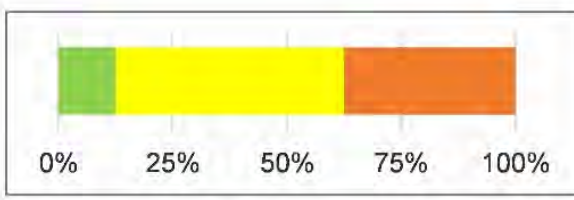

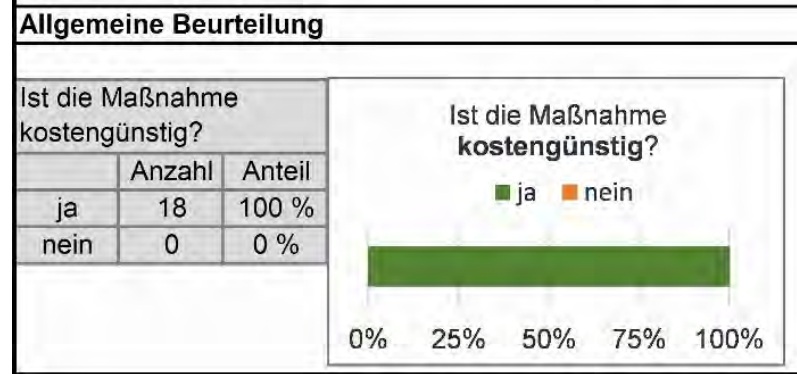

\begin{tabular}{|c|c|c|}
\hline \multicolumn{3}{|c|}{$\begin{array}{l}\text { Ist die Maßnahme } \\
\text { wirksam? }\end{array}$} \\
\hline & Anzahl & Anteil \\
\hline ja & 15 & $88 \%$ \\
\hline nein & 2 & $12 \%$ \\
\hline
\end{tabular}

Ist die Maßnahme wirksam?

m ja mein 
Evaluation der Maßnahmenkategorie: [6], Halte-/Parkverbot anordnen

\begin{tabular}{|c|c|c|c|c|c|}
\hline \multirow{2}{*}{$\begin{array}{l}\text { Einsatzbedingungen und allgemeine Angaben } \\
\text { Örtlichkeit: } \mid \mathrm{OO} \text { Knotenpunkt }\end{array}$} & \multicolumn{5}{|c|}{ Antwortkompetenz (Anzahl Nennungen) } \\
\hline & \multicolumn{2}{|l|}{ Behörde } & \multirow{5}{*}{$\begin{array}{l}\text { Mitglied in Uko sind } \\
25 \text { von } 28 \\
\text { Befragten. } \\
\text { Mittlere UKO-Dienszeit } \\
\text { in Jahren: } 6\end{array}$} & \multicolumn{2}{|c|}{ Zuständigkeit } \\
\hline Regelungsart: VZ/r. vor I. & Polizei: & 6 & & Stadt: & 19 \\
\hline Unfalltyp(en): Einbiegen/Kreuzen & Verkehr: & 9 & & Land: & 5 \\
\hline Befragte: 28 & Straßenbau: & 6 & & Andere: & 5 \\
\hline & Andere: & 7 & & & \\
\hline
\end{tabular}

\section{Bekanntheitsgrad der Maßnahme}

Die Maßnahme ist

25 Befragten bekannt,

2 Befragten nicht bekannt. $\quad$ K. A.: 1 Befragte(r)

Der Bekannheitsgrad der Maßnahme liegt bei ca.: $\quad \mathbf{8 9 \%}$

8 von 25 Befragten, denen die Maßnahme bekannt ist, haben diese Maßnahme ein- oder mehrmals in den letzten 5

Jahren in ihrem Zuständigkeitsbereich umgesetzt.

\section{Beurteilung der Kosten}

\begin{tabular}{|c|c|c|c|c|c|c|}
\cline { 2 - 7 } & \multicolumn{7}{c|}{ Nennungen [Anzahl] nach Kategorie [€] } \\
\cline { 2 - 7 } & Alle & $<2.500$ & $<\mathbf{1 0 . 0 0 0}$ & $<\mathbf{5 0 . 0 0 0}$ & $<100.000$ & $>100.000$ \\
\hline Anzahl: & 23 & 22 & 1 & 0 & 0 & 0 \\
\hline Anteil: & & $96 \%$ & $4 \%$ & $0 \%$ & $0 \%$ & $0 \%$ \\
\hline
\end{tabular}

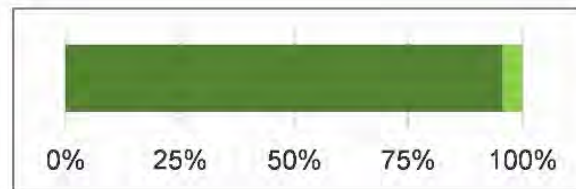

Beurteilung der zeitlichen Umsetzbarkeit

\begin{tabular}{|c|c|c|c|c|c|c|}
\hline \multicolumn{1}{c|}{} & \multicolumn{7}{l|}{ Nennungen [Anzahl] nach Kategorie [Monate] } \\
\cline { 2 - 7 } & Alle & $\leq 3$ & $4-6$ & $7-12$ & $13-24$ & $>24$ \\
\hline Anzahl: & 24 & 24 & 0 & 0 & 0 & 0 \\
\hline Anteil: & & $100 \%$ & $0 \%$ & $0 \%$ & $0 \%$ & $0 \%$ \\
\hline
\end{tabular}

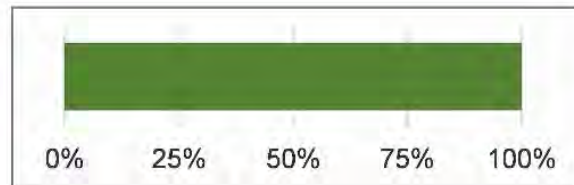

Beurteilung des verwaltungstechnischen Aufwands

\begin{tabular}{|c|c|c|c|c|c|}
\cline { 2 - 6 } & \multicolumn{5}{c|}{ Nennungen [Anzahl] nach Kategorie [Aufwand] } \\
\cline { 2 - 6 } & Alle & gering & $\begin{array}{c}\text { eher } \\
\text { gering }\end{array}$ & eher hoch & hoch \\
\hline Anzahl: & 23 & 21 & 2 & 0 & 0 \\
\hline Anteil: & & $91 \%$ & $9 \%$ & $0 \%$ & $0 \%$ \\
\hline
\end{tabular}

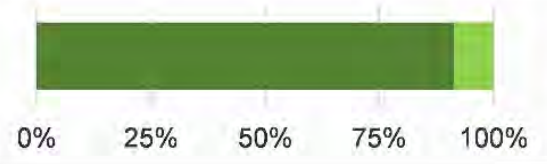

Beurteilung der Wirksamkeit

Wie wird durch die Maßnahme die Schwere der Unfälle reduziert?

\begin{tabular}{|c|c|c|c|c|c|}
\cline { 2 - 6 } & \multicolumn{4}{|c|}{ Nennungen [Anzahl] } & \multicolumn{2}{c|}{} \\
\cline { 2 - 6 } & Alle & stark & mäßig & wenig & nicht \\
\hline Anzahl: & 23 & 3 & 4 & 12 & 4 \\
\hline Anteil: & & $13 \%$ & $17 \%$ & $52 \%$ & $17 \%$ \\
\hline
\end{tabular}

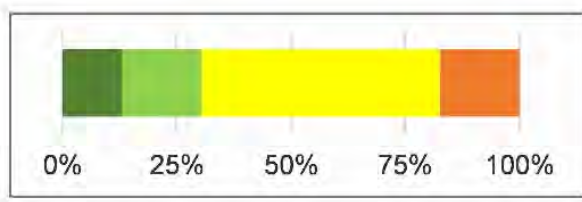

Wie wird durch die Maßnahme die Anzahl der Unfälle reduziert?

\begin{tabular}{|c|c|c|c|c|c|}
\cline { 2 - 6 } & \multicolumn{4}{c|}{ Nennungen [Anzahl] } & \multicolumn{2}{c|}{} \\
\cline { 2 - 6 } & Alle & stark & mäßig & wenig & nicht \\
\hline Anzahl: & 23 & 1 & 8 & 9 & 5 \\
\hline Anteil: & & $4 \%$ & $35 \%$ & $39 \%$ & $22 \%$ \\
\hline
\end{tabular}

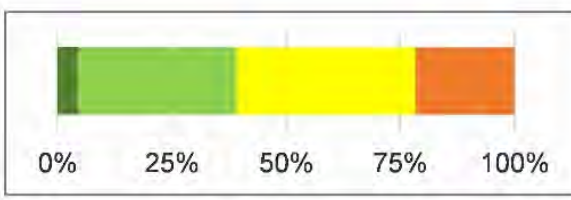

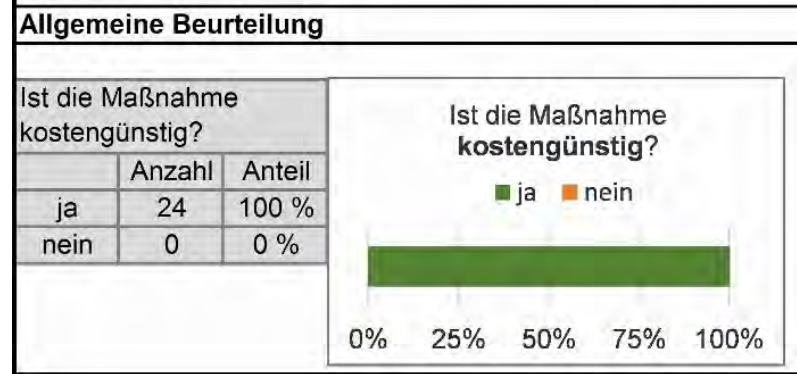

\begin{tabular}{|c|c|c|}
\hline \multicolumn{3}{|c|}{$\begin{array}{l}\text { Ist die Maßnahme } \\
\text { wirksam? }\end{array}$} \\
\hline & Anzahl & Anteil \\
\hline ja & 17 & $71 \%$ \\
\hline nein & 7 & $29 \%$ \\
\hline
\end{tabular}

Ist die Maßnahme wirksam?

mja nein 
Evaluation der Maßnahmenkategorie: [7], Z 625 StVO (Richtungstafel in Kurven) aufstellen

\begin{tabular}{|c|c|c|c|c|c|c|}
\hline \multicolumn{2}{|c|}{ Einsatzbedingungen und allgemeine Angaben } & \multicolumn{5}{|c|}{ |Antwortkompetenz (Anzahl Nennungen) } \\
\hline Örtlichkeit: & $\mathrm{AO}$, Strecke $(300 \mathrm{~m})$ & \multicolumn{2}{|l|}{ Behörde } & \multirow{5}{*}{$\begin{array}{l}\text { Mitglied in Uko sind } \\
18 \text { von } 18 \\
\text { Befragten. } \\
\text { Mittlere UKO-Dienszeit } \\
\text { in Jahren: } 14\end{array}$} & \multicolumn{2}{|l|}{ Zuständigkeit } \\
\hline Regelungsart: & - & \begin{tabular}{r|} 
Polizei: \\
\end{tabular} & 0 & & Stadt: & 8 \\
\hline Unfalltyp(en): & Fahrunfall & Verkehr: 1 & 14 & & Land: & 7 \\
\hline Befragte: & 18 & Straßenbau: & 2 & & Andere: & 3 \\
\hline & & Andere: & 2 & & & \\
\hline
\end{tabular}

\section{Bekanntheitsgrad der Maßnahme}

Die Maßnahme ist

17 Befragten bekannt,

0 Befragten nicht bekannt. $\quad$ K. A.: 1 Befragte(r).

5 von 17 Befragten, denen die Maßnahme bekannt ist, haben diese Maßnahme ein- oder mehrmals in den letzten 5 Jahren in ihrem Zuständigkeitsbereich umgesetzt.

Der Bekannheitsgrad der Maßnahme liegt bei ca.: $\mathbf{9 4 \%}$

\section{Beurteilung der Kosten}

\begin{tabular}{|c|c|c|c|c|c|c|}
\cline { 2 - 7 } & \multicolumn{7}{|c|}{ Nennungen [Anzahl] nach Kategorie [€] } \\
\cline { 2 - 7 } & Alle & $<2.500$ & $<\mathbf{1 0 . 0 0 0}$ & $<\mathbf{5 0 . 0 0 0}$ & $<\mathbf{1 0 0 . 0 0 0}$ & $>100.000$ \\
\hline Anzahl: & 16 & 15 & 1 & 0 & 0 & 0 \\
\hline Anteil: & & $94 \%$ & $6 \%$ & $0 \%$ & $0 \%$ & $0 \%$ \\
\hline
\end{tabular}

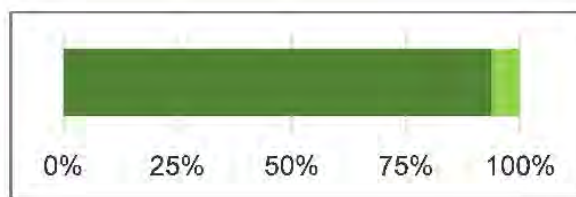

Beurteilung der zeitlichen Umsetzbarkeit

\begin{tabular}{|c|c|c|c|c|c|c|}
\hline & \multicolumn{7}{l|}{ Nennungen [Anzahl] } & nach Kategorie [Monate] \\
\cline { 2 - 7 } & Alle & $\leq 3$ & $\mathbf{4 - 6}$ & $\mathbf{7 - 1 2}$ & $\mathbf{1 3 - 2 4}$ & $>24$ \\
\hline Anzahl: & 17 & 16 & 1 & 0 & 0 & 0 \\
\hline Anteil: & & $94 \%$ & $6 \%$ & $0 \%$ & $0 \%$ & $0 \%$ \\
\hline
\end{tabular}

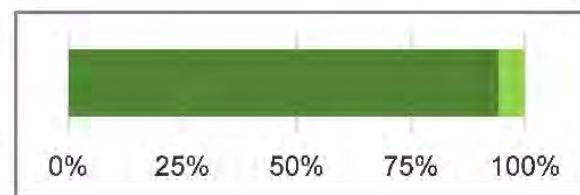

Beurteilung des verwaltungstechnischen Aufwands

\begin{tabular}{|c|c|c|c|c|c|}
\cline { 2 - 6 } & \multicolumn{5}{|c|}{ Nennungen [Anzahl] nach Kategorie [Aufwand] } \\
\cline { 2 - 6 } & Alle & gering & $\begin{array}{c}\text { eher } \\
\text { gering }\end{array}$ & eher hoch & hoch \\
\hline Anzahl: & 17 & 16 & 1 & 0 & 0 \\
\hline Anteil: & & $94 \%$ & $6 \%$ & $0 \%$ & $0 \%$ \\
\hline
\end{tabular}

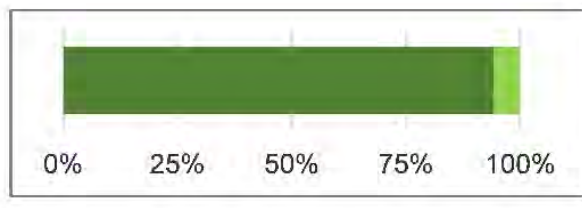

\section{Beurteilung der Wirksamkeit}

Wie wird durch die Maßnahme die Schwere der Unfälle reduziert?

\begin{tabular}{c|c|c|c|c|c|}
\cline { 2 - 6 } & \multicolumn{4}{|c|}{ Nennungen [Anzahl] } & \multicolumn{2}{l|}{} \\
\cline { 2 - 6 } & Alle & stark & mäßig & wenig & nicht \\
\hline Anzahl: & 15 & 3 & 2 & 6 & 4 \\
\hline Anteil: & & $20 \%$ & $13 \%$ & $40 \%$ & $27 \%$ \\
\hline
\end{tabular}

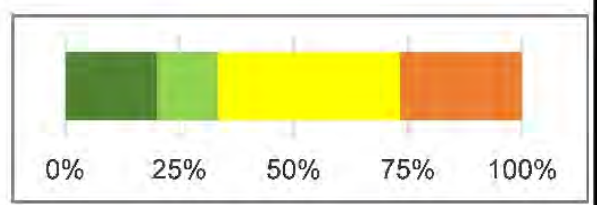

Wie wird durch die Maßnahme die Anzahl der Unfälle reduziert?

\begin{tabular}{|c|c|c|c|c|c|}
\cline { 3 - 6 } & \multicolumn{4}{|c|}{ Nennungen [Anzahl] } & \multicolumn{3}{c|}{} \\
\cline { 2 - 6 } & Alle & stark & mäßig & wenig & nicht \\
\hline Anzahl: & 16 & 0 & 3 & 7 & 6 \\
\hline Anteil: & & $0 \%$ & $19 \%$ & $44 \%$ & $38 \%$ \\
\hline
\end{tabular}

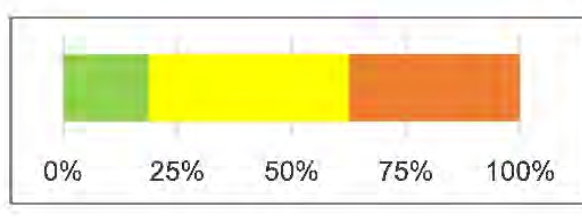

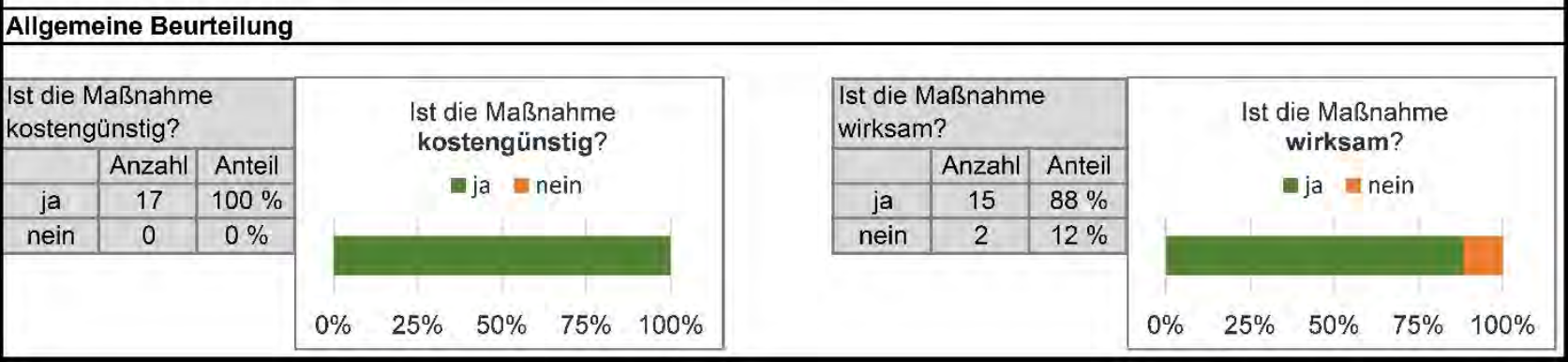


Evaluation der Maßnahmenkategorie: [8/9], Zusatzzeichen ergänzen (Z 1006-36 StVO)

\begin{tabular}{|c|c|c|c|c|c|}
\hline Einsatzbedingu & Ingen und allgemeine Angaben & Antwortkompeter & enz ( & zahl Nennungen) & \\
\hline Örtlichkeit: & IO/AO, Knotenpunkt/Strecke & Behörde & & Mitglied in Uko sind & Zuständigkeit \\
\hline Regelungsart: & beliebig & \begin{tabular}{|r|r|} 
Polizei: 3 \\
\end{tabular} & 38 & 43 von 51 & Stadt: 3 \\
\hline Unfalltyp(en): & Einbiegen/Kreuzen, Unfall im Längsverkehr & Verkehr: & 3 & Befragten. & Land: 2 \\
\hline Befragte: & 51 & Straßenbau: & 4 & Mittlere UKO-Dienszeit & Andere: \\
\hline & & Andere: & 6 & in Jahren: 7 & \\
\hline
\end{tabular}

\section{Bekanntheitsgrad der Maßnahme}

Die Maßnahme ist

40 Befragten bekannt,

10 Befragten nicht bekannt. $\quad$ K. A.: 1 Befragte(r).

Der Bekannheitsgrad der Maßnahme liegt bei ca.: $\mathbf{7 8 \%}$

28 von 40 Befragten, denen die Maßnahme bekannt ist, haben diese Maßnahme ein- oder mehrmals in den letzten 5 Jahren in ihrem Zuständigkeitsbereich umgesetzt.

\section{Beurteilung der Kosten}

\begin{tabular}{|c|c|c|c|c|c|c|}
\cline { 2 - 7 } & \multicolumn{7}{c|}{ Nennungen [Anzahl] nach Kategorie [€] } \\
\cline { 2 - 8 } & Alle & $<2.500$ & $<\mathbf{1 0 . 0 0 0}$ & $<\mathbf{5 0 . 0 0 0}$ & $<\mathbf{1 0 0 . 0 0 0}$ & $>100.000$ \\
\hline Anzahl: & 47 & 42 & 2 & 1 & 0 & 0 \\
\hline Anteil: & & $89 \%$ & $4 \%$ & $2 \%$ & $0 \%$ & $0 \%$ \\
\hline
\end{tabular}

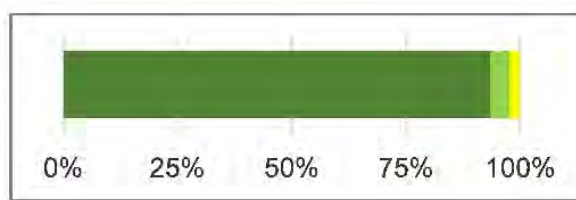

Beurteilung der zeitlichen Umsetzbarkeit

\begin{tabular}{|c|c|c|c|c|c|c|}
\hline \multicolumn{1}{c|}{} & \multicolumn{7}{c|}{ Nennungen [Anzahl] } & nach Kategorie [Monate] \\
\cline { 2 - 7 } & Alle & $\leq 3$ & $\mathbf{4 - 6}$ & $\mathbf{7 - 1 2}$ & $\mathbf{1 3 - 2 4}$ & $>24$ \\
\hline Anzahl: & 47 & 45 & 1 & 0 & 1 & 0 \\
\hline Anteil: & & $96 \%$ & $2 \%$ & $0 \%$ & $2 \%$ & $0 \%$ \\
\hline
\end{tabular}

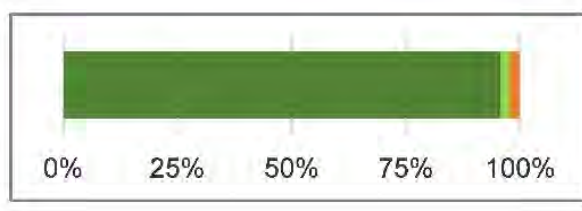

Beurteilung des verwaltungstechnischen Aufwands

\begin{tabular}{|c|c|c|c|c|c|}
\cline { 2 - 6 } & \multicolumn{6}{|c|}{ Nennungen [Anzahl] } & nach Kategorie [Aufwand] & \\
\cline { 2 - 6 } & Alle & gering & $\begin{array}{c}\text { eher } \\
\text { gering }\end{array}$ & eher hoch & hoch \\
\hline Anzahl: & 49 & 45 & 2 & 2 & 0 \\
\hline Anteil: & & $92 \%$ & $4 \%$ & $4 \%$ & $0 \%$ \\
\hline
\end{tabular}

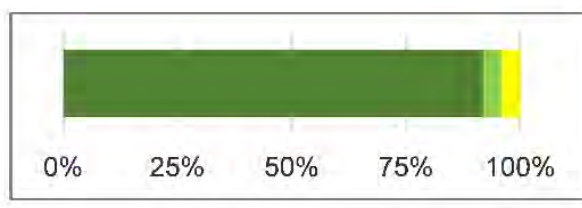

\section{Beurteilung der Wirksamkeit}

Wie wird durch die Maßnahme die Schwere der Unfälle reduziert?

\begin{tabular}{c|c|c|c|c|c|}
\cline { 2 - 6 } & \multicolumn{4}{c|}{ Nennungen [Anzahl] } & \multicolumn{2}{c|}{} \\
\cline { 2 - 6 } & Alle & stark & mäßig & wenig & nicht \\
\hline Anzahl: & 46 & 24 & 17 & 4 & 1 \\
\hline Anteil: & & $52 \%$ & $37 \%$ & $9 \%$ & $2 \%$ \\
\hline
\end{tabular}

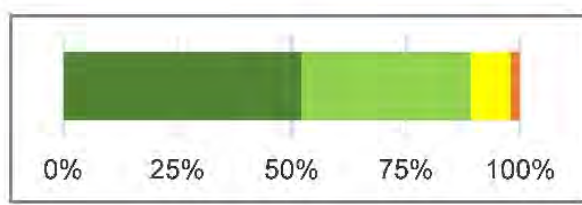

Wie wird durch die Maßnahme die Anzahl der Unfälle reduziert?

\begin{tabular}{|c|c|c|c|c|c|}
\cline { 2 - 6 } & \multicolumn{4}{|c|}{ Nennungen [Anzahl] } & \multicolumn{3}{c|}{} \\
\cline { 2 - 6 } & Alle & stark & mäßig & wenig & nicht \\
\hline Anzahl: & 45 & 20 & 17 & 5 & 3 \\
\hline Anteil: & & $44 \%$ & $38 \%$ & $11 \%$ & $7 \%$ \\
\hline
\end{tabular}

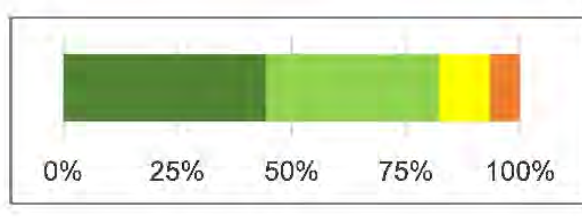

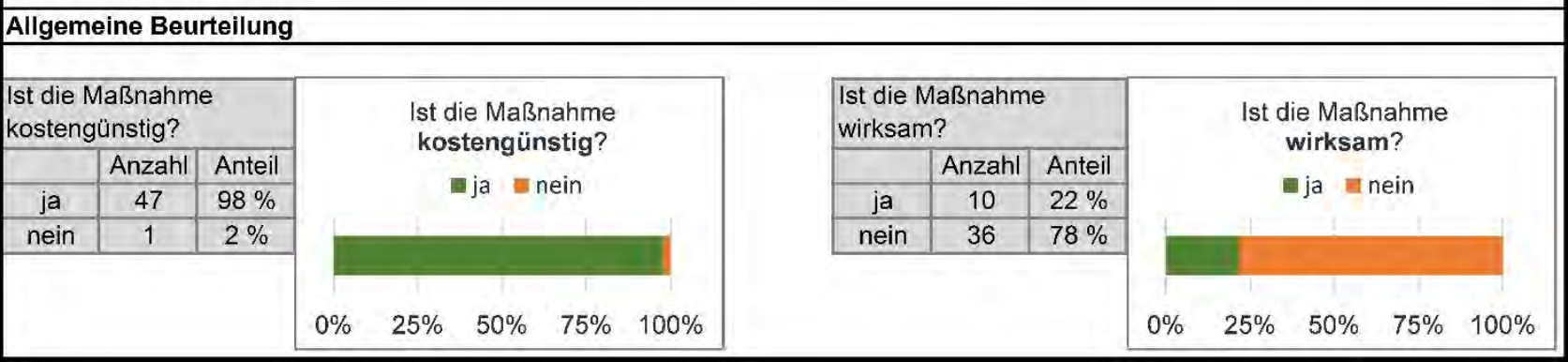


Evaluation der Maßnahmenkategorie: [10/11], Verkehrszeichen anpassen

\begin{tabular}{|c|c|c|c|c|c|c|}
\hline \multicolumn{2}{|c|}{ Einsatzbedingungen und allgemeine Angaben } & \multicolumn{5}{|c|}{ Antwortkompetenz (Anzahl Nennungen) } \\
\hline Örtlichkeit: & IO/AO, Knotenpunkt/Strecke & \multicolumn{2}{|l|}{ Behörde } & \multirow{5}{*}{$\begin{array}{l}\text { Mitglied in Uko sind } \\
18 \text { von } 18 \\
\text { Befragten. } \\
\text { Mittlere UKO-Dienszeit } \\
\text { in Jahren: } 14\end{array}$} & \multicolumn{2}{|l|}{ Zuständigkeit } \\
\hline Regelungsart: & - & $\begin{array}{r}\text { Polizei: } \\
\end{array}$ & 0 & & Stadt: & 8 \\
\hline Unfalltyp(en): & Fahrunfall, Abbiegen, Einbiegen/Kreuzen & Verkehr: & 14 & & Land: & 7 \\
\hline Befragte: & 18 & Straßenbau: & 2 & & Andere: & 3 \\
\hline & & Andere: & 2 & & & \\
\hline
\end{tabular}

\section{Bekanntheitsgrad der Maßnahme}

Die Maßnahme ist

15 Befragten bekannt,

2 Befragten nicht bekannt. $\quad$ K. A.: 1 Befragte(r).

Der Bekannheitsgrad der Maßnahme liegt bei ca.: $\mathbf{8 3} \%$

2 von 15 Befragten, denen die Maßnahme bekannt ist, haben diese Maßnahme ein- oder mehrmals in den letzten 5 Jahren in ihrem Zuständigkeitsbereich umgesetzt.

\section{Beurteilung der Kosten}

\begin{tabular}{|c|c|c|c|c|c|c|}
\cline { 2 - 7 } & \multicolumn{7}{c|}{ Nennungen [Anzahl] nach Kategorie [€] } \\
\cline { 2 - 8 } & Alle & $<2.500$ & $<\mathbf{1 0 . 0 0 0}$ & $<\mathbf{5 0 . 0 0 0}$ & $<\mathbf{1 0 0 . 0 0 0}$ & $>100.000$ \\
\hline Anzahl: & 16 & 14 & 2 & 0 & 0 & 0 \\
\hline Anteil: & & $88 \%$ & $13 \%$ & $0 \%$ & $0 \%$ & $0 \%$ \\
\hline
\end{tabular}

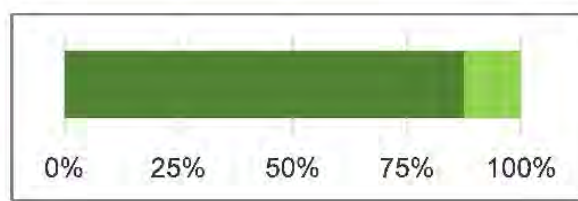

Beurteilung der zeitlichen Umsetzbarkeit

\begin{tabular}{|c|c|c|c|c|c|c|}
\hline & \multicolumn{8}{|c|}{ Nennungen [Anzahl] } & nach Kategorie [Monate] \\
\cline { 2 - 7 } & Alle & $\leq 3$ & $\mathbf{4 - 6}$ & $\mathbf{7 - 1 2}$ & $\mathbf{1 3 - 2 4}$ & $>24$ \\
\hline Anzahl: & 16 & 14 & 1 & 1 & 0 & 0 \\
\hline Anteil: & & $88 \%$ & $6 \%$ & $6 \%$ & $0 \%$ & $0 \%$ \\
\hline
\end{tabular}

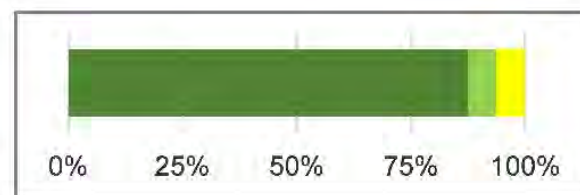

Beurteilung des verwaltungstechnischen Aufwands

\begin{tabular}{|c|c|c|c|c|c|}
\cline { 2 - 6 } & \multicolumn{5}{|c|}{ Nennungen [Anzahl] nach Kategorie [Aufwand] } \\
\cline { 2 - 6 } & Alle & gering & $\begin{array}{c}\text { eher } \\
\text { gering }\end{array}$ & eher hoch & hoch \\
\hline Anzahl: & 17 & 15 & 2 & 0 & 0 \\
\hline Anteil: & & $88 \%$ & $12 \%$ & $0 \%$ & $0 \%$ \\
\hline
\end{tabular}

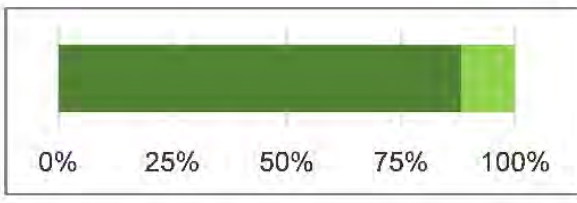

\section{Beurteilung der Wirksamkeit}

Wie wird durch die Maßnahme die Schwere der Unfälle reduziert?

\begin{tabular}{c|c|c|c|c|c|}
\cline { 2 - 6 } & \multicolumn{4}{|c|}{ Nennungen [Anzahl] } & \\
\cline { 2 - 6 } & Alle & stark & mäßig & wenig & nicht \\
\hline Anzahl: & 15 & 1 & 9 & 3 & 2 \\
\hline Anteil: & & $7 \%$ & $60 \%$ & $20 \%$ & $13 \%$ \\
\hline
\end{tabular}

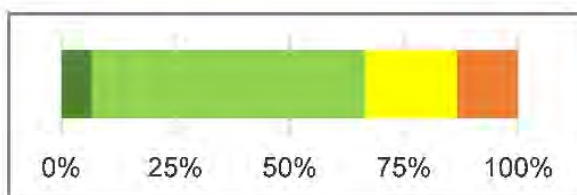

Wie wird durch die Maßnahme die Anzahl der Unfälle reduziert?

\begin{tabular}{|c|c|c|c|c|c|}
\cline { 3 - 6 } & \multicolumn{4}{|c|}{ Nennungen [Anzahl] } & \multicolumn{3}{c|}{} \\
\cline { 2 - 6 } & Alle & stark & mäßig & wenig & nicht \\
\hline Anzahl: & 16 & 0 & 8 & 5 & 3 \\
\hline Anteil: & & $0 \%$ & $50 \%$ & $31 \%$ & $19 \%$ \\
\hline
\end{tabular}

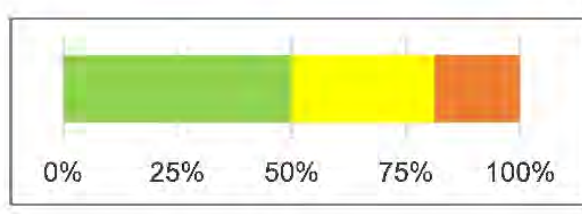

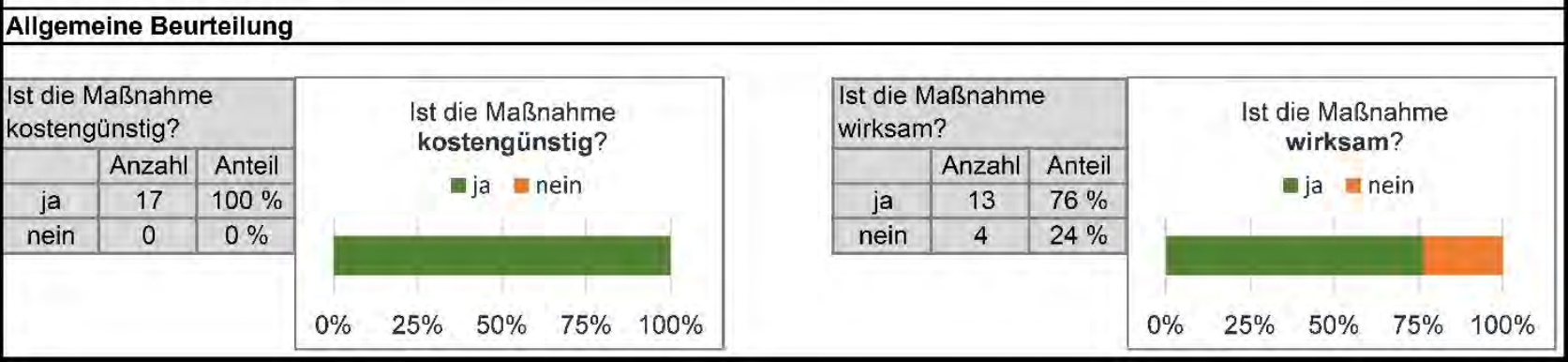


Evaluation der Maßnahmenkategorie: [12], Beschränkung Vzul (auf der Strecke)

\begin{tabular}{|c|c|c|c|c|c|c|}
\hline \multicolumn{2}{|c|}{ Einsatzbedingungen und allgemeine Angaben } & \multicolumn{5}{|c|}{ |Antwortkompetenz (Anzahl Nennungen) } \\
\hline Örtlichkeit: & AO, Strecke $(300 \mathrm{~m})$ & \multicolumn{2}{|l|}{ Behörde } & \multirow{5}{*}{$\begin{array}{l}\text { Mitglied in Uko sind } \\
18 \text { von } 18 \\
\text { Befragten. } \\
\text { Mittlere UKO-Dienszeit } \\
\text { in Jahren: } 14\end{array}$} & \multicolumn{2}{|l|}{ Zuständigkeit } \\
\hline Regelungsart: & - & \begin{tabular}{|r|} 
Polizei: \\
\end{tabular} & 0 & & \begin{tabular}{|r|} 
Stadt: \\
\end{tabular} & 8 \\
\hline Unfalltyp(en): & Fahrunfall & Verkehr: & 14 & & Land: & 7 \\
\hline Befragte: & 18 & Straßenbau: & 2 & & Andere: & 3 \\
\hline & & Andere: & 2 & & & \\
\hline
\end{tabular}

\section{Bekanntheitsgrad der Maßnahme}

Die Maßnahme ist

14 Befragten bekannt,

2 Befragten nicht bekannt. $\quad$ K. A.: 2 Befragte(r).

Der Bekannheitsgrad der Maßnahme liegt bei ca.: $\quad \mathbf{7 8 \%}$

1 von 14 Befragten, denen die Maßnahme bekannt ist, haben diese Maßnahme ein- oder mehrmals in den letzten 5 Jahren in ihrem Zuständigkeitsbereich umgesetzt.

\section{Beurteilung der Kosten}

\begin{tabular}{|c|c|c|c|c|c|c|}
\cline { 2 - 7 } & \multicolumn{7}{c|}{ Nennungen [Anzahl] nach Kategorie [€] } \\
\cline { 2 - 7 } & Alle & $<2.500$ & $<\mathbf{1 0 . 0 0 0}$ & $<\mathbf{5 0 . 0 0 0}$ & $<100.000$ & $>100.000$ \\
\hline Anzahl: & 15 & 15 & 0 & 0 & 0 & 0 \\
\hline Anteil: & & $100 \%$ & $0 \%$ & $0 \%$ & $0 \%$ & $0 \%$ \\
\hline
\end{tabular}

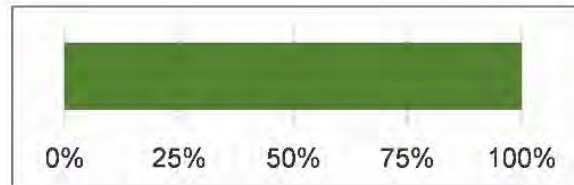

Beurteilung der zeitlichen Umsetzbarkeit

\begin{tabular}{c|c|c|c|c|c|c|}
\hline & \multicolumn{7}{|c|}{ Nennungen [Anzahl] nach Kategorie [Monate] } \\
\cline { 2 - 8 } & Alle & $\leq 3$ & $\mathbf{4 - 6}$ & $\mathbf{7 - 1 2}$ & $\mathbf{1 3 - 2 4}$ & $>24$ \\
\hline Anzahl: & 15 & 14 & 1 & 0 & 0 & 0 \\
\hline Anteil: & & $93 \%$ & $7 \%$ & $0 \%$ & $0 \%$ & $0 \%$ \\
\hline
\end{tabular}

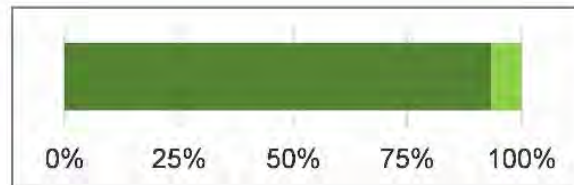

Beurteilung des verwaltungstechnischen Aufwands

\begin{tabular}{|c|c|c|c|c|c|}
\cline { 3 - 6 } & \multicolumn{4}{c|}{ Nennungen [Anzahl] nach Kategorie [Aufwand] } \\
\cline { 2 - 6 } & Alle & gering & $\begin{array}{c}\text { eher } \\
\text { gering }\end{array}$ & eher hoch & hoch \\
\hline Anzahl: & 16 & 15 & 0 & 1 & 0 \\
\hline Anteil: & & $94 \%$ & $0 \%$ & $6 \%$ & $0 \%$ \\
\hline
\end{tabular}

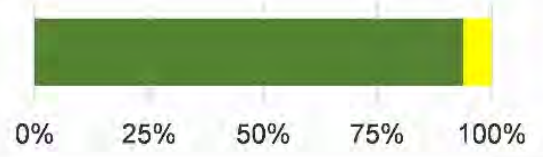

\section{Beurteilung der Wirksamkeit}

Wie wird durch die Maßnahme die Schwere der Unfälle reduziert?

\begin{tabular}{|c|c|c|c|c|c|}
\cline { 2 - 6 } & \multicolumn{4}{|c|}{ Nennungen [Anzahl] } & \multicolumn{2}{c|}{} \\
\cline { 2 - 6 } & Alle & stark & mäßig & wenig & nicht \\
\hline Anzahl: & 16 & 1 & 3 & 10 & 2 \\
\hline Anteil: & & $6 \%$ & $19 \%$ & $63 \%$ & $13 \%$ \\
\hline
\end{tabular}

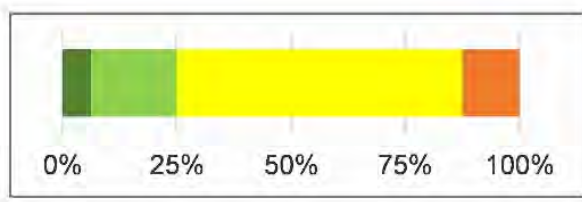

Wie wird durch die Maßnahme die Anzahl der Unfälle reduziert?

\begin{tabular}{|c|c|c|c|c|c|}
\cline { 2 - 6 } & \multicolumn{4}{c|}{ Nennungen [Anzahl] } & \multicolumn{2}{c|}{} \\
\cline { 2 - 6 } & Alle & stark & mäßig & wenig & nicht \\
\hline Anzahl: & 14 & 0 & 5 & 6 & 3 \\
\hline Anteil: & & $0 \%$ & $36 \%$ & $43 \%$ & $21 \%$ \\
\hline
\end{tabular}

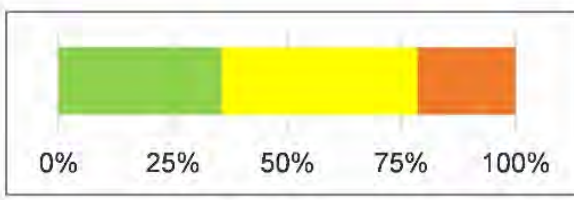

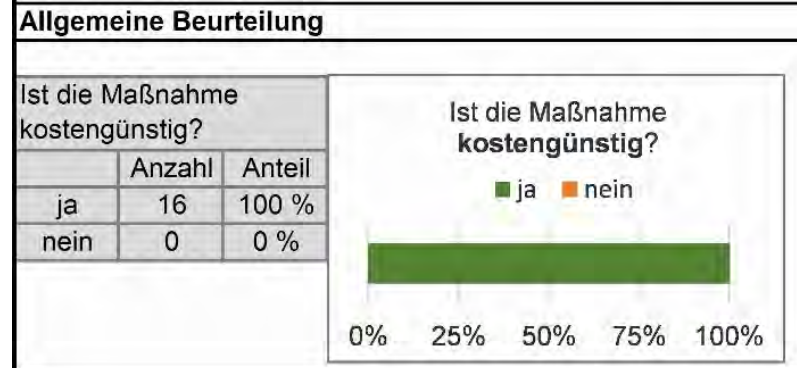

\begin{tabular}{|c|c|c|}
\hline \multicolumn{3}{|c|}{$\begin{array}{l}\text { Ist die Maßnahme } \\
\text { wirksam? }\end{array}$} \\
\hline & Anzahl & Anteil \\
\hline ja & 13 & $81 \%$ \\
\hline nein & 3 & $19 \%$ \\
\hline
\end{tabular}

Ist die Maßnahme wirksam?

m ja mein 
Evaluation der Maßnahmenkategorie: [15], Beschränkung Vzul (am Knotenpunkt)

\begin{tabular}{|c|c|c|c|c|c|c|}
\hline \multicolumn{2}{|c|}{ Einsatzbedingungen und allgemeine Angaben } & \multicolumn{5}{|c|}{ |Antwortkompetenz (Anzahl Nennungen) } \\
\hline Örtlichkeit: & AO, Knotenpunkt & \multicolumn{2}{|c|}{ Behörde } & \multirow{5}{*}{$\begin{array}{l}\text { Mitglied in Uko sind } \\
30 \text { von } 37 \\
\text { Befragten. } \\
\text { Mittlere UKO-Dienszeit } \\
\text { in Jahren: } 8\end{array}$} & \multicolumn{2}{|l|}{ Zuständigkeit } \\
\hline Regelungsart: & VZ & \begin{tabular}{|r|} 
Polizei: \\
\end{tabular} & 34 & & Stadt: & 23 \\
\hline Unfalltyp(en): & Fahrunfall, Einbiegen/Kreuzen & Verkehr: & 0 & & Land: & 23 \\
\hline Befragte: & 37 & Straßenbau: & 0 & & Andere: & 8 \\
\hline & & Andere: & 3 & & & \\
\hline
\end{tabular}

\section{Bekanntheitsgrad der Maßnahme}

Die Maßnahme ist

33 Befragten bekannt,

4 Befragten nicht bekannt. $\quad$ K. A.: 0 Befragte(r).

Der Bekannheitsgrad der Maßnahme liegt bei ca.: $\quad \mathbf{8 9 \%}$

\section{Beurteilung der Kosten}

\begin{tabular}{|c|c|c|c|c|c|c|}
\hline & \multicolumn{2}{c|}{ Nennungen [Anzahl] nach Kategorie [€] } \\
\cline { 2 - 7 } & Alle & $<2.500$ & $<10.000$ & $<\mathbf{5 0 . 0 0 0}$ & $<100.000$ & $>100.000$ \\
\hline Anzahl: & 35 & 30 & 3 & 0 & 0 & 0 \\
\hline Anteil: & & $86 \%$ & $9 \%$ & $0 \%$ & $0 \%$ & $0 \%$ \\
\hline
\end{tabular}

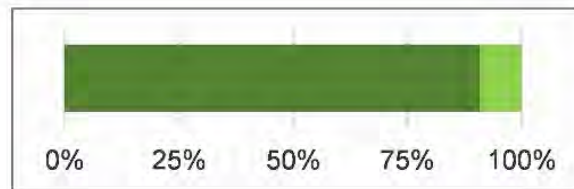

Beurteilung der zeitlichen Umsetzbarkeit

\begin{tabular}{|c|c|c|c|c|c|c|}
\hline & \multicolumn{8}{l|}{} & \multicolumn{2}{l|}{ Nennungen [Anzahl] nach Kategorie [Monate] } \\
\cline { 2 - 7 } & Alle & $\leq 3$ & $4-6$ & $7-12$ & $13-24$ & $>24$ \\
\hline Anzahl: & 34 & 29 & 5 & 0 & 0 & 0 \\
\hline Anteil: & & $85 \%$ & $15 \%$ & $0 \%$ & $0 \%$ & $0 \%$ \\
\hline
\end{tabular}

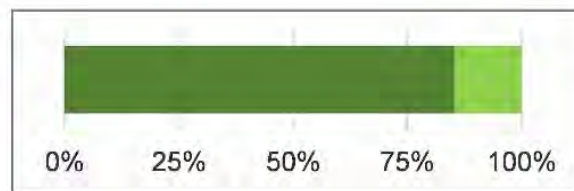

Beurteilung des verwaltungstechnischen Aufwands

\begin{tabular}{|c|c|c|c|c|c|}
\cline { 2 - 6 } & \multicolumn{4}{|c|}{ Nennungen [Anzahl] } & nach Kategorie [Aufwand] \\
\cline { 2 - 6 } & Alle & gering & $\begin{array}{c}\text { eher } \\
\text { gering }\end{array}$ & eher hoch & hoch \\
\hline Anzahl: & 37 & 26 & 10 & 1 & 0 \\
\hline Anteil: & & $70 \%$ & $27 \%$ & $3 \%$ & $0 \%$ \\
\hline
\end{tabular}

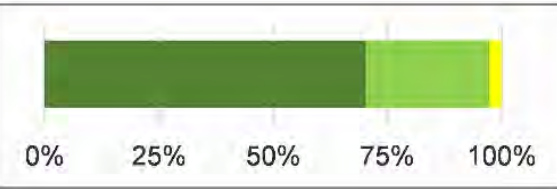

\section{Beurteilung der Wirksamkeit}

Wie wird durch die Maßnahme die Schwere der Unfälle reduziert?

\begin{tabular}{|c|c|c|c|c|c|}
\cline { 2 - 6 } & \multicolumn{2}{|c|}{ Nennungen [Anzahl] } & & \\
\cline { 2 - 6 } & Alle & stark & mäßig & wenig & nicht \\
\hline Anzahl: & 37 & 1 & 14 & 16 & 6 \\
\hline Anteil: & & $3 \%$ & $38 \%$ & $43 \%$ & $16 \%$ \\
\hline
\end{tabular}

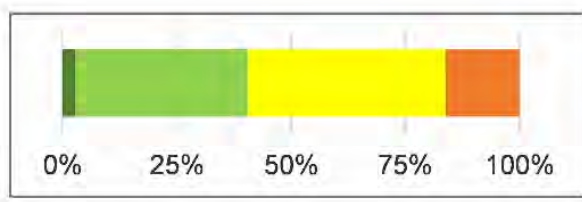

Wie wird durch die Maßnahme die Anzahl der Unfälle reduziert?

\begin{tabular}{|c|c|c|c|c|c|}
\cline { 2 - 6 } & \multicolumn{4}{c}{ Nennungen [Anzahl] } & \multicolumn{2}{c|}{} \\
\cline { 2 - 6 } & Alle & stark & mäßig & wenig & nicht \\
\hline Anzahl: & 37 & 4 & 21 & 10 & 2 \\
\hline Anteil: & & $11 \%$ & $57 \%$ & $27 \%$ & $5 \%$ \\
\hline
\end{tabular}

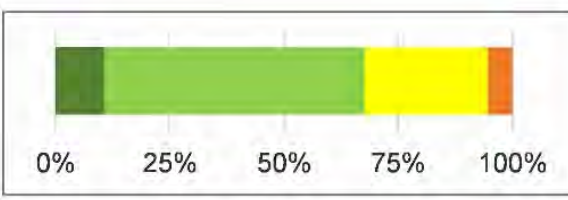

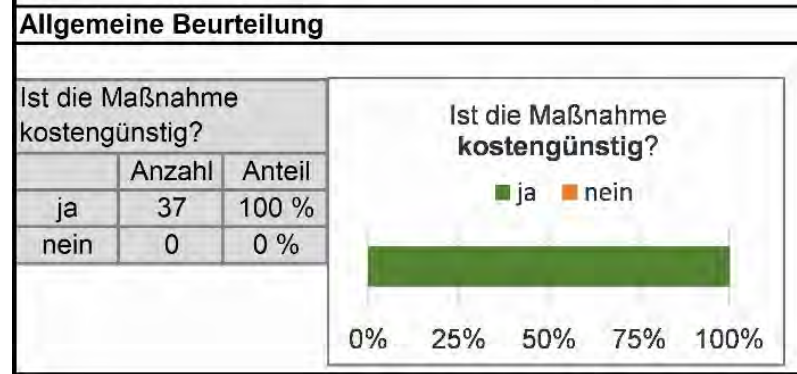

\begin{tabular}{|c|c|c|}
\hline \multicolumn{3}{|c|}{$\begin{array}{l}\text { Ist die Maßnahme } \\
\text { wirksam? }\end{array}$} \\
\hline & Anzahl & Anteil \\
\hline ja & 20 & $53 \%$ \\
\hline nein & 18 & $47 \%$ \\
\hline
\end{tabular}

Ist die Maßnahme wirksam?

m ja mein 
Evaluation der Maßnahmenkategorie: [16/17], Überwachung zulässige Höchstgeschwindigkeit mit OGÜ

\begin{tabular}{|c|c|c|c|c|c|}
\hline Einsatzbedingu & Ingen und allgemeine Angaben & Antwortkompete & nz & Izahl Nennungen) & \\
\hline Örtlichkeit: & AO, Strecke/Knotenpunkt & Behörde & & Mitglied in Uko sind & Zuständigkeit \\
\hline Regelungsart: & - & Polizei: & 8 & 12 von 18 & Stadt: 1 \\
\hline Unfalltyp(en): & Fahrunfall, Einbiegen/Kreuzen & Verkehr: & 6 & Befragten. & Land: \\
\hline Befragte: & 18 & Straßenbau: & 4 & Mittlere UKO-Dienszeit & Andere: \\
\hline & & Andere: & 0 & in Jahren: 5 & \\
\hline
\end{tabular}

\section{Bekanntheitsgrad der Maßnahme}

Die Maßnahme ist

14 Befragten bekannt,

4 Befragten nicht bekannt. $\quad$ K. A.: 0 Befragte(r).

13 von 14 Befragten, denen die Maßnahme bekannt ist, haben diese Maßnahme ein- oder mehrmals in den letzten 5 Jahren in ihrem Zuständigkeitsbereich umgesetzt.

Der Bekannheitsgrad der Maßnahme liegt bei ca.: $\mathbf{7 8 \%}$

\section{Beurteilung der Kosten}

\begin{tabular}{|c|c|c|c|c|c|c|}
\cline { 2 - 7 } & \multicolumn{7}{c|}{ Nennungen [Anzahl] nach Kategorie [€] } \\
\cline { 2 - 8 } & Alle & $<2.500$ & $<\mathbf{1 0 . 0 0 0}$ & $\mathbf{2 5 0 . 0 0 0}$ & $<\mathbf{1 0 0 . 0 0 0}$ & $>100.000$ \\
\hline Anzahl: & 18 & 0 & 8 & 7 & 3 & 0 \\
\hline Anteil: & & $0 \%$ & $\mathbf{4 4} \%$ & $39 \%$ & $17 \%$ & $0 \%$ \\
\hline
\end{tabular}

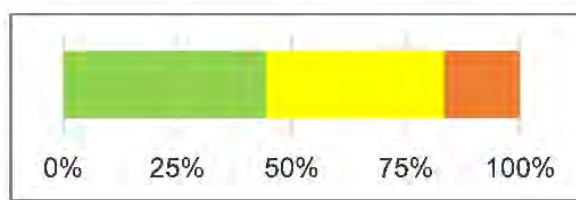

Beurteilung der zeitlichen Umsetzbarkeit

\begin{tabular}{|c|c|c|c|c|c|c|}
\hline & \multicolumn{8}{|c|}{ Nennungen [Anzahl] } & nach Kategorie [Monate] \\
\cline { 2 - 7 } & Alle & $\leq 3$ & $\mathbf{4 - 6}$ & $\mathbf{7 - 1 2}$ & $\mathbf{1 3 - 2 4}$ & $>24$ \\
\hline Anzahl: & 18 & 1 & 6 & 9 & 2 & 0 \\
\hline Anteil: & & $6 \%$ & $33 \%$ & $50 \%$ & $11 \%$ & $0 \%$ \\
\hline
\end{tabular}

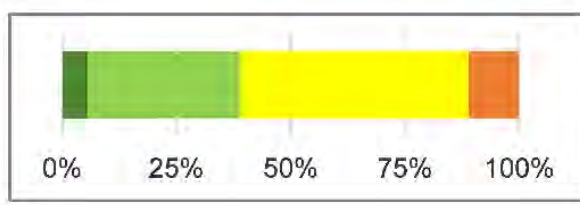

Beurteilung des verwaltungstechnischen Aufwands

\begin{tabular}{|c|c|c|c|c|c|}
\cline { 2 - 6 } & \multicolumn{5}{|c|}{ Nennungen [Anzahl] nach Kategorie [Aufwand] } \\
\cline { 2 - 6 } & Alle & gering & $\begin{array}{c}\text { eher } \\
\text { gering }\end{array}$ & eher hoch & hoch \\
\hline Anzahl: & 18 & 2 & 7 & 8 & 1 \\
\hline Anteil: & & $11 \%$ & $39 \%$ & $44 \%$ & $6 \%$ \\
\hline
\end{tabular}

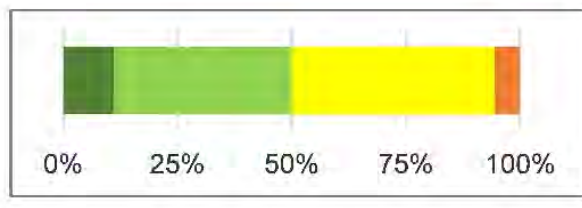

\section{Beurteilung der Wirksamkeit}

Wie wird durch die Maßnahme die Schwere der Unfälle reduziert?

\begin{tabular}{c|c|c|c|c|c|}
\cline { 2 - 6 } & \multicolumn{4}{|c|}{ Nennungen [Anzahl] } & \multicolumn{2}{l|}{} \\
\cline { 2 - 6 } & Alle & stark & mäßig & wenig & nicht \\
\hline Anzahl: & 18 & 1 & 2 & 8 & 7 \\
\hline Anteil: & & $6 \%$ & $11 \%$ & $44 \%$ & $39 \%$ \\
\hline
\end{tabular}

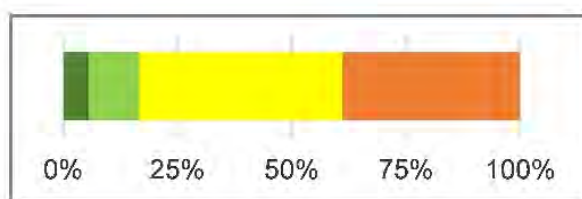

Wie wird durch die Maßnahme die Anzahl der Unfälle reduziert?

\begin{tabular}{|c|c|c|c|c|c|}
\cline { 2 - 6 } & \multicolumn{4}{|c|}{ Nennungen [Anzahl] } & \multicolumn{3}{c|}{} \\
\cline { 2 - 6 } & Alle & stark & mäßig & wenig & nicht \\
\hline Anzahl: & 17 & 0 & 0 & 11 & 6 \\
\hline Anteil: & & $0 \%$ & $0 \%$ & $65 \%$ & $35 \%$ \\
\hline
\end{tabular}

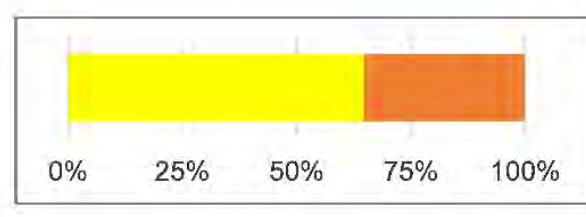

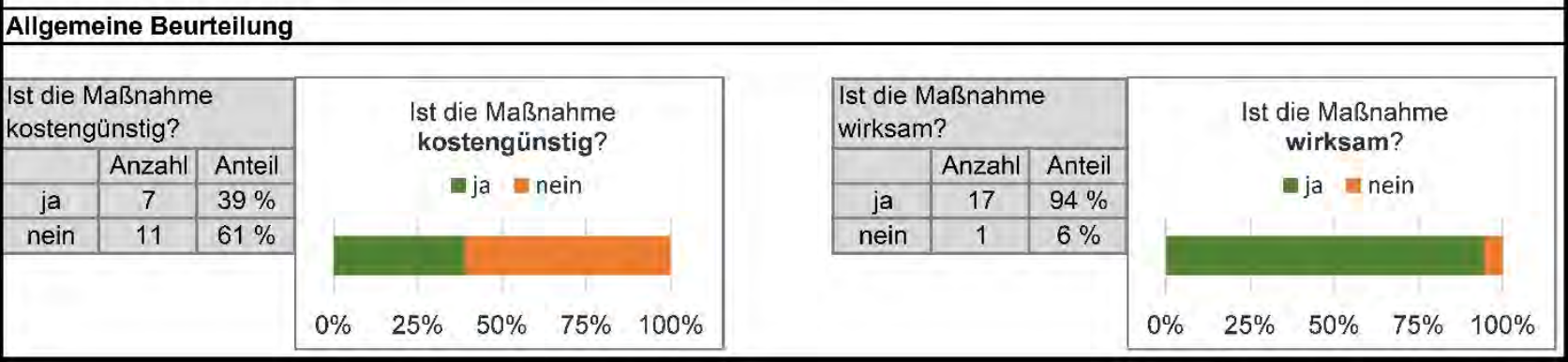


Evaluation der Maßnahmenkategorie: [18], Erneuerung Fahrbahnoberfläche

\begin{tabular}{|c|c|c|c|c|c|c|}
\hline \multicolumn{2}{|c|}{ Einsatzbedingungen und allgemeine Angaben } & \multicolumn{5}{|c|}{ |Antwortkompetenz (Anzahl Nennungen) } \\
\hline Örtlichkeit: & AO, Strecke $(300 \mathrm{~m})$ & \multicolumn{2}{|l|}{ Behörde } & \multirow{5}{*}{$\begin{array}{l}\text { Mitglied in Uko sind } \\
12 \text { von } 14 \\
\text { Befragten. } \\
\text { Mittlere UKO-Dienszeit } \\
\text { in Jahren: } 6\end{array}$} & \multicolumn{2}{|l|}{ Zuständigkeit } \\
\hline Regelungsart: & - & $\begin{array}{r}\text { Polizei: } \\
\end{array}$ & 2 & & Stadt: & 6 \\
\hline Unfalltyp(en): & Fahrunfall, Längsverkehr & Verkehr: & 6 & & Land: & 5 \\
\hline Befragte: & 14 & Straßenbau: & 2 & & Andere: & 4 \\
\hline & & Andere: & 4 & & & \\
\hline
\end{tabular}

\section{Bekanntheitsgrad der Maßnahme}

Die Maßnahme ist

14 Befragten bekannt,

0 Befragten nicht bekannt. $\quad$ K. A.: 0 Befragte( $r$ ).

Der Bekannheitsgrad der Maßnahme liegt bei ca.: $100 \%$

3 von 14 Befragten, denen die Maßnahme bekannt ist, haben diese Maßnahme ein- oder mehrmals in den letzten 5 Jahren in ihrem Zuständigkeitsbereich umgesetzt.

\section{Beurteilung der Kosten}

\begin{tabular}{|c|c|c|c|c|c|c|}
\cline { 2 - 7 } & \multicolumn{7}{c|}{ Nennungen [Anzahl] nach Kategorie [€] } \\
\cline { 2 - 7 } & Alle & $<2.500$ & $<\mathbf{1 0 . 0 0 0}$ & $<\mathbf{5 0 . 0 0 0}$ & $<\mathbf{1 0 0 . 0 0 0}$ & $>100.000$ \\
\hline Anzahl: & 11 & 0 & 1 & 9 & 1 & 0 \\
\hline Anteil: & & $0 \%$ & $9 \%$ & $82 \%$ & $9 \%$ & $0 \%$ \\
\hline
\end{tabular}

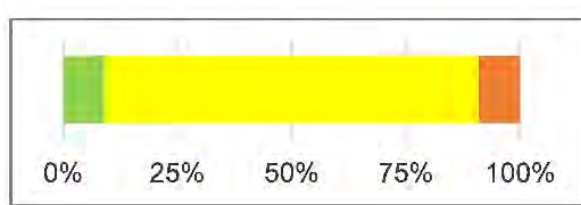

Beurteilung der zeitlichen Umsetzbarkeit

\begin{tabular}{|c|c|c|c|c|c|c|}
\hline & \multicolumn{8}{|c|}{ Nennungen [Anzahl] } & nach Kategorie [Monate] \\
\cline { 2 - 7 } & Alle & $\leq 3$ & $\mathbf{4 - 6}$ & $\mathbf{7 - 1 2}$ & $\mathbf{1 3 - 2 4}$ & $>24$ \\
\hline Anzahl: & 11 & 2 & 1 & 5 & 3 & 0 \\
\hline Anteil: & & $18 \%$ & $9 \%$ & $45 \%$ & $27 \%$ & $0 \%$ \\
\hline
\end{tabular}

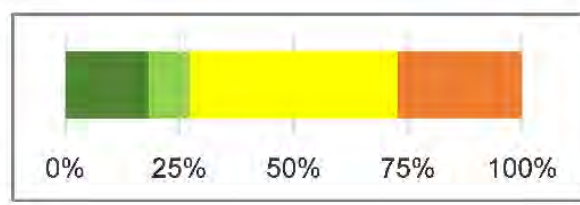

Beurteilung des verwaltungstechnischen Aufwands

\begin{tabular}{|c|c|c|c|c|c|}
\cline { 2 - 6 } & \multicolumn{6}{|c|}{ Nennungen [Anzahl] nach Kategorie [Aufwand] } & \\
\cline { 2 - 6 } & Alle & gering & $\begin{array}{c}\text { eher } \\
\text { gering }\end{array}$ & eher hoch & hoch \\
\hline Anzahl: & 11 & 0 & 5 & 5 & 1 \\
\hline Anteil: & & $0 \%$ & $45 \%$ & $45 \%$ & $9 \%$ \\
\hline
\end{tabular}

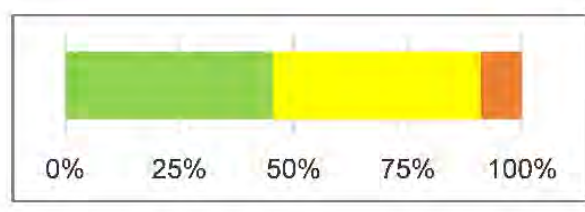

\section{Beurteilung der Wirksamkeit}

Wie wird durch die Maßnahme die Schwere der Unfälle reduziert?

\begin{tabular}{c|c|c|c|c|c|}
\cline { 2 - 6 } & \multicolumn{4}{|c|}{ Nennungen [Anzahl] } & \multicolumn{2}{l|}{} \\
\cline { 2 - 6 } & Alle & stark & mäßig & wenig & nicht \\
\hline Anzahl: & 12 & 0 & 3 & 3 & 6 \\
\hline Anteil: & & $0 \%$ & $25 \%$ & $25 \%$ & $50 \%$ \\
\hline
\end{tabular}

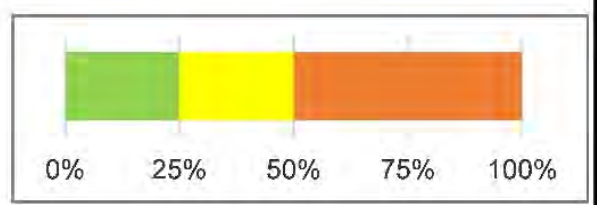

Wie wird durch die Maßnahme die Anzahl der Unfälle reduziert?

\begin{tabular}{|c|c|c|c|c|c|}
\hline & \multicolumn{4}{|c|}{ Nennungen [Anzahl] } & \multicolumn{3}{l|}{} \\
\cline { 2 - 6 } & Alle & stark & mäßig & wenig & nicht \\
\hline Anzahl: & 12 & 0 & 1 & 4 & 7 \\
\hline Anteil: & & $0 \%$ & $8 \%$ & $33 \%$ & $58 \%$ \\
\hline
\end{tabular}

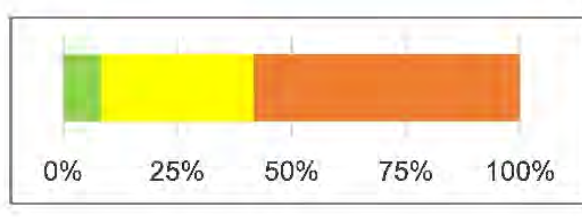

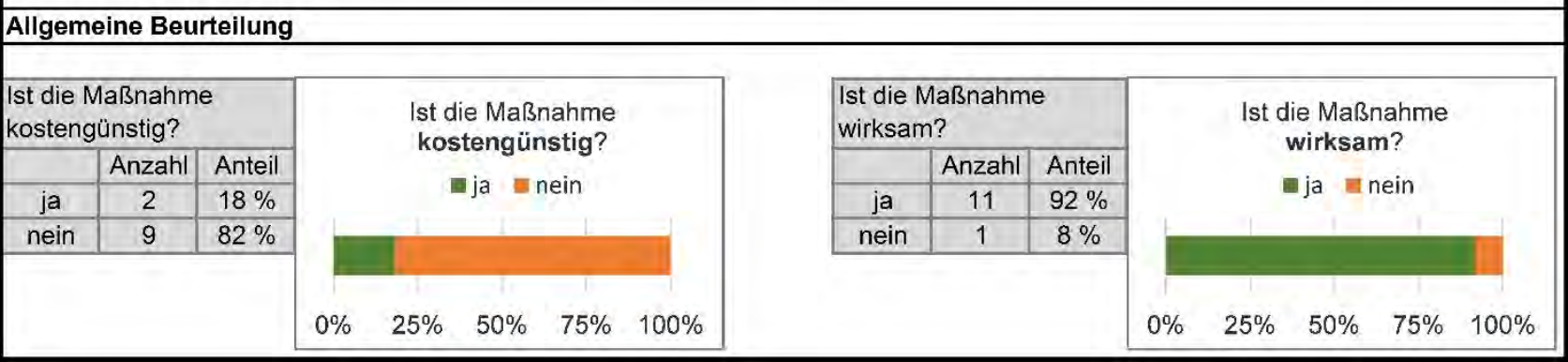


Evaluation der Maßnahmenkategorie: [19], Zusätzlichen Signalgeber anordnen

\begin{tabular}{|c|c|c|c|c|}
\hline Einsatzbedingungen und allgemeine Angaben & Antwortkompetenz & Izahl Nennungen) & & \\
\hline Örtlichkeit: 10, Knotenpunkt & Behörde & Mitglied in Uko sind & Zuständigkeit & \\
\hline Regelungsart: LSA & \begin{tabular}{|l|} 
Polizei: \\
\end{tabular} & 18 von 18 & Stadt: & 8 \\
\hline Unfalltyp(en): Einbiegen/Kreuzen, Längsverkehr & Verkehr: 14 & Befragten. & Land: & 7 \\
\hline Befragte: 18 & Straßenbau: 2 & Mittlere UKO-Dienszeit & Andere: & 3 \\
\hline & Andere: & in Jahren: 14 & & \\
\hline
\end{tabular}

\section{Bekanntheitsgrad der Maßnahme}

Die Maßnahme ist

15 Befragten bekannt,

1 Befragten nicht bekannt. $\quad$ K. A.: 2 Befragte(r).

3 von 15 Befragten, denen die Maßnahme bekannt ist, haben diese Maßnahme ein- oder mehrmals in den letzten 5 Jahren in ihrem Zuständigkeitsbereich umgesetzt.

Der Bekannheitsgrad der Maßnahme liegt bei ca.: $\mathbf{8 3} \%$

\section{Beurteilung der Kosten}

\begin{tabular}{|c|c|c|c|c|c|c|}
\hline \multirow{2}{*}{} & \multicolumn{7}{c|}{ Nennungen [Anzahl] nach Kategorie [€] } \\
\cline { 2 - 8 } & Alle & $<2.500$ & $<\mathbf{1 0 . 0 0 0}$ & $<\mathbf{5 0 . 0 0 0}$ & $<\mathbf{1 0 0 . 0 0 0}$ & $>100.000$ \\
\hline Anzahl: & 15 & 2 & 11 & 2 & 0 & 0 \\
\hline Anteil: & & $13 \%$ & $73 \%$ & $13 \%$ & $0 \%$ & $0 \%$ \\
\hline
\end{tabular}

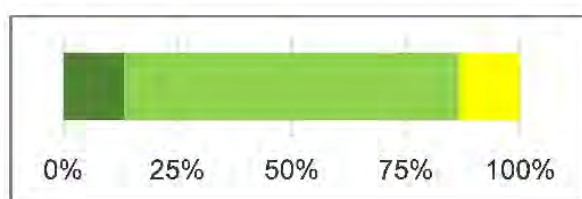

Beurteilung der zeitlichen Umsetzbarkeit

\begin{tabular}{|c|c|c|c|c|c|c|}
\hline & \multicolumn{8}{l|}{ Nennungen [Anzahl] } & nach Kategorie [Monate] \\
\cline { 2 - 7 } & Alle & $\leq \mathbf{3}$ & $\mathbf{4 - 6}$ & $\mathbf{7 - 1 2}$ & $\mathbf{1 3 - 2 4}$ & $>24$ \\
\hline Anzahl: & 16 & 4 & 8 & 4 & 0 & 0 \\
\hline Anteil: & & $25 \%$ & $50 \%$ & $25 \%$ & $0 \%$ & $0 \%$ \\
\hline
\end{tabular}

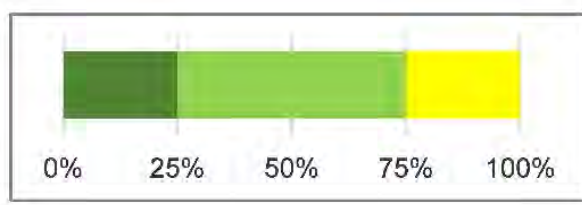

Beurteilung des verwaltungstechnischen Aufwands

\begin{tabular}{|c|c|c|c|c|c|}
\hline \multirow{2}{*}{} & \multicolumn{6}{|c|}{ Nennungen [Anzahl] } & nach Kategorie [Aufwand] & \\
\cline { 2 - 6 } & Alle & gering & $\begin{array}{c}\text { eher } \\
\text { gering }\end{array}$ & eher hoch & hoch \\
\hline Anzahl: & 16 & 4 & 8 & 3 & 1 \\
\hline Anteil: & & $25 \%$ & $50 \%$ & $19 \%$ & $6 \%$ \\
\hline
\end{tabular}

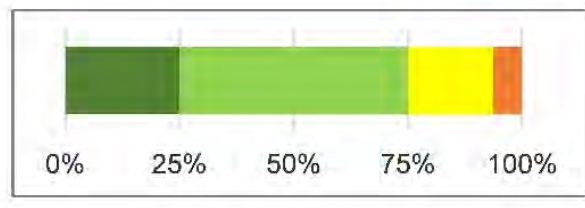

\section{Beurteilung der Wirksamkeit}

Wie wird durch die Maßnahme die Schwere der Unfälle reduziert?

\begin{tabular}{c|c|c|c|c|c|}
\cline { 2 - 6 } & \multicolumn{4}{|c|}{ Nennungen [Anzahl] } & \\
\cline { 2 - 6 } & Alle & stark & mäßig & wenig & nicht \\
\hline Anzahl: & 16 & 1 & 1 & 11 & 3 \\
\hline Anteil: & & $6 \%$ & $6 \%$ & $69 \%$ & $19 \%$ \\
\hline
\end{tabular}

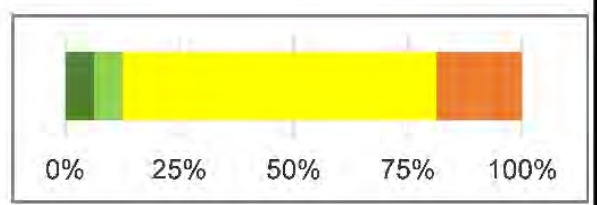

Wie wird durch die Maßnahme die Anzahl der Unfälle reduziert?

\begin{tabular}{|c|c|c|c|c|c|}
\cline { 2 - 6 } & \multicolumn{4}{|c|}{ Nennungen [Anzahl] } & \multicolumn{3}{c|}{} \\
\cline { 2 - 6 } & Alle & stark & mäßig & wenig & nicht \\
\hline Anzahl: & 15 & 0 & 2 & 7 & 6 \\
\hline Anteil: & & $0 \%$ & $13 \%$ & $47 \%$ & $40 \%$ \\
\hline
\end{tabular}

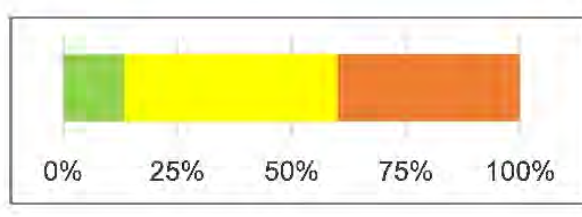

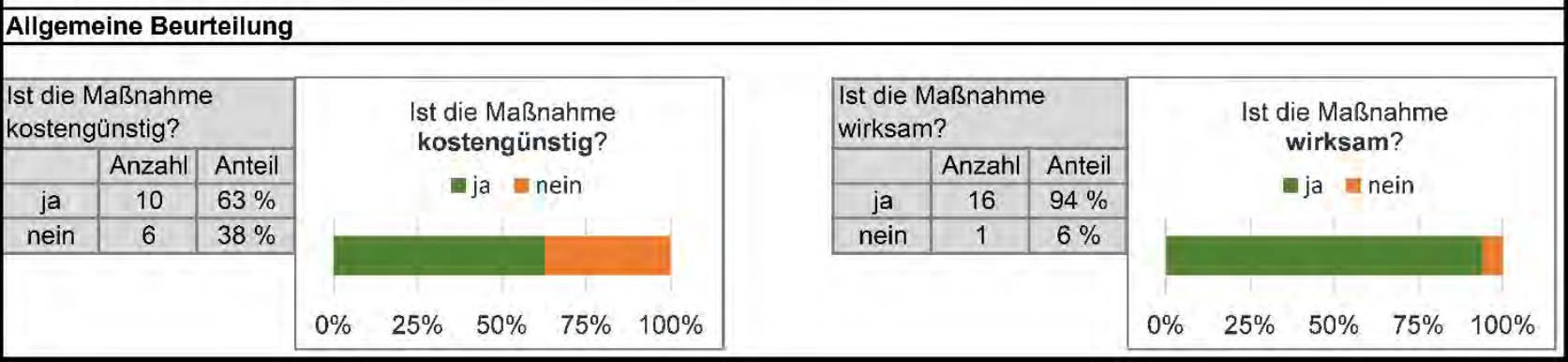


Evaluation der Maßnahmenkategorie: [21], Leuchtmittel Signalgeber verbessern

\begin{tabular}{|c|c|c|c|}
\hline Einsatzbedingungen und allgemeine Angaben & \multicolumn{3}{|c|}{ Antwortkompetenz (Anzahl Nennungen) } \\
\hline Örtlichkeit: 10, Knotenpunkt & Behörde & Mitglied in Uko sind & Zuständigkeit \\
\hline Regelungsart: LSA & 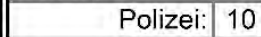 & 22 von 27 & Stadt: 1 \\
\hline Unfalltyp(en): Einbiegen/Kreuzen, Längsverkehr & Verkehr: 7 & Befragten. & Land: \\
\hline Befragte: 27 & Straßenbau: 5 & Mittlere UKO-Dienszeit & Andere: \\
\hline & Andere: 5 & in Jahren: 6 & \\
\hline
\end{tabular}

\begin{tabular}{|c|c|}
\hline Bekanntheitsgrad der Maßnahme & \\
\hline $\begin{array}{l}\text { Die Maßnahme ist } \\
\begin{array}{lll}21 & \text { Befragten bekannt, } \\
5 & \text { Befragten nicht bekannt. } & \text { K. A.: } 1 \text { Befragte(r). }\end{array}\end{array}$ & $\begin{array}{l}14 \text { von } 21 \text { Befragten, denen die Maßnahme bekannt ist, } \\
\text { haben diese Maßnahme ein- oder mehrmals in den letzten } 5 \\
\text { Jahren in ihrem Zuständigkeitsbereich umgesetzt. }\end{array}$ \\
\hline
\end{tabular}

Der Bekannheitsgrad der Maßnahme liegt bei ca.: $\mathbf{7 8 \%}$

\section{Beurteilung der Kosten}

\begin{tabular}{|c|c|c|c|c|c|c|}
\cline { 2 - 7 } & \multicolumn{7}{c|}{ Nennungen [Anzahl] nach Kategorie [€] } \\
\cline { 2 - 7 } & Alle & $<2.500$ & $<\mathbf{1 0 . 0 0 0}$ & $<\mathbf{5 0 . 0 0 0}$ & $<\mathbf{1 0 0 . 0 0 0}$ & $>100.000$ \\
\hline Anzahl: & 23 & 6 & 15 & 2 & 0 & 0 \\
\hline Anteil: & & $26 \%$ & $65 \%$ & $9 \%$ & $0 \%$ & $0 \%$ \\
\hline
\end{tabular}

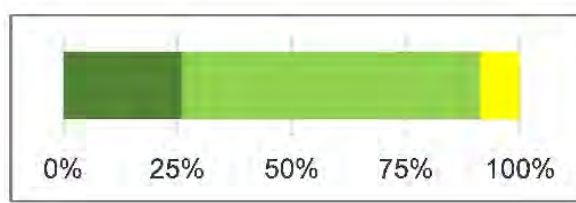

Beurteilung der zeitlichen Umsetzbarkeit

\begin{tabular}{|c|c|c|c|c|c|c|}
\hline & \multicolumn{6}{|c|}{ Nennungen [Anzahl] nach Kategorie [Monate] } \\
\hline & Alle & $\leq 3$ & $4-6$ & $7-12$ & $13-24$ & $>24$ \\
\hline Anzahl: & 24 & 9 & 11 & 2 & 2 & 0 \\
\hline Anteil: & & $38 \%$ & $46 \%$ & $8 \%$ & $8 \%$ & $0 \%$ \\
\hline
\end{tabular}

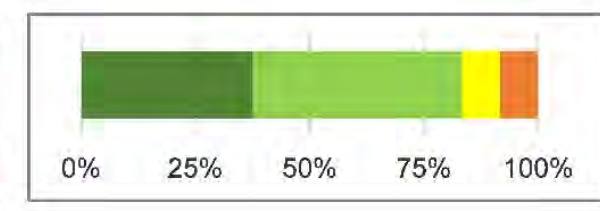

Beurteilung des verwaltungstechnischen Aufwands

\begin{tabular}{|c|c|c|c|c|c|}
\cline { 2 - 6 } & \multicolumn{6}{|c|}{ Nennungen [Anzahl] } & nach Kategorie [Aufwand] & \multicolumn{1}{c|}{ hoch } \\
\cline { 2 - 6 } & Alle & gering & $\begin{array}{c}\text { eher } \\
\text { gering }\end{array}$ & eher hoch & hoch \\
\hline Anzahl: & 24 & 8 & 13 & 3 & 0 \\
\hline Anteil: & & $33 \%$ & $54 \%$ & $13 \%$ & $0 \%$ \\
\hline
\end{tabular}

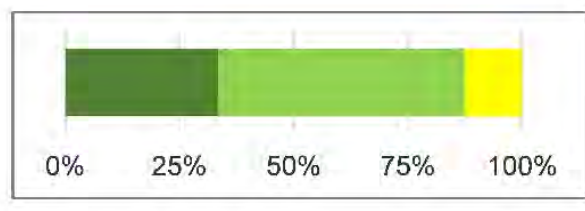

\section{Beurteilung der Wirksamkeit}

Wie wird durch die Maßnahme die Schwere der Unfälle reduziert?

\begin{tabular}{c|c|c|c|c|c|}
\cline { 2 - 6 } & \multicolumn{4}{c|}{ Nennungen [Anzahl] } & \\
\cline { 2 - 6 } & Alle & stark & mäßig & wenig & nicht \\
\hline Anzahl: & 22 & 6 & 3 & 9 & 4 \\
\hline Anteil: & & $27 \%$ & $14 \%$ & $41 \%$ & $18 \%$ \\
\hline
\end{tabular}

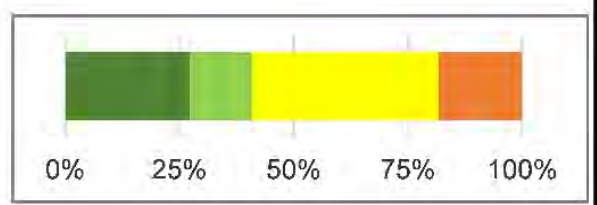

Wie wird durch die Maßnahme die Anzahl der Unfälle reduziert?

\begin{tabular}{|c|c|c|c|c|c|}
\cline { 2 - 6 } & \multicolumn{4}{|c|}{ Nennungen [Anzahl] } & \multicolumn{3}{c|}{} \\
\cline { 2 - 6 } & Alle & stark & mäßig & wenig & nicht \\
\hline Anzahl: & 23 & 0 & 2 & 17 & 4 \\
\hline Anteil: & & $0 \%$ & $9 \%$ & $74 \%$ & $17 \%$ \\
\hline
\end{tabular}

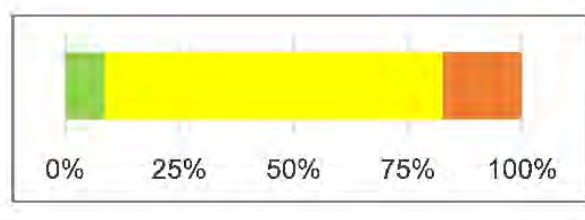

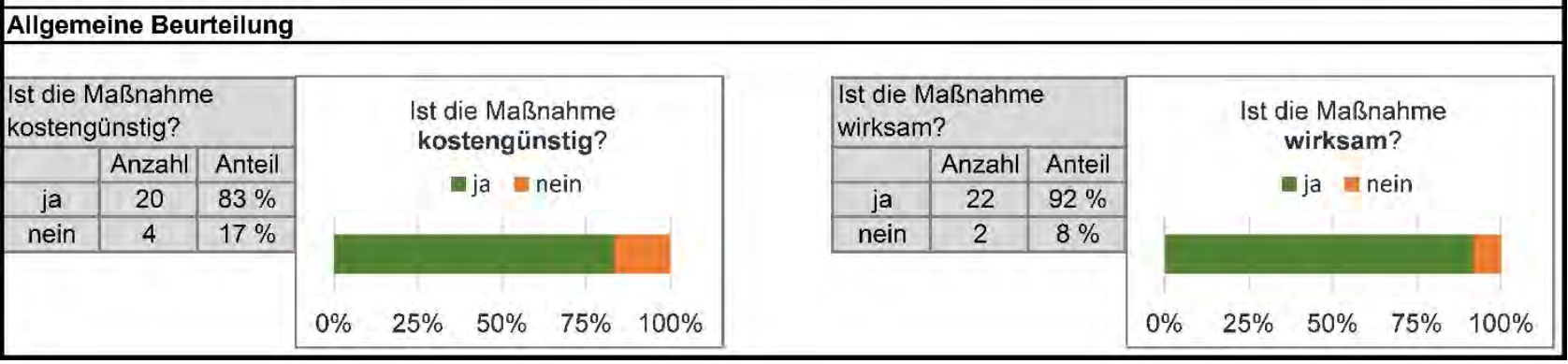


Evaluation der Maßnahmenkategorie: [22/23], Sichthindernisse entfernen

\begin{tabular}{|c|c|c|c|c|c|c|}
\hline \multicolumn{2}{|c|}{ Einsatzbedingungen und allgemeine Angaben } & \multicolumn{5}{|c|}{ Antwortkompetenz (Anzahl Nennungen) } \\
\hline Örtlichkeit:|I & IO/AO, Knotenpunkt & \multicolumn{2}{|l|}{ Behörde } & \multirow{5}{*}{$\begin{array}{l}\text { Mitglied in Uko sind } \\
12 \text { von } 18 \\
\text { Befragten. } \\
\text { Mittlere UKO-Dienszeit } \\
\text { in Jahren: } 5\end{array}$} & \multicolumn{2}{|l|}{ Zuständigkeit } \\
\hline Regelungsart: & VZ & $\begin{array}{r}\text { Polizei: } \\
\end{array}$ & 8 & & Stadt: & 10 \\
\hline Unfalltyp(en): $\mathrm{E}$ & Einbiegen/Kreuzen & Verkehr: & 6 & & Land: & 6 \\
\hline Befragte: & 18 & Straßenbau: & 4 & & Andere: & 5 \\
\hline & & Andere: & 0 & & & \\
\hline
\end{tabular}

\section{Bekanntheitsgrad der Maßnahme}

Die Maßnahme ist

15 Befragten bekannt,

3 Befragten nicht bekannt. $\quad$ K. A.: 0 Befragte(r).

9 von 15 Befragten, denen die Maßnahme bekannt ist, haben diese Maßnahme ein- oder mehrmals in den letzten 5 Jahren in ihrem Zuständigkeitsbereich umgesetzt.

Der Bekannheitsgrad der Maßnahme liegt bei ca.: $\mathbf{8 3} \%$

\section{Beurteilung der Kosten}

\begin{tabular}{|c|c|c|c|c|c|c|}
\cline { 2 - 7 } & \multicolumn{7}{|c|}{ Nennungen [Anzahl] nach Kategorie [€] } \\
\cline { 2 - 7 } & Alle & $<2.500$ & $<\mathbf{1 0 . 0 0 0}$ & $<\mathbf{5 0 . 0 0 0}$ & $<\mathbf{1 0 0 . 0 0 0}$ & $>100.000$ \\
\hline Anzahl: & 16 & 3 & 11 & 2 & 0 & 0 \\
\hline Anteil: & & $19 \%$ & $69 \%$ & $13 \%$ & $0 \%$ & $0 \%$ \\
\hline
\end{tabular}

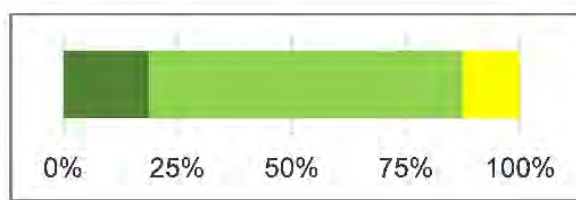

Beurteilung der zeitlichen Umsetzbarkeit

\begin{tabular}{|c|c|c|c|c|c|c|}
\hline & \multicolumn{8}{|c|}{ Nennungen [Anzahl] } & nach Kategorie [Monate] \\
\cline { 2 - 7 } & Alle & $\leq 3$ & $\mathbf{4 - 6}$ & $\mathbf{7 - 1 2}$ & $\mathbf{1 3 - 2 4}$ & $>24$ \\
\hline Anzahl: & 17 & 5 & 10 & 2 & 0 & 0 \\
\hline Anteil: & & $29 \%$ & $59 \%$ & $12 \%$ & $0 \%$ & $0 \%$ \\
\hline
\end{tabular}

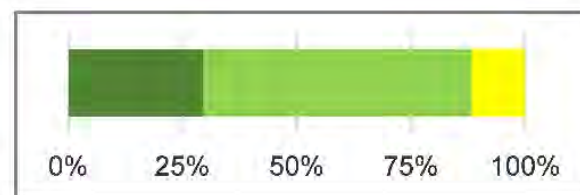

Beurteilung des verwaltungstechnischen Aufwands

\begin{tabular}{|c|c|c|c|c|c|}
\cline { 2 - 6 } & \multicolumn{5}{|c|}{ Nennungen [Anzahl] nach Kategorie [Aufwand] } \\
\cline { 2 - 6 } & Alle & gering & $\begin{array}{c}\text { eher } \\
\text { gering }\end{array}$ & eher hoch & hoch \\
\hline Anzahl: & 17 & 3 & 12 & 2 & 0 \\
\hline Anteil: & & $18 \%$ & $71 \%$ & $12 \%$ & $0 \%$ \\
\hline
\end{tabular}

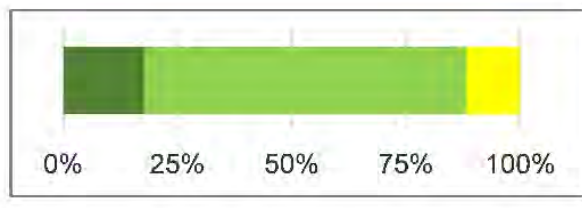

\section{Beurteilung der Wirksamkeit}

Wie wird durch die Maßnahme die Schwere der Unfälle reduziert?

\begin{tabular}{c|c|c|c|c|c|}
\cline { 2 - 6 } & \multicolumn{4}{|c|}{ Nennungen [Anzahl] } & \\
\cline { 2 - 6 } & Alle & stark & mäßig & wenig & nicht \\
\hline Anzahl: & 17 & 1 & 6 & 7 & 3 \\
\hline Anteil: & & $6 \%$ & $35 \%$ & $41 \%$ & $18 \%$ \\
\hline
\end{tabular}

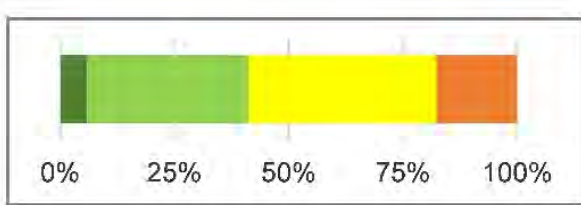

Wie wird durch die Maßnahme die Anzahl der Unfälle reduziert?

\begin{tabular}{|c|c|c|c|c|c|}
\cline { 2 - 6 } & \multicolumn{2}{|c|}{ Nennungen [Anzahl] } & \multicolumn{3}{c|}{} \\
\cline { 2 - 6 } & Alle & stark & mäßig & wenig & nicht \\
\hline Anzahl: & 17 & 0 & 1 & 10 & 6 \\
\hline Anteil: & & $0 \%$ & $6 \%$ & $59 \%$ & $35 \%$ \\
\hline
\end{tabular}

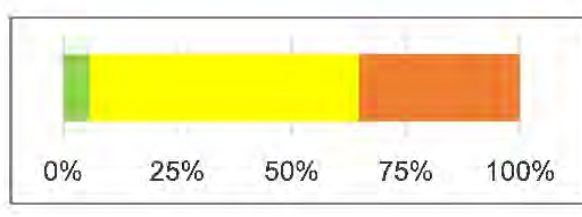

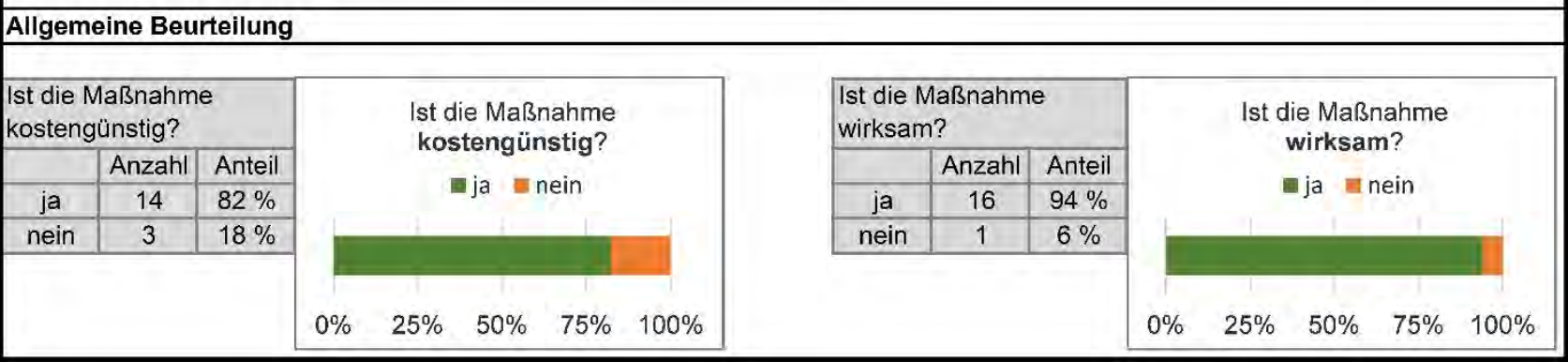


Evaluation der Maßnahmenkategorie: [24], Beleuchtung anpassen/verbessern

\begin{tabular}{|c|c|c|c|c|c|c|}
\hline \multicolumn{2}{|c|}{ Einsatzbedingungen und allgemeine Angaben } & \multicolumn{5}{|c|}{ |Antwortkompetenz (Anzahl Nennungen) } \\
\hline Örtlichkeit: & 10, Knotenpunkt/Strecke & \multicolumn{2}{|l|}{ Behörde } & \multirow{5}{*}{$\begin{array}{l}\text { Mitglied in Uko sind } \\
42 \text { von } 51 \\
\text { Befragten. } \\
\text { Mittlere UKO-Dienszeit } \\
\text { in Jahren: } 7\end{array}$} & \multicolumn{2}{|l|}{ Zuständigkeit } \\
\hline Regelungsart: & - & \begin{tabular}{|r|r|} 
Polizei: 3 \\
\end{tabular} & 36 & & Stadt: & 29 \\
\hline Unfalltyp(en): & beliebig & Verkehr: & 6 & & Land: & 28 \\
\hline Befragte: & 51 & Straßenbau: & 2 & & Andere: & 12 \\
\hline & & Andere: & 7 & & & \\
\hline
\end{tabular}

\section{Bekanntheitsgrad der Maßnahme}

Die Maßnahme ist

47 Befragten bekannt,

3 Befragten nicht bekannt. $\quad$ K. A.: 1 Befragte(r).

Der Bekannheitsgrad der Maßnahme liegt bei ca.: $\mathbf{9 2} \%$

27 von 47 Befragten, denen die Maßnahme bekannt ist, haben diese Maßnahme ein- oder mehrmals in den letzten 5 Jahren in ihrem Zuständigkeitsbereich umgesetzt.

\section{Beurteilung der Kosten}

\begin{tabular}{|c|c|c|c|c|c|c|}
\cline { 2 - 7 } & \multicolumn{7}{c|}{ Nennungen [Anzahl] nach Kategorie [€] } \\
\cline { 2 - 7 } & Alle & $<2.500$ & $<\mathbf{1 0 . 0 0 0}$ & $<\mathbf{5 0 . 0 0 0}$ & $<\mathbf{1 0 0 . 0 0 0}$ & $>100.000$ \\
\hline Anzahl: & 44 & 6 & 20 & 16 & 1 & 1 \\
\hline Anteil: & & $14 \%$ & $\mathbf{4 5} \%$ & $36 \%$ & $2 \%$ & $2 \%$ \\
\hline
\end{tabular}

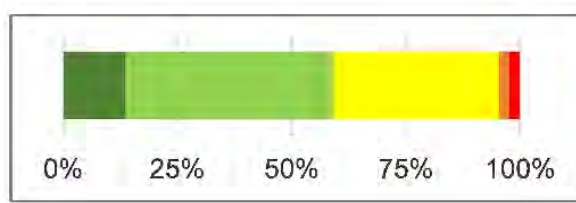

Beurteilung der zeitlichen Umsetzbarkeit

\begin{tabular}{|c|c|c|c|c|c|c|}
\hline & \multicolumn{8}{|c|}{ Nennungen [Anzahl] } & nach Kategorie [Monate] \\
\cline { 2 - 7 } & Alle & $\leq 3$ & $\mathbf{4 - 6}$ & $\mathbf{7 - 1 2}$ & $\mathbf{1 3 - 2 4}$ & $>24$ \\
\hline Anzahl: & 44 & 6 & 22 & 14 & 2 & 0 \\
\hline Anteil: & & $14 \%$ & $50 \%$ & $32 \%$ & $5 \%$ & $0 \%$ \\
\hline
\end{tabular}

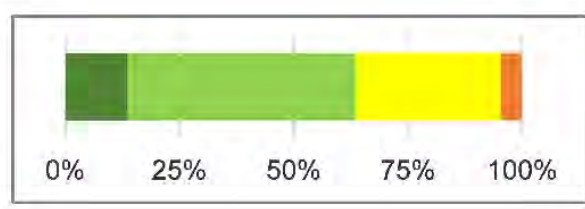

Beurteilung des verwaltungstechnischen Aufwands

\begin{tabular}{|c|c|c|c|c|c|}
\cline { 2 - 6 } & \multicolumn{6}{|c|}{ Nennungen [Anzahl] } & nach Kategorie [Aufwand] & \\
\cline { 2 - 6 } & Alle & gering & $\begin{array}{c}\text { eher } \\
\text { gering }\end{array}$ & eher hoch & hoch \\
\hline Anzahl: & 45 & 3 & 25 & 16 & 1 \\
\hline Anteil: & & $7 \%$ & $56 \%$ & $36 \%$ & $2 \%$ \\
\hline
\end{tabular}

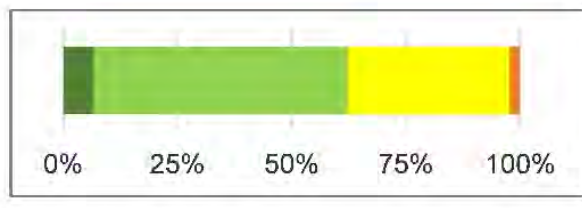

\section{Beurteilung der Wirksamkeit}

Wie wird durch die Maßnahme die Schwere der Unfälle reduziert?

\begin{tabular}{c|c|c|c|c|c|}
\cline { 2 - 6 } & \multicolumn{4}{|c|}{ Nennungen [Anzahl] } & \\
\cline { 2 - 6 } & Alle & stark & mäßig & wenig & nicht \\
\hline Anzahl: & 44 & 7 & 6 & 13 & 18 \\
\hline Anteil: & & $16 \%$ & $14 \%$ & $30 \%$ & $41 \%$ \\
\hline
\end{tabular}

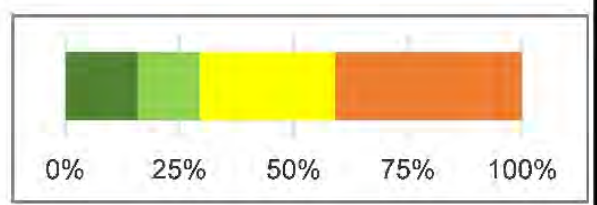

Wie wird durch die Maßnahme die Anzahl der Unfälle reduziert?

\begin{tabular}{|c|c|c|c|c|c|}
\cline { 2 - 6 } & \multicolumn{4}{|c|}{ Nennungen [Anzahl] } & \multicolumn{3}{c|}{} \\
\cline { 2 - 6 } & Alle & stark & mäßig & wenig & nicht \\
\hline Anzahl: & 43 & 1 & 1 & 17 & 24 \\
\hline Anteil: & & $2 \%$ & $2 \%$ & $40 \%$ & $56 \%$ \\
\hline
\end{tabular}

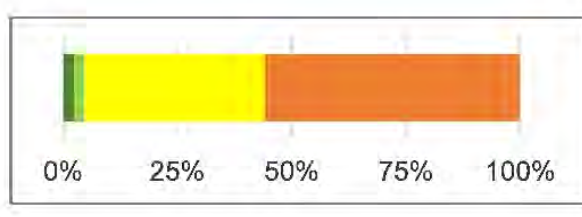

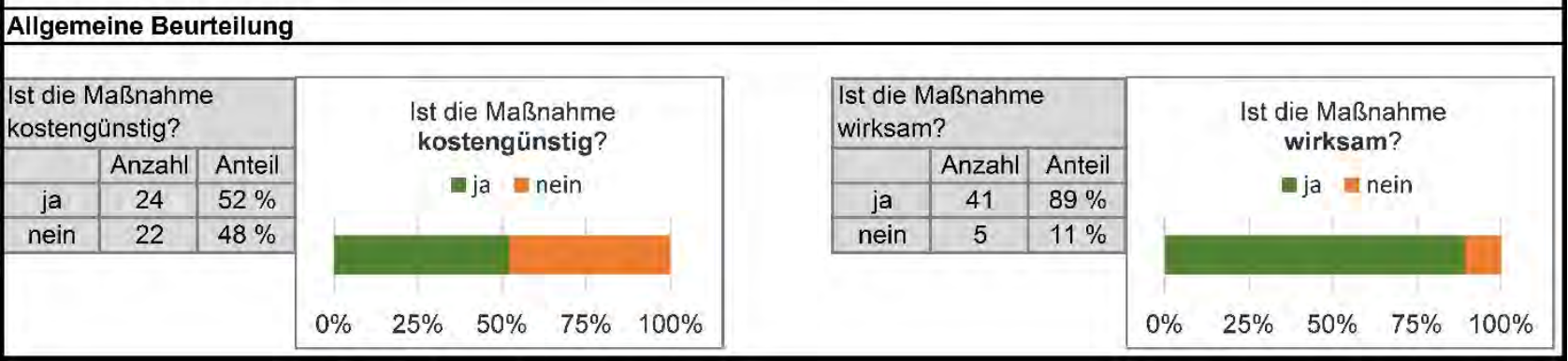


Evaluation der Maßnahmenkategorie: [25], Sichtschutzzaun aufstellen

\begin{tabular}{|c|c|c|c|c|c|c|}
\hline \multicolumn{2}{|c|}{ Einsatzbedingungen und allgemeine Angaben } & \multicolumn{5}{|c|}{ |Antwortkompetenz (Anzahl Nennungen) } \\
\hline Örtlichkeit: & $\mathrm{AO}$, Knotenpunkt & \multicolumn{2}{|c|}{ Behörde } & \multirow{5}{*}{$\begin{array}{l}\text { Mitglied in Uko sind } \\
19 \text { von } 23 \\
\text { Befragten. } \\
\text { Mittlere UKO-Dienszeit } \\
\text { in Jahren: } 5\end{array}$} & \multicolumn{2}{|l|}{ Zuständigkeit } \\
\hline Regelungsart: & VZ & \begin{tabular}{|l|} 
Polizei: \\
\end{tabular} & 5 & & Stadt: & 11 \\
\hline Unfalltyp(en): & Einbiegen/Kreuzen & Verkehr: & 10 & & Land: & 10 \\
\hline Befragte: & 23 & Straßenbau: & 3 & & Andere: & 5 \\
\hline & & Andere: & 5 & & & \\
\hline
\end{tabular}

\section{Bekanntheitsgrad der Maßnahme}

Die Maßnahme ist

8 Befragten bekannt,

14 Befragten nicht bekannt. $\quad$ K. A.: 1 Befragte(r).

Der Bekannheitsgrad der Maßnahme liegt bei ca.: $35 \%$

5 von 8 Befragten, denen die Maßnahme bekannt ist, haben diese Maßnahme ein- oder mehrmals in den letzten 5 Jahren in ihrem Zuständigkeitsbereich umgesetzt.

\section{Beurteilung der Kosten}

\begin{tabular}{|c|c|c|c|c|c|c|}
\cline { 2 - 7 } & \multicolumn{2}{|c|}{ Nennungen [Anzahl] nach Kategorie [€] } \\
\cline { 2 - 7 } & Alle & $<2.500$ & $<\mathbf{1 0 . 0 0 0}$ & $<\mathbf{5 0 . 0 0 0}$ & $<100.000$ & $>100.000$ \\
\hline Anzahl: & 23 & 9 & 10 & 4 & 0 & 0 \\
\hline Anteil: & & $39 \%$ & $\mathbf{4 3} \%$ & $17 \%$ & $0 \%$ & $0 \%$ \\
\hline
\end{tabular}

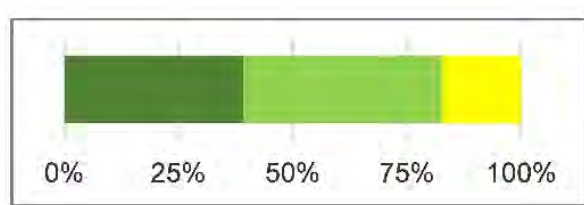

Beurteilung der zeitlichen Umsetzbarkeit

\begin{tabular}{|c|c|c|c|c|c|c|}
\hline & \multicolumn{8}{l|}{ Nennungen [Anzahl] } & nach Kategorie [Monate] \\
\cline { 2 - 7 } & Alle & $\leq 3$ & $\mathbf{4 - 6}$ & $\mathbf{7 - 1 2}$ & $\mathbf{1 3 - 2 4}$ & $>24$ \\
\hline Anzahl: & 22 & 15 & 2 & 2 & 1 & 2 \\
\hline Anteil: & & $68 \%$ & $9 \%$ & $9 \%$ & $5 \%$ & $9 \%$ \\
\hline
\end{tabular}

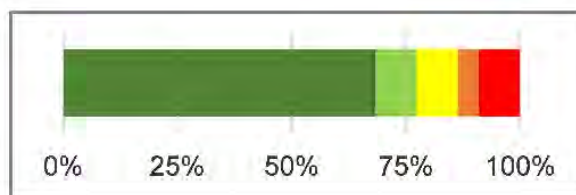

Beurteilung des verwaltungstechnischen Aufwands

\begin{tabular}{|c|c|c|c|c|c|}
\cline { 2 - 6 } & \multicolumn{4}{|c|}{ Nennungen [Anzahl] nach Kategorie [Aufwand] } \\
\cline { 2 - 6 } & Alle & gering & $\begin{array}{c}\text { eher } \\
\text { gering }\end{array}$ & eher hoch & hoch \\
\hline Anzahl: & 22 & 8 & 11 & 3 & 0 \\
\hline Anteil: & & $36 \%$ & $50 \%$ & $14 \%$ & $0 \%$ \\
\hline
\end{tabular}

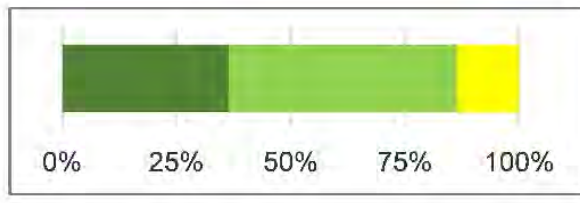

\section{Beurteilung der Wirksamkeit}

Wie wird durch die Maßnahme die Schwere der Unfälle reduziert?

\begin{tabular}{c|c|c|c|c|c|}
\cline { 2 - 6 } & \multicolumn{4}{|c|}{ Nennungen [Anzahl] } & \multicolumn{2}{l|}{} \\
\cline { 2 - 6 } & Alle & stark & mäßig & wenig & nicht \\
\hline Anzahl: & 23 & 5 & 5 & 8 & 5 \\
\hline Anteil: & & $22 \%$ & $22 \%$ & $35 \%$ & $22 \%$ \\
\hline
\end{tabular}

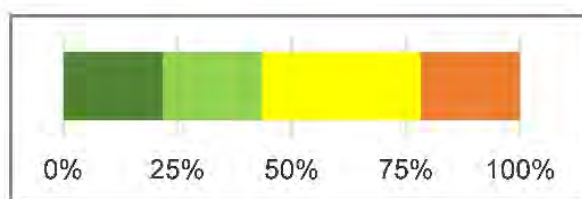

Wie wird durch die Maßnahme die Anzahl der Unfälle reduziert?

\begin{tabular}{|c|c|c|c|c|c|}
\cline { 3 - 6 } & \multicolumn{2}{|c|}{ Nennungen [Anzahl] } & \multicolumn{3}{l|}{} \\
\cline { 2 - 6 } & Alle & stark & mäßig & wenig & nicht \\
\hline Anzahl: & 23 & 2 & 3 & 12 & 6 \\
\hline Anteil: & & $9 \%$ & $13 \%$ & $52 \%$ & $26 \%$ \\
\hline
\end{tabular}

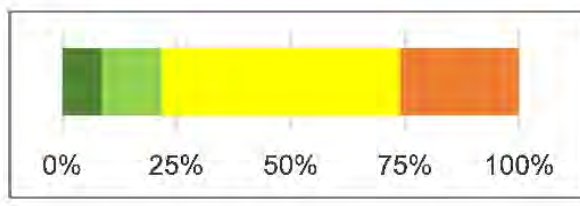

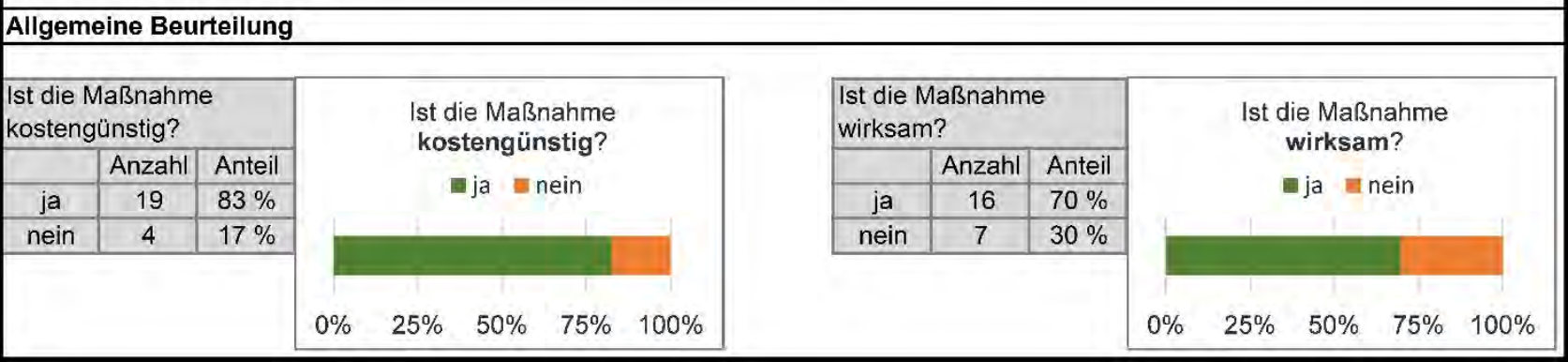


Evaluation der Maßnahmenkategorie: [26], Markierung anpassen/verbessern

\begin{tabular}{|c|c|c|c|c|c|c|}
\hline \multicolumn{2}{|c|}{ Einsatzbedingungen und allgemeine Angaben } & \multicolumn{5}{|c|}{ |Antwortkompetenz (Anzahl Nennungen) } \\
\hline Örtlichkeit: & IO, Knotenpunkt & \multicolumn{2}{|c|}{ Behörde } & \multirow{5}{*}{$\begin{array}{l}\text { Mitglied in Uko sind } \\
55 \text { von } 80 \\
\text { Befragten. } \\
\text { Mittlere UKO-Dienszeit } \\
\text { in Jahren: } 8\end{array}$} & \multicolumn{2}{|l|}{ Zuständigkeit } \\
\hline Regelungsart: & beliebig & \begin{tabular}{|r|} 
Polizei: \\
\end{tabular} & 35 & & Stadt: & 30 \\
\hline Unfalltyp(en): & beliebig & Verkehr: & 18 & & Land: & 36 \\
\hline Befragte: & 80 & Straßenbau: & 5 & & Andere: & 12 \\
\hline & & Andere: & 22 & & & \\
\hline
\end{tabular}

\section{Bekanntheitsgrad der Maßnahme}

Die Maßnahme ist

58 Befragten bekannt,

21 Befragten nicht bekannt. $\quad$ K. A.: 1 Befragte(r).

Der Bekannheitsgrad der Maßnahme liegt bei ca.: $\quad 73 \%$

\section{Beurteilung der Kosten}

\begin{tabular}{|c|c|c|c|c|c|c|}
\hline & \multicolumn{2}{|c|}{ Nennungen [Anzahl] nach Kategorie [€] } \\
\cline { 2 - 7 } & Alle & $<2.500$ & $<\mathbf{1 0 . 0 0 0}$ & $<\mathbf{5 0 . 0 0 0}$ & $<100.000$ & $>100.000$ \\
\hline Anzahl: & 76 & 29 & 35 & 11 & 1 & 0 \\
\hline Anteil: & & $38 \%$ & $\mathbf{4 6} \%$ & $14 \%$ & $1 \%$ & $0 \%$ \\
\hline
\end{tabular}

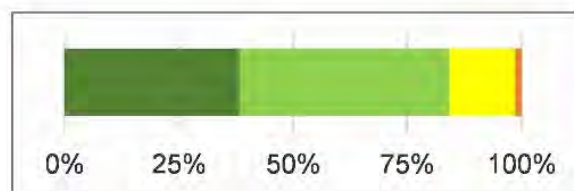

Beurteilung der zeitlichen Umsetzbarkeit

\begin{tabular}{|c|c|c|c|c|c|c|}
\hline & \multicolumn{8}{l|}{} \\
\cline { 2 - 7 } & Nennungen [Anzahl] nach Kategorie [Monate] & \\
\cline { 2 - 7 } & Alle & $\leq 3$ & $4-6$ & $7-12$ & $13-24$ & $>24$ \\
\hline Anzahl: & 79 & 43 & 20 & 14 & 1 & 1 \\
\hline Anteil: & & $54 \%$ & $25 \%$ & $18 \%$ & $1 \%$ & $1 \%$ \\
\hline
\end{tabular}

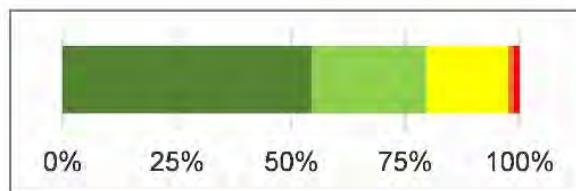

Beurteilung des verwaltungstechnischen Aufwands

\begin{tabular}{|c|c|c|c|c|c|}
\cline { 2 - 6 } & \multicolumn{4}{|c|}{ Nennungen [Anzahl] nach Kategorie [Aufwand] } \\
\cline { 2 - 6 } & Alle & gering & $\begin{array}{c}\text { eher } \\
\text { gering }\end{array}$ & eher hoch & hoch \\
\hline Anzahl: & 78 & 22 & 44 & 12 & 0 \\
\hline Anteil: & & $28 \%$ & $56 \%$ & $15 \%$ & $0 \%$ \\
\hline
\end{tabular}

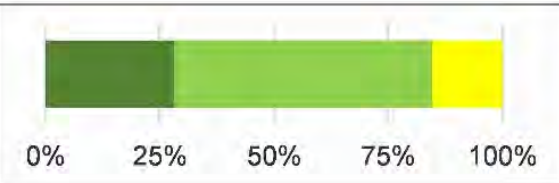

\section{Beurteilung der Wirksamkeit}

Wie wird durch die Maßnahme die Schwere der Unfälle reduziert?

\begin{tabular}{|c|c|c|c|c|c|}
\cline { 2 - 6 } & \multicolumn{4}{|c|}{ Nennungen [Anzahl] } & \\
\cline { 2 - 6 } & Alle & stark & mäßig & wenig & nicht \\
\hline Anzahl: & 75 & 4 & 17 & 31 & 23 \\
\hline Anteil: & & $5 \%$ & $23 \%$ & $41 \%$ & $31 \%$ \\
\hline
\end{tabular}

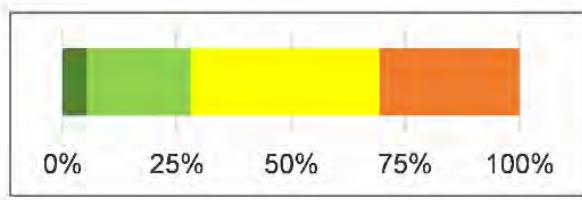

Wie wird durch die Maßnahme die Anzahl der Unfälle reduziert?

\begin{tabular}{|c|c|c|c|c|c|}
\cline { 2 - 6 } & \multicolumn{4}{c}{ Nennungen [Anzahl] } & \multicolumn{2}{c|}{} \\
\cline { 2 - 6 } & Alle & stark & mäßig & wenig & nicht \\
\hline Anzahl: & 73 & 1 & 5 & 41 & 26 \\
\hline Anteil: & & $1 \%$ & $7 \%$ & $56 \%$ & $36 \%$ \\
\hline
\end{tabular}

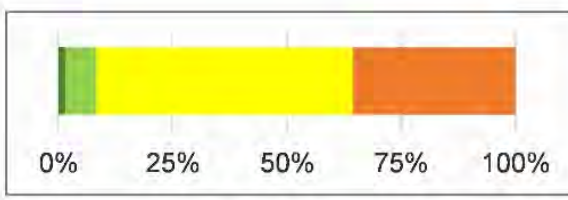

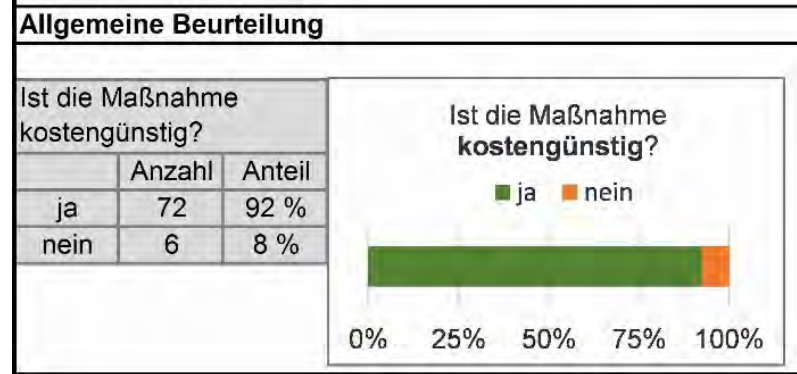

\begin{tabular}{|c|c|c|}
\hline \multicolumn{3}{|c|}{$\begin{array}{l}\text { Ist die Maßnahme } \\
\text { wirksam? }\end{array}$} \\
\hline & Anzahl & Anteil \\
\hline ja & 71 & $93 \%$ \\
\hline nein & 5 & $7 \%$ \\
\hline
\end{tabular}

Ist die Maßnahme wirksam?

aja nein 
Evaluation der Maßnahmenkategorie: [27], Markierung in Stand setzen

\begin{tabular}{|c|c|c|c|c|}
\hline Einsatzbedingu & Ingen und allgemeine Angaben & Antwortkompeten: & Izahl Nennungen) & \\
\hline Örtlichkeit: & IO, Knotenpunkt & Behörde & Mitglied in Uko sind & Zuständigkeit \\
\hline Regelungsart: & beliebig & \begin{tabular}{|l|l} 
Polizei: & 35
\end{tabular} & 55 von 80 & Stadt: 30 \\
\hline Unfalltyp(en): & beliebig & \begin{tabular}{l|l} 
Verkehr: $1 \varepsilon$ & 18
\end{tabular} & Befragten. & Land: 36 \\
\hline Befragte: & 80 & \begin{tabular}{l|l} 
Straßenbau: & 5
\end{tabular} & Mittlere UKO-Dienszeit & Andere: 12 \\
\hline & & Andere: 22 & in Jahren: 8 & \\
\hline
\end{tabular}

\section{Bekanntheitsgrad der Maßnahme}

Die Maßnahme ist

69 Befragten bekannt,

10 Befragten nicht bekannt. $\quad$ K. A.: 1 Befragte(r).

22 von 69 Befragten, denen die Maßnahme bekannt ist, haben diese Maßnahme ein- oder mehrmals in den letzten 5 Jahren in ihrem Zuständigkeitsbereich umgesetzt.

Der Bekannheitsgrad der Maßnahme liegt bei ca.: $86 \%$

\section{Beurteilung der Kosten}

\begin{tabular}{|c|c|c|c|c|c|c|}
\cline { 2 - 7 } & \multicolumn{7}{c|}{ Nennungen [Anzahl] nach Kategorie [€] } \\
\cline { 2 - 7 } & Alle & $<2.500$ & $<\mathbf{1 0 . 0 0 0}$ & $<\mathbf{5 0 . 0 0 0}$ & $<\mathbf{1 0 0 . 0 0 0}$ & $>100.000$ \\
\hline Anzahl: & 77 & 53 & 22 & 2 & 0 & 0 \\
\hline Anteil: & & $69 \%$ & $29 \%$ & $3 \%$ & $0 \%$ & $0 \%$ \\
\hline
\end{tabular}

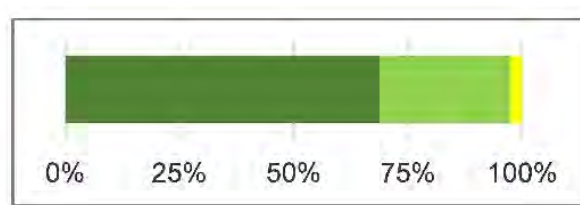

Beurteilung der zeitlichen Umsetzbarkeit

\begin{tabular}{|c|c|c|c|c|c|c|}
\hline & \multicolumn{8}{|c|}{ Nennungen [Anzahl] } & nach Kategorie [Monate] \\
\cline { 2 - 7 } & Alle & $\leq 3$ & $\mathbf{4 - 6}$ & $\mathbf{7 - 1 2}$ & $\mathbf{1 3 - 2 4}$ & $>24$ \\
\hline Anzahl: & 80 & 52 & 20 & 8 & 0 & 0 \\
\hline Anteil: & & $65 \%$ & $25 \%$ & $10 \%$ & $0 \%$ & $0 \%$ \\
\hline
\end{tabular}

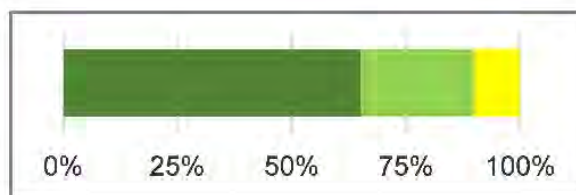

Beurteilung des verwaltungstechnischen Aufwands

\begin{tabular}{|c|c|c|c|c|c|}
\cline { 3 - 6 } & \multicolumn{6}{|c|}{ Nennungen [Anzahl] } & nach Kategorie [Aufwand] & \multicolumn{1}{c|}{ hoch } \\
\cline { 2 - 6 } & Alle & gering & $\begin{array}{c}\text { eher } \\
\text { gering }\end{array}$ & eher hoch & hech \\
\hline Anzahl: & 80 & 51 & 27 & 2 & 0 \\
\hline Anteil: & & $64 \%$ & $34 \%$ & $3 \%$ & $0 \%$ \\
\hline
\end{tabular}

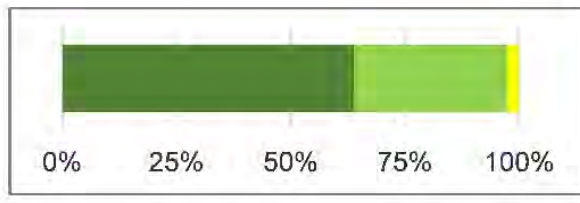

\section{Beurteilung der Wirksamkeit}

Wie wird durch die Maßnahme die Schwere der Unfälle reduziert?

\begin{tabular}{c|c|c|c|c|c|}
\cline { 2 - 6 } & \multicolumn{4}{|c|}{ Nennungen [Anzahl] } & \\
\cline { 2 - 6 } & Alle & stark & mäßig & wenig & nicht \\
\hline Anzahl: & 80 & 7 & 29 & 33 & 11 \\
\hline Anteil: & & $9 \%$ & $36 \%$ & $41 \%$ & $14 \%$ \\
\hline
\end{tabular}

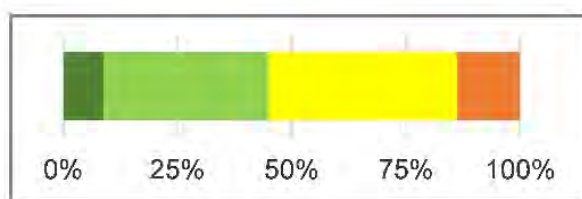

Wie wird durch die Maßnahme die Anzahl der Unfälle reduziert?

\begin{tabular}{|c|c|c|c|c|c|}
\cline { 2 - 6 } & \multicolumn{4}{|c|}{ Nennungen [Anzahl] } & \multicolumn{2}{c|}{} \\
\cline { 2 - 6 } & Alle & stark & mäßig & wenig & nicht \\
\hline Anzahl: & 80 & 1 & 25 & 42 & 12 \\
\hline Anteil: & & $1 \%$ & $31 \%$ & $53 \%$ & $15 \%$ \\
\hline
\end{tabular}

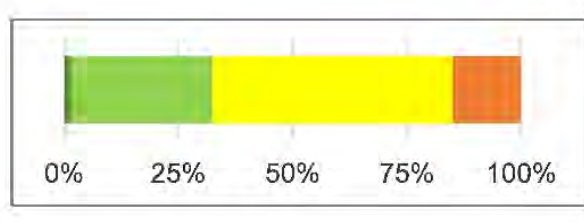

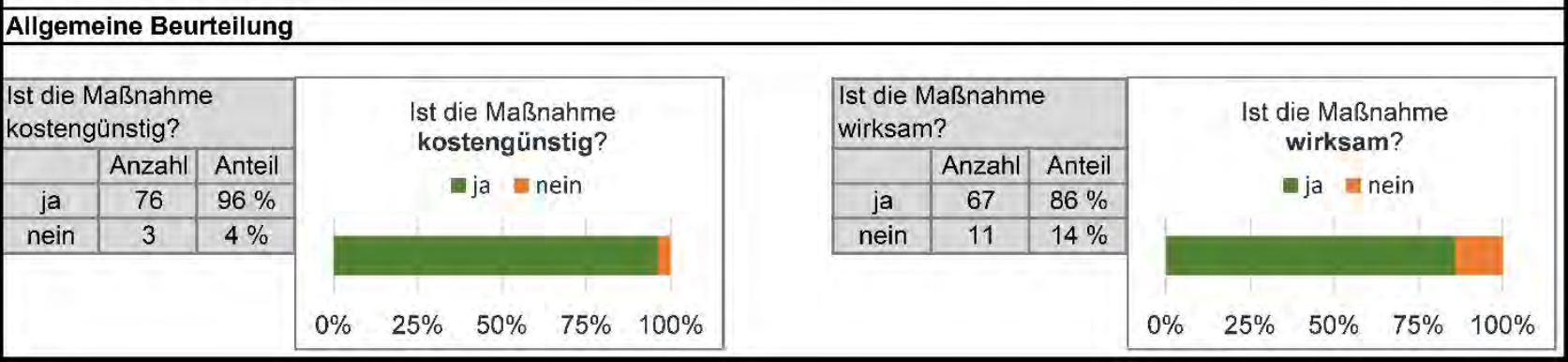


Evaluation der Maßnahmenkategorie: [29], Radverkehrsführung anpassen

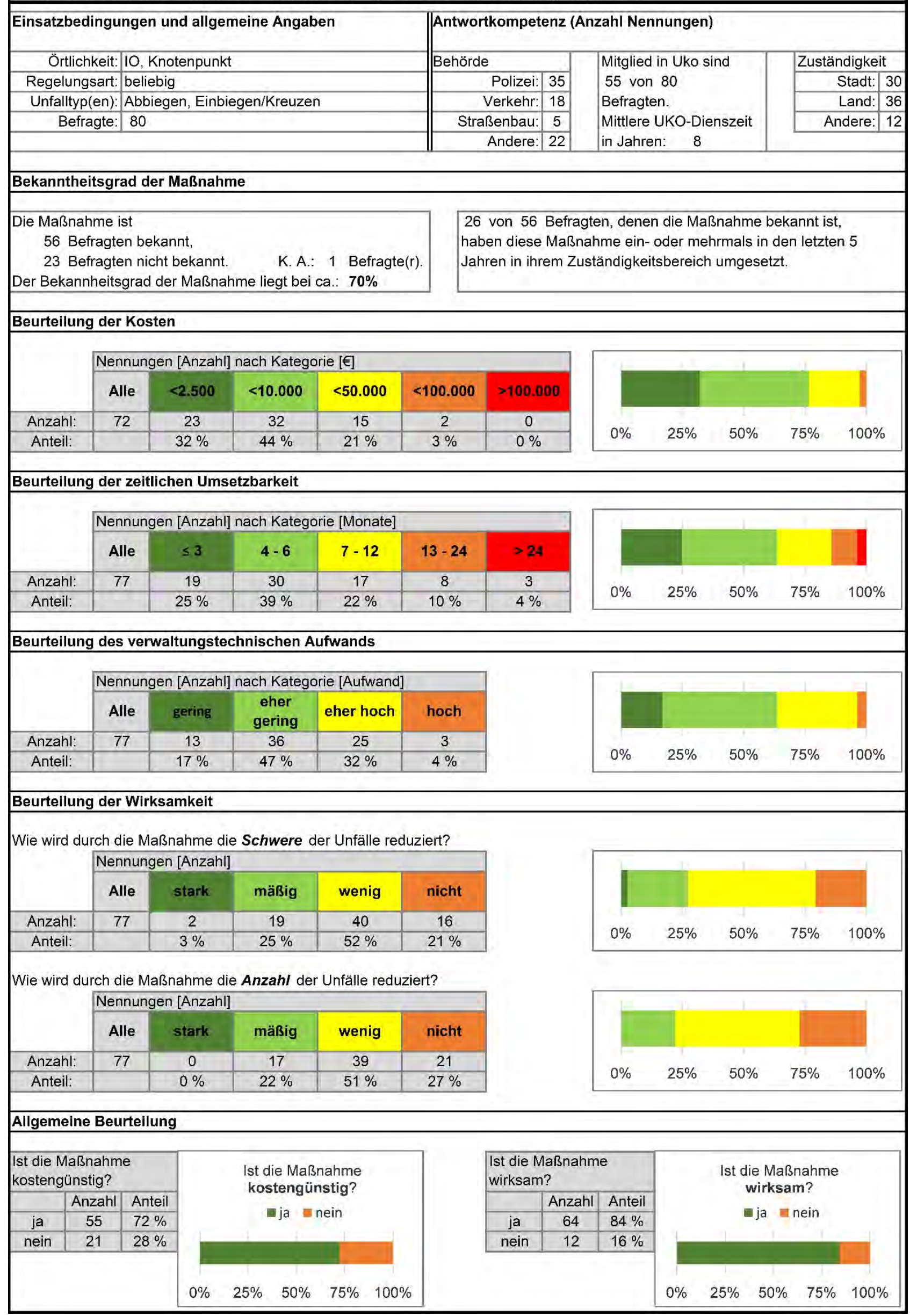


Evaluation der Maßnahmenkategorie: [30], Radfahrerfurt Rot einfärben

\begin{tabular}{|c|c|c|c|c|c|}
\hline Einsatzbedingungen und allgemeine Angaben & Antwortkompeter & enz ( & zahl Nennungen) & & \\
\hline Örtlichkeit: 10, Knotenpunkt & Behörde & & Mitglied in Uko sind & Zuständigkeit & \\
\hline Regelungsart: VZ & \begin{tabular}{|l|} 
Polizei: \\
\end{tabular} & 8 & 21 von 27 & Stadt: & 10 \\
\hline Unfalltyp(en): Einbiegen/Kreuzen & Verkehr: 1 & 10 & Befragten. & Land: & 9 \\
\hline \begin{tabular}{l|l} 
Befragte: 27 \\
\end{tabular} & Straßenbau: & 3 & Mittlere UKO-Dienszeit & Andere: & 8 \\
\hline & Andere: & 6 & in Jahren: 6 & & \\
\hline
\end{tabular}

\section{Bekanntheitsgrad der Maßnahme}

Die Maßnahme ist

25 Befragten bekannt,

1 Befragten nicht bekannt. $\quad$ K. A.: 1 Befragte(r).

11 von 25 Befragten, denen die Maßnahme bekannt ist, haben diese Maßnahme ein- oder mehrmals in den letzten 5 Jahren in ihrem Zuständigkeitsbereich umgesetzt.

Der Bekannheitsgrad der Maßnahme liegt bei ca.: $93 \%$

\section{Beurteilung der Kosten}

\begin{tabular}{|c|c|c|c|c|c|c|}
\cline { 2 - 7 } & \multicolumn{2}{c|}{ Nennungen [Anzahl] nach Kategorie [€] } \\
\cline { 2 - 8 } & Alle & $<2.500$ & $<\mathbf{1 0 . 0 0 0}$ & $<\mathbf{5 0 . 0 0 0}$ & $<\mathbf{1 0 0 . 0 0 0}$ & $>100.000$ \\
\hline Anzahl: & 23 & 15 & 6 & 1 & 0 & 0 \\
\hline Anteil: & & $65 \%$ & $26 \%$ & $4 \%$ & $0 \%$ & $0 \%$ \\
\hline
\end{tabular}

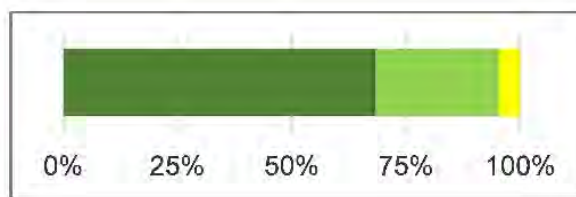

Beurteilung der zeitlichen Umsetzbarkeit

\begin{tabular}{|c|c|c|c|c|c|c|}
\hline & \multicolumn{8}{|c|}{ Nennungen [Anzahl] } & nach Kategorie [Monate] \\
\cline { 2 - 7 } & Alle & $\leq 3$ & $\mathbf{4 - 6}$ & $\mathbf{7 - 1 2}$ & $\mathbf{1 3 - 2 4}$ & $>24$ \\
\hline Anzahl: & 24 & 13 & 10 & 1 & 0 & 0 \\
\hline Anteil: & & $54 \%$ & $42 \%$ & $4 \%$ & $0 \%$ & $0 \%$ \\
\hline
\end{tabular}

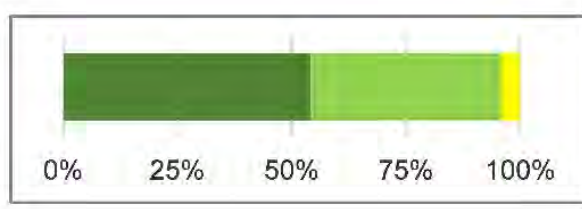

Beurteilung des verwaltungstechnischen Aufwands

\begin{tabular}{|c|c|c|c|c|c|}
\cline { 3 - 6 } & \multicolumn{6}{|c|}{ Nennungen [Anzahl] } & nach Kategorie [Aufwand] & \\
\cline { 2 - 6 } & Alle & gering & $\begin{array}{c}\text { eher } \\
\text { gering }\end{array}$ & eher hoch & hoch \\
\hline Anzahl: & 25 & 14 & 10 & 1 & 0 \\
\hline Anteil: & & $56 \%$ & $40 \%$ & $4 \%$ & $0 \%$ \\
\hline
\end{tabular}

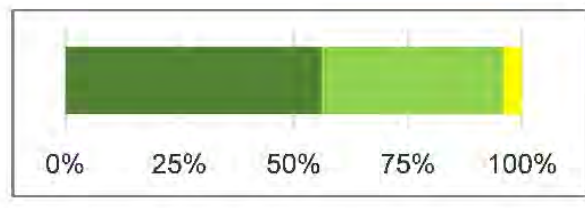

\section{Beurteilung der Wirksamkeit}

Wie wird durch die Maßnahme die Schwere der Unfälle reduziert?

\begin{tabular}{c|c|c|c|c|c|}
\cline { 2 - 6 } & \multicolumn{4}{|c|}{ Nennungen [Anzahl] } & \\
\cline { 2 - 6 } & Alle & stark & mäßig & wenig & nicht \\
\hline Anzahl: & 25 & 6 & 6 & 9 & 4 \\
\hline Anteil: & & $24 \%$ & $24 \%$ & $36 \%$ & $16 \%$ \\
\hline
\end{tabular}

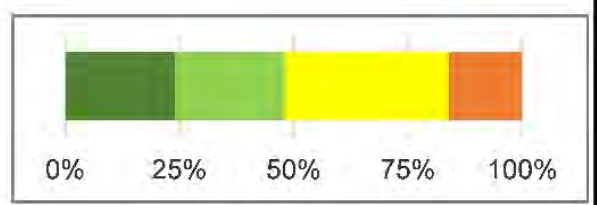

Wie wird durch die Maßnahme die Anzahl der Unfälle reduziert?

\begin{tabular}{|c|c|c|c|c|c|}
\cline { 2 - 6 } & \multicolumn{4}{|c|}{ Nennungen [Anzahl] } & \multicolumn{2}{c|}{} \\
\cline { 2 - 6 } & Alle & stark & mäßig & wenig & nicht \\
\hline Anzahl: & 25 & 0 & 5 & 13 & 7 \\
\hline Anteil: & & $0 \%$ & $20 \%$ & $52 \%$ & $28 \%$ \\
\hline
\end{tabular}

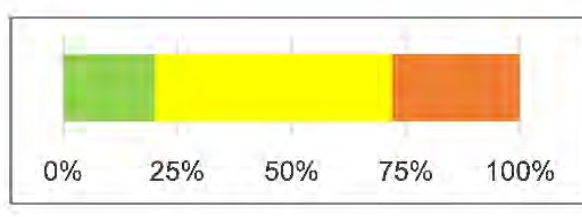

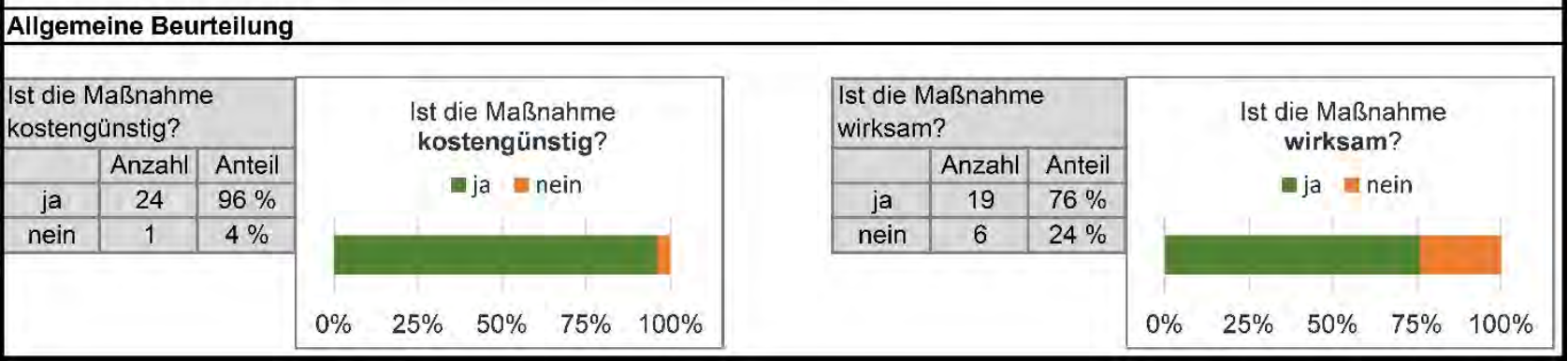


Evaluation der Maßnahmenkategorie: [31], Passive Schutzeinrichtung aufstellen

\begin{tabular}{|c|c|c|c|c|c|c|}
\hline \multicolumn{2}{|c|}{ Einsatzbedingungen und allgemeine Angaben } & \multicolumn{5}{|c|}{ |Antwortkompetenz (Anzahl Nennungen) } \\
\hline Örtlichkeit: & AO, Strecke $(300 \mathrm{~m})$ & \multicolumn{2}{|l|}{ Behörde } & \multirow{5}{*}{$\begin{array}{l}\text { Mitglied in Uko sind } \\
12 \text { von } 18 \\
\text { Befragten. } \\
\text { Mittlere UKO-Dienszeit } \\
\text { in Jahren: } 5\end{array}$} & \multicolumn{2}{|l|}{ Zuständigkeit } \\
\hline Regelungsart: & - & \begin{tabular}{|r|} 
Polizei: \\
\end{tabular} & 8 & & Stadt: & 10 \\
\hline Unfalltyp(en): & Fahrunfall & Verkehr: & 6 & & Land: & 6 \\
\hline Befragte: & 18 & Straßenbau: & 4 & & Andere: & 5 \\
\hline & & Andere: & 0 & & & \\
\hline
\end{tabular}

\section{Bekanntheitsgrad der Maßnahme}

Die Maßnahme ist

15 Befragten bekannt,

3 Befragten nicht bekannt. $\quad$ K. A.: 0 Befragte( $r)$

Der Bekannheitsgrad der Maßnahme liegt bei ca.: $83 \%$

11 von 15 Befragten, denen die Maßnahme bekannt ist, haben diese Maßnahme ein- oder mehrmals in den letzten 5 Jahren in ihrem Zuständigkeitsbereich umgesetzt.

\section{Beurteilung der Kosten}

\begin{tabular}{|c|c|c|c|c|c|c|}
\cline { 2 - 7 } & \multicolumn{7}{c|}{ Nennungen [Anzahl] nach Kategorie [€] } \\
\cline { 2 - 7 } & Alle & $<2.500$ & $<\mathbf{1 0 . 0 0 0}$ & $<\mathbf{5 0 . 0 0 0}$ & $<\mathbf{1 0 0 . 0 0 0}$ & $>100.000$ \\
\hline Anzahl: & 18 & 0 & 8 & 8 & 2 & 0 \\
\hline Anteil: & & $0 \%$ & $44 \%$ & $44 \%$ & $11 \%$ & $0 \%$ \\
\hline
\end{tabular}

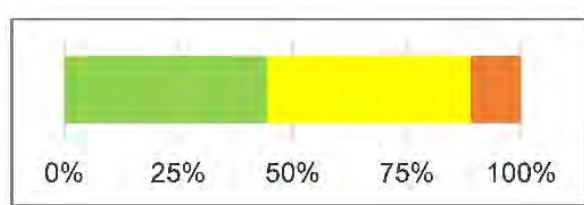

Beurteilung der zeitlichen Umsetzbarkeit

\begin{tabular}{|c|c|c|c|c|c|c|}
\hline & \multicolumn{8}{|c|}{ Nennungen [Anzahl] } & nach Kategorie [Monate] \\
\cline { 2 - 7 } & Alle & $\leq 3$ & $\mathbf{4 - 6}$ & $\mathbf{7 - 1 2}$ & $\mathbf{1 3 - 2 4}$ & $>24$ \\
\hline Anzahl: & 18 & 0 & 11 & 7 & 0 & 0 \\
\hline Anteil: & & $0 \%$ & $61 \%$ & $39 \%$ & $0 \%$ & $0 \%$ \\
\hline
\end{tabular}

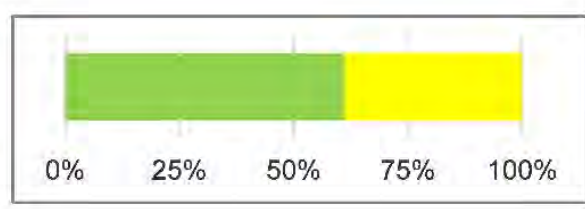

Beurteilung des verwaltungstechnischen Aufwands

\begin{tabular}{|c|c|c|c|c|c|}
\cline { 2 - 6 } & \multicolumn{6}{|c|}{ Nennungen [Anzahl] } & nach Kategorie [Aufwand] & \\
\cline { 2 - 6 } & Alle & gering & $\begin{array}{c}\text { eher } \\
\text { gering }\end{array}$ & eher hoch & hoch \\
\hline Anzahl: & 18 & 0 & 10 & 7 & 1 \\
\hline Anteil: & & $0 \%$ & $56 \%$ & $39 \%$ & $6 \%$ \\
\hline
\end{tabular}

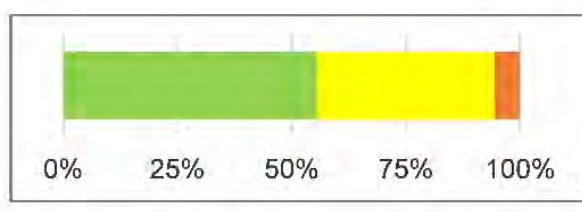

\section{Beurteilung der Wirksamkeit}

Wie wird durch die Maßnahme die Schwere der Unfälle reduziert?

\begin{tabular}{c|c|c|c|c|c|}
\cline { 2 - 6 } & \multicolumn{4}{|c|}{ Nennungen [Anzahl] } & \\
\cline { 2 - 6 } & Alle & stark & mäßig & wenig & nicht \\
\hline Anzahl: & 18 & 0 & 0 & 2 & 16 \\
\hline Anteil: & & $0 \%$ & $0 \%$ & $11 \%$ & $89 \%$ \\
\hline
\end{tabular}

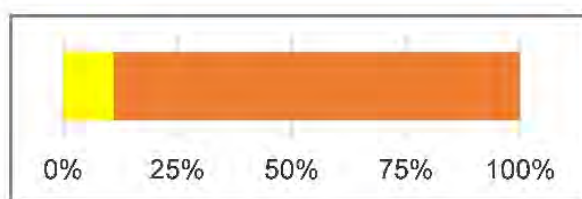

Wie wird durch die Maßnahme die Anzahl der Unfälle reduziert?

\begin{tabular}{|c|c|c|c|c|c|}
\cline { 3 - 6 } & \multicolumn{4}{|c|}{ Nennungen [Anzahl] } & \multicolumn{3}{c|}{} \\
\cline { 2 - 6 } & Alle & stark & mäßig & wenig & nicht \\
\hline Anzahl: & 18 & 0 & 9 & 6 & 3 \\
\hline Anteil: & & $0 \%$ & $50 \%$ & $33 \%$ & $17 \%$ \\
\hline
\end{tabular}

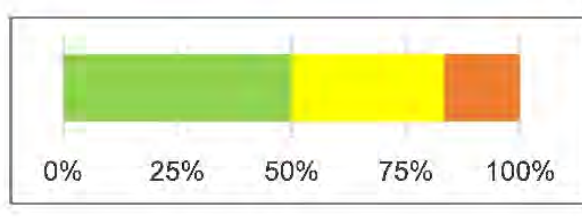

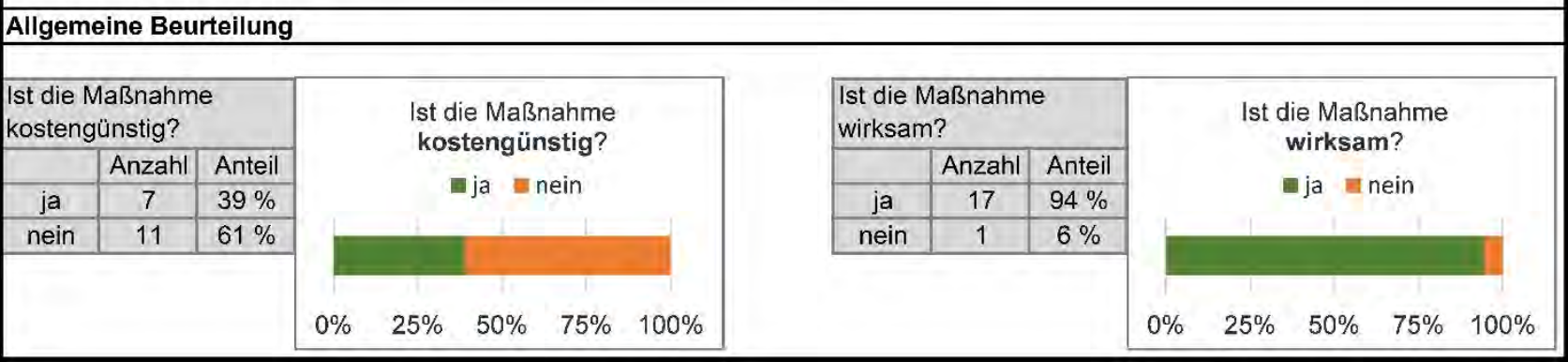


Evaluation der Maßnahmenkategorie: [32], Unterfahrschutz an passiver Schutzeinrichtung anbringen Einsatzbedingungen und allgemeine Angaben

\begin{tabular}{|c|c|c|c|c|}
\hline \multicolumn{5}{|c|}{ Antwortkompetenz (Anzahl Nennungen) } \\
\hline \multicolumn{2}{|c|}{ Behörde } & \multirow{5}{*}{$\begin{array}{l}\text { Mitglied in Uko sind } \\
27 \text { von } 31 \\
\text { Befragten. } \\
\text { Mittlere UKO-Dienszeit } \\
\text { in Jahren: } 11\end{array}$} & \multicolumn{2}{|c|}{ Zuständigkeit } \\
\hline Polizei: & 6 & & Stadt: & 12 \\
\hline Verkehr: & 18 & & Land: & 11 \\
\hline Straßenbau: & 3 & & Andere: & 7 \\
\hline Andere: & 4 & & & \\
\hline
\end{tabular}

\section{Bekanntheitsgrad der Maßnahme}

Die Maßnahme ist

21 Befragten bekannt,

7 Befragten nicht bekannt. $\quad$ K. A.: 3 Befragte( $r$.

Der Bekannheitsgrad der Maßnahme liegt bei ca.: $\mathbf{6 8 \%}$

21 von 21 Befragten, denen die Maßnahme bekannt ist, haben diese Maßnahme ein- oder mehrmals in den letzten 5 Jahren in ihrem Zuständigkeitsbereich umgesetzt.

\section{Beurteilung der Kosten}

\begin{tabular}{|c|c|c|c|c|c|c|}
\cline { 2 - 7 } & \multicolumn{7}{c|}{ Nennungen [Anzahl] nach Kategorie [€] } \\
\cline { 2 - 7 } & Alle & $<2.500$ & $<\mathbf{1 0 . 0 0 0}$ & $<\mathbf{5 0 . 0 0 0}$ & $<\mathbf{1 0 0 . 0 0 0}$ & $>100.000$ \\
\hline Anzahl: & 22 & 2 & 10 & 7 & 2 & 1 \\
\hline Anteil: & & $9 \%$ & $\mathbf{4 5} \%$ & $32 \%$ & $9 \%$ & $5 \%$ \\
\hline
\end{tabular}

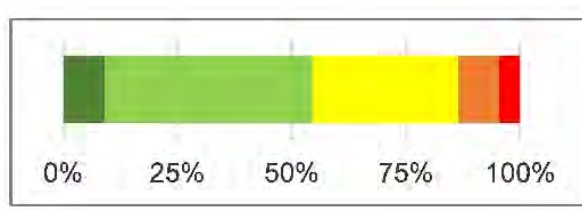

Beurteilung der zeitlichen Umsetzbarkeit

\begin{tabular}{|c|c|c|c|c|c|c|}
\hline \multicolumn{1}{c|}{} & \multicolumn{2}{l|}{ Nennungen [Anzahl] } & nach Kategorie [Monate] \\
\cline { 2 - 7 } & Alle & $\leq 3$ & $\mathbf{4 - 6}$ & $\mathbf{7 - 1 2}$ & $\mathbf{1 3 - 2 4}$ & $>24$ \\
\hline Anzahl: & 23 & 5 & 9 & 7 & 2 & 0 \\
\hline Anteil: & & $22 \%$ & $39 \%$ & $30 \%$ & $9 \%$ & $0 \%$ \\
\hline
\end{tabular}

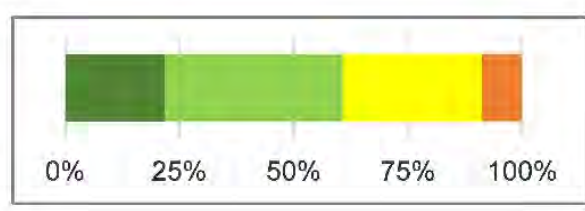

Beurteilung des verwaltungstechnischen Aufwands

\begin{tabular}{|c|c|c|c|c|c|}
\cline { 2 - 6 } & \multicolumn{6}{|c|}{ Nennungen [Anzahl] } & nach Kategorie [Aufwand] & \\
\cline { 2 - 6 } & Alle & gering & $\begin{array}{c}\text { eher } \\
\text { gering }\end{array}$ & eher hoch & hoch \\
\hline Anzahl: & 23 & 6 & 11 & 5 & 1 \\
\hline Anteil: & & $26 \%$ & $48 \%$ & $22 \%$ & $4 \%$ \\
\hline
\end{tabular}

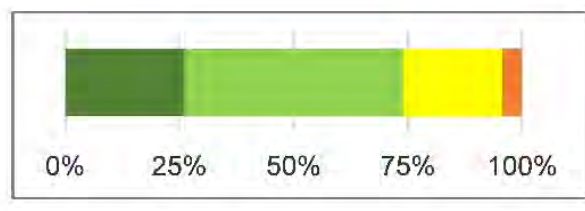

\section{Beurteilung der Wirksamkeit}

Wie wird durch die Maßnahme die Schwere der Unfälle reduziert?

\begin{tabular}{c|c|c|c|c|c|}
\cline { 2 - 6 } & \multicolumn{4}{c|}{ Nennungen [Anzahl] } & \\
\cline { 2 - 6 } & Alle & stark & mäßig & wenig & nicht \\
\hline Anzahl: & 24 & 0 & 1 & 8 & 15 \\
\hline Anteil: & & $0 \%$ & $4 \%$ & $33 \%$ & $63 \%$ \\
\hline
\end{tabular}

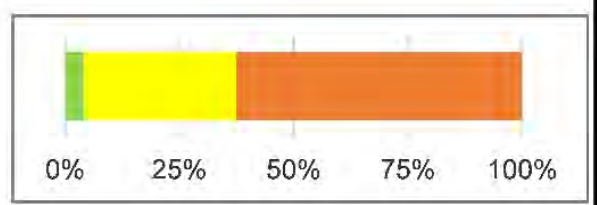

Wie wird durch die Maßnahme die Anzahl der Unfälle reduziert?

\begin{tabular}{|c|c|c|c|c|c|}
\cline { 2 - 6 } & \multicolumn{4}{|c|}{ Nennungen [Anzahl] } & \multicolumn{3}{c|}{} \\
\cline { 2 - 6 } & Alle & stark & mäßig & wenig & nicht \\
\hline Anzahl: & 23 & 12 & 7 & 3 & 1 \\
\hline Anteil: & & $52 \%$ & $30 \%$ & $13 \%$ & $4 \%$ \\
\hline
\end{tabular}

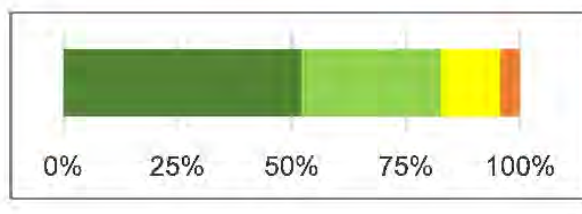

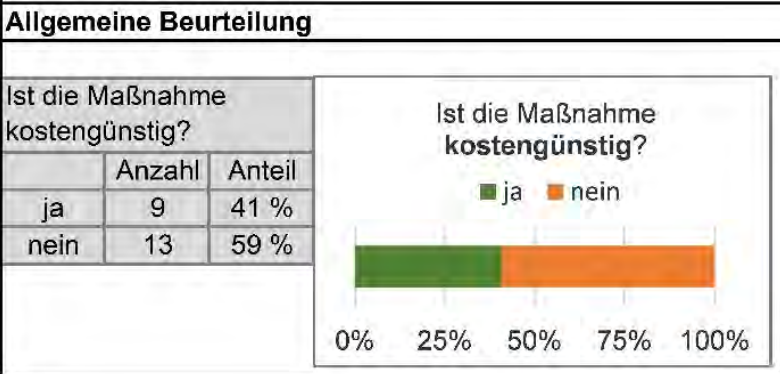

\begin{tabular}{|c|c|c|}
\hline \multicolumn{3}{|c|}{$\begin{array}{l}\text { Ist die Maßnahme } \\
\text { wirksam? }\end{array}$} \\
\hline & Anzahl & Antei \\
\hline ja & 20 & $91 \%$ \\
\hline nein & 2 & $9 \%$ \\
\hline
\end{tabular}

Ist die Maßnahme wirksam?

- ja enein 
Evaluation der Maßnahmenkategorie: [33], Hindernisse im Seitenraum entfernen

\begin{tabular}{|c|c|c|c|c|c|c|}
\hline \multicolumn{2}{|c|}{ Einsatzbedingungen und allgemeine Angaben } & \multicolumn{5}{|c|}{ |Antwortkompetenz (Anzahl Nennungen) } \\
\hline Örtlichkeit: & $\mathrm{AO}$, Strecke $(300 \mathrm{~m})$ & \multicolumn{2}{|l|}{ Behörde } & \multirow{5}{*}{$\begin{array}{l}\text { Mitglied in Uko sind } \\
49 \text { von } 60 \\
\text { Befragten. } \\
\text { Mittlere UKO-Dienszeit } \\
\text { in Jahren: } 6\end{array}$} & \multicolumn{2}{|l|}{ Zuständigkeit } \\
\hline Regelungsart: & - & \begin{tabular}{|r|r|} 
Polizei: 3 \\
\end{tabular} & 39 & & Stadt: & 34 \\
\hline Unfalltyp(en): & Fahrunfall & Verkehr: 1 & 10 & & Land: & 33 \\
\hline Befragte: & 60 & Straßenbau: & 3 & & Andere: & 13 \\
\hline & & Andere: & 8 & & & \\
\hline
\end{tabular}

\section{Bekanntheitsgrad der Maßnahme}

Die Maßnahme ist

55 Befragten bekannt,

5 Befragten nicht bekannt. $\quad$ K. A.: 0 Befragte(r).

33 von 55 Befragten, denen die Maßnahme bekannt ist, haben diese Maßnahme ein- oder mehrmals in den letzten 5 Jahren in ihrem Zuständigkeitsbereich umgesetzt.

Der Bekannheitsgrad der Maßnahme liegt bei ca.: $\mathbf{9 2} \%$

\section{Beurteilung der Kosten}

\begin{tabular}{|c|c|c|c|c|c|c|}
\cline { 2 - 7 } & \multicolumn{2}{c|}{ Nennungen [Anzahl] nach Kategorie [€] } \\
\cline { 2 - 8 } & Alle & $<2.500$ & $<\mathbf{1 0 . 0 0 0}$ & $<\mathbf{5 0 . 0 0 0}$ & $<\mathbf{1 0 0 . 0 0 0}$ & $>100.000$ \\
\hline Anzahl: & 58 & 31 & 26 & 1 & 0 & 0 \\
\hline Anteil: & & $53 \%$ & $\mathbf{4 5 \%}$ & $\mathbf{2} \%$ & $0 \%$ & $0 \%$ \\
\hline
\end{tabular}

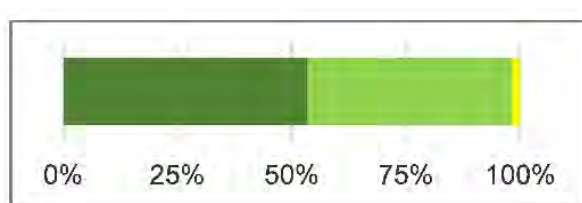

Beurteilung der zeitlichen Umsetzbarkeit

\begin{tabular}{|c|c|c|c|c|c|c|}
\hline & \multicolumn{8}{l|}{ Nennungen [Anzahl] } & nach Kategorie [Monate] \\
\cline { 2 - 7 } & Alle & $\leq 3$ & $\mathbf{4 - 6}$ & $\mathbf{7 - 1 2}$ & $13-24$ & $>24$ \\
\hline Anzahl: & 57 & 28 & 23 & 5 & 1 & 0 \\
\hline Anteil: & & $49 \%$ & $40 \%$ & $9 \%$ & $2 \%$ & $0 \%$ \\
\hline
\end{tabular}

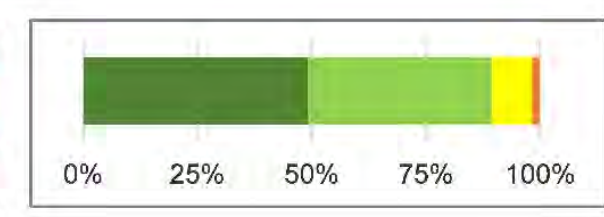

Beurteilung des verwaltungstechnischen Aufwands

\begin{tabular}{|c|c|c|c|c|c|}
\hline & \multicolumn{4}{|c|}{ Nennungen [Anzahl] nach Kategorie [Aufwand] } & \multirow[b]{2}{*}{ hoch } \\
\hline & Alle & gering & $\begin{array}{l}\text { eher } \\
\text { gering }\end{array}$ & eher hoch & \\
\hline Anzahl: & 58 & 23 & 20 & 12 & 3 \\
\hline Anteil: & & $40 \%$ & $34 \%$ & $21 \%$ & $5 \%$ \\
\hline
\end{tabular}

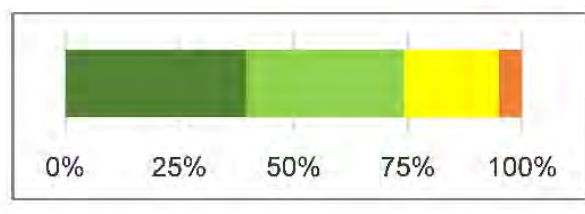

\section{Beurteilung der Wirksamkeit}

Wie wird durch die Maßnahme die Schwere der Unfälle reduziert?

\begin{tabular}{c|c|c|c|c|c|}
\cline { 2 - 6 } & \multicolumn{4}{c|}{ Nennungen [Anzahl] } & \multicolumn{3}{c|}{} \\
\cline { 2 - 6 } & Alle & stark & mäßig & wenig & nicht \\
\hline Anzahl: & 60 & 1 & 3 & 15 & 41 \\
\hline Anteil: & & $2 \%$ & $5 \%$ & $25 \%$ & $68 \%$ \\
\hline
\end{tabular}

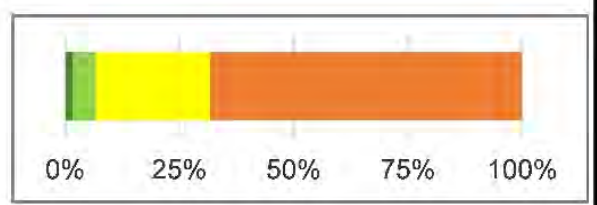

Wie wird durch die Maßnahme die Anzahl der Unfälle reduziert?

\begin{tabular}{|c|c|c|c|c|c|}
\cline { 2 - 6 } & \multicolumn{4}{|c|}{ Nennungen [Anzahl] } & \multicolumn{3}{c|}{} \\
\cline { 2 - 6 } & Alle & stark & mäßig & wenig & nicht \\
\hline Anzahl: & 59 & 20 & 18 & 10 & 11 \\
\hline Anteil: & & $34 \%$ & $31 \%$ & $17 \%$ & $19 \%$ \\
\hline
\end{tabular}

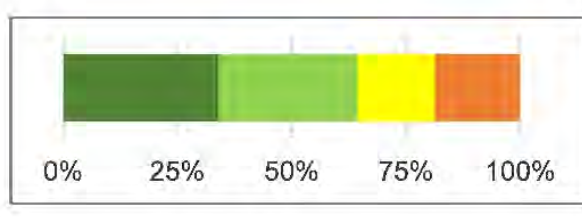

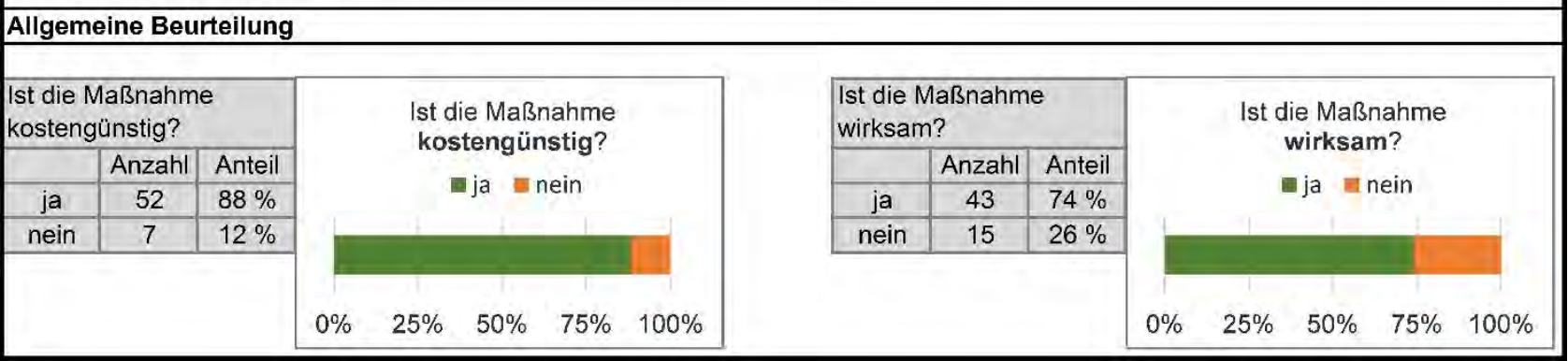


Evaluation der Maßnahmenkategorie: [34], Rüttelstreifen aufbringen

\begin{tabular}{|c|c|c|c|c|c|c|}
\hline \multicolumn{2}{|c|}{ Einsatzbedingungen und allgemeine Angaben } & \multicolumn{5}{|c|}{ |Antwortkompetenz (Anzahl Nennungen) } \\
\hline Örtlichkeit: & AO, Strecke $(300 \mathrm{~m})$ & \multicolumn{2}{|l|}{ Behörde } & \multirow{5}{*}{$\begin{array}{l}\text { Mitglied in Uko sind } \\
55 \text { von } 80 \\
\text { Befragten. } \\
\text { Mittlere UKO-Dienszeit } \\
\text { in Jahren: } 8\end{array}$} & \multicolumn{2}{|l|}{ Zuständigkeit } \\
\hline Regelungsart: & - & |rolizei: & 35 & & Stadt: & 30 \\
\hline Unfalltyp(en): & Fahrunfall & Verkehr: & 18 & & Land: & 36 \\
\hline Befragte: & 80 & Straßenbau: & 5 & & Andere: & 12 \\
\hline & & Andere: & 22 & & & \\
\hline
\end{tabular}

\section{Bekanntheitsgrad der Maßnahme}

Die Maßnahme ist

45 Befragten bekannt,

33 Befragten nicht bekannt. $\quad$ K. A.: 2 Befragte(r).

34 von 45 Befragten, denen die Maßnahme bekannt ist, haben diese Maßnahme ein- oder mehrmals in den letzten 5 Jahren in ihrem Zuständigkeitsbereich umgesetzt.

Der Bekannheitsgrad der Maßnahme liegt bei ca.: $56 \%$

\section{Beurteilung der Kosten}

\begin{tabular}{|c|c|c|c|c|c|c|}
\cline { 2 - 7 } & \multicolumn{2}{c|}{ Nennungen [Anzahl] nach Kategorie [€] } \\
\cline { 2 - 8 } & Alle & $<2.500$ & $<\mathbf{1 0 . 0 0 0}$ & $<\mathbf{5 0 . 0 0 0}$ & $<\mathbf{1 0 0 . 0 0 0}$ & $>100.000$ \\
\hline Anzahl: & 73 & 19 & 31 & 18 & 3 & 1 \\
\hline Anteil: & & $26 \%$ & $\mathbf{4 2} \%$ & $\mathbf{2 5} \%$ & $4 \%$ & $1 \%$ \\
\hline
\end{tabular}

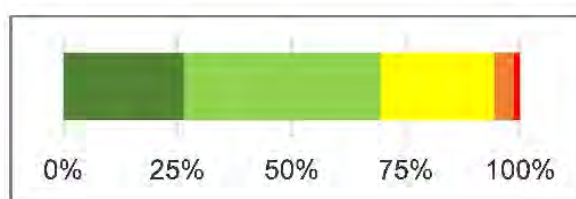

Beurteilung der zeitlichen Umsetzbarkeit

\begin{tabular}{|c|c|c|c|c|c|c|}
\hline & \multicolumn{8}{|c|}{ Nennungen [Anzahl] } & nach Kategorie [Monate] \\
\cline { 2 - 7 } & Alle & $\leq 3$ & $\mathbf{4 - 6}$ & $\mathbf{7 - 1 2}$ & $\mathbf{1 3 - 2 4}$ & $>24$ \\
\hline Anzahl: & 77 & 24 & 21 & 19 & 8 & 5 \\
\hline Anteil: & & $31 \%$ & $27 \%$ & $25 \%$ & $10 \%$ & $6 \%$ \\
\hline
\end{tabular}

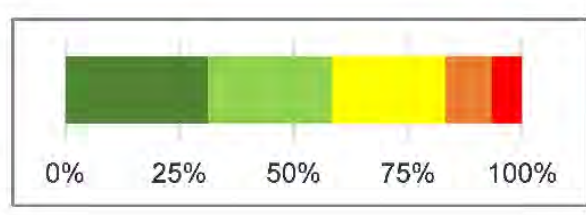

Beurteilung des verwaltungstechnischen Aufwands

\begin{tabular}{|c|c|c|c|c|c|}
\hline \multirow{2}{*}{} & \multicolumn{6}{|c|}{ Nennungen [Anzahl] } & nach Kategorie [Aufwand] & \\
\cline { 2 - 6 } & Alle & gering & $\begin{array}{c}\text { eher } \\
\text { gering }\end{array}$ & eher hoch & hoch \\
\hline Anzahl: & 77 & 16 & 29 & 26 & 6 \\
\hline Anteil: & & $21 \%$ & $38 \%$ & $34 \%$ & $8 \%$ \\
\hline
\end{tabular}

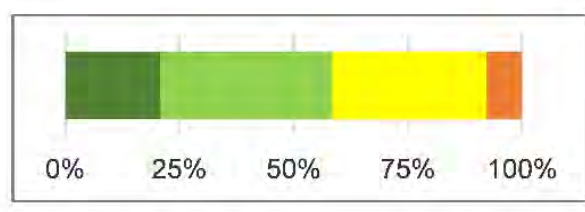

\section{Beurteilung der Wirksamkeit}

Wie wird durch die Maßnahme die Schwere der Unfälle reduziert?

\begin{tabular}{|c|c|c|c|c|c|}
\cline { 3 - 6 } & \multicolumn{4}{c|}{ Nennungen [Anzahl] } & \multicolumn{3}{c|}{} \\
\cline { 2 - 6 } & Alle & stark & mäßig & wenig & nicht \\
\hline Anzahl: & 76 & 4 & 21 & 26 & 25 \\
\hline Anteil: & & $5 \%$ & $28 \%$ & $34 \%$ & $33 \%$ \\
\hline
\end{tabular}

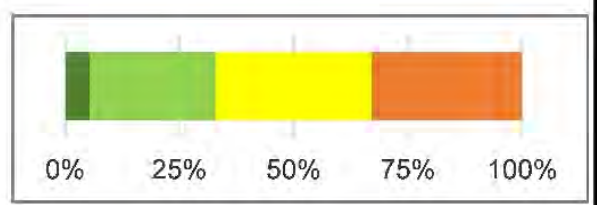

Wie wird durch die Maßnahme die Anzahl der Unfälle reduziert?

\begin{tabular}{|c|c|c|c|c|c|}
\cline { 2 - 6 } & \multicolumn{4}{|c|}{ Nennungen [Anzahl] } & \multicolumn{3}{c|}{} \\
\cline { 2 - 6 } & Alle & stark & mäßig & wenig & nicht \\
\hline Anzahl: & 76 & 3 & 18 & 30 & 25 \\
\hline Anteil: & & $4 \%$ & $24 \%$ & $39 \%$ & $33 \%$ \\
\hline
\end{tabular}

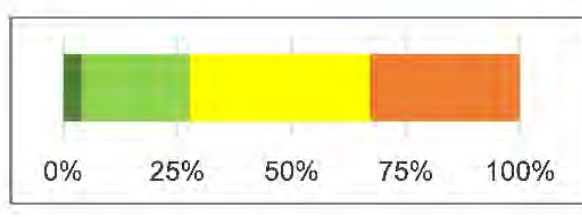

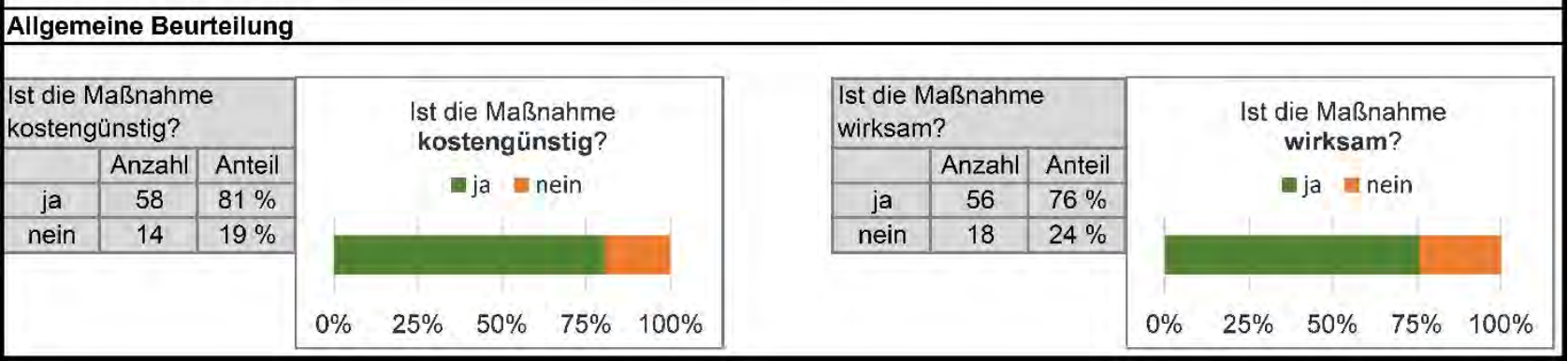


Evaluation der Maßnahmenkategorie: [35], Gummischwellen aufbringen

\begin{tabular}{|c|c|c|c|c|c|c|}
\hline \multicolumn{2}{|c|}{ Einsatzbedingungen und allgemeine Angaben } & \multicolumn{5}{|c|}{ |Antwortkompetenz (Anzahl Nennungen) } \\
\hline Örtlichkeit: & AO, Knotenpunkt & \multicolumn{2}{|c|}{ Behörde } & \multirow{5}{*}{$\begin{array}{l}\text { Mitglied in Uko sind } \\
67 \text { von } 94 \\
\text { Befragten. } \\
\text { Mittlere UKO-Dienszeit } \\
\text { in Jahren: } 8\end{array}$} & \multicolumn{2}{|l|}{ Zuständigkeit } \\
\hline Regelungsart: & beliebig & 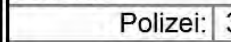 & 37 & & Stadt: & 36 \\
\hline Unfalltyp(en): & Abbiegen, Einbiegen/Kreuzen & Verkehr: & 24 & & Land: & 41 \\
\hline Befragte: & 94 & Straßenbau: & 7 & & Andere: & 16 \\
\hline & & Andere: & 26 & & & \\
\hline
\end{tabular}

\section{Bekanntheitsgrad der Maßnahme}

Die Maßnahme ist

67 Befragten bekannt,

25 Befragten nicht bekannt. $\quad$ K. A.: 2 Befragte(r).

Der Bekannheitsgrad der Maßnahme liegt bei ca.: $\quad 71 \%$

29 von 67 Befragten, denen die Maßnahme bekannt ist haben diese Maßnahme ein- oder mehrmals in den letzten 5 Jahren in ihrem Zuständigkeitsbereich umgesetzt.

\section{Beurteilung der Kosten}

\begin{tabular}{|c|c|c|c|c|c|c|}
\cline { 2 - 7 } & \multicolumn{2}{c|}{ Nennungen [Anzahl] nach Kategorie [€] } \\
\cline { 2 - 7 } & Alle & $<2.500$ & $<10.000$ & $<50.000$ & $<100.000$ & $>100.000$ \\
\hline Anzahl: & 85 & 16 & 48 & 18 & 3 & 0 \\
\hline Anteil: & & $19 \%$ & $56 \%$ & $21 \%$ & $4 \%$ & $0 \%$ \\
\hline
\end{tabular}

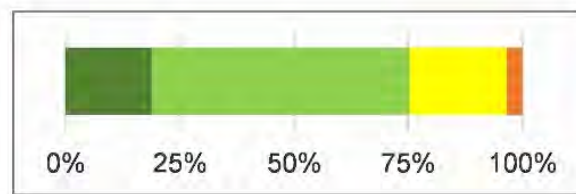

Beurteilung der zeitlichen Umsetzbarkeit

\begin{tabular}{|c|c|c|c|c|c|c|}
\hline & \multicolumn{8}{l|}{} \\
\cline { 2 - 7 } & Nennungen [Anzahl] nach Kategorie [Monate] & \\
\cline { 2 - 7 } & Alle & $\leq 3$ & $4-6$ & $7-12$ & $13-24$ & $>24$ \\
\hline Anzahl: & 86 & 28 & 37 & 12 & 7 & 2 \\
\hline Anteil: & & $33 \%$ & $43 \%$ & $14 \%$ & $8 \%$ & $2 \%$ \\
\hline
\end{tabular}

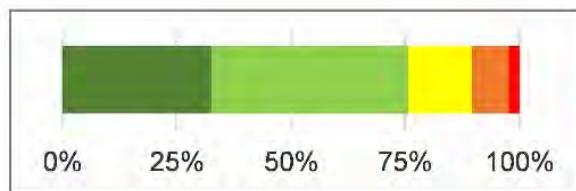

Beurteilung des verwaltungstechnischen Aufwands

\begin{tabular}{|c|c|c|c|c|c|}
\cline { 2 - 6 } & \multicolumn{4}{|c|}{ Nennungen [Anzahl] } & nach Kategorie [Aufwand] \\
\cline { 2 - 6 } & Alle & gering & $\begin{array}{c}\text { eher } \\
\text { gering }\end{array}$ & eher hoch & hoch \\
\hline Anzahl: & 86 & 21 & 44 & 20 & 1 \\
\hline Anteil: & & $24 \%$ & $51 \%$ & $23 \%$ & $1 \%$ \\
\hline
\end{tabular}

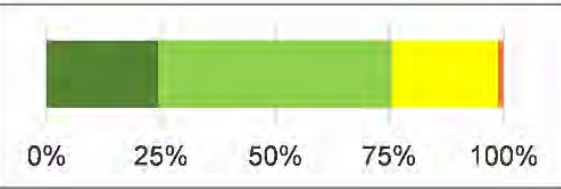

\section{Beurteilung der Wirksamkeit}

Wie wird durch die Maßnahme die Schwere der Unfälle reduziert?

\begin{tabular}{|c|c|c|c|c|c|}
\cline { 3 - 6 } & \multicolumn{4}{|c|}{ Nennungen [Anzahl] } & \multicolumn{2}{c|}{ nicht } \\
\cline { 2 - 6 } & Alle & stark & mäßig & wenig & nich \\
\hline Anzahl: & 84 & 4 & 16 & 49 & 15 \\
\hline Anteil: & & $5 \%$ & $19 \%$ & $58 \%$ & $18 \%$ \\
\hline
\end{tabular}

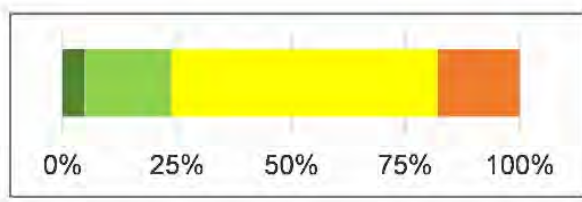

Wie wird durch die Maßnahme die Anzahl der Unfälle reduziert?

\begin{tabular}{|c|c|c|c|c|c|}
\hline & \multicolumn{5}{|c|}{ Nennungen [Anzahl] } \\
\hline & Alle & stark & mäßig & wenig & nicht \\
\hline Anzahl: & 82 & 1 & 17 & 45 & 19 \\
\hline Anteil: & & $1 \%$ & $21 \%$ & $55 \%$ & $23 \%$ \\
\hline
\end{tabular}

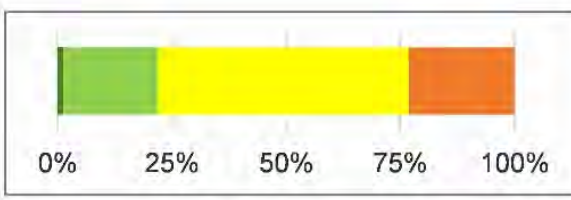

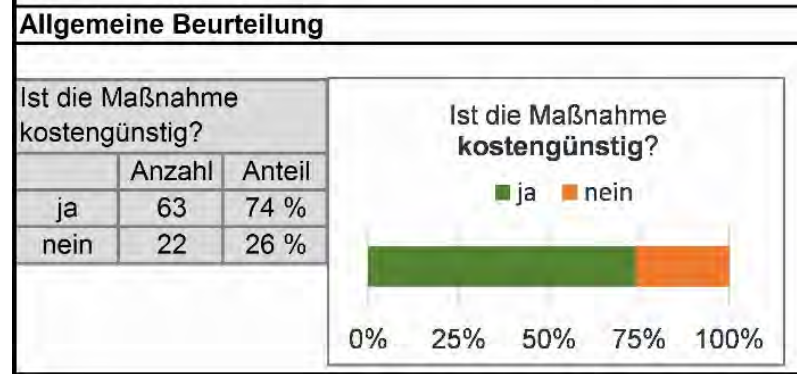

\begin{tabular}{|c|c|c|}
\hline \multicolumn{3}{|c|}{$\begin{array}{l}\text { Ist die Maßnahme } \\
\text { wirksam? }\end{array}$} \\
\hline & Anzahl & Anteil \\
\hline ja & 71 & $84 \%$ \\
\hline nein & 14 & $16 \%$ \\
\hline
\end{tabular}

Ist die Maßnahme wirksam?

aja nein 
Evaluation der Maßnahmenkategorie: [36], Sperrfläche markieren

\begin{tabular}{|c|c|c|c|c|c|}
\hline \multirow{2}{*}{$\begin{array}{l}\text { Einsatzbedingungen und allgemeine Angaben } \\
\text { Örtlichkeit: } \mid \mathrm{O}, \text { Knotenpunkt }\end{array}$} & \multicolumn{5}{|c|}{ ||Antwortkompetenz (Anzahl Nennungen) } \\
\hline & \multicolumn{2}{|c|}{ Behörde } & \multirow{5}{*}{$\begin{array}{l}\text { Mitglied in Uko sind } \\
21 \text { von } 27 \\
\text { Befragten. } \\
\text { Mittlere UKO-Dienszeit } \\
\text { in Jahren: } 6\end{array}$} & \multicolumn{2}{|c|}{ Zuständigkeit } \\
\hline Regelungsart: VZ & || Polizei: & 8 & & Stadt: & 10 \\
\hline Unfalltyp(en): Einbiegen/Kreuzen, Längsverkehr & Verkehr: & 10 & & Land: & 9 \\
\hline Befragte: 27 & Straßenbau: & 3 & & Andere: & 8 \\
\hline & Andere: & 6 & & & \\
\hline
\end{tabular}

\section{Bekanntheitsgrad der Maßnahme}

Die Maßnahme ist

22 Befragten bekannt,

4 Befragten nicht bekannt. $\quad$ K. A.: 1 Befragte(r).

Der Bekannheitsgrad der Maßnahme liegt bei ca.: $\mathbf{8 1 \%}$

12 von 22 Befragten, denen die Maßnahme bekannt ist, haben diese Maßnahme ein- oder mehrmals in den letzten 5 Jahren in ihrem Zuständigkeitsbereich umgesetzt.

\section{Beurteilung der Kosten}

\begin{tabular}{|c|c|c|c|c|c|c|}
\cline { 2 - 7 } & \multicolumn{7}{c|}{ Nennungen [Anzahl] nach Kategorie [€] } \\
\cline { 2 - 8 } & Alle & $<2.500$ & $<\mathbf{1 0 . 0 0 0}$ & $<\mathbf{5 0 . 0 0 0}$ & $<100.000$ & $>100.000$ \\
\hline Anzahl: & 22 & 14 & 8 & 0 & 0 & 0 \\
\hline Anteil: & & $64 \%$ & $36 \%$ & $0 \%$ & $0 \%$ & $0 \%$ \\
\hline
\end{tabular}

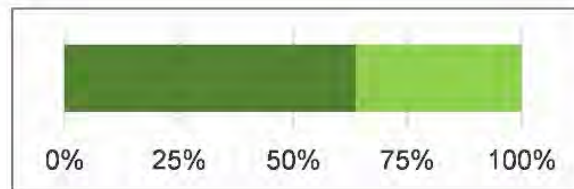

Beurteilung der zeitlichen Umsetzbarkeit

\begin{tabular}{|c|c|c|c|c|c|c|}
\hline \multicolumn{1}{c|}{} & \multicolumn{7}{l|}{ Nennungen [Anzahl] nach Kategorie [Monate] } \\
\cline { 2 - 8 } & Alle & $\leq 3$ & $4-6$ & $7-12$ & $13-24$ & $>24$ \\
\hline Anzahl: & 23 & 15 & 7 & 1 & 0 & 0 \\
\hline Anteil: & & $65 \%$ & $30 \%$ & $4 \%$ & $0 \%$ & $0 \%$ \\
\hline
\end{tabular}

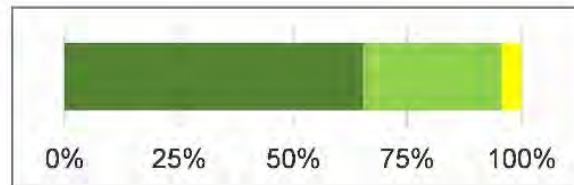

Beurteilung des verwaltungstechnischen Aufwands

\begin{tabular}{|c|c|c|c|c|c|}
\hline & \multicolumn{5}{c|}{ Nennungen [Anzahl] nach Kategorie [Aufwand] } \\
\cline { 2 - 6 } & Alle & gering & $\begin{array}{c}\text { eher } \\
\text { gering }\end{array}$ & eher hoch & hoch \\
\hline Anzahl: & 24 & 12 & 9 & 3 & 0 \\
\hline Anteil: & & $50 \%$ & $38 \%$ & $13 \%$ & $0 \%$ \\
\hline
\end{tabular}

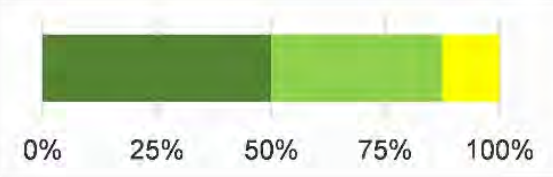

\section{Beurteilung der Wirksamkeit}

Wie wird durch die Maßnahme die Schwere der Unfälle reduziert?

\begin{tabular}{|c|c|c|c|c|c|}
\cline { 2 - 6 } & \multicolumn{3}{|c|}{ Nennungen [Anzahl] } & & \\
\cline { 2 - 6 } & Alle & stark & mäßig & wenig & nicht \\
\hline Anzahl: & 22 & 3 & 6 & 10 & 3 \\
\hline Anteil: & & $14 \%$ & $27 \%$ & $45 \%$ & $14 \%$ \\
\hline
\end{tabular}

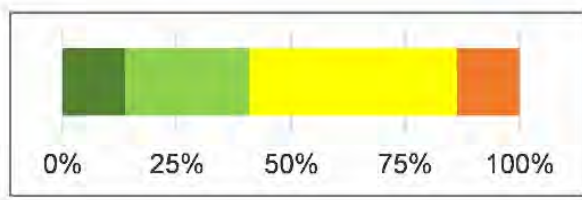

Wie wird durch die Maßnahme die Anzahl der Unfälle reduziert?

\begin{tabular}{|c|c|c|c|c|c|}
\cline { 2 - 6 } & \multicolumn{4}{c|}{ Nennungen [Anzahl] } & \multicolumn{2}{c|}{} \\
\cline { 2 - 6 } & Alle & stark & mäßig & wenig & nicht \\
\hline Anzahl: & 22 & 1 & 5 & 14 & 2 \\
\hline Anteil: & & $5 \%$ & $23 \%$ & $64 \%$ & $9 \%$ \\
\hline
\end{tabular}

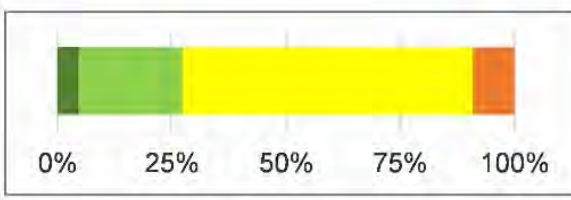

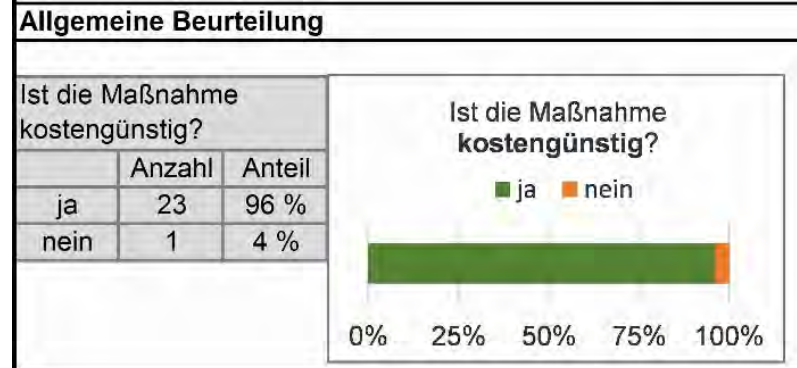

\begin{tabular}{|c|c|c|}
\hline \multicolumn{3}{|c|}{$\begin{array}{l}\text { Ist die Maßnahme } \\
\text { wirksam? }\end{array}$} \\
\hline & Anzahl & Anteil \\
\hline ja & 19 & $83 \%$ \\
\hline nein & 4 & $17 \%$ \\
\hline
\end{tabular}

Ist die Maßnahme wirksam?

m ja mein 
Evaluation der Maßnahmenkategorie: [37], Verkehrszeichen versetzen (Erhöhung Erkennbarkeit)

\begin{tabular}{|c|c|c|c|c|c|}
\hline Einsatzbedingungen und allgemeine Angaben & \multicolumn{5}{|c|}{ |Antwortkompetenz (Anzahl Nennungen) } \\
\hline Örtlichkeit: $\mid \mathrm{AO}$, Knotenpunkt/Strecke & Behörde & & Mitglied in Uko sind & Zuständigkeit & \\
\hline Regelungsart: beliebig & \begin{tabular}{|l|} 
Polizei: \\
\end{tabular} & 6 & 27 von 31 & Stadt: 1 & 12 \\
\hline Unfalltyp(en): Fahrunfall, Abbiegen, Einbiegen/Kreuzen & \begin{tabular}{l|l} 
Verkehr: & 1
\end{tabular} & 18 & Befragten. & Land: & 11 \\
\hline Befragte: 31 & Straßenbau: 3 & 3 & Mittlere UKO-Dienszeit & Andere: & 7 \\
\hline & Andere: 2 & 4 & in Jahren: 11 & & \\
\hline
\end{tabular}

\section{Bekanntheitsgrad der Maßnahme}

Die Maßnahme ist

27 Befragten bekannt,

2 Befragten nicht bekannt. $\quad$ K. A.: 2 Befragte(r).

Der Bekannheitsgrad der Maßnahme liegt bei ca.: $87 \%$

5 von 27 Befragten, denen die Maßnahme bekannt ist, haben diese Maßnahme ein- oder mehrmals in den letzten 5 Jahren in ihrem Zuständigkeitsbereich umgesetzt.

\section{Beurteilung der Kosten}

\begin{tabular}{|c|c|c|c|c|c|c|}
\cline { 2 - 7 } & \multicolumn{7}{c|}{ Nennungen [Anzahl] nach Kategorie [€] } \\
\cline { 2 - 7 } & Alle & $<2.500$ & $<\mathbf{1 0 . 0 0 0}$ & $<\mathbf{5 0 . 0 0 0}$ & $<\mathbf{1 0 0 . 0 0 0}$ & $>100.000$ \\
\hline Anzahl: & 27 & 27 & 0 & 0 & 0 & 0 \\
\hline Anteil: & & $100 \%$ & $0 \%$ & $0 \%$ & $0 \%$ & $0 \%$ \\
\hline
\end{tabular}

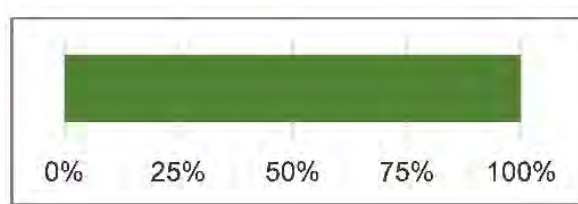

Beurteilung der zeitlichen Umsetzbarkeit

\begin{tabular}{c|c|c|c|c|c|c|}
\hline & \multicolumn{7}{l|}{ Nennungen [Anzahl] } & nach Kategorie [Monate] \\
\cline { 2 - 7 } & Alle & $\leq \mathbf{3}$ & $\mathbf{4 - 6}$ & $\mathbf{7 - 1 2}$ & $\mathbf{1 3 - 2 4}$ & $>24$ \\
\hline Anzahl: & 29 & 27 & 2 & 0 & 0 & 0 \\
\hline Anteil: & & $93 \%$ & $7 \%$ & $0 \%$ & $0 \%$ & $0 \%$ \\
\hline
\end{tabular}

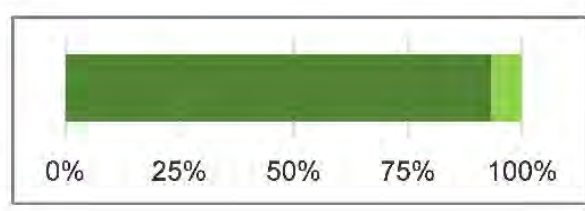

Beurteilung des verwaltungstechnischen Aufwands

\begin{tabular}{|c|c|c|c|c|c|}
\hline & \multicolumn{4}{|c|}{ Nennungen [Anzahl] nach Kategorie [Aufwand] } & \multirow[b]{2}{*}{ hoch } \\
\hline & Alle & gering & $\begin{array}{l}\text { eher } \\
\text { gering }\end{array}$ & eher hoch & \\
\hline Anzahl: & 29 & 27 & 2 & 0 & 0 \\
\hline Anteil: & & $93 \%$ & $7 \%$ & $0 \%$ & $0 \%$ \\
\hline
\end{tabular}

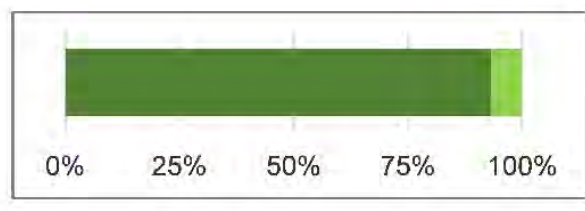

\section{Beurteilung der Wirksamkeit}

Wie wird durch die Maßnahme die Schwere der Unfälle reduziert?

\begin{tabular}{c|c|c|c|c|c|}
\cline { 2 - 6 } & \multicolumn{4}{|c|}{ Nennungen [Anzahl] } & \\
\cline { 2 - 6 } & Alle & stark & mäßig & wenig & nicht \\
\hline Anzahl: & 27 & 5 & 7 & 14 & 1 \\
\hline Anteil: & & $19 \%$ & $26 \%$ & $52 \%$ & $4 \%$ \\
\hline
\end{tabular}

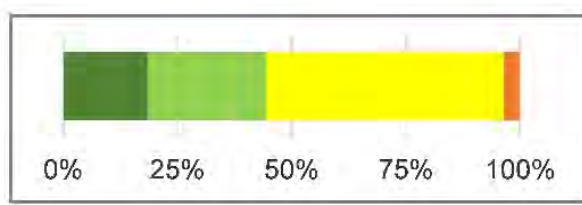

Wie wird durch die Maßnahme die Anzahl der Unfälle reduziert?

\begin{tabular}{|c|c|c|c|c|c|}
\cline { 2 - 6 } & \multicolumn{4}{|c|}{ Nennungen [Anzahl] } & \multicolumn{3}{c|}{} \\
\cline { 2 - 6 } & Alle & stark & mäßig & wenig & nicht \\
\hline Anzahl: & 28 & 1 & 8 & 17 & 2 \\
\hline Anteil: & & $4 \%$ & $29 \%$ & $61 \%$ & $7 \%$ \\
\hline
\end{tabular}

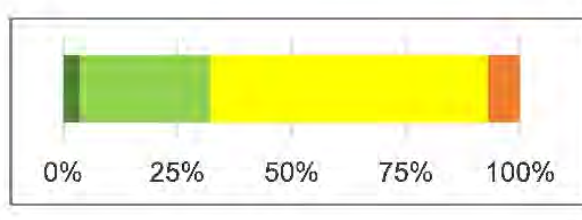

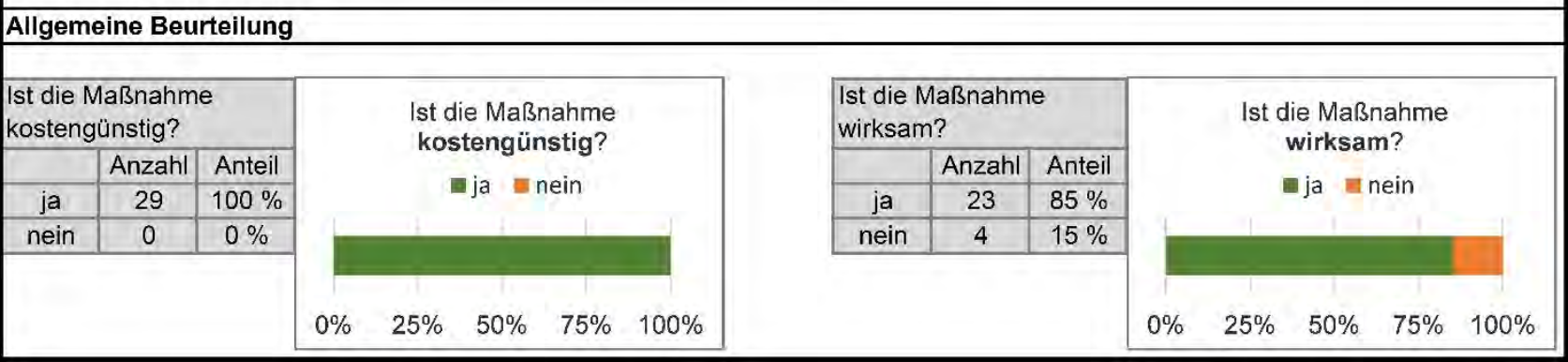


Evaluation der Maßnahmenkategorie: [38], Beschränkung Vzul und Z 625 StVO (Richtungstafel in Kurven)

\begin{tabular}{|c|c|c|c|c|c|c|}
\hline \multicolumn{2}{|c|}{ Einsatzbedingungen und allgemeine Angaben } & \multicolumn{5}{|c|}{ |Antwortkompetenz (Anzahl Nennungen) } \\
\hline Örtlichkeit: & AO, Strecke $(300 \mathrm{~m})$ & \multicolumn{2}{|l|}{ Behörde } & \multirow{5}{*}{$\begin{array}{l}\text { Mitglied in Uko sind } \\
19 \text { von } 23 \\
\text { Befragten. } \\
\text { Mittlere UKO-Dienszeit } \\
\text { in Jahren: } 5\end{array}$} & \multicolumn{2}{|l|}{ Zuständigkeit } \\
\hline Regelungsart: & - & |rolizei: & 5 & & Stadt: & 11 \\
\hline Unfalltyp(en): & Fahrunfall & Verkehr: & 10 & & Land: & 10 \\
\hline Befragte: & 23 & Straßenbau: & 3 & & Andere: & 5 \\
\hline & & Andere: & 5 & & & \\
\hline
\end{tabular}

\section{Bekanntheitsgrad der Maßnahme}

Die Maßnahme ist

22 Befragten bekannt,

1 Befragten nicht bekannt. $\quad$ K. A.: 0 Befragte( $r)$

14 von 22 Befragten, denen die Maßnahme bekannt ist, haben diese Maßnahme ein- oder mehrmals in den letzten 5 Jahren in ihrem Zuständigkeitsbereich umgesetzt.

Der Bekannheitsgrad der Maßnahme liegt bei ca.: $96 \%$

\section{Beurteilung der Kosten}

\begin{tabular}{|c|c|c|c|c|c|c|}
\hline \multirow{2}{*}{} & \multicolumn{7}{c|}{ Nennungen [Anzahl] nach Kategorie [€] } \\
\cline { 2 - 8 } & Alle & $<2.500$ & $<\mathbf{1 0 . 0 0 0}$ & $<\mathbf{5 0 . 0 0 0}$ & $<\mathbf{1 0 0 . 0 0 0}$ & $>100.000$ \\
\hline Anzahl: & 23 & 19 & 4 & 0 & 0 & 0 \\
\hline Anteil: & & $83 \%$ & $17 \%$ & $0 \%$ & $0 \%$ & $0 \%$ \\
\hline
\end{tabular}

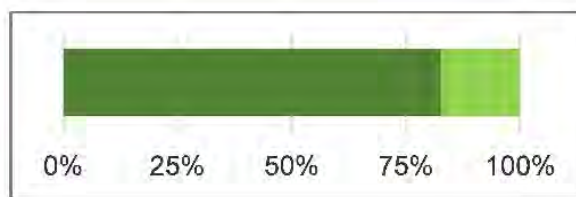

Beurteilung der zeitlichen Umsetzbarkeit

\begin{tabular}{|c|c|c|c|c|c|c|}
\hline & \multicolumn{8}{l|}{ Nennungen [Anzahl] } & nach Kategorie [Monate] \\
\cline { 2 - 7 } & Alle & $\leq 3$ & $\mathbf{4 - 6}$ & $\mathbf{7 - 1 2}$ & $\mathbf{1 3 - 2 4}$ & $>24$ \\
\hline Anzahl: & 23 & 20 & 3 & 0 & 0 & 0 \\
\hline Anteil: & & $87 \%$ & $13 \%$ & $0 \%$ & $0 \%$ & $0 \%$ \\
\hline
\end{tabular}

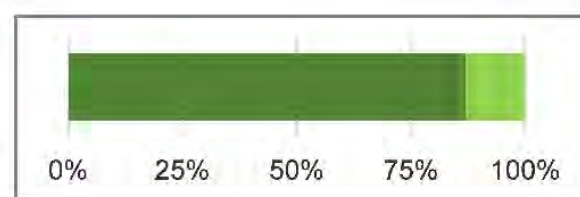

Beurteilung des verwaltungstechnischen Aufwands

\begin{tabular}{|c|c|c|c|c|c|}
\cline { 2 - 6 } & \multicolumn{6}{|c|}{ Nennungen [Anzahl] } & nach Kategorie [Aufwand] & \\
\cline { 2 - 6 } & Alle & gering & $\begin{array}{c}\text { eher } \\
\text { gering }\end{array}$ & eher hoch & hoch \\
\hline Anzahl: & 23 & 16 & 7 & 0 & 0 \\
\hline Anteil: & & $70 \%$ & $30 \%$ & $0 \%$ & $0 \%$ \\
\hline
\end{tabular}

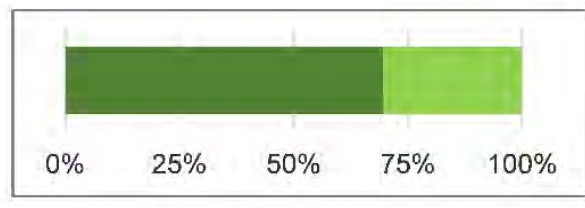

\section{Beurteilung der Wirksamkeit}

Wie wird durch die Maßnahme die Schwere der Unfälle reduziert?

\begin{tabular}{c|c|c|c|c|c|}
\cline { 2 - 6 } & \multicolumn{4}{|c|}{ Nennungen [Anzahl] } & \multicolumn{2}{l|}{} \\
\cline { 2 - 6 } & Alle & stark & mäßig & wenig & nicht \\
\hline Anzahl: & 23 & 3 & 4 & 9 & 7 \\
\hline Anteil: & & $13 \%$ & $17 \%$ & $39 \%$ & $30 \%$ \\
\hline
\end{tabular}

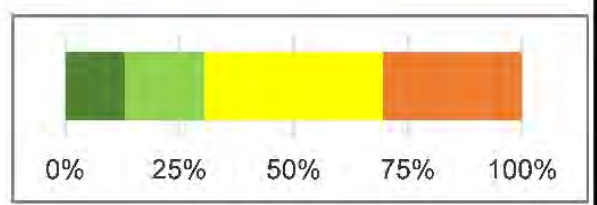

Wie wird durch die Maßnahme die Anzahl der Unfälle reduziert?

\begin{tabular}{|c|c|c|c|c|c|}
\cline { 2 - 6 } & \multicolumn{4}{|c|}{ Nennungen [Anzahl] } & \multicolumn{3}{c|}{} \\
\cline { 2 - 6 } & Alle & stark & mäßig & wenig & nicht \\
\hline Anzahl: & 23 & 2 & 5 & 8 & 8 \\
\hline Anteil: & & $9 \%$ & $22 \%$ & $35 \%$ & $35 \%$ \\
\hline
\end{tabular}

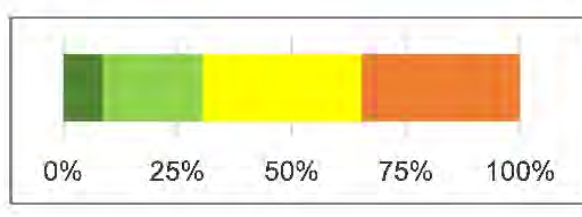

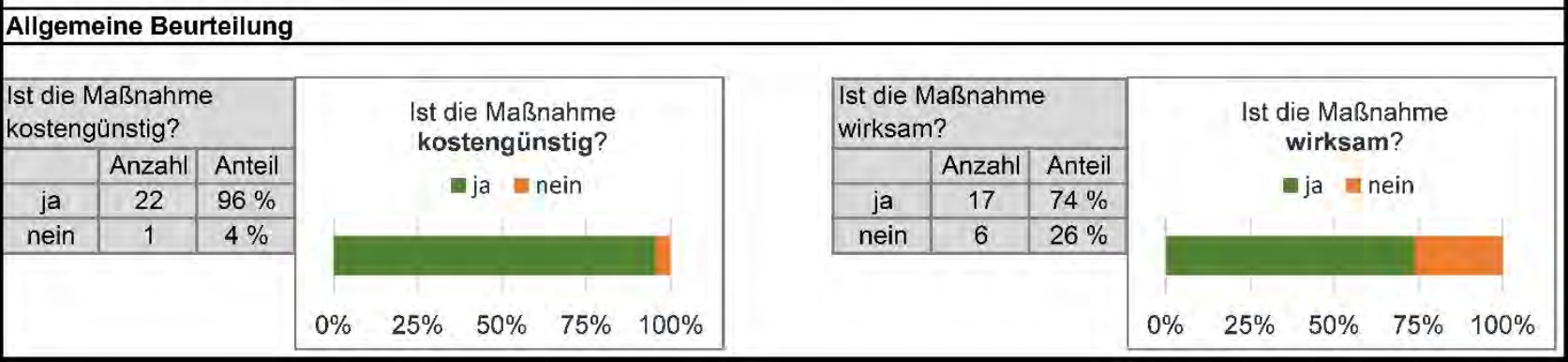


Evaluation der Maßnahmenkategorie: [39], Beschränkung Vzul und sonstige Gefahrenzeichen

\begin{tabular}{|c|c|c|c|c|c|c|}
\hline \multicolumn{2}{|c|}{ Einsatzbedingungen und allgemeine Angaben } & \multicolumn{5}{|c|}{ |Antwortkompetenz (Anzahl Nennungen) } \\
\hline Örtlichkeit: & AO, Strecke $(300 \mathrm{~m})$ & \multicolumn{2}{|l|}{ Behörde } & \multirow{5}{*}{$\begin{array}{l}\text { Mitglied in Uko sind } \\
19 \text { von } 23 \\
\text { Befragten. } \\
\text { Mittlere UKO-Dienszeit } \\
\text { in Jahren: } 5\end{array}$} & \multicolumn{2}{|c|}{ Zuständigkeit } \\
\hline Regelungsart: & - & \begin{tabular}{|l|} 
Polizei: \\
\end{tabular} & 5 & & Stadt: & 11 \\
\hline Unfalltyp(en): & Fahrunfall & Verkehr: & 10 & & Land: & 10 \\
\hline Befragte: & 23 & Straßenbau: & 3 & & Andere: & 5 \\
\hline & & Andere: & 5 & & & \\
\hline
\end{tabular}

\section{Bekanntheitsgrad der Maßnahme}

Die Maßnahme ist

23 Befragten bekannt,

0 Befragten nicht bekannt. $\quad$ K. A.: 0 Befragte(r)

Der Bekannheitsgrad der Maßnahme liegt bei ca.: $100 \%$

12 von 23 Befragten, denen die Maßnahme bekannt ist, haben diese Maßnahme ein- oder mehrmals in den letzten 5 Jahren in ihrem Zuständigkeitsbereich umgesetzt.

\section{Beurteilung der Kosten}

\begin{tabular}{|c|c|c|c|c|c|c|}
\cline { 2 - 7 } & \multicolumn{7}{c|}{ Nennungen [Anzahl] nach Kategorie [€] } \\
\cline { 2 - 7 } & Alle & $<2.500$ & $<\mathbf{1 0 . 0 0 0}$ & $<\mathbf{5 0 . 0 0 0}$ & $<\mathbf{1 0 0 . 0 0 0}$ & $>100.000$ \\
\hline Anzahl: & 23 & 23 & 0 & 0 & 0 & 0 \\
\hline Anteil: & & $100 \%$ & $0 \%$ & $0 \%$ & $0 \%$ & $0 \%$ \\
\hline
\end{tabular}

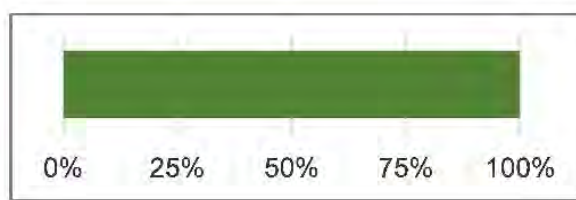

\section{Beurteilung der zeitlichen Umsetzbarkeit}

\begin{tabular}{|c|c|c|c|c|c|c|}
\hline & \multicolumn{7}{|c|}{ Nennungen [Anzahl] } & nach Kategorie [Monate] \\
\cline { 2 - 7 } & Alle & $\leq 3$ & $\mathbf{4 - 6}$ & $\mathbf{7 - 1 2}$ & $\mathbf{1 3 - 2 4}$ & $>24$ \\
\hline Anzahl: & 23 & 22 & 1 & 0 & 0 & 0 \\
\hline Anteil: & & $96 \%$ & $4 \%$ & $0 \%$ & $0 \%$ & $0 \%$ \\
\hline
\end{tabular}

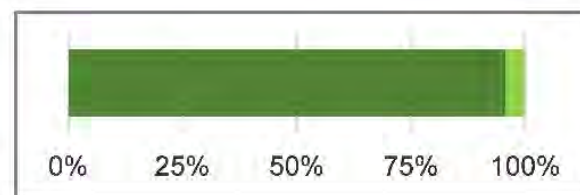

\section{Beurteilung des verwaltungstechnischen Aufwands}

\begin{tabular}{|c|c|c|c|c|c|}
\cline { 3 - 6 } & \multicolumn{6}{c|}{ Nennungen [Anzahl] nach Kategorie [Aufwand] } \\
\cline { 2 - 6 } & Alle & gering & $\begin{array}{c}\text { eher } \\
\text { gering }\end{array}$ & eher hoch & hoch \\
\hline Anzahl: & 23 & 19 & 4 & 0 & 0 \\
\hline Anteil: & & $83 \%$ & $17 \%$ & $0 \%$ & $0 \%$ \\
\hline
\end{tabular}

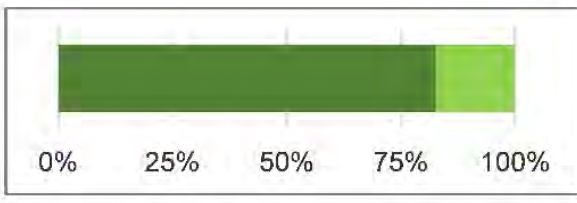

\section{Beurteilung der Wirksamkeit}

Wie wird durch die Maßnahme die Schwere der Unfälle reduziert?

\begin{tabular}{c|c|c|c|c|c|}
\cline { 2 - 6 } & \multicolumn{4}{|c|}{ Nennungen [Anzahl] } & \multicolumn{2}{l|}{} \\
\cline { 2 - 6 } & Alle & stark & mäßig & wenig & nicht \\
\hline Anzahl: & 23 & 3 & 4 & 10 & 6 \\
\hline Anteil: & & $13 \%$ & $17 \%$ & $43 \%$ & $26 \%$ \\
\hline
\end{tabular}

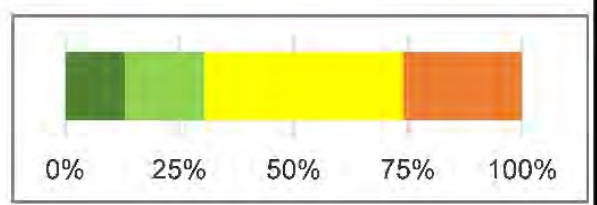

Wie wird durch die Maßnahme die Anzahl der Unfälle reduziert?

\begin{tabular}{|c|c|c|c|c|c|}
\cline { 3 - 6 } & \multicolumn{2}{|c|}{ Nennungen [Anzahl] } & \multicolumn{3}{l|}{} \\
\cline { 2 - 6 } & Alle & stark & mäßig & wenig & nicht \\
\hline Anzahl: & 23 & 1 & 5 & 12 & 5 \\
\hline Anteil: & & $4 \%$ & $22 \%$ & $52 \%$ & $22 \%$ \\
\hline
\end{tabular}

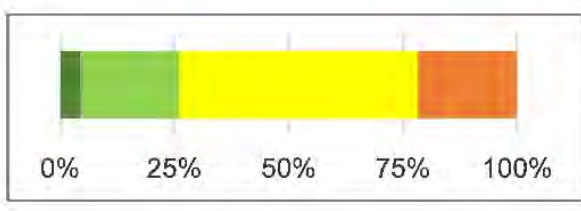

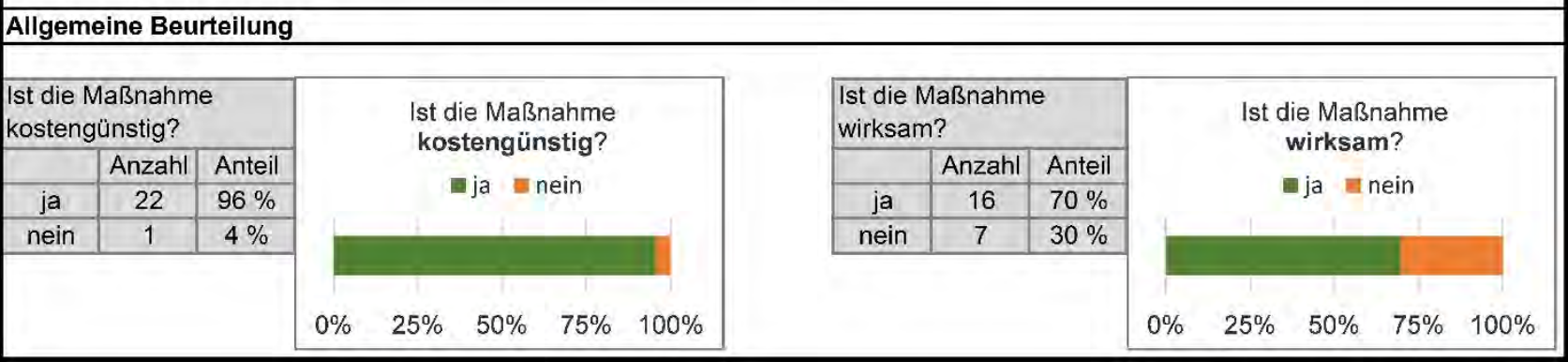




\section{Anhang 7 Faktenblätter Unfalldatenanalyse}

Nachfolgend sind die Faktenblätter zur Unfalldatenanalyse der einzelnen Maßnahmenkategorien aufgeführt. Die Beschreibung des inhaltlichen Aufbaus ist Kapitel 6.2.1 zu entnehmen. Die Kollektivzusammensetzung beinhaltet Tabelle 8 in Kapitel 4.3.3. Die nachstehende Tabelle verweist auf die Seitenzahl der Maßnahmenkategorien. Der beispielhafte Aufbau des Faktenblattes ist Seite A-XLIII dargestellt, die Erläuterung der Inhalte finden sich auf Seite A-XLIV.

\begin{tabular}{|c|c|c|}
\hline Nr. & $\begin{array}{r}\text { Maßnahmenkategorie } \\
\text { Name }\end{array}$ & $\begin{array}{l}\text { Seite im } \\
\text { Anhang }\end{array}$ \\
\hline 1 & Verbesserung der Koordinierung & $\mathrm{XLV}$ \\
\hline 2 & Änderung der Signalisierung & $\mathrm{XLVI}$ \\
\hline 3 & "sonstige" Gefahrenzeichen anbringen & XLVII \\
\hline 4 & HALT Z 206 StVO (anstelle Z 205 StVO) & XLVIII \\
\hline 5 & HALT Z 206 StVO (anstelle Z 205 StVO) & XLIX \\
\hline 6 & Halte-/Parkverbot anordnen & $\mathrm{L}$ \\
\hline 7 & Z 625 StVO (Richtungstafel in Kurven) aufstellen & $\mathrm{LI}$ \\
\hline 8 & Zusatzzeichen Z 1006-36 StVO (Unfallgefahr) ergänzen & LII \\
\hline 9 & Zusatzzeichen Z 1006-36 StVO (Unfallgefahr) ergänzen & LIII \\
\hline 10 & Verkehrszeichen anpassen & LIV \\
\hline 11 & Verkehrszeichen anpassen & LV \\
\hline 12 & Beschränkung Vzul & LVI \\
\hline 15 & Beschränkung Vzul & LVII \\
\hline 16 & Überwachung zulässige Höchstgeschwindigkeit mit OGÜ & LVIII \\
\hline 17 & Überwachung zulässige Höchstgeschwindigkeit mit OGÜ & LIX \\
\hline 18 & Erneuerung Fahrbahnoberfläche & LX \\
\hline 19 & Zusätzlichen Signalgeber anordnen & LXI \\
\hline 21 & Leuchtmittel Signalgeber verbessern & LXII \\
\hline 22 & Sichthindernisse entfernen & LXIII \\
\hline 23 & Sichthindernisse entfernen & LXIV \\
\hline 24 & Beleuchtung anpassen/verbessern & LXV \\
\hline 25 & Sichtschutzzaun aufstellen & LXVI \\
\hline 26 & Markierung anpassen/verbessern & LXVII \\
\hline 27 & Markierung in Stand setzen & LXVIII \\
\hline 29 & Radverkehrsführung anpassen & LXIX \\
\hline 30 & Radfahrfurt Rot einfärben & LXX \\
\hline 31 & Passive Schutzeinrichtung aufstellen & LXXI \\
\hline 32 & Unterfahrschutz an passiver Schutzeinrichtung anbringen & LXXII \\
\hline 33 & Hindernisse im Seitenraum entfernen & LXXIII \\
\hline 34 & Rüttelstreifen aufbringen & LXXIV \\
\hline 35 & Gummischwellen aufbringen & LXXV \\
\hline 36 & Sperrfläche markieren & LXXVI \\
\hline 37 & Verkehrszeichen versetzen (Erhöhung Erkennbarkeit) & LXXVII \\
\hline 38 & Beschränkung Vzul und Z 625 StVO (Richtungstafel in Kurve) & LXXVIII \\
\hline 39 & Beschränkung Vzul und sonst. Gefahrenzeichen & LXXIX \\
\hline
\end{tabular}




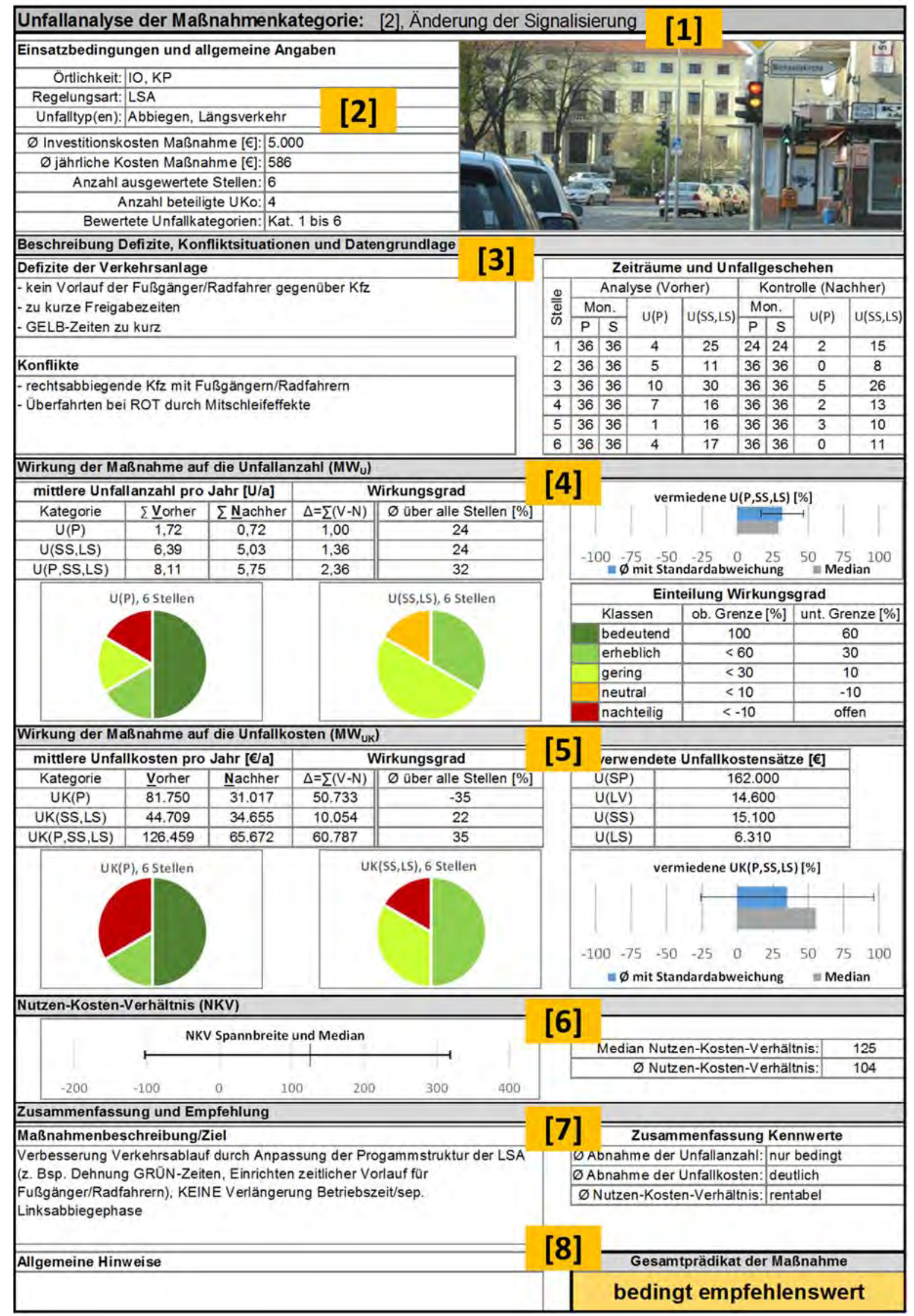

Faktenblatt zur Unfalldatenanalyse einer Maßnahmenkategorie MK, allgemeine Darstellung 


\begin{tabular}{|c|c|c|}
\hline Nummer & Bezeichnung Bereich & Inhalt Bereich \\
\hline [1] & Bezeichnung MK & - Maßnahmenkategorie mit Nummer und Namen \\
\hline [2] & $\begin{array}{l}\text { Einsatzbedingungen und } \\
\text { allgemeine Angaben }\end{array}$ & $\begin{array}{l}\text { - Beschreibung zur Örtlichkeit, Regelungsart des Knotenpunkts (wenn gegeben), } \\
\text { prägende Unfalltypen und Beispielbild (allgemeine Situation) } \\
\text { - Angabe zu Investitionskosten und durchschnittlichen jährlichen Kosten (inkl. } \\
\text { Veränderung Betriebskosten } \\
\text { - Erläuterungen zu Kollektivgröße, Kollektivbeschaffenheit, bewertete Unfallkategorien }\end{array}$ \\
\hline [3] & $\begin{array}{l}\text { Beschreibung Defizite, Kon- } \\
\text { fliktsituationen und Daten- } \\
\text { grundlage }\end{array}$ & $\begin{array}{l}\text { - Defizite der Verkehrsanlage und der daraus resultierenden Konflikte } \\
\text { - Tabellarische Übersicht zum Unfallgeschehen der betrachteten Stellen, Angabe der } \\
\text { betrachteten Monate und Unfallanzahlen für } U(P) \text { und die betrachteten Kategorien } \\
\text { der Unfälle mit Sachschaden für Vorher- und Nachher-Zeitraum }\end{array}$ \\
\hline [4] & $\begin{array}{l}\text { Wirkung der Maßnahme auf } \\
\text { die Unfallanzahl }(U)\end{array}$ & $\begin{array}{l}\text { - Angabe der mittleren Unfallanzahlen im Vorher- und Nachher-Zeitraum sowie der } \\
\text { Differenz }(\Delta) \text { für U(P), betrachtete Kategorien der Unfälle mit Sachschaden und } \\
\text { gesamtes betrachtetes Unfallgeschehen (Unfallzahlen erfüllen nicht zwingend das } \\
\text { Grenzwertkriterium nach M Uko (FGSV 2012]) } \\
\text { - Angabe des durchschnittlichen }(\varnothing) \text { Wirkungsgrades [\%] für die Unfallanzahl nach } \\
\text { betrachteten Unfall-kategorien (dieser kann von der Differenz aufgrund der Mittelwert- } \\
\text { betrachtung des Kollektivs abweichen) } \\
\text { - Darstellung des durchschnittlichen }(\varnothing) \text { Wirkungsgrades [\%]und der Standardab- } \\
\text {.. weichung der MK für die Unfallanzahl (alle betrachteten Unfallkategorien), Angabe } \\
\text { des Median für Kollektive mit mindestens } 5 \text { Stellen) } \\
\text { - Darstellung der Klassifizierung der Wirkungsgrade mit Angabe der Klassengrenzen } \\
\text { - Grafische Darstellung der Maßnahmenwirkung MW } \text { M }_{\cup} \text { (klassifiziert) für die einzelnen } \\
\text { Stellen der MK differenziert nach U(P) und den betrachteten Kategorien der Unfälle } \\
\text { mit Sachschaden }\end{array}$ \\
\hline [5] & $\begin{array}{l}\text { Wirkung der Maßnahme auf } \\
\text { die Unfallkosten (UK) }\end{array}$ & $\begin{array}{l}\text { - Angabe der mittleren Unfallkosten im Vorher- und Nachher-Zeitraum sowie der } \\
\text { Differenz }(\Delta) \text { für U(P), betrachtete Kategorien der Unfälle mit Sachschaden und } \\
\text { gesamtes betrachtetes Unfallgeschehen } \\
\text { - Angabe des durchschnittlichen }(\varnothing) \text { Wirkungsgrades [\%] für die Unfallkosten nach } \\
\text { betrachteten Unfall-kategorien (dieser kann von der Differenz aufgrund der Mittelwert- } \\
\text { betrachtung des Kollektivs abweichen) } \\
\text { - Darstellung des durchschnittlichen }(\varnothing) \text { Wirkungsgrades [\%] und der Standardab- } \\
\text { weichung der MK für die Unfallkosten (alle betrachteten Unfallkategorien), Angabe } \\
\text { des Median für Kollektive mit mindestens } 5 \text { Stellen) } \\
\text { - Tabellarische Darstellung der verwendeten Unfallkostensätze [€] } \\
\text { - Grafische Darstellung der Maßnahmenwirkung MWW (klassifiziert) für die einzelnen } \\
\text { Stellen der MK differenziert nach U(P) und den betrachteten Kategorien der Unfälle } \\
\text { mit Sachschaden }\end{array}$ \\
\hline [6] & Nutzen-Kosten-Verhältnis & $\begin{array}{l}\text { - Grafische Darstellung Median des NKV (Kollektiv) und Spannbreite der Einzelwerte } \\
\text { - Angabe Median und Mittelwert ( } \varnothing) \text { für NKV der Maßnahmenkategorie }\end{array}$ \\
\hline [7] & $\begin{array}{l}\text { Zusammenfassung und } \\
\text { Empfehlung }\end{array}$ & $\begin{array}{l}\text { - Beschreibung der konkreten Maßnahmenumsetzung (Ziele der Maßnahme) } \\
\text { - Angabe von allgemeinen Hinweisen, diese betreffen z. B. Einschränkungen bei der } \\
\text { Interpretation der Ergebnisse oder Besonderheiten des Kollektivs } \\
\text { - Zusammenfassung und Einschätzung der maßgebenden Kennwerte; Wirkung der } \\
\text { Maßnahme auf Unfallanzahl und Unfallkosten sowie Bewertung des NKV } \\
\text { (Da die Größe der mittleren Wirkungsgrade unter Ziffer } 4 \text { und Ziffer } 5 \text { durch } \\
\text { Ausreißer geprägt sein können, richtet sich die Vergabe des Prädikates für die } \\
\text { Abnahme der Unfallanzahl und Unfallkosten nach der Bewertung der MK nach } \\
\text { Tabelle 15.) }\end{array}$ \\
\hline [8] & $\begin{array}{l}\text { Gesamtprädikat der Maß- } \\
\text { nahme }\end{array}$ & - Vergabe des Gesamtprädikats der Maßnahme \\
\hline
\end{tabular}

Erläuterung der Inhalte des Faktenblatts zur Unfalldatenanalyse einer Maßnahmenkategorie MK 


\section{Unfallanalyse der Maßnahmenkategorie: [1], Verbesserung der Koordinierung}

Einsatzbedingungen und allgemeine Angaben

Örtlichkeit: IO, KP

Regelungsart: LSA

Unfalltyp(en): Längsverkehr

$\varnothing$ Investitionskosten Maßnahme [€]: 5.000

$\varnothing$ jährliche Kosten Maßnahme [€]: 586

Anzahl ausgewertete Stellen: 5 Anzahl beteiligte UKo: 1

Bewertete Unfallkategorien:|Kat. 1 bis 6

Beschreibung Defizite, Konfliktsituationen und Datengrundlage

\section{Defizite der Verkehrsanlage}

- kürzere Freigabezeit an einer der LSA (Verengung Grünband)

- Änderung in der Progressionsgeschwindigkeit

- nur gering versetzte Freigabe verschiedener Ströme in der Zufahrt

\section{Konflikte}

- Auffahren auf den Vorausfahrenden

- Überfahrten bei ROT durch Mitschleifeffekte
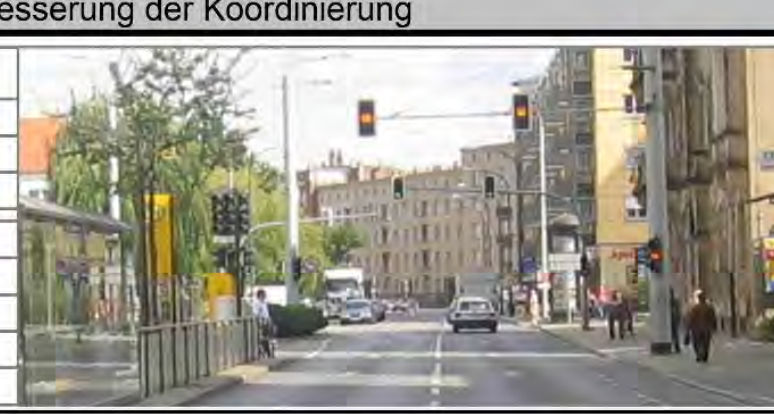

Wirkung der Maßnahme auf die Unfallanzahl (MW

\begin{tabular}{|c|c|c|c||c|}
\hline \hline \multicolumn{2}{|c|}{ mittlere Unfallanzahl pro Jahr [U/a] } & \multicolumn{2}{c|}{ Wirkungsgrad } \\
\hline Kategorie & $\sum \underline{\text { Vorher }}$ & $\sum \underline{\text { Nachher }}$ & $\Delta=\Sigma(\mathrm{V}-\mathrm{N})$ & $\varnothing$ über alle Stellen [\%] \\
\hline $\mathrm{U}(\mathrm{P})$ & 3,00 & 2,60 & 0,40 & 10 \\
\hline $\mathrm{U}(\mathrm{SS}, \mathrm{LS})$ & 8,93 & 10,40 & $-1,47$ & -16 \\
\hline $\mathrm{U}(\mathrm{P}, \mathrm{SS}, \mathrm{LS})$ & 11,93 & 13,00 & $-1,07$ & -7 \\
\hline
\end{tabular}
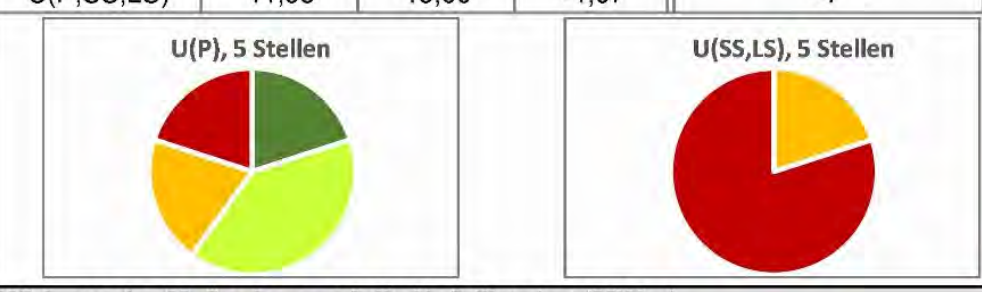

\begin{tabular}{|c|c|c|c|c|c|c|c|c|}
\hline \multicolumn{9}{|c|}{ Zeiträume und Unfallgeschehen } \\
\hline \multirow{3}{*}{ 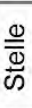 } & \multicolumn{4}{|c|}{ Analyse (Vorher) } & \multicolumn{4}{|c|}{ Kontrolle (Nachher) } \\
\hline & \multicolumn{2}{|c|}{ Mon. } & \multirow{2}{*}{$U(P)$} & \multirow{2}{*}{$\mathrm{U}(\mathrm{SS}, \mathrm{LS})$} & \multicolumn{2}{|c|}{ Mon. } & \multirow{2}{*}{$U(P)$} & \multirow{2}{*}{ U(SS, LS } \\
\hline & $\mathrm{P}$ & $\mathrm{S}$ & & & $\mathrm{P}$ & S & & \\
\hline 1 & 36 & 36 & 5 & 35 & 36 & 36 & 8 & 42 \\
\hline 2 & 36 & 36 & 17 & 32 & 36 & 36 & 18 & 37 \\
\hline 3 & 36 & 36 & 7 & 20 & 36 & 36 & 5 & 23 \\
\hline 4 & 36 & 36 & 6 & 23 & 36 & 36 & 5 & 22 \\
\hline 5 & 36 & 36 & 10 & 24 & 36 & 36 & 3 & 32 \\
\hline 6 & & & & & & & & \\
\hline
\end{tabular}

Wirkung der Maßnahme auf die Unfallkosten ( $\mathrm{MW}_{\mathrm{UK}}$ )

\begin{tabular}{|c|c|c|c||c|}
\hline \hline \multicolumn{2}{|c|}{ mittlere Unfallkosten pro Jahr [€/a] } & \multicolumn{2}{|c|}{ Wirkungsgrad } \\
\hline Kategorie & $\underline{\text { Vorher }}$ & $\underline{\text { Nachher }}$ & $\Delta=\Sigma(\mathrm{V}-\mathrm{N})$ & $\varnothing$ über alle Stellen [\%] \\
\hline UK(P) & 112.587 & 136.227 & -23.640 & -69 \\
\hline UK(SS,LS) & 62.815 & 76.172 & -13.357 & -19 \\
\hline UK(P,SS,LS) & 175.402 & 212.399 & -36.997 & -31 \\
\hline
\end{tabular}

UK(P,SS,LS

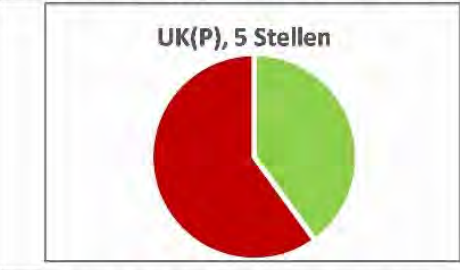

UK(SS,LS), 5 Stellen

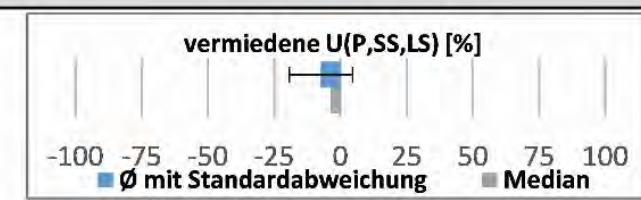

\section{$\emptyset$ mit Standardabweichung Median}

\begin{tabular}{|l|c|c|}
\hline \hline \multicolumn{3}{|c|}{ Einteilung Wirkungsgrad } \\
\hline Klassen & ob. Grenze [\%] & unt. Grenze [\%] \\
\hline bedeutend & 100 & 60 \\
\hline erheblich & $<60$ & 30 \\
\hline gering & $<30$ & 10 \\
\hline neutral & $<10$ & -10 \\
\hline nachteilig & $<-10$ & offen \\
\hline
\end{tabular}

\section{nac}

\begin{tabular}{|c|c|}
\hline \multicolumn{2}{|c|}{ verwendete Unfallkostensätze [€] } \\
\hline $\mathrm{U}(\mathrm{SP})$ & 162.000 \\
\hline $\mathrm{U}(\mathrm{LV})$ & 14.600 \\
\hline $\mathrm{U}(\mathrm{SS})$ & 15.100 \\
\hline $\mathrm{U}(\mathrm{LS})$ & 6.310 \\
\hline \hline
\end{tabular}
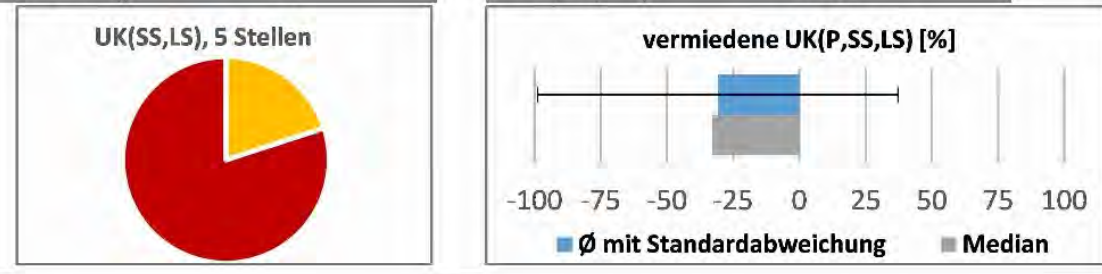

$\varpi$ mit Standardabweichung $=$ Median

Nutzen-Kosten-Verhältnis (NKV)

\begin{tabular}{|lllllll}
\hline \multicolumn{7}{|c|}{ NKV Spannbreite und Median } \\
\hline
\end{tabular}

Zusammenfassung und Empfehlung

\section{Maßnahmenbeschreibung/Ziel}

Überarbeitung der LSA-Programme mit dem Ziel, eine Verbesserung des Verkehrsablaufs zu erreichen, u. a. durch das Vermeiden von

"Mitschleifeffekten" oder Auffahrunfällen
Zusammenfassung Kennwerte

$\varnothing$ Abnahme der Unfallanzahl: nicht gegeben

$\varnothing$ Abnahme der Unfallkosten: nur bedingt

$\varnothing$ Nutzen-Kosten-Verhältnis: bedingt rentabel 


\section{Unfallanalyse der Maßnahmenkategorie: [2], Änderung der Signalisierung}

\begin{tabular}{|c|c|c|c|c|c|c|c|c|c|}
\hline \multicolumn{10}{|l|}{ Einsatzbedingungen und allgemeine Angaben } \\
\hline \multicolumn{10}{|l|}{ Örtlichkeit: $10, \mathrm{KP}$} \\
\hline \multicolumn{10}{|l|}{ Regelungsart: LSA } \\
\hline \multicolumn{10}{|l|}{ Unfalltyp(en): Abbiegen, Längsverkehr } \\
\hline \multicolumn{10}{|l|}{$\varnothing$ Investitionskosten Maßnahme [€]: 5.000} \\
\hline \multicolumn{10}{|l|}{$\varnothing$ jährliche Kosten Maßnahme [€]: 586} \\
\hline \multicolumn{10}{|l|}{ Anzahl ausgewertete Stellen: 6} \\
\hline \multicolumn{10}{|l|}{ Anzahl beteiligte UKo: 4} \\
\hline \multicolumn{10}{|l|}{ Bewertete Unfallkategorien:|Kat. 1 bis 6} \\
\hline \multicolumn{10}{|c|}{ Beschreibung Defizite, Konfliktsituationen und Datengrundlage } \\
\hline \multirow{4}{*}{$\begin{array}{l}\text { Defizite der Verkehrsanlage } \\
\text { - kein Vorlauf der Fußgänger/Radfahrer gegenüber Kfz } \\
\text { - zu kurze Freigabezeiten } \\
\text { - GELB-Zeiten zu kurz } \\
\end{array}$} & \multicolumn{9}{|c|}{ Zeiträume und Unfallgeschehen } \\
\hline & \multirow{3}{*}{$\frac{\frac{\omega}{\Phi}}{\omega}$} & \multicolumn{4}{|c|}{ Analyse (Vorher) } & \multicolumn{4}{|c|}{ Kontrolle (Nachher) } \\
\hline & & \multicolumn{2}{|c|}{ Mon. } & \multirow{2}{*}{$\mathrm{U}(\mathrm{P})$} & \multirow{2}{*}{$\mathrm{U}(\mathrm{SS}, \mathrm{LS})$} & \multicolumn{2}{|c|}{ Mon. } & \multirow{2}{*}{$U(P)$} & \multirow{2}{*}{$\mathrm{U}(\mathrm{SS}, \mathrm{LS}$} \\
\hline & & $\mathrm{P}$ & S & & & $\mathrm{P}$ & $S$ & & \\
\hline & 1 & 36 & 36 & 4 & 25 & 24 & 24 & 2 & 15 \\
\hline Konflikte & 2 & 36 & 36 & 5 & 11 & 36 & 36 & 0 & 8 \\
\hline \multirow{4}{*}{$\begin{array}{l}\text { - rechtsabbiegende Kfz mit Fußgängern/Radfahrern } \\
\text { - Überfahrten bei ROT durch Mitschleifeffekte }\end{array}$} & 3 & 36 & 36 & 10 & 30 & 36 & 36 & 5 & 26 \\
\hline & 4 & 36 & 36 & 7 & 16 & 36 & 36 & 2 & 13 \\
\hline & 5 & 36 & 36 & 1 & 16 & 36 & 36 & 3 & 10 \\
\hline & 6 & 36 & 36 & 4 & 17 & 36 & 36 & 0 & 11 \\
\hline
\end{tabular}

Wirkung der Maßnahme auf die Unfallanzahl $\left(\mathrm{MW}_{\mathrm{U}}\right)$

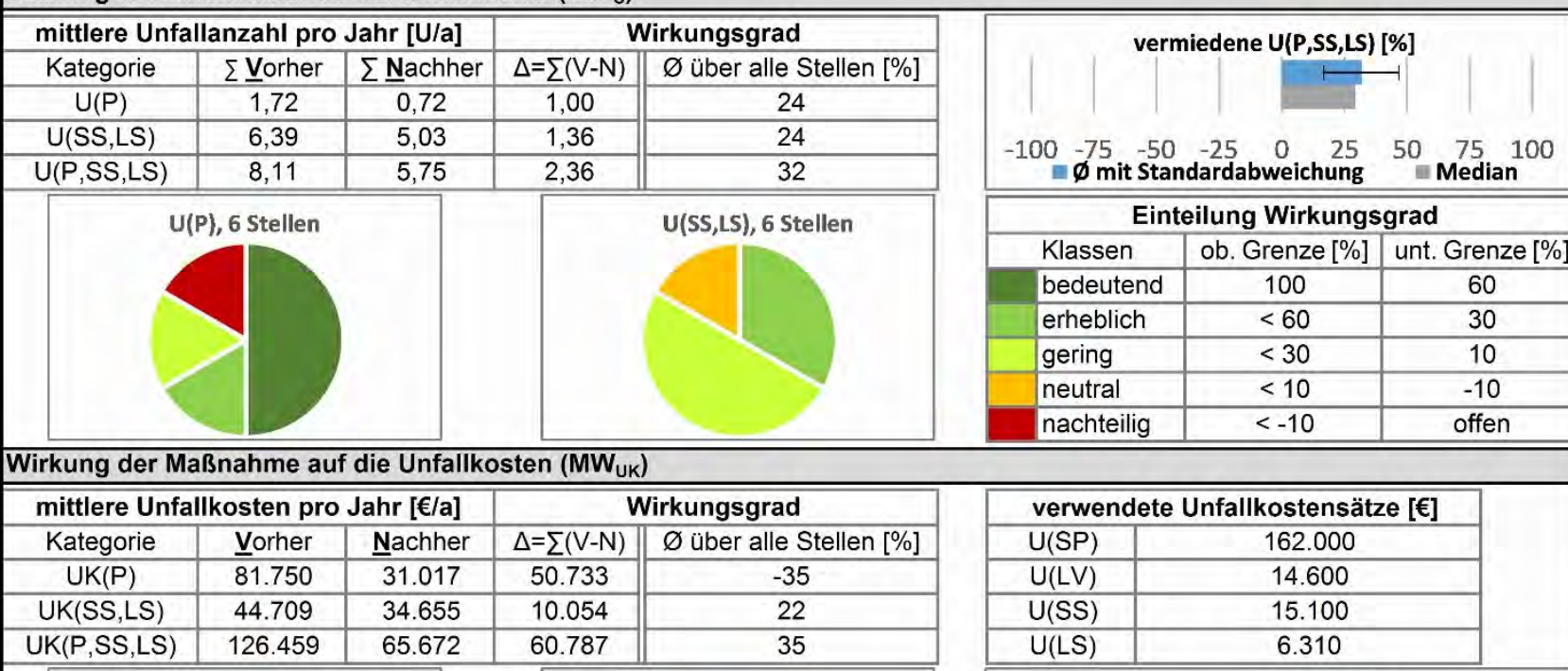

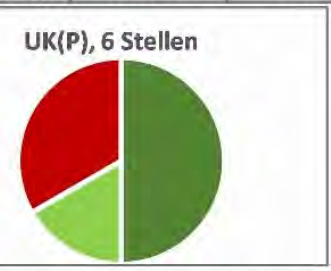

UK(SS,LS), 6 Stellen

vermiedene UK(P,SS,LS) [\%]

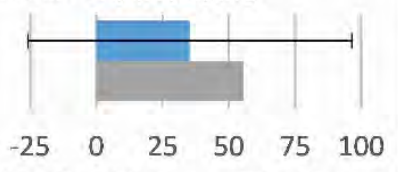

$\begin{array}{lllllllll}-100 & -75 & -50 & -25 & 0 & 25 & 50 & 75 & 100\end{array}$

$₫ \emptyset$ mit Standardabweichung $=$ Median

Nutzen-Kosten-Verhältnis (NKV)

\begin{tabular}{|c|c|c|c|c|c|c|}
\hline \multicolumn{7}{|c|}{ NKV Spannbreite und Median } \\
\hline-200 & -100 & 0 & 100 & 200 & 300 & 400 \\
\hline
\end{tabular}

Zusammenfassung und Empfehlung

Maßnahmenbeschreibung/Ziel

Verbesserung Verkehrsablauf durch Anpassung der Progammstruktur der LSA

(z. Bsp. Dehnung GRÜN-Zeiten, Einrichten zeitlicher Vorlauf für

Fußgänger/Radfahrern), KEINE Verlängerung Betriebszeit/sep.

Linksabbiegephase

Allgemeine Hinweise

\begin{tabular}{r|r}
\hline Median Nutzen-Kosten-Verhältnis: & 125 \\
$\varnothing$ Nutzen-Kosten-Verhältnis: & 104 \\
\hline
\end{tabular}

Zusammenfassung Kennwerte

$\varnothing$ Abnahme der Unfallanzahl: nur bedingt

Ø Abnahme der Unfallkosten: deutlich

$\varnothing$ Nutzen-Kosten-Verhältnis: rentabel

Gesamtprädikat der Maßnahme

bedingt empfehlenswert 


\section{Unfallanalyse der Maßnahmenkategorie: [3], Anbringen "sonstiger" Gefahrenzeichen}

Einsatzbedingungen und allgemeine Angaben

Örtlichkeit: AO, Strecke (300 m)

Regelungsart:-

Unfalltyp(en): Fahrunfall

$\varnothing$ Investitionskosten Maßnahme [€]: 1.000

$\varnothing$ jährliche Kosten Maßnahme [€]: 66

Anzahl ausgewertete Stellen: 2

Anzahl beteiligte UKo: 2

Bewertete Unfallkategorien:|Kat. 1 bis 4

Beschreibung Defizite, Konfliktsituationen und Datengrundlage

Defizite der Verkehrsanlage

- mangelnde Erkennbarkeit des Streckenverlaufs

- schlecht/spät erkennbare Gefahrenstellen

- Relationstrassierung nicht eingehalten

\section{Konflikte}

- Fahrunfälle mit Abkommen von der Fahrbahn

- u. a. Konflikte mit Gegenverkehr

Wirkung der Maßnahme auf die Unfallanzahl $\left(\mathrm{MW}_{\mathrm{U}}\right)$

\begin{tabular}{|c|c|c|c||c|}
\hline \multicolumn{2}{|c|}{ mittlere Unfallanzahl pro Jahr [U/a] } & \multicolumn{2}{c|}{ Wirkungsgrad } \\
\hline Kategorie & $\sum \underline{\text { Vorher }}$ & $\sum$ Nachher & $\Delta=\sum(\mathrm{V}-\mathrm{N})$ & $\varnothing$ über alle Stellen [\%] \\
\hline $\mathrm{U}(\mathrm{P})$ & 1,50 & 0,50 & 1,00 & 70 \\
\hline $\mathrm{U}(\mathrm{SS})$ & 0,67 & 0,50 & 0,17 & -17 \\
\hline $\mathrm{U}(\mathrm{P}, \mathrm{SS})$ & 2,17 & 1,00 & 1,17 & 51 \\
\hline
\end{tabular}
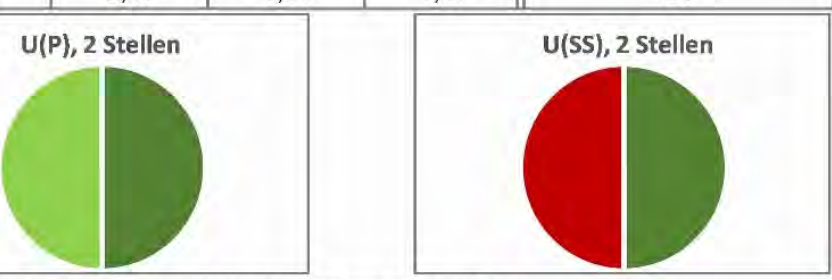

Wirkung der Maßnahme auf die Unfallkosten ( $\left(\mathrm{MW}_{\mathrm{UK}}\right)$

\begin{tabular}{|c|c|c|c||c|}
\hline \hline \multicolumn{2}{|c|}{ mittlere Unfallkosten pro Jahr [€/a] } & \multicolumn{2}{|c|}{ Wirkungsgrad } \\
\hline Kategorie & $\underline{\text { Vorher }}$ & $\underline{\text { Nachher }}$ & $\Delta=\Sigma(\mathrm{V}-\mathrm{N})$ & $\varnothing$ über alle Stellen [\%] \\
\hline UK(P) & 197.917 & 52.567 & 145.350 & 74 \\
\hline UK(SS) & 11.933 & 8.950 & 2.983 & -17 \\
\hline UK(P,SS) & 209.850 & 61.517 & 148.333 & 70 \\
\hline
\end{tabular}

UK(P,SS

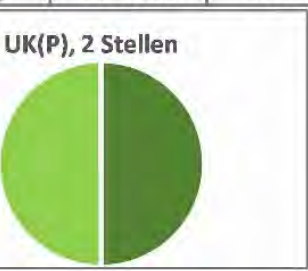

UK(SS), 2 Stellen

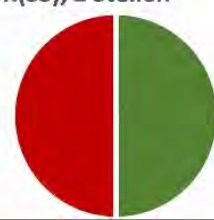

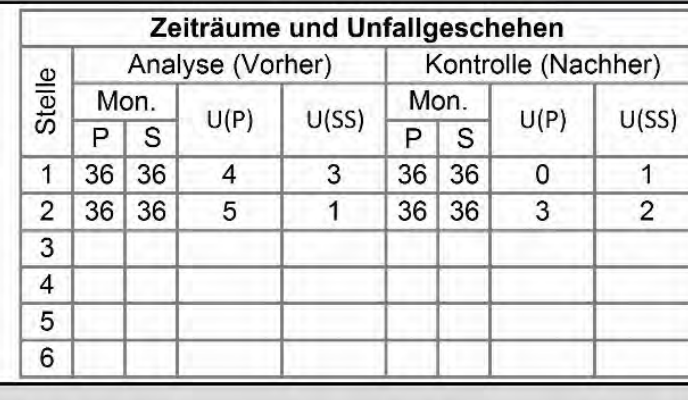

(r)

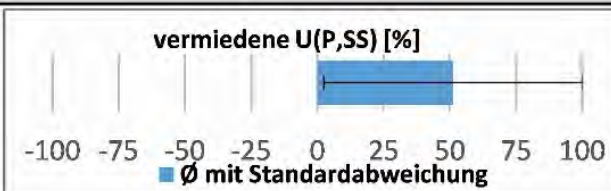

Einteilung Wirkungsgrad

\begin{tabular}{l|l|l|}
\hline Klassen & ob. Grenze [\%] & unt. Grenze [\%]
\end{tabular}

\begin{tabular}{|c|c|c|}
\hline bedeutend & 100 & 60 \\
\hline
\end{tabular}

\begin{tabular}{l|l|l}
\hline erheblich & $<60$ & 30
\end{tabular}

gering

neutral

nachteilig

$<10-10$

\begin{tabular}{l|l}
$<10$ & -10 \\
\hline$<-10$ & offen
\end{tabular}

ffen

\begin{tabular}{|l|c|}
\hline \multicolumn{2}{|c|}{ verwendete Unfallkostensätze [€] } \\
\hline$U(S P)$ & 266.000 \\
\hline$U(L V)$ & 24.700 \\
\hline$U(S S)$ & 17.900 \\
\hline
\end{tabular}

vermiedene UK(P,SS) [\%]

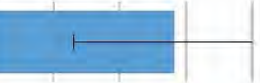

$\begin{array}{lllllllll}-100 & -75 & -50 & -25 & 0 & 25 & 50 & 75 & 100\end{array}$

$\emptyset$ mit Standardabweichung

Nutzen-Kosten-Verhältnis (NKV)

\begin{tabular}{|c|c|c|c|c|c|}
\hline \multicolumn{6}{|c|}{ NKV Spannbreite und Median } \\
\hline 0 & 1000 & 2000 & 3000 & 4000 & 5000 \\
\hline
\end{tabular}

Median Nutzen-Kosten-Verhältnis:

$\varnothing$ Nutzen-Kosten-Verhältnis:

Zusammenfassung und Empfehlung

Maßnahmenbeschreibung/Ziel

Verdeutlichung Streckenverlauf durch das Aufstellen von hinweisenden

Gefahrenzeichen

Zusammenfassung Kennwerte

Ø Abnahme der Unfallanzahl: nur bedingt

$\varnothing$ Abnahme der Unfallkosten: nur bedingt

$\varnothing$ Nutzen-Kosten-Verhältnis: rentabel 


\section{Unfallanalyse der Maßnahmenkategorie: [4], HALT (Z 206 StVO anstelle Z 205 StVO)}

\begin{tabular}{|c|c|c|c|c|c|c|c|c|c|c|c|c|c|}
\hline \multicolumn{14}{|c|}{ Einsatzbedingungen und allgemeine Angaben } \\
\hline \multicolumn{14}{|c|}{ Örtlichkeit: $10, \mathrm{KP}$} \\
\hline Regelungsart: & \multicolumn{13}{|l|}{ VZ } \\
\hline \multirow{2}{*}{\multicolumn{14}{|c|}{$\begin{array}{l}\text { Unfalltyp(en): Einbiegen/Kreuzen } \\
\varnothing \text { Investitionskosten Maßnahme [€]: } 1.800\end{array}$}} \\
\hline & & & & & & & & & & & & & \\
\hline \multicolumn{14}{|c|}{$\varnothing$ jährliche Kosten Maßnahme [€]: 211} \\
\hline \multicolumn{14}{|c|}{ Anzahl ausgewertete Stellen: 5} \\
\hline \multicolumn{14}{|c|}{ Anzahl beteiligte UKo: 4} \\
\hline \multicolumn{14}{|c|}{ Bewertete Unfallkategorien: Kat. 1 bis 6} \\
\hline \multicolumn{14}{|c|}{ Beschreibung Defizite, Konfliktsituationen und Datengrundlage } \\
\hline \multicolumn{5}{|c|}{ Defizite der Verkehrsanlage } & \multicolumn{9}{|c|}{ Zeiträume und Unfallgeschehen } \\
\hline \multirow{4}{*}{\multicolumn{5}{|c|}{$\begin{array}{l}\text { - mangelnde Erkennbarkeit } \\
\text { - optischer Eindruck, dass untergeordnete Straße übergeordnet ist } \\
\text { - schlechte Sichtbeziehung durch bauliche Elemente }\end{array}$}} & \multirow{3}{*}{$\frac{\stackrel{\Phi}{\bar{D}}}{\omega}$} & \multicolumn{4}{|c|}{ Analyse (Vorher) } & \multicolumn{4}{|c|}{ Kontrolle (Nachher) } \\
\hline & & & & & & \multicolumn{2}{|c|}{ Mon. } & \multirow{2}{*}{$\mathrm{U}(\mathrm{P})$} & \multirow{2}{*}{$\mathrm{U}(\mathrm{SS}, \mathrm{LS})$} & \multicolumn{2}{|c|}{ Mon. } & \multirow{2}{*}{$U(P)$} & \multirow{2}{*}{$\mathrm{U}(\mathrm{SS}, \mathrm{LS})$} \\
\hline & & & & & & $\mathrm{P}$ & S & & & $\mathrm{P}$ & S & & \\
\hline & & & & & 1 & 36 & 36 & 4 & 6 & 36 & 36 & 0 & 5 \\
\hline \multirow{5}{*}{\multicolumn{5}{|c|}{$\begin{array}{l}\text { Konflikte } \\
\text { - Einbiegen/Kreuzen-Unfälle } \\
\text { - Bevorrechtigte werden übersehen }\end{array}$}} & 2 & 36 & 36 & 3 & 5 & 36 & 36 & 3 & 7 \\
\hline & & & & & 3 & 36 & 36 & 5 & 6 & 36 & 36 & 2 & 1 \\
\hline & & & & & 4 & 36 & 36 & 2 & 7 & 36 & 36 & 0 & 9 \\
\hline & & & & & 5 & 36 & 36 & 1 & 7 & 36 & 36 & 0 & 1 \\
\hline & & & & & 6 & & & & & & & & \\
\hline Wirkung der $\mathrm{Ma}$ & ßßnahme au & die Unfalla & $\mathrm{zahl}\left(\mathrm{MW}_{\mathrm{u}}\right)$ & & & & & & & & & & \\
\hline mittlere Unfal & llanzahl pro & Jahr [U/a] & & irkungsgrad & & & & vern & niedene $U$ & $J(P, S S$ & S,LS) & [\%] & \\
\hline Kategorie & $\sum$ Vorher & $\sum \underline{\text { Nachher }}$ & $\Delta=\Sigma(\mathrm{V}-\mathrm{N})$ & $\varnothing$ über alle Stellen [\%] & & & & & & & & & -1 \\
\hline$U(P)$ & 1,00 & 0,33 & 0,67 & 72 & & & & & & & & & \\
\hline U(SS,LS) & 2,07 & 1,53 & 0,53 & 23 & & & $00-7-7-3-1$ & $5-50$ & -25 & 0 & 25 & 507 & $5 \quad 100$ \\
\hline $\mathrm{U}(\mathrm{P}, \mathrm{SS}, \mathrm{LS})$ & 3,07 & 1,87 & 1,20 & 37 & & & & mit Sta & idardabw & reichs & ung & $\mathrm{Me}$ & dian \\
\hline & P), 5 Stellen & & & $\mathbf{U}(\mathbf{S S}, \mathrm{LS}), 5$ Stellen & & & & Eint & eilung $\mathrm{V}$ & Virku & ungs & grad & \\
\hline & & & & & & & Klas & sen & ob. Gre & enze & {$[\%]$} & unt. $\mathrm{Gr}$ & enze [\%] \\
\hline & & & & & & & bede & utend & & 00 & & & 50 \\
\hline & & & & & & & erhe & blich & & 60 & & & 30 \\
\hline & & & & & & & gerir & & & 30 & & & 10 \\
\hline & & & & & & & neut & & & 10 & & & 10 \\
\hline & & & & & & & nach & teilig & & -10 & & & fen \\
\hline Wirkung der Ma & ßnahme au & die Unfallk & sten $\left(\mathrm{MW}_{\mathrm{UK}}\right.$ & & & & & & & & & & \\
\hline mittlere Unfal & Ilkosten pro & Jahr [€/a] & & irkungsgrad & & & verw & endete & Unfallko & sten & nsätz & e $[€]$ & \\
\hline Kategorie & Vorher & Nachher & $\Delta=\Sigma(\mathrm{V}-\mathrm{N})$ & $\varnothing$ über alle Stellen [\%] & & & U(SP & & & 62.0 & & & \\
\hline UK(P) & 73.560 & 14.693 & 58.867 & 89 & & & $\mathrm{U}(\mathrm{LV})$ & & & 14.60 & & & \\
\hline UK(SS,LS) & 18.315 & 11.433 & 6.881 & 34 & & & U(SS & & & 15.10 & & & \\
\hline UK(P,SS,LS) & 91.875 & 26.127 & 65.748 & 67 & & & $\mathrm{U}(\mathrm{LS})$ & & & 6.31 & & & \\
\hline
\end{tabular}

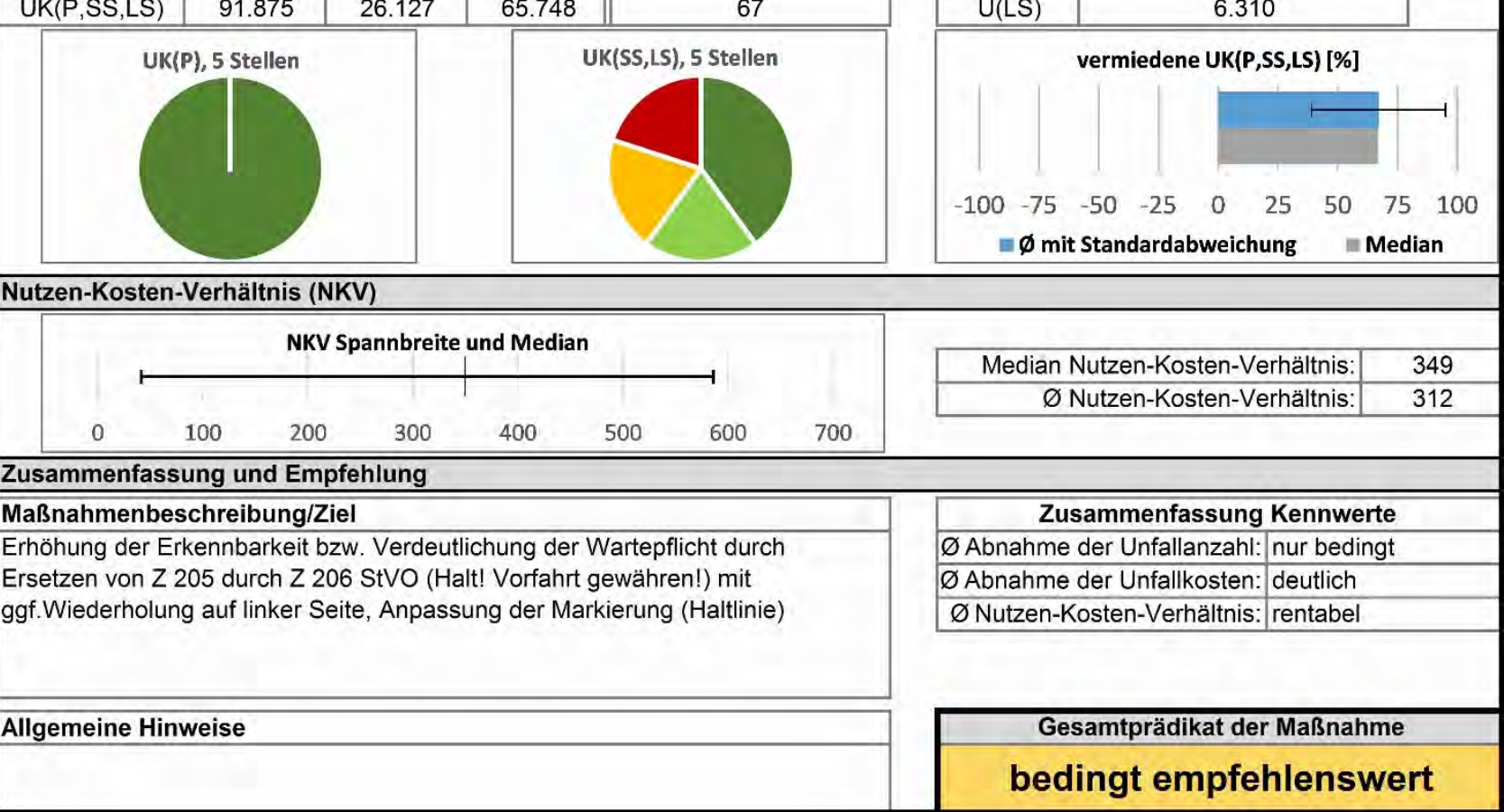




\section{Unfallanalyse der Maßnahmenkategorie: [5], HALT (Z 206 StVO anstelle Z 205 StVO)}

Einsatzbedingungen und allgemeine Angaben

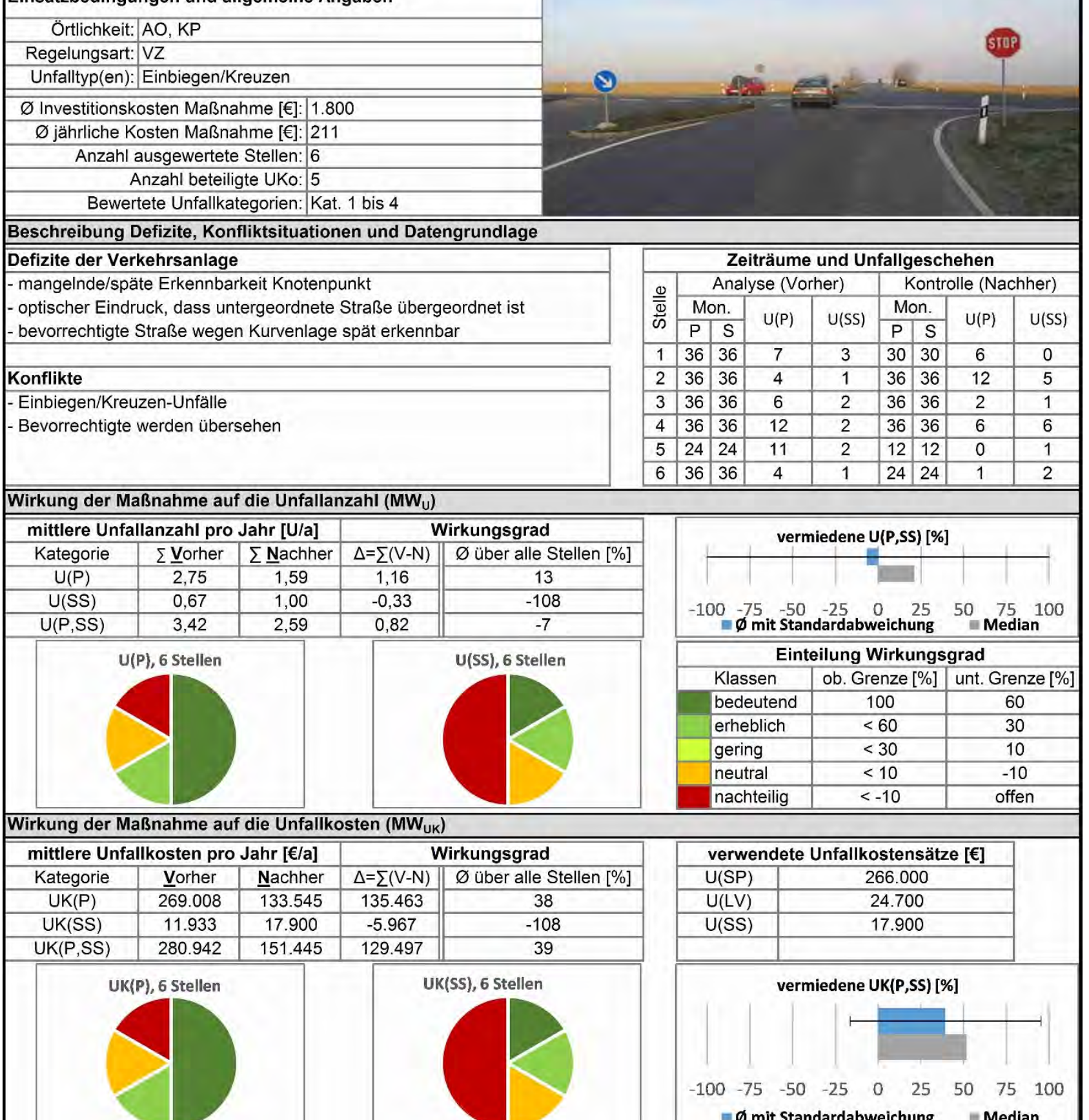

Nutzen-Kosten-Verhältnis (NKV)

NKV Spannbreite und Median

\begin{tabular}{|c|c|c|c|c|c|c|}
\hline \multicolumn{7}{|c|}{ NKV Spannbreite und Median } \\
\hline-1000 & -500 & 0 & 500 & 1000 & 1500 & 2000 \\
\hline
\end{tabular}

$\approx \emptyset$ mit Standardabweichung $\equiv$ Median

Zusammenfassung und Empfehlung

Maßnahmenbeschreibung/Ziel

Erhöhung der Erkennbarkeit bzw. Verdeutlichung der Wartepflicht durch Ersetzen von Z 205 durch Z 206 StVO (Halt! Vorfahrt gewähren!) mit

ggf.Wiederholung auf linker Seite/überkopf, Anpassung der Markierung (Haltlinie)
Median Nutzen-Kosten-Verhältnis: $\varnothing$ Nutzen-Kosten-Verhältnis:

Zusammenfassung Kennwerte

$\varnothing$ Abnahme der Unfallanzahl: nur bedingt

$\varnothing$ Abnahme der Unfallkosten: deutlich

$\varnothing$ Nutzen-Kosten-Verhältnis: rentabel 


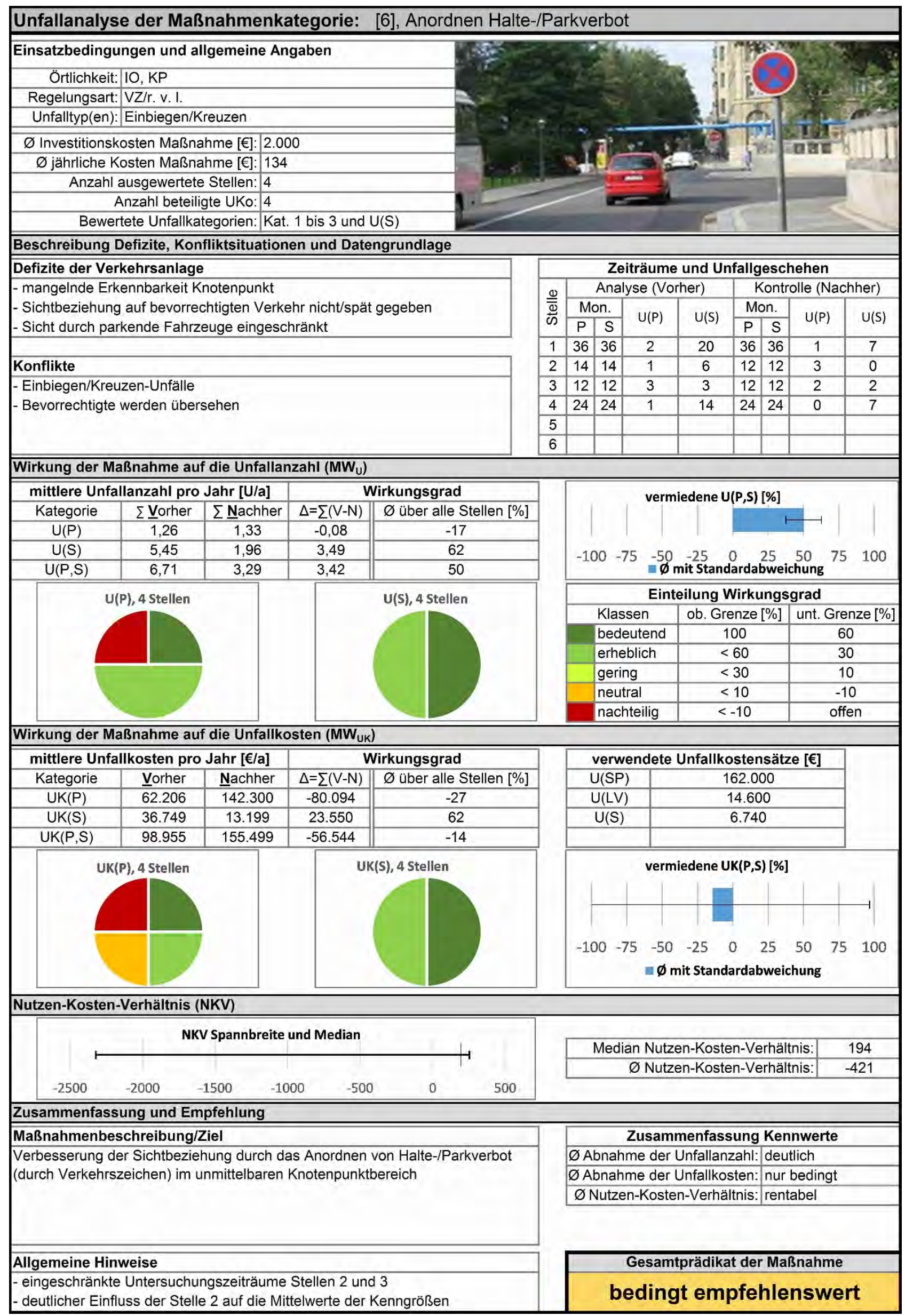




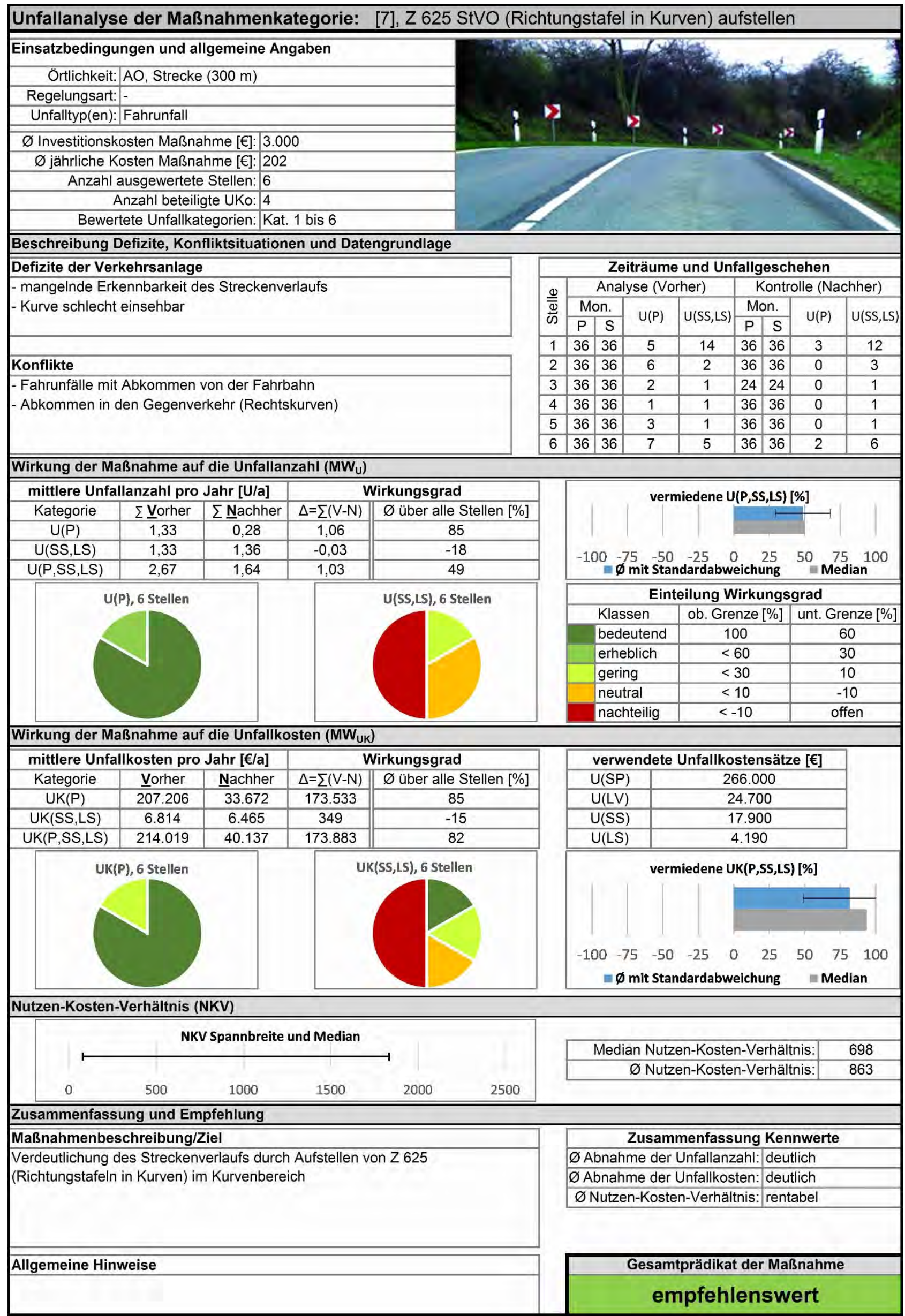


Unfallanalyse der Maßnahmenkategorie: [8], Zusatzzeichen Z 1006-36 StVO (Unfallgefahr) ergänzen Einsatzbedingungen und allgemeine Angaben

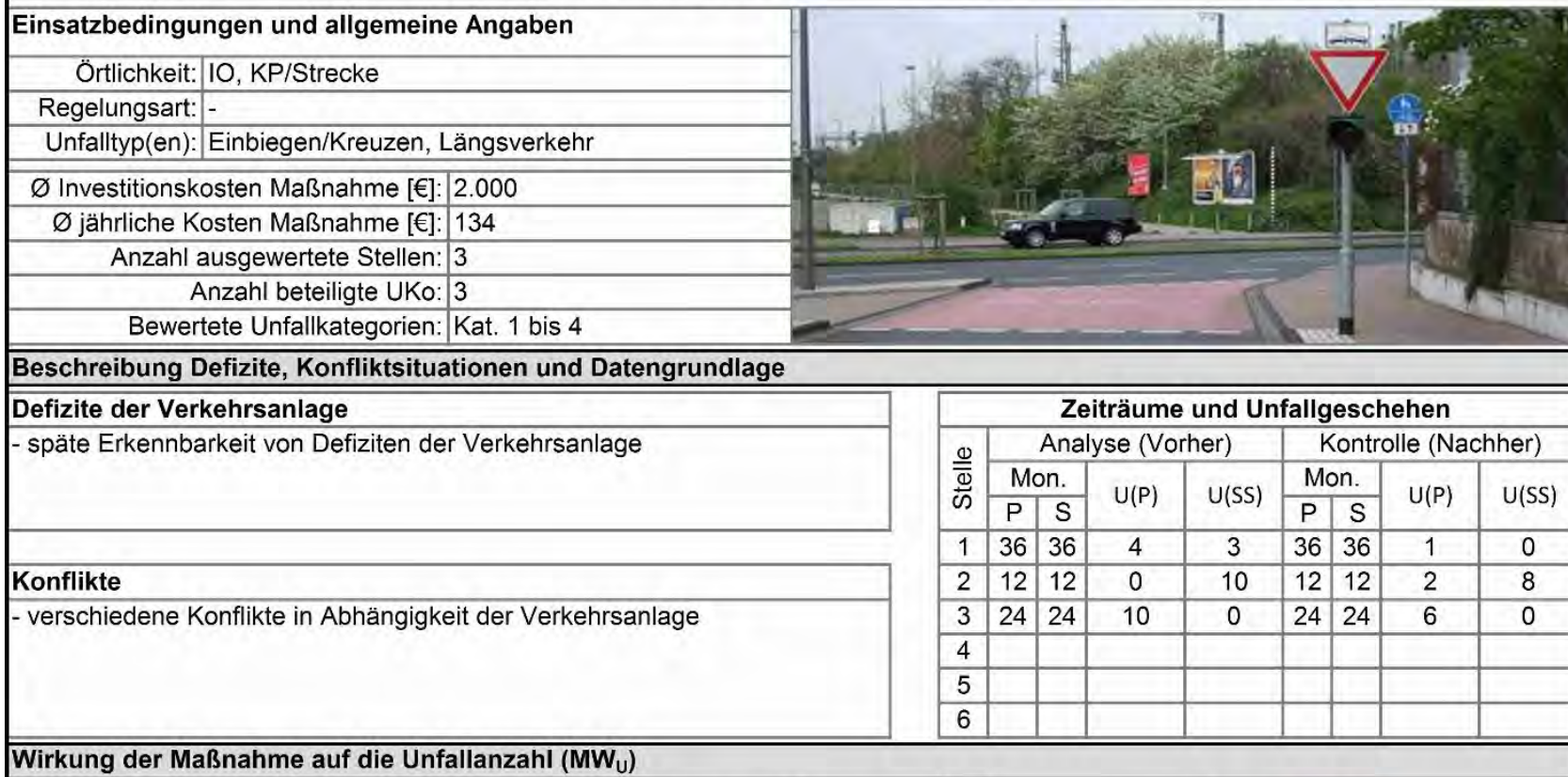

Wirkung der Maßnahme auf die Unfallanzahl $\left(\mathrm{MW}_{\mathrm{U}}\right)$

\begin{tabular}{|c|c|c|c||c|}
\hline \multicolumn{2}{|c|}{ mittlere Unfallanzahl pro Jahr [U/a] } & \multicolumn{2}{c|}{ Wirkungsgrad } \\
\hline Kategorie & $\sum \underline{\text { Vorher }}$ & $\sum$ Nachher & $\Delta=\sum(\mathrm{V}-\mathrm{N})$ & $\varnothing$ über alle Stellen [\%] \\
\hline$U(\mathrm{P})$ & 2,11 & 1,78 & 0,33 & 58 \\
\hline $\mathrm{U}(\mathrm{SS})$ & 3,67 & 2,67 & 1,00 & 40 \\
\hline $\mathrm{U}(\mathrm{P}, \mathrm{SS})$ & 5,78 & 4,44 & 1,33 & 42 \\
\hline
\end{tabular}
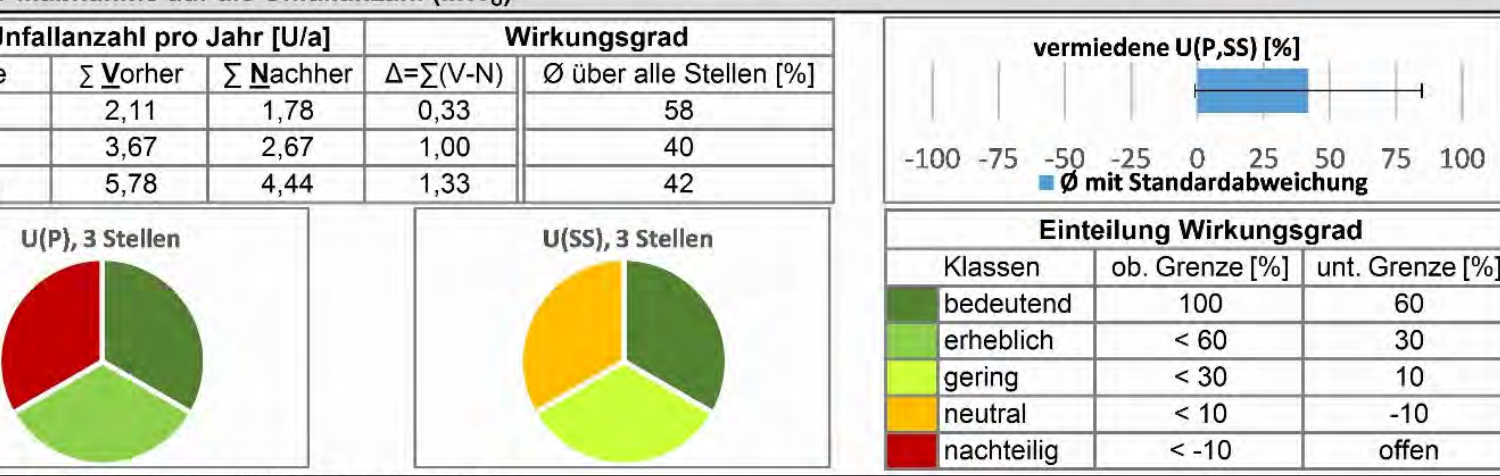

U(SS), 3 Stellen

Einteilung Wirkungsgrad

\begin{tabular}{|l|c|c|}
\hline Klassen & ob. Grenze [\%] & unt. Grenze [\%] \\
\hline bedeutend & 100 & 60 \\
\hline erheblich & $<60$ & 30 \\
\hline gering & $<30$ & 10 \\
\hline neutral & $<10$ & -10 \\
\hline nachteilig & $<-10$ & offen \\
\hline
\end{tabular}

Wirkung der Maßnahme auf die Unfallkosten ( $\left.\mathrm{MW}_{\mathrm{UK}}\right)$

\begin{tabular}{|c|c|c|c||c|}
\hline \hline \multicolumn{2}{|c|}{ mittlere Unfallkosten pro Jahr [€/a] } & \multicolumn{2}{|c|}{ Wirkungsgrad } \\
\hline Kategorie & $\underline{\text { Vorher }}$ & $\underline{\underline{N} a c h h e r}$ & $\Delta=\sum(\mathrm{V}-\mathrm{N})$ & $\varnothing$ über alle Stellen [\%] \\
\hline UK(P) & 63.578 & 25.956 & 37.622 & 68 \\
\hline UK(SS) & 55.367 & 40.267 & 15.100 & 40 \\
\hline UK(P,SS) & 118.944 & 66.222 & 52.722 & 46 \\
\hline
\end{tabular}

\begin{tabular}{l|l} 
UK(P,SS $)$ & 118.944 \\
\hline
\end{tabular}

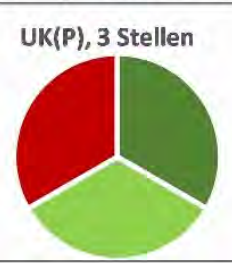

UK(SS), 3 Stellen

\begin{tabular}{|c|c|}
\hline \multicolumn{2}{|c|}{ verwendete Unfallkostensätze [€] } \\
\hline $\mathrm{U}(\mathrm{SP})$ & 162.000 \\
\hline $\mathrm{U}(\mathrm{LV})$ & 14.600 \\
\hline $\mathrm{U}(\mathrm{SS})$ & 15.100 \\
\hline & \\
\hline
\end{tabular}

vermiedene UK(P,SS) [\%]

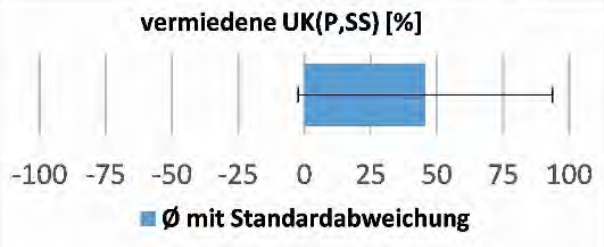

Nutzen-Kosten-Verhältnis (NKV)

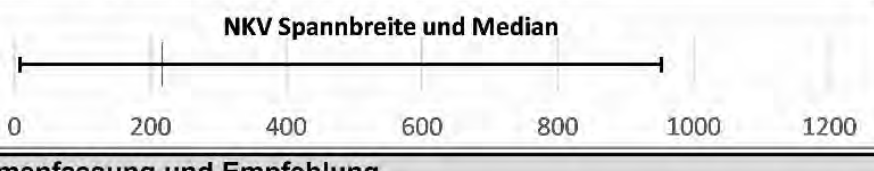

Median Nutzen-Kosten-Verhältnis: 217

$\varnothing$ Nutzen-Kosten-Verhältnis:

Zusammenfassung und Empfehlung

\section{Maßnahmenbeschreibung/Ziel}

(lediglich) Hinweis auf Gefahren durch Anbringen von Verkehrszeichen in der Örtlichkeit, wie z. Bsp. Z 1000-32 StVO (querende Radfahrer aus beiden

Richtungen) oder Z 1006-36 StVO (Unfallgefahr) zur allgemeinen

Aufmerksamkeitserhöhung, weitere Maßnamen sind umzusetzen

Zusammenfassung Kennwerte

$\varnothing$ Abnahme der Unfallanzahl: nur bedingt $\varnothing$ Abnahme der Unfallkosten: nur bedingt

$\varnothing$ Nutzen-Kosten-Verhältnis: rentabel

\section{Allgemeine Hinweise}

- eingeschränkter Analysezeitraum Stelle 2

- Rückschlüsse auf die Wirksamkeit der Maßnahme nicht möglich 


\section{Unfallanalyse der Maßnahmenkategorie: [9], Zusatzzeichen Z 1006-36 StVO (Unfallgefahr) ergänzen}

Einsatzbedingungen und allgemeine Angaben

Örtlichkeit: $A O, K P /$ Strecke

Regelungsart:-

Unfalltyp(en): Einbiegen/Kreuzen, Längsverkehr

$\varnothing$ Investitionskosten Maßnahme [€]: 2.000

$\varnothing$ jährliche Kosten Maßnahme [€]: 134

Anzahl ausgewertete Stellen: 4

Anzahl beteiligte UKo: 3

Bewertete Unfallkategorien:|Kat. 1 bis 4

Beschreibung Defizite, Konfliktsituationen und Datengrundlage

Defizite der Verkehrsanlage

- späte Erkennbarkeit von Defiziten der Verkehrsanlage

Konflikte

- verschiedene Konflikte in Abhängigkeit der Verkehrsanlage

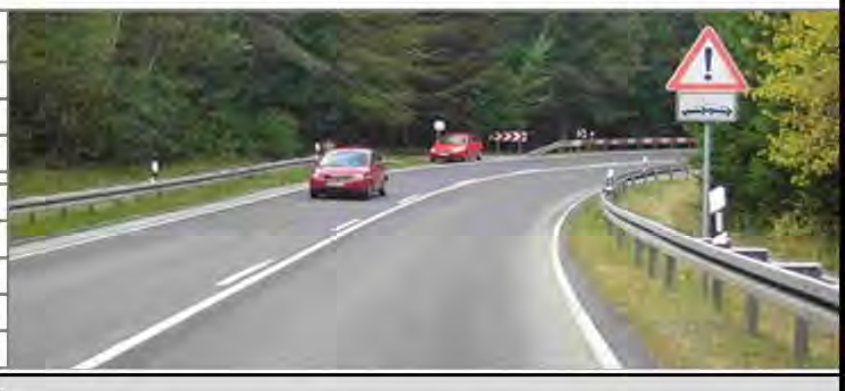

Wirkung der Maßnahme auf die Unfallanzahl $\left(\mathrm{MW}_{\mathrm{U}}\right)$

\begin{tabular}{|c|c|c|c||c|}
\hline \hline \multicolumn{2}{|c|}{ mittlere Unfallanzahl pro Jahr [U/a] } & \multicolumn{2}{|c|}{ Wirkungsgrad } \\
\hline Kategorie & $\Sigma$ Vorher & $\sum$ Nachher & $\Delta=\Sigma(\mathrm{V}-\mathrm{N})$ & $\varnothing$ über alle Stellen [\%] \\
\hline$U(\mathrm{P})$ & 2,53 & 2,34 & 0,19 & -3 \\
\hline $\mathrm{U}(\mathrm{SS})$ & 0,46 & 0,64 & $-0,18$ & -25 \\
\hline $\mathrm{U}(\mathrm{P}, \mathrm{SS})$ & 2,99 & 2,97 & 0,02 & -17 \\
\hline
\end{tabular}

\begin{tabular}{|r|r|}
\hline$U(P), 4$ Stellen \\
\hline
\end{tabular}
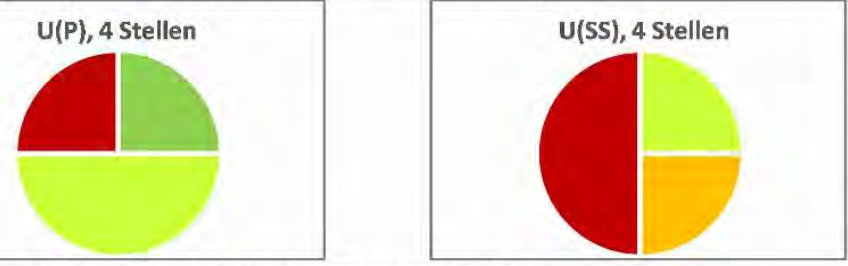

\begin{tabular}{|c|c|c|c|c|c|c|c|c|}
\hline \multicolumn{9}{|c|}{ Zeiträume und Unfallgeschehen } \\
\hline \multirow{3}{*}{$\frac{\frac{\omega}{\Phi}}{\frac{\omega}{\omega}}$} & \multicolumn{4}{|c|}{ Analyse (Vorher) } & \multicolumn{4}{|c|}{ Kontrolle (Nachher) } \\
\hline & \multicolumn{2}{|c|}{ Mon. } & \multirow[b]{2}{*}{$U(P)$} & \multirow[b]{2}{*}{$\mathrm{U}(\mathrm{SS})$} & \multicolumn{2}{|c|}{ Mon. } & \multirow[b]{2}{*}{$U(P)$} & \multirow[b]{2}{*}{$\mathrm{U}(\mathrm{SS})$} \\
\hline & $\mathrm{P}$ & $\mathrm{s}$ & & & $\mathrm{P}$ & $\mathrm{s}$ & & \\
\hline 1 & 36 & 36 & 5 & 0 & 36 & 36 & 2 & 0 \\
\hline 2 & 36 & 36 & 10 & 4 & 24 & 24 & 6 & 2 \\
\hline 3 & 24 & 24 & 8 & 1 & 24 & 24 & 7 & 2 \\
\hline 4 & 32 & 32 & 3 & 0 & 22 & 22 & 4 & 1 \\
\hline 5 & & & & & & & & \\
\hline 6 & & & & & & & & \\
\hline
\end{tabular}

6

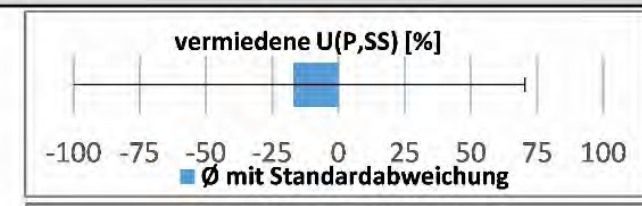

\section{Einteilung Wirkungsgrad}

\begin{tabular}{|l|c|c|}
\hline Klassen & ob. Grenze [\%] & unt. Grenze [\%] \\
\hline bedeutend & 100 & 60 \\
\hline erheblich & $<60$ & 30 \\
\hline gering & $<30$ & 10 \\
\hline neutral & $<10$ & -10 \\
\hline nachteilig & $<-10$ & offen \\
\hline
\end{tabular}

Wirkung der Maßnahme auf die Unfallkosten ( $\mathrm{MW}_{\mathrm{UK}}$ )

\begin{tabular}{|c|c|c|c||c|}
\hline \hline \multicolumn{2}{|c|}{ mittlere Unfallkosten pro Jahr [€/a] } & \multicolumn{2}{c|}{ Wirkungsgrad } \\
\hline Kategorie & $\underline{\text { Vorher }}$ & $\underline{\text { Nachher }}$ & $\Delta=\Sigma(\mathrm{V}-\mathrm{N})$ & $\varnothing$ über alle Stellen [\%] \\
\hline UK(P) & 208.307 & 138.160 & 70.147 & 19 \\
\hline UK(SS) & 8.204 & 11.391 & -3.187 & -19 \\
\hline UK(P,SS) & 216.511 & 149.551 & 66.960 & 20 \\
\hline
\end{tabular}

UK(P,SS

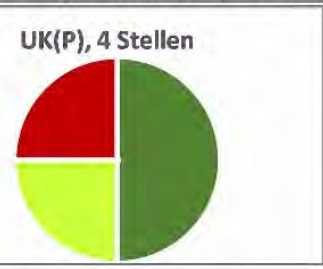

UK(SS), 4 Stellen

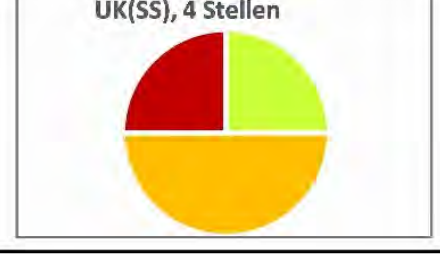

\begin{tabular}{|c|c|}
\hline \multicolumn{2}{|c|}{ verwendete Unfallkostensätze [€] } \\
\hline $\mathrm{U}(\mathrm{SP})$ & 266.000 \\
\hline $\mathrm{U}(\mathrm{LV})$ & 24.700 \\
\hline $\mathrm{U}(\mathrm{SS})$ & 17.900 \\
\hline & \\
\hline
\end{tabular}

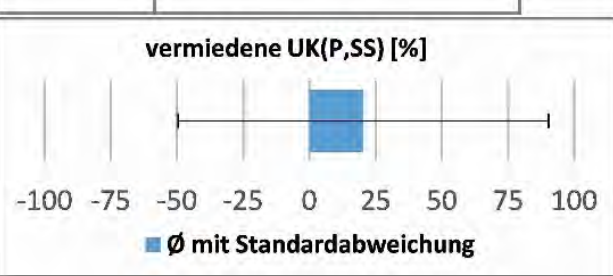

Nutzen-Kosten-Verhältnis (NKV)

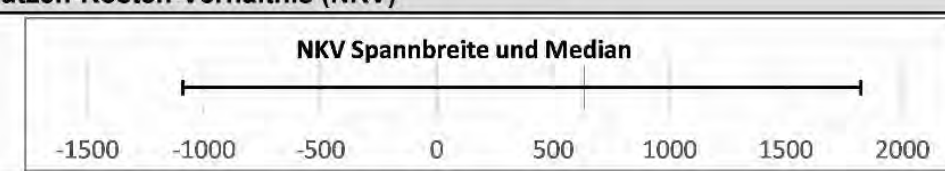

Zusammenfassung und Empfehlung

\section{Maßnahmenbeschreibung/Ziel}

(lediglich) Hinweis auf allgemeine Gefahren, die als solches nicht unmittelbar wahrnehmbar sind, durch Aufstellen Z 1006-36 StVO (Unfallgefahr), weitere

Maßnahmen sind umzusetzen

\begin{tabular}{r|r}
\hline Median Nutzen-Kosten-Verhältnis: & 632 \\
$\varnothing$ Nutzen-Kosten-Verhältnis: & 498 \\
\hline
\end{tabular}

\section{Allgemeine Hinweise}

- deutlicher Einfluss der Stelle 4 auf den $\varnothing$ Wirkungsgrad U(P,SS)

- Umsetzung als einzelne Maßnahme wird nicht empfohlen

Zusammenfassung Kennwerte

$\varnothing$ Abnahme der Unfallanzahl: nicht gegeben

$\varnothing$ Abnahme der Unfallkosten: nur bedingt

$\varnothing$ Nutzen-Kosten-Verhältnis: rentabel 


\section{Unfallanalyse der Maßnahmenkategorie: [10], Verkehrszeichen anpassen}

Einsatzbedingungen und allgemeine Angaben

Örtlichkeit: $10, \mathrm{KP} /$ Strecke

Regelungsart:-

Unfalltyp(en): Fahrunfall, Abbiegen, Einbiegen/Kreuzen

$\varnothing$ Investitionskosten Maßnahme [€]: 1.000

$\varnothing$ jährliche Kosten Maßnahme [ $€$ : 67 Anzahl ausgewertete Stellen: 4 Anzahl beteiligte UKo: 2

Bewertete Unfallkategorien:|Kat. 1 bis 4

Beschreibung Defizite, Konfliktsituationen und Datengrundlage

Defizite der Verkehrsanlage

Defizite in Zusammenhang mit der Verkehr

- überhöhte Geschwindigkeit

- späte Erkennbarkeit der Verkehrsführung

\section{Konflikte}

- verschiedene Konflikte in Abhängigkeit der Verkehrsanlage

- u. a. Fahrunfall, Abbiegen, Einbiegen/Kreuzen

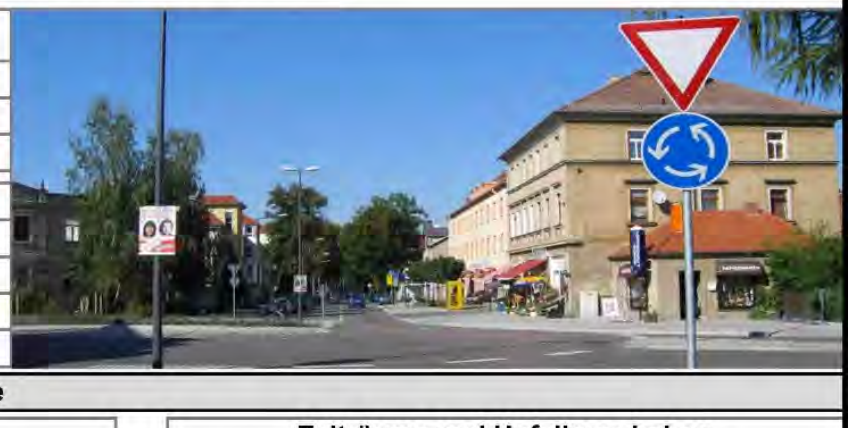

Wirkung der Maßnahme auf die Unfallanzahl (MW

mittlere Unfallanzahl pro Jahr [U/a]

\begin{tabular}{|c|c|c|c||c|}
\hline \multicolumn{2}{|c|}{ mittlere Unfallanzahl pro Jahr [U/a] } & \multicolumn{2}{|c|}{ Wirkungsgrad } \\
\hline Kategorie & $\Sigma$ Vorher & $\sum$ Nachher & $\Delta=\sum(\mathrm{V}-\mathrm{N})$ & $\varnothing$ über alle Stellen [\%] \\
\hline $\mathrm{U}(\mathrm{P})$ & 1,33 & 0,67 & 0,67 & 40 \\
\hline $\mathrm{U}(\mathrm{SS})$ & 0,79 & 0,13 & 0,67 & 88 \\
\hline $\mathrm{U}(\mathrm{P}, \mathrm{SS})$ & 2,13 & 0,79 & 1,33 & 59 \\
\hline
\end{tabular}

\begin{tabular}{|c|c|c|c|c|c|c|c|c|}
\hline \multirow{3}{*}{$\frac{\stackrel{0}{\bar{D}}}{\bar{\omega}}$} & \multicolumn{4}{|c|}{ Analyse (Vorher) } & \multicolumn{4}{|c|}{ Kontrolle (Nachher) } \\
\hline & \multicolumn{2}{|c|}{ Mon. } & \multirow{2}{*}{$U(P)$} & \multirow{2}{*}{$\mathrm{U}(\mathrm{SS})$} & \multicolumn{2}{|c|}{ Mon. } & \multirow{2}{*}{$U(P)$} & \multirow{2}{*}{$\mathrm{U}(\mathrm{SS})$} \\
\hline & $\mathrm{P}$ & S & & & $P$ & $\mathrm{~S}$ & & \\
\hline 1 & 36 & 36 & 3 & 1 & 36 & 36 & 0 & 0 \\
\hline 2 & 36 & 36 & 4 & 4 & 36 & 36 & 3 & 0 \\
\hline 3 & 24 & 24 & 2 & 1 & 36 & 36 & 5 & 0 \\
\hline 4 & 24 & 24 & 4 & 2 & 24 & 24 & 0 & 1 \\
\hline 5 & & & & & & & & \\
\hline 6 & & & & & & & & \\
\hline
\end{tabular}
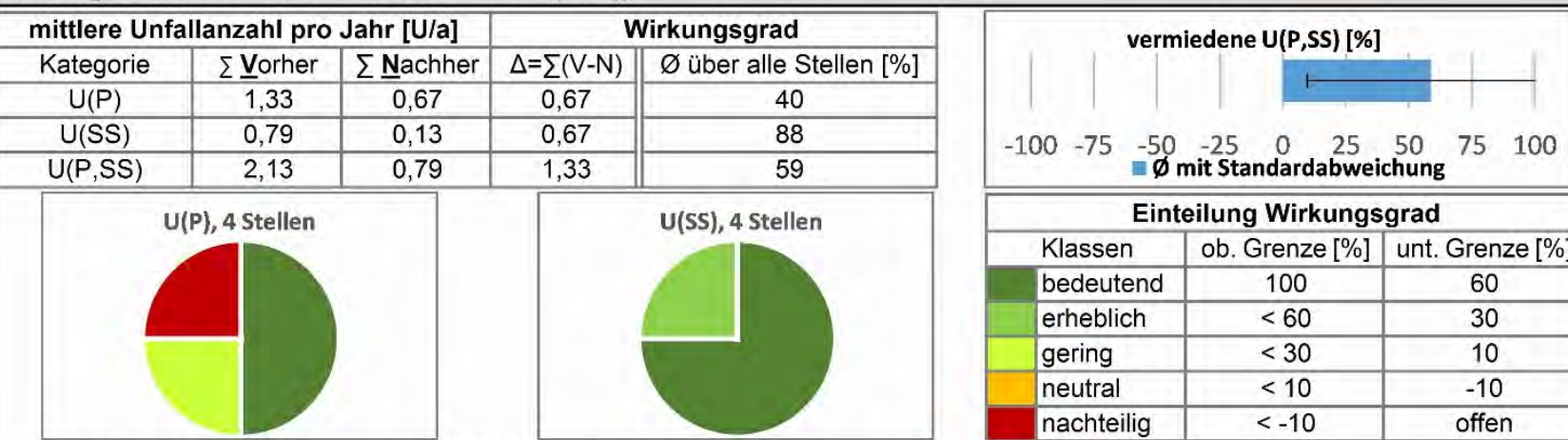

$\mathrm{U}(\mathrm{SS}), 4$ Stellen

Einteilung Wirkungsgrad

\begin{tabular}{|l|c|c|}
\hline \hline \multicolumn{3}{|c|}{ Einteilung Wirkungsgrad } \\
\hline Klassen & ob. Grenze [\%] & unt. Grenze [\%] \\
\hline bedeutend & 100 & 60 \\
\hline erheblich & $<60$ & 30 \\
\hline gering & $<30$ & 10 \\
\hline neutral & $<10$ & -10 \\
\hline nachteilig & $<-10$ & offen \\
\hline
\end{tabular}

Wirkung der Maßnahme auf die Unfallkosten ( $\left(\mathrm{MW}_{\mathrm{UK}}\right)$

\begin{tabular}{|c|c|c|c||c|}
\hline \hline \multicolumn{2}{|c|}{ mittlere Unfallkosten pro Jahr [€/a] } & \multicolumn{2}{c|}{ Wirkungsgrad } \\
\hline Kategorie & Vorher & $\underline{\text { Nachher }}$ & $\Delta=\Sigma(\mathrm{V}-\mathrm{N})$ & $\varnothing$ über alle Stellen [\%] \\
\hline UK(P) & 56.317 & 22.017 & 34.300 & 11 \\
\hline UK(SS) & 11.954 & 1.888 & 10.067 & 88 \\
\hline UK(P,SS $)$ & 68.271 & 23.904 & 44.367 & 52 \\
\hline
\end{tabular}

UK(P,SS
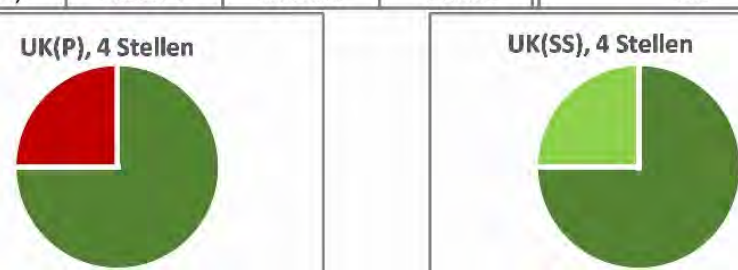

\begin{tabular}{|l|c|}
\hline \multicolumn{2}{|c|}{ verwendete Unfallkostensätze [€] } \\
\hline $\mathrm{U}(\mathrm{SP})$ & 162.000 \\
\hline $\mathrm{U}(\mathrm{LV})$ & 14.600 \\
\hline $\mathrm{U}(\mathrm{SS})$ & 15.100 \\
\hline & \\
\hline
\end{tabular}

vermiedene UK(P,SS) [\%]

Nutzen-Kosten-Verhältnis (NKV)

\section{NKV Spannbreite und Median}

\begin{tabular}{lrrrrr} 
& \multicolumn{5}{c}{ NKV Spannbreite und Median } \\
\hline & 0 & 500 & 1000 & 1500 & 2000 \\
\hline
\end{tabular}

Zusammenfassung und Empfehlung

\section{Maßnahmenbeschreibung/Ziel}

Verbesserung der Erkennbarkeit unterstützender Verkehrszeichen durch größere Form, Ausstattung mit stärker reflektierender Folie oder Aufbringen auf weißer

Trägertafel, im Vorfeld sind die Rahmenbedingungen zu prüfen

Zusammenfassung Kennwerte

$\varnothing$ Abnahme der Unfallanzahl: nur bedingt

$\varnothing$ Abnahme der Unfallkosten: nur bedingt

$\varnothing$ Nutzen-Kosten-Verhältnis: rentabel 


\section{Unfallanalyse der Maßnahmenkategorie: [11], Verkehrszeichen anpassen}

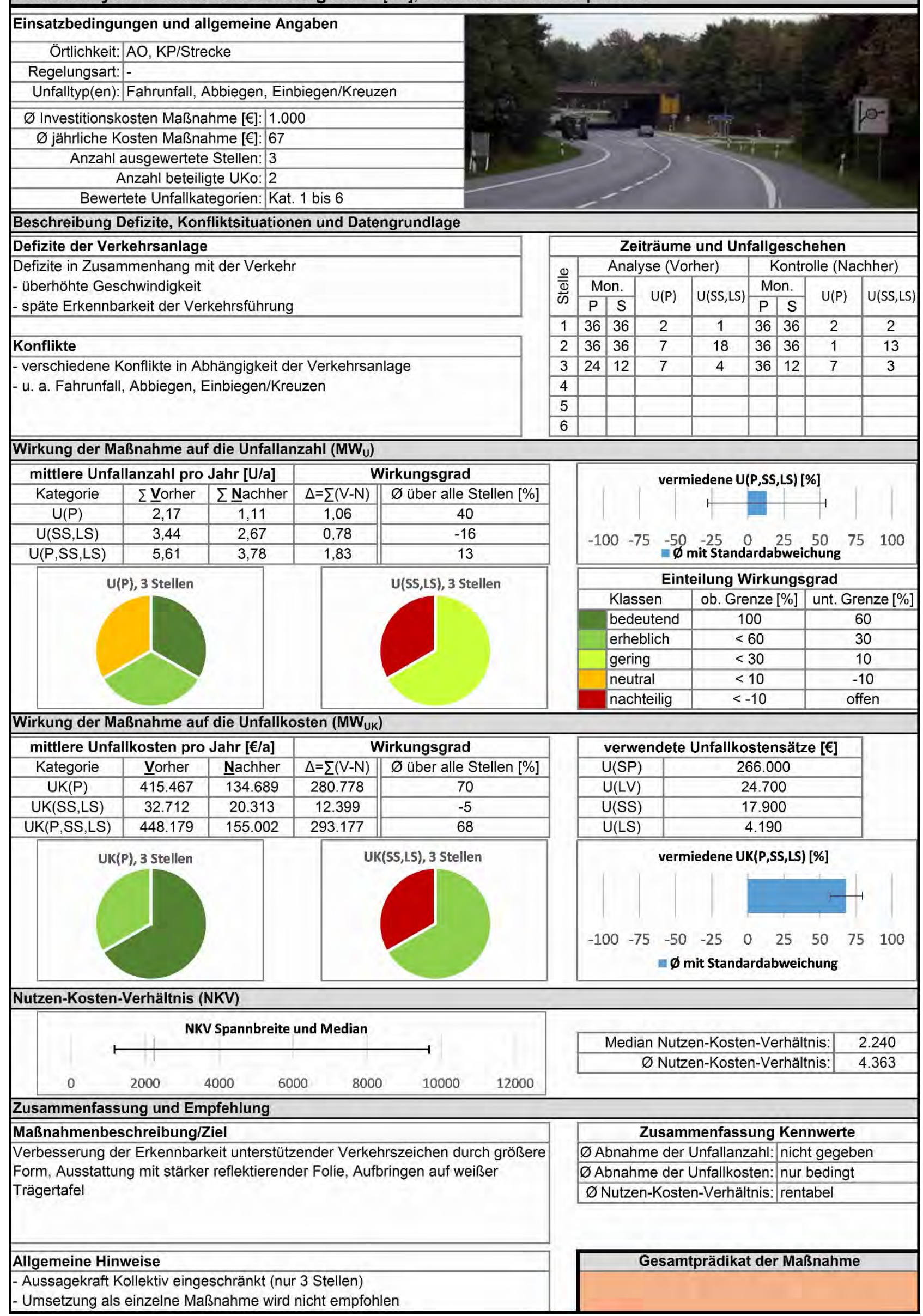




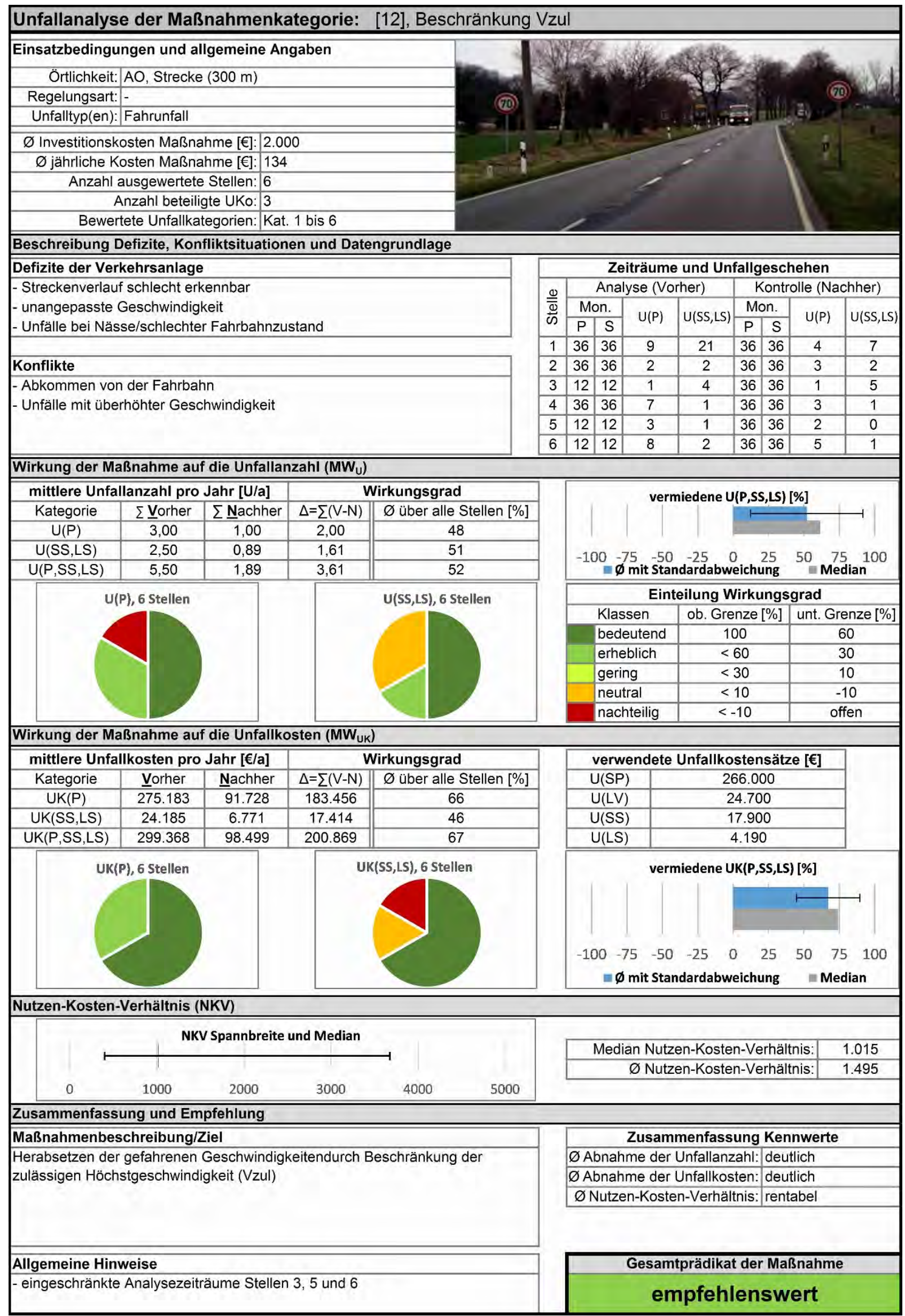




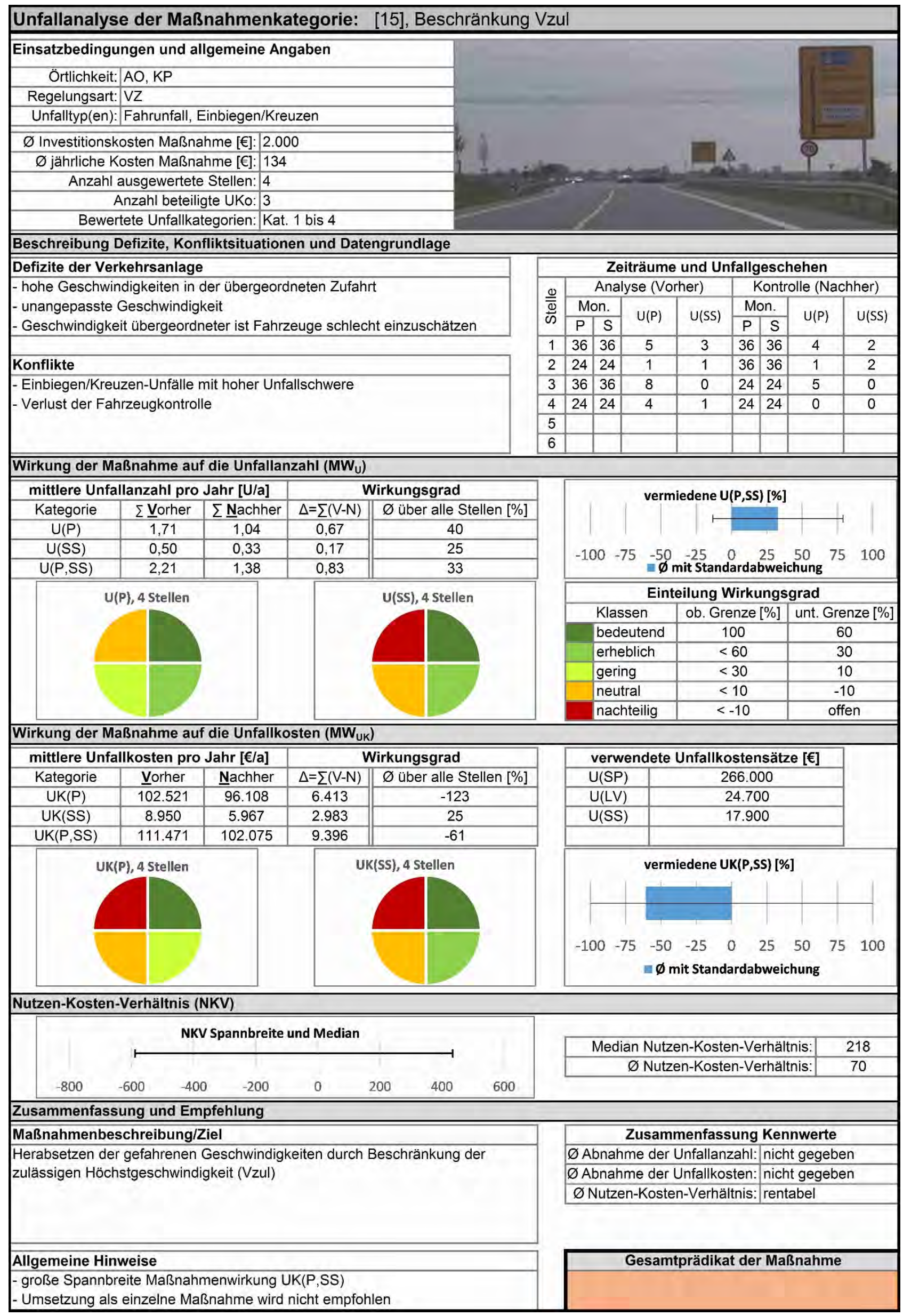


Unfallanalyse der Maßnahmenkategorie: [16], Überwachung zulässige Höchstgeschwindigkeit mit OGÜ Einsatzbedingungen und allgemeine Angaben

\begin{tabular}{|r|l}
\hline Örtlichkeit: & AO, KP \\
\hline Regelungsart: & VZ \\
\hline Unfalltyp(en): & Abbiegen, Einbiegen/Kreuzen \\
\hline$\varnothing$ Investitionskosten Maßnahme [€]: & 70.000 \\
\hline$\varnothing$ jährliche Kosten Maßnahme [€]: 8.204 \\
\hline Anzahl ausgewertete Stellen: & 2 \\
\hline Anzahl beteiligte UKo: & 2 \\
\hline Bewertete Unfallkategorien: & Kat. 1 bis 4 \\
\hline
\end{tabular}

Beschreibung Defizite, Konfliktsituationen und Datengrundlage Defizite der Verkehrsanlage

- hohe Geschwindigkeiten entgegenkommender Fahrzeuge

- zulässige Höchstgeschwindigkeit wird häufig überschritten

- kurviger Streckenverlauf

\section{Konflikte \\ - Einbiegen/Kreuzen \\ - Abbiege-Unfälle mit Entgegenkommenden}

Wirkung der Maßnahme auf die Unfallanzahl (MW $\mathrm{MW}_{\mathrm{U}}$

\begin{tabular}{|c|c|c|c||c|}
\hline \hline \multicolumn{2}{|c|}{ mittlere Unfallanzahl pro Jahr [U/a] } & \multicolumn{2}{|c|}{ Wirkungsgrad } \\
\hline Kategorie & $\Sigma$ Vorher & $\Sigma$ Nachher & $\Delta=\Sigma(\mathrm{V}-\mathrm{N})$ & $\varnothing$ über alle Stellen [\%] \\
\hline $\mathrm{U}(\mathrm{P})$ & 1,42 & 1,50 & $-0,08$ & 19 \\
\hline $\mathrm{U}(\mathrm{SS})$ & 1,76 & 0,17 & 1,59 & 91 \\
\hline $\mathrm{U}(\mathrm{P}, \mathrm{SS})$ & 3,18 & 1,67 & 1,51 & 55 \\
\hline
\end{tabular}
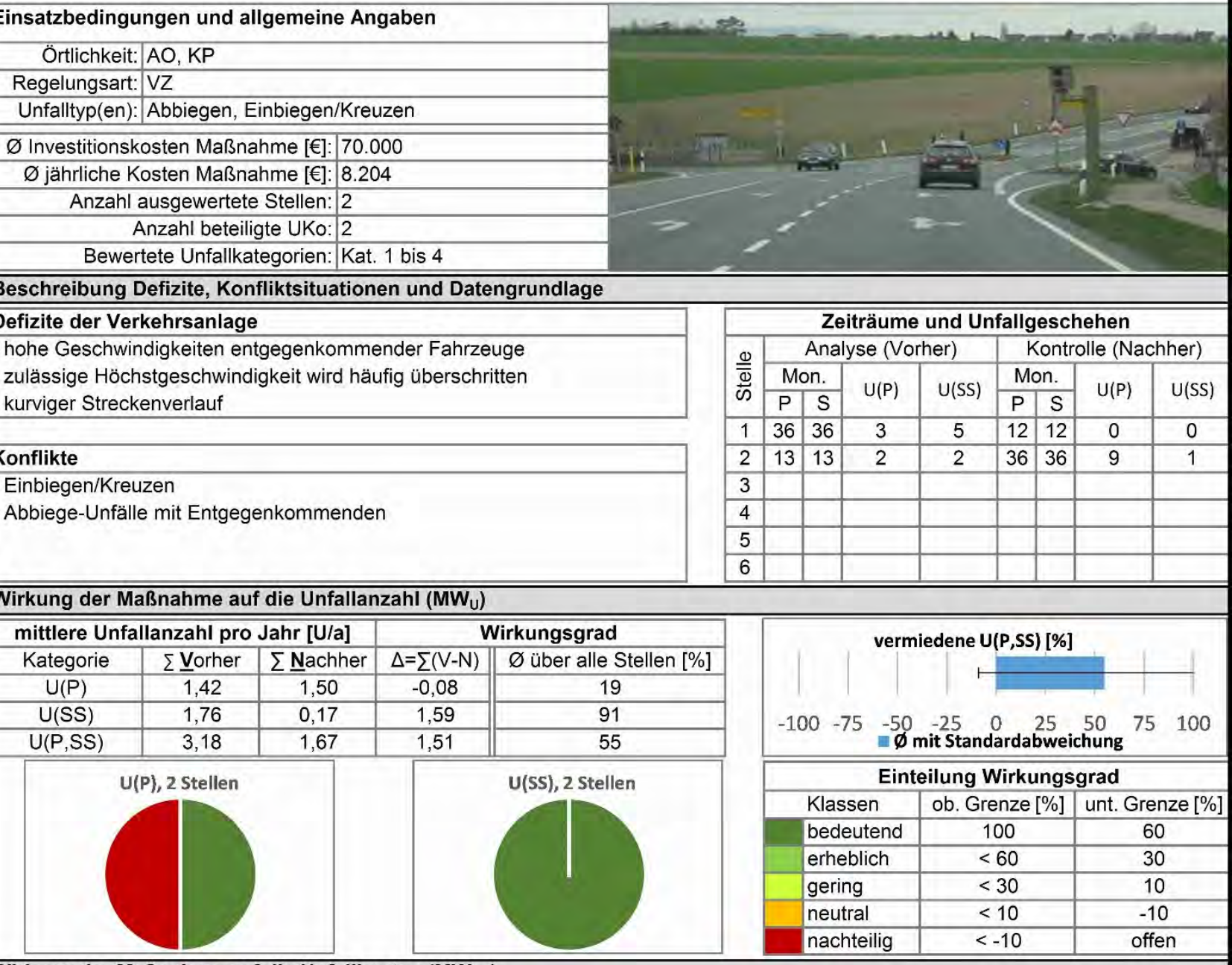

Wirkung der Maßnahme auf die Unfallkosten ( $\left.\mathrm{MW}_{\mathrm{UK}}\right)$

\begin{tabular}{|c|c|c|c||c|}
\hline \hline \multicolumn{2}{|c|}{ mittlere Unfallkosten pro Jahr [€/a] } & \multicolumn{2}{|c|}{ Wirkungsgrad } \\
\hline Kategorie & $\underline{\text { Vorher }}$ & $\underline{\text { Nachher }}$ & $\Delta=\Sigma(\mathrm{V}-\mathrm{N})$ & $\varnothing$ über alle Stellen [\%] \\
\hline UK(P) & 146.519 & 37.050 & 109.469 & 86 \\
\hline UK(SS) & 31.440 & 2.983 & 28.456 & 91 \\
\hline UK(P,SS) & 177.959 & 40.033 & 137.926 & 87 \\
\hline
\end{tabular}

UK(P,SS

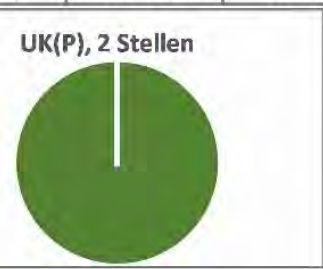

UK(SS), 2 Stellen

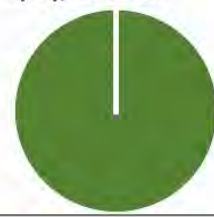

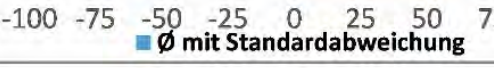

Einteilung Wirkungsgrad

\begin{tabular}{|l|c|c|}
\hline Klassen & ob. Grenze [\%] & unt. Grenze [\%] \\
\hline bedeutend & 100 & 60 \\
\hline erheblich & $<60$ & 30 \\
\hline gering & $<30$ & 10 \\
\hline neutral & $<10$ & -10 \\
\hline nachteilig & $<-10$ & offen \\
\hline
\end{tabular}

\begin{tabular}{|l|c|}
\hline \multicolumn{2}{|c|}{ verwendete Unfallkostensätze [€] } \\
\hline$U(S P)$ & 266.000 \\
\hline$U(L V)$ & 24.700 \\
\hline$U(S S)$ & 17.900 \\
\hline & \\
\hline \hline
\end{tabular}

vermiedene UK(P,SS) [\%]

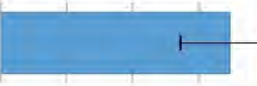

$\begin{array}{lllllllll}-100 & -75 & -50 & -25 & 0 & 25 & 50 & 75 & 100\end{array}$

$\emptyset$ mit Standardabweichung

Nutzen-Kosten-Verhältnis (NKV)

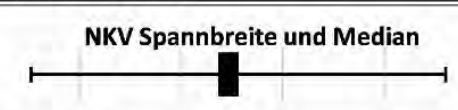

$\begin{array}{lllllllll}0 & 5 & 10 & 15 & 20 & 25 & 30 & 35 & 40\end{array}$

Zusammenfassung und Empfehlung

Maßnahmenbeschreibung/Ziel

Durchsetzen der zulässigen Höchstgeschwindigkeit durch ortsfeste

Geschwindigkeitsüberwachung (OGÜ)

Zusammenfassung Kennwerte

$\varnothing$ Abnahme der Unfallanzahl: nur bedingt

$\varnothing$ Abnahme der Unfallkosten: nur bedingt

$\varnothing$ Nutzen-Kosten-Verhältnis: rentabel 
Unfallanalyse der Maßnahmenkategorie: [17], Überwachung zulässige Höchstgeschwindigkeit mit OGÜ Einsatzbedingungen und allgemeine Angaben

\begin{tabular}{|r|l|}
\hline Örtlichkeit: & $\mathrm{AO}$, Strecke $(300 \mathrm{~m})$ \\
\hline Regelungsart: & - \\
\hline Unfalltyp(en): & Fahrunfall \\
\hline$\varnothing$ Investitionskosten Maßnahme [€]: & 70.000 \\
\hline$\varnothing$ jährliche Kosten Maßnahme [€]: & 8.204 \\
\hline Anzahl ausgewertete Stellen: & 4 \\
\hline Anzahl beteiligte UKo: & 3 \\
\hline Bewertete Unfallkategorien: & Kat. 1 bis 3 \\
\hline
\end{tabular}

Beschreibung Defizite, Konfliktsituationen und Datengrundlage

Defizite der Verkehrsanlage

- hohe Geschwindigkeiten

- zulässige Höchstgeschwindigkeit wird häufig überschritten

- u. a. kurviger Streckenverlauf

Konflikte

- Verlust der Fahrzeugkontrolle

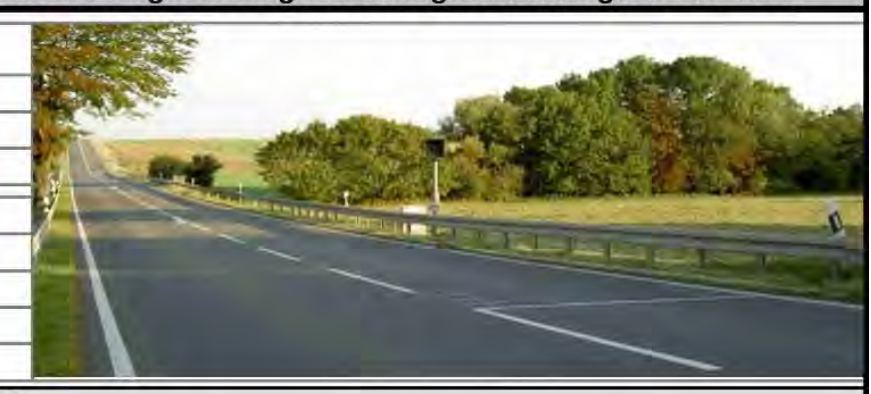

\begin{tabular}{|c|c|c|c|c|}
\hline \multicolumn{5}{|c|}{ Wirkung der Maßnahme auf die Unfallanzahl $\left(\mathrm{MW}_{\mathrm{U}}\right)$} \\
\hline \multicolumn{3}{|c|}{ mittlere Unfallanzahl pro Jahr [U/a] } & \multicolumn{2}{|c|}{ Wirkungsgrad } \\
\hline Kategorie & $\sum \underline{\text { Vorher }}$ & $\sum \underline{\text { Nachher }}$ & $\Delta=\Sigma(\mathrm{V}-\mathrm{N})$ & $\varnothing$ über alle Stellen [\%] \\
\hline$U(P)$ & 3,71 & 1,96 & 1,75 & 48 \\
\hline & & & & 91 \\
\hline $\mathrm{U}(\mathrm{P}, \mathrm{SS})$ & 3,71 & 1,96 & 1,75 & 48 \\
\hline
\end{tabular}

\begin{tabular}{|c|c|c|c|c|c|c|}
\hline \multicolumn{7}{|c|}{ Zeiträume und Unfallgeschehen } \\
\hline \multirow{3}{*}{$\frac{\stackrel{\Phi}{\Phi}}{\omega}$} & \multicolumn{3}{|c|}{ Analyse (Vorher) } & \multicolumn{3}{|c|}{ Kontrolle (Nachher) } \\
\hline & \multicolumn{2}{|c|}{ Mon. } & \multirow{2}{*}{$U(P)$} & Mon. & \multirow{2}{*}{$U(P)$} & \\
\hline & $P$ & $S$ & & S & & \\
\hline 1 & 36 & 36 & 12 & 36 & 12 & \\
\hline 2 & 24 & 24 & 5 & 36 & 3 & \\
\hline 3 & 36 & 36 & 15 & 24 & 3 & \\
\hline 4 & 36 & 0 & 10 & 36 & 4 & \\
\hline 5 & & & & & & \\
\hline 6 & & & & & & \\
\hline
\end{tabular}

Wirkung der Maßnahme auf die Unfallanzahl $\left(\mathrm{MW}_{\mathrm{U}}\right)$

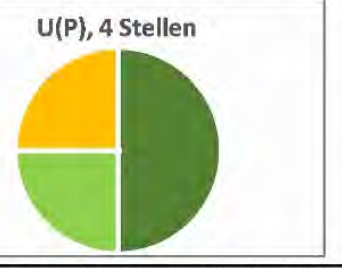

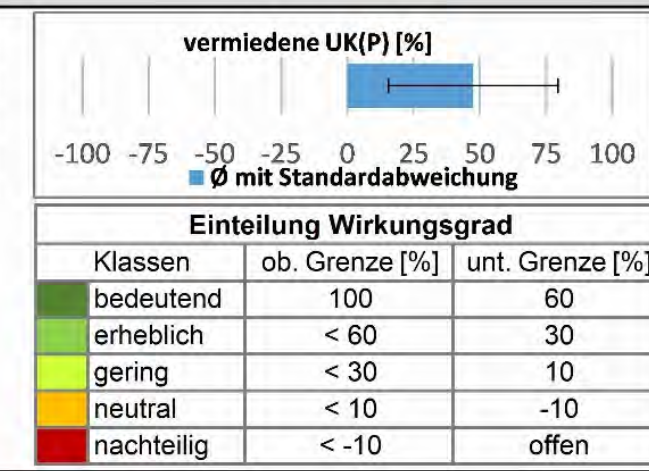

Wirkung der Maßnahme auf die Unfallkosten ( $\mathrm{MW}_{\mathrm{UK}}$ )

\begin{tabular}{|c|c|c|c||c|}
\hline \hline \multicolumn{2}{|c|}{ mittlere Unfallkosten pro Jahr [€/a] } & \multicolumn{2}{c|}{ Wirkungsgrad } \\
\hline Kategorie & $\underline{\text { Vorher }}$ & $\underline{\text { Nachher }}$ & $\Delta=\Sigma(\mathrm{V}-\mathrm{N})$ & $\varnothing$ über alle Stellen [\%] \\
\hline UK(P) & 433.438 & 339.942 & 93.496 & 32 \\
\hline & & & & 91 \\
\hline UK(P) & 433.438 & 339.942 & 93.496 & 32 \\
\hline
\end{tabular}

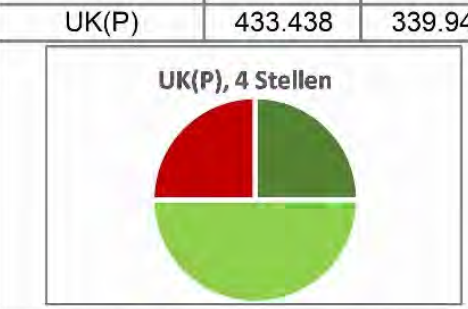

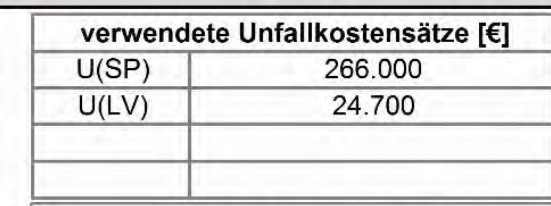

vermiedene UK(P) [\%]

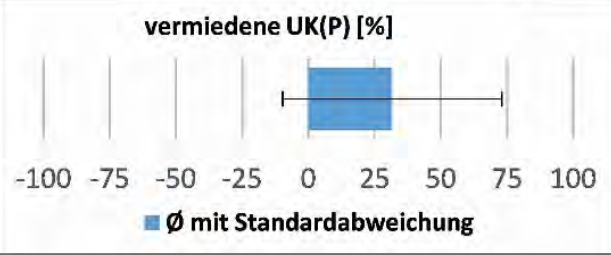

Nutzen-Kosten-Verhältnis (NKV)

Nutzen-Kosten-Verhältnis (NKV)
NKV Spannbreite und Median

$-30$

$-10$

0

10

20

30

40

Zusammenfassung und Empfehlung

Maßnahmenbeschreibung/Ziel

Durchsetzen der zulässigen Höchstgeschwindigkeit durch ortsfeste

Geschwindigkeitsüberwachung (OGÜ)

Zusammenfassung Kennwerte

$\varnothing$ Abnahme der Unfallanzahl: nur bedingt

$\varnothing$ Abnahme der Unfallkosten: nur bedingt

$\varnothing$ Nutzen-Kosten-Verhältnis: rentabel 


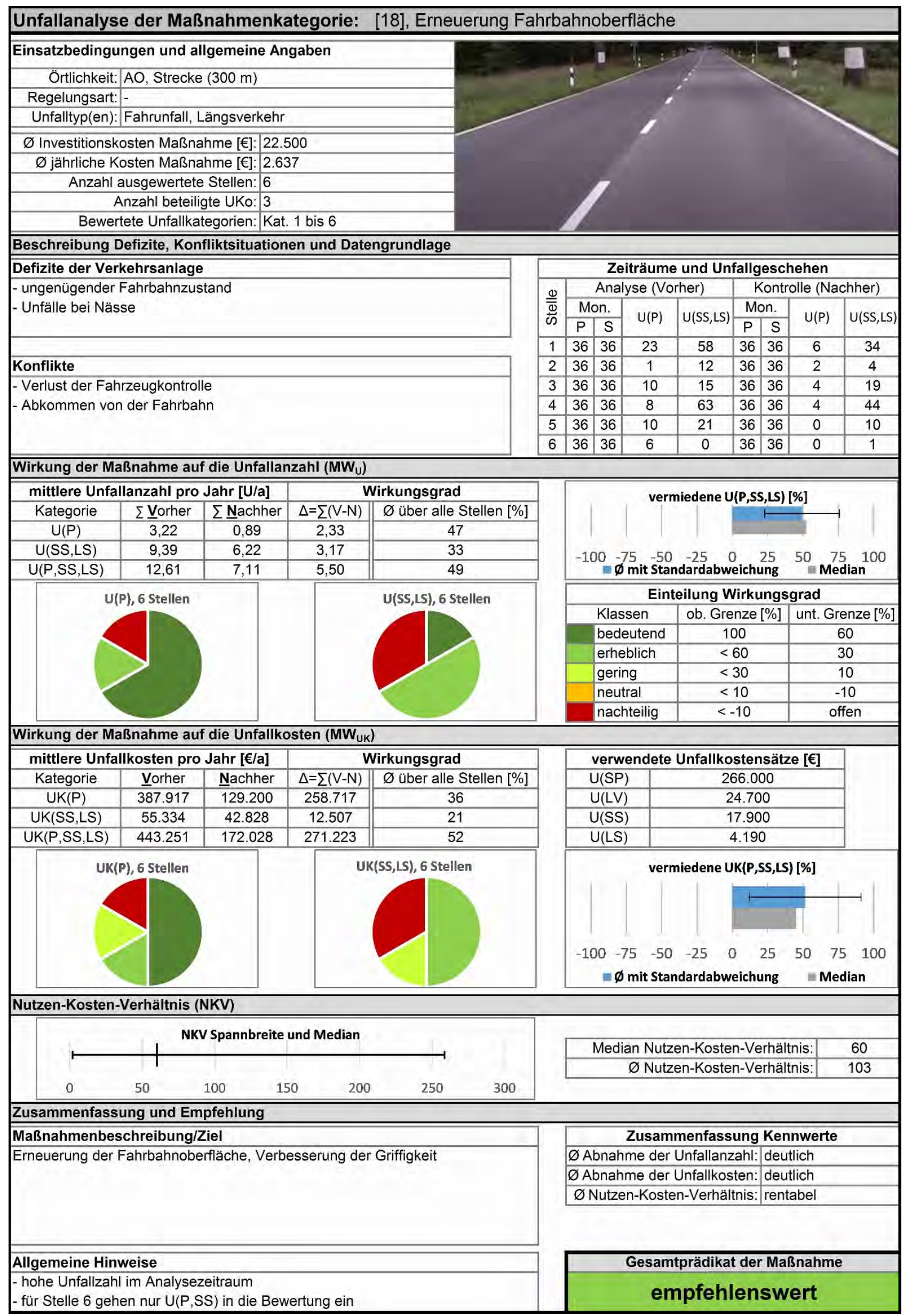




\section{Unfallanalyse der Maßnahmenkategorie: [19], Zusätzlichen Signalgeber anordnen}

Einsatzbedingungen und allgemeine Angaben

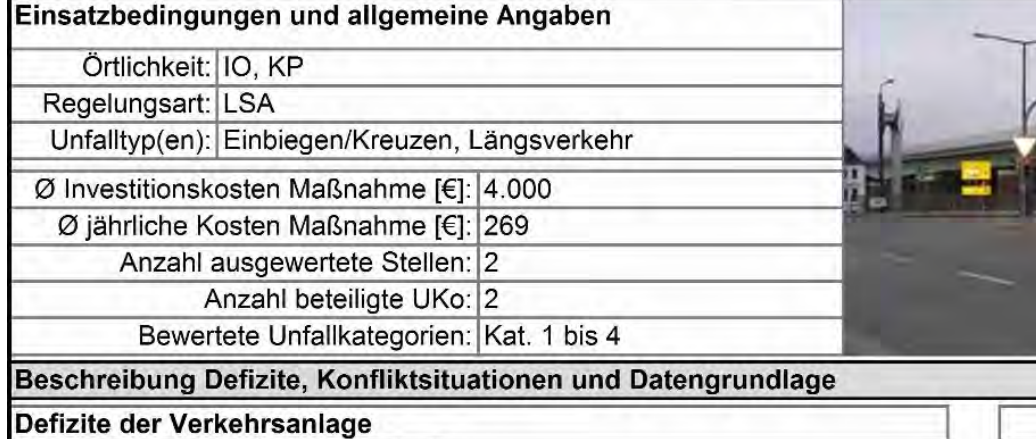
(2)

- häufige Verdeckung der Signalgeber

- keine Wiederholung Signalgeber

\section{Konflikte}

- Auffahrunfälle in der Zufahrt

- Überfahrten bei ROT

Wirkung der Maßnahme auf die Unfallanzahl $\left(\mathrm{MW}_{\mathrm{U}}\right)$

\begin{tabular}{|c|c|c|c||c|}
\hline \hline \multicolumn{2}{|c|}{ mittlere Unfallanzahl pro Jahr [U/a] } & \multicolumn{2}{|c|}{ Wirkungsgrad } \\
\hline Kategorie & $\Sigma \underline{\text { Vorher }}$ & $\Sigma \underline{\text { Nachher }}$ & $\Delta=\sum(\mathrm{V}-\mathrm{N})$ & $\varnothing$ über alle Stellen [\%] \\
\hline $\mathrm{U}(\mathrm{P})$ & 2,50 & 0,83 & 1,67 & 54 \\
\hline $\mathrm{U}(\mathrm{SS})$ & 0,00 & 0,17 & $-0,17$ & 0 \\
\hline $\mathrm{U}(\mathrm{P}, \mathrm{SS})$ & 2,50 & 1,00 & 1,50 & 38 \\
\hline
\end{tabular}

\begin{tabular}{|c|c|c|c|c|c|c|c|c|}
\hline \multicolumn{9}{|c|}{ Zeiträume und Unfallgeschehen } \\
\hline \multirow{3}{*}{$\frac{\stackrel{0}{\bar{d}}}{\frac{\Phi}{\omega}}$} & \multicolumn{4}{|c|}{ Analyse (Vorher) } & \multicolumn{4}{|c|}{ Kontrolle (Nachher) } \\
\hline & \multicolumn{2}{|c|}{ Mon. } & \multirow{2}{*}{$U(P)$} & \multirow{2}{*}{$\mathrm{U}(\mathrm{SS})$} & \multicolumn{2}{|c|}{ Mon. } & \multirow{2}{*}{$U(P)$} & \multirow{2}{*}{$\mathrm{U}(\mathrm{SS})$} \\
\hline & $\mathrm{P}$ & $S$ & & & $\mathrm{P}$ & S & & \\
\hline 1 & 36 & 36 & 3 & 0 & 36 & 36 & 2 & 1 \\
\hline 2 & 12 & 12 & 4 & 0 & 12 & 12 & 1 & 0 \\
\hline 3 & & & & & & & & \\
\hline 4 & & & & & & & & \\
\hline 5 & & & & & & & & \\
\hline 6 & & & & & & & & \\
\hline
\end{tabular}

$U(P), 2$ Stellen

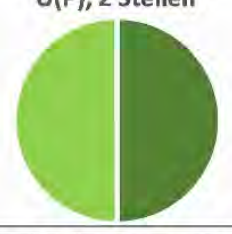

$\mathrm{U}(\mathrm{SS}), 2$ Stellen

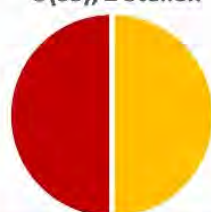

Wirkung der Maßnahme auf die Unfallkosten ( $\left(\mathrm{MW}_{\mathrm{UK}}\right)$

\begin{tabular}{|c|c|c|c||c|}
\hline \hline \multicolumn{2}{|c|}{ mittlere Unfallkosten pro Jahr [€/a] } & \multicolumn{2}{c|}{ Wirkungsgrad } \\
\hline Kategorie & $\underline{\text { Vorher }}$ & $\underline{\text { Nachher }}$ & $\Delta=\Sigma(\mathrm{V}-\mathrm{N})$ & $\varnothing$ über alle Stellen [\%] \\
\hline UK(P) & 61.067 & 36.733 & 24.333 & 41 \\
\hline UK(SS) & 0 & 2.517 & -2.517 & 0 \\
\hline UK(P,SS $)$ & 61.067 & 39.250 & 21.817 & 37 \\
\hline
\end{tabular}

UK(P,SS $)$

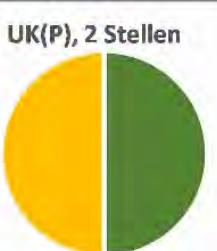

UK(SS), 2 Stellen

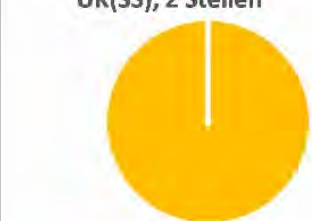

Nutzen-Kosten-Verhältnis (NKV)

\section{NKV Spannbreite und Median}

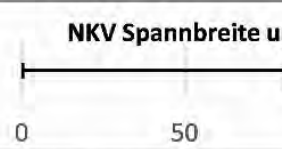

$-50$

0

50

100

150

200

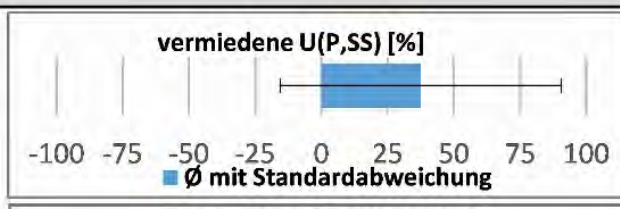

Einteilung Wirkungsgrad

\begin{tabular}{l|l|l|}
\hline Klassen & ob. Grenze [\%] & unt. Grenze [\%]
\end{tabular}

\begin{tabular}{|c|c|c|}
\hline bedeutend & 100 & 60 \\
\hline
\end{tabular}

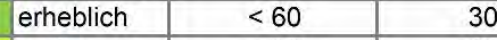

gering

neutral

nachteilig

\begin{tabular}{l|r}
$<30$ & 10 \\
$<10$ & -10 \\
\hline
\end{tabular}

$<-10 \quad$ offen

$\frac{10}{10}$

Zusammenfassung und Empfehlung

Maßnahmenbeschreibung/Ziel

Verbesserung Erkennbarkeit der Signalanlage durch Anbringen weiterer Signalgeber

\begin{tabular}{|c|c|}
\hline \multicolumn{2}{|c|}{ verwendete Unfallkostensätze [€] } \\
\hline $\mathrm{U}(\mathrm{SP})$ & 162.000 \\
\hline $\mathrm{U}(\mathrm{LV})$ & 14.600 \\
\hline $\mathrm{U}(\mathrm{SS})$ & 15.100 \\
\hline & \\
\hline
\end{tabular}

vermiedene UK(P,SS) [\%]

$\begin{array}{lllllllll}-100 & -75 & -50 & -25 & 0 & 25 & 50 & 75 & 100\end{array}$

$\emptyset$ mit Standardabweichung

\begin{tabular}{|c|c|}
\hline Signalgeber & $\begin{array}{l}\text { Ø Abnahme der Unfallkosten: } \\
\varnothing \text { nur bedingt } \\
\varnothing \text { Nutzen-Kosten-Verhältnis: }\end{array}$ \\
\hline Allgemeine Hinweise & Gesamtprädikat der Maßnahme \\
\hline $\begin{array}{l}\text { - Aussagekraft Kollektiv deutlich eingeschränkt (nur } 2 \text { Stellen) } \\
\text { - eingeschränkter Untersuchungszeitraum Stelle } 2\end{array}$ & bedingt empfehlenswer \\
\hline
\end{tabular}


Unfallanalyse der Maßnahmenkategorie: [21], Leuchtmittel Signalgeber verbessern

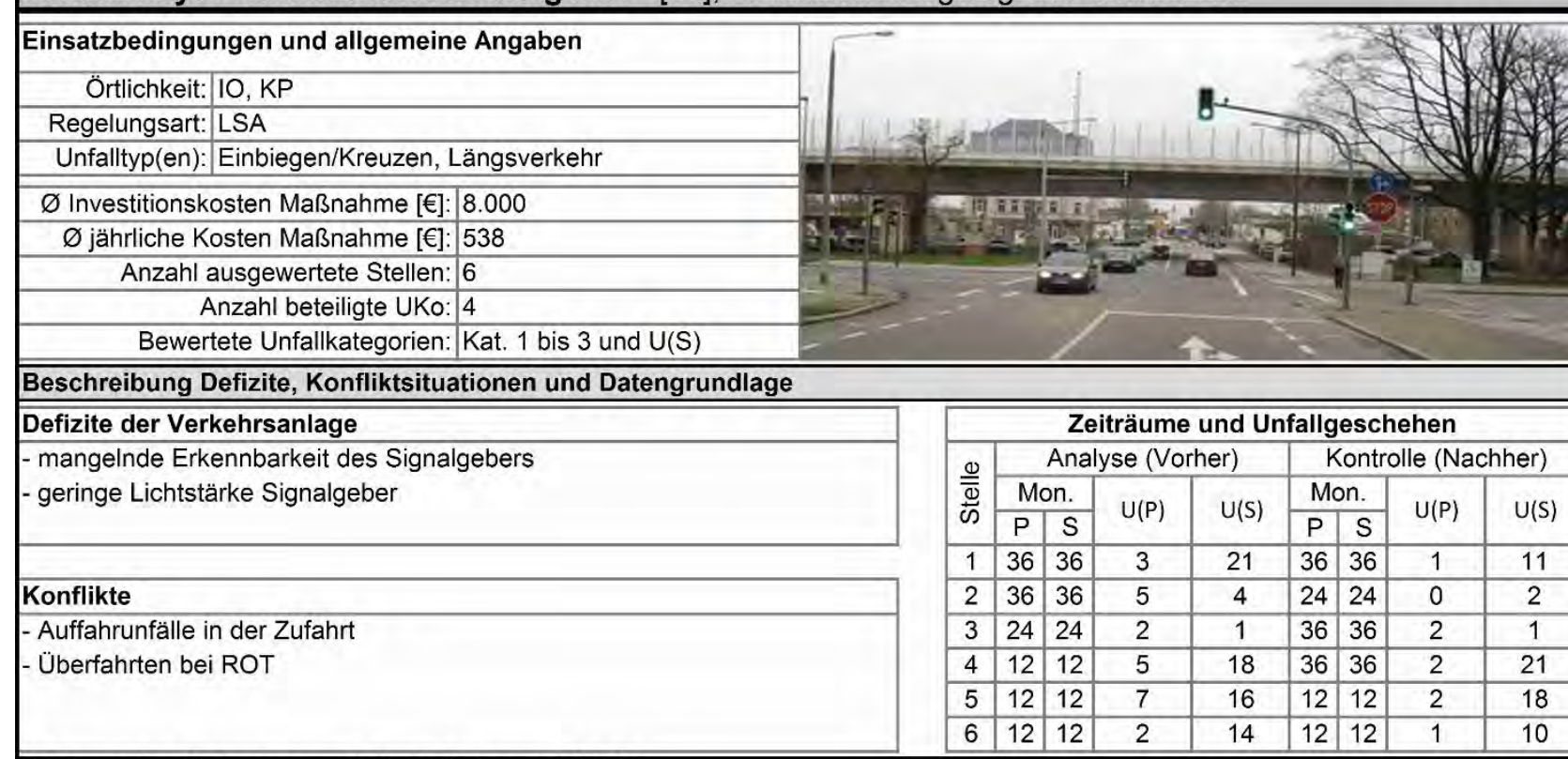

Wirkung der Maßnahme auf die Unfallanzahl $\left(\mathrm{MW}_{\mathrm{U}}\right)$

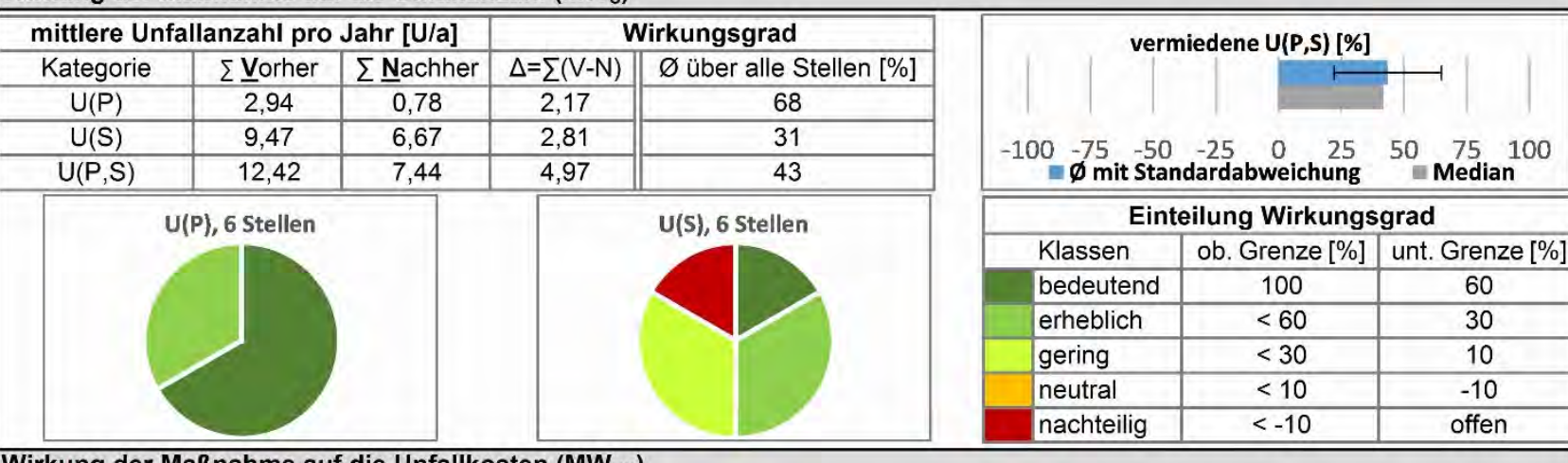

Wirkung der Maßnahme auf die Unfallkosten $\left(\mathrm{MW}_{\mathrm{UK}}\right)$

\begin{tabular}{|c|c|c|c||c|}
\hline \hline \multicolumn{2}{|c|}{ mittlere Unfallkosten pro Jahr [€/a] } & \multicolumn{2}{|c|}{ Wirkungsgrad } \\
\hline Kategorie & Vorher & $\underline{\text { Nachher }}$ & $\Delta=\Sigma(\mathrm{V}-\mathrm{N})$ & $\varnothing$ über alle Stellen [\%] \\
\hline UK(P) & 141.256 & 14.344 & 126.911 & 74 \\
\hline UK(S) & 63.843 & 44.933 & 18.909 & 31 \\
\hline UK(P,S) & 205.098 & 59.278 & 145.821 & 63 \\
\hline
\end{tabular}
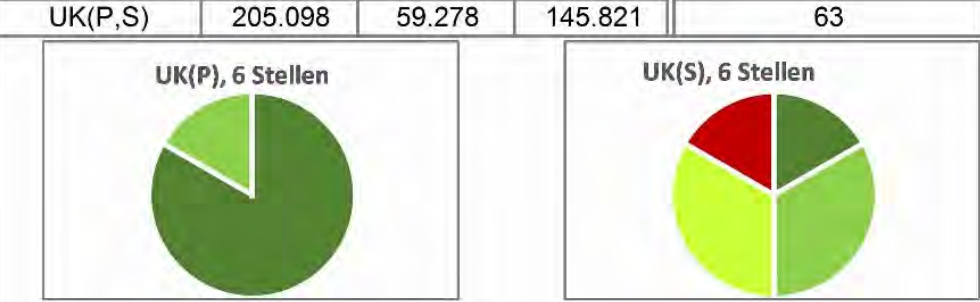

\begin{tabular}{|c|c|}
\hline \multicolumn{2}{|c|}{ verwendete Unfallkostensätze [€] } \\
\hline $\mathrm{U}(\mathrm{SP})$ & 162.000 \\
\hline $\mathrm{U}(\mathrm{LV})$ & 14.600 \\
\hline $\mathrm{U}(\mathrm{S})$ & 6.740 \\
\hline & \\
\hline
\end{tabular}

vermiedene UK(P,S) [\%]

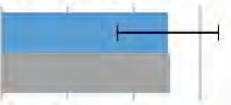

$\begin{array}{lllllllll}-100 & -75 & -50 & -25 & 0 & 25 & 50 & 75 & 100\end{array}$

$\approx \varnothing$ mit Standardabweichung $\equiv$ Median

Nutzen-Kosten-Verhältnis (NKV)

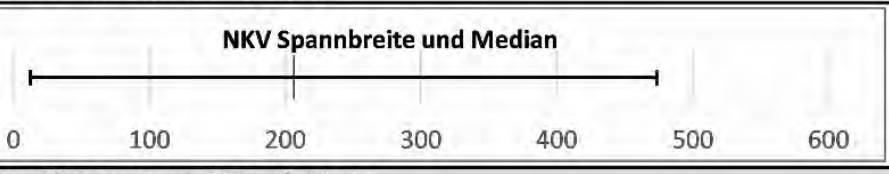

\begin{tabular}{r|r} 
Median Nutzen-Kosten-Verhältnis: & 206 \\
$\varnothing$ Nutzen-Kosten-Verhältnis: & 222
\end{tabular}

Zusammenfassung und Empfehlung

\section{Maßnahmenbeschreibung/Ziel}

Verbesserung Erkennbarkeit bestehender Signalgeber durch technische Aufwertung der Signalgeber (bspw. durch LED)
Zusammenfassung Kennwerte

$\varnothing$ Abnahme der Unfallanzahl: deutlich

$\varnothing$ Abnahme der Unfallkosten: deutlich

$\varnothing$ Nutzen-Kosten-Verhältnis: rentabel 


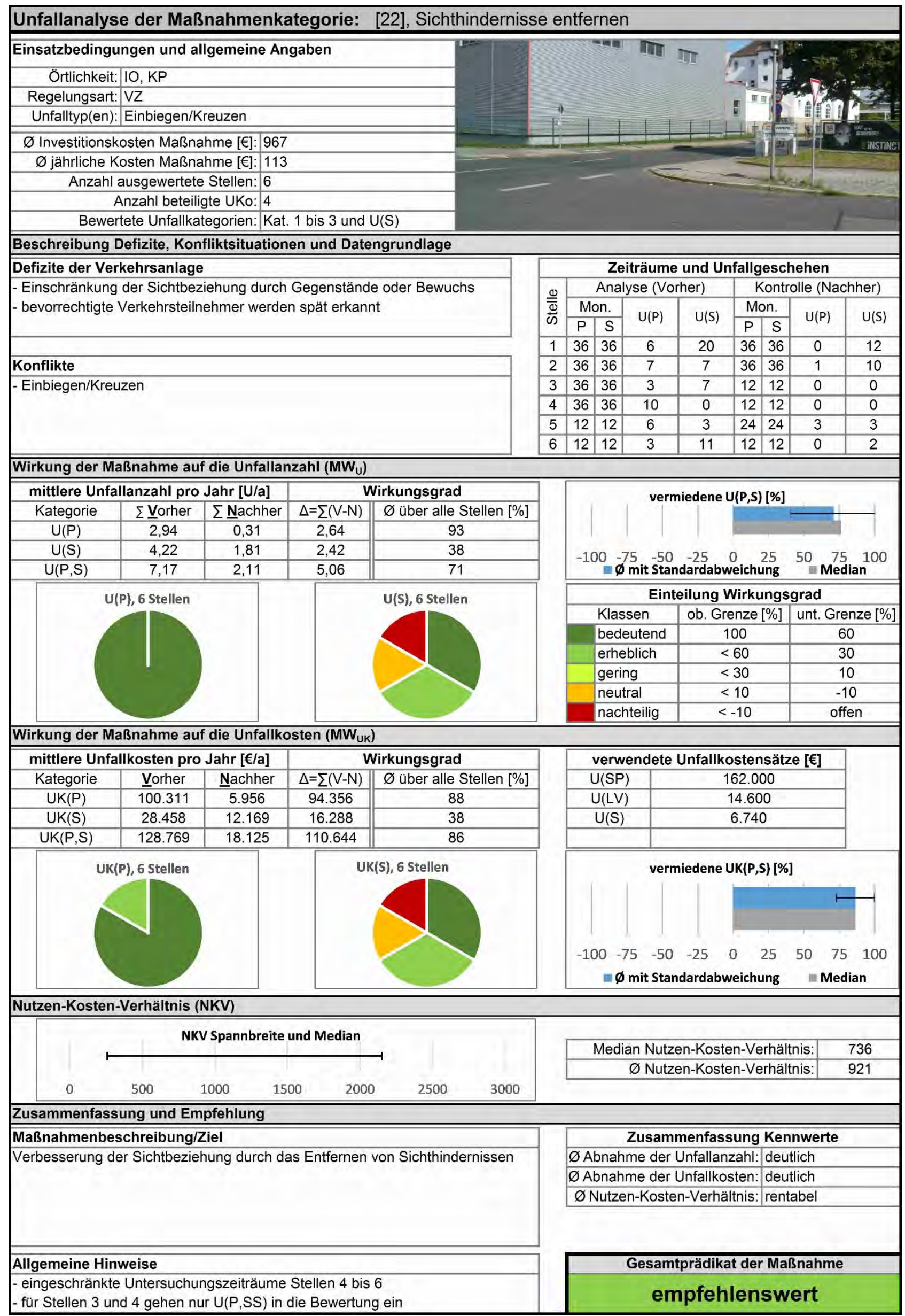


Unfallanalyse der Maßnahmenkategorie: [23], Sichthindernisse entfernen

Einsatzbedingungen und allgemeine Angaben

Örtlichkeit: $A O, K P$

Regelungsart: $\mathrm{VZ}$

Unfalltyp(en): Einbiegen/Kreuzen

$\varnothing$ Investitionskosten Maßnahme [€]: 5.183

$\varnothing$ jährliche Kosten Maßnahme [€]: 607

Anzahl ausgewertete Stellen: 6

Anzahl beteiligte UKo: 6

Bewertete Unfallkategorien:|Kat. 1 bis 3 und U(S)

Beschreibung Defizite, Konfliktsituationen und Datengrundlage

Defizite der Verkehrsanlage

- Einschränkung der Sichtbeziehung durch Gegenstände oder Bewuchs

- bevorrechtigte Verkehrsteilnehmer werden spät erkannt

Konflikte

- Einbiegen/Kreuzen

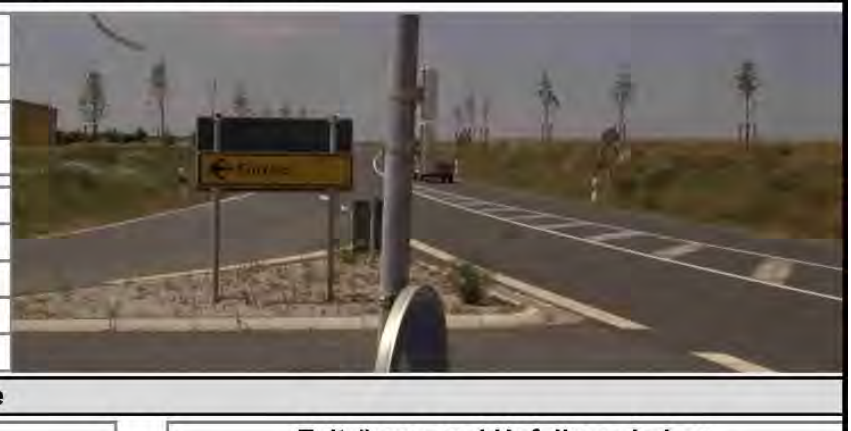

Wirkung der Maßnahme auf die Unfallanzahl $\left(\mathrm{MW}_{\mathrm{U}}\right)$

\begin{tabular}{|c|c|c|c||c|}
\hline \multicolumn{2}{|c|}{ mittlere Unfallanzahl pro Jahr [U/a] } & \multicolumn{2}{c|}{ Wirkungsgrad } \\
\hline Kategorie & $\Sigma$ Vorher & $\sum \underline{\text { Nachher }}$ & $\Delta=\sum(\mathrm{V}-\mathrm{N})$ & $\varnothing$ über alle Stellen [\%] \\
\hline $\mathrm{U}(\mathrm{P})$ & 1,94 & 1,22 & 0,72 & 54 \\
\hline $\mathrm{U}(\mathrm{S})$ & 3,22 & 2,28 & 0,94 & -49 \\
\hline $\mathrm{U}(\mathrm{P}, \mathrm{S})$ & 5,17 & 3,50 & 1,67 & 32 \\
\hline
\end{tabular}

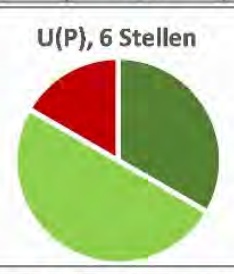

$\mathrm{U}(\mathrm{S}), 6$ Stellen

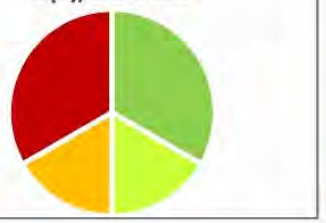

\begin{tabular}{|c|c|c|c|c|c|c|c|c|}
\hline \multicolumn{9}{|c|}{ Zeiträume und Unfallgeschehen } \\
\hline \multirow{3}{*}{ 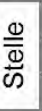 } & \multicolumn{4}{|c|}{ Analyse (Vorher) } & \multicolumn{4}{|c|}{ Kontrolle (Nachher) } \\
\hline & \multicolumn{2}{|c|}{ Mon. } & \multirow[b]{2}{*}{$U(P)$} & \multirow{2}{*}{$\mathrm{U}(\mathrm{S})$} & \multicolumn{2}{|c|}{ Mon. } & \multirow{2}{*}{$U(P)$} & \multirow{2}{*}{$\mathrm{U}(\mathrm{S})$} \\
\hline & $P$ & $\mathrm{~S}$ & & & $P$ & $S$ & & \\
\hline 1 & 36 & 36 & 4 & 6 & 36 & 36 & 0 & 4 \\
\hline 2 & 36 & 36 & 4 & 0 & 36 & 36 & 2 & 0 \\
\hline 3 & 36 & 36 & 5 & 0 & 36 & 36 & 2 & 1 \\
\hline 4 & 36 & 36 & 5 & 2 & 24 & 24 & 2 & 1 \\
\hline 5 & 36 & 36 & 5 & 2 & 12 & 12 & 0 & 3 \\
\hline 6 & 12 & 12 & 4 & 16 & 24 & 24 & 10 & 17 \\
\hline
\end{tabular}

\begin{tabular}{l|l|l|l|l}
6 & 12 & 12 & 4 \\
\hline
\end{tabular}

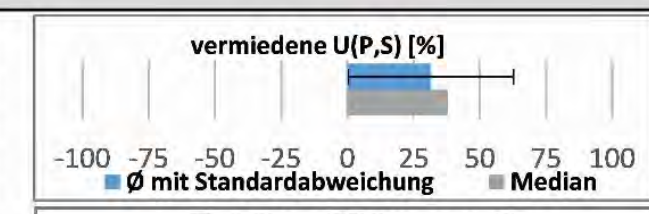

$\emptyset$ mit Standardabweichung Median

\begin{tabular}{|l|c|c|}
\hline \multicolumn{3}{|c|}{ Einteilung Wirkungsgrad } \\
\hline Klassen & ob. Grenze [\%] & unt. Grenze [\%] \\
\hline bedeutend & 100 & 60 \\
\hline erheblich & $<60$ & 30 \\
\hline gering & $<30$ & 10 \\
\hline neutral & $<10$ & -10 \\
\hline nachteilig & $<-10$ & offen \\
\hline
\end{tabular}

Wirkung der Maßnahme auf die Unfallkosten ( $\mathrm{MW}_{\mathrm{UK}}$ )

\begin{tabular}{|c|c|c|c||c|}
\hline \hline \multicolumn{2}{|c|}{ mittlere Unfallkosten pro Jahr [€/a] } & \multicolumn{2}{|c|}{ Wirkungsgrad } \\
\hline Kategorie & $\underline{\text { Vorher }}$ & $\underline{\underline{N}}$ achher & $\Delta=\sum(\mathrm{V}-\mathrm{N})$ & $\varnothing$ über alle Stellen [\%] \\
\hline UK(P) & 249.111 & 103.919 & 145.192 & 77 \\
\hline UK(S) & 16.723 & 11.822 & 4.902 & -41 \\
\hline UK(P,S) & 265.834 & 115.741 & 150.093 & 71 \\
\hline
\end{tabular}

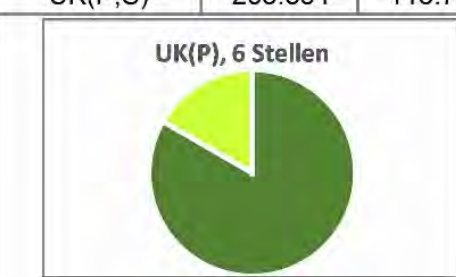

UK(S), 6 Stellen

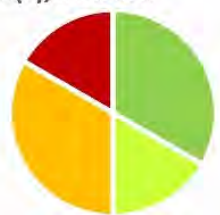

\begin{tabular}{|l|c|}
\hline \multicolumn{2}{|c|}{ verwendete Unfallkostensätze [€] } \\
\hline$U(S P)$ & 266.000 \\
\hline$U(L V)$ & 24.700 \\
\hline$U(S)$ & 5.190 \\
\hline & \\
\hline
\end{tabular}

vermiedene UK(P,S) [\%]

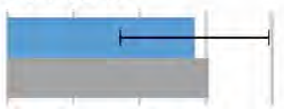

$\begin{array}{lllllllll}-100 & -75 & -50 & -25 & 0 & 25 & 50 & 75 & 100\end{array}$

$₫ \emptyset$ mit Standardabweichung $=$ Median

Nutzen-Kosten-Verhältnis (NKV)

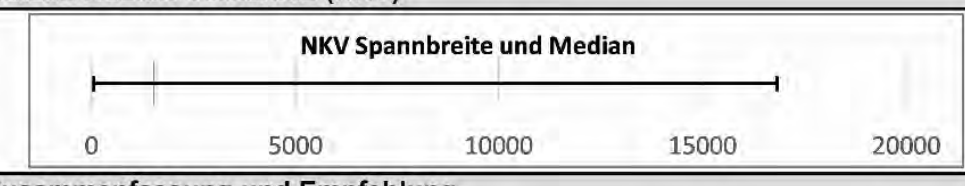

Zusammenfassung und Empfehlung

Maßnahmenbeschreibung/Ziel

Verbesserung der Sichtbeziehung durch das Entfernen von Sichthindernissen
Zusammenfassung Kennwerte

$\varnothing$ Abnahme der Unfallanzahl: deutlich

$\varnothing$ Abnahme der Unfallkosten: deutlich

$\varnothing$ Nutzen-Kosten-Verhältnis: rentabel 


\section{Unfallanalyse der Maßnahmenkategorie: [24], Beleuchtung anpassen/verbessern}

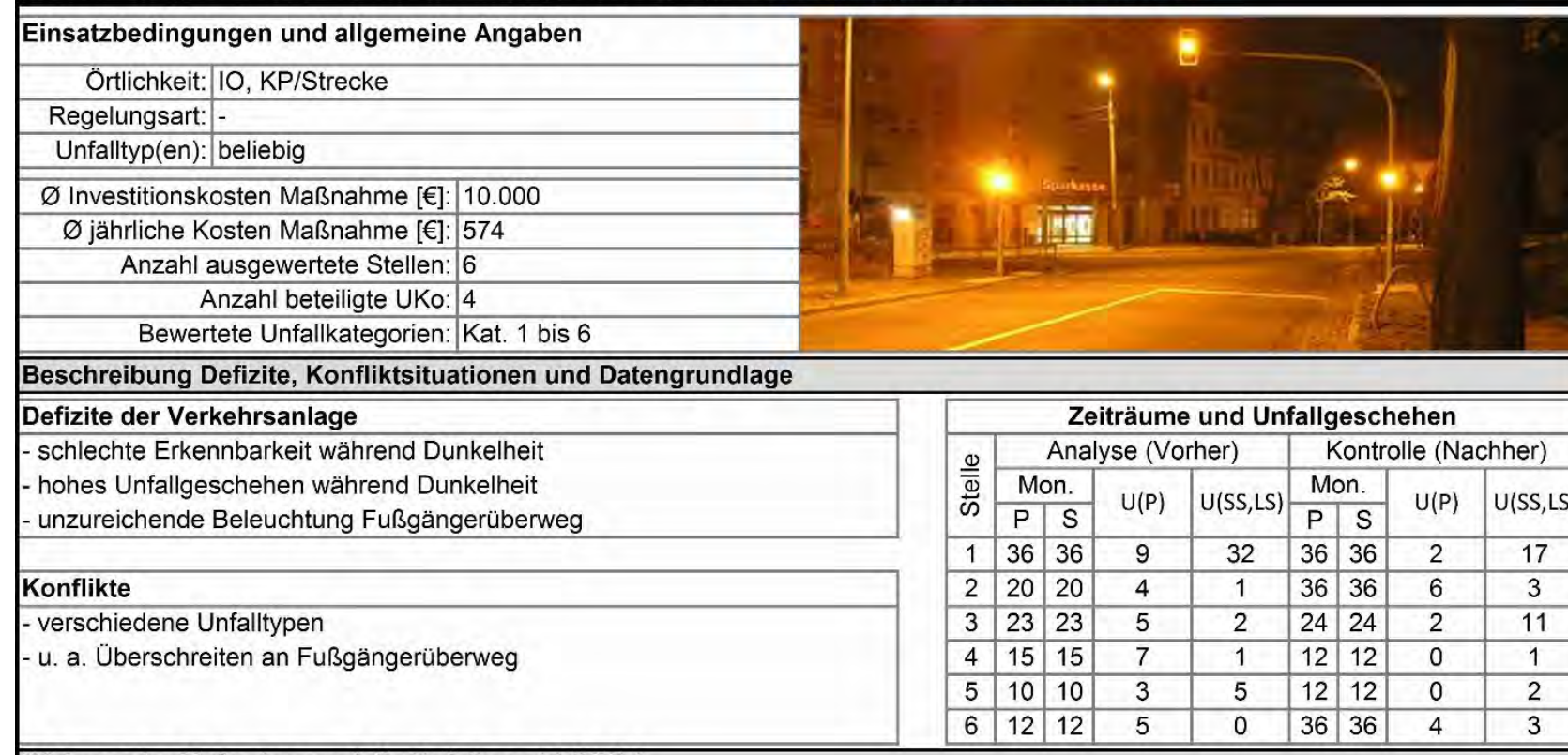

Wirkung der Maßnahme auf die Unfallanzahl $\left(\mathrm{MW}_{\mathrm{U}}\right)$

\begin{tabular}{|c|c|c|c||c|}
\hline \multicolumn{2}{|c|}{ mittlere Unfallanzahl pro Jahr [U/a] } & \multicolumn{2}{c|}{ Wirkungsgrad } \\
\hline Kategorie & $\Sigma \underline{\text { Vorher }}$ & $\Sigma \underline{\text { Nachher }}$ & $\Delta=\Sigma(\mathrm{V}-\mathrm{N})$ & $\varnothing$ über alle Stellen [\%] \\
\hline $\mathrm{U}(\mathrm{P})$ & 3,70 & 0,83 & 2,87 & 72 \\
\hline $\mathrm{U}(\mathrm{SS}, \mathrm{LS})$ & 3,19 & 2,69 & 0,49 & -81 \\
\hline $\mathrm{U}(\mathrm{P}, \mathrm{SS}, \mathrm{LS})$ & 6,89 & 3,53 & 3,36 & 32 \\
\hline
\end{tabular}
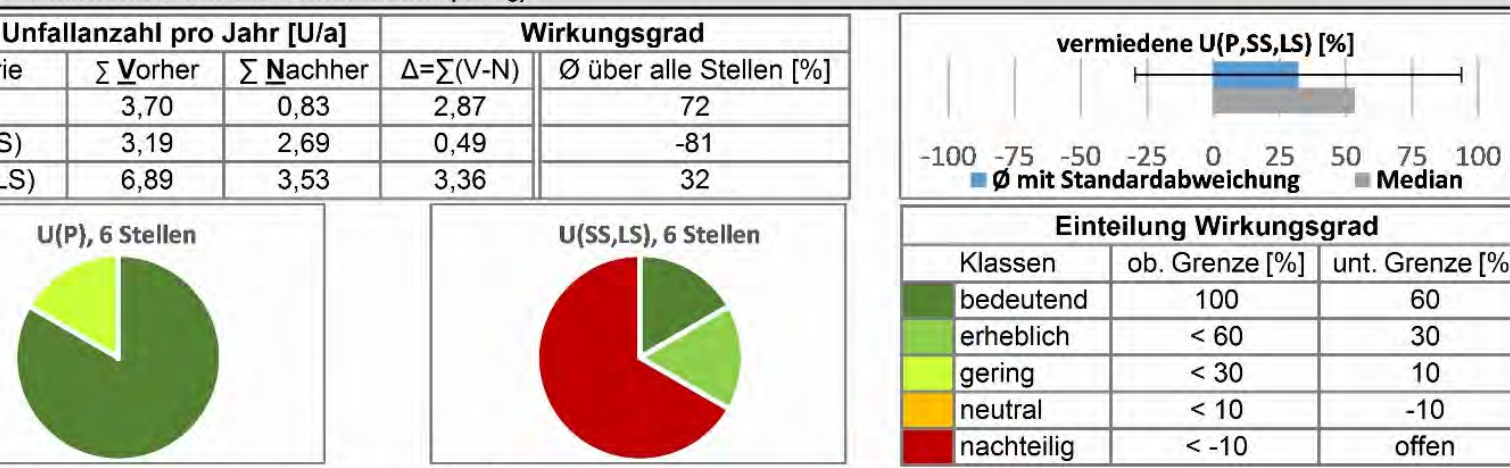

(

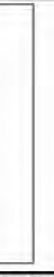

\begin{tabular}{|l|c|c|}
\hline \hline \multicolumn{3}{|c|}{ Einteilung Wirkungsgrad } \\
\hline Klassen & ob. Grenze [\%] & unt. Grenze [\%] \\
\hline bedeutend & 100 & 60 \\
\hline erheblich & $<60$ & 30 \\
\hline gering & $<30$ & 10 \\
\hline neutral & $<10$ & -10 \\
\hline nachteilig & $<-10$ & offen \\
\hline
\end{tabular}

Wirkung der Maßnahme auf die Unfallkosten ( $\left.\mathrm{MW}_{\mathrm{UK}}\right)$

\begin{tabular}{|c|c|c|c||c|}
\hline \hline \multicolumn{2}{|c|}{ mittlere Unfallkosten pro Jahr [€/a] } & \multicolumn{2}{|c|}{ Wirkungsgrad } \\
\hline Kategorie & $\underline{\text { Vorher }}$ & $\underline{\text { Nachher }}$ & $\Delta=\sum(\mathrm{V}-\mathrm{N})$ & $\varnothing$ über alle Stellen [\%] \\
\hline UK(P) & 175.885 & 61.300 & 114.585 & 24 \\
\hline UK(SS,LS) & 22.832 & 19.444 & 3.389 & -83 \\
\hline UK(P,SS,LS) & 198.717 & 80.744 & 117.974 & 51 \\
\hline
\end{tabular}

UK(P,SS,LS

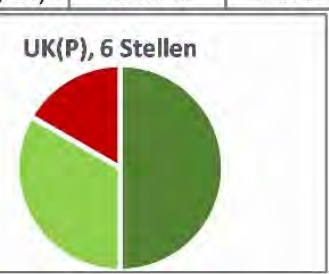

UK(SS,LS), 6 Stellen

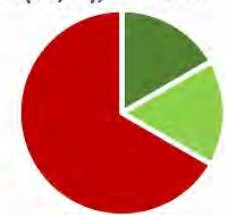

\begin{tabular}{|l|c|}
\hline \multicolumn{2}{|c|}{ verwendete Unfallkostensätze [€] } \\
\hline$U(S P)$ & 162.000 \\
\hline$U(L V)$ & 14.600 \\
\hline$U(S S)$ & 15.100 \\
\hline$U(L S)$ & 6.310 \\
\hline
\end{tabular}

vermiedene UK(P,SS,LS) [\%]

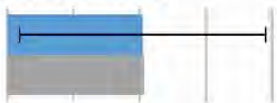

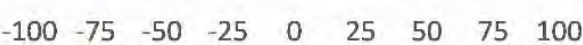

$\varpi$ mit Standardabweichung $=$ Median

Nutzen-Kosten-Verhältnis (NKV)

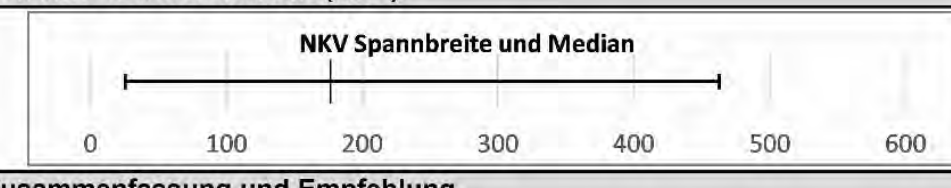

Zusammenfassung und Empfehlung

\section{Maßnahmenbeschreibung/Ziel}

Verbesserung der Sichtverhältnisse während der Dunkelheit durch Anpassung der Beleuchtung
Zusammenfassung Kennwerte

$\varnothing$ Abnahme der Unfallanzahl: deutlich

Ø Abnahme der Unfallkosten: deutlich

$\varnothing$ Nutzen-Kosten-Verhältnis: rentabel 


\section{Unfallanalyse der Maßnahmenkategorie: [25], Sichtschutzzaun aufstellen}

Einsatzbedingungen und allgemeine Angaben

Örtlichkeit: $\mathrm{AO}, \mathrm{KP}$

Regelungsart: $\mathrm{VZ}$

Unfalltyp(en): Einbiegen/Kreuzen

$\varnothing$ Investitionskosten Maßnahme [€]: 2.833

$\varnothing$ jährliche Kosten Maßnahme [€]: 332

Anzahl ausgewertete Stellen: 6

Anzahl beteiligte UKo: 3

Bewertete Unfallkategorien:|Kat. 1 bis 4

Beschreibung Defizite, Konfliktsituationen und Datengrundlage

Defizite der Verkehrsanlage

- Knotenpunkt aus weiter Entfernung einsehbar

- Fehleinschätzung von Fahrzeuggeschwindigkeiten

\section{Konflikte}

- Einbiegen/Kreuzen

- u. a. hohe Unfallschwere

Wirkung der Maßnahme auf die Unfallanzahl $\left(\mathrm{MW}_{\mathrm{U}}\right)$

\begin{tabular}{|c|c|c|c||c|}
\hline \hline \multicolumn{2}{|c|}{ mittlere Unfallanzahl pro Jahr [U/a] } & \multicolumn{2}{c|}{ Wirkungsgrad } \\
\hline Kategorie & $\Sigma \underline{\text { Vorher }}$ & $\Sigma \underline{\text { Nachher }}$ & $\Delta=\Sigma(\mathrm{V}-\mathrm{N})$ & $\varnothing$ über alle Stellen [\%] \\
\hline $\mathrm{U}(\mathrm{P})$ & 2,00 & 1,17 & 0,83 & 32 \\
\hline $\mathrm{U}(\mathrm{SS})$ & 0,17 & 0,11 & 0,06 & 17 \\
\hline $\mathrm{U}(\mathrm{P}, \mathrm{SS})$ & 2,17 & 1,28 & 0,89 & 31 \\
\hline
\end{tabular}
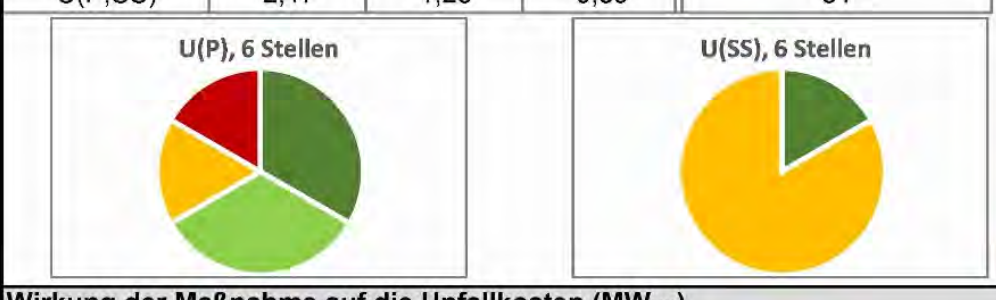

Wirkung der Maßnahme auf die Unfallkosten $\left(\mathrm{MW}_{\mathrm{UK}}\right)$

\begin{tabular}{|c|c|c|c||c|}
\hline \hline \multicolumn{3}{|c|}{ mittlere Unfallkosten pro Jahr [€/a] } & \multicolumn{2}{|c|}{ Wirkungsgrad } \\
\hline Kategorie & $\underline{\text { Vorher }}$ & $\underline{\text { Nachher }}$ & $\Delta=\Sigma(\mathrm{V}-\mathrm{N})$ & $\varnothing$ über alle Stellen [\%] \\
\hline UK(P) & 290.700 & 109.250 & 181.450 & 65 \\
\hline UK(SS) & 2.983 & 1.989 & 994 & 17 \\
\hline UK(P,SS) & 293.683 & 111.239 & 182.444 & 65 \\
\hline
\end{tabular}

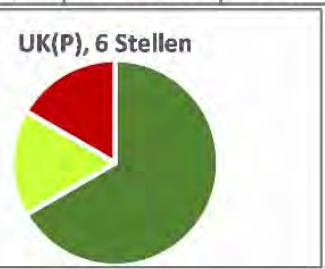

UK(SS), 6 Stellen

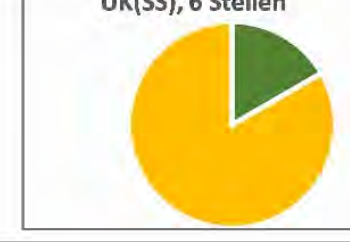

Nutzen-Kosten-Verhältnis (NKV)

\section{NKV Spannbreite und Median}

$-500$

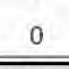

500

1000

1500

2000

2500

Zusammenfassung und Empfehlung

Maßnahmenbeschreibung/Ziel

Reduzierung der Fahrgeschwindigkeit und Erhöhung Aufmerksamkeit wartepflichtiger Fahrzeuge durch Errichten Sichtschutzaun in untergeordneter Zufahrt
Zeiträume und Unfallgeschehen

\begin{tabular}{|c|c|c|c|c|c|c|c|c|}
\hline \multirow{3}{*}{$\frac{\frac{\omega}{\Phi}}{\bar{\omega}}$} & \multicolumn{4}{|c|}{ Analyse (Vorher) } & \multicolumn{4}{|c|}{ Kontrolle (Nachher) } \\
\hline & \multicolumn{2}{|c|}{ Mon. } & \multirow{2}{*}{$U(P)$} & \multirow{2}{*}{$U(\mathrm{SS})$} & \multicolumn{2}{|c|}{ Mon. } & \multirow{2}{*}{$U(P)$} & \multirow{2}{*}{$\mathrm{U}(\mathrm{SS}$} \\
\hline & $P$ & $S$ & & & $P$ & $S$ & & \\
\hline 1 & 36 & 36 & 9 & 2 & 36 & 36 & 5 & 2 \\
\hline 2 & 36 & 36 & 6 & 1 & 27 & 27 & 0 & 0 \\
\hline 3 & 36 & 36 & 5 & 0 & 36 & 36 & 10 & 0 \\
\hline 4 & 36 & 36 & 5 & 0 & 36 & 36 & 2 & 0 \\
\hline 5 & 36 & 36 & 8 & 0 & 36 & 36 & 1 & 0 \\
\hline 6 & 36 & 36 & 3 & 0 & 36 & 36 & 3 & 0 \\
\hline
\end{tabular}

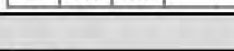

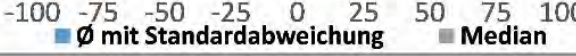

vermiedene $\mathrm{U}(\mathrm{P}, \mathrm{SS})[\%]$

\section{Einteilung Wirkungsgrad}

\begin{tabular}{|l|c|c|}
\hline Klassen & ob. Grenze [\%] & unt. Grenze [\%] \\
\hline bedeutend & 100 & 60 \\
\hline erheblich & $<60$ & 30 \\
\hline gering & $<30$ & 10 \\
\hline neutral & $<10$ & -10 \\
\hline nachteilig & $<-10$ & offen \\
\hline
\end{tabular}

\begin{tabular}{|c|c|}
\hline \multicolumn{2}{|c|}{ verwendete Unfallkostensätze [€] } \\
\hline $\mathrm{U}(\mathrm{SP})$ & 266.000 \\
\hline $\mathrm{U}(\mathrm{LV})$ & 24.700 \\
\hline $\mathrm{U}(\mathrm{SS})$ & 17.900 \\
\hline & \\
\hline \hline
\end{tabular}

vermiedene UK(P,SS) [\%]

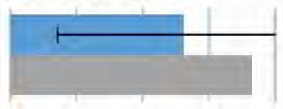

$\begin{array}{lllllllll}-100 & -75 & -50 & -25 & 0 & 25 & 50 & 75 & 100\end{array}$

$=\varnothing$ mit Standardabweichung $=$ Median

\begin{tabular}{|c|c|}
\hline Zutanrt & $\varnothing$ Nutzen-Kosten-Verhältnis:| rentabel \\
\hline Allgemeine Hinweise & Gesamtprädikat der Maßnahme \\
\hline & empfehlenswert \\
\hline
\end{tabular}




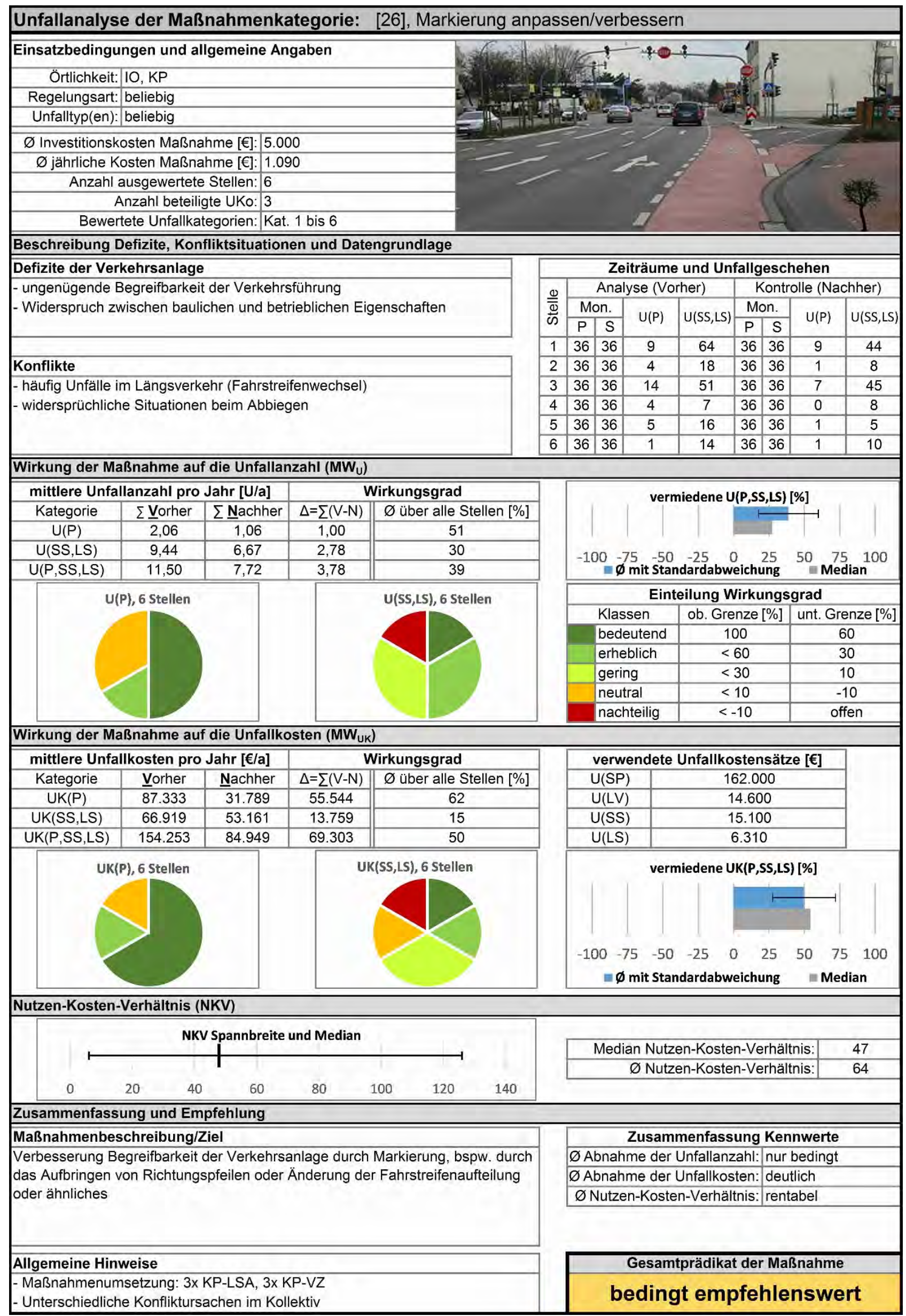




\section{Unfallanalyse der Maßnahmenkategorie: [27], Markierung in Stand setzen}

\begin{tabular}{|c|c|c|c|c|c|c|c|c|c|c|c|c|}
\hline \multicolumn{13}{|c|}{ Einsatzbedingungen und allgemeine Angaben } \\
\hline \multicolumn{13}{|c|}{ Örtlichkeit: $10, \mathrm{KP}$} \\
\hline Regelungsart: & \multicolumn{12}{|l|}{ beliebig } \\
\hline \multirow{2}{*}{\multicolumn{13}{|c|}{$\begin{array}{l}\text { Unfalltyp(en): beliebig } \\
\varnothing \text { Investitionskosten Maßnahme [€]: } 5.000\end{array}$}} \\
\hline & & & & & & & & & & & & \\
\hline \multicolumn{13}{|c|}{$\varnothing$ jährliche Kosten Maßnahme [€]: 1.090} \\
\hline \multicolumn{13}{|c|}{ Anzahl ausgewertete Stellen: 6} \\
\hline \multicolumn{13}{|c|}{ Anzahl beteiligte UKo: 4} \\
\hline \multicolumn{13}{|c|}{ Bewertete Unfallkategorien: Kat. 1 bis 6} \\
\hline \multicolumn{13}{|c|}{ Beschreibung Defizite, Konfliktsituationen und Datengrundlage } \\
\hline \multirow{4}{*}{\multicolumn{5}{|c|}{$\begin{array}{l}\text { Defizite der Verkehrsanlage } \\
\text { - ungenügende Begreifbarkeit der Verkehrsführung } \\
\text { - schlechte Erkennbarkeit der Verkehrsführung }\end{array}$}} & \multicolumn{8}{|c|}{ Zeiträume und Unfallgeschehen } \\
\hline & & & & & \multirow{3}{*}{$\frac{\stackrel{\Xi}{\bar{\Phi}}}{\omega}$} & \multicolumn{4}{|c|}{ Analyse (Vorher) } & \multicolumn{3}{|c|}{ Kontrolle (Nachher) } \\
\hline & & & & & & \multicolumn{2}{|c|}{ Mon. } & \multirow{2}{*}{$U(P)$} & \multirow{2}{*}{$\mathrm{U}(\mathrm{SS}, \mathrm{LS})$} & Mon. & \multirow{2}{*}{$\mathrm{U}(\mathrm{P})$} & \multirow{2}{*}{$\mathrm{U}(\mathrm{SS}, \mathrm{LS})$} \\
\hline & & & & & & $\mathrm{P}$ & S & & & \begin{tabular}{l|l}
$\mathrm{P}$ & $\mathrm{S}$ \\
\end{tabular} & & \\
\hline & & & & & 1 & 36 & 36 & 8 & 11 & $36 \quad 36$ & 2 & 6 \\
\hline \multicolumn{5}{|l|}{ Konflikte } & 2 & 36 & 36 & 4 & 7 & $36 \quad 36$ & 3 & 15 \\
\hline \multirow{4}{*}{\multicolumn{5}{|c|}{$\begin{array}{l}\text { - Unfälle im Längsverkehr (Fahrstreifenwechsel) } \\
\text { - widersprüchliche Situationen beim Abbiegen }\end{array}$}} & 3 & 36 & 36 & 8 & 29 & \begin{tabular}{|l|l|}
36 & 36 \\
\end{tabular} & 3 & 37 \\
\hline & & & & & 4 & 36 & 36 & 4 & 3 & $24 \quad 24$ & 2 & 3 \\
\hline & & & & & 5 & 36 & 36 & 8 & 2 & $24 \quad 24$ & 7 & 2 \\
\hline & & & & & 6 & 36 & 36 & 1 & 11 & \begin{tabular}{|l|l|}
24 & 24 \\
\end{tabular} & 2 & 7 \\
\hline Wirkung der Ma & ßnahme au & die Unfallan & zahl $\left(\mathrm{MW}_{\mathrm{U}}\right)$ & & & & & & & & & \\
\hline mittlere Unfal & llanzahl pro & Jahr [U/a] & & lirkungsgrad & & & & vern & niedene U & $(\mathrm{P}, \mathrm{SS}, \mathrm{LS})$ & [\%] & \\
\hline Kategorie & $\sum \underline{\text { Vorher }}$ & $\sum \underline{\text { Nachher }}$ & $\Delta=\Sigma(\mathrm{V}-\mathrm{N})$ & $\varnothing$ über alle Stellen [\%] & & & & & & ${ }^{-1}$ & & \\
\hline$U(P)$ & 1,83 & 1,36 & 0,47 & -7 & & & & & & & & \\
\hline U(SS,LS) & 3,50 & 4,22 & $-0,72$ & -32 & & & & & & 25 & & 100 \\
\hline $\mathrm{U}(\mathrm{P}, \mathrm{SS}, \mathrm{LS})$ & 5,33 & 5,58 & $-0,25$ & -11 & & & & mit Star & idardabw & eichung & 鲜 & dian \\
\hline$U(F$ & P), 6 Stellen & & & U(SS,LS), 6 Stellen & & & & Eint & eilung $\mathrm{V}$ & lirkungs & grad & \\
\hline & & & & & & & Klas & $\operatorname{sen}$ & ob. Gre & nze [\%] & unt. Gr & enze [\%] \\
\hline & & & & & & & bede & utend & & 00 & & 30 \\
\hline & & & & & & & erhe & olich & & 60 & & 30 \\
\hline & & & & & & & gerin & & & 30 & & 10 \\
\hline & & & & & & & neut & & & 10 & & 10 \\
\hline & & & & & & & nach & teilig & & 10 & & fen \\
\hline Wirkung der Ma & ßßnahme al & die Unfallko & sten $\left(\mathrm{MW}_{\mathrm{UK}}\right.$ & & & & & & & & & \\
\hline mittlere Unfal & Ilkosten pro & Jahr [€/a] & & irkungsgrad & & & verw & andete & Unfallko & stensätz & ee [€] & \\
\hline Kategorie & Vorher & Nachher & $\Delta=\Sigma(\mathrm{V}-\mathrm{N})$ & $\varnothing$ über alle Stellen [\%] & & & $\mathrm{U}(\mathrm{SP})$ & & & 52.000 & & \\
\hline UK(P) & 67.711 & 40.344 & 27.367 & -2 & & & $\mathrm{U}(\mathrm{LV})$ & & & 4.600 & & \\
\hline UK(SS,LS) & 26.968 & 29.816 & -2.848 & -21 & & & $\mathrm{U}(\mathrm{SS})$ & & & 5.100 & & \\
\hline UK(P,SS,LS) & 94.679 & 70.161 & 24.519 & 13 & & & $\mathrm{U}(\mathrm{LS})$ & & & 6.310 & & \\
\hline
\end{tabular}

UK(P), 6 Stellen

Nutzen-Kosten-Verhältnis (NKV)

\begin{tabular}{|c|c|c|c|c|c|c|c|c|c|c|}
\hline \multirow{2}{*}{\multicolumn{8}{|c|}{ NKV Spannbreite und Median }} & \multirow{3}{*}{100} & \multirow{2}{*}{$\begin{array}{r}\text { Median Nutzen-Kosten-Verhältnis: } \\
\varnothing \text { Nutzen-Kosten-Verhältnis: }\end{array}$} & \\
\hline & & & & & & & & & & 19 \\
\hline-60 & -40 & -20 & 0 & 20 & 40 & 60 & 80 & & & \\
\hline
\end{tabular}

Zusammenfassung und Empfehlung

Maßnahmenbeschreibung/Ziel

Verbesserung Begreifbarkeit/Erkennbarkeit der Verkehrsführung durch

Erneuerung der Markierung

Zusammenfassung Kennwerte

$\varnothing$ Abnahme der Unfallanzahl: nicht gegeben

$\varnothing$ Abnahme der Unfallkosten: nur bedingt

$\varnothing$ Nutzen-Kosten-Verhältnis: rentabel 


\section{Unfallanalyse der Maßnahmenkategorie: [29], Radverkehrsführung anpassen}

\begin{tabular}{|c|c|c|c|c|c|c|c|c|}
\hline \multicolumn{9}{|l|}{ Einsatzbedingungen und allgemeine Angaben } \\
\hline \multicolumn{9}{|l|}{ Örtlichkeit: IO, KP } \\
\hline \multicolumn{9}{|l|}{ Regelungsart: beliebig } \\
\hline \multicolumn{9}{|l|}{ Unfalltyp(en): Abbiegen, Einbiegen/Kreuzen } \\
\hline \multicolumn{9}{|l|}{$\varnothing$ Investitionskosten Maßnahme [€]: 15.800} \\
\hline \multicolumn{9}{|l|}{$\varnothing$ jährliche Kosten Maßnahme [€]: 3.444} \\
\hline \multicolumn{9}{|l|}{ Anzahl ausgewertete Stellen: 5} \\
\hline \multicolumn{9}{|l|}{ Anzahl beteiligte UKo: 4} \\
\hline \multicolumn{9}{|l|}{ Bewertete Unfallkategorien: Kat. 1 bis 3 und U(S) } \\
\hline \multicolumn{9}{|l|}{ Beschreibung Defizite, Konfliktsituationen und Datengrundlage } \\
\hline \multirow{4}{*}{$\begin{array}{l}\text { Defizite der Verkehrsanlage } \\
\text { - ungenügende Erkennbarkeit der Radverkehrsführung } \\
\text { - Radverkehrsführung schlecht begreifbar }\end{array}$} & \multicolumn{8}{|c|}{ Zeiträume und Unfallgeschehen } \\
\hline & \multirow{3}{*}{$\frac{\varrho}{\frac{\Phi}{\omega}}$} & \multicolumn{3}{|c|}{ Analyse (Vorher) } & \multicolumn{4}{|c|}{ Kontrolle (Nachher) } \\
\hline & & Mon. & \multirow{2}{*}{$\mathrm{U}(\mathrm{P})$} & \multirow{2}{*}{$\mathrm{U}(\mathrm{S})$} & \multicolumn{2}{|c|}{ Mon. } & \multirow{2}{*}{$U(P)$} & \multirow{2}{*}{$\mathrm{U}(\mathrm{S})$} \\
\hline & & \begin{tabular}{l|l|}
$P$ & $S$ \\
\end{tabular} & & & $\mathrm{P}$ & S & & \\
\hline & 1 & $36 \quad 36$ & 5 & 5 & 36 & 36 & 0 & 1 \\
\hline Konflikte & 2 & $36 \quad 36$ & 22 & 19 & 18 & 18 & 2 & 4 \\
\hline \multirow{4}{*}{$\begin{array}{l}\text { - Unfälle mit Radverkehrsbeteiligung } \\
\text { - Kfz mit parallel oder entgegengesetzt fahrenden Radfahrern }\end{array}$} & 3 & $24 \quad 24$ & 4 & 0 & 36 & 36 & 2 & 0 \\
\hline & 4 & \begin{tabular}{|l|l|}
41 & 41 \\
\end{tabular} & 6 & 6 & 34 & 34 & 6 & 4 \\
\hline & 5 & \begin{tabular}{|l|l|}
36 & 36 \\
\end{tabular} & 5 & 25 & 21 & 21 & 1 & 6 \\
\hline & 6 & & & & & & & \\
\hline
\end{tabular}

Wirkung der Maßnahme auf die Unfallanzahl (MW

\begin{tabular}{|c|c|c|c||c|}
\hline \multicolumn{2}{|c|}{ mittlere Unfallanzahl pro Jahr [U/a] } & \multicolumn{2}{c|}{ Wirkungsgrad } \\
\hline Kategorie & $\Sigma$ Vorher & $\sum \underline{\text { Nachher }}$ & $\Delta=\Sigma(\mathrm{V}-\mathrm{N})$ & $\varnothing$ über alle Stellen [\%] \\
\hline $\mathrm{U}(\mathrm{P})$ & 2,88 & 0,94 & 1,95 & 59 \\
\hline $\mathrm{U}(\mathrm{S})$ & 3,62 & 1,57 & 2,05 & 43 \\
\hline $\mathrm{U}(\mathrm{P}, \mathrm{S})$ & 6,50 & 2,51 & 4,00 & 57 \\
\hline
\end{tabular}
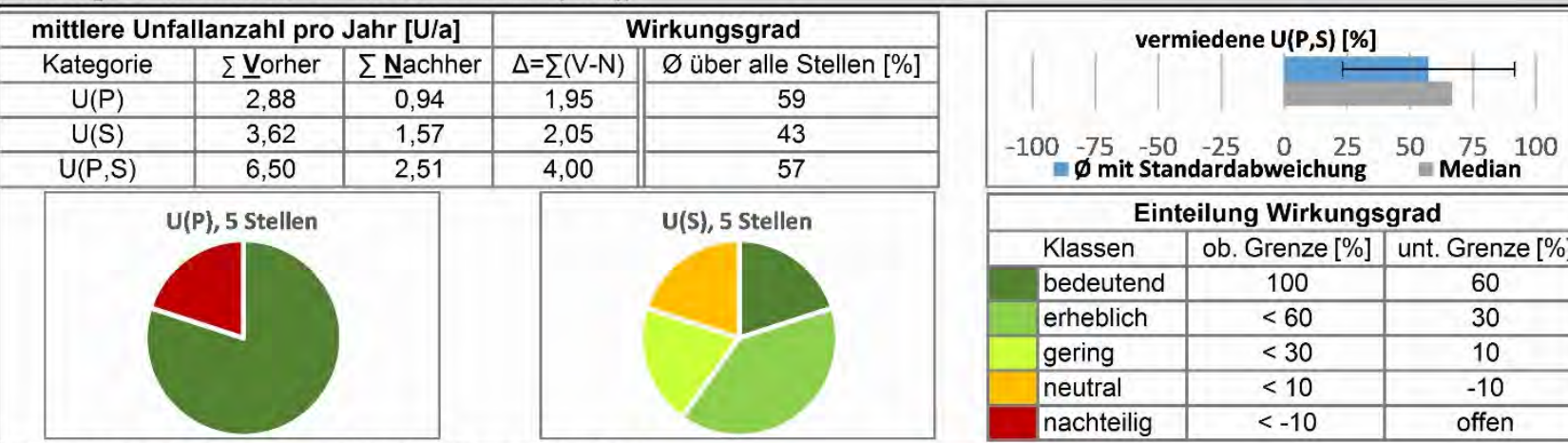

\begin{tabular}{|c|c|c|c||c|}
\hline \multicolumn{4}{|c|}{ Wirkung der Maßnahme auf die Unfallkosten $\left(\mathrm{MW}_{\text {UK }}\right)$} \\
\hline \hline \multicolumn{2}{|c|}{ mittlere Unfallkosten pro Jahr [€/a] } & \multicolumn{2}{|c|}{ Wirkungsgrad } \\
\hline Kategorie & Vorher & Nachher & $\Delta=\Sigma(\mathrm{V}-\mathrm{N})$ & $\varnothing$ über alle Stellen [\%] \\
\hline UK(P) & 86.454 & 54.733 & 31.721 & 31 \\
\hline UK(S) & 24.385 & 10.569 & 13.816 & 43 \\
\hline UK(P,S) & 110.839 & 65.302 & 45.537 & 28 \\
\hline
\end{tabular}
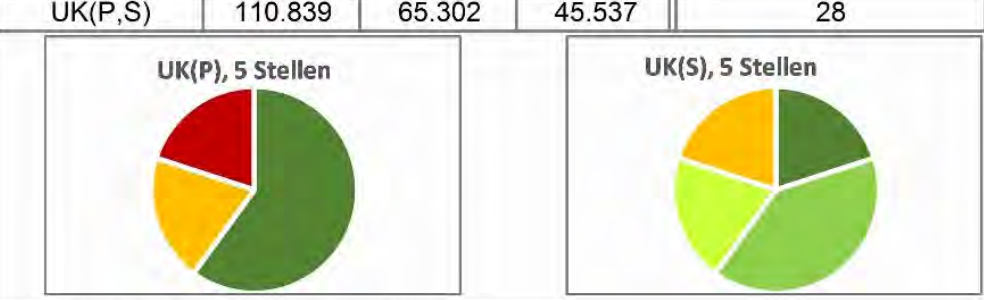

\begin{tabular}{|l|c|c|}
\hline \hline \multicolumn{3}{|c|}{ Einteilung Wirkungsgrad } \\
\hline Klassen & ob. Grenze [\%] & unt. Grenze [\%] \\
\hline bedeutend & 100 & 60 \\
\hline erheblich & $<60$ & 30 \\
\hline gering & $<30$ & 10 \\
\hline neutral & $<10$ & -10 \\
\hline nachteilig & $<-10$ & offen \\
\hline
\end{tabular}

Nutzen-Kosten-Verhältnis (NKV)

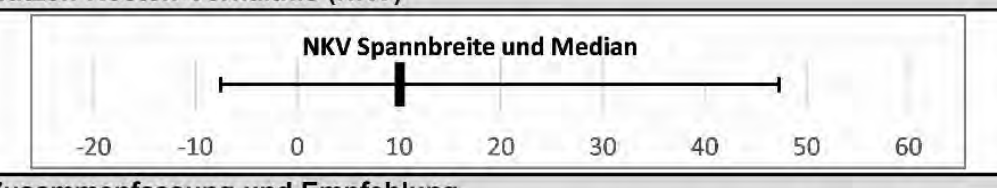

\begin{tabular}{|c|c|}
\hline \multicolumn{2}{|c|}{ verwendete Unfallkostensätze [€] } \\
\hline $\mathrm{U}(\mathrm{SP})$ & 162.000 \\
\hline $\mathrm{U}(\mathrm{LV})$ & 14.600 \\
\hline $\mathrm{U}(\mathrm{S})$ & 6.740 \\
\hline & \\
\hline \hline
\end{tabular}

vermiedene UK(P,S) [\%]

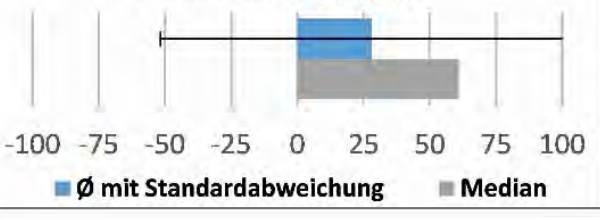
$\varpi$ mit Standardabweichung
Median

Zusammenfassung und Empfehlung

\section{Maßnahmenbeschreibung/Ziel}

Verbesserung/Anpassung der Radverkehrsführung u. a. durch:

Anlegen/Änderung von Radfurten, Heranrücken Radverkehrsanlage in

Fahrbahnnähe, Markiern von Piktogrammen auf Radfahrstreifen oder Anlegen

von Aufstellflächen vor Lichtsignalanlagen

Median Nutzen-Kosten-Verhältnis: $\quad 10$

$\varnothing$ Nutzen-Kosten-Verhältnis:

13

Zusammenfassung Kennwerte

Ø Abnahme der Unfallanzahl: deutlich

$\varnothing$ Abnahme der Unfallkosten: nur bedingt

$\varnothing$ Nutzen-Kosten-Verhältnis: rentabel 


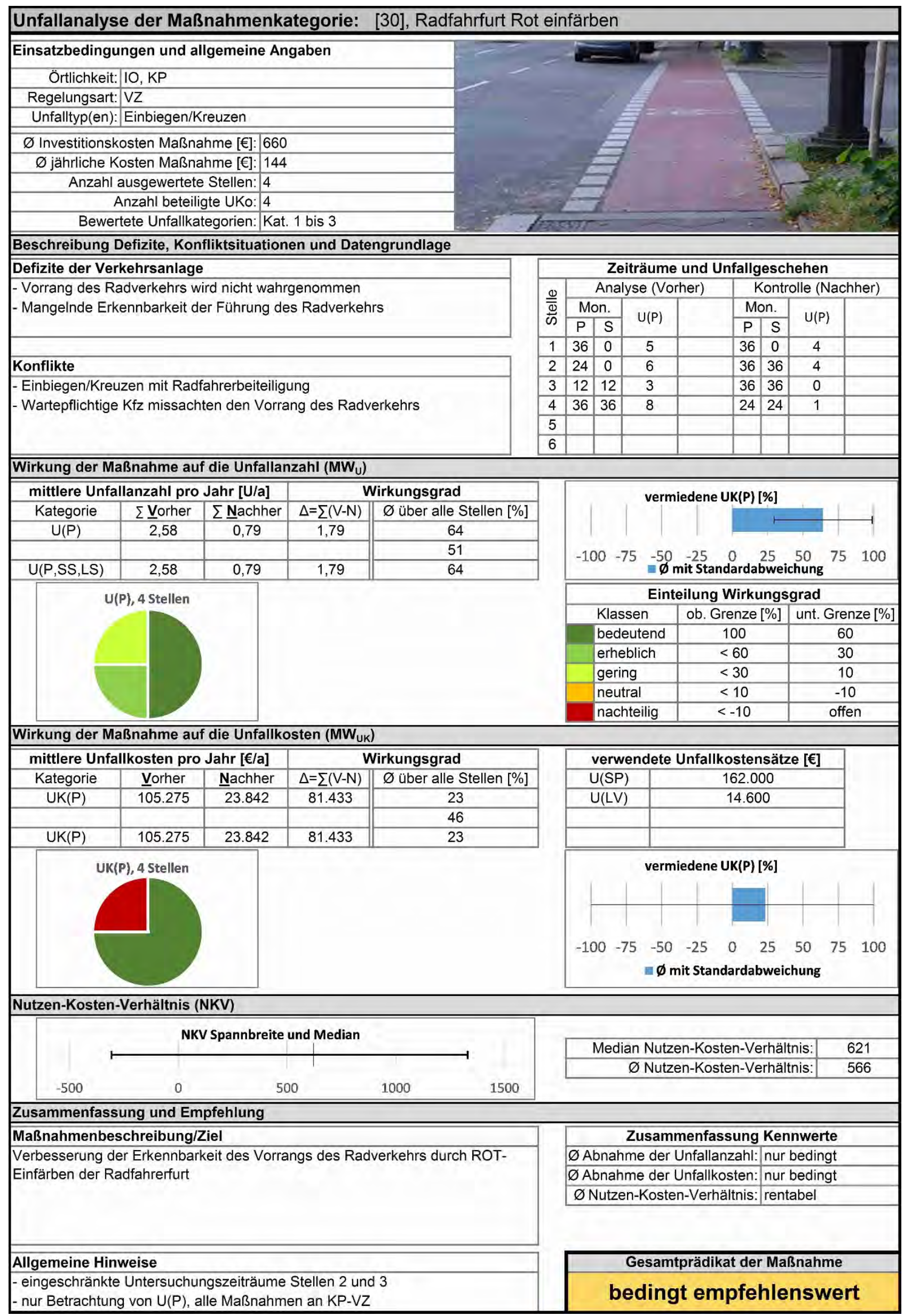




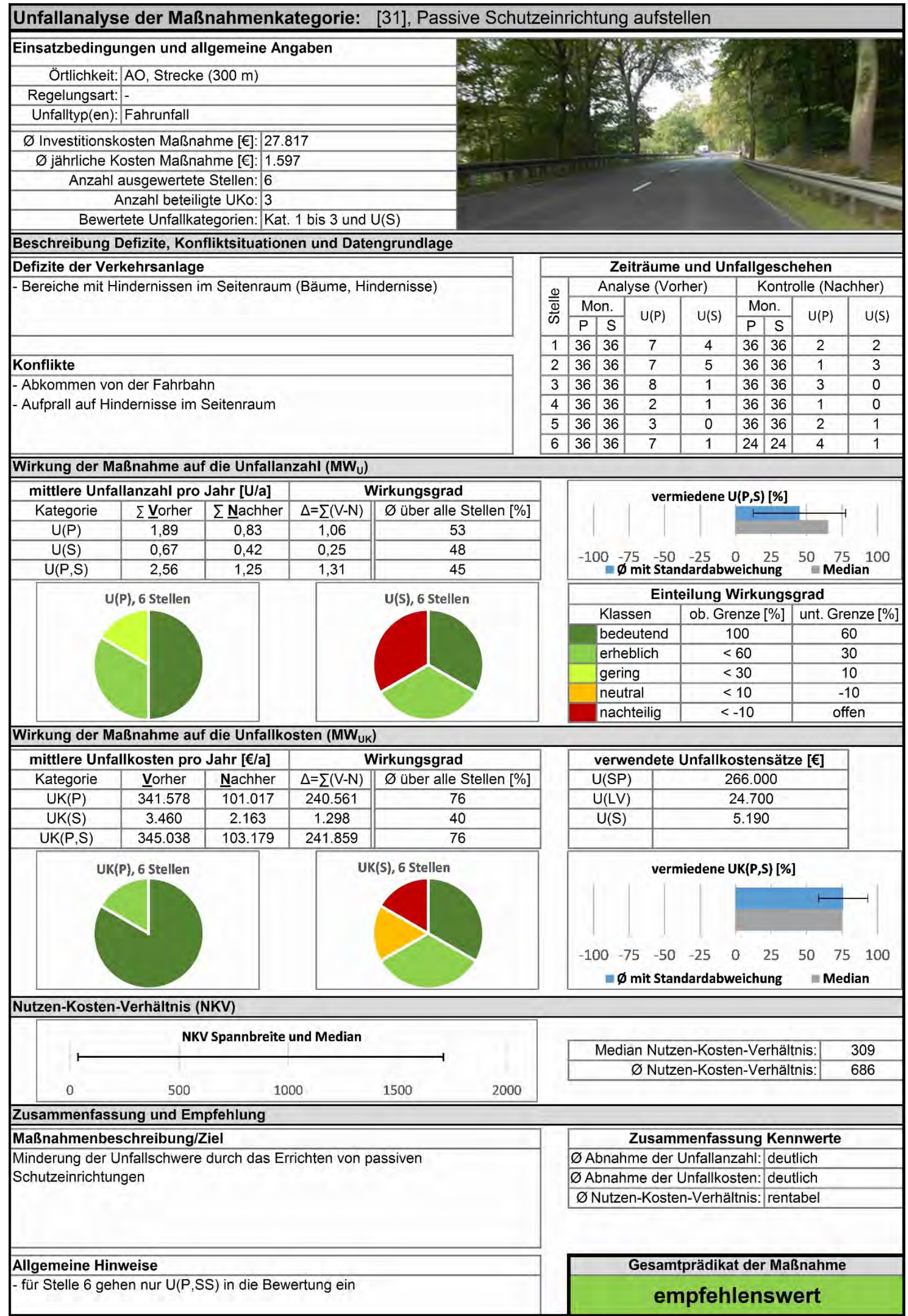


Unfallanalyse der Maßnahmenkategorie: [32], Unterfahrschutz an passiver Schutzeinrichtung anbringen Einsatzbedingungen und allgemeine Angaben

Örtlichkeit: AO, Strecke (300 m)

Regelungsart:-

Unfalltyp(en): Fahrunfall

$\varnothing$ Investitionskosten Maßnahme [€]: 13.300

$\varnothing$ jährliche Kosten Maßnahme [€]: 894

Anzahl ausgewertete Stellen: 5

Anzahl beteiligte UKo: 5

Bewertete Unfallkategorien:|Kat. 1 bis 6

Beschreibung Defizite, Konfliktsituationen und Datengrundlage

Defizite der Verkehrsanlage

- Bereiche mit Hindernissen im Seitenraum (Bäume, Hindernisse)

- passive Schutzeinrichtung ohne Unterfahrschutz

\section{Konflikte}

- Abkommen von der Fahrbahn

- Aufprall auf Hindernisse/passive Schutzeinrichtung im Seitenraum

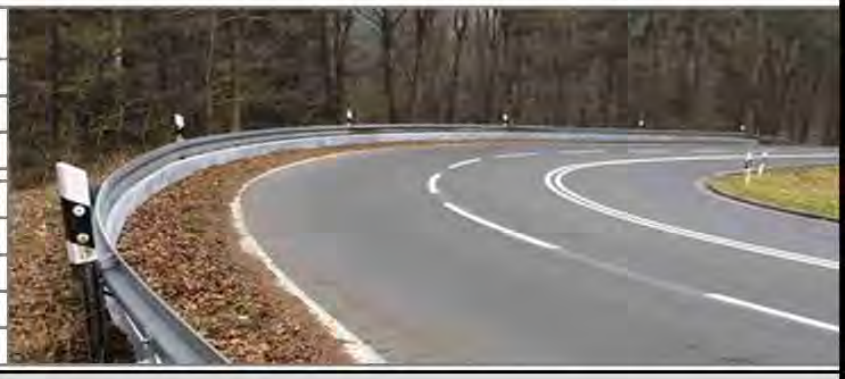

Wirkung der Maßnahme auf die Unfallanzahl (MW

\begin{tabular}{|c|c|c|c||c|}
\hline \hline \multicolumn{2}{|c|}{ mittlere Unfallanzahl pro Jahr [U/a] } & \multicolumn{2}{|c|}{ Wirkungsgrad } \\
\hline Kategorie & $\Sigma$ Vorher & $\Sigma$ Nachher & $\Delta=\Sigma(\mathrm{V}-\mathrm{N})$ & $\varnothing$ über alle Stellen [\%] \\
\hline $\mathrm{U}(\mathrm{P})$ & 1,56 & 1,54 & 0,02 & -4 \\
\hline $\mathrm{U}(\mathrm{SS}, \mathrm{LS})$ & 0,99 & 0,20 & 0,79 & 60 \\
\hline $\mathrm{U}(\mathrm{P}, \mathrm{SS}, \mathrm{LS})$ & 2,55 & 1,74 & 0,81 & 33 \\
\hline
\end{tabular}

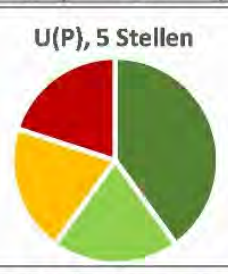

$\mathrm{U}(\mathrm{SS}, \mathrm{LS}), 5$ Stellen

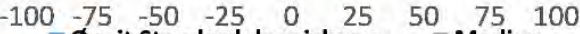

Zeiträume und Unfallgeschehen

\begin{tabular}{|c|c|c|c|c|c|c|c|c|}
\hline & \multicolumn{4}{|c|}{ Analyse (Vorher) } & \multicolumn{4}{|c|}{ Kontrolle (Nachher) } \\
\hline & \multicolumn{2}{|c|}{ Mon. } & \multirow{2}{*}{$U(P)$} & \multirow{2}{*}{$\mathrm{U}(\mathrm{SS}, \mathrm{LS})$} & \multicolumn{2}{|c|}{ Mon. } & \multirow{2}{*}{$U(P)$} & \multirow{2}{*}{$\mathrm{U}(\mathrm{SS}, \mathrm{L}$} \\
\hline & $P$ & $\mathrm{~S}$ & & & $P$ & $\mathrm{~s}$ & & \\
\hline 1 & 36 & 36 & 2 & 3 & 36 & 36 & 7 & 3 \\
\hline 2 & 36 & 36 & 3 & 0 & 36 & 36 & 1 & 0 \\
\hline 3 & 46 & 46 & 3 & 5 & 34 & 34 & 1 & 0 \\
\hline 4 & 36 & 36 & 15 & 2 & 18 & 18 & 7 & 0 \\
\hline 5 & 36 & 36 & 1 & 6 & 12 & 12 & 0 & 0 \\
\hline & & & & & & & & \\
\hline
\end{tabular}

6

vermiedene $\mathrm{U}(\mathrm{P}, \mathrm{SS}, \mathrm{LS})[\%]$

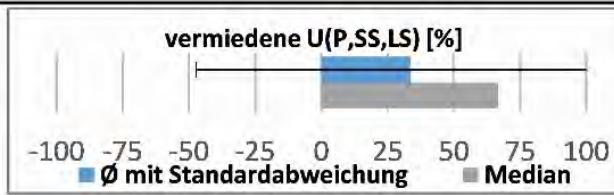

\section{Einteilung Wirkungsgrad}

Klassen $\quad$ ob. Grenze [\%] unt. Grenze [\%]

\begin{tabular}{|c|c|c|}
\hline bedeutend & 100 & 60 \\
\hline
\end{tabular}

\begin{tabular}{l|l|l}
\hline erheblich & $<60$ & 30
\end{tabular}

gering

neutral

nachteilig

\begin{tabular}{r|r}
$<30$ & 10 \\
$<10$ & -10
\end{tabular}

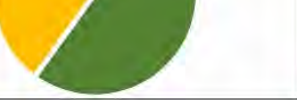

Wirkung der Maßnahme auf die Unfallkosten ( $\left(\mathrm{MW}_{\mathrm{UK}}\right)$

\begin{tabular}{|c|c|c|c||c|}
\hline \hline \multicolumn{2}{|c|}{ mittlere Unfallkosten pro Jahr [€/a] } & \multicolumn{2}{|c|}{ Wirkungsgrad } \\
\hline Kategorie & $\underline{\text { Vorher }}$ & $\underline{\text { Nachher }}$ & $\Delta=\sum(\mathrm{V}-\mathrm{N})$ & $\varnothing$ über alle Stellen [\%] \\
\hline UK(P) & 260.162 & 231.010 & 29.152 & -21 \\
\hline UK(SS,LS) & 8.736 & 838 & 7.898 & 60 \\
\hline UK(P,SS,LS) & 268.898 & 231.848 & 37.050 & -17 \\
\hline
\end{tabular}

\begin{tabular}{|r|r|}
\hline UK(P,SS,LS) & 268.898 \\
\hline UK $(P), 5$ Stellen
\end{tabular}

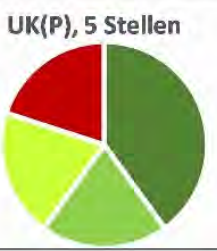

UK(SS,LS), 5 Stellen

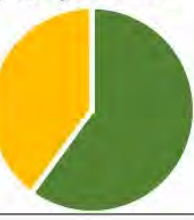

verwendete Unfallkostensätze [€]

\begin{tabular}{|l|c|}
\hline $\mathrm{U}(\mathrm{SP})$ & 266.000 \\
\hline $\mathrm{U}(\mathrm{LV})$ & 24.700 \\
\hline $\mathrm{U}(\mathrm{SS})$ & 17.900 \\
\hline $\mathrm{U}(\mathrm{LS})$ & 4.190 \\
\hline
\end{tabular}

\section{vermiedene UK(P,SS,LS) [\%]}

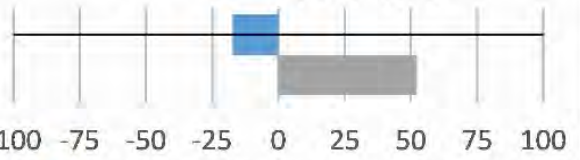

$\begin{array}{lllllllll}-100 & -75 & -50 & -25 & 0 & 25 & 50 & 75 & 100\end{array}$

$₫ \emptyset$ mit Standardabweichung $=$ Median

Nutzen-Kosten-Verhältnis (NKV)

\section{NKV Spannbreite und Median}

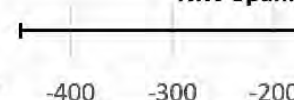

$-100$

0

100

200

300

Median Nutzen-Kosten-Verhältnis:

$\varnothing$ Nutzen-Kosten-Verhältnis:

Zusammenfassung und Empfehlung

Maßnahmenbeschreibung/Ziel

Minderung der Unfallschwere für Motorradfahrer durch das Anbringen von

Unterfahrschutz an passive Schutzeinrichtung

Zusammenfassung Kennwerte

$\varnothing$ Abnahme der Unfallanzahl: nur bedingt

$\varnothing$ Abnahme der Unfallkosten: deutlich

$\varnothing$ Nutzen-Kosten-Verhältnis: rentabel 


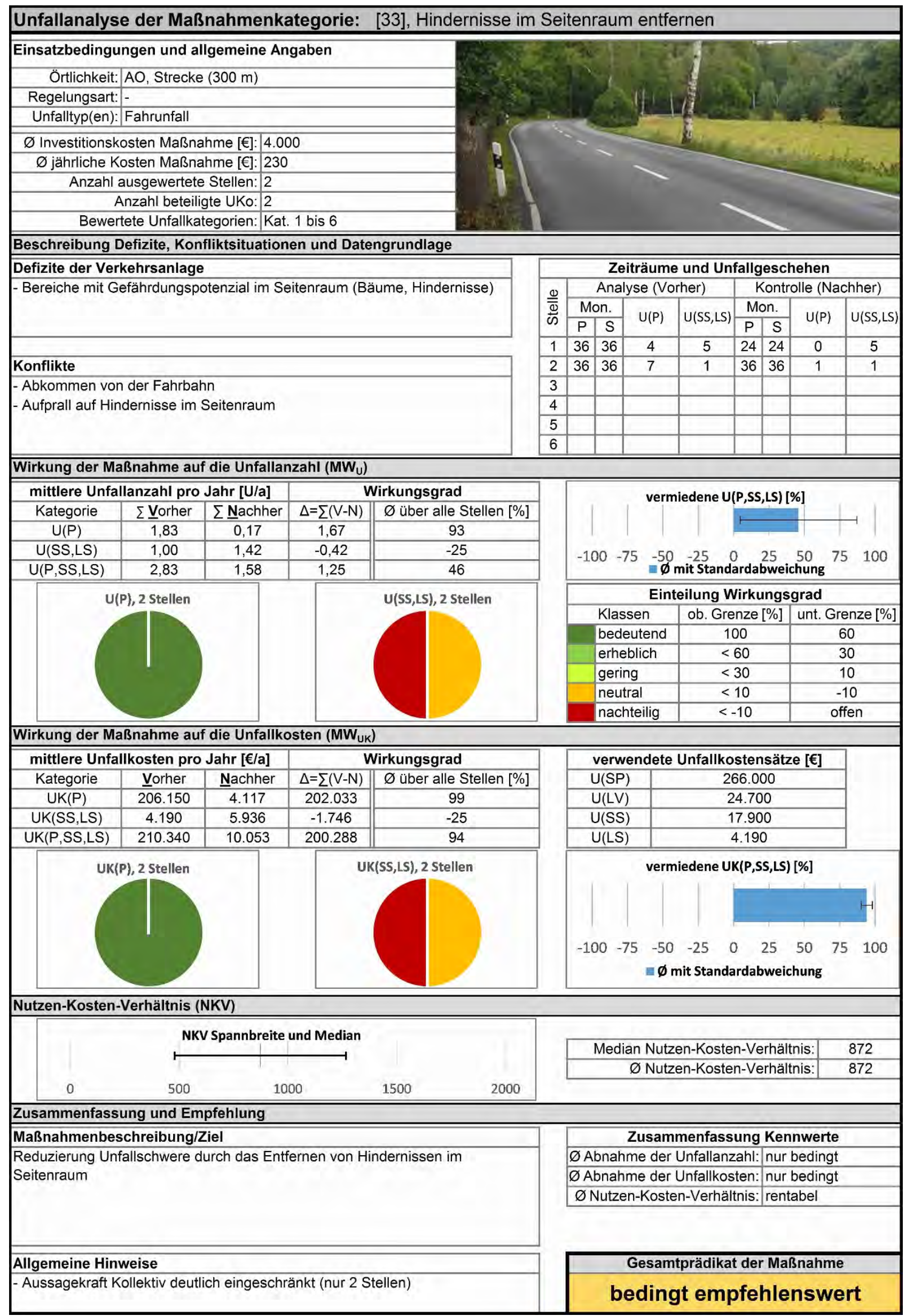




\section{Unfallanalyse der Maßnahmenkategorie: [34], Rüttelstreifen aufbringen}

Einsatzbedingungen und allgemeine Angaben

Örtlichkeit: $A O$, Strecke $(300 \mathrm{~m})$

Regelungsart:-

Unfalltyp(en): Fahrunfall

$\varnothing$ Investitionskosten Maßnahme [€]: 2.400

$\varnothing$ jährliche Kosten Maßnahme [€]: 523

Anzahl ausgewertete Stellen: 5

Anzahl beteiligte UKo: 3

Bewertete Unfallkategorien: Kat. 1 bis 6

Beschreibung Defizite, Konfliktsituationen und Datengrundlage

Defizite der Verkehrsanlage

- kurvige Strecke

- Streckenverlauf schwer einschätzbar

- hohe Geschwindigkeiten von Motorradfahrern

\section{Konflikte}

- Abkommen von der Fahrbahn

- sehr hoher Anteil verunfallter Motorradfahrer

Wirkung der Maßnahme auf die Unfallanzahl $\left(\mathrm{MW}_{\mathrm{U}}\right)$

\begin{tabular}{|c|c|c|c||c|}
\hline \multicolumn{2}{|c|}{ mittlere Unfallanzahl pro Jahr [U/a] } & \multicolumn{2}{c|}{ Wirkungsgrad } \\
\hline Kategorie & $\sum \underline{\text { Vorher }}$ & $\sum$ Nachher & $\Delta=\sum(\mathrm{V}-\mathrm{N})$ & $\varnothing$ über alle Stellen [\%] \\
\hline $\mathrm{U}(\mathrm{P})$ & 2,07 & 0,67 & 1,40 & 71 \\
\hline $\mathrm{U}(\mathrm{SS}, \mathrm{LS})$ & 2,93 & 1,80 & 1,13 & 35 \\
\hline $\mathrm{U}(\mathrm{P}, \mathrm{SS}, \mathrm{LS})$ & 5,00 & 2,47 & 2,53 & 56 \\
\hline
\end{tabular}

$\mathrm{U}(\mathrm{P}, \mathrm{SS}, \mathrm{LS})$
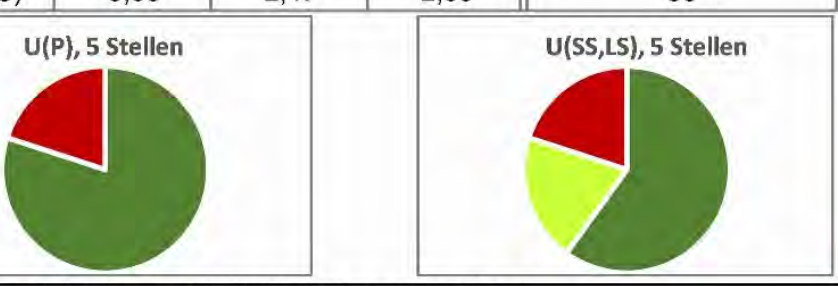

Wirkung der Maßnahme auf die Unfallkosten $\left(\mathrm{MW}_{\mathrm{UK}}\right)$

\begin{tabular}{|c|c|c|c||c|}
\hline \hline \multicolumn{2}{|c|}{ mittlere Unfallkosten pro Jahr [€/a] } & \multicolumn{2}{|c|}{ Wirkungsgrad } \\
\hline Kategorie & $\underline{\text { Vorher }}$ & $\underline{\text { Nachher }}$ & $\Delta=\sum(\mathrm{V}-\mathrm{N})$ & $\varnothing$ über alle Stellen [\%] \\
\hline UK(P) & 356.693 & 48.640 & 308.053 & 88 \\
\hline UK(SS,LS) & 22.345 & 7.542 & 14.803 & 65 \\
\hline UK(P,SS,LS) & 379.038 & 56.182 & 322.856 & 87 \\
\hline
\end{tabular}

UK(P,SS,LS

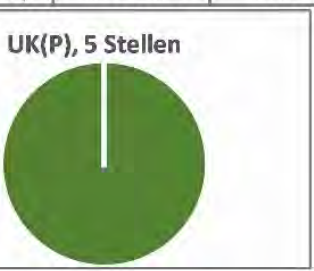

UK(SS,LS), 5 Stellen

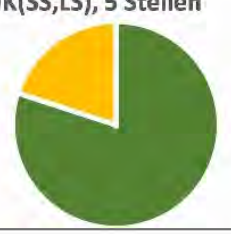

Nutzen-Kosten-Verhältnis (NKV)

NKV Spannbreite und Median

0

200

400

600

800

1000

1200

1400

\begin{tabular}{|c|c|c|c|c|c|c|c|c|}
\hline \multicolumn{9}{|c|}{ Zeiträume und Unfallgeschehen } \\
\hline \multirow{3}{*}{$\frac{\frac{0}{\overline{0}}}{\dot{\omega}}$} & \multicolumn{4}{|c|}{ Analyse (Vorher) } & \multicolumn{4}{|c|}{ Kontrolle (Nachher) } \\
\hline & \multicolumn{2}{|c|}{ Mon. } & \multirow{2}{*}{$U(P)$} & \multirow{2}{*}{$\mathrm{U}(\mathrm{SS}, \mathrm{LS})$} & \multicolumn{2}{|c|}{ Mon. } & \multirow{2}{*}{$U(P)$} & \multirow{2}{*}{$\mathrm{U}(\mathrm{SS}, \mathrm{LS})$} \\
\hline & $\mathrm{P}$ & $\mathrm{S}$ & & & $\mathrm{P}$ & $\mathrm{s}$ & & \\
\hline 1 & 36 & 36 & 9 & 2 & 36 & 36 & 0 & 0 \\
\hline 2 & 36 & 36 & 7 & 4 & 36 & 36 & 2 & 7 \\
\hline 3 & 36 & 36 & 4 & 17 & 36 & 36 & 0 & 6 \\
\hline 4 & 36 & 36 & 7 & 16 & 36 & 36 & 8 & 12 \\
\hline 5 & 36 & 36 & 4 & 5 & 18 & 18 & 0 & 1 \\
\hline 6 & & & & & & & & \\
\hline
\end{tabular}

vermiedene $U(P, S S, L S)[\%]$

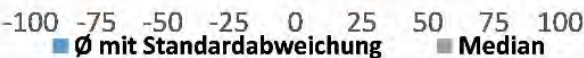

\begin{tabular}{|l|c|c|}
\hline \hline \multicolumn{3}{|c|}{ Einteilung Wirkungsgrad } \\
\hline Klassen & ob. Grenze [\%] & unt. Grenze [\%] \\
\hline bedeutend & 100 & 60 \\
\hline erheblich & $<60$ & 30 \\
\hline gering & $<30$ & 10 \\
\hline neutral & $<10$ & -10 \\
\hline nachteilig & $<-10$ & offen \\
\hline
\end{tabular}

Zusammenfassung und Empfehlung

Maßnahmenbeschreibung/Ziel

Reduktion der gefahrenen Geschwindigkeiten durch Motorradfahrer durch das

Aufbringen von Rüttelstreifen quer zur Fahrtrichtung

Zusammenfassung Kennwerte

$\varnothing$ Abnahme der Unfallanzahl: nur bedingt

$\varnothing$ Abnahme der Unfallkosten: deutlich

$\varnothing$ Nutzen-Kosten-Verhältnis: rentabel 


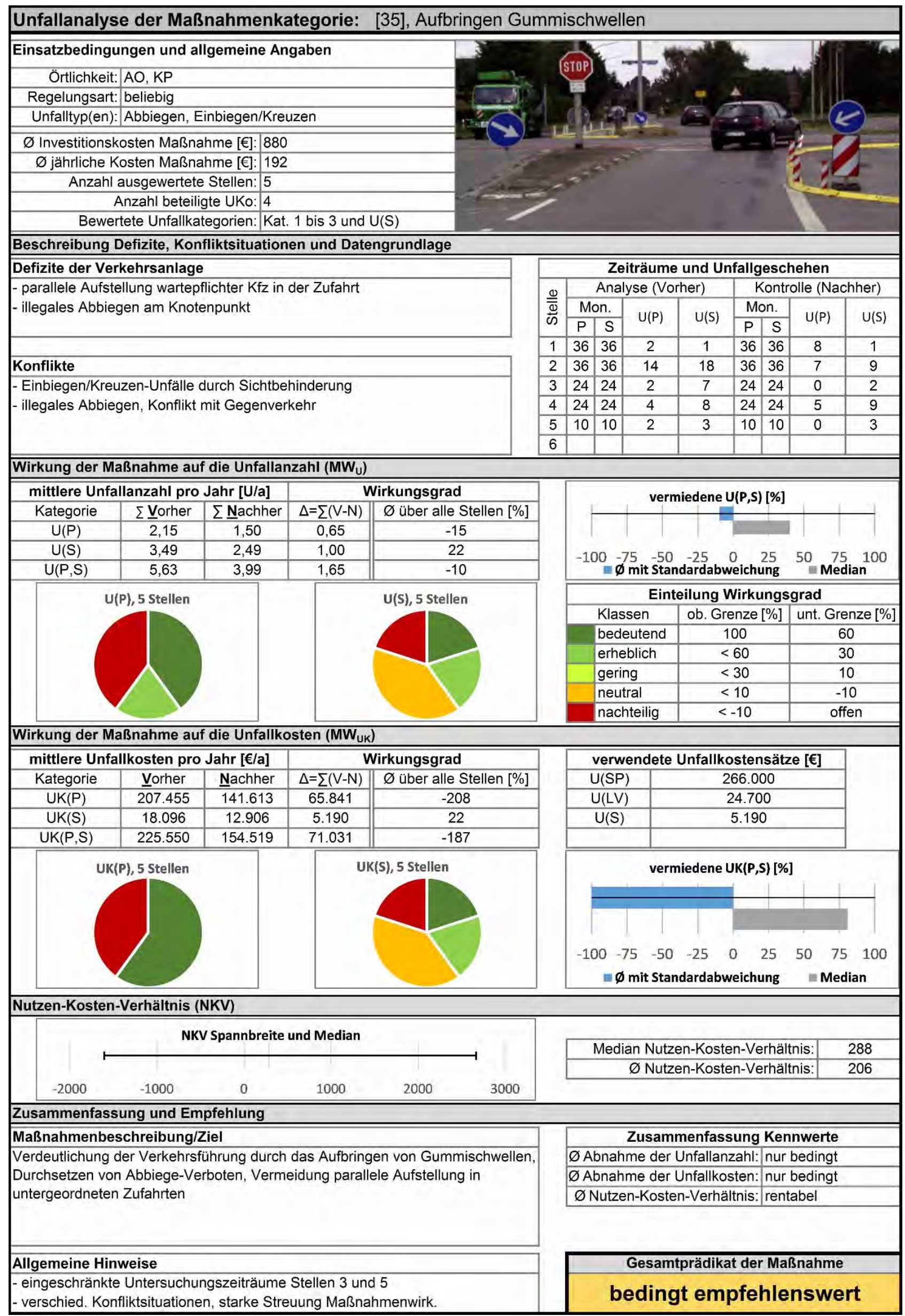




\section{Unfallanalyse der Maßnahmenkategorie: [36], Markierung Sperrfläche}

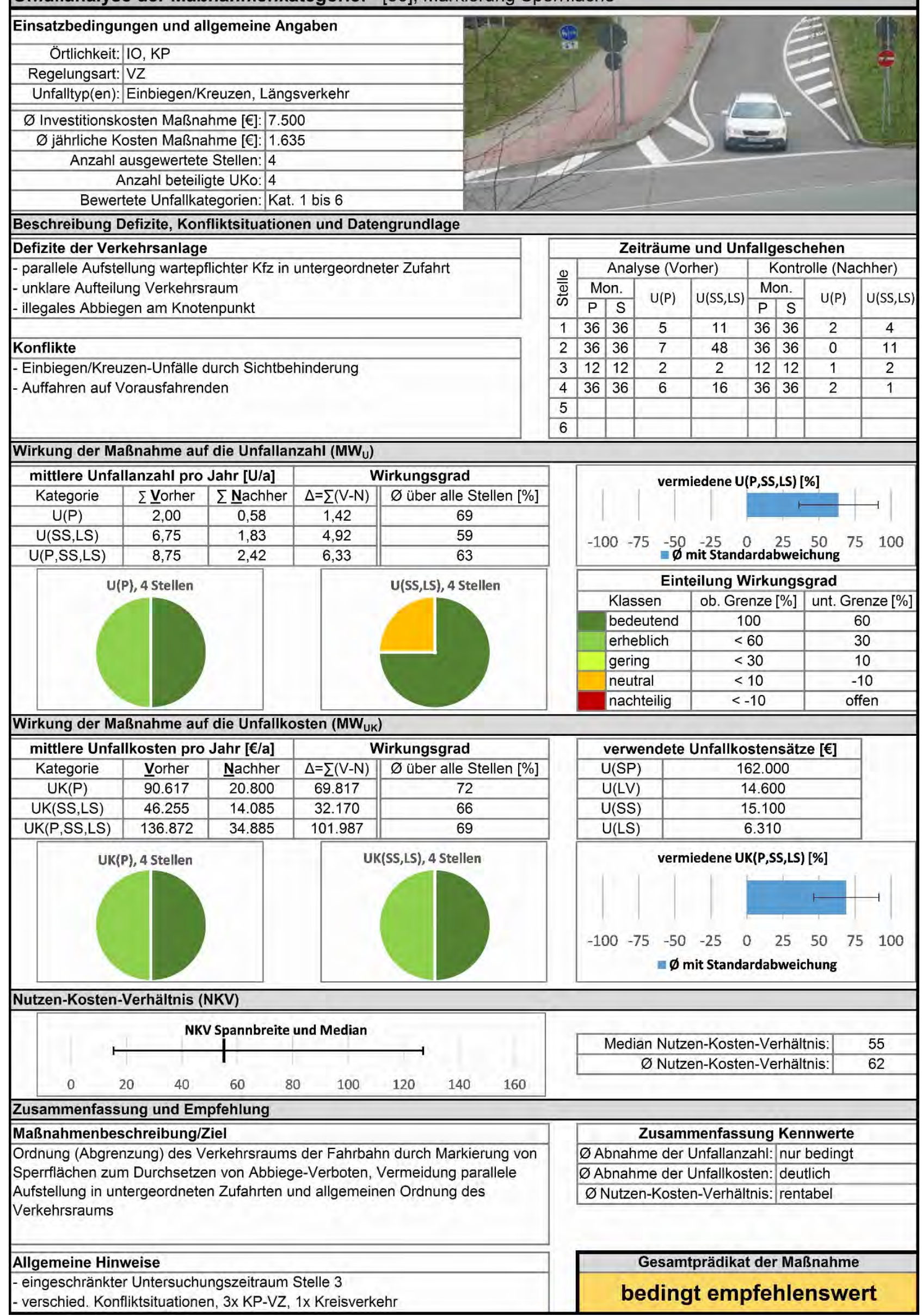




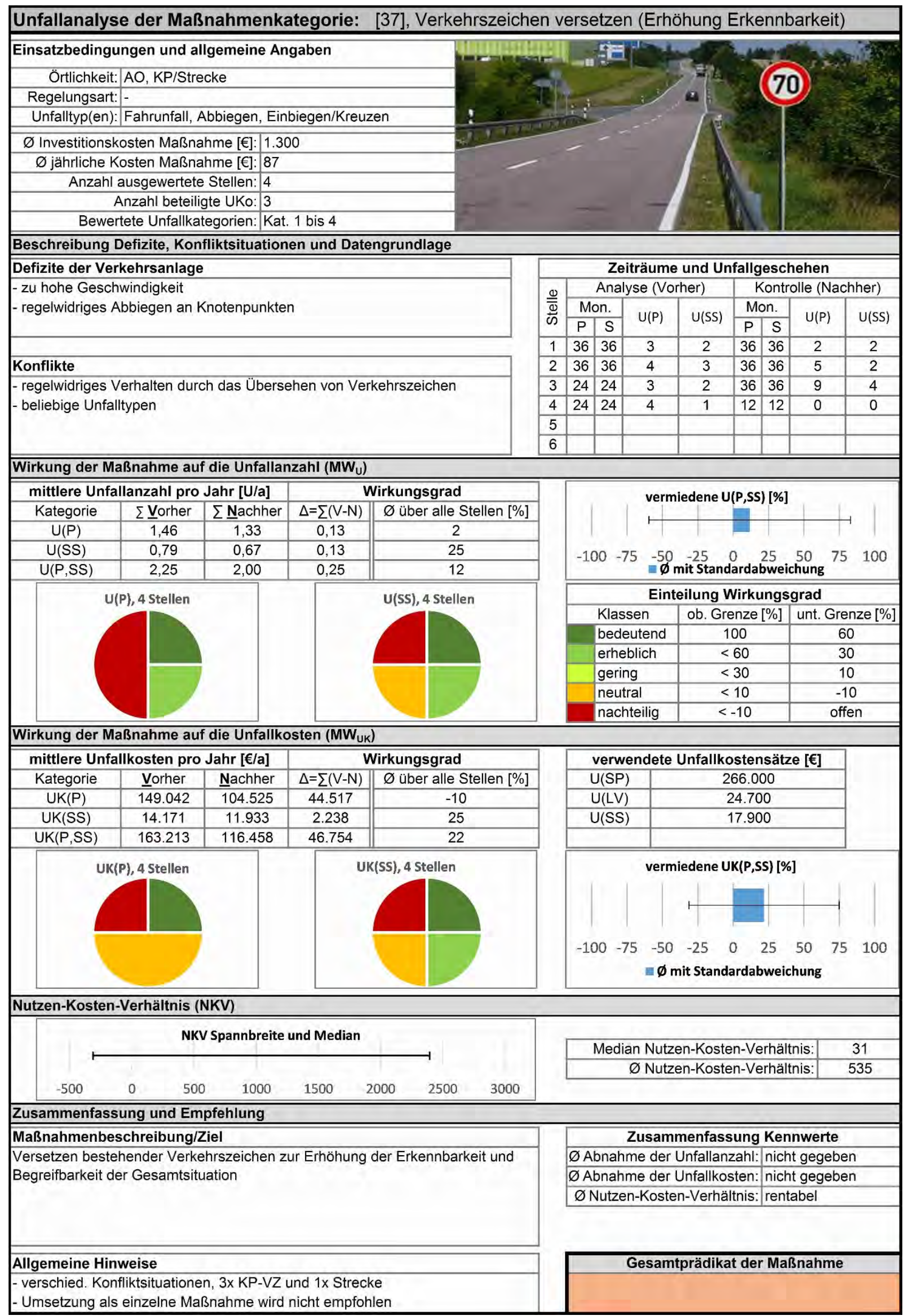


Unfallanalyse der Maßnahmenkategorie: [38], Beschränkung Vzul und Z 625 StVO (Richt.-tafel in Kurve)

Einsatzbedingungen und allgemeine Angaben

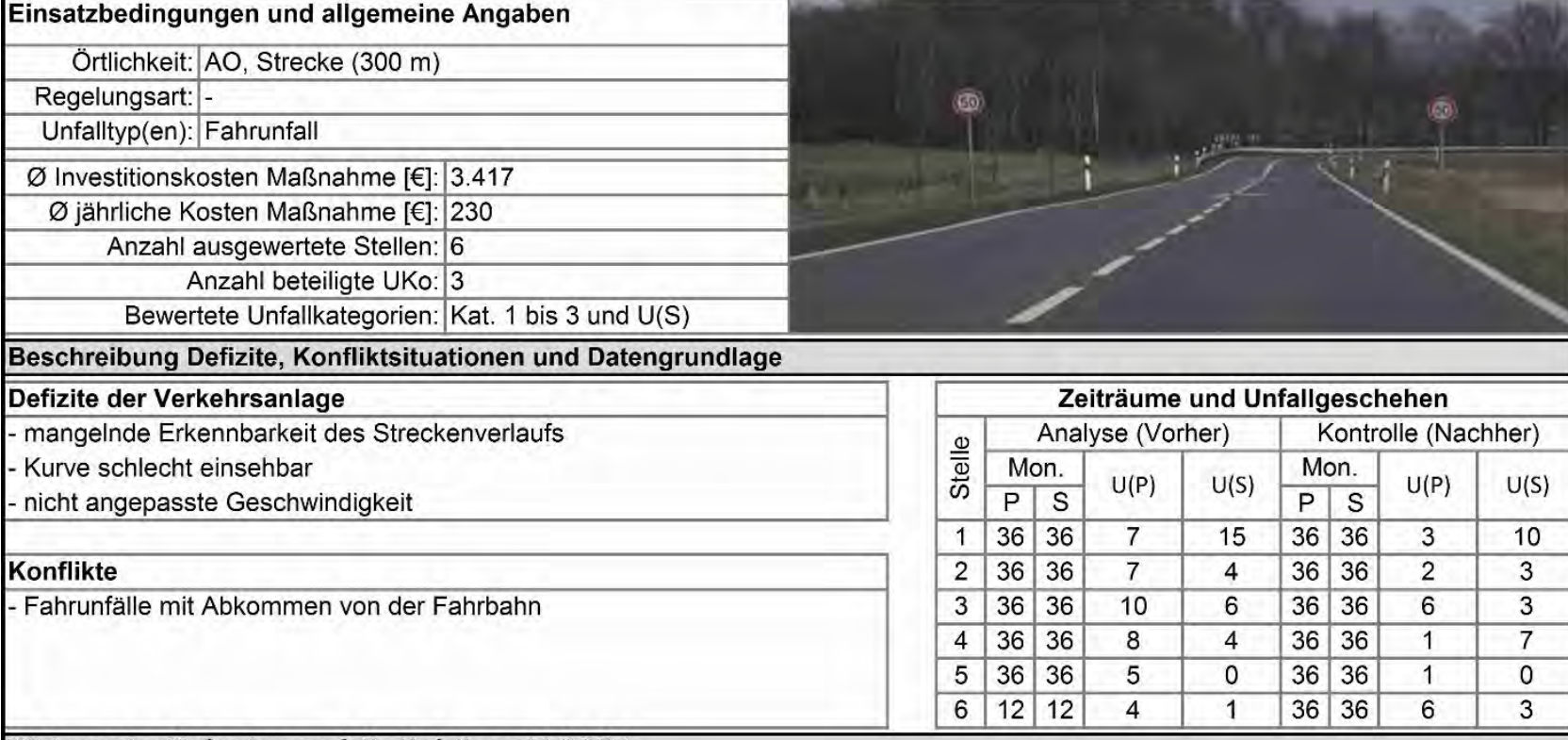

Wirkung der Maßnahme auf die Unfallanzahl (MW $\left.{ }_{\mathrm{U}}\right)$

\begin{tabular}{|c|c|c|c||c|}
\hline \multicolumn{2}{|c|}{ mittlere Unfallanzahl pro Jahr [U/a] } & \multicolumn{2}{|c|}{ Wirkungsgrad } \\
\hline Kategorie & $\Sigma$ Vorher & $\sum$ Nachher & $\Delta=\Sigma(\mathrm{V}-\mathrm{N})$ & $\varnothing$ über alle Stellen [\%] \\
\hline $\mathrm{U}(\mathrm{P})$ & 2,72 & 1,06 & 1,67 & 64 \\
\hline $\mathrm{U}(\mathrm{S})$ & 1,78 & 1,44 & 0,33 & 6 \\
\hline $\mathrm{U}(\mathrm{P}, \mathrm{S})$ & 4,50 & 2,50 & 2,00 & 49 \\
\hline
\end{tabular}

$U(P), 6$ Stellen

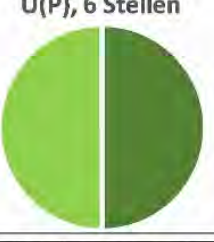

U(S), 6 Stellen

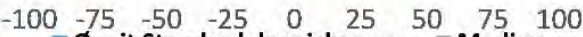

vermiedene $U(P, S)[\%]$

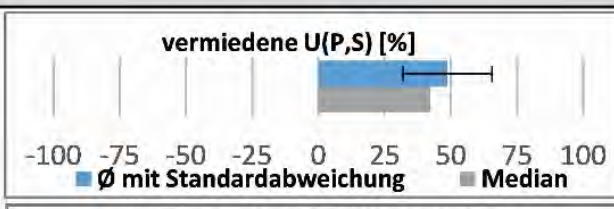

\section{Einteilung Wirkungsgrad}

Klassen $\quad$ ob. Grenze [\%] unt. Grenze [\%]

\begin{tabular}{|c|c|c|}
\hline bedeutend & 100 & 60 \\
\hline
\end{tabular}

\begin{tabular}{l|l|l}
\hline erheblich & $<60$ & 30
\end{tabular}

gering

neutral

nachteilig

\begin{tabular}{l|r}
$<30$ & 10 \\
$<10$ & -10
\end{tabular}

\begin{tabular}{c|c}
$<10$ & -10 \\
\hline$<-10$ & offen
\end{tabular}

Wirkung der Maßnahme auf die Unfallkosten ( $\left(\mathrm{MW}_{\mathrm{UK}}\right)$

\begin{tabular}{|c|c|c|c||c|}
\hline \multicolumn{2}{|c|}{ mittlere Unfallkosten pro Jahr [€/a] } & \multicolumn{2}{c|}{ Wirkungsgrad } \\
\hline Kategorie & $\underline{\text { Vorher }}$ & $\underline{\text { Nachher }}$ & $\Delta=\Sigma(\mathrm{V}-\mathrm{N})$ & $\varnothing$ über alle Stellen [\%] \\
\hline UK(P) & 402.378 & 79.694 & 322.683 & 80 \\
\hline UK(S) & 9.227 & 7.497 & 1.730 & 6 \\
\hline UK(P,S) & 411.604 & 87.191 & 324.413 & 79 \\
\hline
\end{tabular}

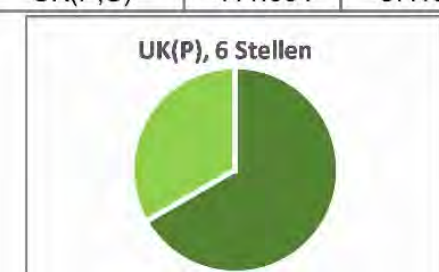

UK(S), 6 Stellen

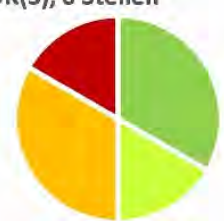

\begin{tabular}{|l|c|}
\hline \multicolumn{2}{|c|}{ verwendete Unfallkostensätze [€] } \\
\hline$U(S P)$ & 266.000 \\
\hline$U(L V)$ & 24.700 \\
\hline$U(S)$ & 5.190 \\
\hline & \\
\hline
\end{tabular}

vermiedene UK(P,S) [\%]

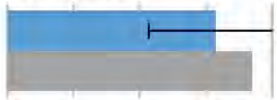

$\begin{array}{lllllllll}-100 & -75 & -50 & -25 & 0 & 25 & 50 & 75 & 100\end{array}$

$\approx \emptyset$ mit Standardabweichung $\equiv$ Median

Nutzen-Kosten-Verhältnis (NKV)

\begin{tabular}{|lllllllll|}
\hline & \multicolumn{9}{|c|}{ NKV Spannbreite und Median } & & \\
& & 500 & 1000 & 1500 & 2000 & 2500 & 3000 & 3500 \\
\hline & 50 & & & & & \\
\hline
\end{tabular}

Zusammenfassung und Empfehlung

Maßnahmenbeschreibung/Ziel

Verdeutlichung Streckenverlauf durch das Aufstellen von Z 625 (Richtungstafeln

in Kurven) im Kurvenbereich und Beschränkung der zulässigen

Höchstgeschwindigkeit

\begin{tabular}{r|r} 
Median Nutzen-Kosten-Verhältnis: & 1.196 \\
$\varnothing$ Nutzen-Kosten-Verhältnis: & 1.536
\end{tabular}

Zusammenfassung Kennwerte

$\varnothing$ Abnahme der Unfallanzahl: deutlich

Ø Abnahme der Unfallkosten: deutlich

$\varnothing$ Nutzen-Kosten-Verhältnis: rentabel 


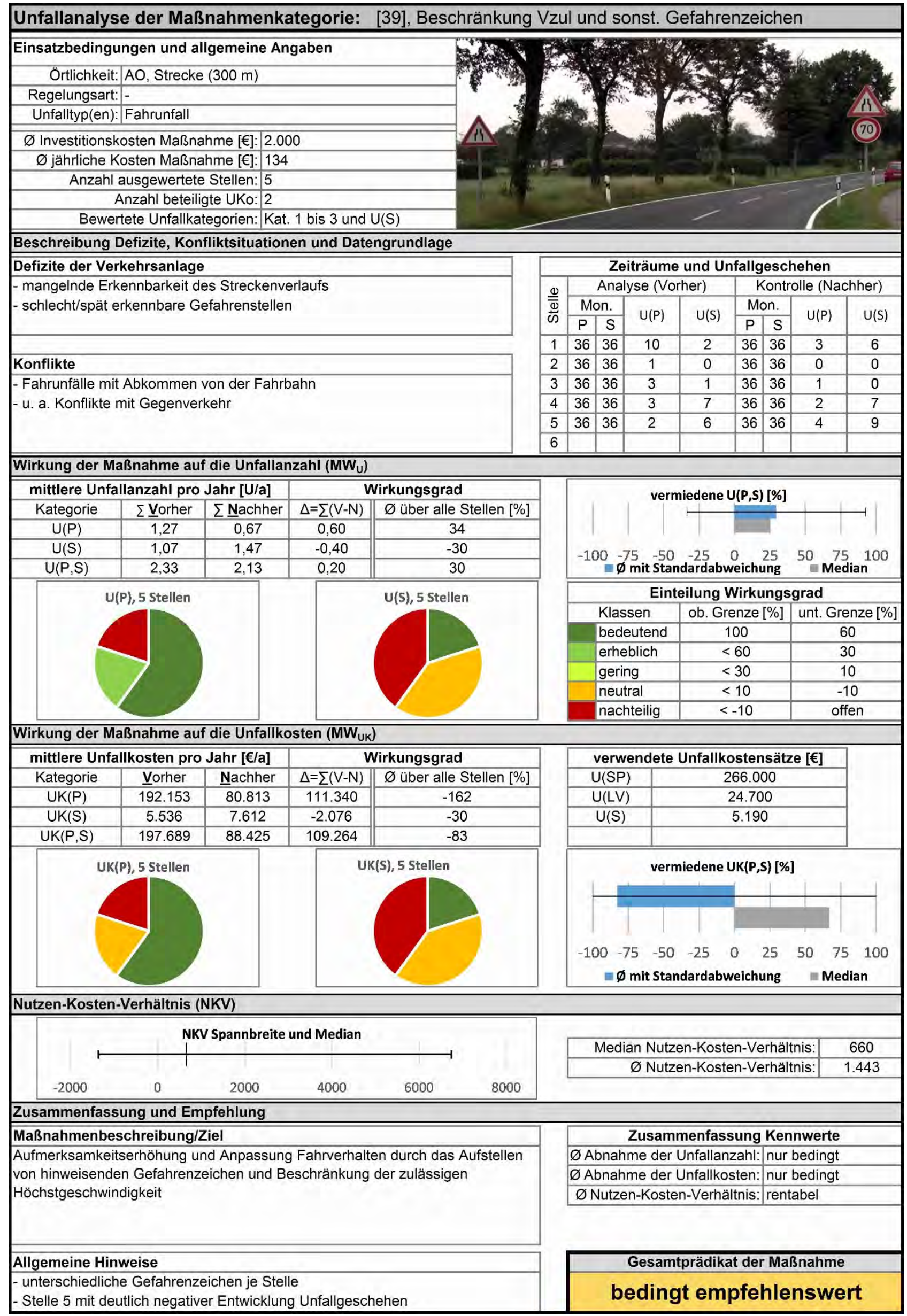




\section{Anhang 8 Darstellung Präsentationsbeispiele Maßnahmenkategorien}

Nachfolgend enthalten sind die Aufbereitungen der Maßnahmenkategorien, wie sie den Unfallkommissionen während der Evaluation vorgestellt wurden. Die nachstehende Tabelle verweist auf die Seitenzahl der jeweiligen Maßnahmenkategorien.

\begin{tabular}{|c|c|c|}
\hline \multicolumn{2}{|r|}{ Maßnahmenkategorie } & \multirow{2}{*}{$\begin{array}{l}\text { Seite im } \\
\text { Anhang }\end{array}$} \\
\hline Nr. & Name & \\
\hline 1 & Verbesserung der Koordinierung & LXXXI \\
\hline 2 & Änderung der Signalisierung & LXXXI \\
\hline 3 & "sonstige" Gefahrenzeichen anbringen & LXXXII \\
\hline 4 & HALT Z 206 StVO (anstelle Z 205 StVO) & \multirow{2}{*}{ LXXXII } \\
\hline 5 & HALT Z 206 StVO (anstelle Z 205 StVO) & \\
\hline 6 & Halte-/Parkverbot anordnen & LXXXIII \\
\hline 7 & Z 625 StVO (Richtungstafel in Kurven) aufstellen & LXXXIII \\
\hline 8 & Zusatzzeichen Z 1006-36 StVO (Unfallgefahr) ergänzen & \multirow{2}{*}{ LXXXIV } \\
\hline 9 & Zusatzzeichen Z 1006-36 StVO (Unfallgefahr) ergänzen & \\
\hline 10 & Verkehrszeichen anpassen & \multirow{2}{*}{ LXXXIV } \\
\hline 11 & Verkehrszeichen anpassen & \\
\hline 12 & Beschränkung Vzul & LXXXV \\
\hline 15 & Beschränkung Vzul & LXXXV \\
\hline 16 & Überwachung zulässige Höchstgeschwindigkeit mit OGÜ & \multirow{2}{*}{ LXXXVI } \\
\hline 17 & Überwachung zulässige Höchstgeschwindigkeit mit OGÜ & \\
\hline 18 & Erneuerung Fahrbahnoberfläche & LXXXVI \\
\hline 19 & Zusätzlichen Signalgeber anordnen & LXXXVII \\
\hline 21 & Leuchtmittel Signalgeber verbessern & LXXXVII \\
\hline 22 & Sichthindernisse entfernen & \multirow{2}{*}{ LXXXVIII } \\
\hline 23 & Sichthindernisse entfernen & \\
\hline 24 & Beleuchtung anpassen/verbessern & LXXXVIII \\
\hline 25 & Sichtschutzzaun aufstellen & LXXXIX \\
\hline 26 & Markierung anpassen/verbessern & LXXXIX \\
\hline 27 & Markierung in Stand setzen & $\mathrm{XC}$ \\
\hline 29 & Radverkehrsführung anpassen & $\mathrm{XC}$ \\
\hline 30 & Radfahrfurt Rot einfärben & $\mathrm{XCl}$ \\
\hline 31 & Passive Schutzeinrichtung aufstellen & $\mathrm{XCl}$ \\
\hline 32 & Unterfahrschutz an passiver Schutzeinrichtung anbringen & $\mathrm{XClI}$ \\
\hline 33 & Hindernisse im Seitenraum entfernen & $\mathrm{XCll}$ \\
\hline 34 & Rüttelstreifen aufbringen & XCIII \\
\hline 35 & Gummischwellen aufbringen & $\mathrm{XCIII}$ \\
\hline 36 & Sperrfläche markieren & XCIV \\
\hline 37 & Verkehrszeichen versetzen (Erhöhung Erkennbarkeit) & XCIV \\
\hline 38 & Beschränkung Vzul und Z 625 StVO (Richtungstafel in Kurve) & $\mathrm{XCV}$ \\
\hline 39 & Beschränkung Vzul und sonst. Gefahrenzeichen & $\mathrm{XCV}$ \\
\hline
\end{tabular}




\section{Konflikt}

Abbiegen (mit Entgegenkommenden)

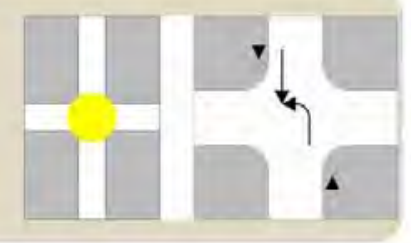

Örtlichkeit

Kotenpunkt Innerorts

\section{Regelungsart}

Lichtsignalanlage

\section{Maßnahme: Verbesserung der Koordinierung}

Anpassung Koordinierung um "Mitschleifen" zu unterbinden
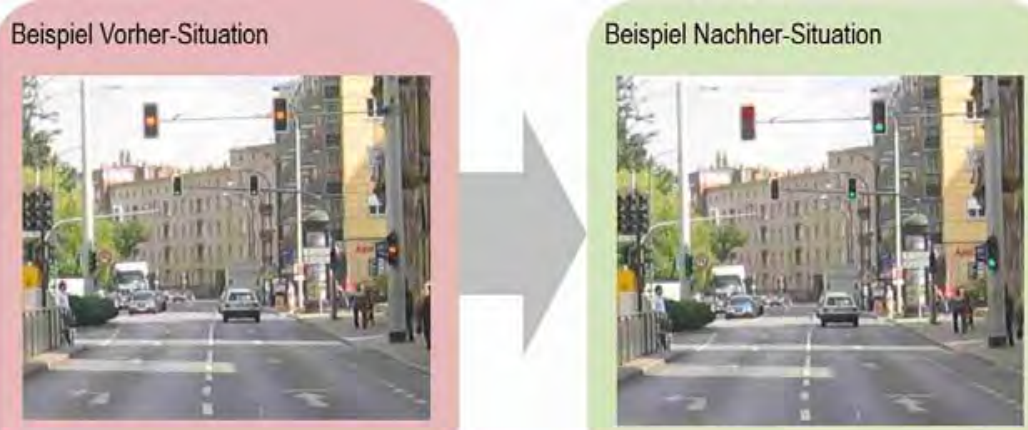

Maßnahmenkategorie 1

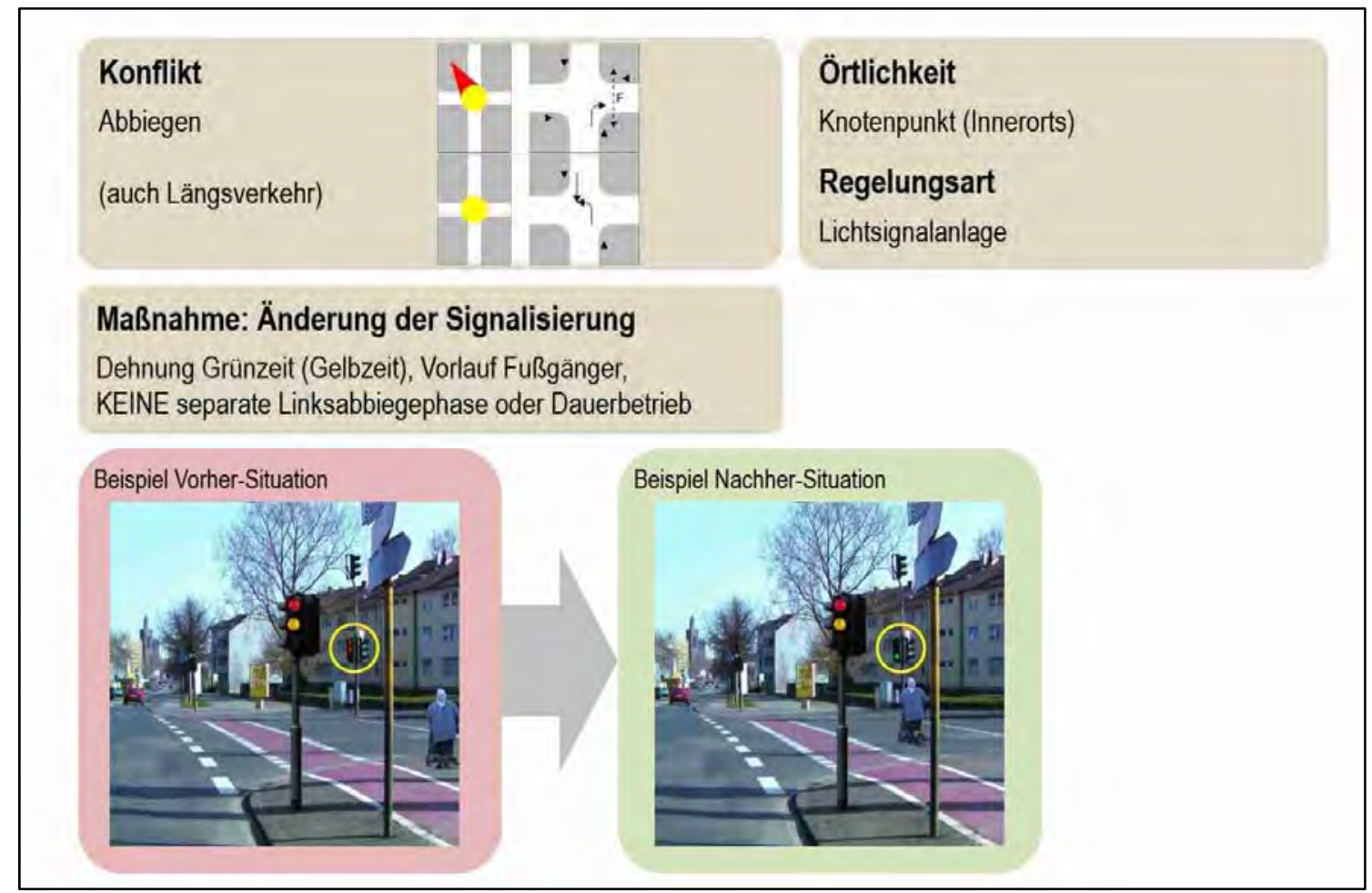

Maßnahmenkategorie 2 


\section{Konflikt}

Fahrunfall

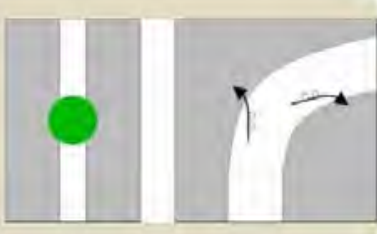

Maßnahme: „sonst.“ Gefahrenzeichen anbringen

Allgemeine Gefahrenzeichen,

KEINE Zeichen die Streckenverlauf (Kurve o. a.) zeigen

\section{Örtlichkeit}

Strecke Außerorts

Regelungsart

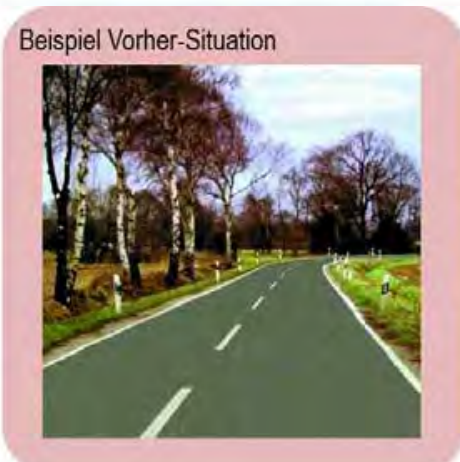

Beispiel Nachher-Situation
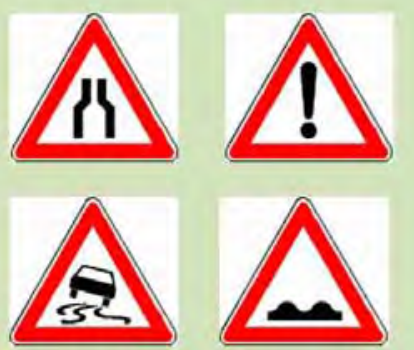

Maßnahmenkategorie 3

\section{Konflikt}

Wartepflichtiger mit Bevorrechtigtem von links oder rechts

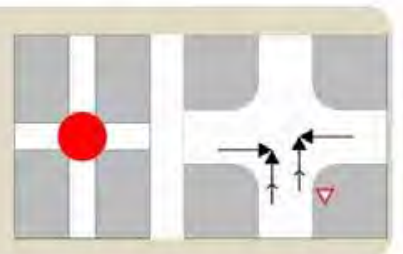

Örtlichkeit

Knotenpunkt (10 oder AO)

\section{Regelungsart}

durch Verkehrszeichen

Maßnahme: Halt Z 206 StVO (anstelle Z 205 StVO)

Ersetzen Z 205 durch Z 206 (Halt! Vorfahrt gewähren), Markierung einer Haltlinie

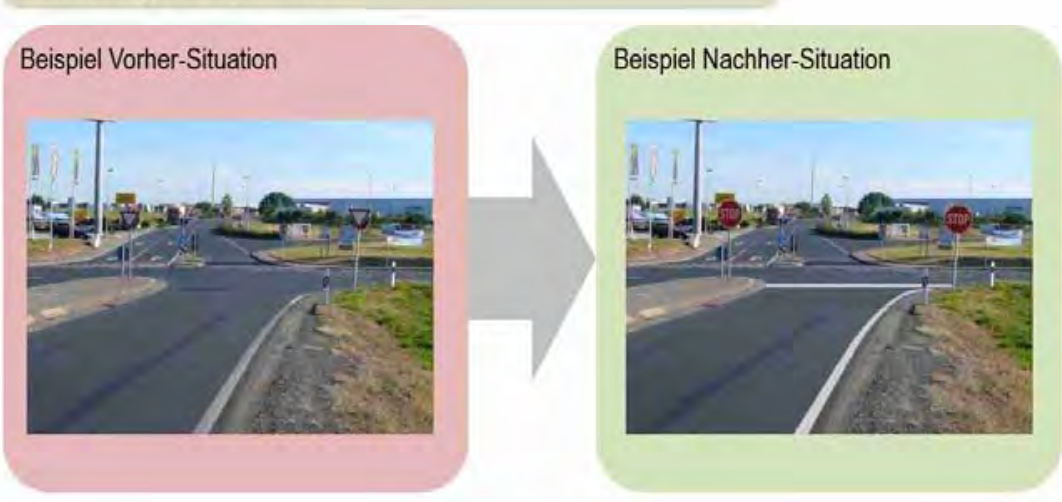

Maßnahmenkategorie 4 und 5 


\section{Konflikt}

Wartepflichtiger mit Bevorrechtigtem von links oder rechts

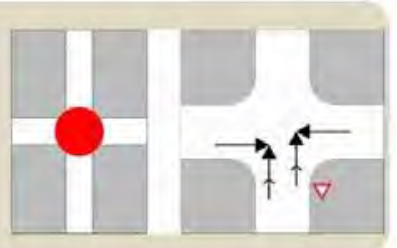

Örtlichkeit

Knotenpunkt Innerorts

Regelungsart

durch Verkehrszeichen / rechts-vor-links

\section{Maßnahme: Halte-/Parkverbot anordnen}

Anordnung Halte-IParkverbot ( durch Verkehrszeichen)

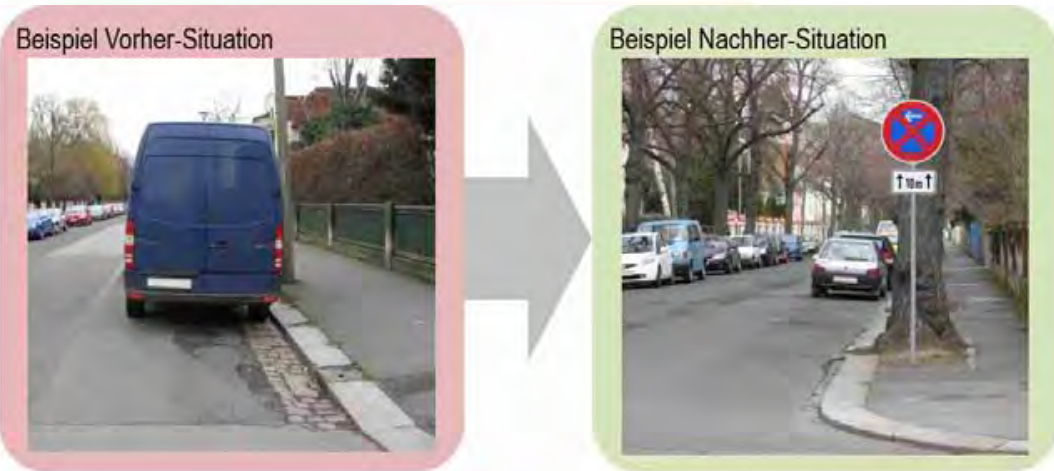

Maßnahmenkategorie 6

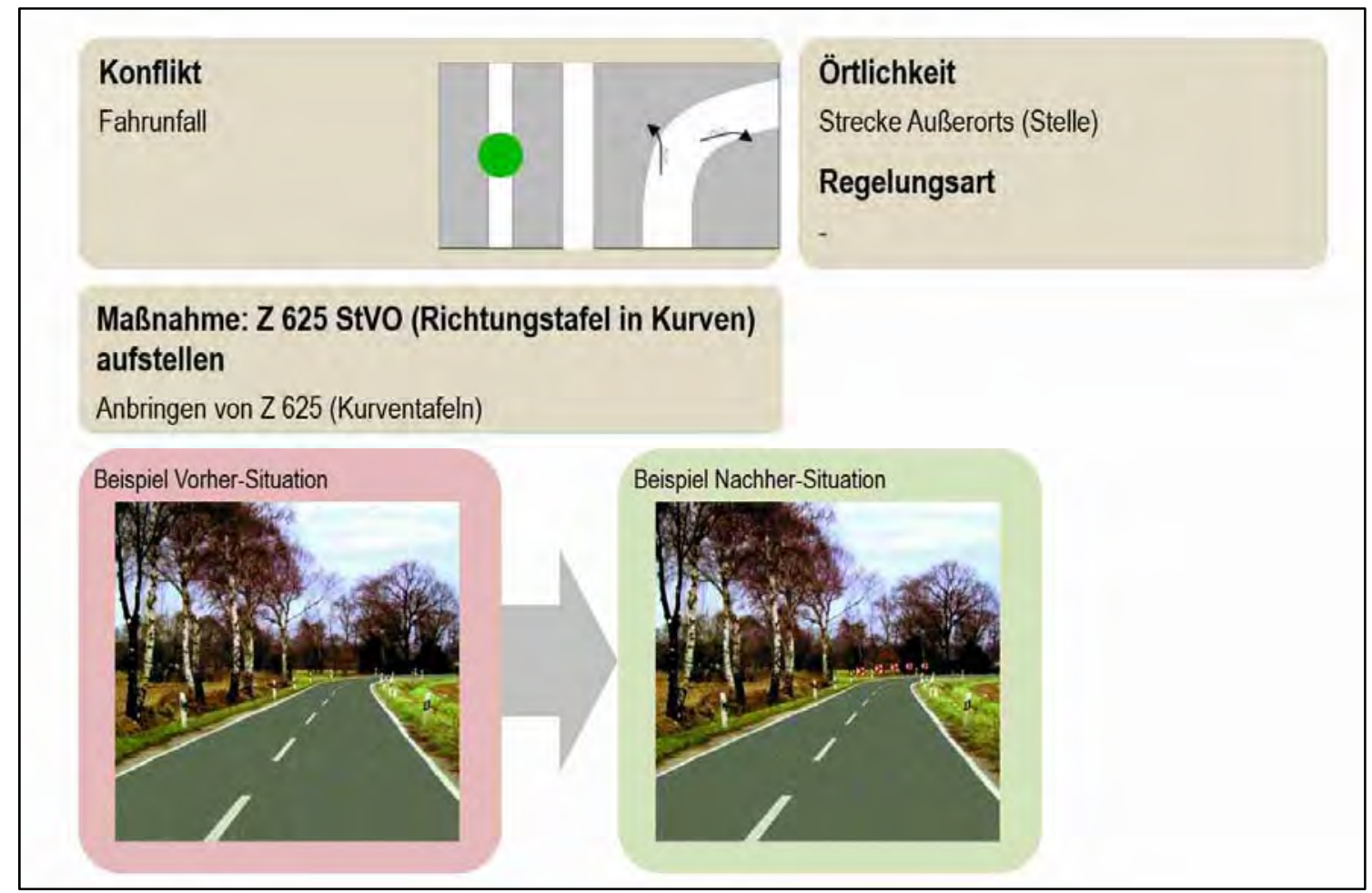

Maßnahmenkategorie 7 


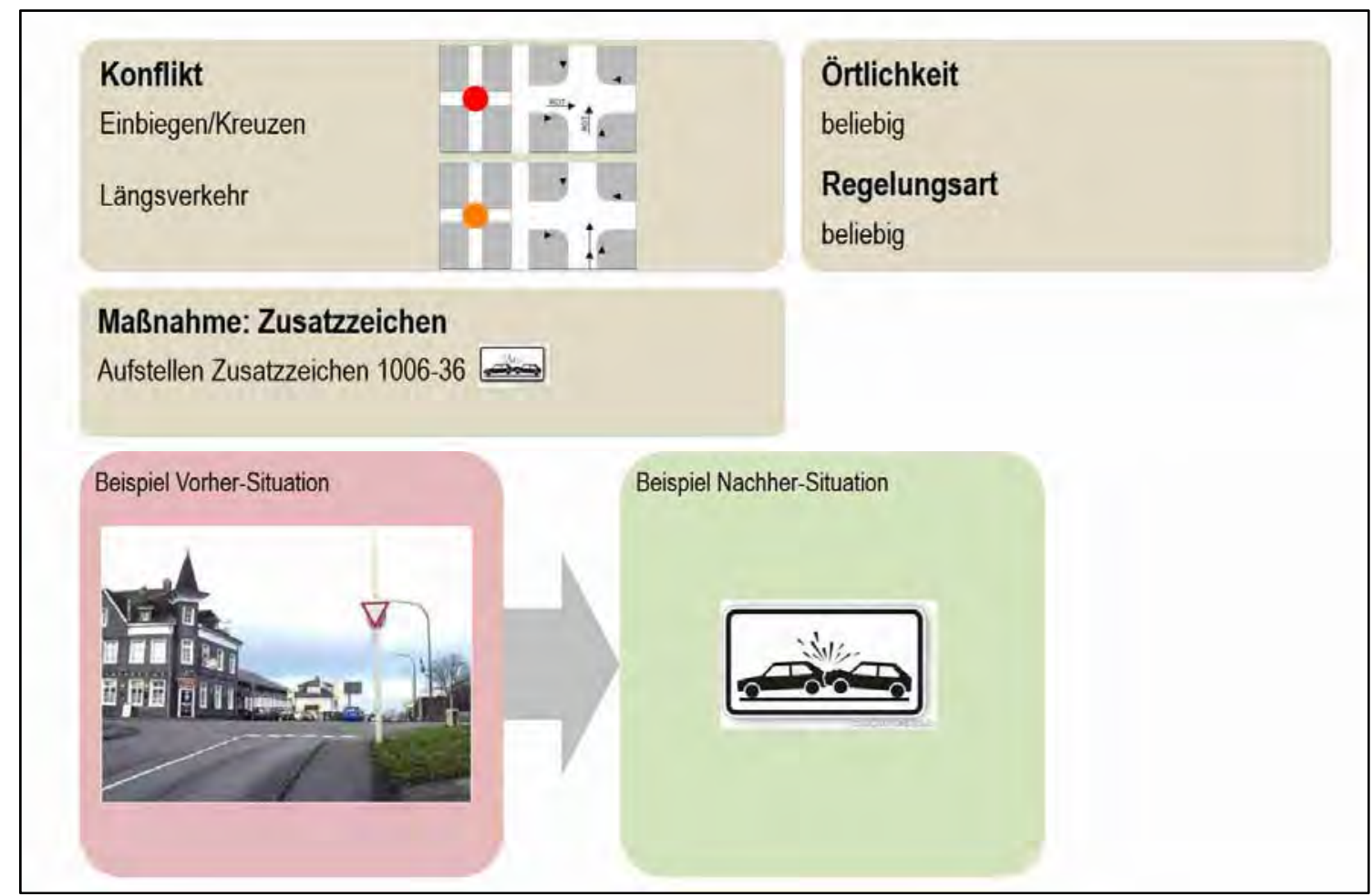

Maßnahmenkategorie 8 und 9

\section{Konflikt}

Fahrunfall

Abbiegen

Einbiegen/Kreuzen

Längsverkehr

\section{Örtlichkeit}

beliebig (häufig vor Knotenpunkten)

Regelungsart

\section{Maßnahme: Verkehrszeichen anpassen}

Verkehrszeichen zur besseren Erkennbarkeit vergrößern, verkleinern, mit reflektierender Folie ausstatten
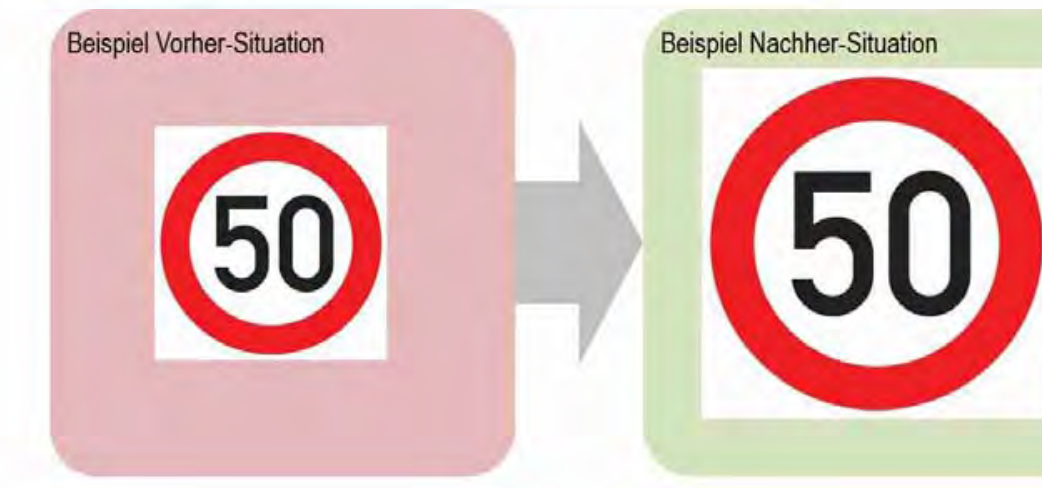

Maßnahmenkategorie 10 und 11 


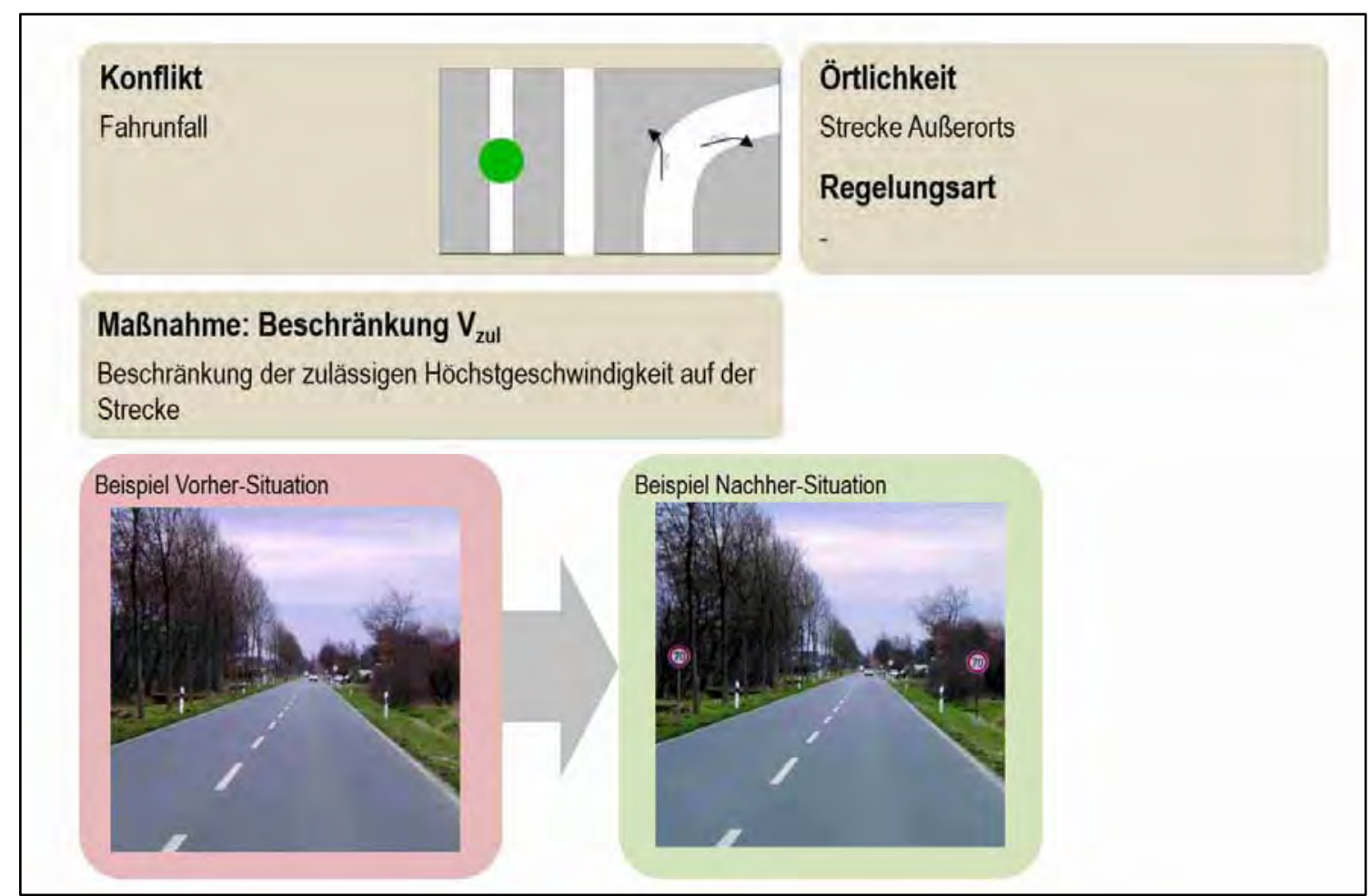

Maßnahmenkategorie 12

\section{Konflikt}

Wartepflichtiger mit Bevorrechtigtem von links oder rechts

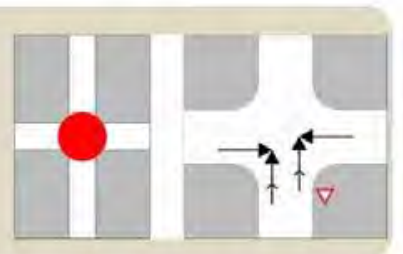

Örtlichkeit

Knotenpunkt Außerorts

Regelungsart

durch Verkehrszeichen

\section{Maßnahme: Beschränkung $\mathrm{V}_{z u l}$}

Beschränkung der zulässigen Höchstgeschwindigkeit in der Zufahrt zum Knotenpunkt
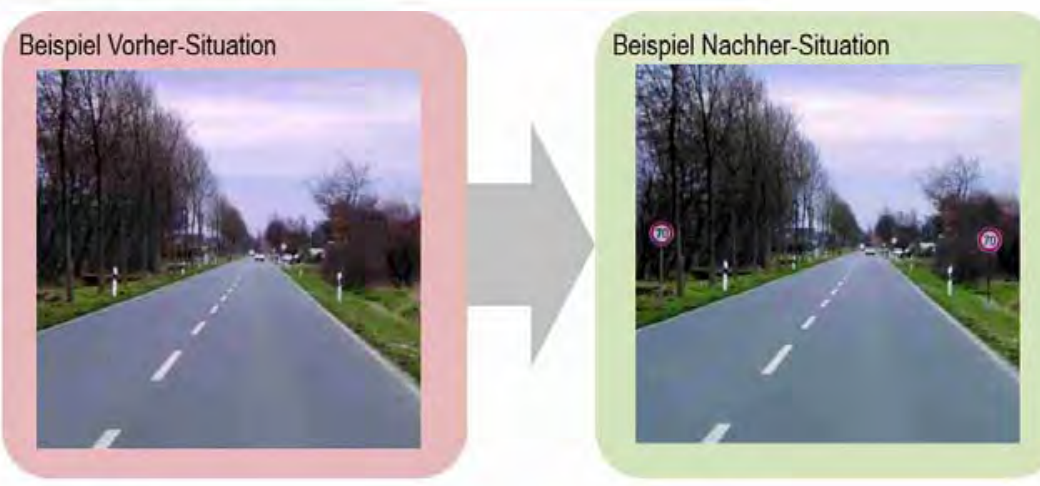

Maßnahmenkategorie 15 


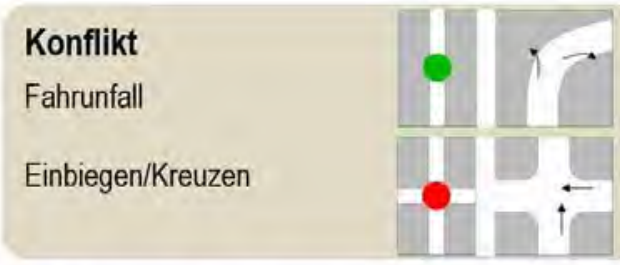

Maßnahme: Ortsfeste Geschwindigkeitsüberwach.

Überwachung der zulässigen Höchstgeschwindigkeit durch örtliche Geschwindigkeitskontrolle (OGU)

\section{Örtlichkeit}

Strecke / Knotenpunkt Außerorts

\section{Regelungsart}

i. d. R. durch VZ

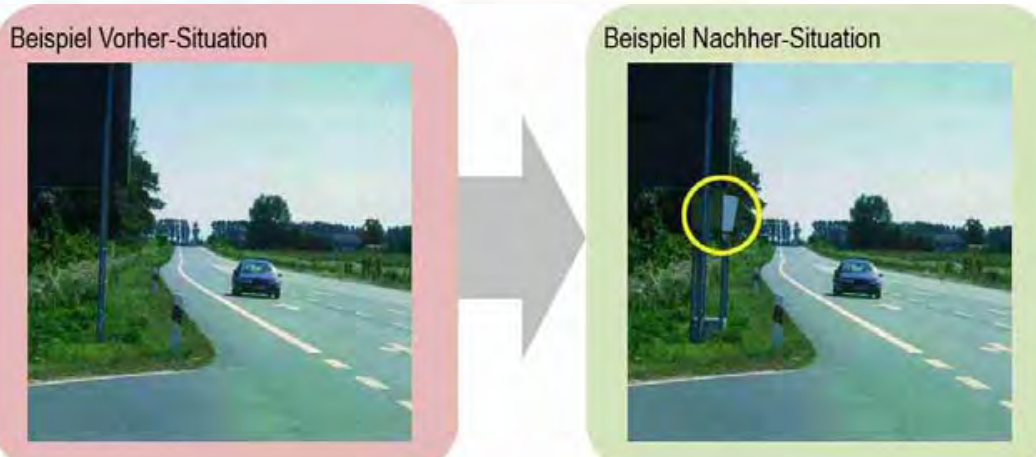

Maßnahmenkategorie 16 und 17

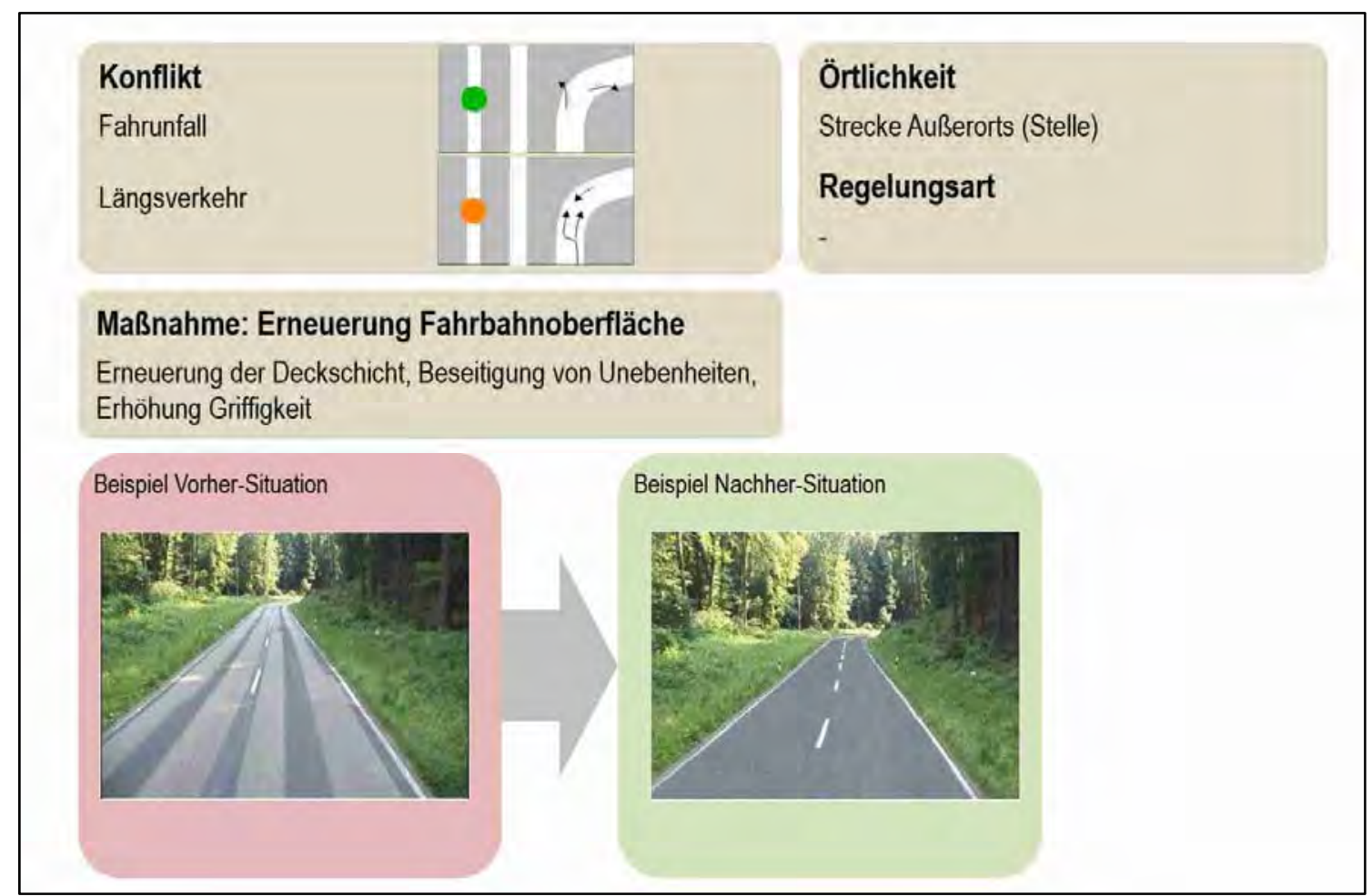

Maßnahmenkategorie 18 


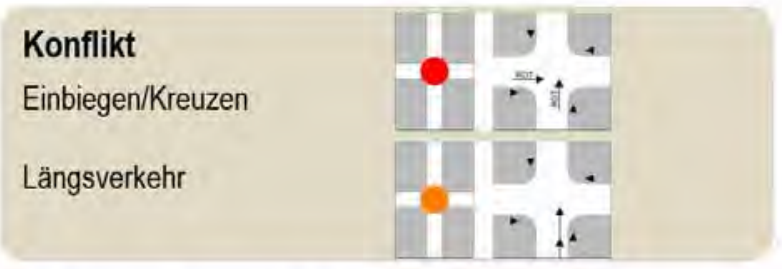

\section{Örtlichkeit}

Knotenpunkt (Innerorts)

\section{Regelungsart}

Lichtsignalanlage

\section{Maßnahme: zusätzlichen Signalgeber anordnen}

Einrichten zusätzlicher Signalgeber

KEIN Einrichten einer neuen Phase oder Stromsignalisierung
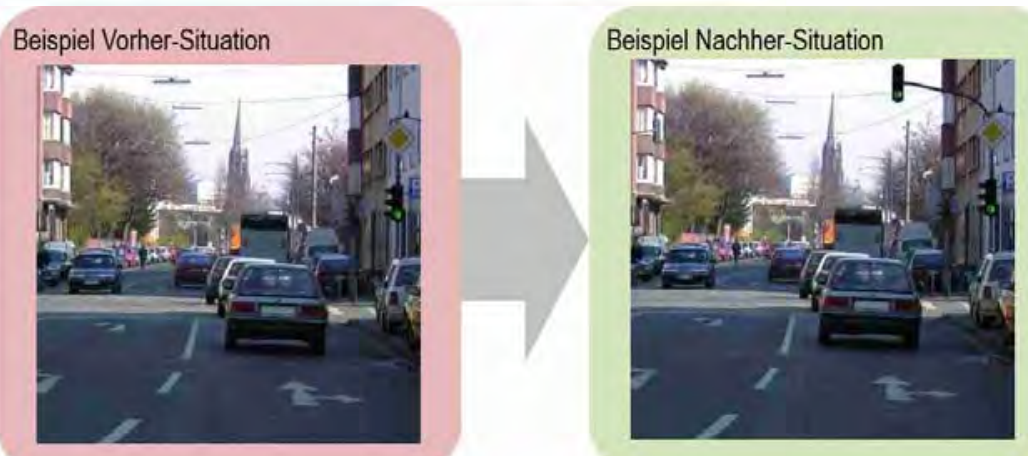

Maßnahmenkategorie 19

\section{Konflikt \\ Einbiegen/Kreuzen \\ Längsverkehr}

\section{Maßnahme: Leuchtmittel Signalgeber verbessern}

Technische Verbesserung Sichtbarkeit Signalgeber, bspw. LED

\section{Örtlichkeit}

Knotenpunkt (Innerorts)

\section{Regelungsart}

Lichtsignalanlage

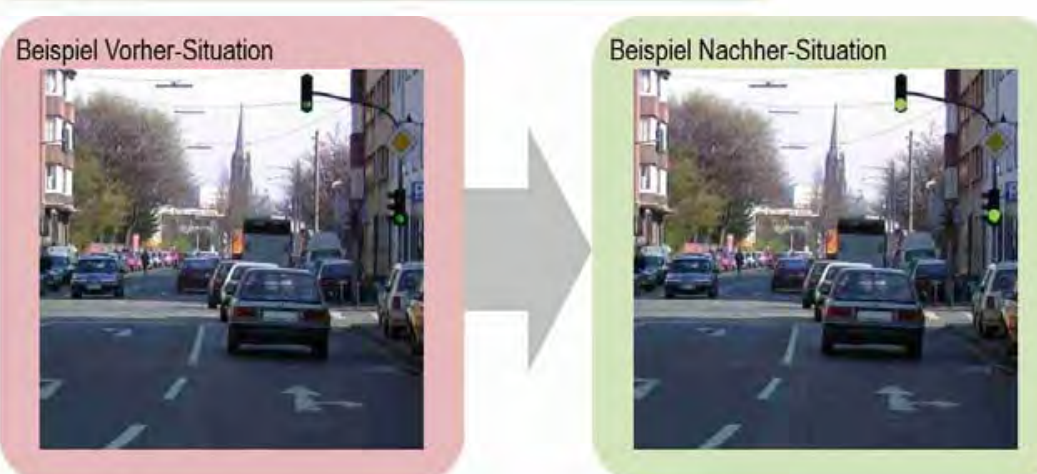

Maßnahmenkategorie 21 


\section{Konflikt}

Wartepflichtiger mit Bevorrechtigtem von links oder rechts

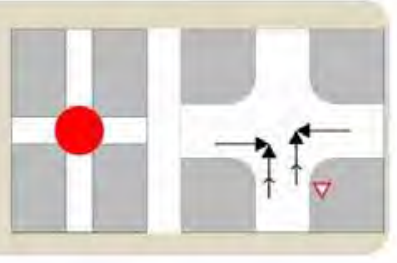

Örtlichkeit

Knotenpunkt $(10, \mathrm{AO})$

Regelungsart

durch Verkehrszeichen

\section{Maßnahme: Sichthindernisse entfernen}

Sichthindernisse entfernen, Verkehrszeichen versetzen

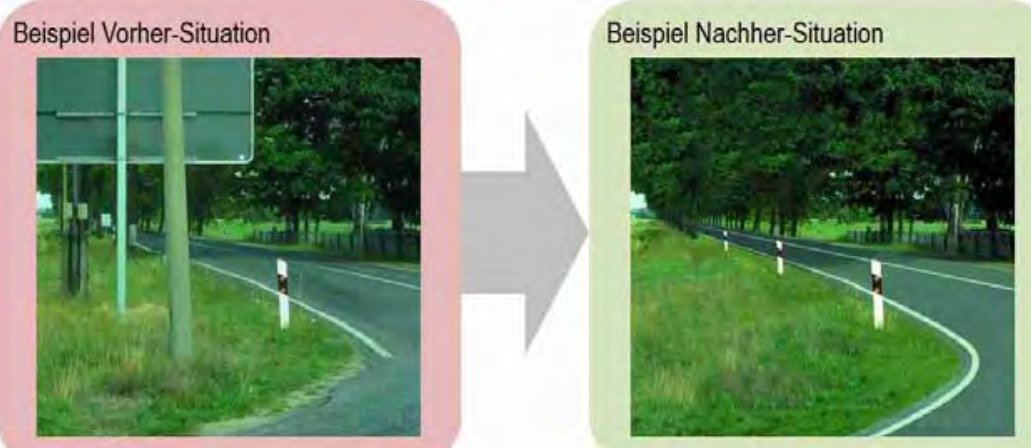

Maßnahmenkategorie 22 und 23

\section{Konflikt \\ Beliebig, \\ häufig Überschreiten}

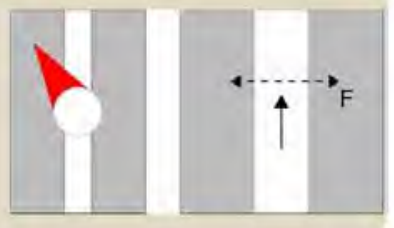

Örtlichkeit

Innerorts (Knoten oder Strecke)

\section{Regelungsart}

\section{Maßnahme: Beleuchtung verbessern}

Beleuchtung anpassen/verbessern
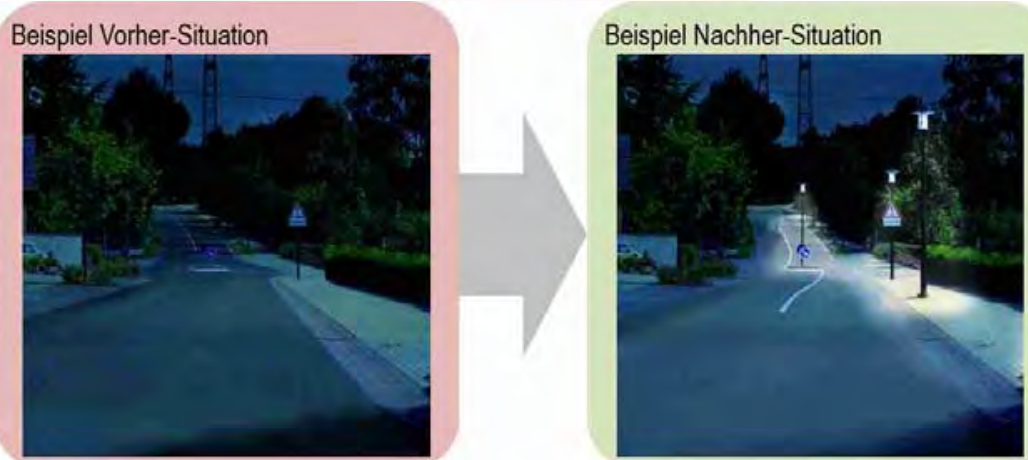

Maßnahmenkategorie 24 


\section{Konflikt}

Wartepflichtiger mit Bevorrechtigtem von links oder rechts

\section{Örtlichkeit}

Knotenpunkt (AO)

\section{Regelungsart}

durch Verkehrszeichen

\section{Maßnahme: Sichtschutzzaun aufstellen}

Verhinderung von Fehleinschătzung übergeordneter Fahrzeuge durch zu große Sichtweiten, Aufmerksamkeitserhöhung

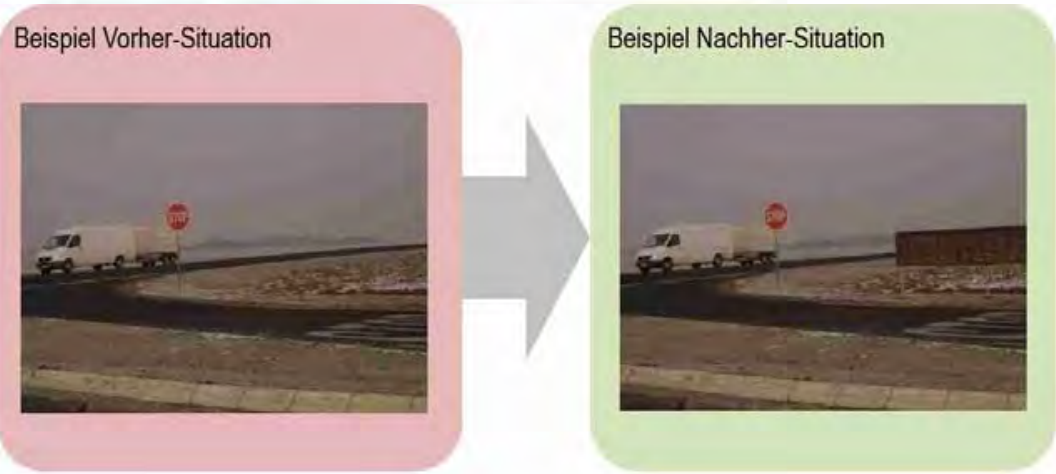

Maßnahmenkategorie 25

\section{Konflikt}

Linksabbiegender mit Nachfolgenden,

Linksabbiegender mit Gegenverkehr

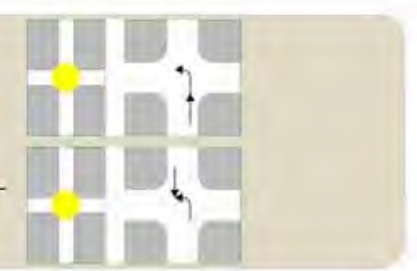

\section{Örtlichkeit}

Knotenpunkt Innerorts

\section{Regelungsart}

beliebig

\section{Maßnahme: Markierung anpassen/verbessern}

Markierung im Knoten verbessern, Pfeilmarkierung aufbringen, Veränderung von Markierung

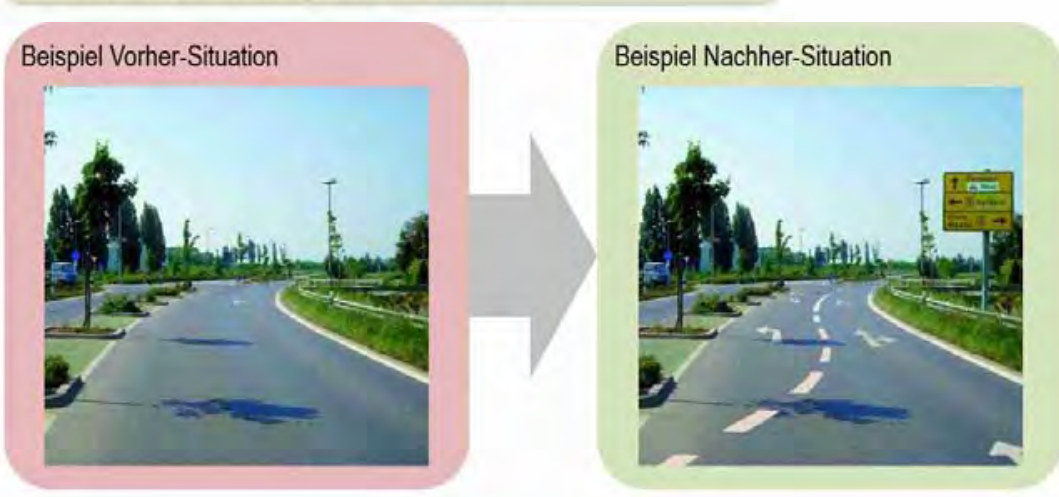




\section{Konflikt}

Einbiegen/Kreuzen

Abbiegen

Maßnahme: Markierung in Stand setzen

Aufwertung/instand Setzung bestehender Markierung

\section{Örtlichkeit}

Knotenpunkt Innerorts

Regelungsart

beliebig

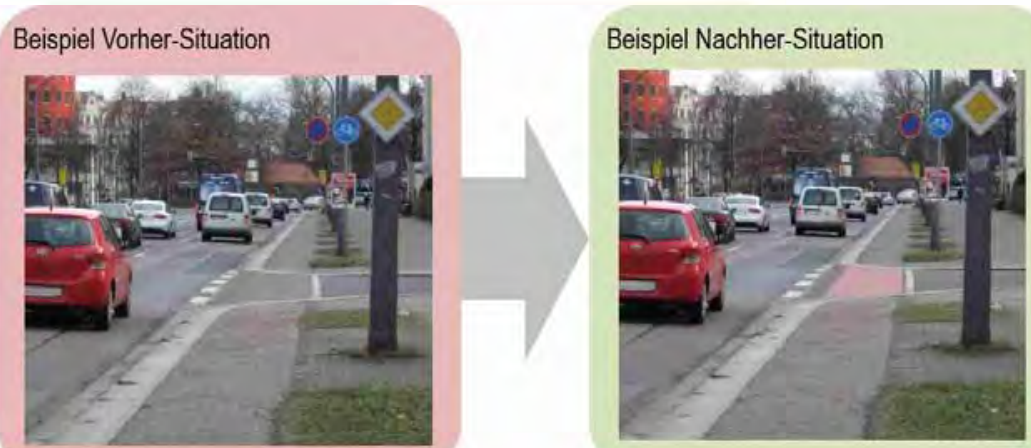

Maßnahmenkategorie 27

Konflikt
Einbiegen/Kreuzen
Abbiegen

\section{Maßnahme: Radverkehrsführung anpassen}

Furt für Radfahrer anlegen, Piktogramm Radfahrer markieren, Aufstellfläche vor Lichtsignalanlage anlegen

\section{Örtlichkeit}

Knotenpunkt Innerorts

\section{Regelungsart}

beliebig
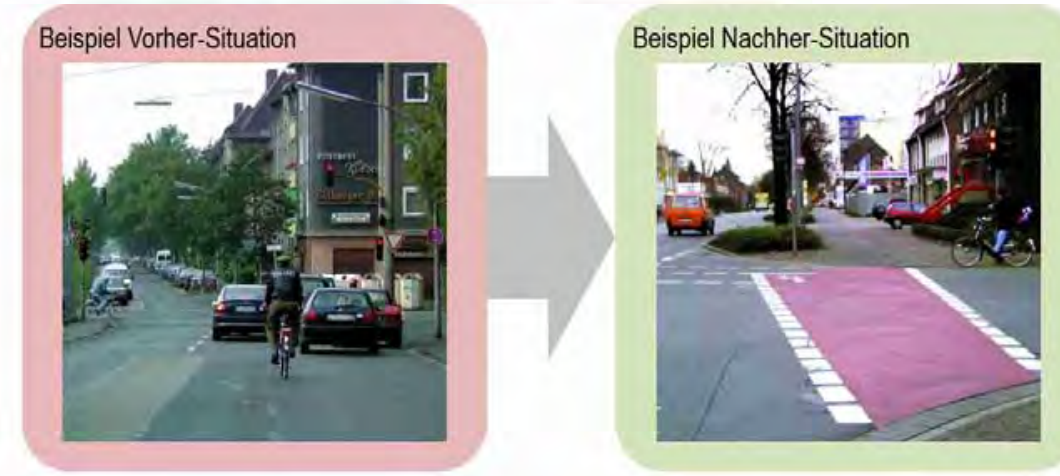

Maßnahmenkategorie 29 


\section{Konflikt}

Wartepflichtiger mit querendem Radfahrer

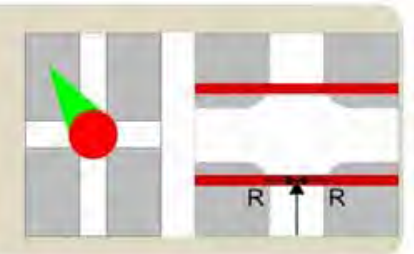

\section{Örtlichkeit}

Knotenpunkt Innerorts

\section{Regelungsart}

durch Verkehrszeichen

\section{Maßnahme: Radfahrerfurt Rot einfärben}

Einfärben/Markierung der Radfahrerfurt in Rot

(Breite der Radverkehrsanlage)

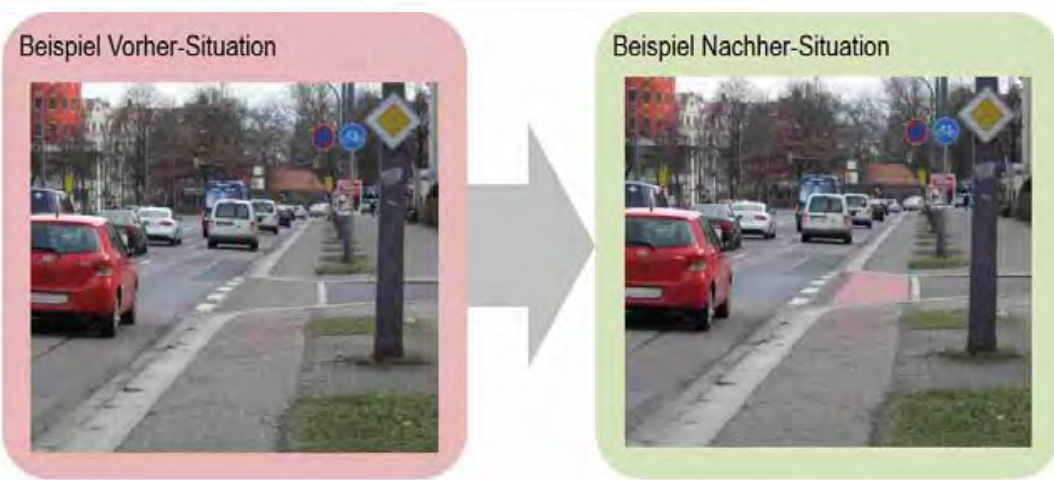

Maßnahmenkategorie $\mathbf{3 0}$

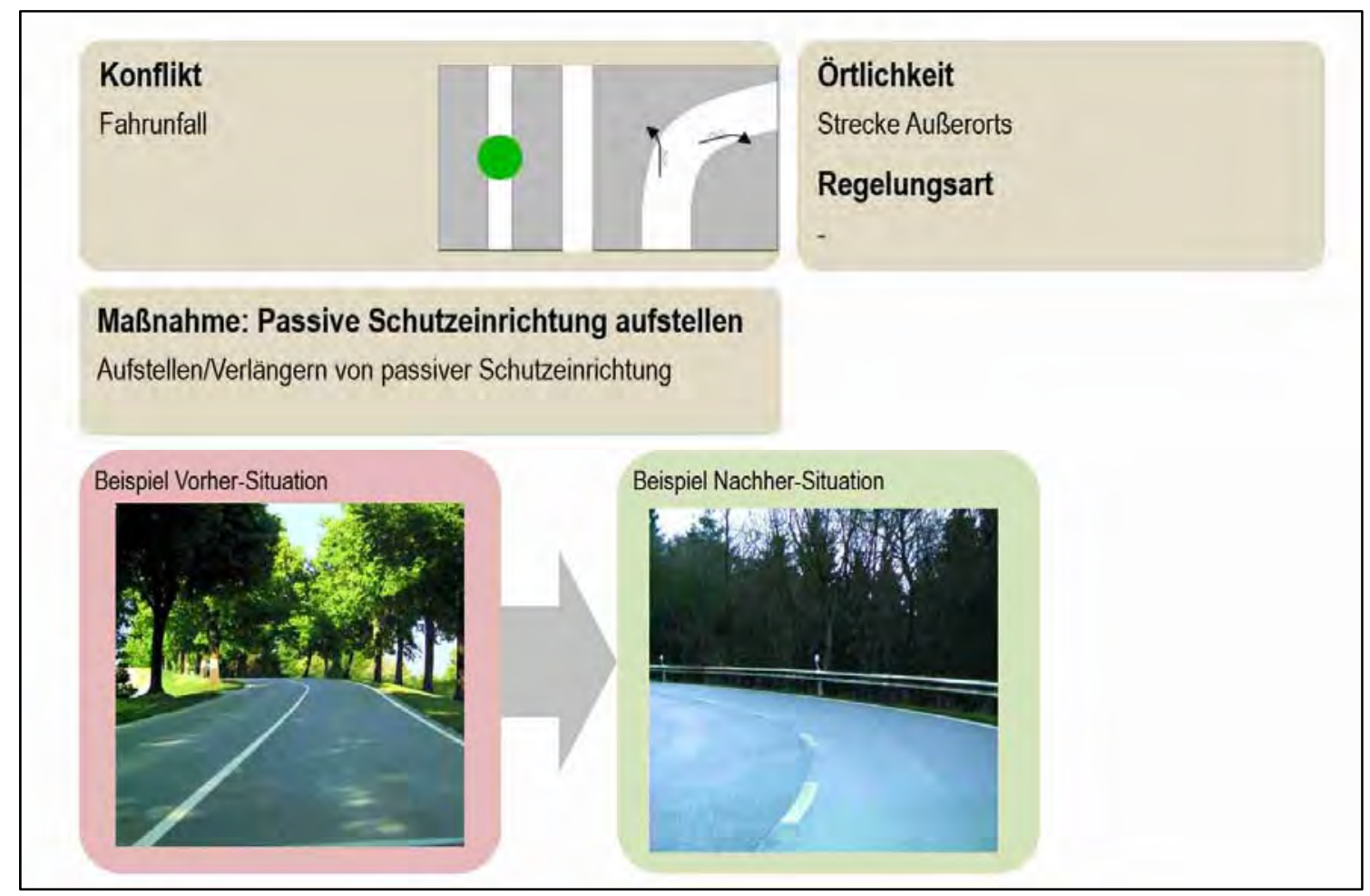

Maßnahmenkategorie 31 


\section{Konflikt}

Fahrunfall (motorisiertes Zweirad)

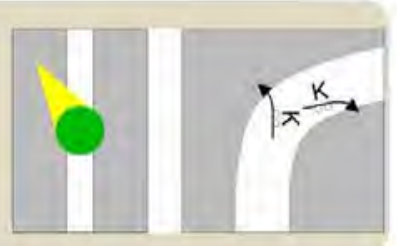

\section{Örtlichkeit}

Strecke Außerorts

Regelungsart

\section{Maßnahme: Unterfahrschutz an PSE anbringen}

Anbringen/Ergänzen von Unterfahrschutz für Motorradfahrer an passiver Schutzeinrichtung

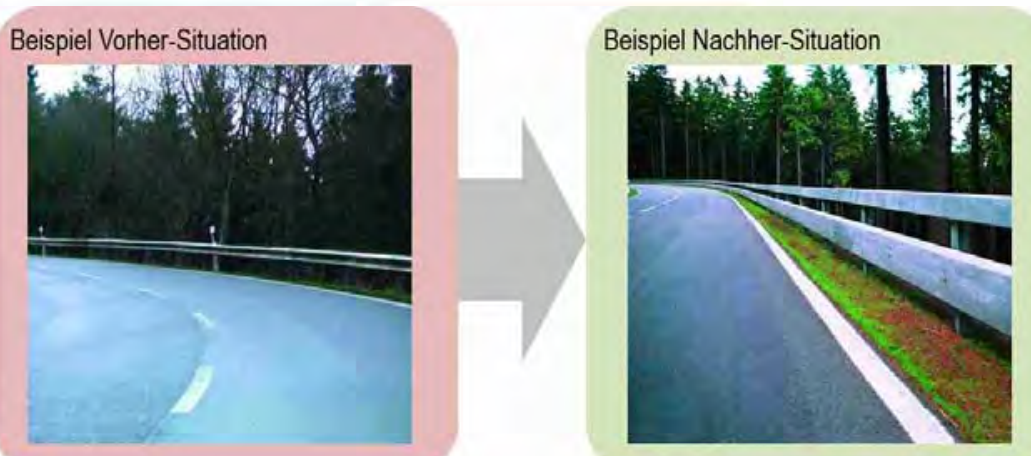

Maßnahmenkategorie 32

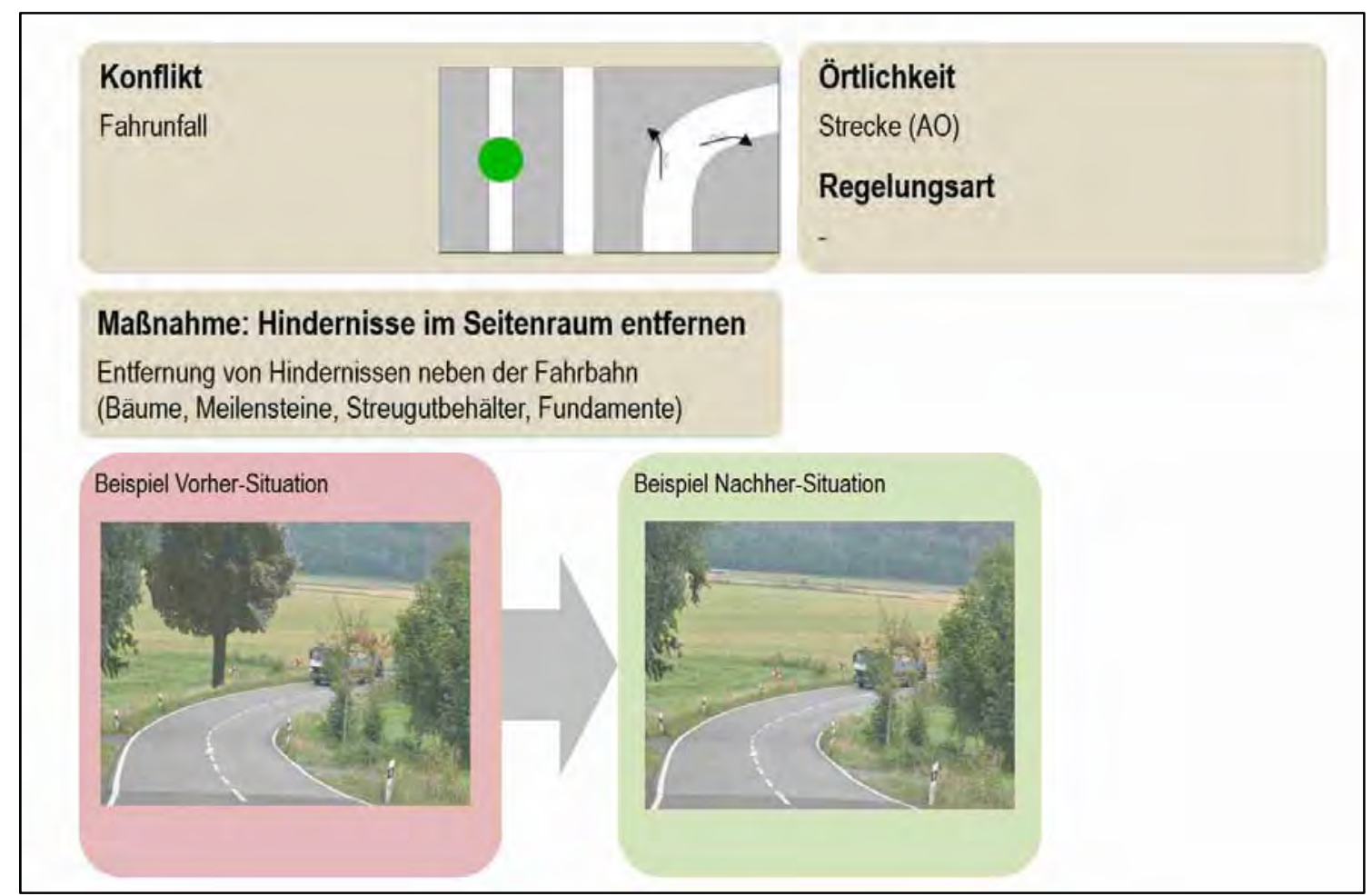

Maßnahmenkategorie 33 


\section{Konflikt}

Fahrunfall (motorisiertes Zweirad)

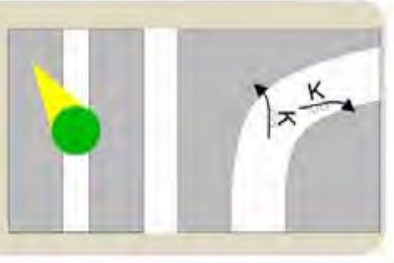

\section{Örtlichkeit}

Strecke Außerorts

Regelungsart

\section{Maßnahme: Rüttelstreifen aufbringen}

Aufbringen von profilierter Quermarkierung (Rüttelstreifen) zur Senkung Geschwindigkeit motorisierter Zweiräder

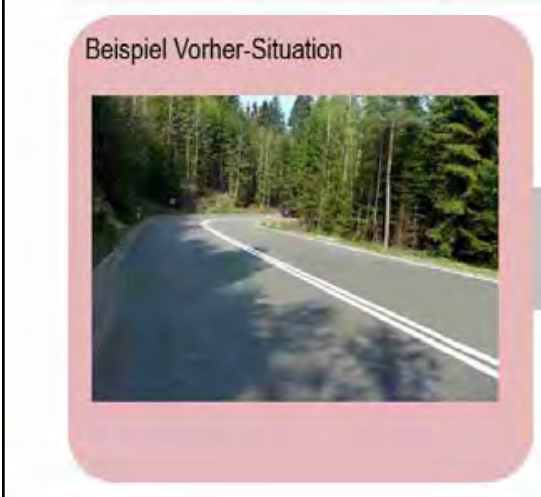

\section{Beispiel Nachher-Situation}

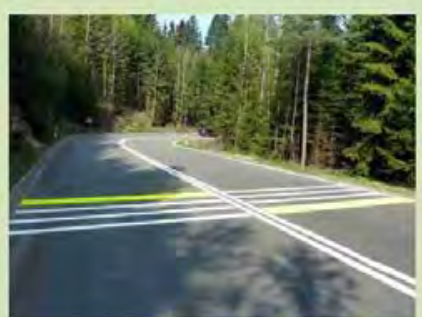

Maßnahmenkategorie 34

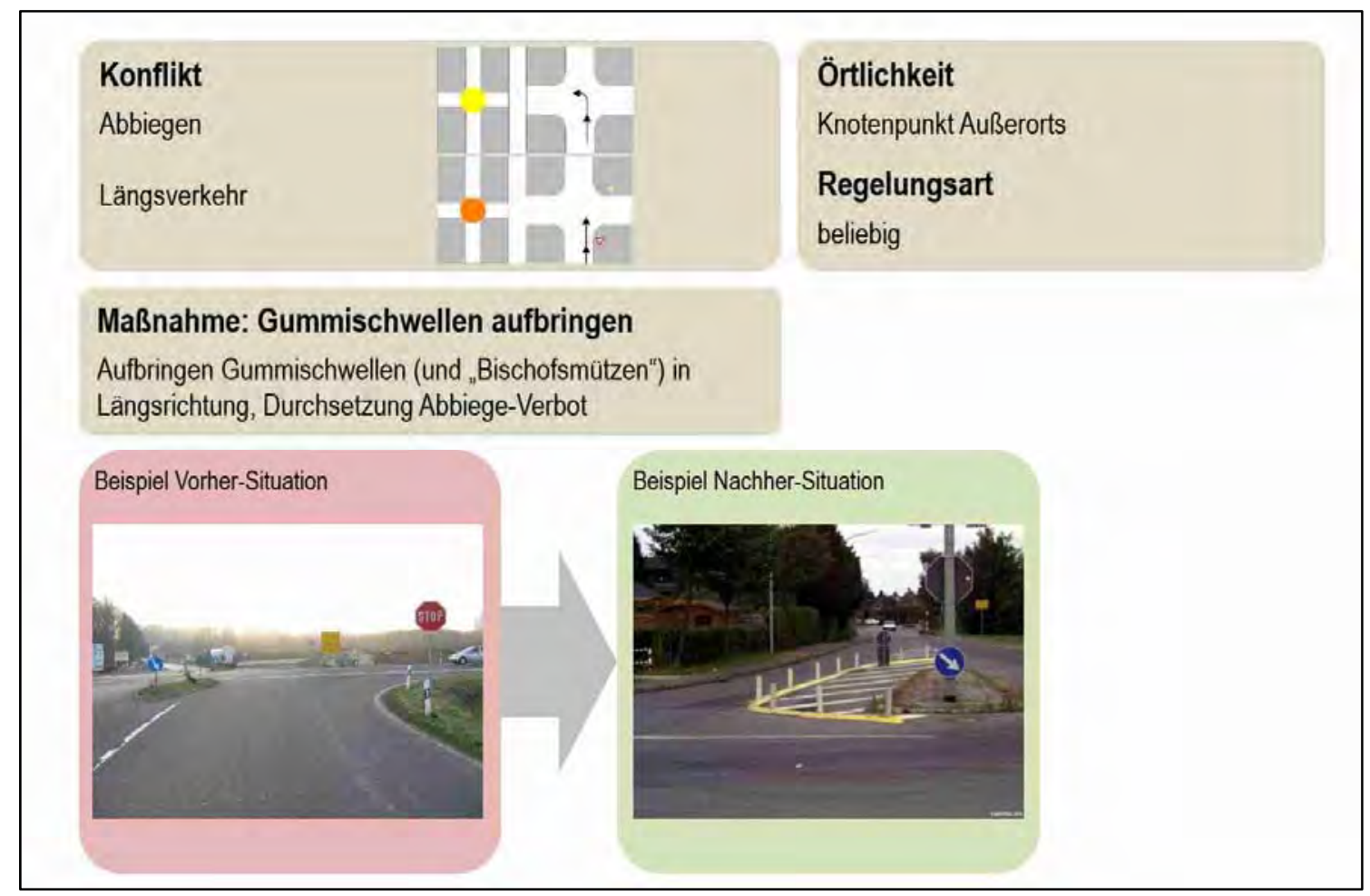

Maßnahmenkategorie 35 


\section{Konflikt}

Einbiegen /Kreuzen

Abbiegen

\section{Maßnahme: Sperrfläche markieren}

Fahrbeziehung unterbinden

(Fahrstreifen zurücknehmen/durch Sperrfläche ersetzen)

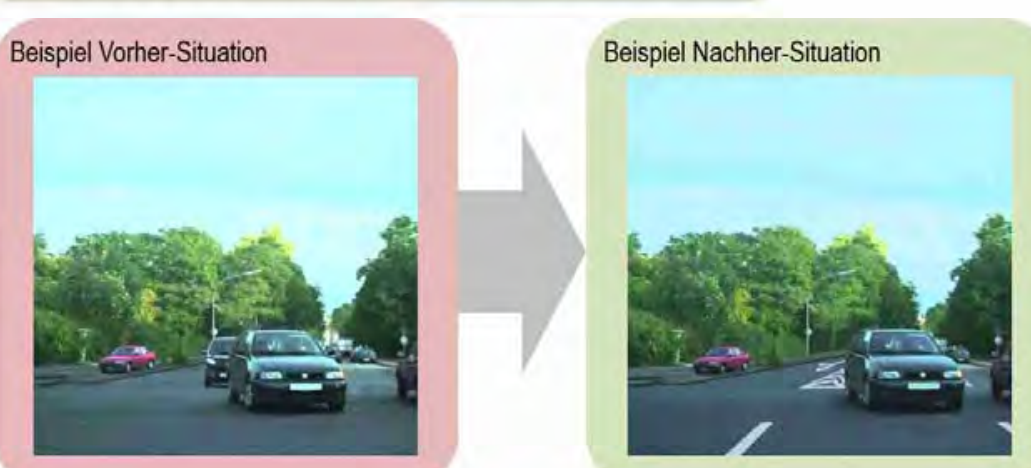

\section{Örtlichkeit}

Knotenpunkt Innerorts

\section{Regelungsart}

durch Verkehrszeichen

Maßnahmenkategorie 36

\section{Konflikt \\ Fahrunfall \\ Abbiegen \\ Einbiegen/Kreuzen \\ Längsverkehr}

\section{Maßnahme: Verkehrszeichen versetzen}

Verkehrszeichen zur besseren Erkennbarkeit versetzen

\section{Örtlichkeit}

beliebig (häufig vor Knotenpunkten)

\section{Regelungsart}

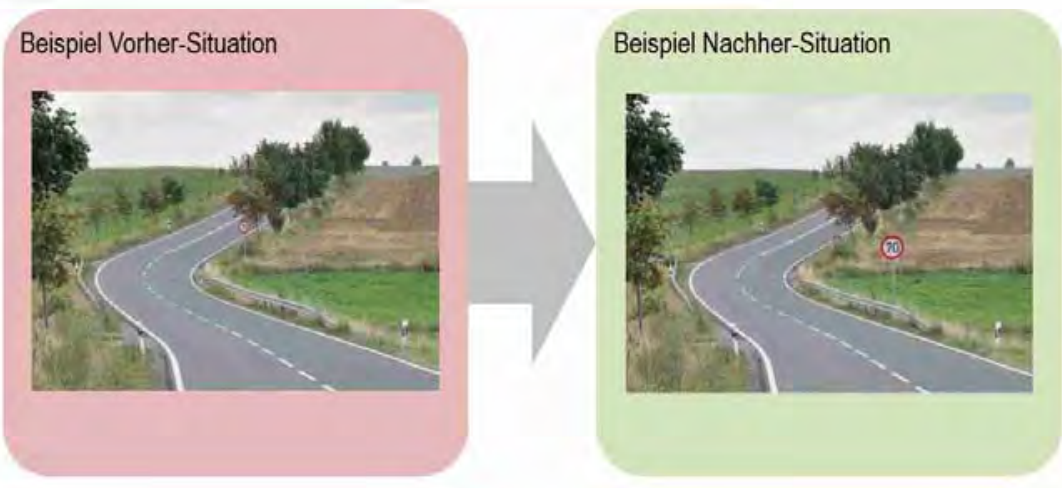

Maßnahmenkategorie 37 


\section{Konflikt}

Fahrunfall in Kurven

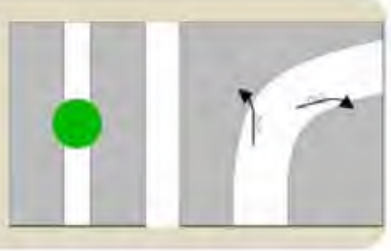

Örtlichkeit

Strecke AO

Regelungsart

\section{Maßnahme: Beschränkung $\mathrm{v}_{z u l}+\mathrm{Z} 625$ StVo}

Beschränkung der $\mathrm{v}_{\text {zul }}$ im Kurvenbereich in Kombination mit Z 625 StVO (Richtungstafel in Kurven)

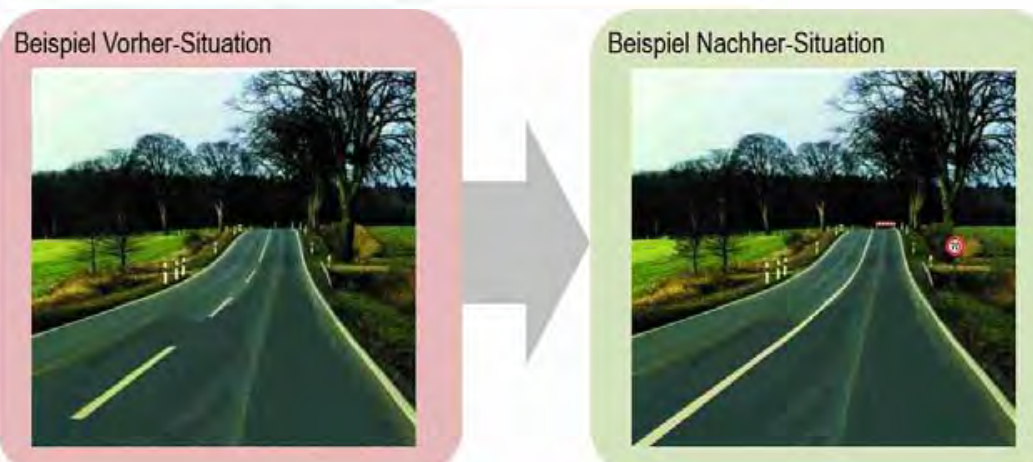

Maßnahmenkategorie 38

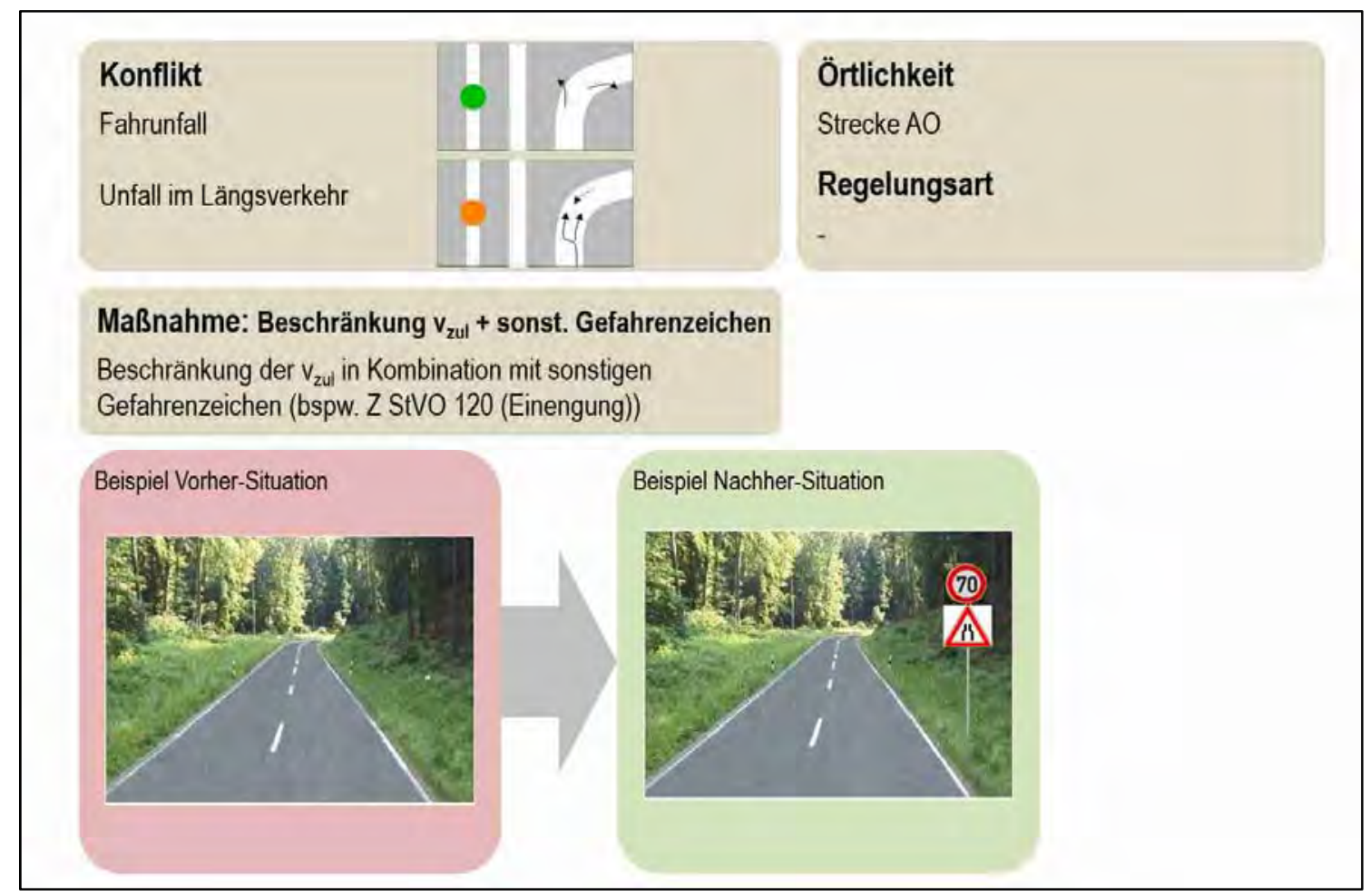

Maßnahmenkategorie 39 


\section{Anhang 9 Übersicht alle Maßnahmenkategorien}

\begin{tabular}{|c|c|c|c|c|c|c|}
\hline \multirow{2}{*}{\multicolumn{2}{|c|}{ Maßnahme }} & \multicolumn{5}{|c|}{ Gesamt } \\
\hline & & Anzaht & $1-\mathrm{JK}$ & $3-1 \mathrm{k}$ & $1+3-\mathrm{JK}$ & nicht möglich \\
\hline Aufnahme-Nr. & Gesamt & 1093 & 134 & 8 & 167 & 784 \\
\hline 1 & FG-Gitter & 3 & 0 & 0 & 0 & 3 \\
\hline 2 & FGÜ & 2 & 0 & 0 & 0 & 2 \\
\hline 3 & Mittelinsel einrichten & 3 & 0 & 0 & 1 & 2 \\
\hline 4 & FG-LSA & 3 & 0 & 0 & 0 & 3 \\
\hline 5 & Betriebszeit LSA & 26 & 4 & 0 & 2 & 20 \\
\hline 6 & separate Linksabbiegerphase & 47 & 10 & 0 & 5 & 32 \\
\hline 7 & Verbesserung Koordinierung & 8 & 0 & 0 & 5 & 3 \\
\hline 8 & freier RA mit signalisieren & 1 & 0 & 0 & 0 & 1 \\
\hline 9 & sonstige Änderung Signalisierung & 60 & 7 & 1 & 10 & 42 \\
\hline 10 & Gefahrenzeichen Kurve & 4 & 1 & 0 & 1 & 2 \\
\hline 11 & Gefahrenzeichen $r-v-1$ & 4 & 1 & 0 & 0 & 3 \\
\hline 12 & sonst. Gefahrenzeichen & 16 & 1 & 0 & 2 & 13 \\
\hline 13 & Gefahrenzeichen FG & 1 & 0 & 0 & 0 & 1 \\
\hline 14 & Gefahrenzeichen RF & 2 & 0 & 0 & 0 & 2 \\
\hline 15 & Wild & 8 & 0 & 0 & 1 & 7 \\
\hline $15 a$ & Wildabhaltende Maßnahmen & 4 & 0 & 0 & 0 & 4 \\
\hline 16 & HALT & 62 & 6 & 0 & 13 & 43 \\
\hline 17 & vorgeschriebene $F R$ & 2 & 0 & 0 & 0 & 2 \\
\hline 18 & Fahrverbotszeichen & 0 & 0 & 0 & 0 & 0 \\
\hline 19 & Wendeverbot & 0 & 0 & 0 & 0 & 0 \\
\hline 20 & Überholverbot (VZ) & 0 & 0 & 0 & 0 & 0 \\
\hline 21 & Park-/ / Halteverbot & 18 & 3 & 0 & 1 & 14 \\
\hline 22 & Z620 (Leitpfosten) & 2 & 0 & 0 & 0 & 2 \\
\hline 23 & Z625 (Kurventafel) & 43 & 3 & 0 & 13 & 27 \\
\hline 24 & Zusatzzeichen ergänzen (sonst) & 1 & 0 & 0 & 1 & 0 \\
\hline $24 a$ & Zusatzzeichen ergänzen (1006-36) & 11 & 1 & 0 & 3 & 7 \\
\hline $24 \mathrm{~b}$ & Zusatzzeichen ergânzen (1000-32) & 9 & 1 & 0 & 0 & 8 \\
\hline 25 & Wegweisung & 5 & 0 & 0 & 1 & 4 \\
\hline 26 & VZ anpassen & 27 & 4 & 0 & 7 & 16 \\
\hline 27 & VZ entfernen & 6 & 2 & 0 & 1 & 3 \\
\hline 28 & sonst. VZ & 7 & 0 & 0 & 1 & 6 \\
\hline 29 & Beschränkung vzul (Strecke, io) & 1 & 0 & 0 & 0 & 1 \\
\hline 30 & Beschränkung vzul (Strecke, ao) & 37 & 6 & 0 & 5 & 26 \\
\hline 31 & Beschränkung vzul $(K P$, io $)$ & 10 & 1 & 0 & 2 & 7 \\
\hline 32 & Beschränkung vzul (KP, LSA, ao) & 3 & 0 & 0 & 0 & 3 \\
\hline $32 a$ & Beschränkung vzul (KP_VZ, ao) & 16 & 1 & 0 & 5 & 10 \\
\hline 33 & OGÜ & 23 & 3 & 1 & 6 & 13 \\
\hline 34 & temp. Geschwindigkeitsüberwachungen & 20 & 1 & 0 & 3 & 16 \\
\hline 35 & sonst. Überwachungsmaßnahmen & 15 & 3 & 0 & 2 & 10 \\
\hline 36 & Rotlichtüberwachung & 2 & 0 & 0 & 2 & 0 \\
\hline 37 & FBB erneuern & 112 & 11 & 0 & 19 & 82 \\
\hline 38 & Griffigkeit verbessern & 2 & 0 & 0 & 0 & 2 \\
\hline 39 & Aufpflasterung/Schwellen & 3 & 0 & 0 & 1 & 2 \\
\hline 40 & Fahrbahn einengen (baulich) & 4 & 0 & 0 & 0 & 4 \\
\hline 41 & Rückbau Fahrstreifen & 9 & 2 & 1 & 2 & 4 \\
\hline 43 & zus. Signalgeber anordnen & 9 & 3 & 0 & 0 & 6 \\
\hline $43 a$ & Signalgeber anpassen & 3 & 0 & 0 & 0 & 3 \\
\hline $43 \mathrm{~b}$ & Leuchtmittel Signalgeber verbessern & 19 & 3 & 0 & 4 & 12 \\
\hline $43 c$ & Installation Kontrastblenden & 8 & 0 & 0 & 1 & 7 \\
\hline 44 & Maststandort anpassen & 1 & 0 & 0 & 0 & 1 \\
\hline 45 & Verkehrsspiegel & 1 & 0 & 0 & 1 & 0 \\
\hline 46 & Sichthindernisse entfernen & 84 & 12 & 0 & 6 & 66 \\
\hline 47 & Beleuchtung verbessern & 7 & 2 & 0 & 0 & 5 \\
\hline 48 & Sichtschutzzaun aufstellen & 4 & 0 & 0 & 1 & 3 \\
\hline 49 & Markierung anpassen & 42 & 10 & 1 & 4 & 27 \\
\hline $49 a$ & Markierung instand setzen & 77 & 9 & 2 & 13 & 53 \\
\hline 50 & Beschleunigungsfahrstreifen & 5 & 0 & 0 & 0 & 5 \\
\hline 51 & Minikreis einrichten & 6 & 1 & 0 & 0 & 5 \\
\hline 52 & Tropfen bauen & 3 & 0 & 0 & 0 & 3 \\
\hline 53 & Umbau Eckausrundung & 6 & 0 & 0 & 2 & 4 \\
\hline 54 & Zufahrt baulich schließen & 3 & 0 & 0 & 0 & 3 \\
\hline 55 & Linksabbiegefahrstreifen & 7 & 0 & 0 & 1 & 6 \\
\hline 56 & Rechtsabbiegefahrstreifen & 6 & 1 & 0 & 1 & 4 \\
\hline 57 & Fahrbeziehung/Vorfahrtregelung & 14 & 5 & 0 & 1 & 8 \\
\hline 58 & Zweirichtungsverkehr aufheben (RF) & 0 & 0 & 0 & 0 & 0 \\
\hline 59 & Radverkehrsanlage einrichten & 3 & 0 & 0 & 1 & 2 \\
\hline 60 & Radverkehrsführung im KP anpassen & 12 & 4 & 0 & 1 & 7 \\
\hline $60 a$ & Radfahrerfurt rot einfärben & 19 & 4 & 1 & 0 & 14 \\
\hline 61 & Zweirichtungsverkehr einrichten (RF) & 1 & 0 & 0 & 0 & 1 \\
\hline 62 & PSE (aufstellen/verlängern) & 13 & 0 & 0 & 0 & 13 \\
\hline $62 a$ & PSE (instand setzen) & 0 & 0 & 0 & 0 & 0 \\
\hline 63 & PSE (Mot.) & 11 & 2 & 0 & 3 & 6 \\
\hline 64 & Hindernisse im Seitenraum entfernen & 3 & 0 & 0 & 1 & 2 \\
\hline 65 & Überholverbot (Mark.) & 21 & 2 & 0 & 2 & 17 \\
\hline 66 & Rüttelstreifen/profilierte Markierung & 10 & 2 & 1 & 5 & 2 \\
\hline 67 & Bischofsmützen/Schwellen (längs) & 14 & 0 & 0 & 1 & 13 \\
\hline 68 & Fahrstreifenbreite anpassen & 4 & 1 & 0 & 0 & 3 \\
\hline 69 & $Z 205$ & 5 & 0 & 0 & 0 & 5 \\
\hline 70 & Markierung Sperrfläche & 10 & 0 & 0 & 3 & 7 \\
\hline 71 & VZ versetzen & 18 & 1 & 0 & 0 & 17 \\
\hline 73 & Unterbinden Parken/ Halten (Durchsetzen) & 2 & 0 & 0 & 0 & 2 \\
\hline neu & Reflektoren aufbringen & 0 & 0 & 0 & 0 & 0 \\
\hline
\end{tabular}


Gesamtverband der Deutschen Versicherungswirtschaft e. V.

Wilhelmstraße 43 / 43G, 10117 Berlin

Postfach 0802 64, 10002 Berlin

Telefon 030 / 2020 - 50 00, Fax 030 / 2020 - 6000

Internet: www.gdv.de, www.udv.de 